Andreas Siefke

\title{
Zufriedenheit mit Dienstleistungen
}




\section{Andreas Siefke}

\section{Zufriedenheit mit Dienstleistungen}

Sowohl in der Wissenschaft als auch in der Unternehmenspraxis wird Kundenzufriedenheit vornehmlich als ein Nachkaufphänomen charakterisiert. Eine solche Auffassung von Kundenzufriedenheit greift jedoch insbesondere im Dienstleistungsbereich zu kurz. So bedingt der Prozeßcharakter des Dienstleistungskonsums, daß die Zufriedenheit von Kunden aus der Beurteilung zeitlich aufeinanderfolgender Teilerlebnisse entsteht. Vor diesem Hintergrund wird in der Arbeit ein prozeßorientierter Ansatz zur Operationalisierung und Erklärung der Kundenzufriedenheit mit Dienstleistungen entwickelt. Dabei werden insbesondere der Episodencharakter des Dienstleistungskonsums und die damit einhergehende Dynamik der Zufriedenheitsbildung berücksichtigt. Das theoriegestützte Konzept erfährt zudem eine fundierte empirische Überprüfung auf der Basis einer Befragung von 603 Fahrgästen der Deutschen Bahn AG im innerdeutschen Personenfernverkehr.

Andreas Siefke wurde 1967 in Münster geboren. Von 1987 bis 1993 studierte er Betriebswirtschaftslehre an der Universität Münster mit den Schwerpunkten Marketing und Statistik. 1993 erfolgte der Abschluß zum Diplom-Kaufmann. Seitdem ist er wissenschaftlicher Mitarbeiter am Institut für Marketing der Universität Münster und übt seit 1996 leitende Funktionen in der Forschungsstelle Bahnmarketing in der Wissenschaftlichen Gesellschaft für Marketing und Unternehmensführung e.V. aus. 
Zufriedenheit mit Dienstleistungen 


\section{SCHRIFTEN ZU \\ MARKETING UND MANAGEMENT \\ Herausgegeben von Prof. Dr. Dr. h.c. Heribert Meffert}

Band 35



Frankfurt am Main - Berlin - Bern. New York - Paris - Wien

Andreas Siefke and Universität Münster - 978-3-631-75103-9

Downloaded from PubFactory at 01/11/2019 08:18:16AM

via free access 


\section{Andreas Siefke}

\section{Zufriedenheit mit Dienstleistungen}

Ein phasenorientierter Ansatz zur Operationalisierung und Erklärung der Kundenzufriedenheit im Verkehrsbereich auf empirischer Basis



Europäischer Verlog der Wissenschaften 
Die Deutsche Bibliothek - CIP-Einheitsaufnahme

Siefke, Andreas:

Zufriedenheit mit Dienstleistungen : ein phasenorientierter Ansatz zur Operationalisierung und Erklärung der Kundenzufriedenheit im Verkehrsbereich auf empirischer Basis / Andreas Siefke. - Frankfurt am Main ; Berlin ; Bern ; New York ; Paris ; Wien : Lang, 1998

(Schriften zu Marketing und Management ; Bd. 35)

Zugl.: Münster (Westfalen), Univ., Diss., 1997 ISBN 3-631-32885-0

Open Access: The online version of this publication is published on www.peterlang.com and www.econstor.eu under the international Creative Commons License CC-BY 4.0. Learn more on how you can use and share this work: http://creativecommons.org/licenses/ by/4.0.

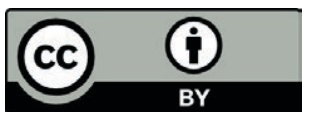

This book is available Open Access thanks to the kind support of ZBW - Leibniz-Informationszentrum Wirtschaft.

\author{
D 6 \\ ISSN 0176-2729 \\ ISBN 3-631-32885-0 \\ ISBN 978-3-631-75103-9 (eBook) \\ (C) Peter Lang $\mathrm{GmbH}$ \\ Europäischer Verlag der Wissenschaften \\ Frankfurt am Main 1998 \\ Alle Rechte vorbehalten.
}

Das Werk einschließlich aller seiner Teile ist urheberrechtlich geschützt. Jede Verwertung außerhalb der engen Grenzen des Urheberrechtsgesetzes ist ohne Zustimmung des Verlages unzulässig und strafbar. Das gilt insbesondere für

Vervielfältigungen, Übersetzungen, Mikroverfilmungen und die Einspeicherung und Verarbeitung in elektronischen Systemen.

Printed in Germany 124567 


\section{Meinen Eltern}

und

Corinna 
Andreas Siefke and Universität Münster - 978-3-631-75103-9

Downloaded from PubFactory at 01/11/2019 08:18:16AM

via free access 


\section{Vorwort des Herausgebers}

Wenngleich die hohe Bedeutung von Kundenzufriedenheit für den Unternehmungserfolg in Wissenschaft und Praxis unbestritten ist, dokumentiert die kaum noch überschaubare Anzahl an Veröffentlichungen zu diesem Themengebiet, daß zentrale konzeptionelle und methodische Aspekte der Zufriedenheitsforschung nach wie vor umstritten sind. Dabei werden in der jüngeren Vergangenheit in sehr unterschiedlichen Bereichen neue Akzente in der Zufriedenheitsforschung gesetzt. So ist zum einen eine intensive Diskussion über die Bedeutung der Kundenzufriedenheit unter ökonomischen Gesichtspunkten festzustellen. Wurde bisher ein positiver Einfluß einer hohen Kundenzufriedenheit auf den Unternehmungserfolg in vielen Veröffentlichungen als gegeben vorausgesetzt, sind nun verstärkt Forschungsbemühungen hinsichtlich einer Ökonomisierung der Zufriedenheitsforschung zu beobachten. Zum anderen erfährt die Zufriedenheitsforschung eine Spezialisierung unter sektoralen Aspekten. Während lange Zeit der Schwerpunkt der Forschungsbemühungen auf dem Konsumgüterbereich lag, finden seit Beginn der achtziger Jahre die Besonderheiten von Dienstleistungen und Industriegütern Eingang in die wissenschaftliche Erforschung der Kundenzufriedenheit. So setzt sich im Bereich des Investitionsgütermarketing zunehmend die Erkenntnis durch, daß z.B. die mit der Beschaffung und Verwendung von Investitionsgütern einhergehende Multipersonalität einer entsprechenden Berücksichtigung bei der Operationalisierung des Zufriedenheitskonstruktes bedarf.

Ebenso ist insbesondere im Dienstleistungsbereich eine neue Forschungsrichtung zu beobachten, die sich mit der Entwicklung dynamischer Modelle der Kundenzufriedenheit beschäftigt und damit die bislang häufig rein statische Sichtweise der Kundenzufriedenheit kritisch hinterfragt. So weisen interessanterweise viele der bisherigen Untersuchungen die Gemeinsamkeit auf, sich vornehmlich mit der Zufriedenheit von Kunden nach dem Kauf bzw. am Ende des Dienstleistungsprozesses auseinanderzusetzen. Diese Sichtweise der Kundenzufriedenheit als ein reines Nachkaufphänomen ist jedoch insbesondere im Dienstleistungsbereich nicht ausreichend. Vielmehr hat der Prozeßcharakter des Dienstleistungskonsums zur Folge, daß Kundenzufriedenheit aus der Wahrnehmung und Beurteilung zeitlich aufeinanderfolgender Teilerlebnisse resultiert. Hieraus wird die Notwendigkeit deutlich, die ergebnisorientierte Betrachtung von Kundenzufriedenheit um eine am Dienstleistungsprozeß orientierte Sichtweise zu ergänzen. 
In diesem Forschungszweig liegt auch der Schwerpunkt der vorliegenden Arbeit, die sich insbesondere mit zwei zentralen Problembereichen auseinandersetzt, die in der Vergangenheit nur vereinzelt Eingang in die wissenschaftliche Forschung gefunden haben. So gilt es auf der einen Seite, bei der Messung der Kundenzufriedenheit den Prozeßcharakter von Dienstleistungen explizit zu berücksichtigen. Auf der anderen Seite ist der Dynamik und damit der Entwicklung der Kundenzufriedenheit im Zeitablauf differenziert Rechnung zu tragen. Als empirisches Beispiel dient dem Verfasser in diesem Zusammenhang der schienengebundene Personeniernverkehr der Deutschen Bahn AG.

Der in der wissenschaftlichen Diskussion festzustellende Dissens über das konzeptionelle Grundverständnis von Kundenzufriedenheit und über deren Abgrenzung von den Konstrukten Dienstleistungsqualität und Einstellung führt dazu, daß der Diskussion zentraler Erklärungsansätze der Kundenzufriedenheit eine Analyse des konzeptionellen Differenzierungsspektrums und der kausalen Beziehungen dieser Konstrukte vorangestellt wird.

Auf dieser Basis werden grundlegende Theorien und Modellansätze der Kundenzufriedenheit erörtert und das weitverbreitete Disconfirmation-Modell den weiteren Ausführungen zugrunde gelegt. Die Analyse dieses Modells sowie die Darstellung der psychologischen Grundlagen des Entstehungsprozesses von Kundenzufriedenheit haben dabei zum Ergebnis, daß der Modellansatz in seiner ursprünglichen Form den Prozeßcharakter von Dienstleistungen nur unzureichend berücksichtigt. Im Rahmen der hierauf aufbauenden Diskussion der Möglichkeiten einer prozeßorientierten Messung von Kundenzufriedenheit zeigt sich, daß der isolierte Einsatz der traditionellen Ansätze der Zielsetzung einer prozeßorientierten Erfassung der Kundenzufriedenheit nur unzureichend gerecht und damit der kombinierte Einsatz der Meßkonzepte notwendig wird.

Diese Erkenntnisse aufgreifend wird ein phasenorientierter Ansatz zur Erfassung und Erklärung der Kundenzufriedenheit mit Verkehrsdienstleistungen formuliert, der die Grundlage für die empirische Analyse darstellt. In diesem Zusammenhang leitet der Verfasser eine Kundenprozeßhierarchie der Verkehrsdienstleistung "Bahnreise" ab, die den konzeptionellen Rahmen für die empirische Analyse der Kundenzufriedenheit mit Verkehrsdienstleistungen bildet. Zur Analyse der Struktur und Dynamik der Kundenzufriedenheit auf den unterschiedlichen Ebenen der Kundenprozeßhierarchie wird der EQS-Ansatz der Kausalanalyse als hypothesenprüfendes Verfahren eingesetzt. 
Als zentrales Ergebnis der empirischen Untersuchung ist festzuhalten, daß auf allen Ebenen der aufgestellten Kundenprozeßhierarchie dynamische Effekte der Kundenzufriedenheit zu beobachten sind. Unter Berücksichtigung der Stärke der ermittelten Wirkungsbeziehungen stellt der Verfasser gleichwohl vereinzelt eine vergleichsweise eigenständige Wahrnehmung der verschiedenen Abschnitte einer Bahnreise fest. Ebenso werden die in der Zufriedenheitsforschung postulierten Primacy- und Recency-Effekte der Kundenzufriedenheit auf der Grundlage dieser Studie kontextspezifisch relativiert.

Dabei begnügt sich der Verfasser nicht mit der Analyse der Struktur und Dynamik der Kundenzufriedenheit, sondern hinterfragt darüber hinaus die Erklärungskraft unterschiedlicher personen- und reisebezogener Bestimmungsfaktoren der Kundenzufriedenheit mit einer Bahnreise. In den Ergebnissen zeigt sich, daß insbesondere psychographische und reise- bzw. verkehrsdienstleistungsspezifische Determinanten einen vergleichsweise starken Einfluß auf die Kundenzufriedenheit ausüben.

Insgesamt stellt die vorliegende Untersuchung eine deutliche Bereicherung der wissenschaftlichen Arbeiten auf dem Gebiet der Zufriedenheitsforschung dar. Die besondere Leistung liegt dabei in der systematischen Entwicklung und theoretischen Fundierung eines prozeßorientierten Grundverständnisses der Kundenzufriedenheit mit Dienstleistungen, das darüber hinaus auf methodisch anspruchsvolle Weise einer empirischen Überprüfung unterzogen wird. Vor diesem Hintergrund liefert die Arbeit wertvolle Anregungen für weiterührende Meßansätze der Kundenzufriedenheit in der Marketingwissenschaft und in der Verkehrsdienstleistungspraxis.

Das empirische Datenmaterial der vorliegenden Arbeit beruht auf einem Forschungsprojekt der Forschungsstelle Bahnmarketing in der Wissenschaftlichen Gesellschaft für Marketing und Unternehmensführung e.V. und ist ein Beleg für die erfolgreiche Verzahnung von Wissenschaft und Praxis. Ohne die Unterstützung durch die Deutsche Bahn AG und die Bereitschaft ihrer Mitarbeiter, den Dialog zwischen Wissenschaft und Praxis zu fördern, wäre die Realisierung der Untersuchung in dieser Form nicht möglich gewesen. Dafür gilt mein besonderer Dank. 
Andreas Siefke and Universität Münster - 978-3-631-75103-9

Downloaded from PubFactory at 01/11/2019 08:18:16AM

via free access 


\section{Vorwort des Verfassers}

Der verstärkte Wettbewerbsdruck auf der Angebotsseite sowie ein gestiegenes Anspruchsniveau an die Qualität von Dienstleistungen auf der Nachfragerseite führen dazu, daß auch Verkehrsdienstleistungsunternehmen in hohem Maße gefordert sind, ihr Leistungsangebot im Wettbewerbsumfeld zu profilieren und die Zufriedenheit ihrer Kunden sicherzustellen. In diesem Zusammenhang kommt einer regelmäßigen Messung der Kundenzufriedenheit eine zentrale Bedeutung zu. Der Prozeßcharakter von Dienstleistungen stellt dabei besondere Anforderungen an die Operationalisierung des Zufriedenheitskonstruktes. So hat das prozessuale Erleben einer Dienstleistung zur Folge, daß Zufriedenheit aus der Beurteilung sequentiell verlaufender Ereignisse entsteht. Diesem Phänomen wurde in der Vergangenheit jedoch nur ansatzweise Rechnung getragen. Die in jüngerer Zeit zunehmend in der Wissenschaft diskutierten und in ersten empirischen Arbeiten bestätigten Wirkungseffekte einer im Zeitablauf zu beobachtenden Dynamik der Kundenzufriedenheit belegen gleichwohl die Notwendigkeit einer differenzierten Auseinandersetzung mit diesem Themenkomplex.

Vor diesem Hintergrund verfolgt die vorliegende Arbeit das Ziel, einen Beitrag zur Erfassung und Erklärung der Zufriedenheit mit Verkehrsdienstleistungen zu erbringen. Hier interessiert insbesondere, wie aus einer prozeßbezogenen Sichtweise die Kundenzufriedenheit mit personenbezogenen Verkehrsdienstleistungen zu operationalisieren ist und welche Bestimmungsfaktoren die Zufriedenheit von Reisenden determinieren. Demzufolge wird der Episoden- und Schnittstellencharakter von Verkehrsdienstleistungen bei der Entwicklung eines Operationalisierungs- und Erklärungsansatzes der Kundenzufriedenheit in expliziter Weise berücksichtigt. Der theoriegestützte Ansatz erfährt dabei eine empirische Validierung auf der Basis einer Befragung von Verkehrsdienstleistungskunden.

Angesichts der Heterogenität des Dienstleistungssektors sowie der Komplexität des Zufriedenheitskonstruktes konzentriert sich die empirische Analyse der vorliegenden Arbeit auf den Personenfernverkehr der Deutschen Bahn AG. Der historische Kontext dieses Unternehmens als Hauptanbieter von schienengebundenen Verkehrsdienstleistungen und die Herausforderungen, mit denen sich die Deutsche Bahn AG aktuell und in der Zukunft konfrontiert sieht, machen dabei die Analyse der Kundenzufriedenheit mit dem Angebot dieses Dienstleistungsunternehmens zu einem besonders lohnenden Forschungsfeld. 
Die Anfertigung der vorliegenden Arbeit war nur durch die Unterstützung zahlreicher Personen möglich. Mein besonderer Dank gebührt zunächst meinem akademischen Lehrer, Herrn Professor Dr. Dr. h.c. Heribert Meffert, der bereits frühzeitig die Themenstellung anregte und die Erstellung der Arbeit in allen Phasen ihrer Entstehung umfassend förderte. Ebenso danke ich Herrn Professor Meffert für das Vertrauen, das er mir mit der Übertragung leitender Funktionen im Rahmen der Forschungsstelle Bahnmarketing in der Wissenschaftlichen Gesellschaft für Marketing und Unternehmensführung e.V. entgegengebracht hat. Auf diese Weise gab er mir die Möglichkeit, mich mit den Besonderheiten von Verkehrsdienstleistungen in unterschiedlichsten Forschungsprojekten vertraut zu machen. Herrn Professor Dr. Klaus Backhaus danke ich herzlich für die Übernahme des Zweitgutachtens.

Der empirische Teil der vorliegenden Arbeit stützt sich auf eine Befragung von mehr als 600 Fahrgästen der Deutschen Bahn AG im innerdeutschen Personenfernverkehr. Die Durchführung dieser Studie wäre in diesem Umfang ohne die ausgezeichnete Zusammenarbeit mit der Deutschen Bahn AG nicht realisierbar gewesen. Daher möchte ich an dieser Stelle allen Mitarbeitern des Geschäftsbereichs Fernverkehr - Marketing, die zum positiven Verlauf dieses Forschungsprojektes beigetragen haben, nachdrücklich danken. Dabei gilt mein Dank auch den Interviewern dieses Forschungsprojektes, die durch ihren unermüdlichen Einsatz die Grundlage für die empirische Untersuchung geschaffen haben.

Dank schulde ich nicht zuletzt auch allen aktuellen und ehemaligen Kolleginnen und Kollegen am Institut für Marketing der Universität Münster, die mich während der Abfassung der Arbeit in vielfältiger Weise unterstützt und von anderweitigen Aufgaben entlastet haben. Ganz besonders danken möchte ich meinem Freund und Kollegen Dipl.-Kfm. Jesko Perrey, der mir jederzeit ein motivierender und kompetenter Ansprechpartner war. Ferner gilt mein Dank Herrn Dr. Manfred Kirchgeorg und Herrn Dr. Christoph Burmann, die mir nicht nur während der Erstellung der Arbeit immer wieder mit ihrer Fachkompetenz hilfreich zur Seite standen und mir den Zugang in die komplexe Welt der Kausalanalyse erleichterten. Herrn Dipl.-Kfm. Thomas Schwetje danke ich für die wertvollen Anregungen bei der Durchsicht des Manuskriptes. Den Herren cand. rer. pol. Dirk Vollmann und Dipl.-Wirt.Inform. Michael zur Mühlen sei an dieser Stelle herzlich für die perfekte Erstellung der Abbildungen gedankt, wobei letzterem zudem mein spezieller Dank für die exzellente und geduldige Abwicklung der Manuskripterstellung zukommt. 
Darüber hinaus bin ich meinen Eltern zu tiefem Dank verpflichtet, die mich in allen - manchmal auch schwierigen - Phasen meiner Ausbildung in vielerlei Hinsicht liebevoll unterstützt haben. Dabei haben sie mir immer die Freiheit eigener Entscheidungen gelassen und mir zusammen mit meiner Schwester Judith ihr Verständnis und ihre Hilfe entgegengebracht. Meinem Vater bin ich dabei nicht zuletzt für die sorgfältige Schlußdurchsicht des Manuskriptes sehr dankbar.

Schließlich ist es mir ein besonderes Anliegen, meiner großartigen Corinna zu danken. Trotz eigener Examensbelastung war sie mir mit ihrer Ausgeglichenheit, ihrem Zuspruch und ihrer Hilfsbereitschaft nicht nur in der Zeit der Promotion stets eine unentbehrliche Ratgeberin und Partnerin. 
Andreas Siefke and Universität Münster - 978-3-631-75103-9

Downloaded from PubFactory at 01/11/2019 08:18:16AM

via free access 


\section{Inhaltsverzeichnis}

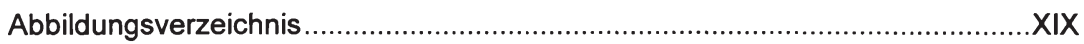

Abkürzungsverzeichnis...........................................................................

A. Kundenzufriedenheit als Herausforderung an das Marketing von Dienstleistungsunternehmen ...................................................... 1

1. Bedeutung der Kundenzufriedenheit im Dienstleistungsbereich für die marktorientierte Unternehmensführung........................................................ 1

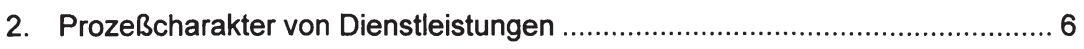

3. Begriff der Kundenzufriedenheit mit Dienstleistungen .................................. 21

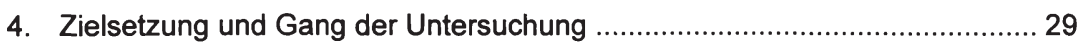

B. Konzeptionelle Grundlagen zur Analyse der Kundenzufriedenheit mit Dienstleistungen

1. Anforderungen an einen Operationalisierungsansatz zur Erfassung und Erklärung der Kundenzufriedenheit mit Dienstleistungen ............................... 33

1.1 Allgemeine Anforderungen an einen Operationalisierungsansatz ........... 34

1.2 Dienstleistungsspezifische Anforderungen ............................................ 37

1.21 Generelle dienstleistungsspezifische Anforderungen...................... 37

1.22 Aspekte des spezifischen Charakters von Verkehrsdienst-

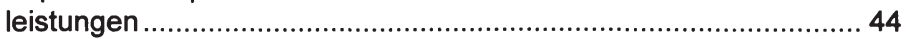

2. Theoretische Konzeption von Kundenzufriedenheit im Dienstleistungs-

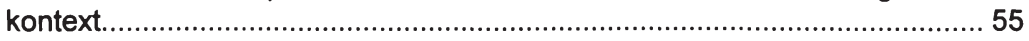

2.1 Beziehung der Kundenzufriedenheit zu verwandten Konstrukten ........... 55

2.11 Konzeptionelle Differenzierungspotentiale .................................... 55

2.12 Analyse der kausalen Beziehungen der Konstrukte........................ 61

2.2 Erklärungsansätze der Kundenzufriedenheit .......................................... 64

2.21 Grundlegende Theorien und Modellansätze ................................... 64

2.22 Analyse der Konstruktkomponenten und deren Einflußfaktoren im Disconfirmation-Paradigma 
2.3 Erscheinungsformen der Kundenzufriedenheit ......................................73

2.31 Psychologische Grundlagen der Wahrnehmung von Kunden-

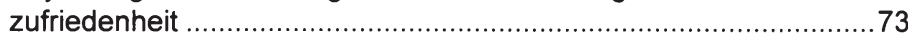

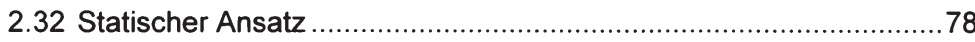

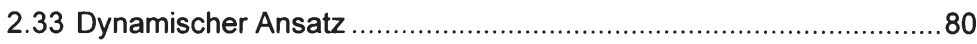

2.331 Wirkungseffekte der Zufriedenheitsdynamik ........................82

2.332 Dynamisch-konsumaktbezogene Betrachtung...................... 86

2.333 Dynamisch-konsumphasenbezogene Betrachtung................92

2.4 Würdigung der Erklärungsansätze und Erscheinungsformen der

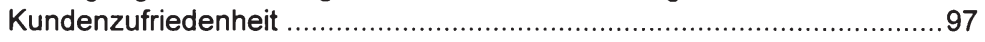

3. Ansätze zur prozeßorientierten Strukturanalyse und Messung von Kundenzufriedenheit im Dienstleistungsbereich ........................................99

3.1 Strukturanalyse und Visualisierung der Prozeßkomponente von

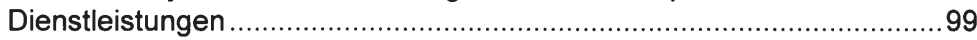

3.2 Verfahren zur Messung der Kundenzufriedenheit .................................104





3.221 Merkmalsgestützte Verfahren .........................................109

3.222 Ereignisgestützte Verfahren ..........................................118

3.3 Würdigung der Meßansätze zur Kundenzufriedenheit ..........................124

4. Phasenorientierter Ansatz zur Erfassung und Erklärung der Kundenzufriedenheit mit Verkehrsdienstleistungen ................................................126

4.1 Erfassung der Kundenzufriedenheit mit Verkehrsdienstleistungen ........126

4.11 Strukturanalyse und Visualisierung der untersuchten Verkehrs-

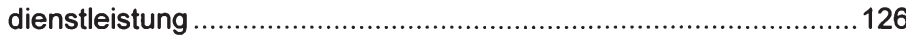

4.12 Ebenen und Indikatoren der Kundenzufriedenheit mit Verkehrs-

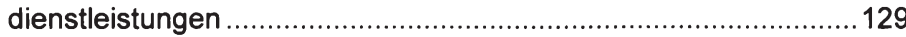

4.121 Struktur und Dynamik der Transaktionszufriedenheit ..........130

4.122 Struktur und Dynamik der Episodenzufriedenheiten .............132

4.2 Bestimmungsfaktoren der Kundenzufriedenheit mit Verkehrsdienst-



4.21 Personenbezogene Bestimmungsfaktoren.................................. 136

4.22 Reisebezogene Bestimmungsfaktoren .........................................140

5. Bezugsrahmen der empirischen Untersuchung .........................................142 
C. Empirische Analyse zur phasenorientierten Erfassung und Erklärung der Zufriedenheit mit Verkehrsdienstleistungen

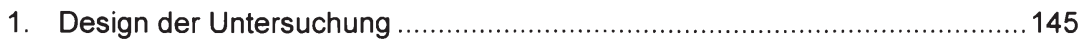

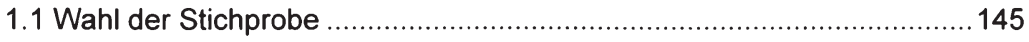

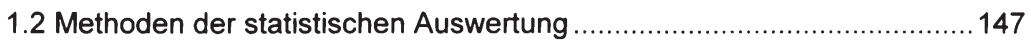

2. Phasenbezogene Analyse der Zufriedenheit mit Verkehrsdienst-

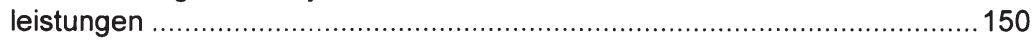

2.1 Überprüfung zentraler Operationalisierungsanforderungen ..................151

2.2 Intraphasenspezifische Analyse der Kundenzufriedenheit.....................153

2.21 Analyse der Vor-Reisezufriedenheit.......................................... 153

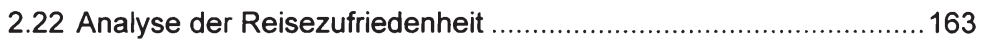



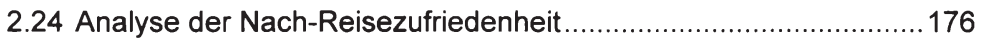

2.3 Interphasenspezifische Analyse der Kundenzufriedenheit.................... 180

2.31 Struktur und Dynamik der Transaktionszufriedenheit ....................181

2.32 Einfluß auf die Beziehungszufriedenheit.....................................188

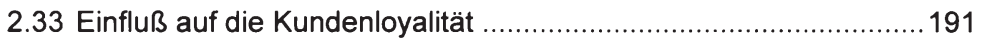

2.4 Analyse der Bestimmungsfaktoren der Zufriedenheit mit Verkehrs-

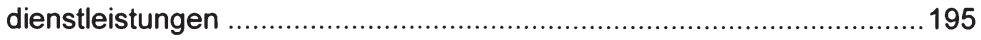

2.41 Personenbezogene Bestimmungsfaktoren ................................... 195

2.411 Soziodemographische Bestimmungsfaktoren ..................... 196

2.412 Psychographische Bestimmungsfaktoren ...........................205

2.42 Reisebezogene Bestimmungsfaktoren ......................................214 
D. Zusammenfassung und Implikationen ............................................ 225

1. Zusammenfassung und Würdigung der Untersuchungsergebnisse ............225

2. Implikationen für das Management von Verkehrsdienstleistungs-

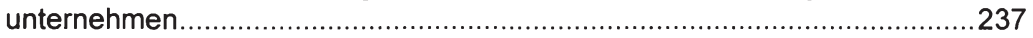

3. Implikationen für die Zufriedenheitsforschung im Dienstleistungsbereich ....240

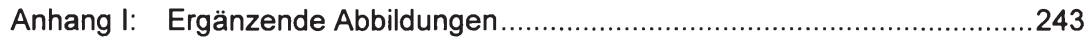

Anhang II: Fragebogen der empirischen Untersuchung ..............................247

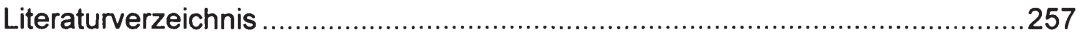




\section{Abbildungsverzeichnis}

Abb. 1: Klassifizierung von Dienstleistungen ....................................... 10

Abb. 2: Charakter des Dienstleistungsprozesses ....................................... 15

Abb. 3: Hierarchie von Dienstleistungsprozessen ...................................20

Abb. 4: Vereinfachte Darstellung einer Reisekette ...................................50

Abb. 5: Determinanten der Verkehrsmittelwahl .........................................53

Abb. 6: Anforderungen an einen Operationalisierungsansatz der Kundenzufriedenheit mit Verkehrsdienstleistungen.

Abb. 7: Differenzierungspotentiale von Kundenzufriedenheit und Dienstleistungsqualität

Abb. 8: Kausalzusammenhang von Kundenzufriedenheit und Dienstleistungsqualität

Abb. 9: Erklärungsmodell der Kundenzufriedenheit .....................................72

Abb. 10: Hierarchie der Kundenzufriedenheit.............................................. 79

Abb. 11: Integrierte Betrachtung der Erscheinungsformen von Kundenzufriedenheit.

Abb. 12: Dynamisches Disconfirmation-Modell 82

Abb. 13: Richtungseffekte der Kundenzufriedenheit 84

Abb. 14: Prozeßmodell der Dienstleistungsqualität nach Boulding et al. 87

Abb. 15: Modell der Beziehungsqualität nach Strandvik / Liljander 90

Abb. 16: Vereinfachte Service Map am Beispiel einer Flugreise 102

Abb. 17: Ansätze zur Messung der Kundenzufriedenheit. 106

Abb. 18: Ausschnitt aus dem Kundenpfad einer Cluburlaubsreise. 123

Abb. 19: Vergleichende Bewertung der Ansätze zur Messung der Kundenzufriedenheit.

Abb. 20: Hierarchie des Kundenprozesses bei schienengebundenen Verkehrsdienstleistungen

Abb. 21: Bezugsrahmen der empirischen Untersuchung der Kundenzufriedenheit mit Verkehrsdienstleistungen.... 


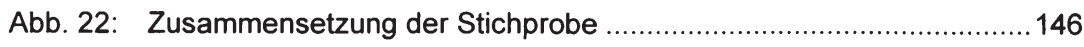



Abb. 24: Kritische Ereignisse der Vor-Reisephase ..........................................156

Abb. 25: Kausalmodell zur Struktur und Dynamik der Vor-Reise-





Abb. 27: Kritische Ereignisse der Reisephase ……………………..................165

Abb. 28: Kausalmodell zur Struktur und Dynamik der Reisezufriedenheit .......167

Abb. 29: Zufriedenheitsprofil der Umsteigephase ...........................................172

Abb. 30: Kritische Ereignisse der Umsteigephase...........................................173

Abb. 31: Kausalmodell zur Struktur und Dynamik der Umsteige-



Abb. 32: Zufriedenheitsprofil der Nach-Reisephase ......................................176

Abb. 33: Kritische Ereignisse der Nach-Reisephase .....................................178

Abb. 34: Kausalmodell zur Struktur und Dynamik der Nach-Reisezufriedenheit.

Abb. 35: Profil der Kundenzufriedenheit auf der Episoden- und Transaktionsebene.

Abb. 36: Episodenübergreifende Betrachtung der kritischen Ereignisse ..........183

Abb. 37: Zusammenhang von kritischen Ereignissen und der Zufriedenheit auf der Episodenebene.

Abb. 38: Konfirmatorische Faktorenanalyse zur Struktur und Dynamik der Transaktionszufriedenheit

Abb. 39: Konfirmatorische Faktorenanalyse zur Beziehungszufriedenheit.......189

Abb. 40: Konfirmatorische Faktorenanalyse zur Kundenloyalität .....................192

Abb. 41: Kundenloyalität in Abhängigkeit vom Niveau der Transaktionszufriedenheit.

Abb. 42: Einfluß demographischer Bestimmungsfaktoren auf die Kundenzufriedenheit. 
Abb. 43: Beziehungszusammenhänge der Kundenzufriedenheit in Abhängigkeit vom Alter.

Abb. 44: Beziehungszusammenhänge der Kundenzufriedenheit in Abhängigkeit vom Geschlecht.

Abb. 45: Einfluß sozioökonomischer Bestimmungsfaktoren auf die Kundenzufriedenheit.

Abb. 46: Beziehungszusammenhänge der Kundenzufriedenheit in Abhängigkeit von sozioökonomischen Bestimmungsfaktoren. 204

Abb. 47: Einstellungen gegenüber dem Verkehrsdienstleister Deutsche Bahn AG 206

Abb. 48: Ausprägungen des wahrgenommenen Risikos und des Involvement von Bahnreisenden.

Abb. 49: Kausalmodell zum Einfluß psychographischer Bestimmungsfaktoren auf die Kundenzufriedenheit

Abb. 50: Beziehungszusammenhänge der Kundenzufriedenheit in Abhängigkeit vom Reiseanlaß

Abb. 51: Differenzen in der Kundenzufriedenheit in Abhängigkeit vom Zugtyp

Abb. 52: Zufriedenheitsprofile in Abhängigkeit von der Reiseklasse.

Abb. 53: Zufriedenheitsprofile in Abhängigkeit vom Abteiltyp.

Abb. 54: Zufriedenheitsprofile in Abhängigkeit vom Auslastungsgrad der Züge

Abb. 55: Zufriedenheitsprofile in Abhängigkeit von der Inanspruchnahme einer Sitzplatzreservierung.

Abb. 56: Zusammenfassende Übersicht zur Prüfung der Untersuchungshypothesen zur Struktur und Dynamik der Kundenzufriedenheit mit Verkehrsdienstleistungen

Abb. 57: Zusammenfassende Übersicht zur Prüfung der Untersuchungshypothesen zum Einfluß personen- und reisebezogener Bestimmungsfaktoren auf die Kundenzufriedenheit. 


\section{Abkürzungsverzeichnis}

a.a.O.

Abb.

AGFI

AGLS

AMA

asw

Aufl.

$\mathrm{Bd}$.

Bhf.

bspw.

BWL

bzw.

ca.

d.h.

DB

DBB

DBW

DDB

e.V.

ed.

EJoM

EQS

et al.

etc.

ETR

f., ff.

FAZ

FWW

GFI

$\mathrm{H}$.

HBM

HBR

$\mathrm{HM}$

Hrsg.

Hyp

i.d.R.

i.e.S. am angegebenen Ort

Abbildung

Adjusted Goodness of Fit Index

Arbitrary Generalized Least Squares

American Marketing Association

Absatzwirtschaft

Auflage

Band

Bahnhof

beispielsweise

Betriebswirtschaftslehre

beziehungsweise

circa

das heißt

Deutsche Bundesbahn, Deutsche Bahn

Die Bundesbahn

Die Betriebswirtschaft

Die Deutsche Bahn

eingetragener Verein

Edition

European Journal of Marketing

Equations based Structural Program

et alii, et alia

et cetera

Eisenbahntechnische Rundschau

folgende, fortfolgende

Frankfurter Allgemeine Zeitung

Fremdenverkehrswirtschaft International

Goodness of Fit Index

Heft

Harvard Business Manager

Harvard Business Review

Harvard Manager

Herausgeber

Hypothese

in der Regel

im engeren Sinne 
i.S.

i.w.S.

IJoQRM

IJoRiM

IJoSIM

Inc.

insbes.

IVW

J.

JAMS

JdAV

$\mathrm{Jg}$.

JoBR

JoCR

JoCS/D\&CB

JoM

JoMR

JoR

JoSM

Kap.

KKV

LISREL

It.

M\&M

Mass.

MIV

N.J.

n.s.

No.

Nr.

o.Jg.

o.V.

OPNV

ÖV

P\&A

PC

Pkw

QZ im Sinne

im weiteren Sinne

International Journal of Quality \& Reliability Management

International Journal of Research in Marketing

International Journal of Service Industry Management

incorporated

insbesondere

Internationales Verkehrswesen

Jahre

Journal of the Academy of Marketing Science

Jahrbuch der Absatz- und Verbrauchsforschung

Jahrgang

Journal of Business Research

Journal of Consumer Research

Journal of Consumer Satisfaction, Dissatisfaction and

Complaining Behavior

Journal of Marketing

Journal of Marketing Research

Journal of Retailing

Journal of Services Marketing

Kapitel

Komparativer Konkurrenzvorteil

Linear Structural Relations System

laut

Marktforschung \& Management

Massachusetts

Motorisierter Individualverkehr

New Jersey

nicht signifikant

Number

Nummer

ohne Jahrgang

ohne Verfasser

Offentlicher Personennahverkehr

Öffentlicher Personenverkehr

Planung und Analyse

Personal Computer

Personenkraftwagen

QZ - Zeitschrift für industrielle Qualitätssicherung 
RMR

$S$.

s.o.

s.u.

SMR

sog.

SPSS

TCD

TQM

u.ä.

u.a.

u.U.

usw.

vgl.

VM

Vol.

vs.

WiSt

WISU

WiWo

z.B.

z.T.

$\mathrm{ZfB}$

$\mathrm{ZfbF}$

ZFP

ZögU
Root Mean Square Residuals

Seite

siehe oben

siehe unten

Sloan Management Review

sogenannte

Superior Performing Software Systems

Total Coefficient of Determination

Total Quality Management

und ähnliche

und andere, unter anderem

unter Umständen

und so weiter

vergleiche

Verkehrsmittel

Volume

versus

Wirtschaftswissenschaftliches Studium

Das Wirtschaftsstudium

Wirtschaftswoche

zum Beispiel

zum Teil

Zeitschrift für Betriebswirtschaft

Zeitschrift für betriebswirtschaftliche Forschung

Zeitschrift für Forschung und Praxis

Zeitschrift für öffentliche und gemeinwirtschaftliche

Unternehmen 


\section{A. Kundenzufriedenheit als Herausforderung an das Marketing von Dienstleistungsunternehmen}

\section{Bedeutung der Kundenzufriedenheit im Dienstleistungsbereich für die marktorientierte Unternehmensführung}

Vor dem Hintergrund eines sich verschärfenden dynamischen Wettbewerbs auch und insbesondere im Dienstleistungsbereich werden Markt- und Kundenorientierung zunehmend als zentrale Erfolgsfaktoren im Wettbewerb angesehen. ${ }^{1}$ Als Maßstab für die erreichte Kundenorientierung und -bindung wird häufig das Konstrukt der Kundenzufriedenheit herangezogen. Viele der aktuell diskutierten Managementkonzepte wie Total Quality Management (TQM), Business Reengineering oder Lean Management orientieren sich an der Zufriedenheit der Kunden als oberster Verhaltensmaxime. ${ }^{2}$ Jedoch ist die diesen Konzepten zugrunde liegende Sichtweise nicht als neu anzusehen. ${ }^{3}$ Vielmehr sind Kundenorientierung und Kundenzufriedenheit integrale Bestandteile des modernen Marketingkonzeptes. Die Entwicklung vom Verkäufer- zum Käufermarkt hat eine MarketingPhilosophie begründet, die „die Probleme, Wünsche und Bedürfnisse aktueller und potentieller Kunden ..." an den „Anfang aller Überlegungen“ stellt. ${ }^{4}$ Im Zuge dieser Überlegungen wird insbesondere die Bedeutung der Kundenzufriedenheit für den langfristigen Unternehmenserfolg hervorgehoben. Die besondere Betonung der Kundenzufriedenheit als Erfolgsfaktor ist darüber hinaus auf Entwicklungen und Herausforderungen zurückzuführen, mit denen sich die marktorientierte Unternehmensführung aktuell und in der Zukunft in verstärktem Maße auch im Dienstleistungsbereich konfrontiert sieht.

1 Vgl. Schütze, R., Kundenzufriedenheit: After-Sales-Marketing auf industriellen Märkten, Wiesbaden 1992, S. 1 ff.; Herrmann, A., Produktqualităt, Kundenzufriedenheit und Unternehmensrentabilität: Eine branchenübergreifende Analyse, in: Wege des Marketing: Festschrift zum 60. Geburtstag von Erwin Dichtl, Bauer, H.H., Diller, H. (Hrsg.), Berlin 1995, S. 237 und die dort angegebene Literatur.

Zu dem Verhältnis der Konstrukte Markt- und Kundenorientierung sowie Kundenzufriedenheit $\mathrm{vgl}$. Meffert, H., Kundenorientierung und Kundenzufriedenheit - Zwei Seiten einer Medaille?, Manuskript, Februar 1997, S. 7 und 11 f.

2 Vgl. Meffert, H., Siefke, A., Lean Marketing - mehr als ein Schlagwort?, Arbeitspapier Nr. 88 der Wissenschaftlichen Gesellschaft für Marketing und Unternehmensführung e.V., Meffert, $H$., Wagner, H., Backhaus, K. (Hrsg.), Münster 1994, S. 9.; Stauss, B., Total Quality Management und Marketing, in: Marketing ZFP, 16. Jg., H. 3, 1994, S. 149.

3 So betonte McCarthy bereits Anfang der 60er Jahre, daß "the marketing job ... is one of trying to satisfy a particular group ... with a particular good or service", zit. nach McNeal, J., Consumer Satisfaction: The Measure of Marketing Effectiveness, in: MSU Business Topics, Summer 1969, S. 31.

4

Meffert, H., Marketing: Grundlagen der Absatzpolitik, 7. Aufl., Wiesbaden 1986, S. 31. 
Der vielzitierte "Marsch in die Dienstleistungsgesellschaft"5 ist auf unterschiedliche Ursachen zurückzuführen. Gesellschaftliche und demographische Veränderungen, wie verkürzte Arbeitszeiten oder eine steigende Lebenserwartung der Bevölkerung, stellen dabei die zentralen Ursachen für die dynamische Zunahme der Dienstleistungsnachfrage dar.

Diese Entwicklungen lassen sich auch als Begründung für die steigende Nachfrage nach Verkehrsdienstleistungen in den westlichen Industriestaaten heranziehen. Darüber hinaus sind in diesem Zusammenhang die zunehmende Globalisierung der Märkte sowie die Integrationsentwicklung in Europa einhergehend mit einem wachsenden Freizeit- und Mobilitätsbedürfnis der Bevölkerung zu nennen, die zu einer "Ent-Lokalisierung" von geschäftlichen und privaten Interaktionen führen. ${ }^{6}$ Die zentrale geographische Lage in Europa verstärkt diese Tendenzen in Deutschland zusätzlich durch die Bedeutung als Transitland.

Dabei ist sowohl auf der inter- als auch der intramodalen Betrachtungsebene ${ }^{7}$ der Anbieter von Verkehrsdienstleistungen ein intensiver Wettbewerb festzustellen bzw. noch zu erwarten. ${ }^{8}$ Ursache hierfür stellen Deregulierungs- und Privatisierungsmaßnahmen bei den ehemals staatlichen Flug- und Bahngesellschaften dar. Die steigende Wettbewerbsintensität äußerte sich in den letzten Jahren insbesondere auf dem Luftverkehrsmarkt. Hier waren sowohl auf dem innerdeutschen als auch auf dem internationalen Markt zunehmend aggressive Preisstrategien von Fluggesellschaften zu beobachten. ${ }^{9}$

Darüber hinaus wird mit der Liberalisierung des europäischen Eisenbahnverkehrsmarktes durch die angestrebte Öfnung der nationalen Schienennetze auch ausländischen Eisenbahngesellschaften sowie inländischen Drittanbietern

5 Vgl. Fourastié, J., Die große Hoffnung des zwanzigsten Jahrhunderts, Köln 1954, S. 133 ff.

6 Vgl. Litzenroth, H., Dem Verbraucher auf der Spur. Quantitative und qualitative Konsumtrends, in: JdAF, 41. Jg. H. 3, 1995, S. 242 und 259; Laakmann, K., Mobilităts-Marketing, in: Lexikon der aktuellen Marketingbegriffe, Meffert, H. (Hrsg.), Wien 1994, S. 161; Meffert, H., Euromarketing im Spannungsfeld zwischen nationalen Bedürfnissen und globalem Wettbewerb, in: Europa 1992 - Chancen und Risiken für das Marketing, Bruhn, M., Wehrle, F. (Hrsg.), 2. Aufl., Münster 1990, S. $14 \mathrm{ff}$.

7 Während die intermodale Sichtweise verkehrsträgerübergreifend zu verstehen ist, wird der Wettbewerb auf Verkehrsträgerebene (Flugzeug, Bahn, Pkw) als intramodal bezeichnet.

8 So ist im Rahmen der Bahnreform auch der Zugang Dritter als Anbieter von Schienenverkehrsleistungen vorgesehen. Vgl. Laaser, C.-F., Die Bahnstrukturreform: Richtige Weichenstellung oder Fahrt aufs Abstellgleis?, Kieler Diskussionsbeitrăge Nr. 239, Kiel 1994, S. 10.

9 Vgl. Klein, H., Qualitătsmanagement der Deutschen Lufthansa AG, in: Dienstleistungsqualităt: Konzepte, Methoden, Erfahrungen, Bruhn, M., Stauss, B. (Hrsg.), 2. Aufl., Wiesbaden 1995, S. 479. 
die Möglichkeit eröffnet, am deutschen Verkehrsmarkt zu partizipieren. Neben dem Luft- und motorisierten Individualverkehr wird die Deutsche Bahn AG als Hauptanbieter dieser Leistungen daher mit weiterer Konkurrenz und damit mit einem zu erwartenden Verdrängungswettbewerb konfrontiert. ${ }^{10}$ Die aufgezeigten Entwicklungen werden überdies von einem spürbar gestiegenem Anspruchsniveau der Konsumenten an die Qualität von Verkehrsdienstleistungen begleitet. ${ }^{11}$

In diesem Zusammenhang ist festzustellen, daß es der Deutschen Bahn AG bisher nicht ausreichend gelungen ist, am Wachstum des Personenfernverkehrsmarktes in der Bundesrepublik zu partizipieren. Während die Wettbewerber der DB AG - Pkw, Flugzeug und Reisebus - ihre Marktanteile steigern konnten, hat die Bahn in den letzten Jahrzehnten z.T. deutliche Marktanteilsverluste hinnehmen müssen. ${ }^{12}$ Auch wenn das Straßennetz stetig gewachsen ist, während sich das Schienennetz zurückentwickelte, muß die Ursachenforschung für die Marktanteilsentwicklung über die Analyse rechtlich-politischer Rahmenbedingungen hinausgehen. ${ }^{13}$ Ein Grund für die aktuelle Marktstellung besteht darin, daß der Deutschen Bahn AG auch einige Jahre nach ihrer Privatisierung in großen Teilen der Bevölkerung immer noch das Image eines "bürokratischen Staatsbetriebes" anlastet und eine damit einhergehende nur mangelhafte Preisakzeptanz festzustellen ist. Sowohl die Ergebnisse bahneigener Erhebungen als auch die Berichterstattung in den Medien sind Beweis dafür, daß die Bahn in der Vergangenheit aus Kundensicht Fortschritte machen konnte, jedoch sowohl innerhalb des Verkehrssektors als auch branchenübergreifend weiterhin ein starker Aufholbedarf in

Vgl. Institut der deutschen Wirtschaft Köln (Hrsg.), EU-Verkehr - Freie Fahrt für die Eisenbahn, in: iwd Informationsdienst des Instituts der deutschen Wirtschaft, Nr. 41 vom 10. Oktober 1996, S. 7 .

11 Vgl. u.a. Meffert, H., Bruhn, M., Dienstleistungsmarketing: Grundlagen, Konzepte, Methoden; mit Fallbeispielen, 2. Aufl., Wiesbaden 1997, S. 3 und $7 \mathrm{f}$.

12 Betrug der Marktanteil der Deutschen Bundesbahn in den fünfziger und Anfang der sechziger Jahre etwa ein Drittel der gesamten Personenfernverkehrsnachfrage, verlor die Bahn in den nachfolgenden Jahren kontinuierlich Anteile an die Wettbewerber Pkw, Flugzeug und Reisebus. So belief sich der Anteil der Bahn am Fernverkehr im Jahre 1992 auf unter 10\%. Vgl. Garre, K.-H., InterRegio: Ein neues Leistungsangebot der Deutschen Bundesbahn im Schienenpersonenfernverkehr, in: DBB, Jg. 64, H. 9, 1988, S. 775; Weigand, W., Planungsund Prognosemethoden für das Angebot im Hochgeschwindigkeitsverkehr der Zukunft, in: ETR, Jg. 43, H. 5, 1994, S. 279.

13 Vgl. Laaser, C.-F., Die Bahnstrukturreform: Richtige Weichenstellung oder Fahrt aufs Abstellgleis?, a.a.O., S. 3 . 
der Imagewahrnehmung bei aktuellen und potentiellen Kunden besteht und deutliche Defizite in der Zufriedenheit mit Bahnreisen auszumachen sind. ${ }^{14}$

Bedingt durch die wettbewerbsbezogene Reaktionsverbundenheit und einhergehend mit der hohen Austauschbarkeit der originären Grundleistung, der Beförderung von $A$ nach $B$, nimmt die Profilierung der eigenen Leistung im Wettbewerb und die optimale Ausgestaltung des Leistungsprogrammes zur Befriedigung der Mobilitätsbedürfnisse und Sicherstellung der Kundenzufriedenheit eine zentrale Stellung ein. ${ }^{15}$

Ist die Gewinnung von Neukunden weiterhin von hoher Bedeutung, wird es auch für Verkehrsdienstleistungsunternehmen immer wichtiger, aktuelle Kunden zu halten und diese in ihrem Ertragspotential für das Unternehmen noch zu steigern. ${ }^{16}$ Der Ertragswert eines Kunden ist bei ausschließlicher Betrachtung des Erstkaufs nur relativ gering. Sein Ertragspotential kann erst durch langfristige Kundenbindung ausgeschöpft werden. ${ }^{17}$ Findet darüber hinaus die Tatsache Berücksichtigung, daß die Kosten der Neukundenakquisition im Schnitt etwa das Fünffache jener Kosten betragen, die für die Pflege von Altkunden aufzubringen sind, ${ }^{18}$ tritt die Bedeutung einer dauerhaften Kundenbindung durch die Schaffung einer möglichst hohen Kundenzufriedenheit in allen Phasen des Konsums besonders zu Tage.

14 Vgl. Antrecht, R., Claßen, W., Friese, U., Wem die Deutschen trauen, in: Capital, H. 3, 1996, S. 38ff.; Stiftung Warentest (Hrsg.), Service der Bahn - Zügig informiert, zuviel kassiert, in: Test, H. 5, 1995, S. 80 ff. In den Ergebnissen des seit 1992 jährlich durchgeführten Deutschen Kundenbarometers, das auf repräsentativer Basis die Kundenzufriedenheit mit über 700 Anbietern aus über 40 Branchen in Deutschland untersucht, rangiert die Deutsche Bahn im Branchenranking deutlich im letzten Drittel. Meyer, A., Dornach, F., Das Deutsche Kundenbarometer: Qualität und Zufriedenheit, in: Simon, H., Homburg, Ch. (Hrsg.), Kundenzufriedenheit: Konzepte, Methoden, Erfahrungen, Wiesbaden 1995, S. $170 \mathrm{ff}$.

Vgl. Burmann, Ch., Touristik-Marketing, in: Lexikon der aktuellen Marketingbegriffe, Meffert, H. (Hrsg.), Wien 1994, S. 236.

16 Hierin spiegelt sich auch ein zentraler Ansatzpunkt der in der aktuellen Diskussion stehenden Konzepte des sog. Beziehungsmanagement bzw. Relationship Marketing sowie des Kundenbindungsmarketing bzw. Retention Marketing wider, die eine Abkehr von einem reinen transaktions- hin zu einem beziehungsorientierten Marketing postulieren. Vgl. hierzu ausführlich Bruhn, M., Bunge, B., Beziehungsmarketing: Neuorientierung für Marketingwissenschaft und praxis?, in: Marktorientierte Führung im Umbruch: Effizienz und Flexibilităt als Herausforderungen des Marketing, Bruhn, M., Meffert, H., Wehrle, F. (Hrsg.), Stuttgart 1994, S. $41 \mathrm{ff}$.; Diller, H., Kundenbindung als Marketingziel, in: Marketing ZFP, Jg. 18, H. 2, 1996, S. 81 ff.

17 Vgl. Reichheld, F.F., Sasser, W.E., Zero-Migration: Dienstleister im Sog der Qualitătsrevolution, in: HM, Jg. 13, H. 4, 1991, S. $108 \mathrm{ff}$.

18 Vgl. Müller, W., Riesenbeck, H.-J., Wie aus zufriedenen auch anhängliche Kunden werden, in: HM, Jg. 13, H. 3, 1991, S. 69. 
Wird in Anlehnung an MEFFERT/BRUHN in einer ersten Definition unter Kundenzufriedenheit die Übereinstimmung zwischen den subjektiven Erwartungen an die Qualität von Produkten oder Dienstleistungen und der tatsächlich erlebten Motivbefriedigung verstanden, sind vor allem die Verhaltenskonsequenzen von Interesse, die die Konsumenten aufgrund der wahrgenommenen Disparität zwischen Erwartungen und tatsächlich Erreichtem ziehen. ${ }^{19}$ Hohe Zufriedenheit stellt die Basis für eine Kundenbindung bzw. Kundentreue an die Marke oder Unternehmung dar. Unzufriedenheit von Konsumenten kann unterschiedliche Reaktionsformen auf die nicht erlebte Bedürfnisbefriedigung zur Folge haben. Die Kunden können beispielsweise still zu anderen Unternehmen abwandern (sog. Unvoiced Complaints) und/oder ihr Mißfallen gegenüber den Marktbeteiligten oder anderen Personen und Institutionen zum Ausdruck bringen (z.B. durch Beschwerden). ${ }^{20}$

Es ist daher überraschend, daß eine ausreichend konkretisierte Aufnahme der Kundenzufriedenheit als Erfolgsindikator und Steuerungsgröße des langfristigen Unternehmenserfolges im Zielsystem von Dienstleistungsunternehmen eher die Ausnahme bildet bzw. erst in jüngerer Zeit stattfindet. ${ }^{21}$ Dies verwundert um so mehr, als ihre Relevanz als zentrales psychographisches Ziel, bedingt durch die Erkenntnis ihres hohen Einflusses auf ökonomische Zielgrößen, überwiegend anerkannt wird. $^{22}$

Vgl. Meffert, H., Bruhn, M., Beschwerdeverhalten und Zufriedenheit von Konsumenten, in: DBW, Jg. 41, H. 4, 1981, S. 597

20 Vgl. Hirschmann, A.O., Abwanderung und Widerspruch - Reaktionen auf Leistungsabfall bei Unternehmungen, Organisationen und Staaten, Tübingen 1974. Neben der Diskussion der Kundenzufriedenheit als Indikator der Kundenbindung werden in der wissenschaftlichen Literatur u.a. Wechselbarrieren, Variety Seeking, also der Wunsch nach Abwechslung, sowie die Attraktivität von Konkurrenzangeboten als weitere Determinanten der Kundenloyalität genannt. Vgl. Dichtl, E., Peter, S., Kundenzufriedenheit und Kundenbindung in der Automobilindustrie: Ergebnisse einer empirischen Untersuchung, in: Automobilmarktforschung: Nutzenorientierung von Pkw-Herstellern, Bauer, H.H., Dichtl, E., Herrmann, A. (Hrsg.), München 1996, S. 24 ff.; Stauss, B., Neuhaus, P., Das Qualitative Zufriedenheitsmodell (QZM), Diskussionsbeitrăge der Wirtschaftswissenschaftlichen Fakultăt Ingolstadt Nr. 66, Ingolstadt 1995, S. 4 ff.; Bänsch, A., Variety Seeking: Marketingfolgerungen aus Überlegungen und Untersuchungen zum Abwechslungsbedürfnis von Konsumenten, in: JdAV, Jg. 41, H. 4, 1995, S. 342 ff.

21 Befunde der Erfolgsfaktorenforschung bestätigen die (bisherige) Dominanz finanzieller und marketingpolitischer Indikatoren. Vgl. Dichtl, E., Schneider, W., Kundenzufriedenheit im Zeitalter des Beziehungsmanagement, in: Lean Management und Lean Marketing, Belz, C., Schögel, M., Kramer, M. (Hrsg.), St. Gallen 1994, S. 6; Stauss, B., Friege, Ch., Zehn Lektionen in TQM, in: HBM, H. 2, 1996, S. 26 ff.; Nader, G., Zufriedenheit mit Finanzdienstleistungen: Erfolgswirksamkeit, Messung und Modellierung, Wien, New York 1995, S. 2; Kube, Ch., Erfolgsfaktoren in Filialsystemen - Diagnose und Umsetzung im strategischen Controlling, Wiesbaden 1991, S. 57.

Während sich in der Vergangenheit die Diskussion um die Erfolgswirksamkeit von Kundenzufriedenheit vorwiegend auf theoretisch-konzeptionelle Überlegungen stützte, sind seit Ende der 80 er Jahre Bemühungen erkennbar, den Einfluß von Kundenzufriedenheit auf ökonomi-

(Fortsetzung der Fußnote auf der nächsten Seite) 


\section{Prozeßcharakter von Dienstleistungen}

Die Vielzahl an Veröffentlichungen zum Dienstleistungsbegriff dokumentiert das Bestreben einer präzisen Abgrenzung und Systematisierung von Dienstleistungen, wobei deutlich unterschiedliche Begriffsauffassungen ausgemacht werden können, die letztendlich dazu führen, daß bis dato kein Konsens hinsichtlich einer einheitlichen Definition des Dienstleistungsbegriffes erzielt werden konnte. ${ }^{23}$

Breiten Raum nehmen solche Definitionsansätze ein, deren Ziel darin besteht, Dienstleistungen über die Erarbeitung konstitutiver Merkmale zu bestimmen. ${ }^{24}$ Regelmäßig werden dabei Merkmale wie Immaterialität, Intangibilität, Unteilbarkeit, Vergänglichkeit, Standortgebundenheit, Individualität sowie Integration des externen Faktors als charakteristisch für Dienstleistungen angesehen. ${ }^{25}$ Wenngleich bezüglich der Zusammensetzung und Anzahl eines solchen Merkmalskataloges unterschiedliche Meinungen anzutreffen sind, erfahren mit der Immate-

sche Erfolgsgrößen näher zu quantifizieren. Vgl. u.a. Anderson, E.W., Fornell, C., Lehmann, D.R., Customer Satisfaction, Market Share, and Profitability: Findings from Sweden, in: JoM, Vol. 58, July 1994, S. 53 ff.; Zeithaml, V.A., Berry, L.L., Parasuraman, A., The Behavioral Consequences of Service Quality, in: JoM, Vol. 60, April 1996, S. $31 \mathrm{ff}$;; Nader, G., Zufriedenheit mit Finanzdienstleistungen: Erfolgswirksamkeit, Messung und Modellierung, a.a.O., S. 4 f., Rust, R.T., Zahorik, A.J., Keiningham, T.L., Return on Quality (ROQ): Making Service Quality Financially Accountable, Marketing Science Institute (Report No. 94-106), Cambridge, Mass. 1994; Marcati, A., Cecere, F., Marzocchi, G.L., Towards a Better Understanding of the Economics of Customer Satisfaction. An Analysis of Customer Satisfaction, Spending Levels and Spending Patterns, in: Marketing Today and for the 21st Century, Proceedings of the 24th Annual Conference of the European Marketing Academy, Bergadaa, M. (Hrsg.), Cergy 1995, S. $1871 \mathrm{ff}$.

23 Vgl. Meffert, H., Marktorientierte Führung von Dienstleistungsunternehmen - neuere Entwicklungen in Theorie und Praxis, in: DBW, Jg. 54, H. 4, 1994, S. $521 \mathrm{ff}$.

24 Des weiteren sind Versuche zu nennen, eine Erfassung des Dienstleistungsbegriffes durch Aufzăhlung von Beispielen im Sinne einer enumerativen Definition vorzunehmen sowie solche, die eine Abgrenzung über eine Negativdefinition zu Sachleistungen zum Gegenstand haben. Vgl. Meffert, H., Bruhn, M., Dienstleistungsmarketing: Grundlagen, Konzepte, Methoden; mit Fallbeispielen, a.a.O., S. 23 und die dort angegebene Literatur.

In der aktuellen Diskussion werden darüber hinaus Typologien von Absatzobjekten zur Überwindung der Definitionsproblematik herangezogen. Vgl. Engelhardt, W.H., Kleinaltenkamp, M., Reckenfelderbäumer, M., Leistungsbündel als Absatzobjekte: Ein Ansatz zur Überwindung der Dichotomie von Sach- und Dienstleistungen, in: ZfbF, Jg. 45, H. 5, 1993, S. $404 \mathrm{ff}$;; Meffert, H., Marktorientierte Führung von Dienstleistungsunternehmen - neuere Entwicklungen in Theorie und Praxis, a.a.O., S. $522 \mathrm{ff}$;; Woratschek, H., Die Typologie von Dienstleistungen aus informationsökonomischer Sicht, in: Der Markt, Jg. 35, H. 1, 1996 , S. 59 ff.; Knoblich, H., Oppermann, R., Dienstleistung: Ein Produkttyp - Eine Erfassung und Abgrenzung des Dienstleistungsbegriffes auf produkttypologischer Basis, in: Der Markt, Jg. 35 , H. 1, 1996, S. $13 \mathrm{ff}$.

25 Vgl. Bruhn, M., Qualitätsmanagement für Dienstleistungen: Grundlagen, Konzepte, Methoden, Berlin u.a. 1996, S. 11. Diese Merkmale werden im Rahmen der Diskussion der dienstleistungsspezifischen Besonderheiten der Zufriedenheitsmessung in Kap. B 1.2 dieser Arbeit erörtert und im Hinblick auf ihre Auswirkungen auf Kundenzufriedenheit mit Verkehrsdienstleistungen analysiert. 
rialität und der Integration des externen Faktors zwei dieser Merkmale in der Diskussion besondere Berücksichtigung. ${ }^{26}$ Dabei bezieht sich der Immaterialitätsgrad primär auf das Leistungsergebnis, während der Integrationsgrad auf den Prozeß der Leistungserstellung Bezug nimmt.

Darüber hinaus wird häufig auf einer übergeordneten Ebene eine phasenorientierte Definition von Dienstleistungen vorgenommen, die zwischen der Potential-, der Prozeß- und der Ergebnisphase einer Dienstleistung unterscheidet und den Wesensmerkmalen einer Dienstleistung in geeigneter Weise Rechnung trägt. ${ }^{27}$ Dabei ist zu beachten, daß diese Dimensionen ${ }^{28}$ nicht als sich gegenseitig ausschließend angesehen werden dürfen, sondern vielmehr einer integrierten Betrachtung unterzogen werden müssen. ${ }^{29}$ Diese Sichtweise aufgreifend können Dienstleistungsdefinitionen als Kombination dieser Leistungsdimensionen mit den als konstitutiv angesehenen Merkmalen abgeleitet und systematisiert werden. In Anlehnung an MEFFERT/BRUHN sind Dienstleistungen dementsprechend

„selbständige, marktfähige Leistungen, die mit der Bereitstellung und/oder dem Einsatz von Leistungsfähigkeiten verbunden sind (Potentialorientierung). Interne und externe Faktoren werden im Rahmen des Erstellungsprozesses kombiniert (Prozeßorientierung). Die Faktorenkombination des Dienstleistungsanbieters wird mit dem Ziel eingesetzt, an den externen Faktoren, an Menschen oder deren Objekten, nutzenstiftende Wirkungen zu erzielen (Ergebnisorientierung). ${ }^{\text {"30 }}$

Vgl. Stauss, B., Kundenprozeßorientiertes Qualitătsmanagement im Dienstleistungsbereich, in: Total Quality Management II, Preßmar, D.B. (Hrsg.), Wiesbaden 1995, S. 28; Stauss, B., Friege, Ch., Zehn Lektionen in TQM, a.a.O., S. 26 ff.; Nader, G., Zufriedenheit mit Finanzdienstleistungen: Erfolgswirksamkeit, Messung und Modellierung, a.a.O., S. 8. So betonte Berekoven bereits im Jahre 1966 den Prozeßcharakter von Dienstleistungen und den synchronen Kontakt zwischen Dienstleistungsanbieter und -nachfrager. Vgl. Berekoven, L., Der Begriff "Dienstleistung" und seine Bedeutung für eine Analyse der Dienstleistungsbetriebe, in: JdAV, Jg. 12, H. 4, 1966, S. 314 ff.

27 Vgl. dazu insbes. Hilke, W., Grundprobleme und Entwicklungstendenzen des DienstleistungsMarketing, in: Dienstleistungs-Marketing, Schriften zur Unternehmensführung, Bd. 35, Jacob, H. et al. (Hrsg.), Wiesbaden 1989, S. $10 \mathrm{ff}$.; Büker, B., Qualitätsbeurteilung investiver Dienstleistungen: Operationalisierungsansătze an einem Beispiel zentraler EDV-Dienste, Frankfurt am Main u.a. 1991, S. $28 \mathrm{ff}$.

Die Begriffe Phasen und Dimensionen werden hier als synonym betrachtet, da eine Dienstleistung im Idealfall chronologisch in der Reihenfolge Produkt - Prozeß - Ergebnis verlăuft. Zu einer ăhnlichen Argumentation vgl. Haller, S., Beurteilung von Dienstleistungsqualităt: Dynamische Betrachtung des Qualitătsurteils im Weiterbildungsbereich, Wiesbaden 1995, S. 57.

Vgl. Knoblich, H., Oppermann, R., Dienstleistung: Ein Produkttyp - Eine Erfassung und Abgrenzung des Dienstleistungsbegriffes auf produkttypologischer Basis, a.a.O., S. 15.

30 Meffert, H., Bruhn, M., Dienstleistungsmarketing: Grundlagen, Konzepte, Methoden; mit Fallbeispielen, a.a.O., S. 27. Im Rahmen dieser Definition verzichten die Autoren auf eine Unterscheidung zwischen den Begriffen Service und Dienstleistung. Dies geschieht auch vor dem Hintergrund, begriffliche Unklarheiten bei der Berücksichtigung anglo-amerikanischer Literatur zu vermeiden. 
Obwohl diese Dreiteilung von einigen Autoren modifiziert bzw. erweitert worden ist, hat sich das folgende Grundverständnis über den Inhalt der Dimensionen weitgehend durchgesetzt: ${ }^{31}$

Gegenstand der Potentialdimension sind die Fähigkeit und Bereitschaft des Dienstleistungsunternehmens und seiner Mitarbeiter zur Dienstleistungserstellung, die technische Ausrüstung sowie die Zugangs- und Nutzungsmöglichkeit durch den Nachfrager.

Die Prozeßdimension bezieht sich auf den Tätigkeitsvollzug. Sie umfaßt die Gesamtheit aller Aktivitäten, die im Verlauf der tatsächlichen Dienstleistungserstellung stattfinden.

Inhalt der Ergebnisdimension schließlich ist der Grad der Erreichung der Leistungsziele. Sie stellt eine abgeschlossene Tätigkeit und damit den beendeten Vollzug eines Dienstleistungspaketes dar.

Die bisher genannten Merkmale und Dimensionen von Dienstleistungen haben definitionsgemäß für alle Dienstleistungen Geltung und sind daher nur bedingt geeignet, die Komplexität und Heterogenität des Dienstleistungssektors zu erfassen und Ansatzpunkte zur Ableitung geeigneter Marketingstrategien und -maßnahmen für einzelne Dienstleistungen zu liefern. Dieser Umstand führte zur Entwicklung einer Vielzahl von z.T. sehr unterschiedlichen Systematisierungsansätzen von Dienstleistungen, die auf der Kombination der als charakteristisch betrachteten Merkmale von Dienstleistungen oder hieraus abgeleiteter Kriterien beruhen.$^{32}$ Da diese Ansätze häufig ein- bzw. zweidimensionaler Natur sind, sind sie auf der einen Seite sehr anschaulich. Auf der anderen Seite hat die geringe Anzahl berücksichtigter Dimensionen allerdings den großen Nachteil, daß nur Teilaspekte einer Dienstleistung beleuchtet werden. Derartigen Ansätzen stehen

31 Vgl. Schmitz, G., Qualitätsmanagement im Privatkundengeschäft von Banken: Konzeption und aufbauorganisatorische Verankerung, Wiesbaden 1996, S. 36 ff. So erweitert z.B. Mengen das Konzept um eine vierte Phase, die Nutzenphase. Vgl. Mengen, A., Konzeptgestaltung von Dienstleistungsprodukten: Eine Conjoint-Analyse im Luftfrachtmarkt unter Berücksichtigung der Qualitătsunsicherheit beim Dienstleistungskauf, Stuttgart 1993, S. 14 ff.

32 Einen Überblick über verschiedene Ansătze bieten Corsten, H., Betriebswirtschaftslehre der Dienstleistungsunternehmen, München 1988, S. 23 ff. sowie Meffert, H., Bruhn, M., Dienstleistungsmarketing: Grundlagen, Konzepte, Methoden; mit Fallbeispielen, a.a.O., S. 35 ff.; Lovelock, Ch.H., Classifying Services to Gain Strategic Marketing Insights, in: JoM, Vol. 47, Summer 1983, S. $9 \mathrm{ff}$. 
Bemühungen gegenüber, die eine Mehrzahl von Dimensionen zur Klassifizierung von Dienstleistungen heranziehen.

Abbildung 1 gibt ein derartiges Klassifizierungsschema wieder. ${ }^{33}$ Dieser Ansatz ist das Ergebnis einer Metaanalyse existierender Systematisierungsansätze von Dienstleistungen, die durch eine empirische Untersuchung bei elf Dienstleistungsunternehmen gestützt wurde. Die Autoren legen ihren Ausführungen sechs Dimensionen zugrunde, die sich wie folgt charakterisieren lassen: ${ }^{34}$

- Intensität der ausrüstungsbezogenen bzw. personalbezogenen Bestandteile einer Dienstleistung (Faktordominanz).

- Länge der Kundenkontaktzeit bei einmaliger Dienstleistungserstellung.

- Individualisierungs- versus Standardisierungsgrad der Dienstleistung.

- Autonomiegrad bzw. Freiheitsraum des Personals hinsichtlich der individuellen Ausrichtung auf den Konsumenten. Dieser fällt um so höher aus, je mehr das Kundenkontaktpersonal befugt und in der Lage ist, ohne Rücksprache mit Vorgesetzten auf die individuellen Wünsche des Kunden einzugehen.

- Quelle der Nutzenstiftung, die im „Front Office“ bzw. „Back Office“-Bereich liegt. Bei einer „Back Office“-orientierten Dienstleistung ist das Verhältnis des Personals mit direktem Kundenkontakt in Relation zur Gesamtbelegschaft vergleichsweise klein.

- Ergebnis- versus Prozeßorientierung. Während bei ergebnisorientierten Dienstleistungen im Vordergrund steht, "was“ der Kunde erhält, dominiert bei prozeßorientierten Dienstleistungen das "wie" des Leistungserstellungsprozesses.

Wenngleich auch diese Dimensionen nicht unbedingt als überschneidungsfrei und zwingend vollständig anzusehen sind, erleichtern sie doch das Grundverständnis über den Charakter einer Dienstleistung sowie über die damit einhergehenden Probleme und Aufgaben, die sich aus dem Angebot einer solchen Dienstleistung für ein Unternehmen ergeben.

33 Vgl. Silvestro, R. et al., Towards a Classification of Service Processes, in: IJoSIM, Vol. 3, No. 3,1992, S. $63 \mathrm{ff}$.

34 Dabei werden die verschiedenen Ausprägungen der einzelnen Dimensionen als Endpunkte eines Kontinuums betrachtet, und es wird somit nicht der Anspruch auf eine klare Klassenabgrenzung erhoben. 


\begin{tabular}{|c|c|c|c|c|c|c|c|}
\hline Unternehmen & * & $\begin{array}{c}\text { Ausrūstungs- } \\
\text { vs. } \\
\text { Personal- } \\
\text { orientierung }\end{array}$ & $\begin{array}{l}\text { Kunden- } \\
\text { kontakt- } \\
\text { zeit }\end{array}$ & $\begin{array}{l}\text { Indivi- } \\
\text { duali- } \\
\text { sierungs- } \\
\text { grad }\end{array}$ & $\begin{array}{c}\text { Auto- } \\
\text { nomie- } \\
\text { grad }\end{array}$ & $\begin{array}{l}\text { Quelle } \\
\text { der } \\
\text { Nutzen- } \\
\text { stiftung }\end{array}$ & $\begin{array}{l}\text { Prozeß- } \\
\text { vs. } \\
\text { Ergebnis- } \\
\text { Orientierung }\end{array}$ \\
\hline $\begin{array}{l}\text { Unternehmens- } \\
\text { beratung }\end{array}$ & 1 & Personal & Hoch & Hoch & Hoch & $\begin{array}{l}\text { Front } \\
\text { Office } \\
\end{array}$ & Prozeß \\
\hline $\begin{array}{l}\text { Markt- } \\
\text { forschung }\end{array}$ & 2 & Personal & Hoch & Hoch & Hoch & $\begin{array}{l}\text { Front } \\
\text { Office }\end{array}$ & Prozeß \\
\hline $\begin{array}{l}\text { Bank } \\
\text { (Geschäfts- } \\
\text { kunden) }\end{array}$ & 3 & Personal & Mittel & Hoch & Hoch & $\begin{array}{c}\text { Front u. } \\
\text { Back Office }\end{array}$ & Mix \\
\hline Hotel & 4 & Mix & Hoch & Mittel & Mittel & $\begin{array}{c}\text { Front u. } \\
\text { Back Office }\end{array}$ & Prozeß \\
\hline $\begin{array}{l}\text { Elektrogerăte- } \\
\text { verleih }\end{array}$ & 5 & Mix & Mittel & Mittel & Mittel & $\begin{array}{c}\text { Front u. } \\
\text { Back Office }\end{array}$ & Mix \\
\hline Einzelhandel & 6 & Mix & Mittel & Mittel & Mittel & $\begin{array}{c}\text { Front u. } \\
\text { Back Office }\end{array}$ & Mix \\
\hline $\begin{array}{l}\text { Bäckereien, } \\
\text { Tabakgeschăfte, } \\
\text { Zeitschriftenhandel }\end{array}$ & 7 & Personal & Niedrig & Niedrig & Niedrig & $\begin{array}{l}\text { Front } \\
\text { Office }\end{array}$ & Ergebnis \\
\hline $\begin{array}{l}\text { Bank } \\
\text { (Privatkunden) }\end{array}$ & 8 & Mix & Niedrig & Mittel & Mittel & $\begin{array}{c}\text { Front u. } \\
\text { Back Office }\end{array}$ & Mix \\
\hline Paketdienst & 9 & Mix & Niedrig & Mittel & Mittel & $\begin{array}{l}\text { Front } \\
\text { Office }\end{array}$ & Ergebnis \\
\hline $\begin{array}{l}\text { Personentransport- } \\
\text { unternehmen }\end{array}$ & 10 & Ausrūstung & Hoch & Niedrig & Niedrig & $\begin{array}{l}\text { Back } \\
\text { Office }\end{array}$ & Ergebnis \\
\hline $\begin{array}{l}\text { Verkehrsdienst- } \\
\text { leistungszentrum }\end{array}$ & 11 & Ausrüstung & Hoch & Niedrig & Niedrig & $\begin{array}{l}\text { Back } \\
\text { Office }\end{array}$ & Ergebnis \\
\hline
\end{tabular}

* Ranking nach Kundenvolumen pro Tag (aufsteigend)



\section{Abb. 1: Klassifizierung von Dienstleistungen}

(Quelle: In Anlehnung an Silvestro, R. et al., Towards a Classification of Service Processes, a.a.O., S. 69 ff.) 
Dem obigen Schema folgend zeichnen sich Verkehrsdienstleistungen, die den Schwerpunkt der vorliegenden Arbeit bilden, durch eine hohe Intensität ausrüstungs- und einrichtungsbezogener Faktoren aus ${ }^{35}$ Hier sind beispielsweise die Bahnhöfe oder Flughäfen sowie das Flug- bzw. Zugmaterial zu nennen. Ebenso wird das Ausmaß der Kundenkontaktzeit als vergleichsweise hoch eingestuft, da die Kunden häufig mehrere Stunden in Kontakt mit dem Anbieter stehen. Dagegen werden die Möglichkeiten einer individuellen Dienstleistungserstellung und der Autonomiegrad des Kundenkontaktpersonals als eher gering eingestuft. So besteht z.B. zumindest in kurzfristiger Hinsicht keine Möglichkeit, Verbindungen über das aktuelle Streckennetz hinaus anzubieten. Der primäre Ursprung der Nutzenstiftung wird dem „Back Office“-Bereich zugewiesen und der Dienstleistung eine hauptsächlich ergebnisorientierte Ausrichtung zugeordnet. ${ }^{36}$

Die oben angeführten Merkmale aufgreifend wurde von den Autoren der Versuch unternommen, diese einem Maß gegenüberzustellen, das dem Produktionsvolumen von Herstellerunternehmen nahekommt. Hierzu wurde als Maßstab die Anzahl an Kunden pro Tag und Dienstleistung gewählt, um somit die Möglichkeit zu schaffen, das Angebot unterschiedlicher Dienstleistungsunternehmen zu Grundtypen zusammenzufassen und eine Integration bisher getrennt betrachteter Systematisierungsansätze zu erreichen.

In Anlehnung an eine Terminologie von LOVELOCK identifizieren die Autoren drei Dienstleistungstypen. ${ }^{37}$ Während "Professional Services" personalintensive und kundenindividuelle Leistungen (z.B. Unternehmensberatungen) umfassen, sind "Mass Services" durch einen geringen Grad an Individualität und eine primär aus dem „Back Office“-Bereich stammende Nutzenstiftung gekennzeichnet. „Service Shops" (z.B. Hotels) bilden einen Mittelpol zwischen diesen beiden Extremtypen. Verkehrsdienstleistungen werden dem Dienstleistungstyp "Mass Services" subsumiert, wenngleich bereits die Zuordnung im Hinblick auf die Kundenkontaktzeit als nur bedingt gelungen anzusehen ist (vgl. Abbildung 1). Insbesondere aber die

Dabei liegt der Schwerpunkt dieser Arbeit auf personenbezogenen Verkehrsdienstleistungen, also auf dem Angebot von Personentransportunternehmen.

Eine detaillierte Analyse der Besonderheiten von Verkehrsdienstleistungen erfolgt in Kap. B 1.22 dieser Arbeit.

Die ursprünglich von Lovelock vorgeschlagene Klassifikation enthallt als vierten Dienstleistungstypen die "Service Factory". Vgl. Lovelock, Ch.H., Classifying Services to Gain Strategic Marketing Insights, a.a.O., S. 9 ff. Nach Ansicht von Silvestro et al. fallen serviceorientierte Herstellerunternehmen, also Unternehmen, die keine "reinen" Dienstleistungsunternehmen darstellen, unter diese Rubrik. Vgl. Silvestro, R. et al., Towards a Classification of Service Processes, a.a.O., S. 74. 
rein ergebnisorientierte Sichtweise von Verkehrsdienstleistungen gilt es, im folgenden kritisch zu hinterfragen.

So haben die bisherigen Ausführungen gezeigt, daß für die Analyse von Dienstleistungen nicht nur das Ergebnis der Leistungserstellung, sondern auch der Leistungserstellungsproze $B$ und die damit einhergehenden Leistungspotentiale eine zentrale Stellung einnehmen. War in der Vergangenheit noch eine deutlich auf das Ergebnis verengte Sichtweise bei Unternehmen festzustellen, ${ }^{38}$ so zeugen die zahlreichen Bemühungen im Rahmen von Reengineering-Programmen von der zunehmenden Einsicht, die Erstellung von Produkten und Dienstleistungen als Prozeß zu interpretieren. ${ }^{39}$ Dabei ist jedoch festzustellen, daß solche Maßnahmen in erster Linie auf die Optimierung innerbetrieblicher Prozesse abzielen.

Die nähere Betrachtung des Prozeßbegriffes zeigt jedoch, daß eine solche, auf interne Tätigkeiten des Unternehmens ausgerichtete Sichtweise insbesondere im Dienstleistungsbereich zu kurz greift. Die Definition eines Prozesses als „Zusammenwirken von Menschen, Maschinen, Material und Verfahren, das darauf ausgerichtet ist, eine bestimmte Dienstleistung zu erbringen ...", 40 verdeutlicht, daß neben den unternehmensbezogenen Dienstleistungserstellungsprozeß der Prozeß des kundenseitigen Dienstleistungserlebens tritt. Während die prozeßorientierte Analyse des unternehmerischen Erstellungsprozesses in den Vordergrund gerückt ist, wird der Ermittlung und Erforschung des kundenseitigen Nutzungsprozesses ${ }^{41}$ im Rahmen des Dienstleistungskonsums jedoch deutlich weniger Aufmerksamkeit gewidmet. ${ }^{42}$ Obwohl Unternehmens- und Kundenprozeß

Vgl. Schmitz, G., Qualitätsmanagement im Privatkundengeschäft von Banken: Konzeption und aufbauorganisatorische Verankerung, a.a.O., S. 38; Büker, B., Qualitătsbeurteilung investiver Dienstleistungen: Operationalisierungsansätze an einem Beispiel zentraler EDV-Dienste, a.a.O., S. 31 .

Vgl. Muffatto, M., Panizzolo, R., A Process-Based View for Customer Satisfaction, in: IJoQRM, Vol. 12, No. 9, 1995, S. 155; Norling, P., Service Design - A Frame of Reference and Some Empirical Results, in: QUiS 3: Quality in Services Conference - Proceedings, Scheuing, E.E. et al. (Hrsg.), New York 1994, S. 361.

Haist, F., Fromm, H., Qualităt im Unternehmen: Prinzipien - Methoden - Techniken, 2. Aufl., München, Wien 1991, S. 93.

41 Stauss bezeichnet den unternehmerischen Erstellungsprozeß als Unternehmensprozeß, während er unter dem Kundenprozeß den kundenseitigen Nutzungsprozeß versteht. Vgl. Stauss, B., Kundenprozeßorientiertes Qualitätsmanagement im Dienstleistungsbereich, a.a.O., S. 27.

Vgl. Gummesson, E., Kingman-Brundage, J., Service Design and Quality: Applying Service Blueprinting and Service Mapping to Railroad Services, in: Quality Management in Services, Kunst, P., Lemmink, J. (Hrsg.), Assen 1992, S. 103; Voss, C.A., Where should Service Quality be measured? Conclusions from a Process Based Model, in: QUiS 2, Quality in Service Conference - Selected Papers, Scheuing, E., Gummesson, E., Little, C.H. (Hrsg.), New York 1992, S. 30. 
teilweise zeitgleich ablaufen und selbstverständlich einer Abstimmung bedürfen, wird häufig nicht erkannt, daß diese sich in ihrem Charakter nachhaltig unterscheiden. ${ }^{43}$ So beinhaltet der Unternehmensprozeß einerseits Tätigkeiten, die für den Kunden nicht sichtbar sind. Andererseits enthält der Kundenprozeß Aktivitäten, die über den Unternehmensprozeß hinausgehen. So kommt ein Kunde nicht mit allen Tätigkeiten und Teilprozessen des Unternehmens in Kontakt und nimmt diese entsprechend nicht bzw. höchstens deren Ergebnis wahr. Ebenso kann der kundenseitig wahrgenommene Prozeß auch Elemente enthalten, die sich dem eigentlichen Verantwortungs- und Kontrollbereich des Anbieters entziehen. Das Beispiel einer Flugreise zeigt, daß ein Kunde bereits vor Inanspruchnahme der Kerndienstleistung, nämlich der Beförderung von $A$ nach $B$, mit einer großen Zahl von Komponenten einer Reise in Kontakt kommt, die einen nachhaltigen Einfluß auf die Wahrnehmung folgender Abschnitte und der gesamten Dienstleistung ausüben können. Der Kunde muß sich zunächst um sein Flugticket kümmern, dann seinen Transfer zum Flughafen organisieren und im Flughafen für die Aufgabe seines Gepäcks sorgen. Werden in dieser Zeit negative Erfahrungen gemacht, kann dies die Qualitätsbeurteilung nachteilig beeinflussen. Ebenso gilt es Kontakte zu berücksichtigen, die nach der Erstellung der Kerndienstleistung die Wahrnehmung des Kunden beeinflussen können. So kann eine verzögerte Abfertigung durch Zollbeamte oder die Nichtverfügbarkeit von öffentlichen Verkehrsmitteln den Gesamteindruck verschlechtern.

Das als für Dienstleistungen konstitutiv erachtete Merkmal „Integration des externen Faktors“44 bedingt, daß eine Dienstleistung meist nur dann erstellt werden kann, wenn der Kunde sich oder eines seiner Güter in den Dienstleistungserstellungsprozeß einbringt. Der Kunde bzw. sein Gut stellen somit selbst Prozeßelemente dar. Insbesondere im Fall personenbezogener Dienstleistungen ist davon auszugehen, daß nicht nur das Ergebnis, sondern auch der Prozeßcharakter einer Dienstleistung entsprechend wahrgenommen wird. "The process, as described by the customer, may well differ greatly from that perceived by the firm. “45 Damit kommt aber neben der Betrachtung des Unternehmensprozesses der Analyse des Kundenprozesses eine zentrale Bedeutung zu, da die Qualitäts-

Vgl. Hinterhuber, H.H., Handlbauer, G., Matzler, K., Kundenzufriedenheit durch Kernkompetenzen: eigene Potentiale erkennen - entwickeln - umsetzen, München, Wien 1997, S. 69.

Vgl. z.B. Meyer, A., Dienstleistungsmarketing: Erkenntnisse und praktische Beispiele, 6. Aufl., München 1994, S. $17 \mathrm{ff}$.

45 Bateson, J.E.G., Managing Services Marketing: Text and Readings, 3. Aufl., Fort Worth u.a. 1995, S. 214. 
beurteilung und somit auch die Entstehung von Zufriedenheit mit einer Dienstleistung im Rahmen des Nutzungsprozesses stattfindet. ${ }^{46}$

Die Interaktionen ${ }^{47}$ des Kunden mit dem Dienstleistungsanbieter und die damit verbundenen Prozesse können in Art und Intensität sehr unterschiedlich ausfallen. So unterscheidet LOVELOCK zwischen vier verschiedenen Prozeßarten, die auf einer Differenzierung nach berührbaren und unberührbaren Prozessen sowie nach dem Personen- bzw. Objektbezug der Dienstleistung beruhen (vgl. Abbildung 2). ${ }^{48}$

Während für die Erstellung z.B. von personenbezogenen Verkehrsdienstleistungen die dauerhafte physische Präsenz des Kunden zwingend erforderlich ist („People Processing“), reicht für die Inanspruchnahme von z.B. Reparaturdienstleistungen die Abgabe und das Abholen des zu reparierenden Objektes aus („Possession Processing“). Die Prozeßart „Mental Stimulus Processing“ zielt auf Dienstleistungen $a b$, die auf den Intellekt des Kunden gerichtet sind und sich durch einen unberührbaren Charakter auszeichnen. Derartige Prozesse machen die geistige, aber nicht zwingend die physische Präsenz des Kunden erforderlich. Dies ist z.B. bei Ausbildungsmaßnahmen der Fall. Der vierten Kategorie werden Dienstleistungsprozesse zugeordnet, die auf unberührbare Vermögensgegenstände abstellen und nur eine zeitweise geistige Präsenz des Kunden erfordern („Information Processing“). Unter diese Rubrik fallen Dienstleistungen wie die von Banken oder Rechtsanwälten. Obwohl diese Prozeßarten nicht als überschneidungsfrei anzusehen sind, läßt sich in den meisten Fällen der Kernprozeß einer Dienstleistung einer dieser vier Prozeßarten zuordnen und damit die grundsätzliche „Natur der Dienstleistung" feststellen. Somit besteht die Möglichkeit, Rückschlüsse auf das notwendige Ausmaß an physischer und/oder geistiger Präsenz

Vgl. Grönroos, Ch., Service Management and Marketing: Managing the Moment of Truth in Service Competition, Lexington, Mass. 1990, S. 201; Klaus, P.G., Auf dem Weg zu einer Betriebswirtschaftslehre der Dienstleistungen: Der Interaktionsansatz, in: DBW, Jg. 44, H. 3 , 1984, S. 474.

Unter Interaktionen sollen in diesem Zusammenhang die "gegenseitigen Abhängigkeiten und Beeinflussungen in menschlichen Austauschbeziehungen" verstanden werden. Jeschke, K., Nachkaufmarketing: Kundenzufriedenheit und Kundenbindung auf Konsumgütermärkten, Frankfurt am Main u.a. 1995, S. 149. Zu einer ausführlichen Diskussion des Interaktionsbegriffes in der Marketingtheorie vgl. Backhaus, K., Investitionsgütermarketing, 4. Aufl., München 1995, S. 107 ff.; Kern, E., Der Interaktionsansatz im Investitionsgütermarketing: Eine konfirmatorische Analyse, Berlin 1990, S. $7 \mathrm{ff}$.

48 Vgl. Lovelock, Ch., Product Plus: How Product + Service $=$ Competitive Advantage, New York u.a. 1994, S. $10 \mathrm{ff}$. 


\begin{tabular}{|c|c|c|}
\hline \multirow{2}{*}{$\begin{array}{l}\text { Charakter des } \\
\text { Dienstleistungs- } \\
\text { Prozesses }\end{array}$} & \multicolumn{2}{|c|}{ Empfänger der Dienstleistung } \\
\hline & Person & Objekt \\
\hline berührbar & $\begin{array}{l}\text { People Processing: } \\
\begin{aligned} \text { z.B.: - } & \text { personenbezogene } \\
& \text { Verkehrsdienst- } \\
& \text { leistungen } \\
\text { - } & \text { Gesundheitswesen }\end{aligned}\end{array}$ & $\begin{array}{l}\text { Possession Processing: } \\
\begin{aligned} \text { z.B.: - } & \text { Fracht- / Transport- } \\
& \text { dienstleistungen } \\
\text { - } & \text { Reparaturdienst- } \\
& \text { leistungen }\end{aligned}\end{array}$ \\
\hline unberührbar & $\begin{array}{l}\text { Mental Stimulus Processing: } \\
\begin{aligned} \text { z.B.: } & \text { - Ausbildung } \\
& \text { - Rundfunk / TV }\end{aligned}\end{array}$ & $\begin{array}{l}\text { Information Processing: } \\
\begin{aligned} \text { z.B.: - Rechtsberatung } \\
\text { - Bankwesen }\end{aligned}\end{array}$ \\
\hline
\end{tabular}

Abb. 2: Charakter des Dienstleistungsprozesses

(Quelle: Lovelock, Ch., Product Plus: How Product + Service = Competitive Advantage, a.a.O., S. 11)

des Kunden bei der Dienstleistungserstellung zu ziehen und zu überprüfen, inwieweit Ansatzpunkte bestehen, z.B. mit Hilfe von Kommunikationstechnologien die geistige Präsenz eines Kunden sicherzustellen und gleichzeitig die Notwendigkeit der physischen Präsenz aufzuheben.

Für die im Rahmen der Dienstleistungserstellung und -nutzung stattfindenden Interaktionen zwischen Nachfrager und Anbieter werden in der Literatur unterschiedliche Bezeichnungen genannt. Während sich in der anglo-amerikanischen Literatur Begriffe wie "Moments of Truth" oder „Service Encounter" durchgesetzt haben, finden diese ihr deutschsprachiges Pendant in Ausdrücken wie „Augenblicke der Wahrheit" oder auch „Kontaktpunkt" bzw. „Kundenkontaktpunkt“. ${ }^{49}$

Vgl. Albrecht, K., Zemke, R., Service-Strategien, Hamburg u.a. 1987, S. 31 f.; Shostack, G.L., Planning the Service Encounter, in: The Service Encounter: Managing Employee/Customer Interaction in Service Businesses, Czepiel, J.A., Solomon, M.R., Surprenant, C.F. (Hrsg.), Lexington 1985, S. 243 ff.; Stauss, B., "Augenblicke der Wahrheit" in der Dienstleistungserstellung, in: Dienstleistungsqualităt: Konzepte, Methoden, Erfahrungen, Bruhn, M., Stauss, B. (Hrsg.), 2. Aufl., Wiesbaden 1995, S. 382. 
Eine nähere Betrachtung der Definitionen dieser Begriffe zeigt die Notwendigkeit der Konkretisierung der innen zugrunde liegenden Interaktionsperioden. ${ }^{50}$ Die von SHOSTACK ${ }^{51}$ gewählte Definition des "Service Encounter" als "a period of time during which a customer directly interacts with a service" läßt ebenso wie die Begriffsauslegung von ALBRECHT ${ }^{52}$ für die "Moments of Truth“, die er mit "any episode in which the customer comes into contact with any aspect of the organisation and gets an impression of the quality of its service" umschreibt, eine sehr unterschiedliche Auslegung des Episodenbegriffes nach Art und Inhalt $\mathrm{zu}^{53}$ Es wird nicht deutlich, ob unter einer Episode das einfache oder mehrmalige Erbringen einer Dienstleistung oder gar nur ein Teilprozeß hieraus verstanden werden soll und welcher Art diese Episoden sind. STAUSS konkretisiert den Begriff der "Augenblicke der Wahrheit" dahingehend, daß er unter diesen personal- und nicht personalbezogene sowie gewöhnliche und außergewöhnliche Kontakterlebnisse versteht. ${ }^{54}$ Personalbezogene Kontaktpunkte umfassen die Interaktionen des Kunden mit dem Personal des Dienstleisters während der Erstellung der Dienstleistung. Nicht personalbezogene Kontaktpunkte beinhalten dagegen die tangiblen Elemente des Dienstleistungsumfeldes (z.B. der Bahnhof oder das Zugmaterial). Ebenso können die Interaktionen mit anderen Nachfragern zu den nicht personalbezogenen Kontaktpunkten gezählt werden. Während gewöhnliche Kontakterlebnisse das übliche Erleben von Kontaktsituationen abbilden, stellen ungewöhnliche Kontakterlebnisse auf die von Kunden als besonders (positiv oder negativ) empfundenen „kritischen Augenblicke der Wahrheit" ab.

Je größer die Zahl an Interaktionen während des Dienstleistungskonsums ist, desto deutlicher wird der Prozeßcharakter des Erlebens einer Dienstleistung. Die Abfolge von Interaktionen, die während des Konsums einer Dienstleistung zu beobachten ist, wird in diesem Zusammenhang als Dienstleistungstransaktion

Vgl. Stauss, B., Seidel, W., Prozessuale Zufriedenheitsermittlung und Zufriedenheitsdynamik bei Dienstleistungen, in: Kundenzufriedenheit: Konzepte, Methoden, Erfahrungen, Simon, $H$., Homburg, Ch. (Hrsg.), Wiesbaden 1995, S. 185.

Shostack, G.L., Planning the Service Encounter, a.a.O., S. 243.

52 Albrecht, K., At America's Service - How Corporations can Revolutionize the Way they Treat their Customers, Homewood 1988, S. 26.

53 Im Investitionsgütermarketing werden unter einer Episode alle mit der Anbahnung, Vereinbarung und Realisation einer Investitionsgütertransaktion verbundenen Aktivitäten und Interaktionen verstanden. Vgl. Kirsch, W., Kutschker, M., Das Marketing von Investitionsgütern Theoretische und empirische Perspektiven, Wiesbaden 1978, S. 34 ff.; Kirsch, W., Kutschker, M., Lutschewitz, H., Ansătze und Entwicklungstendenzen im Investitionsgütermarketing, 2. Aufl., Stuttgart 1980, S. 5 ff.

54 Vgl. hierzu und im folgenden Stauss, B., "Augenblicke der Wahrheit" in der Dienstleistungserstellung, a.a.O., S. $382 \mathrm{ff}$. 
bezeichnet und stellt eine aus Sicht des Kunden spezifische und vollständige Dienstleistungsnutzung mit fixierbarem Beginn und Ende dar. ${ }^{55}$

Ähnlich wie im Konzept des Geschäftsprozeß-Managements ist es daher sinnvoll, den aus Kundensicht wahrgenommenen Gesamtprozeß einer Dienstleistung in Teil- oder Subprozesse zu zerlegen und diese in weitere Subprozesse aufzuspalten, bis eine weitere Zerlegung nicht mehr möglich oder sinnvoll erscheint. Dieses Vorgehen führt zu einer „Hierarchie von Prozessen“, deren verschiedene Ebenen sich durch einen unterschiedlich hohen Detaillierungsgrad auszeichnen. ${ }^{56}$ Für eine Analyse des Kundenprozesses hat diese Prozeßzerlegung zwingend aus Sicht des Kunden zu erfolgen und erfordert daher eine möglichst umfassende Identifikation und Dokumentation der von einem Kunden während der Inanspruchnahme einer Dienstleistung wahrgenommenen Konsumphasen und Kontaktpunkte. ${ }^{57}$ Die grundsätzliche Annahme einer solchen Zerlegung des Kundenprozesses besteht darin, daß die Einteilung in zeitlich abgeschlossene Leistungssequenzen sachlogisch vertretbar ist und die einzelnen Phasen auch vom Kunden als solche erlebt und verarbeitet werden. ${ }^{58}$ Eine Reihe von Autoren vertritt dabei die Ansicht, daß besonders im Dienstleistungsbereich diese Voraussetzungen als erfüllt anzusehen sind. ${ }^{59}$ Die theoretischen Grundlagen einer solchen Prozeßzerlegung bilden insbesondere das „Konzept der episodischen Informationsverarbeitung"60 sowie das maßgeblich von SOLOMON et al. und SMITH/HOUSTON entwickelte "Service Script Concept" bzw. die Rollentheorie. ${ }^{61}$ Ausgangspunkt

Vgl. Stauss, B., Seidel, W., Prozessuale Zufriedenheitsermittlung und Zufriedenheitsdynamik bei Dienstleistungen, a.a.O., S. 186.

56 Vgl. Haist, F., Fromm, H., Qualităt im Unternehmen: Prinzipien - Methoden - Techniken, a.a.O. S. 96.; Schmitz, G., Qualitătsmanagement im Privatkundengeschäft von Banken: Konzeption und aufbauorganisatorische Verankerung, a.a.O., S. 117.

57 Eine Darstellung der in diesem Zusammenhang relevanten theoretischen Ansätze und die Analyse geeigneter Techniken zur Strukturierung und Visualisierung des Kundenprozesses erfolgen in Kap. B 2.2 bzw. B 3.1 dieser Arbeit.

Vgl. Meyer, A., Westerbarkey, P., Zufriedenheit von Hotelgästen - Entwurf eines selbstregulierenden Systems, in: Kundenzufriedenheit: Konzepte, Methoden, Erfahrungen, Simon, H., Homburg, Ch. (Hrsg.), Wiesbaden 1995, S. 389 f.; Jeschke, K., Nachkaufmarketing: Kundenzufriedenheit und Kundenbindung auf Konsumgütermärkten, a.a.O., S. 29.

Vgl. z.B. Güthoff, J., Qualităt komplexer Dienstleistungen: Konzeption und empirische Analyse der Wahrnehmungsdimensionen, Wiesbaden 1995, S. 34; Geva, A., Goldman, A., Changes in the Perception of a Service during its Consumption: A Case of Organised Tours, in: EJoM, Vol. 23, No. 12, 1989, S. 45 ff.; Hentschel, B., Dienstleistungsqualităt aus Kundensicht: Vom merkmals- zum ereignisorientierten Ansatz, Wiesbaden 1992, S. $158 \mathrm{ff}$; vgl. auch die Ausführungen in Kap. B 2.3 dieser Arbeit.

Vgl. Hentschel, B., Dienstleistungsqualităt aus Kundensicht: Vom merkmals- zum ereignisorientierten Ansatz, a.a.O., S. 158 und die dort angegebenen Literaturquellen.

61 Vgl. Solomon, M.R., Surprenant, C., Czepiel, J.A., Gutman, E.G., A Role Theory Perspective on Dyadic Interactions: The Service Encounter, in: JoM, Vol. 49, Winter 1985, S. 99 ff.; Smith,

(Fortsetzung der Fußnote auf der nächsten Seite) 
dieser Ansätze ist die psychologische Fundierung der Annahme, daß Dienstleistungen prozeßbezogen wahrgenommen und kognitiv (gedanklich) verarbeitet werden. ${ }^{62}$

Wird eine Phase als „zeitlicher Abschnitt eines übergeordneten Gesamtprozesses" interpretiert, ${ }^{63}$ so ist der interpretatorische Spielraum im Hinblick auf die Festlegung der Länge und Anzahl einzelner Phasen offenkundig. Als ein erstes Grobraster kann eine Unterscheidung in Vornutzungsphase, Nutzungsphase und Nachnutzungsphase dienen und damit der häufig anzutreffenden verkürzten Sichtweise von Unternehmen entgegenwirken, sich auf den Kern ihrer Dienstleistung zu beschränken und Erfahrungen, die der Kunde im Vorfeld und nach dem Dienstleistungskonsum macht, außer acht zu lassen. ${ }^{64}$

Im Mittelpunkt der vorliegenden Untersuchung stehen personenbezogene Verkehrsdienstleistungen und hier insbesondere die Personenbeförderung im innerdeutschen schienengebundenen Fernverkehr. Es stellt sich folgerichtig die Frage, inwieweit eine Übertragung des Prozeßansatzes auf diese Dienstleistungen sinnvoll und notwendig bzw. möglich ist. Zunächst erscheint eine erste Unterteilung des Kundenprozesses in die Bereiche der Vor-Reise-, Reise- und Nach-Reisephase dem Charakter des Konsums dieser Dienstleistungsart zu entsprechen. Diese Argumentation aufgreifend kommt JESCHKE für den Bereich des Schienenpersonenverkehrs zu folgendem Ergebnis: „Die Wahrnehmung der Leistungsqualität der personenbezogenen Dienstleistung wird seitens der Konsumenten durch das Zusammenwirken der drei Dienstleistungsebenen Potential, Prozeß und Ergebnis determiniert. Der eigentliche Transport der Kunden steht nicht mehr im Mittelpunkt der Leistungserstellung. Er ist nur noch Teilelement eines umfangreichen Beziehungsgeflechts zwischen Service, Komfort, Zuver-

R.A., Houston, M.J., Script-Based Evaluations of Satisfaction with Services, in: Emerging Perspectives on Services Marketing, Proceedings Series, AMA, Berry, L.L., Shostack, G.L., Upah, G.D. (Hrsg.), Chicago 1983, S. 59 ff.

62 Zu einer ausführlichen Diskussion dieser Konzepte vgl. Kap. B 2.3 dieser Arbeit.

63 Jeschke, K., Nachkaufmarketing: Kundenzufriedenheit und Kundenbindung auf Konsumgütermärkten, a.a.O., S. 25.

64 Wăhrend Fisk eine Einteilung in "Preconsumption", "Consumption" und "Postconsumption" wăhlt und Walker von "Peripheral Service Performance", die vor und nach der "Core Service Performance" stattfindet, spricht, unterscheidet Grönroos zwischen „Joining Phase", "Intensive Consumption" und "Detachment Phase". Vgl. Fisk, R.P., Toward a Consumption/Evaluation Process Model for Services, in: Marketing of Services, Proceedings Series, AMA, Donnelly, J.H., George, W.R. (Hrsg.), Chicago 1981, S. 192; Grönroos, Ch., Service Management and Marketing: Managing the Moment of Truth in Service Competition, a.a.O., S. 206; Walker, J.L., Service Encounter Satisfaction: Conceptualized, in: JoSM, Vol. 9, No. 1, 1995, S. 7. 
lässigkeit und technischer Leistungsfähigkeit im Bahnhof und Zug sowie vor, während und nach einer Bahnreise."65

In Abhängigkeit vom Untersuchungszweck kann jedoch der Wunsch nach einer differenzierten Analyse des Kundenprozesses oder eines Ausschnittes hieraus bestehen. $\mathrm{Zu}$ einer Systematisierung von Kundenprozessen kann zum einen auf den bereits erwähnten Begriff der Dienstleistungstransaktion zurückgegriffen werden. Darüber hinaus erscheint im Rahmen des Aufbaus einer Prozeßhierarchie eine Unterscheidung in Dienstleistungsepisode und Dienstleistungskontakt bzw. -punkt sinnvoll (vgl. Abbildung 3). ${ }^{66}$

Eine erste Aufteilung einer Dienstleistungstransaktion in sequentielle Teilprozesse führt zu Dienstleistungsepisoden, die abgrenzbare Teilleistungen einer Dienstleistung darstellen, grundsätzlich aber vom Kunden nicht als eigenständige Dienstleistung wahrgenommenen werden. STAUSSMEINLICH verdeutlichen dieses Vorgehen am Beispiel einer Clubreise, bei der sie auf einer ersten Prozeßebene zwischen den Episoden Informationsbeschaffung, Buchung, Anreise bzw. Empfang der Gäste, Clubaufenthalt und Abreise unterscheiden. ${ }^{67}$ Eine Untergliederung einer solchen Dienstleistungsepisode führt zu der Ermittlung von Dienstleistungskontakten, die sich im obigen Beispiel in einer Zerlegung der Episode „Empfang der Gäste“ in Kontaktpunkte wie Empfang am Flughafen, Transfer

Jeschke, K., Institutionalisierungsformen der Kundenorientierung in offentlichen Unternehmen am Beispiel der Deutschen Bundesbahn, Lehr- und Forschungsberichte des Lehrstuhls Markt und Konsum der Universităt Hannover, Nr. 12, Hannover 1988, S. 24 f.; vgl. auch Klein, H., Deutsche Bundesbahn - Dienstleistung als Marke, in: FAZ vom 26.5.1987, S. B 18.

In diesem Sinne formuliert Jeschke für das Leistungsangebot der Deutschen Bundesbahn: „Der Personennah- und Personenfernverkehr der DB (Deutschen Bundesbahn, der Verf.) "sowie sämtliche sich daran anknüpfende Serviceleistungen sind als spezifische, auf Personen oder Sachen bezogene Dienstleistungen aufzufassen. Dabei ist im Rahmen der personenbezogenen Dienstleistungen vor allem die Komplexitat des Potentials sowie das hohe Maß an persönlicher Interaktion im Erstellungsprozeß von Bedeutung." Jeschke, K., Institutionalisierungsformen der Kundenorientierung in offentlichen Unternehmen am Beispiel der Deutschen Bundesbahn, a.a.O., S. 24.

Vgl. Stauss, B., Seidel, W., Prozessuale Zufriedenheitsermittlung und Zufriedenheitsdynamik bei Dienstleistungen, a.a.O., S. $186 \mathrm{f}$. In der Bezeichnung Kontaktpunkt manifestiert sich der lokalisierbare Ort eines Dienstleistungskontaktes. Woodside et al. unterscheiden in diesem Zusammenhang zwischen dem "Service Encounter", der dem Begriff der Dienstleistungstransaktion entspricht, sowie zwischen "Service Acts“ und „Service Events“. Vgl. Woodside, A.G., Frey, L.L., Daly, R.T., Linking Service Quality, Customer Satisfaction, and Behavioral Intention, in: Journal of Health Care Marketing, Vol. 9, No. 4, 1989, S. 6 f.

67 Vgl. Stauss, B., Weinlich, B., Die Sequentielle Ereignismethode - ein Instrument der prozeßorientierten Messung von Dienstleistungsqualităt, in: Der Markt, Jg. 35, H. 1, 1996, S. 52 f. 


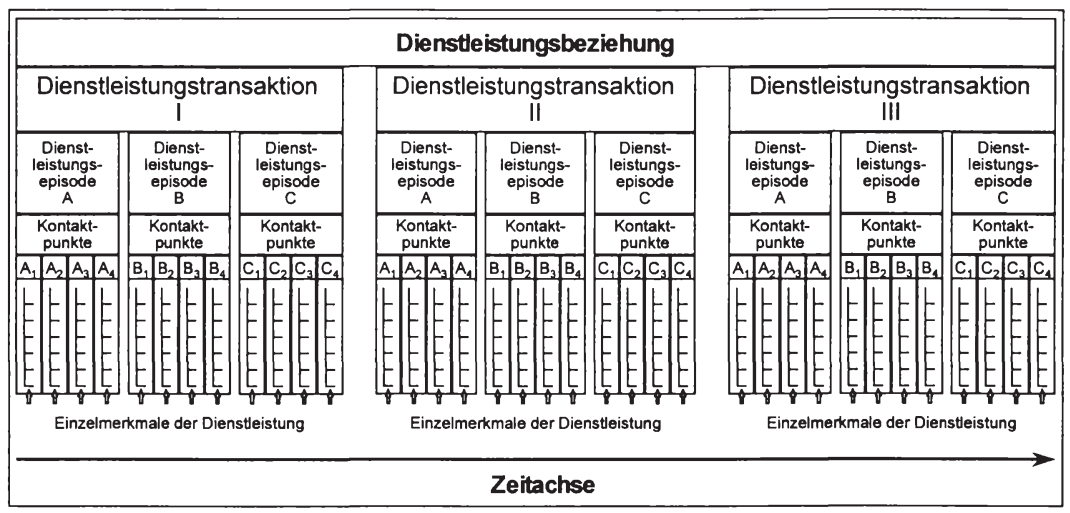

Abb. 3: Hierarchie von Dienstleistungsprozessen

(Quelle: In Anlehnung an Woodside, A.G. et al., Linking Service Quality, Customer Satisfaction, and Behavioral Intention, a.a.O., S. 7)

zur Clubanlage, Ankunft im Club, Einchecken sowie Aufsuchen des Zimmers darstellt. Wenngleich eine weitere Untergliederung eines Kundenkontaktpunktes nach weiteren Merkmalen grundsätzlich denkbar ist, soll hier die Ermittlung eines solchen Kontaktpunktes als „kleinste“ Einheit eines Kundenprozesses verstanden werden, die sich ihrerseits aus verschiedenen Merkmalen einer Dienstleistung zusammensetzt. An dem genannten Beispiel wird gleichzeitig deutlich, daß neben der Ermittlung der Episoden und Kontaktpunkte die Analyse der Reihenfolge dieser Prozeßelemente von zentraler Bedeutung ist, da erst deren jeweilige zeitliche Abfolge den Kundenprozeß konstituiert. $^{68}$

Während der Gegenstand der bisherigen Ausführungen in der Analyse einer einzelnen Dienstleistungstransaktion bestand, zeigt die aktuelle Diskussion um Konzepte des Beziehungsmarketing, daß auch die Untersuchung des Beziehungsgeflechtes aufeinanderfolgender Transaktionen einen zentralen Stellenwert für die marktorientierte Unternehmensführung einnimmt. Die Summe der bisherigen Erfahrungen mit einem Dienstleistungsanbieter und deren Beurteilung, die sich mit dem Begriff der wahrgenommenen Dienstleistungsbeziehung umschreiben läßt, kann sowohl die Wahrnehmung der aktuellen Dienstleistungstransaktion und die bei Dienstleistungen, a.a.O., S. 187. 
hieraus resultierende Kundenzufriedenheit als auch das künftige Verhalten eines Kunden gegenüber diesem Anbieter in entscheidender Weise beeinflussen. ${ }^{69}$

Daher gilt es, bei Kundenzufriedenheitsuntersuchungen auch und insbesondere bei der Analyse der kundenseitigen Wahrnehmung einer Verkehrsdienstleistung sowohl auf der Transaktions- als auch auf der Beziehungsebene eine entsprechende prozeßorientierte Sichtweise einzunehmen. ${ }^{70}$ Voraussetzung hierfür ist eine Abgrenzung und Erläuterung des Begriffsverständnisses der Kundenzufriedenheit mit Dienstleistungen.

\section{Begriff der Kundenzufriedenheit mit Dienstleistungen}

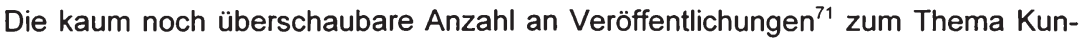
denzufriedenheit belegt zum einem den hohen Stellenwert, der diesem Forschungszweig beigemessen wird, dokumentiert aber auf der anderen Seite auch, daß bis heute keine Einigkeit über das konzeptionelle Verständnis dieses Konstrukts erzielt werden konnte. So ist eine ebenso intensiv wie kontrovers geführte Diskussion um die Abgrenzung der Kundenzufriedenheit von anderen Konstrukten wie der Dienstleistungsqualität und dem Konstrukt der Einstellung zu beobachten. "In literature these concepts are treated in different ways which has lead to confusion and even to obstruction of ... knowledge. "72 Eine wesentliche Ursache für diese Konfusion liegt in der lange Zeit voneinander unabhängigen, nicht aufeinander abgestimmten Entwicklung der Forschung um diese Konzepte. ${ }^{73}$ Erst

Vgl. Stauss, B., Seidel, W., Prozessuale Zufriedenheitsermittlung und Zufriedenheitsdynamik bei Dienstleistungen, a.a.O., S. 188.

Vgl. Bitner, M.J., Hubbert, A.R., Encounter Satisfaction Versus Overall Satisfaction Versus Quality: The Customer's Voice, in: Service Quality: New Directions in Theory and Practice, Rust, R.T., Oliver, R.L. (Hrsg.), Thousand Oaks u.a. 1994, S. 73; Bruhn, M., Bunge, B., Beziehungsmarketing: Neuorientierung für Marketingwissenschaft und -praxis?, a.a.O., S. 49 und die dort angegebene Literatur.

71 Während Bruhn Mitte der achtziger Jahre bereits von ca. 6.000 Arbeiten vornehmlich zum Thema Arbeitszufriedenheit sprach, die Forschung im Bereich der Kundenzufriedenheit aber als vergleichsweise jungen Forschungszweig ansah, schätzen Peterson und Wilson Anfang der neunziger Jahre die Anzahl an Veröffentlichungen allein für die anglo-amerikanische Literatur auf über 15.000 Artikel und Beiträge zum Thema Kundenzufriedenheit. Vgl. Bruhn, M., Marketing und Konsumentenzufriedenheit, in: WISU, Jg. 14, H. 6, 1985, S. 300; Peterson, R.A., Wilson, W.R., Measuring Customer Satisfaction: Fact and Artifact, in: JAMS, Vol. 20, No. 1, 1992, S. 61.

Koelemeijer, K., Roest, H., Verhallen, T., An Integrative Framework of Perceived Service Quality and its Relations to Satisfaction/Dissatisfaction, Attitude and Repurchase Intention, in: Marketing for the New Europe: Dealing with Complexity, Chias, J., Sureda, J. (Hrsg.), Barcelona 1993, S. 696.

73 So konstatiert Liljander einen „surprising lack of interest" der einzelnen Forschungsrichtungen hinsichtlich der jeweils anderen Schulen. Liljander, V., Introducing Deserved Service and

(Fortsetzung der Fußnote auf der nächsten Seite) 
in jüngerer Zeit sind Bemühungen einer gleichzeitigen gedanklichen Auseinandersetzung mit den verschiedenen Konstrukten festzustellen. ${ }^{74}$

Eine Betrachtung der zeitlichen Entwicklung der verschiedenen Forschungsrichtungen zeigt, daß bereits zu Beginn des zwanzigsten Jahrhunderts Einstellungen Bestandteil psychologisch-soziologischer Theorien waren. Mitte der fünfziger Jahre wurden Einstellungen insbesondere in der US-amerikanischen Sozialpsychologie eine zentrale Rolle im Rahmen der empirischen Forschung der Verhaltenswissenschaften beigemessen. Die postulierten Einstellungs-Verhaltensbeziehungen führten dazu, daß Einstellungen einen relativ schnellen Eingang auch in die Marketingforschung fanden. ${ }^{75}$

Die Zufriedenheitsforschung hingegen hat ihre Wurzeln in umfangreichen Untersuchungen zur Arbeitszufriedenheit im Rahmen der Organisationstheorie und -psychologie der sechziger Jahre. ${ }^{76}$ Die Konsumerismusbewegung ${ }^{77}$ in den Vereinigten Staaten führte $\mathrm{zu}$ einer Erweiterung dieser Forschung auf eine gesamtwirtschaftliche Perspektive. Hier stand die allgemeine Versorgungszufriedenheit mit Ge- und Verbrauchsgütern im Mittelpunkt des Interesses. Mitte der siebziger Jahre fand die Erforschung der Zufriedenheit mit konkreten Leistungen bzw. im Falle von Unzufriedenheit die Analyse des Beschwerdeverhaltens und der Beschwerdezufriedenheit des Individuums Kunde als eigenständige Disziplin Eingang in die amerikanische Marketingwissenschaft. ${ }^{78}$ Dem Forschungszweig der sog. CS/D \& CB Forschung ${ }^{79}$ können wiederum unterschiedliche Forschungsrichtungen zugeordnet werden, die sich durch unterschiedliche Betrachtungsebenen und die ihnen zugrunde liegenden Theorien unterscheiden. ${ }^{80}$ Den verschiedenen Ansätzen der Konsumentenzufriedenheit ist

Equity into Service Quality Models, in: Dienstleistungsmarketing: Konzeptionen und Anwendungen, Kleinaltenkamp, M. (Hrsg.), Wiesbaden 1995, S. 143.

Vgl. Westerbarkey, P., Methoden zur Messung und Beeinflussung der Dienstleistungsqualităt: Feedback- und Anreizsysteme in Beherbergungsunternehmen, Wiesbaden 1996, S. 31.

Vgl. Geise, W., Einstellung und Marktverhalten: Analyse der theoretisch-empirischen Bedeutung des Einstellungskonzeptes im Marketing und Entwicklung eines alternativen Forschungsprogramms aus alltagstheoretischer Sicht, Thun, Frankfurt 1984, S. 27.

Vgl. Bruhn, M., Marketing und Konsumentenzufriedenheit, a.a.O., S. 300.

Vgl. Meffert, H., Marketing und Konsumerismus, in: ZfB, Jg. 45, H. 2, 1975, S. 69 ff.

Vgl. Meffert, H., Bruhn, M., Beschwerdeverhalten und Zufriedenheit von Konsumenten, a.a.O., S. $597 \mathrm{ff}$.

CS/D \& CB steht in diesem Zusammenhang für Consumer Satisfaction/Dissatisfaction and Complaining Behavior.

80 Zur zeitlichen und inhaltlichen Entwicklung der verschiedenen Forschungsrichtungen vgl. Riemer, M., Beschwerdemanagement, Frankfurt am Main, New York 1986, S. 47 ff.; Bruhn, M.,

(Fortsetzung der Fußnote auf der nächsten Seite) 
die Auffassung gemeinsam, daß Zufriedenheit als ein nicht beobachtbares Phänomen aufzufassen ist und als intervenierende Variable zwischen Stimuli und Verhaltensreaktionen wirkt. ${ }^{81}$ Damit wurde die Zufriedenheit als ein weiteres theoretisches Konstrukt neben anderen hypothetischen Konstrukten, wie z.B. Einstellungen zur Erklärung des Konsumentenverhaltens, herangezogen.

Die intensive Auseinandersetzung mit dem Zufriedenheitskonstrukt in den Vereinigten Staaten, die mit der Etablierung regelmäßiger Tagungen und eigenständiger Veröffentlichungen einherging, ${ }^{82}$ führte Anfang der achtziger Jahre in Deutschland zu einem verstärkten Interesse an der Zufriedenheitsforschung. Dabei ist festzustellen, daß lange Zeit der Schwerpunkt der Veröffentlichungen zur Zufriedenheitsforschung auf dem Konsumgüterbereich lag. Erst seit Beginn der achtziger Jahre finden die Besonderheiten von Dienstleistungen und Industriegütern explizit Berücksichtigung in der wissenschaftlichen Erforschung der Kundenzufriedenheit. ${ }^{83}$

Während der Begriff der Verbraucherzufriedenheit verstärkt auf den institutionellen Rahmen von Kundenzufriedenheit abzielt und in der Tradition wohlstandsorientierter Ansätze der Zufriedenheitsforschung steht, wurde die Bezeichnung Konsumentenzufriedenheit vor allem im Zusammenhang mit Produkten verwendet. Ist dagegen die Zufriedenheit mit Dienstleistungen Gegenstand des Interesses, wird insbesondere der weiter gefaßte Begriff Kundenzufriedenheit verwendet. ${ }^{84}$ Die beiden letztgenannten Begriffe sind damit dem Bereich der marktorientierten Ansätze der Zufriedenheitsforschung zuzuordnen. ${ }^{85}$

Konsumentenzufriedenheit und Beschwerden: Erklärungsansätze und Ergebnisse einer empirischen Untersuchung in ausgewählten Konsumbereichen, Frankfurt am Main 1982, S. 8 ff.; Schütze, R., Kundenzufriedenheit: After-Sales-Marketing auf industriellen Märkten, a.a.O., S. $120 \mathrm{ff}$.

81 Vgl. Bruhn, M., Marketing und Konsumentenzufriedenheit, a.a.O., S. 301.

82 Hier sei beispielhaft auf das seit 1988 jährlich erscheinende "Journal of Consumer Satisfaction, Dissatisfaction and Complaining Behavior" und weitere Veröffentlichungen der School of Business, Bloomington, verwiesen. Vgl. Woodruff, R.B., Developing and Applying Consumer Satisfaction Knowledge: Implications for Future Research, in: JoCS/D\&CB, Vol. 6, 1993, S. 1.

$83 \mathrm{Vgl}$. Schütze, R., Kundenzufriedenheit: After-Sales-Marketing auf industriellen Märkten, a.a.O., S. 123; Eckert, St., Rentabilitătssteigerung durch Kundenbindung, Hammer 1995, S. 62 f.

84 Zu einer Abgrenzung der Begriffe "Kunde“, "Konsument" und "Verbraucher" vgl. Riemer, M., Beschwerdemanagement, a.a.O., S. $44 \mathrm{ff}$.

$85 \mathrm{Zu}$ der Unterscheidung in wohlstands- und marktorientierte Ansätze vgl. Scherhorn, G., Die Funktionsfähigkeit von Konsumgütermärkten, in: Marktpsychologie als Sozialwissenschaft, Irle, M. (Hrsg.), Göttingen 1983, S. 97. 
In der Literatur ist gelegentlich ein fließender Übergang vom Begriff der Kundenzufriedenheit zu dem Begriff der Dienstleistungsqualität festzustellen. Während die Qualität materieller Güter seit geraumer Zeit Gegenstand intensiver Forschungsarbeiten ist, wurde der Qualität von Dienstleistungen vergleichsweise wenig Aufmerksamkeit gewidmet. Allgemein wird Qualität zunächst in Verbindung mit einem Objekt gesehen und erst dann mit einem Subjekt, das die Qualität wahrnimmt. Vor diesem Hintergrund stellt HENTSCHEL fest, daß im Rahmen der Forschung zum Konsumentenverhalten keine „umfangreiche eigenständige Forschungstradition unter der Überschrift Qualität existiert, sondern in der Vergangenheit das Thema in der Regel als Randbereich der Einstellungsforschung" Berücksichtigung fand. ${ }^{86}$ Mit der zunehmenden Bedeutung, die dem Dienstleistungsbereich attestiert wurde, entstand Anfang der achtziger Jahre eine Diskussion bezüglich der Übertragbarkeit der Erkenntnisse der Qualitätsforschung im warenproduzierenden Gewerbe auf den Dienstleistungssektor. Hier wurde vor allem der Frage nachgegangen, inwieweit die Besonderheiten und Charakteristika von Dienstleistungen eine Anpassung des durch die Forschungen im Konsumgütermarketing geprägten Qualitätsverständnisses erforderlich machen. ${ }^{87}$

Ungeachtet der Unterschiede im Ursprung und der zeitlichen Entwicklung der verschiedenen Forschungsrichtungen werden potentielle Unterschiede und postulierte Kausalzusammenhänge zwischen den verschiedenen Konstrukten sehr unterschiedlich beurteilt. ${ }^{88}$ Solche Betrachtungen werden dabei zusätzlich durch den Umstand erschwert, daß auch innerhalb der einzelnen Disziplinen sehr heterogene Vorstellungen über den jeweiligen Forschungsgegenstand anzutreffen sind.

Die Durchsicht der Forschungsliteratur zeigt dabei insbesondere für die Zufriedenheitsforschung, daß DAY immer noch zuzustimmen ist, wenn er feststellt, daß "no consensus has been achieved with respect to a core theory of satis-

86 Hentschel, B., Dienstleistungsqualităt aus Kundensicht: Vom merkmals- zum ereignisorientierten Ansatz, a.a.O., S. 120.

87 Mittlerweile hat sich eine eigenstăndige Schule der Dienstleistungsqualităt etabliert, die, ăhnlich wie die Zufriedenheitsforschung, von einer Vielzahl an Kongressen und damit einhergehenden Veróffentlichungen begleitet wird. Hier sind z.B. die regelmäßig stattfindenden Tagungen der International Service Quality Association, New York bzw. die hierzu erscheinenden umfangreichen Tagungsberichte „Quality in Services Conference" zu nennen.

88 Eine differenzierte Analyse der Beziehungen der verschiedenen Konstrukte zueinander erfolgt in Kap. B 2.1 dieser Arbeit. 
faction/dissatisfaction." ${ }^{89}$ Vielmehr existiert eine Vielzahl divergierender Zufriedenheitsmodelle mit ebenso vielen Begriffsbestimmungen und damit einhergehenden Ansätzen zur Messung von Kundenzufriedenheit.

Beispielhaft werden hier verschiedene Definitionen angeführt, die verdeutlichen, welch unterschiedliche Sachverhalte unter den Begriff Kundenzufriedenheit subsumiert werden: ${ }^{90}$

- HOWARD/SHETH legen einen Schwerpunkt auf den kognitiven Charakter der Kundenzufriedenheit, wenn sie diese als "the buyer's cognitive state of being adequately or inadequately rewarded in a buying situation for the sacrifices he has undergone" definieren. ${ }^{91}$

- Oliver betont die affektive Komponente der Kundenzufriedenheit, wenn er in Anlehnung an HUNT von einer „evaluation of an emotion" spricht und dementsprechend Kundenzufriedenheit als "summary psychological state resulting when the emotion surrounding disconfirmed expectations is coupled with the consumer's prior feelings about the consumption experience" bezeichnet. $^{92}$

Trotz unterschiedlich vorgenommener Gewichtung besteht mittlerweile in der Wissenschaft weitgehend Einigkeit darin, daß das Konstrukt stets von mehr oder minder starken affektiven Faktoren beeinflußt wird und somit neben kognitiven auch affektive Elemente enthält. ${ }^{93}$

Day, R.L., The Next Step: Commonly Accepted Constructs for Satisfaction, Paper Presented at the Seventh Annual Conference of Consumer Satisfaction/Dissatisfaction and Complaining Behavior, Knoxville 1982, S. 3.

90 Zu einer Übersicht unterschiedlicher Definitionen der Kundenzufriedenheit vgl. u.a. Nader, G. Zufriedenheit mit Finanzdienstleistungen: Erfolgswirksamkeit, Messung, Modellierung, a.a.O., S. $17 \mathrm{ff}$; Korte, Ch., Customer Satisfaction Measurement: Kundenzufriedenheitsmessung als Informationsgrundlage des Hersteller- und Handelsmarketing am Beispiel der Automobilwirtschaft, Frankfurt am Main u.a. 1995, S. 27 f.; Stauss, B., Neuhaus, P., Das Qualitative Zufriedenheitsmodell (QZM), a.a.O., S. $12 \mathrm{ff}$.

Howard, J.H., Sheth, J.N., The Theory of Buyer Behavior, New York 1969, S. 145.

Oliver, R.L., Measurement and Evaluation of Satisfaction Processes in Retail Settings, in: JoR, Vol. 57, No. 3, 1981, S. 27.

Vgl. Homburg, Ch., Rudolph, B., Theoretische Perspektiven zur Kundenzufriedenheit, in: Kundenzufriedenheit: Konzepte, Methoden, Erfahrungen, Simon, H., Homburg, Ch. (Hrsg.), Wiesbaden 1995, S. 31. So kommen auch Mano und Oliver zu dem Ergebnis, daß „..., satisfaction is positioned as a consequence of both affective and cognitive (i.e., utilitarian) judgement, ... ". Mano, H., Oliver, R.L., Assessing the Dimensionality and Structure of the Consumption Experience: Evaluation, Feeling, and Satisfaction, in: JoCR, Vol. 20, December 1993, S. 464. Zu der Diskussion der intentionalen bzw. konativen Komponente von Kundenzufriedenheit im Sinne eines dem Konstrukt inhärenten Verhaltenselementes vgl. Stauss, B., Neuhaus, P., Das Qualitative Zufriedenheitsmodell (QZM), a.a.O., S. 14 f. 
Während in den beiden ersten Definitionen eine mehr ergebnisorientierte Sicht von Kundenzufriedenheit zu erkennen ist, kommt z.B. in der Begriffsauslegung von TSEMILTON eine verstärkt prozeßorientierte Betrachtung von Kundenzufriedenheit zum Ausdruck: "Consumer satisfaction/dissatisfaction (CS/D) can be defined as the consumer's response to the evalution of the perceived discrepancy between prior expectations (or some other norm of performance) and the actual performance of the product as perceived after its consumption." ${ }^{44}$ Definitionen dieser Art betonen den der Zufriedenheitsbildung zugrunde liegenden Bewertungsprozeß und stellen dabei auf das gesamte Konsumerlebnis des Kunden ab. ${ }^{95}$

Wird der Versuch unternommen, die den unterschiedlichen Ansätzen zugrunde liegenden Gemeinsamkeiten herauszufiltern, kann auf einem vergleichsweise hohen Abstraktionsniveau festgestellt werden, daß sich Definitionen der Kundenzufriedenheit in vielen Fällen aus vier zentralen Bausteinen zusammensetzen, wie dies die Definition von KAAS/RUNOW anschaulich zeigt. Hier wird unter Kundenzufriedenheit das „Ergebnis eines psychischen Soll-Ist-Vergleiches über Konsumerlebnisse" verstanden. ${ }^{96}$ Während die Soll-Komponente überwiegend als die Erwartung eines Kunden an ein Produkt bzw. eine Dienstleistung interpretiert wird, bezieht sich die Ist-Komponente auf die subjektiv wahrgenommene Leistung aus Kundensicht. Das Objekt, auf das sich der Soll-Ist-Vergleich bezieht, stellt das Konsumerlebnis dar, und das Ergebnis des Vergleichs spricht schließlich das Konstrukt der Kundenzufriedenheit selbst an. ${ }^{97}$ Dabei wird generell davon ausgegangen, daß sich das globale Zufriedenheitsurteil eines Konsumenten als Ergebnis der Bewertung von einzeln wahrgenommenen Merkmalen eines Sachgutes bzw. einer Dienstleistung ergibt (Multiattributansatz), wobei jedoch die Meinungen über den Aggregationsalgorithmus, der dieser Annahme zugrunde liegt, z.T. deutlich differieren. ${ }^{98}$

94 Tse, D.K., Wilton, P.C., Models of Consumer Satisfaction Formation: An Extension, in: JoMR, Vol. 24, May 1988, S. 204.

95

Vgl. Nader, G., Zufriedenheit mit Finanzdienstleistungen: Erfolgswirksamkeit, Messung, Modellierung, a.a.O., S. 18 und die dort angegebenen Literaturquellen.

96 Kaas, K.P., Runow, H., Wie befriedigend sind die Ergebnisse der Forschung zur Verbraucherzufriedenheit?, in: DBW, Jg. 44, H. 3, 1984, S. 452.

97 Eine ausführliche Betrachtung der verschiedenen Komponenten des Zufriedenheitskonstrukts erfolgt in Kap. B 2.2 dieser Arbeit.

98

Vgl. Korte, Ch., Customer Satisfaction Measurement: Kundenzufriedenheitsmessung als Informationsgrundlage des Hersteller- und Handelsmarketing am Beispiel der Automobilwirtschaft, a.a.O., S. $35 \mathrm{ff}$. und die dort angegebene Literatur. 
Die bisher sehr allgemein gehaltene Sichtweise des Konstruktes veranschaulicht deutlich, daß Kundenzufriedenheit in erster Linie als ein Nachkaufphänomen im Sinne des Ergebnisses einer ex-post-Beurteilung verstanden wird, deren notwendige Voraussetzung in einem konkreten selbsterfahrenen Konsumerlebnis besteht. $^{99}$

Unter Berücksichtigung der Ausführungen zum Prozeßcharakter von Dienstleistungen wird jedoch die Notwendigkeit deutlich, die Auffassung der Kundenzufriedenheit als Nachkaufphänomen zu erweitern. ${ }^{100}$ Bedingt durch die aufgezeigten Besonderheiten des Dienstleistungskonsums ist es erforderlich, die ergebnisorientierte Betrachtung von Kundenzufriedenheit um eine am Kundenprozeß orientierte Sichtweise zu ergänzen. Da das Konsumerlebnis auf der Wahrnehmung unterschiedlicher Teilabschnitte einer Dienstleistung basiert, liegt die Vermutung nahe, daß sich das Zufriedenheitsurteil eines Kunden aus der Beurteilung der einzelnen, zeitlich aufeinander folgenden Phasen zusammensetzt. ${ }^{101}$ Darüber hinaus deuten die Ergebnisse der wenigen empirischen Untersuchungen zu diesem Themenkomplex darauf hin, daß die Zufriedenheit mit einer zeitlich vorgelagerten Phase Einfluß auf die Zufriedenheit mit den darauf folgenden Phasen ausübt. ${ }^{102}$

In diesem Zusammenhang soll daher der Begriff der Zufriedenheit in Anlehnung an die bereits aufgezeigte Prozeßhierarchie von Dienstleistungen untergliedert werden. ${ }^{103}$ Eine solche Sichtweise führt somit zu der Unterscheidung von Transaktionszufriedenheit, die den Zufriedenheitsgrad mit einer einzelnen Dienstleistungstransaktion widerspiegelt, und Episodenzufriedenheit, die die Zufriedenheit mit einer einzelnen Dienstleistungsepisode beinhaltet. Sind einzelne Kon-

99

Vgl. Stauss, B., Seidel, W., Prozessuale Zufriedenheitsermittlung und Zufriedenheitsdynamik bei Dienstleistungen, a.a.O., S. 182.

100 Vgl. ebenda, S. 190.

101 Vgl. Güthoff, J., Qualität komplexer Dienstleistungen: Konzeption und empirische Analyse der Wahrnehmungsdimensionen, a.a.O., S. 34; Hentschel, B., Dienstleistungsqualităt aus Kundensicht: Vom merkmals- zum ereignisorientierten Ansatz, a.a.O., S. 151; Westerbarkey, P., Methoden zur Messung und Beeinflussung der Dienstleistungsqualităt: Feedback- und Anreizsysteme in Beherbergungsunternehmen, a.a.O., S. 63.

Vgl. z.B. Danaher, P.J., Mattson, J., Customer Satisfaction during the Service Delivery Process, in: EJoM, Vol. 28, No. 5, 1994, S. 5 ff.; Danaher, P.J., Mattson, J., Cumulative Encounter Satisfaction in the Hotel Conference Process, in: IJoSIM, Vol. 5, No. 4, 1994, S. $69 \mathrm{ff}$;; Burmann, Ch., Konsumentenzufriedenheit als Determinante der Marken- und Händlerloyalität, in: Marketing ZFP, Jg. 13, H. 4, 1991, S. 252 ff.

$103 \mathrm{Vgl}$. hierzu und im folgenden Stauss, B., Seidel, W., Prozessuale Zufriedenheitsermittlung und Zufriedenheitsdynamik bei Dienstleistungen, a.a.O., S. 191. 
taktpunkte Gegenstand der Betrachtung, ist dementsprechend der Begriff Kontaktpunktzufriedenheit zu wählen. Auf einer übergreifenden, die Summe der bisher erlebten Transaktionen berücksichtigenden Ebene kann darüber hinaus eine Beziehungszufriedenheit oder Globalzufriedenheit unterschieden werden.

Damit ergeben sich für die Zufriedenheitsforschung zwei zentrale Herausforderungen, die in einem engen Verhältnis zueinander stehen. ${ }^{104}$ Zum einen gilt es bei der Messung der Kundenzufriedenheit insbesondere den Prozeßcharakter des Dienstleistungskonsums zu berücksichtigen. Zum anderen ist die Dynamik und damit die Entwicklung der Kundenzufriedenheit im Zeitablauf zu analysieren. ${ }^{105}$ Auch hier kann zwischen einer transaktions- und einer beziehungsorientierten Sichtweise unterschieden werden.

Angesichts der besonderen Heterogenität des Dienstleistungssektors und der hohen Komplexität des Zufriedenheitskonstruktes erscheint es notwendig, sich bei der Analyse auf spezifische Bedingungskonstellationen zu beschränken. ${ }^{106}$ Während z.B. die Kundenzufriedenheit mit Finanzdienstleistungen oder der Gaststätten- bzw. Hotelbranche Gegenstand zahlreicher Untersuchungen ist, wird der Zufriedenheit mit Verkehrsdienstleistungen vergleichsweise wenig Aufmerksamkeit zuteil. Wenngleich die Aussage von GUMmESSON und KINGMAN-BRUNDAGE „In the thousands of articles and conference papers on services produced during the past fifteen years, we have not found a single one on railroad services"107 zumindest für die deutschsprachige Literatur in dieser Form nicht zutrifft, so ergibt sich doch eine deutliche Dominanz an Veröffentlichungen, die sich mit dem Angebot von Fluggesellschaften beschäftigen. Der historische Kontext der Deutschen Bahn AG als Hauptanbieter schienengebundener Verkehrsdienstleistungen und die

104 Vgl. Johnston, R., The Zone of Tolerance: Exploring the Relationship Between Service Transactions and Satisfaction with the Overall Service, in: IJoSIM, Vol. 6, No. 2, 1995, S. $47 \mathrm{f}$.; von Lingen, Th., Zufriedenheitsmanagement, in: P\&A, H. 1, 1994, S. 8; Stauss, B., Seidel, W., Prozessuale Zufriedenheitsermittlung und Zufriedenheitsdynamik bei Dienstleistungen, a.a.O., S. 191.

In der aktuellen wissenschaftlichen Diskussion wird insbesondere auf Defizite in diesem Bereich der Zufriedenheitsforschung hingewiesen. Vgl. z.B. Woodruff, R.B., Developing and Applying Consumer Satisfaction Knowledge: Implications for Future Research, a.a.O., S. 5; Gronroos, Ch., Toward a Third Phase in Service Quality Research: Challenges and Future Directions, in: Advances in Service Marketing and Management: Research and Practice, Swartz, T.A., Bowen, D.E., Brown, St.W. (Hrsg.), 2. Aufl., Greenwich, London 1993, S. 49 ff.

Womit vor allem die Beschränkung auf bestimmte Dienstleistungen angesprochen ist. Vgl. Runow, H., Zur Theorie und Messung der Verbraucherzufriedenheit, Frankfurt am Main 1982, S. $66 \mathrm{f}$.

107 Gummesson, E., Kingman-Brundage, J., Service Design and Quality: Applying Service Blueprinting and Service Mapping to Railroad Services, a.a.O., S. 102. 
besonderen Herausforderungen, mit denen sich dieses Unternehmen aktuell und in der Zukunft konfrontiert sieht, machen aber gerade die Analyse der Kundenzufriedenheit mit dem Angebot dieses Dienstleisters zu einem besonders interessanten Forschungsfeld.

Die auf der Grundlage der speziellen Marktsituation schienengebundener Verkehrsträger gewonnenen Erkenntnisse über die Kundenzufriedenheit sollten dabei nicht allein für Anbieter solcher Dienstleistungen von Interesse sein. Vielmehr sollten die Ergebnisse darüber hinaus zumindest insoweit verallgemeinerungsfähig sein, als sie auf das Angebot anderer Wettbewerber der Verkehrsbranche oder Branchen übertragen werden können, die durch eine vergleichbare Bedingungskonstellation gekennzeichnet sind.

\section{Zielsetzung und Gang der Untersuchung}

Ausgehend von dem dargestellten Problemhintergrund besteht die generelle Zielsetzung der Arbeit darin, einen Beitrag zur Erfassung und Erklärung der Zufriedenheit mit Verkehrsdienstleistungen zu erarbeiten. Dabei stehen im Mittelpunkt der Untersuchung die Fragen, wie aus einer prozeßbezogenen Perspektive Zufriedenheit mit personenbezogenen Verkehrsdienstleistungen zu operationalisieren ist und welche Einflußfaktoren die Zufriedenheit determinieren. In diesem Zusammenhang soll insbesondere dem Episodencharakter von Verkehrsdienstleistungen Rechnung getragen werden. Als zentraler Untersuchungsfall dient in der vorliegenden Untersuchung die Dienstleistung „innerdeutscher schienengebundener Personenfernverkehr". Im Zentrum der Arbeit steht die theoriegeleitete Entwicklung eines Operationalisierungs- und Erklärungsansatzes der Zufriedenheit mit personenbezogenen Verkehrsdienstleistungen und dessen empirische Überprüfung. Die Analyse zentraler personaler und reise- bzw. verkehrsdienstleistungsspezifischer Bestimmungsfaktoren soll einen Einblick in die individuelle Zufriedenheitswahrnehmung eines Fernreisenden vermitteln. Darüber hinaus sind auf der Grundlage der empirischen Ergebnisse Schlußfolgerungen abzuleiten, die Anhaltspunkte für eine Verbesserung des Zufriedenheitsmanagements von Verkehrsdienstleistern bereithalten. 
Aus der generellen Zielsetzung leiten sich folgende Forschungsschwerpunkte $a b$ :

- Ausarbeitung einer Grundkonzeption zur Erfassung der Zufriedenheit mit personenbezogenen Verkehrsdienstleistungen. Hierbei soll der zu entwickelnde operationale Ansatz den Prozeßcharakter des Dienstleistungskonsums in expliziter Weise berücksichtigen. Damit ist zum einen die Strukturierung und ggf. die Visualisierung des Kundenprozesses verbunden. Zum anderen muß das zu erstellende Meßinstrumentarium in der Lage sein, die Zufriedenheitsbildung im Rahmen des zugrunde liegenden Konsumprozesses in geeigneter Weise zu erfassen.

- Systematisierung und Operationalisierung relevanter Konsumprozeßphasen von Verkehrsdienstleistungen auf unterschiedlichen Hierarchieebenen.

- Systematisierung und Operationalisierung relevanter Bestimmungsfaktoren der Kundenzufriedenheit mit Verkehrsdienstleistungen.

- Empirische Analyse der Interdependenzen der verschiedenen Konsumprozeßphasen auf unterschiedlichen Aggregationsniveaus sowie deren Einfluß auf die Gesamtzufriedenheit und der damit einhergehenden Auswirkungen auf das künftige Kundenverhalten auf der Grundlage eines hypothesengestützten Bezugsrahmens.

- Empirische Überprüfung des Bezugsrahmens zur Ableitung von Aussagen über den Einfluß zentraler Bestimmungsfaktoren der Kundenzufriedenheit mit Verkehrsdienstleistungen.

- Ableitung von Implikationen aus der empirischen Untersuchung für das Zufriedenheitsmanagement von Verkehrsdienstleistungsunternehmen und die Zufriedenheitsforschung im Dienstleistungsbereich.

Die vorliegende Problemstruktur und die eingenommene Kundenperspektive beeinflussen das theoretisch-methodische Vorgehen der Untersuchung. Die Auswahl relevanter Einfluß- und Bestimmungsfaktoren der Kundenzufriedenheit mit Verkehrsdienstleistungen basiert auf den Erkenntnissen verhaltenswissenschaftlicher und informationstheoretischer Ansätze. Methodisch ist die Arbeit als konzeptionell-literaturgestützt einzuordnen, wobei die konzeptionellen Überlegungen mit Erkenntnissen aus Expertengesprächen sowie mit Ergebnissen einer empirischen Untersuchung unterlegt werden. 
Mit der Zielsetzung ist bereits der Gang der Untersuchung vorgezeichnet. In Teil B erfolgt die Erörterung der konzeptionellen Grundlagen zur Analyse der Zufriedenheit mit Dienstleistungen. Zunächst werden die Anforderungen an einen Operationalisierungsansatz zur Erfassung und Erklärung der Zufriedenheit mit Dienstleistungen erarbeitet und die aus dem spezifischen Charakter von Verkehrsdienstleistungen resultierenden Besonderheiten aufgezeigt. Diese dienen als Grundlage zur Diskussion der in der wissenschaftlichen Literatur erörterten Erklärungsansätze zur Kundenzufriedenheit, wobei ein besonderes Augenmerk auf die Berücksichtigung des Prozeßcharakters von Dienstleistungen gerichtet wird. Dabei wird die Betrachtung nicht auf die Erklärungsansätze der Kundenzufriedenheit beschränkt, sondern um die Erkenntnisse der Forschung über verwandte Konstrukte ergänzt. Daher findet vor der Darstellung der verschiedenen Ansätze zunächst eine Untersuchung der Beziehungen zwischen den Konstrukten Kundenzufriedenheit, Dienstleistungsqualität und Einstellungen statt.

Daran anschließend erfolgen eine Darstellung und Analyse der verschiedenen Erscheinungsformen der Kundenzufriedenheit mit Dienstleistungen, die die inhaltliche Nähe der Kundenzufriedenheit zum Konstrukt der Dienstleistungsqualität aufdecken. Der zumindest bedingte Verwandtschaftsgrad der Konstrukte zeigt sich auch in der Tatsache, daß diese häufig mit sehr ähnlichen Methoden gemessen werden, welche ebenfalls differenziert analysiert und im Hinblick auf ihre Eignung zur Messung des Prozeßcharakters des Dienstleistungskonsums und der Kundenzufriedenheit mit Verkehrsdienstleistungen diskutiert werden. Dabei geht der Diskussion der verschiedenen Meßansätze die Beschreibung geeigneter Instrumente zur Strukturanalyse und Visualisierung der Prozeßkomponente von Dienstleistungen voraus.

Darauf aufbauend werden ein Ansatz zur Erfassung der Zufriedenheit mit Verkehrsdienstleistungen entwickelt, die zugrunde liegende Hierarchie des Konsumprozesses ermittelt, Indikatoren sowie zentrale Bestimmungsfaktoren der Kundenzufriedenheit erarbeitet und der gewählte Meßansatz dargestellt. Die Wirkungsbeziehungen innerhalb der Konsumprozeßhierarchie werden durch Untersuchungshypothesen spezifiziert und im Rahmen der sich anschließenden empirischen Untersuchung überprüft.

Gegenstand der empirischen Analyse des Teils $\mathbf{C}$ der Arbeit bildet zunächst die Beschreibung des Untersuchungsdesigns und der Stichprobenstruktur. Daran anschließend erfolgt eine phasenbezogene Analyse der Kundenzufriedenheit anhand der ermittelten Ausprägungsformen der Konsumprozeßhierarchie. Im 
Mittelpunkt der Untersuchungen steht die Überprüfung der Einflußstrukturen innerhalb der einzelnen Konsumprozeßphasen sowie die interphasenspezifische Betrachtung der Beziehungszusammenhänge. Des weiteren erfolgt eine Betrachtung der Bedeutung ausgewählter Bestimmungsfaktoren zur Erklärung von Abweichungen in den Ausprägungen der Kundenzufriedenheit und deren Beziehungszusammenhängen.

Aufgrund der ermittelten Wirkungszusammenhänge werden in Teil D Implikationen der Intersuchung für das Zufriedenheitsmanagement im Verkehrsdienstleistungsbereich abgeleitet sowie offene Forschungsfelder aufgezeigt. 


\section{B. Konzeptionelle Grundlagen zur Analyse der Kunden- zufriedenheit mit Dienstleistungen}

Innerhalb der folgenden Ausführungen werden zunächst Anforderungen und Kriterien zur Beurteilung von Operationalisierungsansätzen der Kundenzufriedenheit erarbeitet. Daran anschließend wird ein phasenorientierter Ansatz zur Erfassung und Erklärung der Kundenzufriedenheit mit Verkehrsdienstleistungen entwickelt. Dieser bildet die Grundlage zur Ableitung von Untersuchungshypothesen, die aufzeigen sollen, welche Ausprägungsformen und Einflußfaktoren der Zufriedenheit mit Verkehrsdienstleistungen zu beachten sind.

\section{Anforderungen an einen Operationalisierungsansatz zur Erfassung und Erklärung der Kundenzufriedenheit mit Dienstleistungen}

Unabhängig davon, mit welchem hypothetischen Konstrukt sich ein Forscher beschäftigt, sieht er sich mit dem Problem konfrontiert, nicht beobachtbare, qualitative Variablen einer Quantifizierung und intensitätsmäßigen Erfassung zuzuführen. ${ }^{1}$ Zentrale Voraussetzung für das Messen solcher Variablen ist die Operationalisierung des zu untersuchenden theoretischen Konstruktes. ${ }^{2}$ In diesem Zusammenhang gilt es, zwei Teilaufgaben zu lösen. Zum einen bedarf es einer Operationalisierung auf der theoretischen Sprachebene. Hiermit ist der Anspruch verbunden, unter Hinzuziehung zentraler Erklärungsansätze das Konstrukt verhaltenstheoretisch zu untermauern und semantisch wie auch inhaltlich zu konkretisieren. ${ }^{3}$ Zum anderen erfordert die Operationalisierung auf der empirischen Sprachebene die Festlegung einer Meßvorschrift, die so ausgestaltet sein muß, daß Schlußfolgerungen auf das untersuchte Konstrukt nicht nur möglich, sondern auch valide sind. ${ }^{4}$ Während damit die Konstruktoperationalisierung auf der Ebene der theoretischen Sprache die Voraussetzungen für eine intersubjektive Verständigung über den Inhalt von Konstrukten schaffen soll, besteht die

Vgl. Meffert, H., Marketingforschung und Käuferverhalten, 2. Aufl., Wiesbaden 1992, S. 183.

Dabei wird unter einem theoretischen Konstrukt ${ }_{2} .$. an abstract entity which represents the ,true', nonobservable state or nature of a phenomen" verstanden. Bagozzi, R.P., Fornell, C., Theoretical Concepts, Measurement, and Meaning, in: A Second Generation of Multivariate Analysis, Fornell, C. (Hrsg.), Bd. 2, New York 1982, S. 24.

$3 \mathrm{Vgl}$. Andritzky, K., Die Operationalisierbarkeit von Theorien zum Konsumentenverhalten, Berlin 1976, S. 14.

4 Vgl. Berekoven, L., Eckert, W., Ellenrieder, P., Marktforschung: methodische Grundlagen und praktische Anwendung, 4. Aufl., Wiesbaden 1989, S. $192 \mathrm{f}$. 
Zielsetzung der Operationalisierung auf der Ebene der empirischen Sprache in der eigentlichen Messung des zuvor theoretisch spezifizierten Konstruktes. ${ }^{5}$

Zur Ableitung eines Operationalisierungsansatzes der Kundenzufriedenheit mit Verkehrsdienstleistungen werden daher zunächst Überlegungen zu generellen Anforderungen an einen Operationalisierungsansatz angestellt. Darauf aufbauend folgt eine Betrachtung der allgemeinen Besonderheiten von Dienstleistungen sowie von Aspekten, die sich aus dem spezifischen Charakter von Verkehrsdienstleistungen ergeben und im Rahmen der Operationalisierung der Kundenzufriedenheit mit Verkehrsdienstleistungen zu berücksichtigen sind.

\subsection{Allgemeine Anforderungen an einen Operationalisierungsansatz}

Die in der Literatur intensiv geführte Diskussior um die Abgrenzung und Messung kaufverhaltenstheoretischer Konstrukte bestätigt die Schwierigkeit der Ermittlung allgemeingültiger und verläßlicher Maßstäbe, Meßeinheiten und Indikatoren. ${ }^{6}$ Es stellt sich daher die Frage, wann ein Konstrukt als ausreichend operationalisiert anzusehen ist. Nach OpP ist diese Forderung erfüllt, wenn alle Personen, die der (Fach-)Sprache mächtig sind, die semantischen Regeln des Begriffes kennen und dementsprechend in der Lage sind, zu entscheiden, ob ein beliebiges Ereignis zu den Designata des betreffenden Begriffes zugehörig ist oder nicht. ${ }^{7}$ Auf der theoretischen Sprachebene werden häufig Modelle ${ }^{8}$ herangezogen, die eine möglichst vollständige Erfassung und Strukturierung komplexer Sachverhalte garan-

5 In diesem Zusammenhang spricht Andritzky auch von der „Identifizierung von Begriffen der theoretischen mit jenen der empirischen Sprache." Andritzky, K., Die Operationalisierbarkeit von Theorien zum Konsumentenverhalten, a.a.O., S. 14.

6 Vgl. Meffert, H., Marketingforschung und Käuferverhalten, a.a.O., S. 183.

7 Vgl. Opp, K.-D., Methodologie der Sozialwissenschaften: Einführung in Probleme ihrer Theorienbildung, Reinbeck 1970, S. 135 f.

$8 \quad \mathrm{Zu}$ einem Überblick über Modelle im Marketing sowie im enweiterten betriebswirtschaftlichen Kontext vgl. z.B. Meffert, H., Steffenhagen, H., Marketing-Prognosemodelle. Quantitative Grundlagen des Marketing, Stuttgart 1977, S. 29 ff. Gelegentlich ist festzustellen, daß in der wissenschaftlichen Literatur die Begriffe Theorie und Modell synonym verwendet werden, wobei generell an Modelle im Vergleich zu Theorien geringere wissenschaftliche Anforderungen z.B. im Hinblick auf ihren Geltungsbereich sowie ihren normativen Anspruch gestellt werden. Vgl. hierzu Zimmermann, H.-J., Zum Nutzen empirischer Untersuchungen, in: Der praktische Nutzen empirischer Forschung, Witte, E. (Hrsg.), Tübingen 1981, S. 277 ff. 
tieren sollen. ${ }^{9}$ Um dem Kriterium der Vollständigkeit zu genügen, muß der Ansatz sämtliche relevanten Einflußfaktoren berücksichtigen bzw. adaptieren können. ${ }^{10}$

Über die Vollständigkeit hinaus sind bei der Ausgestaltung eines Operationalisierungsansatzes der Kundenzufriedenheit mit Verkehrsdienstleistungen einige weitere generelle Anforderungen zu beachten. ${ }^{11}$ Zum einen hat die Formulierung des Ansatzes so präzise zu erfolgen, daß er den Kriterien der Einfachheit und Handhabbarkeit genügt. Damit wird die Forderung aufgestellt, daß durch das Streben nach Vollständigkeit die Verständlichkeit des Ansatzes nicht beeinträchtigt wird und eine Konzentration auf wesentliche Aspekte erfolgt. ${ }^{12}$ Zum anderen sollte ein Operationalisierungsansatz intersubjektiv überprüfbar und nachvollziehbar sein sowie die wissenschaftliche Diskussion befruchten. ${ }^{13}$

Auch an die Operationalisierung auf der empirischen Sprachebene sind verschiedene Anforderungen zu stellen. Im Rahmen der Festlegung einer Meßvorschrift ist verbindlich vorzugeben, wie das zu untersuchende Konstrukt zu messen ist. Da sich hypothetische Konstrukte wie Kundenzufriedenheit oder Dienstleistungsqualität einer direkten Beobachtung und damit einer unmittelbaren Messung entziehen, bedarf es der Bestimmung von Konstruktindikatoren, die eine indirekte Erfassung des untersuchten Begriffes eindeutig und zweckmäßig ermöglichen. Für die Erfassung der Kundenzufriedenheit mit Verkehrsdienstleistungen ist bei der Auswahl geeigneter Indikatoren daher der explizite Bezug zu der untersuchten Verkehrsdienstleistung herzustellen. ${ }^{14}$ Ist die Entscheidung über die zu berücksichtigenden Merkmale getroffen, bestehen die nächsten Schritte in

9 Vgl. Büker, B., Qualitătsbeurteilung investiver Dienstleistungen: Operationalisierungsansătze an einem Beispiel zentraler EDV-Dienste, a.a.O., S. 24.

10 Die Adaptivität eines Operationalisierungsansatzes kennzeichnet die Anpassungsfähigkeit an reale, sich verăndernde Umweltsituationen. Vgl. Meffert, H., Steffenhagen, H., MarketingPrognosemodelle. Quantitative Grundlagen des Marketing, a.a.O., S. $55 \mathrm{ff}$.

$\mathrm{Zu}$ den generellen Anforderungen an Operationalisierungsansătze in der Betriebswirtschaftslehre vgl. Opp, K.-D., Methodologie der Sozialwissenschaften: Einführung in Probleme ihrer Theorienbildung, a.a.O., S. 135 ff.; Andritzky, K., Die Operationalisierbarkeit von Theorien zum Konsumentenverhalten, a.a.O., S. $20 \mathrm{ff}$.

Vgl. Birkelbach, R., Qualitătsmanagement in Dienstleistungscentern: Konzeption und typenspezifische Ausgestaltung unter besonderer Berücksichtigung von Verkehrsflughäfen, Frankfurt am Main u.a. 1993, S. 28.

Vgl. Büker, B., Qualitătsbeurteilung investiver Dienstleistungen: Operationalisierungsansătze an einem Beispiel zentraler EDV-Dienste, a.a.O., S. 26.

Die Bestimmung der Merkmale zur Erfassung der Kundenzufriedenheit mit Verkehrsdienstleistungen erfolgt im Rahmen dieser Arbeit einerseits auf der Grundlage vermuteter UrsacheWirkungs-Zusammenhänge, andererseits auf der Basis des korrelativen Zusammenhangs der Indikatoren zum untersuchten Konstrukt der Kundenzufriedenheit (vgl. Kap. B 4 dieser Arbeit). Zu den Ergebnissen der empirischen Untersuchung vgl. Kap. C 2 dieser Arbeit. 
der Auswahl eines Meßverfahrens sowie in der Festlegung der Skalierungstechnik. ${ }^{15}$ Darüber hinaus ist eine Entscheidung über die Dimensionalität des Konstruktes und damit über die Anzahl der zu untersuchenden Merkmale zu treffen. ${ }^{16}$

Die Beurteilung unterschiedlicher Meßverfahren erfolgt auf Basis der allgemeinen Gütekriterien Objektivität, Reliabilität und Validität. ${ }^{17}$ Die Objektivität beschreibt die Unabhängigkeit des Meßergebnisses von der Person, die die Messung durchführt. Ein Meßverfahren ist dementsprechend dann objektiv, wenn verschiedene Anwender zum gleichen Ergebnis gelangen und somit Verzerrungen durch z.B. Interviewereinflüsse oder Ermessensspielräume bei der Auswertung und Interpretation der Ergebnisse weitestgehend ausgeschlossen sind ${ }^{18}$ Als reliabel bzw. zuverlässig gilt eine Methode, wenn diese bei wiederholter oder paralleler Messung unter gleichen Voraussetzungen zu gleichen Ergebnissen führt und damit die Reproduzierbarkeit einer Messung garantiert. ${ }^{19}$ Während das Kriterium der Reliabilität auf die formale Genauigkeit eines Meßansatzes abzielt, beschreibt die Validität oder Gültigkeit eines Verfahrens die materielle (inhaltliche) Genauigkeit der Merkmalserfassung ${ }^{20}$ Hiermit ist die Frage verbunden, inwieweit eine Meßmethode das zu messende Konstrukt tatsächlich wiedergibt. Das Kriterium der Validität stellt dabei das zentrale, gleichzeitig aber am schwersten zu erfassende Gütekriterium zur Beurteilung eines Meßansatzes dar. ${ }^{21}$ Es gilt dabei zu beachten,

Skalierung bedeutet im praktischen Sprachgebrauch "die technische Vorbereitung der Messung, d.h. die Konstruktion einer Skala zur Messung der Merkmalsausprägungen bei den jeweiligen Untersuchungseinheiten." Berekoven, L., Eckert, W., Ellenrieder, P., Marktforschung: methodische Grundlagen und praktische Anwendung, a.a.O., S. 69.

Vgl. Meffert, H., Marketingforschung und Käuferverhalten, a.a.O., S. 185 f.

17 Im Rahmen der Diskussion von Meßverfahren im Hinblick auf ihre Eignung zur Messung der Kundenzufriedenheit werden über die genannten Gütekriterien hinaus weitere Beurteilungskriterien herangezogen, die die Wahl eines Meßverfahrens erleichtern sollen. Vgl. hierzu Kap. B 3 dieser Arbeit. Im Mittelpunkt der vorliegenden Untersuchung steht die Erfassung der Kundenzufriedenheit auf der Basis eines multiattributiven Ansatzes im Rahmen einer Primärerhebung im schienengebundenen Personenfernverkehr. Aussagen bezüglich der Güte dieses Vorgehens erfolgen auf der Grundlage der empirischen Ergebnisse in Kap. C 2 dieser Arbeit.

Vgl. Schmidt, R., Marktorientierte Konzeptfindung für langlebige Gebrauchsgüter: Messung und QFD-gestützte Umsetzung von Kundenanforderungen und Kundenurteilen, Wiesbaden 1996, S. 107.

Zur Reliabilităt und zu den Verfahren ihrer Bestimmung vgl. Hildebrandt, L., Kausalanalytische Validierung in der Marketingforschung, in: Marketing ZFP, Jg. 6, H. 1, 1984, S. 41 f.

Vgl. Berekoven, L., Eckert, W., Ellenrieder, P., Marktforschung: methodische Grundlagen und praktische Anwendung, a.a.O., S. 85 f.

21 In der wissenschaftlichen Literatur findet sich eine Vielzahl an unterschiedlichen Kategorisierungsansätzen der Validität und der Verfahren zu ihrer Bestimmung. Beispielhaft sei hier auf Schmidt, R., Marktorientierte Konzeptfindung für langlebige Gebrauchsgüter: Messung und QFD-gestützte Umsetzung von Kundenanforderungen und Kundenurteilen, a.a.O., S. $106 \mathrm{ff}$. sowie Homburg. Ch., Giering, A., Konzeptualisierung und Operationalisierung komplexer (Fortsetzung der Fußnote auf der năchsten Seite) 
daß ein valides Ergebnis als reliabel angesehen werden kann, diese Beziehung aber umgekehrt nicht zwingend gilt. Daher wird die Reliabilität als notwendige, aber nicht hinreichende Voraussetzung der Validität angesehen. ${ }^{22}$

Die bisher aufgeführten allgemeinen Anforderungskriterien an einen Operationalisierungsansatz der Kundenzufriedenheit mit Verkehrsdienstleistungen sollen im folgenden um die Besonderheiten ergänzt werden, die sich aus dem spezifischen Charakter von Dienstleistungen ergeben.

\subsection{Dienstleistungsspezifische Anforderungen}

\subsection{Generelle dienstleistungsspezifische Anforderungen}

Aus der Definition von Dienstleistungen nach MEFFERT/BRUHN ${ }^{23}$ können weitere Anforderungen abgeleitet werden, die im Rahmen eines Operationalisierungsansatzes der Kundenzufriedenheit mit Verkehrsdienstleistungen Beachtung finden müssen. Diese Anforderungen ergeben sich insbesondere aus der Immaterialität einer Dienstleistung, der Leistungsfähigkeit des Dienstleistungsanbieters sowie der Integration eines externen Faktors in den Dienstleistungserstellungsprozeß . $^{24}$ Die genannten Merkmale einer Dienstleistung üben einen entscheidenden Einfluß auf den mentalen Prozeß der Zufriedenheitsbildung aus und sind daher zwingend bei der Entwicklung eines Operationalisierungsansatzes zu berücksichtigen. ${ }^{25}$

Die Immaterialität ${ }^{26}$ bezieht sich auf den Umstand, daß eine Dienstleistung im Gegensatz zu einem Sachgut nicht physisch präsent ist. Auch Verkehrsdienst-

Konstrukte, in: Marketing ZFP, Jg. 18, H. 1, 1996, S. 6 ff. und die dort angegebene Literatur verwiesen.

Vgl. Churchill, G.A., A Paradigm for Developing Better Measures of Marketing Constructs, in: JoMR, Vol. 16, February, 1979, S. 65; Kuß, A., Valide Daten: Die Grundlage für aussagefähige Marktforschung, in: Marktforschung, Tomczak, T., Reinecke, S. (Hrsg.), St. Gallen 1994, S. 20. $\mathrm{Vgl}$. die Ausführungen in Kap. A 2 dieser Arbeit.

Vgl. ebenda.

Vgl. Koelemeijer, K., Roest, H., Verhallen, T., An Integrative Framework of Perceived Service Quality and its Relations to Satisfaction/Dissatisfaction, Attitude and Repurchase Intention, a.a.O., S. 685 .

Hinsichtlich der kontroversen Diskussion um den konstitutiven Charakter des Merkmals Immaterialität kommt Hentschel zu folgendem Ergebnis: „Offensichtlich läßt sich dieser Konflikt nur pragmatisch und im Hinblick auf den Untersuchungszweck lösen. ... Es kommt insofern aus Marketingsicht weniger darauf an, daß sich faktisch stoffliche Veränderungen finden lassen, sondern ob aus Kundensicht am Ende einer Transaktion etwas Greifbares steht, ..." Hentschel, B., Dienstleistungsqualität aus Kundensicht: Vom merkmals- zum ereignisorientierten Ansatz, a.a.O., S. 25. In diesem Zusammenhang präferiert er den Begriff Intangibilität, um zu verdeutlichen, daß eine Dienstleistung „... nicht gesehen, gefühlt oder geschmeckt werden kann ...", ebenda. 
leistungen sind dadurch gekennzeichnet, daß sie eine abstrakte, immaterielle Leistung darstellen. Die eigentliche Kernleistung "Beförderung" ist grundsätzlich immateriell, wenngleich eine Verbindung mit Sachgütern, z.B. in Form des Flugbzw. Zugmaterials, besteht. Dieser Umstand führt dazu, daß ein Kunde eine Prüfung der Qualität der eigentlichen Leistung vor der Inanspruchnahme nur in sehr begrenztem Umfang vornehmen kann. Statt dessen ist er darauf angewiesen, auf Substitute in Form erkennbar materieller Faktoren des physischen Leistungsumfeldes als Indikatoren zurückzugreifen. Im Verkehrsdienstleistungsbereich sind dies z.B. die Ausstattung des Verkehrsmittels oder der Bahnhof bzw. der Flughafen. Dienstleistungen entziehen sich häufig sowohl vor als auch nach ihrer Inanspruchnahme einer faktischen Beurteilbarkeit durch den Kunden. In Ermangelung ausreichender Sucheigenschaften gewinnen bei Dienstleistungen damit Erfahrungs- bzw. Vertrauenseigenschaften für den Kunden an Bedeutung, ${ }^{27}$ die gleichzeitig die Vergleichbarkeit von Dienstleistungen erschweren. Erfahrungseigenschaften stellen z.B. die Höflichkeit des Schalterpersonals oder der mit dem Fahrscheinerwerb verbundene Zeitaufwand dar. Das tatsächliche Leistungsvermögen oder die Sicherheit eines Flugzeugs bzw. eines Hochgeschwindigkeitszuges sind als Vertrauenseigenschaften zu kennzeichnen, da ein Nachfrager diese auch nach der Inanspruchnahme zumeist aufgrund mangelnder Sachkenntnis nicht beurteilen kann.

Aus der Immaterialität leiten sich als Submerkmale die Nichtlagerfähigkeit sowie die Nichttransportfähigkeit von Verkehrsdienstleistungen ab. ${ }^{28}$ Dieser Umstand ist auf die Tatsache zurückzuführen, daß eine Simultaneität von Produktion und Konsumtion bei einer Bahn- oder Flugreise vorliegt (Uno-actu-Prinzip). Erst die Inanspruchnahme führt zu einer Produktion der Dienstleistung für den Kunden. Nicht besetzte Sitzplätze bedeuten damit einen „Verfall“ von Leistungspotentialen. Gleichzeitig leitet sich aus dem Uno-actu-Prinzip aber auch ab, daß eine wie im Sachgüterbereich mögliche Nachbesserung, Aussortierung oder der Umtausch

27 Sucheigenschaften beschreiben alle Eigenschaften einer Sach- oder Dienstleistung, die von dem Kunden vor dem eigentlichen Kauf bzw. der Inanspruchnahme zur Beurteilung herangezogen werden können. Im Vergleich zu Sachleistungen ist der Anteil an Sucheigenschaften bei Dienstleistungen gering. Während Erfahrungseigenschaften Sachverhalte darstellen, die dem Kunden erst während bzw. nach der Dienstleistungserstellung einer Beurteilung zugänglich werden, sind Vertrauens- bzw. Glaubenseigenschaften dadurch gekennzeichnet, daß sie selbst nach dem Kauf bzw. Konsum kaum vom Kunden sicher beurteilt werden können. Vgl. Zeithaml, V.A., How Consumer Evaluation Processes Differ between Goods and Services, in: Services Marketing, Lovelock, Ch.H. (Hrsg.), 2. Aufl., Englewood Cliffs, N.J. 1991, S. 39 ff.

Vgl. Laakmann, K., Value-Added Services als Profilierungsinstrument im Wettbewerb: Analyse, Generierung und Bewertung, Frankfurt am Main 1995, S. 79. 
einer Verkehrsdienstleistung nahezu unmöglich sind und damit der Leistungsfähigkeit des Unternehmens während der Dienstleistungserstellung besondere Bedeutung zukommt. ${ }^{29}$ Diesem Sachverhalt ist bei der Entwicklung eines Operationalisierungsansatzes der Kundenzufriedenheit mit Verkehrsdienstleistungen entsprechend Rechnung zu tragen.

Mit der Leistungsfähigkeit des Dienstleistungsanbieters ist der Umstand angesprochen, daß in Abhängigkeit von der Art der Dienstleistung ein bestimmtes Know-how sowie Fähigkeiten des Personals oder der eingesetzten Sachmittel erforderlich sind. ${ }^{30}$ Aus diesem Kriterium ergibt sich in Verbindung mit dem Merkmal der Immaterialität ein im Vergleich zum Konsumgüterbereich höher wahrgenommenes Kaufrisiko. ${ }^{31}$ Dieses wiederum kann Auswirkungen auf das Involvement des Kunden zur Folge haben. ${ }^{32}$ Wird unter dem wahrgenommenen Kaufrisiko die Nichtvorhersehbarkeit als nachteilig empfundener Folgen des Verhaltens verstanden, ${ }^{33}$ kann eine Reihe unterschiedlicher Teilrisiken identifiziert werden. Während in der Literatur regelmäßig zwischen finanziellen, funktionalen, sozialen, physischen und psychischen Risiken unterschieden wird, sind im Kontext von Verkehrsdienstleistungen auch zeitliche Risiken wie z.B. die Pünktlichkeit von Bedeutung. ${ }^{34}$ Aus diesen Risiken resultiert ein höherer Informationsbedarf, der angesichts der „Informationsarmut" von Dienstleistungen zu einer verstärkten

Vgl. Meyer, A., Mattmüller, R., Qualităt von Dienstleistungen - Entwurf eines praxisorientierten Qualitätsmodells, in: Marketing ZFP, Jg. 9, H. 3, 1987, S. 189.

Vgl. Meffert, H., Bruhn, M., Dienstleistungsmarketing: Grundlagen, Konzepte, Methoden; mit Fallbeispielen, a.a.O., S. 63 f.

31 Vgl. Holzmüller, H.H., Nentwich, A., Ansätze zur Entwicklung eines Marketing-Informationssystems (MAIS) im Bahnbetrieb, in: Der Markt, Jg. 29, H. 1, 1990, S. 18; Zeithaml, V.A., How Consumer Evaluation Processes Differ between Goods and Services, a.a.O., S. 43 ff.; Meffert, H., Bruhn, M., Dienstleistungsmarketing: Grundlagen, Konzepte, Methoden; mit Fallbeispielen, a.a.O., S. 82 .

Vgl. Johnston, R., The Zone of Tolerance: Exploring the Relationship Between Service Transactions and Satisfaction with the Overall Service, a.a.O., 1995, S. 49.

Unter Involvement ist der "Aktivierungsgrad bzw. die Motivstärke zur objektgerichteten Informationssuche, -aufnahme, -verarbeitung und -speicherung" zu verstehen. Trommsdorff, V., Konsumentenverhalten, 2. Aufl., Stuttgart, Berlin, Köln 1993, S. 49. Dabei kann hohes Involvement auf der einen Seite aus der empfundenen Wichtigkeit einer Dienstleistung entstehen, auf der anderen Seite eine Folge des wahrgenommenen Kaufrisikos darstellen. Vgl. hierzu Güthoff, J., Qualităt komplexer Dienstleistungen: Konzeption und empirische Analyse der Wahrnehmungsdimensionen, a.a.O., S. $38 \mathrm{ff}$.

Vgl. Meffert, H., Marketingforschung und Käuferverhalten, a.a.O., S. 69 ff.

Zu den verschiedenen Risikoarten vgl. Hentschel, B., Dienstleistungsqualität aus Kundensicht: Vom merkmals- zum ereignisorientierten Ansatz, a.a.O., S. 62 ff.; Güthoff, J., Qualität komplexer Dienstleistungen: Konzeption und empirische Analyse der Wahrnehmungsdimensionen, a.a.O., S. $36 \mathrm{ff}$. 
Suche nach Surrogatinformationen führt. ${ }^{35}$ Auch bei Kaufentscheidungen, die durch ein tendenziell hoch empfundenes Kaufrisiko gekennzeichnet sind, wird davon ausgegangen, daß zwar relativ viele Produkt- bzw. Dienstleistungseigenschaften wahrgenommen werden, ${ }^{36}$ die letztendliche Kaufentscheidung und -beurteilung jedoch auf der Basis einiger weniger Schlüsselinformationen beruht. ${ }^{37}$ Ein Grund hierfür liegt in der nur begrenzten menschlichen Informationsverarbeitungskapazität. Gerade wenn ein Nachfrager ein hohes Kaufrisiko wahrnimmt, wird er versuchen, dieses zu senken. Hierzu gibt es unterschiedliche Möglichkeiten. Eine davon ist, daß er versucht, seine Informationsbasis zu erweitern, wobei er aber, gerade bei komplexen Entscheidungen, Gefahr läuft, mehr Informationen aufzunehmen als er zu verarbeiten in der Lage ist. Dieser Umstand wird als „information overload“ bezeichnet. ${ }^{38}$ Um diesen zu vermeiden, ist der Nachfrager gezwungen, Informationen zu Blöcken zusammenzufassen. Dieser Vorgang wird als "chunking" bezeichnet und führt zu sog. Schlüsselinformationen. ${ }^{39}$ Eine Analyse entsprechender Schlüsselinformationen ist daher für die Untersuchung der Kundenzufriedenheit von großer Bedeutung.

Neben der Anzahl an Merkmalen, die ein Nachfrager zur Beurteilung heranzieht, ist die Frage nach der Menge in Erwägung gezogener Dienstleistungsalternativen von besonderem Interesse. Dieses „Evoked Set“ ist das Ergebnis eines Lernprozesses, seine Existenz setzt also ein Mindestmaß an Erfahrung und Produktbzw. Dienstleistungskenntnis voraus. In Abhängigkeit von diesen Größen, dem an die einzelne Leistung gestellten Anspruchsniveau und der individuellen Informationsverarbeitungskapazität variieren Umfang und Zusammensetzung des Evoked Set im Zeitablauf. ${ }^{40}$ Da der menschlichen Fähigkeit, Informationen zu verarbeiten, enge Grenzen gesetzt sind, neigen Nachfrager dazu, eine Kaufent-

Vgl. Schmitz, G., Qualitătsmanagement im Privatkundengeschäft von Banken: Konzeption und aufbauorganisatorische Verankerung, a.a.O., S. 54; Hentschel, B., Dienstleistungsqualităt aus Kundensicht: Vom merkmals- zum ereignisorientierten Ansatz, a.a.O., S. 64.

Vgl. Bänsch, A., Käuferverhalten, München, Wien 1983, S. 76.

Vgl. Backhaus, K., Weiber, R., Entwicklung einer Marketing-Konzeption mit SPSS/PC+, Berlin u.a. 1989, S. 41 ff.; Trommsdorff, V., Konsumentenverhalten, a.a.O., S. 96.

Vgl. Jacoby, J., Information Load and Decision Quality: Some Contest Issues, in: JoMR, Vol. 14, November 1977, S. 569.

39 Vgl. Miller, G.A., The Magical Number Seven, Plus or Minus Two: Some Limits on Our Capacity for Processing Information, in: Psychological Review, Vol. 63, March 1956, S. 92 f.; Trommsdorff, V., Konsumentenverhalten, a.a.O., S. $253 \mathrm{ff}$.

40

Vgl. Jarvis, L.P., Wilcox, J.B., Evoked Set Size - Some Theoretical and Empirical Evidence, in: Combined Proceedings Fall Conference of the American Marketing Association, Greer, Th.V. (Hrsg.), No. 35, Chicago 1973, S. $236 \mathrm{ff}$. 
scheidung dahingehend zu vereinfachen, daß hierbei nur eine relativ kleine Menge an Alternativen berücksichtigt wird. In diesem Zusammenhang wird in der Regel die Ansicht vertreten, daß das Evoked Set im Dienstleistungsbereich im Vergleich zum Sachgüterbereich kleiner ausfällt, dafür aber die Selbsterstellung der Leistung als Alternative eher in Erwägung gezogen wird. ${ }^{41} \mathrm{Im}$ Kontext von personenbezogenen Verkehrsdienstleistungen ist es naheliegend, daß der Pkw als Vergleichsmaßstab herangezogen wird. Die Größe des Evoked Set dient daher auch als Indikator, inwieweit ein Konsument seinen Kaufentscheidungsprozeß vereinfacht. ${ }^{42}$ Im Rahmen eines Ansatzes zur Erfassung und Erklärung der Kundenzufriedenheit mit Verkehrsdienstleistungen sind damit neben der Anzahl und Verfügbarkeit in Erwägung gezogener Alternativen auch die Erfahrungen der Kunden mit unterschiedlichen Verkehrsdienstleistungen einzubeziehen.

Auf der Suche nach Informationen zieht ein Konsument interne und externe Informationsquellen heran. ${ }^{43}$ Während im Sachgüterbereich Güter tangibel sind und somit eine Beurteilung (auch) anhand von Produktmerkmalen stattfinden kann, wird ein solches Vorgehen durch die weitgehende Immaterialität von Dienstleistungen erschwert. Im Dienstleistungskontext gewinnen daher insbesondere die persönliche Kommunikation und der private Erfahrungsaustausch bei der Suche nach „derivaten" Informationsquellen an Bedeutung. ${ }^{44}$ Ebenso dienen die eigenen persönlichen Erfahrungen mit der Dienstleistung bzw. dem Dienstleistungsanbieter in der Vergangenheit als Informationsquelle, die sich in den Erwartungen an den nächsten Dienstleistungsprozeß niederschlagen. Die verschiedenen Stufen des Informationsverhaltens werden dabei im wesentlichen vom Involvement und dem wahrgenommenen Risiko des Kunden bestimmt. Eine tiefergehende Analyse der Verarbeitung und Speicherung von Informationen wird angesichts der Unterschiedlichkeit der in der Literatur vorgeschlagenen Ansätze sowie der hohen Bedeutung dieser Teilbereiche des Informationsverhaltens für

41 Vgl. Zeithaml, V.A., How Consumer Evaluation Processes Differ between Goods and Services, a.a.O., S. 39 ff.; Hentschel, B., Dienstleistungsqualität aus Kundensicht: Vom merkmals- zum ereignisorientierten Ansatz, a.a.O., S. 66.

42 Vgl. Weinberg, P., Das Entscheidungsverhalten der Konsumenten, Paderborn U.a. 1981, S. 116.

$43 \mathrm{Zu}$ dieser Unterscheidung und einer weiteren Differenzierung relevanter Informationsquellen vgl. Hentschel, B., Dienstleistungsqualităt aus Kundensicht: Vom merkmals- zum ereignisorientierten Ansatz, a.a.O., S. $68 \mathrm{ff}$.

44

Vgl. Meffert, H., Die Beurteilung und Nutzung von Informationsquellen beim Kauf von Konsumgütern, in: Konsumentenverhalten und Information, Meffert, H., Steffenhagen, H., Freter, H. (Hrsg.), Wiesbaden 1979, S. 46 f. 
die Bildung des Zufriedenheitsurteils im weiteren Verlauf der Ausführungen detailliert vorgenommen. ${ }^{45}$

Ein weiteres zentrales Merkmal von Dienstleistungen besteht in der Integration eines externen Faktors in den Dienstleistungserstellungsprozeß. Unabhängig davon, ob der externe Faktor in der Ausprägung Person oder Objekt vorliegt, verbleibt er jedoch vor, während und nach dem Erstellungsprozeß zumindest teilweise in der Verfügungsgewalt des Nachfragers. HENTSCHEL unterscheidet in diesem Zusammenhang zwischen einer räumlichen, zeitlichen, funktionalen und sozialen Ebene des Kundenkontaktes. ${ }^{46}$ So kann die Beförderung eines Flugoder Bahnreisenden nur erfolgen, wenn dieser persönlich anwesend ist. Systembedingt muß sich der Kunde zur Leistungserstellung zum Standort des Unternehmens - dem Flughafen oder Bahnhof - begeben (räumliche Kontaktebene). Die dauerhafte Präsenz des Dienstleistungsnachfragers während der Leistungserstellung bedingt eine „marketingorientierte Ausrichtung des Dienstleistungsprozesses"47 und damit eine explizite Berücksichtigung der Bedürfnisse des Nachfragers vor, während und nach der Erbringung der eigentlichen Dienstleistung "Beförderung" und eine dementsprechende besondere Sorgfalt bei der Ausführung der Dienstleistungserstellung auch über den Bereich der Kerndienstleistung „Beförderung“ hinaus (zeitliche Kontaktebene).

Aufgrund der Heterogenität des externen Faktors, aber auch des Dienstleistungsanbieters selbst, werden Dienstleistungen mitunter als doppelt individuell bezeichnet. ${ }^{48}$ Der Kunde beeinflußt als mehr oder weniger passiver Produktionsfaktor den Leistungserstellungsprozeß als auch das Dienstleistungsergebnis und damit teilweise selbst seine Zufriedenheit mit der Dienstleistung (funktionale Kontaktebene). Je stärker die Integration vom Kunden wahrgenommen wird, desto schwerer fällt inm die Eindeutigkeit der Ursachenattribution im Falle von Unzufriedenheit. ${ }^{49}$ Leistungsschwankungen des Anbieters führen ebenfalls zu individuell unterschiedlichen Leistungen. Solche Schwankungen können sich z.B.

\footnotetext{
$45 \mathrm{Vgl}$. hierzu insbesondere die Ausführungen in Kap. B 2.3 dieser Arbeit.

46 Vgl. Hentschel, B., Dienstleistungsqualităt aus Kundensicht: Vom merkmals- zum ereignisorientierten Ansatz, a.a.O., S. $29 \mathrm{ff}$.

47 Meffert, H., Bruhn, M., Dienstleistungsmarketing: Grundlagen, Konzepte, Methoden; mit Fallbeispielen, a.a.O., S. 66.

Vgl. Nader, G., Zufriedenheit mit Finanzdienstleistungen: Erfolgswirksamkeit, Messung, Modellierung, a.a.O., S. 12.

49 Vgl. Büker, B., Qualitătsbeurteilung investiver Dienstleistungen: Operationalisierungsansätze an einem Beispiel zentraler EDV-Dienste, a.a.O., S. 35.
} 
in der Stimmung, der Tagesverfassung oder den Sympathien der verschiedenen Mitarbeiter des Unternehmens, mit denen der Kunde in Kontakt kommt, äußern. Das Auftreten des Personals und die Kontaktqualität sind wichtige Einflußgrößen der Kundenzufriedenheit auf der sozialen Kontaktebene. ${ }^{50}$ So zeigen z.B. Untersuchungen im Finanzdienstleistungsbereich, daß Bankkunden möglichst von demselben Mitarbeiter bedient werden möchten. ${ }^{51}$ Die Bedeutung einer hohen persönlichen Bindung zwischen Mitarbeiter und Kunde hat ihre Ursache in dem kundenseitigen Bestreben, ein etwaig wahrgenommenes Risiko durch den Aufbau einer langfristigen Beziehung $\mathrm{zu}$ minimieren. ${ }^{52}$ Insbesondere Verkehrsdienstleistungen sind jedoch durch vielfältige Personalkontakte gekennzeichnet, und die angesprochene Risikoreduktionsstrategie ist für einen Reisenden dementsprechend keine Alternative. Darüber hinaus werden Verkehrsdienstleistungen häufig als kollektive Dienstleistungen erstellt, also für die gleichzeitige und gemeinsame Nutzung für eine Vielzahl von Reisenden. Die Anzahl und physische Nähe der Mitreisenden sowie deren (Interaktions-) Verhalten können einen maßgeblichen Einfluß auf die Zufriedenheit ausüben und stellen so weitere wichtige Komponenten der sozialen Kontaktebene dar. ${ }^{53}$

Verkehrsdienstleistungen verkörpern damit oft eine individualisierte Leistung und sind daher nur bedingt standardisierbar. ${ }^{54}$ Auch im Falle einer Flug- oder Bahnreise wird die Dienstleistung jeweils neu erstellt und kann somit im Umfang sowie in der Qualität individuell verschieden sein. Die beschriebene Heterogenität des externen Faktors wird im Verkehrsdienstleistungsbereich dazu genutzt, einzelne Nachfragersegmente durch das Angebot unterschiedlicher Reiseklassen mit damit einhergehenden preislichen Abstufungen sowie unterschiedlichen Ausstattungen und Serviceangeboten gezielt ansprechen zu können. ${ }^{55}$

Vgl. Lovelock, Ch.H., Developing Frameworks for Understanding Service Marketing, in: Services Marketing, Lovelock, Ch.H. (Hrsg.), 2. Aufl., Englewood Cliffs, N.J. 1991, S. 34 ff.

51 Vgl. Nader, G., Zufriedenheit mit Finanzdienstleistungen: Erfolgswirksamkeit, Messung, Modellierung, a.a.O., S. 12.

Vgl. Güthoff, J., Qualităt komplexer Dienstleistungen: Konzeption und empirische Analyse der Wahrnehmungsdimensionen, a.a.O., S. 33.

53 Vgl. Pompl, W., Touristikmanagement 2: Qualităts-, Produkt-, Preismanagement, Berlin u.a. 1996, S. 38.

54 Vgl. Holzmüller, H.H., Nentwich, A., Ansătze zur Entwicklung eines Marketing-Informationssystems (MAIS) im Bahnbetrieb, a.a.O., S. 18.

55 Vgl. Laakmann, K., Value-Added Services als Profilierungsinstrument im Wettbewerb: Analyse Generierung und Bewertung, a.a.O., S. $82 \mathrm{ff}$. und die dort aufgeführten Beispiele im Bereich von Flugdienstleistungen. 
Zusammenfassend kann festgehalten werden, daß bei der Ableitung von Indikatoren der Zufriedenheit mit personenbezogenen Verkehrsdienstleistungen unterschiedliche Aspekte zu berücksichtigen sind, die sich insbesondere aus der Immaterialität der Dienstleistung sowie der Integration des externen Faktors und dem damit verbundenen Prozeßcharakter von Dienstleistungen ergeben. So ist beispielsweise die Notwendigkeit der Berücksichtigung von Vertrauens- und Erfahrungseigenschaften evident. Ebenso liefern diese Merkmale wichtige Hinweise im Hinblick auf abzuleitende Bestimmungsfaktoren wie z.B. die bisherigen Erfahrungen eines Kunden mit dem Verkehrsdienstleister, die in einen Erklärungsansatz der Kundenzufriedenheit mit Verkehrsdienstleistungen einzubeziehen sind. Über die aus den generellen Charakteristika von Dienstleistungen resultierenden Besonderheiten hinaus lassen sich weitere Anforderungen an einen Operationalisierungsansatz der Kundenzufriedenheit ableiten, die sich aus dem spezifischen Charakter von Verkehrsdienstleistungen ergeben.

\subsection{Aspekte des spezifischen Charakters von Verkehrsdienstleistungen}

Bevor die Besonderheiten von Verkehrsdienstleistungen erörtert werden können, ist es notwendig, den der Untersuchung zugrunde liegenden Verkehrsdienstleistungsbegriff zu konkretisieren. Sowohl im privaten als auch im wirtschaftlichen L.eben besteht das Bedürfnis, Güter, Personen oder Nachrichten in ihrem Aufenthaltsort zu verändern. Verkehr im weiteren Sinne umfaßt nach PIRATH den regelmäßigen Austausch wirtschaftlicher Güter und Leistungen. ${ }^{56}$ Verkehr im engeren Sinne wird als die Überwindung von räumlichen Distanzen bezeichnet. Im Mittelpunkt der folgenden Ausführungen steht der Verkehr im Sinne einer Ortsveränderung von Personen. Unternehmen, deren Ziel es ist, Bedürfnisse nach Ortsveränderungen zu befriedigen, werden im folgenden als Verkehrsdienstleister bzw. Verkehrsträger bezeichnet. Verkehrsträger stellen insbesondere Flug-, Bahn, Schiffahrts- und Busreiseunternehmen dar. ${ }^{57}$ Die Verkehrsbedienung kann dabei sowohl in der Form des Gelegenheits- bzw. Charterverkehrs als auch in der Form

Vgl. Pirath, C., Die Grundlagen der Verkehrswirtschaft, 2. Aufl., Berlin, Göttingen, Heidelberg 1949, S. 1. Beispielhaft sei hier auf den Zahlungsverkehr im Finanzdienstleistungsbereich hingewiesen.

57

Wenngleich diese Definition im übertragenen Sinne auch auf Automobilhersteller zutrifft, sollen diese hier ausgeklammert werden, da die Primärleistung dieser Unternehmen in der Fertigung und dem Verkauf der zur Befriedigung des Mobilitätsbedürfnisses von Konsumenten notwendigen "Endgeräte" und nicht in der eigentlichen Beförderung von Personen besteht. 
des Linienverkehrs erfolgen. ${ }^{58}$ Während das Verkehrsdienstleistungsunternehmen im Gelegenheitsverkehr gezielt auf eine Nachfrage reagiert, legt das Unternehmen im Linienverkehr in Erwartung einer bestimmten Nachfrage im Vorhinein Relationen bzw. Linien (Strecken) fest, die zu festgelegten Zeiten bedient werden sollen. Beim schienengebundenen Personenverkehr werden dabei in Abhängigkeit von den räumlichen Distanzen die Bereiche Nah- und Fernverkehr unterschieden. ${ }^{59}$

Die Unterscheidung in potential-, prozess- und ergebnisorientierte Dimensionen einer Dienstleistung findet ebenfalls in der Literatur zur Verkehrsbetriebslehre Anwendung. Auch hier sind unterschiedliche Begriffsauffassungen festzustellen. ${ }^{60}$ Beispielhaft werden einige Definitionen aufgeführt. DIEDERICH definiert eine Verkehrsdienstleistung ausschließlich über das immaterielle Ergebnis des Leistungserstellungsprozesses. Im Rahmen dieses ergebnisorientierten Ansatzes läßt sich eine Verkehrsdienstleistung als die vollzogene Ortsveränderung definieren. ${ }^{61}$ Demgegenüber steht die prozeßorientierte Definition von GROßE, der den Prozeß der Beförderung in den Mittelpunkt der Betrachtung stellt. Hier werden die Kriterien Schnelligkeit, Pünktlichkeit, Sicherheit und Bequemlichkeit des Transportprozesses betont. $^{62}$ ILLETSCHKO wiederum nimmt eine potentialorientierte Sichtweise ein, wenn er neben der eigentlichen Beförderungsaufgabe auch Weg-

Vgl. Diederich, H., Verkehrsbetriebslehre, Wiesbaden 1977, S. 115 ff.; Schörcher, U., Marketing im Luftverkehr, in: Tourismus-Management: Tourismus-Marketing und Fremdenverkehrsplanung, Haedrich, G., Kaspar, C., Kleinert, H., Klemm, K. (Hrsg.), Berlin, New York 1983, S. 135 f.; Pompl, W., Luftverkehr: Eine ökonomische Einführung, 2. Aufl., Berlin u.a. 1991, S. $21 \mathrm{ff}$.

Unter Schienenpersonenfernverkehr werden hier Reisen mit einer Länge von mehr als 100 Kilometer einfache Entfernung verstanden. Die Legaldefinition des SPNV, die in $\S 2$ Abs. 5 des Allgemeinen Eisenbahngesetzes (AEG) vom 27.12.1993 geregelt ist, bezieht sich dagegen auf Reisen mit einer Länge bis zu 50 Kilometer: "Schienenpersonennahverkehr ist die allgemein zugängliche Beförderung von Personen in Zügen, die überwiegend dazu bestimmt sind, die Verkehrsnachfrage im Stadt-, Vorort- oder Regionalverkehr zu befriedigen. Das ist im Zweifel der Fall, wenn in der Mehrzahl der Beförderungsfälle eines Zuges die gesamte Reiseweite 50 Kilometer oder die gesamte Reisezeit eine Stunde nicht übersteigt", Das Deutsche Bundesrecht 1995, Allgemeines Eisenbahngesetz (AEG), 730. Lieferung, S. 2.

Vgl. hierzu ausführlich Ihde, G.B., Transport, Verkehr, Logistik: gesamtwirtschaftliche Aspekte und einzelwirtschaftliche Handhabung, 2. Aufl., München 1991, S. $5 \mathrm{ff}$.

Vgl. Diederich, H., Verkehrsbetriebslehre, a.a.O., S. $30 \mathrm{ff}$.

Vgl. Große, K.-H., Der außertarifliche Wettbewerb der Unternehmen des Personen- und Güterverkehrs, in: Veröffentlichungen der Akademie für Wirtschaft und Politik Hamburg, Ortlieb, H.-D. (Hrsg.), Tübingen 1963, S. 22. 
sicherungs-, Abfertigungs- und Hilfsaufgaben und die dafür notwendigen Faktoren unter eine Verkehrsdienstleistung subsumiert. ${ }^{63}$

Wie die bisherigen Ausführungen gezeigt haben, ist die isolierte Betrachtung der einzelnen Dimensionen durch eine integrierte Sichtweise zu ersetzen. Somit sollen unter einer Verkehrsdienstleistung sowohl die eigentliche Beförderungsleistung als auch die mit der Beförderungsleistung verbundenen ergänzenden Leistungsprozesse und die zu deren Erstellung erforderlichen Potentiale verstanden werden; eine Auffassung, die inzwischen sowohl von der Wissenschaft als auch von der Unternehmenspraxis vertreten wird. ${ }^{64}$ Die zusätzlichen Leistungskomponenten basieren auf Prozeßvariationen und ermöglichen eine mehr oder minder starke Ausrichtung der vergleichsweise austauschbaren Beförderungsleistung auf Kundenwünsche. Diese Teilprozesse stehen dabei in einem komplementären Verhältnis zur eigentlichen Beförderungsleistung und können dieser vorgeschaltet (z.B. Gepäckträgerservice), mit ihr verbunden (z.B. Bordverpflegung) oder nachgeschaltet (z.B. Taxiservice) sein. ${ }^{65}$

Während diese ergänzenden Leistungen in der Regel ortsfest erstellt werden ${ }^{66}$ und sich somit nicht von anderen Dienstleistungen unterscheiden, ergeben sich aus der originären Beförderungsleistung einige Besonderheiten von Verkehrsdienstleistungen. Der Zweck dieser Leistung besteht in der Ortsveränderung. Im Gegensatz zu anderen Dienstleistungsarten (z.B. Hotelbranche), bei denen Leistungserstellung und -konsum örtlich und zeitlich zusammenfallen, ist dies bei Beförderungsleistungen nicht der Fall, da sie keinen festen Standort der Produktion haben. ${ }^{67}$ Dieser Umstand hat zur Folge, daß sich das Management von Ver-

Vgl. Illetschko, L.L., Transportbetriebswirtschaft im Grundriss, Wien 1957, S. 8 ff.; Illetschko, L.L., Betriebswirtschaftliche Probleme der Verkehrswirtschaft, Wiesbaden 1959, S. 55.

Vgl. Stabenau, H., Verkehrsbetriebslehre, a.a.O., S. 51; Ihde, G.B., Transport, Verkehr, Logistik: gesamtwirtschaftliche Aspekte und einzelwirtschaftliche Handhabung, a.a.O., S. $8 \mathrm{ff}$. Die Notwendigkeit einer solch umfassenden Begriffsauslegung wird (mittlerweile) auch von der Seite der Unternehmenspraxis erkannt. Stellvertretend für die Deutsche Lufthansa AG vgl. Bruhn, M., Schneider, M., Fallstudie Deutsche Lufthansa AG, in: Dienstleistungsmarketing: Grundlagen, Konzepte, Methoden; mit Fallbeispielen, Meffert, H., Bruhn, M. (Hrsg.), 2. Aufl., Wiesbaden 1997, S. 603 sowie für die Deutsche Bahn AG vgl. Bretthauer, I., Deutsche Bahn AG: Von der Behörde zum marktorientierten Touristikanbieter, in: Touristik-Marketing: das Marketing der Tourismus-Organisationen, Verkehrsträger, Reiseveranstalter und Reisebüros, Roth, P., Schrand, A. (Hrsg.), 2. Aufl., München 1995, S. 250.

Vgl. Inde, G.B., Transport, Verkehr, Logistik: gesamtwirtschaftliche Aspekte und einzelwirtschaftliche Handhabung, a.a.O., S. 8 und 10; Pompl, W., Luftverkehr: Eine ökonomische Einführung, a.a.O., S. 34 .

Auch die während der Beförderung erbrachten Nebenleistungen können dahingehend als ortsfest betrachtet werden, als sie innerhalb des Verkehrsmittels erbracht werden.

Vgl. Stabenau, H., Verkehrsbetriebslehre, a.a.O., S. 51. 
kehrsdienstleistungsunternehmen mit spezifischen Herausforderungen konfrontiert sieht:

Da nur wenige Reisende sofort die Rückreise antreten, liegt eine Unpaarigkeit der Verkehrsbeziehung vor, die, bedingt durch die mit der Reise erfolgte Ortsveränderung, zur Folge hat, daß die Dienstleistung an zwei verschiedenen Orten zu erbringen ist. ${ }^{68}$ Damit ist ein Verkehrsdienstleister vor die Aufgabe gestellt, Nachfrage an zwei verschiedenen Orten zu unterschiedlichen Zeitpunkten zu aktivieren und zu koordinieren. ${ }^{69}$ In diesem Zusammenhang sind insbesondere zeitliche Schwankungen in der Nachfrage zu berücksichtigen. So sind im Tagesablauf, regional als auch saisonal, deutliche Nachfrageschwankungen festzustellen. Ein gewisser Teil dieser Unterschiede ist darauf zurückzuführen, daß den Reisen unterschiedliche Anlässe zugrunde liegen, die zu einer unterschiedlichen Nutzung der verschiedenen Verkehrsträger sowie heterogenen Anforderungen der Konsumenten an ein bestimmtes Verkehrsmittel führen ${ }^{70}$ und die es daher im Rahmen eines Operationalisierungsansatzes der Kundenzufriedenheit zu berücksichtigen gilt.

Darüber hinaus ist für Verkehrsträger allgemein ein strukturell bedingter, hoher Anteil an fixen Kosten kennzeichnend. Schwach ausgelastete Kapazitäten führen zu einem unproduktivem Einsatz beschränkter Ressourcen, während gerade im Falle des Personenverkehrs überausgelastete Kapazitäten einen nicht unerheblichen negativen Einfluß auf die Zufriedenheit der Kunden ausüben können. ${ }^{71}$

Vgl. Schörcher, U., Marketing im Luftverkehr, a.a.O., S. 132.

Vgl. zu diesem Problemkreis ausführlich Stabenau, H., Verkehrsbetriebslehre, a.a.O., S. 52 ff.

Vgl. Pompl, W., Luftverkehr: Eine ökonomische Einführung, a.a.O., S. 35 f.

In der Literatur werden der mit den speziellen "Produktionsverfahren" von Verkehrsdienstleistungen verbundene sehr hohe Anteil fixer Kosten an den Gesamtkosten und die hieraus resultierende geringe Angebotselastizität besonders betont. Diese Aspekte haben zur Folge, daß sich Verkehrsdienstleister durch ein kapazitätsorientiertes Verkaufsverhalten auszeichnen, um Leerkosten zu vermeiden. Vgl. hierzu ausführlich Schneider, J., Die Privatisierung der Deutschen Bundes- und Reichsbahn: institutioneller Rahmen - Wertkettenorientiertes Synergiekonzept - Analyse der Infrastrukturgesellschaft, Wiesbaden 1995, S. $120 \mathrm{ff}$. In bezug auf die Produktionsverfahren stellt Stabenau fest: "In keinem anderen Wirtschaftszweig werden vergleichbare Leistungen mit solch differenzierten Verfahren produziert wie dies im Verkehr der Fall ist." Stabenau, H., Verkehrsbetriebslehre, a.a.O., S. 55.

Des weiteren sehen sich insbesondere Fluggesellschaften im grenzüberschreitenden Verkehr einer Vielzahl politischer und rechtlicher (z.B. Landerechte, Verkehrsführung) Restriktionen ausgesetzt. Vgl. hierzu Schorcher, U., Marketing im Luftverkehr, a.a.O., S. 132; Pompl, W., Luftverkehr: Eine okonomische Einführung, a.a.O., S. $37 \mathrm{ff}$. 
Der Ablauf einer Verkehrsdienstleistung kann des weiteren durch externe, vom Unternehmen nicht steuerbare Faktoren beeinflußt werden. Schlechte Wetterverhältnisse oder die in jüngerer Zeit häufiger zu beobachtenden Anschläge auf Gleisanlagen können zu Verspätungen oder Streichungen von Zugverbindungen führen und damit maßgeblich die Zufriedenheit von Kunden beeinflussen.

Eine besondere Stellung nimmt das Schnittstellenmanagement innerhalb der Wertschöpfungskette von Verkehrsdienstleistern ein. ${ }^{72}$ Dabei kann zwischen dem internen und dem externen Schnittstellenmanagement unterschieden werden. Die Aufgabe des internen Schnittstellenmanagements besteht in der Sicherstellung des reibungslosen Verlaufs der selbst erstellten Beförderungsleistungen und der hiermit verknüpften Nebenleistungen. Existiert z.B. auf der von einem Kunden gewünschten Reiseroute keine Direktverbindung, sieht er sich mit dem Problem konfrontiert, einen oder mehr Umsteigevorgänge in Kauf nehmen zu müssen, deren problemloser Ablauf vom Verkehrsdienstleister sicherzustellen ist.

Darüber hinaus kann die Reisekette ${ }^{73}$ aber auch Bestandteile enthalten, die sich einer direkten Kontrolle des Verkehrsdienstleisters entziehen. ${ }^{74}$ In diesem Zusammenhang ist es Aufgabe des externen Schnittstellenmanagements, die eigene Leistung mit dem Angebot weiterer Leistungsträger in qualifizierter Weise zu koordinieren. Diese Problematik wird insbesondere für Fluggesellschaften evident, die i.d.R. nicht Eigentümer der Flughäfen sind, sondern sich diese vielmehr mit ihren Konkurrenten teilen. Der Check-In am Flughafen oder die Gepäckverladung werden häufig von Personal durchgeführt, das nicht im Dienst der jeweiligen Fluggesellschaft steht. Auch Dienste, die nur mittelbar zu einer Flugreise gehören, wie z.B. die Paß- oder Zollkontrolle, können einen nachhaltigen Einfluß auf die Zufriedenheit mit einer Flugdienstleistung ausüben. Ebenso kön-

Vgl. Burmann, Ch., Touristik-Marketing, in: Lexikon der aktuellen Marketingbegriffe, Meffert, H. (Hrsg.), Wien 1994, S. 236; Schorcher, U., Marketing im Luftverkehr, a.a.O., S. $137 \mathrm{ff}$.

73 Die Begriffe Reise-, Kontakt- bzw. Service(prozeß)kette sowie (Verkehrs-)Dienstleistungssequenz bzw. -kette werden im folgenden synonym verwandt und sollen hier alle vom Verkehrsdienstleister bzw. von seinen Absatzmittlern erbrachten Einzelleistungen umfassen, die in den Zeitraum vom ersten bis zum letzten Kontakt einer Dienstleistungstransaktion fallen. Vgl. z.B. Simon, H., Homburg, Ch., Kundenzufriedenheit als strategischer Erfolgsfaktor: Einführende Überlegungen, in: Kundenzufriedenheit: Konzepte, Methoden, Erfahrungen, Simon, H., Homburg, Ch. (Hrsg.), Wiesbaden 1995, S. $15 \mathrm{ff}$.; Pompl. W., Touristikmanagement 2: Qualităts-, Produkt-, Preismanagement, Berlin u.a. 1996, S. 40.

74 Vgl. Meffert, H., Birkelbach, R., Qualitätsmanagement in Dienstleistungszentren: Konzeptionelle Grundlagen und typenspezifische Ausgestaltung, in: Dienstleistungsqualităt: Konzepte, Methoden, Erfahrungen, Bruhn, M., Stauss, B. (Hrsg.), 2. Aufl., Wiesbaden 1995, S. 213 ff.; Laakmann, K., Value-Added Services als Profilierungsinstrument im Wettbewerb: Analyse, Generierung und Bewertung, a.a.O., S. 48 f. 
nen aus Kooperationen von Verkehrsträgern bzw. der kombinierten Nutzung von Verkehrsmitteln (z.B. Bahn/Flugzeug und öffentlicher Nahverkehr) ähnliche Probleme resultieren. Gleichermaßen ist der Einfluß von Reisemittlern auf die Kundenzufriedenheit mit einer Verkehrsdienstleistung in die Überlegungen mit einzubeziehen. ${ }^{75}$ Ein Operationalisierungsansatz der Kundenzufriedenheit ist damit vor die Aufgabe gestellt, sowohl die interne als auch die externe Schnittstellenproblematik von Verkehrsdienstleistungen geeignet aufzugreifen und die Reisekette möglichst vollständig abzubilden.

Dabei ist die Tendenz festzustellen, daß ein Kunde sich auf das schwächste Glied innerhalb eines Leistungsprozesses konzentriert und die hiermit empfundene Unzufriedenheit auf sein Gesamturteil überträgt. ${ }^{76}$ Hier ist insbesondere zu beachten, daß die Verbundeffekte, die sich aus dem Zusammenspiel der Beförderungsleistung mit anderen Leistungselementen ergeben, häufig als Leistungsbündel wahrgenommen werden, unabhängig davon, ob der Verkehrsdienstleister die ergänzenden Teilleistungen beeinflussen kann oder nicht. ${ }^{77}$ Die wenigen Beispiele belegen den Episodencharakter von Verkehrsdienstleistungen und bestätigen damit die Ausführungen über den Prozeßcharakter von Dienstleistungen. Die Analyse der Wahrnehmung der von einem Konsumenten erlebten Reisekette ist damit zwingend erforderlich, um Erkenntnisse über die Entstehung der Kundenzufriedenheit zu erhalten. Die vereinfachte Betrachtung einer Verkehrsdienstleistung als Dienstleistungssequenz ist in Abbildung 4 beispielhaft dargestellt.

Diese vergleichsweise abstrakte Darstellung des Ablaufs veranschaulicht bereits, daß Verkehrsdienstleistungen aus einer Abfolge von Teilprozessen bestehen, die in einem interdependenten Verhältnis zueinander stehen und in Abhängigkeit einer konkreten Verkehrsdienstleistung in Anzahl und Länge sehr unterschiedlich

75 Beispielsweise verkauft die Deutsche Lufthansa AG 85 Prozent ihrer Leistungen über selbständige Reisebüros, auf die sie keinen bzw. nur geringen Einfluß ausüben kann. Vgl. Bruhn, M., Schneider, M., Fallstudie Deutsche Lufthansa AG, a.a.O., S. $605 \mathrm{f}$.

Vgl. Klein, H., Qualitätsmanagement der Deutschen Lufthansa AG, a.a.O., S. 486; Lehmann, A., Dienstleistungsmanagement: Strategien und Ansatzpunkte zur Schaffung von Servicequalität, 2. Aufl., Stuttgart 1995, S. 58.

77 Vgl. Laakmann, K., Value-Added Services als Profilierungsinstrument im Wettbewerb: Analyse, Generierung und Bewertung, a.a.O., S. 48 f.; Klein, H., Management von Kundenzufriedenheit bei der Deutschen Lufthansa AG, in: Kundenzufriedenheit: Konzepte, Methoden, Erfahrungen, Simon, H., Homburg, Ch. (Hrsg.), Wiesbaden 1995, S. 377. 


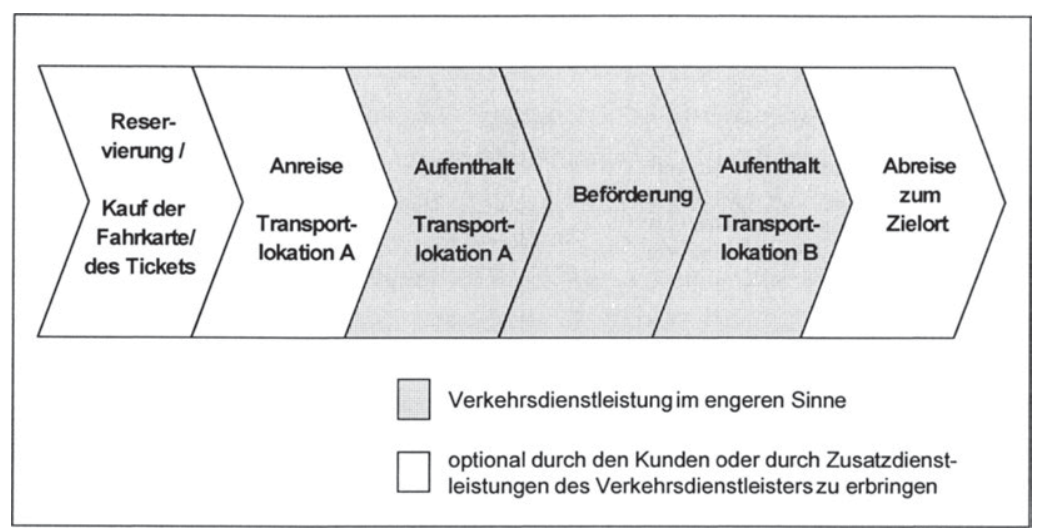

\section{Abb. 4: Vereinfachte Darstellung einer Reisekette}

ausfallen können. ${ }^{78}$ Während der Begriff der Reise- bzw. Servicekette eher die Unternehmensperspektive umfaßt und auf den Leistungserstellungsprozeß abzielt, soll der kundenseitige Nutzungsprozeß, der Kundenprozeß, hier weiter gefaßt werden. So beinhaltet dieser neben den vom Kunden wahrgenommenen Serviceleistungen des Verkehrsdienstleisters auch die vom Kunden selbst vor, während und nach einer Reise erbrachten Teilleistungen. ${ }^{79}$ In diesem Sinne stellt die der eigentlichen Verkehrsdienstleistung vorgelagerte Verkehrsmittelwahl oder die mit dem eigenen Pkw vollzogene Anreise zum Flughafen oder Bahnhof einen selbst erbrachten Teilprozeß dar, deren Ablauf der Verkehrsdienstleister durch unterschiedlichste Optionen erleichtern kann (z.B. Informationsdienste, ShuttleService, Parkmöglichkeiten oder Gepäckwagen). Hieraus ergibt sich die Notwen-

Güthoff zieht als Kriterien zur Charakterisierung komplexer Dienstleistungen u.a. die Merkmale Lănge der Dienstleistungserstellung sowie Heterogenităt und Anzahl einzelner Teilleistungen heran. Vgl. Güthoff, J., Qualităt komplexer Dienstleistungen: Konzeption und empirische Analyse der Wahrnehmungsdimensionen, a.a.O., S. $31 \mathrm{ff}$. und die dort angegebene Literatur. In diesem Zusammenhang argumentiert die Autorin, daß eine Dienstleistung „in Abhängigkeit von der Gesamtlänge bzw. Dauer ihrer Erstellung in Teilabschnitte untergliedert und ihre Qualităt somit phasenweise beurteilt wird. Entsprechend setzt sich das Gesamtqualitätsurteil aus den einzelnen Phasenbeurteilungen zusammen." Ebenda S. 34. Vgl. hieru auch die Ausführungen in Kap. B 2.3 dieser Arbeit.

79 Vgl. Pompl, W., Touristikmanagement 2: Qualitäts-, Produkt-, Preismanagement, a.a. O., S. 45. Böhm unterscheidet im Zusammenhang mit Urlaubsreisen zwischen der faktischen und der psychischen Reisedauer, wobei letztere bereits mit den ersten Reiseüberlegungen beginnt und etwaige Auswirkungen nach der Rückkehr umfaßt. Vgl. Böhm, W., Zur Motivation junger Auslandsreisender, in: Jahrbuch für Jugendreisen und Internationaler Jugendaustausch, Bonn 1962, S. 117. 
digkeit, auch die von einem Kunden selbst erbrachten Leistungen in einen Ansatz zur Erfassung und Erklärung der Kundenzufriedenheit zu integrieren.

Aus Konsumentensicht ergeben sich aus dem Charakter von Verkehrsdienstleistungen spezifische Beurteilungskriterien, anhand derer er die Verkehrswertigkeit einer Verkehrsdienstleistung im Vergleich zu anderen Verkehrsmitteln bemißt. $^{80} \mathrm{Zu}$ diesen zählen u.a. die Massenleistungsfähigkeit, die die Fähigkeit einer flexiblen Anpassung an eine kurzfristig schwankende Nachfrage und somit die Forderung nach flexiblen Buchungsmöglichkeiten beschreibt, sowie der mit der Nutzung eines bestimmten Verkehrsträgers insgesamt verbundene Zeitbedarf. ${ }^{81}$ Darüber hinaus spielen die Sicherheit (Unversehrtheit des Transportobjektes), der Preis sowie die Zahl der Transportlokationen ${ }^{82}$ eine wichtige Rolle. Ebenso sind die Zuverlässigkeit (Pünktlichkeit, Regelmäßigkeit, Häufigkeit), die über die Beförderung hinaus offerierten Serviceleistungen als auch das Prestige sowie die Umweltverträglichkeit einer Verkehrsdienstleistung von hoher Bedeutung. Diese Faktoren beeinflussen die Verkehrsmittelwahl und die hieraus resultierende Zufriedenheit mit einer in Anspruch genommenen Verkehrsdienstleistung. Dabei kann eine Vielzahl von Faktoren die Gewichtung der einzelnen Leistungskriterien im Rahmen der konkreten Verkehrsmittelwahl beeinflussen.

Marktorientiertes Handeln im Verkehrsbereich setzt daher eine sorgfältige Analyse sowie Kenntnis der Determinanten der Verkehrsmittelwahl und Dienstleistungsbeurteilung von aktuellen und potentiellen Konsumenten voraus. Die Kenntnis der Bedürfnisse von Reisenden und deren Anforderungen an die offe-

Zu verschiedenen Kriterienkatalogen vgl. Leonhardt-Weber, B., Die Entwicklung der Qualitätsmerkmale im Verkehr: Eine Analyse vor dem Hintergrund der technischen, wirtschaftlichen und gesellschaftlichen Entwicklung, München 1990, S. 12 ff.; Pompl, W., Luftverkehr: Eine Ökonomische Einführung, a.a.O., S. 34; Inde, G.B., Transport, Verkehr, Logistik: gesamtwirtschaftliche Aspekte und einzelwirtschaftliche Handhabung, a.a.O., S. $91 \mathrm{ff}$; Laakmann, K., Value-Added Services als Profilierungsinstrument im Wettbewerb: Analyse, Generierung und Bewertung, a.a.O., S. 73 ff.; Stabenau, H., Verkehrsbetriebslehre, a.a.O., S. $109 \mathrm{f}$

Dabei sind über die eigentliche Beförderungsdauer hinaus die mit der Nutzung eines Verkehrsmittels verbundenen Wartezeiten (z.B. am Ticketschalter), Ein-, Aus- und Umsteigezeiten sowie die Vor- und Nachlaufzeiten, also die An- und Abreise zur bzw. von der jeweiligen Transportlokation (Bahnhof oder Flughafen), zu berücksichtigen. Vgl. hierzu ausführlich Leonhardt-Weber, B., Die Entwicklung der Qualitătsmerkmale im Verkehr: Eine Analyse vor dem Hintergrund der technischen, wirtschaftlichen und gesellschaftlichen Entwicklung, a.a.O., S. 24 ff.; Stauss, B., Dienstleister und die vierte Dimension, in: HM, Jg. 13, H. 2, 1991, S. 81 ff. und Zielort herzustellen, umschrieben. Diese stellt die Voraussetzung für einen flächendeckenden Verkehr dar (sog. Netzbildungsfähigkeit). 
rierte Leistung stellen zentrale Voraussetzungen für eine zielgruppengerechte Ausrichtung eines Verkehrsunternehmens dar.

So kann beispielsweise die aktuelle Pkw-Verfügbarkeit einen entscheidenden Einfluß auf die Verkehrsmittelwahl ausüben. „Wer sich einen Personenkraftwagen angeschafft hat, ist in bezug auf seine Verkehrsmittel weitgehend festgelegt. ${ }^{\text {"83 }}$ In dieser Aussage offenbart sich der Problemkreis des Substitutionswettbewerbs, der in besonderer Weise auf den Schienenverkehr zutrifft. So zeigen empirische Analysen, daß bei $94 \%$ der Bahnreisen, bei $65 \%$ der Pkw-Reisen und bei $48 \%$ der Flugreisen ein Wechsel zu anderen Verkehrsmitteln grundsätzlich für möglich gehalten wird. ${ }^{84}$ Obwohl die Bahn in diversen empirischen Analysen bei den als am wichtigsten eingestuften Merkmalen Sicherheit, Pünktlichkeit und Umweltverträglichkeit durchgehend besser als das Auto beurteilt wird, zeigen nicht zuletzt die aktuellen Marktanteile der Verkehrsträger, daß bei der letztendlichen Verkehrsmittelwahl weitere Merkmale herangezogen werden, bei denen insbesondere der Pkw als besser eingestuft wird. Aspekte wie die Flexibilität, der Gesamtzeitaufwand oder das Preisniveau sind in diesem Zusammenhang zu nennen. ${ }^{85}$ Diese Erkenntnisse sind entsprechend bei der Entwicklung eines Operationalisierungsansatzes der Kundenzufriedenheit zu berücksichtigen.

Insbesondere für Bahnreisende, die über einen Pkw verfügen, existieren vergleichsweise geringe Wechselbarrieren. Wie gezeigt, ist die Verkehrsmittelwahl zudem sehr situationsabhängig. Daher ist davon auszugehen, daß nur ausgesprochen zufriedene Kunden ein höheres Maß an Loyalität gegenüber der Bahn zeigen und daher die in anderen Dienstleistungsbereichen (z.B. Finanzsektor) zu beobachtende Verhaltensweise „Risikoreduktion durch Loyalität" ${ }^{486}$ nur eingeschränkt gegeben ist.

Schmidt, F.K., Das Verkehrsangebot „Bahn“, in: Tourismus-Management: Tourismus-Marketing und Fremdenverkehrsplanung, Haedrich, G., Kaspar, C., Kleinert, H., Klemm, K. (Hrsg.), Berlin, New York 1983, S. 160. an einem Beispiel zentraler EDV-Dienste, a.a.O., S. 32 f. 


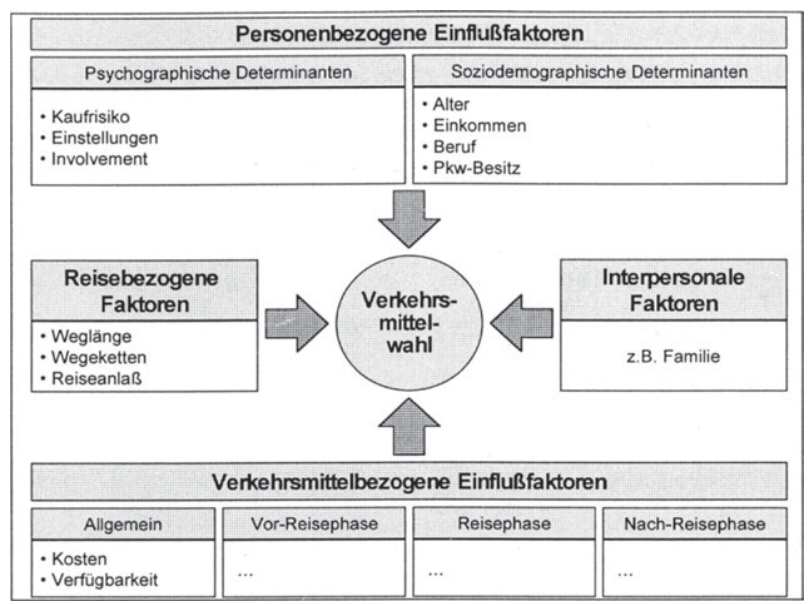

\section{Abb. 5: Determinanten der Verkehrsmittelwahl}

Bedingt durch die Komplexität von Verkehrsdienstleistungen ist das Verkehrsmittelwahlverhalten, die Frequenz der spezifischen Verkehrsmittelnutzung und die hieraus resultierende Kundenzufriedenheit mit einer Verkehrsdienstleistung nur durch das Zusammenwirken verschiedener Einflußfaktoren und deren unterschiedliche Gewichtung durch die Konsumenten zu erklären (vgl. Abbildung 5). Wie die bisherigen Ausführungen gezeigt haben, spielen personen- und reisebzw. verkehrsmittelbezogene Aspekte bei der Entscheidung für ein Verkehrsmittel ebenso eine zentrale Rolle wie für die Zufriedenheit mit diesem Verkehrsmittel. Während unter personenbezogenen Bestimmungsfaktoren psychographische (z.B. Einstellungen zu einem bestimmten Verkehrsmittel) und soziodemographische Determinanten (z.B. Alter und Einkommen der Konsumenten) zu verstehen sind, beziehen sich reisebezogene Einflußfaktoren auf Aspekte wie den Reiseanlaß oder die Länge der Fahrtstrecke. Verkehrsmittelbezogene Einflußfaktoren stellen z.B. der Preis, die Verfügbarkeit oder die mit der Wahl für ein bestimmtes Verkehrsmittel verbundene Reisezeit dar. ${ }^{87}$

87 Vgl. Bamberg, S., Zeit und Geld: Empirische Verhaltenserklärung mittels Restriktionen am Beispiel der Verkehrsmittelwahl, in: ZUMA-Nachrichten, Jg. 38, 20. Mai 1996, S. 10; Bamberg, S., Bien, W., Angebot (des OV) nach Wunsch (des MIV-Nutzers), in: IWW, Jg. 47, H. 3, 1995, S. 111; Bamberg, S., Schmidt, P., Verkehrsmittelwahl: Eine Anwendung der Theorie des geplanten Verhaltens, in: Zeitschrift für Sozialpsychologie, Jg. 24, 1993, S. 28; Wilken, D., Kriterien der Verkehrsmittelwahl im Personenfernverkehr und ihre Bedeutung in Verkehrsnachfragemodellen, in: IVW, Jg. 26, H. 1, 1974, S. 4 f. 
Aus der Analyse des spezifischen Charakters von Verkehrsdienstleistungen lassen sich wichtige Anforderungen an einen Ansatz zur Analyse der Kundenzufriedenheit mit Verkehrsdienstleistungen ableiten. So gilt es insbesondere, dem Episodencharakter und den damit verbundenen Schnittstellenproblemen explizit Rechnung zu tragen. Ebenso ist eine möglichst vollständige Erfassung des Kundenprozesses und der Determinanten der Verkehrsmittelwahl anzustreben.

Die Ausführungen haben gezeigt, daß die Bereiche Personal, tangibles Umfeld, die Kunden selbst sowie die unterschiedlichen Zeitkomponenten des Dienstleistungserstellungsprozesses die Basis zur Ableitung von Erklärungs- und Bestimmungsfaktoren der Kundenzufriedenheit mit Verkehrsdienstleistungen darstellen. Bevor im folgenden theoretische Konzeptionen der Kundenzufriedenheit im Dienstleistungsbereich einer eingehenden Analyse unterzogen werden, gibt Abbildung 6 die ermittelten Anforderungen an einen Operationalisierungsansatz der Kundenzufriedenheit in einem Überblick wieder.

\begin{tabular}{|c|c|c|c|}
\hline \multicolumn{2}{|c|}{ Allgemeine Anforderungen } & \multirow{2}{*}{$\begin{array}{l}\text { Generelle dienstleistungs- } \\
\text { spezifische Anforderungen }\end{array}$} & \multirow{2}{*}{$\begin{array}{l}\text { Verkehrsdienstleistungs- } \\
\text { spezifische Anforderungen }\end{array}$} \\
\hline $\begin{array}{l}\text { auf der theoretischen } \\
\text { Sprachebene }\end{array}$ & $\begin{array}{l}\text { auf der empirischen } \\
\text { Sprachebene }\end{array}$ & & \\
\hline $\begin{array}{l}\text { - Vollständigkeit } \\
\text { - Adaptionsfähigkeit } \\
\text { - Einfachheit/ } \\
\text { Handhabbarkeit } \\
\text { - Prüfbarkeit } \\
\text { - wissenschaftliche } \\
\text { Bewährung }\end{array}$ & $\begin{array}{l}\text { - Eindeutigkeit und } \\
\text { Zweckmäßigkeit } \\
\text { der Indikatoren } \\
\text { und Konstrukte } \\
\text { - Begründete Fest- } \\
\text { legung der } \\
\text { Indikatoren } \\
\text { (Adäquanz) } \\
\text { - Bestimmung eines } \\
\text { geeigneten Meß- } \\
\text { verfahrens } \\
\text { - Objektivităt, } \\
\text { Reliabilität, } \\
\text { Validităt des } \\
\text { Meßverfahrens }\end{array}$ &  & $\begin{array}{l}\text { - Umfassende Abbildung der aus } \\
\text { Kundensicht wahrgenommenen } \\
\text { Reisekette (Kundenprozeß) } \\
\text { - Berücksichtigung des Schnittstellen- } \\
\text { bzw. Episodencharakters } \\
\text { (intern/extern) } \\
\text { - Erfassung der Determinanten } \\
\text { der Verkehrswertigkeit und der } \\
\text { Verkehrsmittelwahl } \\
\text { - Berücksichtigung der spezifischen } \\
\text { Reisesituation }\end{array}$ \\
\hline
\end{tabular}

\section{Abb. 6: Anforderungen an einen Operationalisierungsansatz der Kunden- zufriedenheit mit Verkehrsdienstleistungen}




\section{Theoretische Konzeption von Kundenzufriedenheit im Dienst- leistungskontext}

\subsection{Beziehung der Kundenzufriedenheit zu verwandten Konstrukten}

In der wissenschaftlichen Literatur werden des öfteren die Begriffe Dienstleistungsqualität, Kundenzufriedenheit und Einstellungen als spezielle Form des jeweils anderen Konstrukts interpretiert und zuweilen sogar synonym verwandt. Ebenso finden sich zahlreiche Meinungen, welche die Selbständigkeit der Konstrukte betonen ${ }^{88}$ Es stellt sich also die Frage, inwiefern die Konzepte der Kundenzufriedenheit, der Dienstleistungsqualität und der Einstellungen verschiedenartig, überlappend oder deckungsgleich sind.

\subsection{Konzeptionelle Differenzierungspotentiale}

Unabhängig vom Dienstleistungsbezug ist eine Vielzahl von Qualitätsbegriffen entwickelt worden, die ihren Ursprung in sehr unterschiedlichen Forschungsrichtungen haben. ${ }^{89}$ Besondere Beachtung hat im Dienstleistungsbereich der kundenorientierte Qualitätsbegriff gefunden, der die Anforderungen des Kunden in den Mittelpunkt der Betrachtung stellt. ${ }^{90}$ In diesem Zusammenhang wird Dienstleistungsqualität häufig als die wahrgenommene Differenz zwischen den Erwartungen und der subjektiv erlebten Leistung interpretiert. ${ }^{91}$ Hier zeigt sich bereits die inhaltliche Nähe zum Begriff der Kundenzufriedenheit, die ebenso als das Ergebnis eines Soll-Ist-Vergleiches aufgefaßt wird. ${ }^{92}$ Diese Sichtweise weist

Zu einem Überblick über dieses Problemfeld vgl. Schütze, R., Kundenzufriedenheit: AfterSales-Marketing auf industriellen Märkten, a.a.O., S. $145 \mathrm{ff}$.; Haller, S., Beurteilung von Dienstleistungsqualităt: Dynamische Betrachtung des Qualitătsurteils im Weiterbildungsbereich, a.a.O., S. $43 \mathrm{ff}$.

89 Eine hăufig zitierte Systematisierung des Qualitătsbegriffes stellt der Ansatz von Garvin dar. Vgl. Garvin, D.A., What does "Product Quality" Really Mean?, in: SMR, Vol. 25, Fall 1984, S. $25 \mathrm{ff}$. Garvin unterscheidet in philosophisch/absolute („transcedent approach"), produktorientierte („product-based approach"), kundenorientierte ("user-based approach“), fertigungsbzw. herstellungsorientierte ("manufacturing-based approach") sowie wertorientierte ("valuebased approach") Ansätze. Zu einer Diskussion dieser Begriffe vgl. z.B. Haller, S., Beurteilung von Dienstleistungsqualităt: Dynamische Betrachtung des Qualitătsurteils im Weiterbildungsbereich, a.a.O., S. 7 ff.; Stauss, B., Hentschel, 8., Dienstleistungsqualităt, in: WiSt, Jg. 20, H. 5, 1991, S. 238 f.

90 Vgl. Benkenstein, M., Dienstleistungsqualităt: Ansătze zur Messung und Implikationen für die Steuerung, in: ZfB, Jg. 63, H. 11, 1993, S. 1100 f.; Bruhn, M., Hennig, K., Selektion und Strukturierung von Qualitătsmerkmalen - auf dem Weg zu einem umfassenden Qualitătsmanagement für Kreditinstitute (Teil 1), in: JdAV, Jg. 39, H. 3, 1993, S. 216 f.

91 Vgl. Lewis, R.C., Booms, B.H., The Marketing Aspects of Service Quality, in: Emerging Perspectives on Services Marketing, Berry, L.L., Shostack, L., Upah, G. (Hrsg.), Chicago 1983, S. 99; Parasuraman, A., Zeithaml, A., Berry, L.L., SERVQUAL: A Multiple-Item Scale for Measuring Consumer Perceptions of Service Quality, in: JoR, Vol. 64, No. 1, 1988, S. 17.

Vgl. hierzu die Ausführungen in Kap. A 3 dieser Arbeit. 
wiederum eine hohe Übereinstimmung mit der Auslegung des Einstellungskonstruktes nach dem bekannten TROMMSDORFF-Modell auf. ${ }^{93}$ Einstellungen werden hier ebenfalls als eine Differenzgröße gemessen. ${ }^{94}$

Zur Abgrenzung der Kundenzufriedenheit von den Konstrukten Dienstleistungsqualität und Einstellung werden in der Literatur verschiedene Argumente herangezogen. So wird der Kundenzufriedenheit eine größere Nähe zur Kaufentscheidung (Verhaltenswirksamkeit), eine größere Umsetzungsnähe, ein höherer Konkretisierungsgrad sowie eine geringere zeitliche Stabilität und vor allem die Notwendigkeit eines konkreten Konsumerlebnisses zugeschrieben, während diese Unterschiede zwischen der Dienstleistungsqualität und dem Einstellungskonzept nicht gesehen werden ${ }^{95}$ Hierin zeigt sich bereits, daß Einstellungen und Dienstleistungsqualität als in hohem Grad identische Konstrukte behandelt werden. Dies kommt auch in der Aussage von TROMMSDORFF zum Ausdruck, daß „aus Marketingsicht Qualität und Einstellungen eigentlich gleichgesetzt werden können. ${ }^{966}$ Damit erweist sich eine Abgrenzung der Kundenzufriedenheit von den beiden anderen Konstrukten auf der theoretischen Sprachebene als vergleichsweise unproblematisch. Eine nähere Analyse des Begriffes der Dienstleistungsqualität macht jedoch deutlich, daß die Grenzen zum Konstrukt der Kundenzufriedenheit in Abhängigkeit von der gewählten Operationalisierung auf der empirischen Sprachebene verschwimmen. So wird in der Literatur regelmäßig zwischen einstellungs- und zufriedenheitsorientierten Verfahren zur Messung der Dienstleistungsqualität unterschieden.$^{97}$ Während die genannten Unterschiede im

Vgl. Trommsdorff, V., Konsumentenverhalten, a.a.O., S. $137 \mathrm{ff}$.

Einstellungen werden in diesem Zusammenhang als innere, relativ dauerhafte Bereitschaften eines Individuums, sich gegenüber einem Objekt in einer bestimmten Weise konsistent positiv oder negativ zu verhalten, definiert. Vgl. Meffert, $H$., Marketingforschung und Käuferverhalten, a.a.O., S. $55 \mathrm{ff}$.

Vgl. z.B. Meyer, A., Ertl, R., Nationale Barometer zur Messung von Kundenzufriedenheit: Ein Vergleich zwischen dem "Deutschen Kundenbarometer - Qualităt und Zufriedenheit" und dem "American Customer Satisfaction Index (ASCl)", in: Grundsatzfragen und Herausforderungen des Dienstleistungsmarketing, Meyer, A. (Hrsg.), Wiesbaden 1996, S. 208; Eckert, St., Rentabilitätssteigerung durch Kundenbindung, a.a.O., S.69; Taylor, St.A., Baker, Th., An Assessment of the Relationship Between Service Quality and Customer Satisfaction in the Formation of Consumers' Purchase Intention, in: JoR, Vol. 70, No. 2, 1994, S. 165.

Trommsdorff, V., Konsumentenverhalten, a.a.O., S. 154. Hentschel präzisiert das Verhältnis von Einstellung und Qualităt, indem er argumentiert, daß der Qualitătsbegriff stets durch inhaltliche Vorgaben in Form einer spezifischen Zweck- oder Güteorientierung gekennzeichnet ist; eine Begrenzung, die das Konstrukt der Einstellung nicht erfăhrt. Vgl. Hentschel, B., Dienstleistungsqualităt aus Kundensicht: Vom merkmals- zum ereignisorientierten Ansatz, a.a.O., S. 120.

Vgl. z.B. Benkenstein, M., Dienstleistungsqualităt: Ansătze zur Messung und Implikationen für die Steuerung, a.a.O., S. $1101 \mathrm{f}$. 
Hinblick auf den einstellungsorientierten Begriff der Dienstleistungsqualität bestehen bleiben, stellt sich die Frage, inwiefern sich die zufriedenheitsorientierte Variante der Dienstleistungsqualität vom Konstrukt der Kundenzufriedenheit unterscheidet. HENTSCHEL rechtfertigt die Existenz dieser Begriffe mit dem Hinweis, daß auf diese Weise "die sinnvolle Unterscheidung zwischen der Informationsseite (Einstellungen, Zufriedenheit) und der Aktionsseite des Marketing (Qualitätsgestaltung der Dienst- oder Sachleistung) beibehalten werden kann. “98 Diese Argumentation erscheint jedoch nicht befriedigend. Vielmehr sind die zuweilen diffuse Verwendung der Begriffe Kundenzufriedenheit und Dienstleistungsqualität und die Ableitung z.T. widersprüchlicher Kausalzusammenhänge zwischen den Konstrukten auf die unterschiedliche Auslegung möglicher Differenzierungskriterien zurückzuführen. Diese bestehen u.a. in dem unterstellten Aggregationsniveau, der Unterscheidung der den Konstrukten inhärenten affektiven sowie kognitiven Elemente und der angenommenen unterschiedlichen Dimensionalität der Konstrukte. ${ }^{99}$

Traditionell wird Kundenzufriedenheit in Zusammenhang mit einer spezifischen Transaktion, Dienstleistungsqualität dagegen aus einer Globalperspektive betrachtet. ${ }^{100}$ Bei dieser Interpretation beruht Dienstleistungsqualität nicht auf einem bestimmten Konsumerlebnis, sondern auf der Summe aller bisherigen Konsumerlebnisse. ${ }^{101}$ Dementsprechend wäre ein Qualitätsurteil nur bei Vorliegen mehrerer Konsumerlebnisse möglich. HALLER weist in Anlehnung an HENTSCHEL jedoch darauf hin, daß Dienstleistungsqualität auch wahrgenommen werden kann, wenn nur ein oder auch überhaupt kein konkretes Verwendungserlebnis vorliegt und bezeichnet ein Qualitätsurteil in diesem Zusammenhang als situationsübergreifend. ${ }^{102}$ Beide Autoren argumentieren, daß ein Konsument z.B. ein Qualitäts-

98

Hentschel, B., Dienstleistungsqualităt aus Kundensicht: Vom merkmals- zum ereignisorientierten Ansatz, a.a.O., S. 120.

99 Ebenso wird der in beiden Konstrukten verwandte Erwartungsbegriff uneinheitlich verwendet. Vgl. hierzu die Ausführungen in Kap. B 2.2 dieser Arbeit.

100 Vgl. Parasuraman, A., Zeithaml, A., Berry, L.L., SERVQUAL: A Multiple-Item Scale for Measuring Consumer Perceptions of Service Quality, a.a.O., S. 16.

101

Vgl. Nader, G., Zufriedenheit mit Finanzdienstleistungen: Erfolgswirksamkeit, Messung, Modellierung, a.a.O., S. 21.

102 Vgl. Haller, S., Beurteilung von Dienstleistungsqualität: Dynamische Betrachtung des Qualitătsurteils im Weiterbildungsbereich, a.a.O., S. 46; Hentschel, B., Dienstleistungsqualităt aus Kundensicht: Vom merkmals- zum ereignisorientierten Ansatz, a.a.O., S. 121.

Hier wird erneut die inhaltliche Nähe der Dienstleistungsqualität zum Konstrukt der Einstellung deutlich, da auch zu deren Bildung kein konkretes Konsumerlebnis vorliegen muß. Vgl. Runow, H., Zur Theorie und Messung der Verbraucherzufriedenheit, a.a.O., S. 86. 
urteil über eine Fluggesellschaft fällen kann, ohne jemals ihre Angebote in Anspruch genommen zu haben und mit ihr geflogen zu sein. Um ein solches Qualitätsurteil von der Kundenzufriedenheit abzugrenzen, wird in der Literatur der Dienstleistungsqualität eine "outsider perspective" zugeordnet, während Kundenzufriedenheit aus einer "insider perspective" resultiert. ${ }^{103}$ Als problematisch enweist sich jedoch, daß auch jeweils umgekehrte Sichtweisen vertreten werden. So finden sich in der Literatur sowohl transaktionsbezogene Definitionen der Dienstleistungsqualität als auch eine Interpretation der Kundenzufriedenheit auf globaler Ebene. Ebenso sind Ansätze anzutreffen, innerhalb derer das gleiche Konstrukt auf beiden Ebenen betrachtet wird. ${ }^{104}$ Interessanterweise wird ein solcher Standpunkt inzwischen auch von renommierten Vertretern der Dienstleistungsqualität wie Parasuraman, ZeithamL, und Berry vertreten. ${ }^{105}$ Insgesamt kann festgehalten werden, daß beide Sichtweisen sowohl für den Begriff der Kundenzufriedenheit als auch für das Konzept der Dienstleistungsqualität eingenommen werden können, dieser Umstand aber die Abgrenzung deutlich erschwert. Da empirische Studien belegen, daß Kunden kaum zwischen Kundenzufriedenheit und Dienstleistungsqualität auf globaler Ebene unterscheiden (können), ${ }^{106}$ soll im folgenden Kundenzufriedenheit der klassischen Zufriedenheitsforschung folgend primär aus einer transaktionalen Perspektive betrachtet werden.

In der Wissenschaft besteht weitgehend Einigkeit darin, daß sowohl Dienstleistungsqualität als auch Kundenzufriedenheit je nach dem Grad des Involvements von mehr oder minder starken affektiven Komponenten durchsetzt sind und damit sowohl kognitive als auch affektive Elemente enthalten. ${ }^{107}$ So konnte in diversen empirischen Studien die affektive Komponente bei der Zufriedenheitsbildung, die von einigen Autoren als die zentrale Größe des Zufriedenheits-

Vgl. Strandvik, T., Liljander, V., A Comparison of Episode Performance and Relationship Performance for a Discrete Service, in: Dienstleistungsmarketing: Konzeptionen und Anwendungen, Kleinaltenkamp, M. (Hrsg.), Wiesbaden 1995, S. 119.

Vgl. Bitner, M.J., Hubbert, A.R., Encounter Satisfaction Versus Overall Satisfaction Versus Quality: The Customer's Voice, a.a.O., S. 76 f.; Anderson, E.W., Fornell, C., Lehmann, D.R., Customer Satisfaction, Market Share, and Profitability: Findings from Sweden, a.a.O., S. $54 \mathrm{ff}$.

Vgl. Parasuraman, A., Zeithaml, V.A., Berry, L.L., Reassessment of Expectations as a Comparison Standard in Measuring Service Quality: Implications for Further Research, in: JoM, Vol. 58, January, 1994, S. 122.

Vgl. Bitner, M.J., Hubbert, A.R., Encounter Satisfaction Versus Overall Satisfaction Versus Quality: The Customer's Voice, a.a.O., S. 78 ff.; Spreng, R.A., Singh, A.K., An Empirical Assessment of the Servqual Scale and the Relationship Between Service Quality and Satisfaction, in: AMA, Summer 1993, S. $1 \mathrm{ff}$.

$\mathrm{Vgl}$. die Ausführungen in Kap. A 3 dieser Arbeit. 
konstruktes angesehen wird, ${ }^{108}$ nachgewiesen werden. Da die gängige Definition der Dienstleistungsqualität ebenfalls auf einer kundenseitigen Evaluation beruht, werden auch diesem Konstrukt affektive Elemente zugestanden. Ex definitione sind hier aber die kognitiven Elemente stärker ausgeprägt. Dies ist auch darauf zurückzuführen, daß Dienstleistungsqualität in erster Linier aus einer globalen Perspektive interpretiert wird und somit eine „objektivere", kognitive Beurteilung unterstellt wird. ${ }^{109}$ Es ist jedoch $\mathrm{zu}$ beachten, daß die hier dargestellte Unterscheidung an Trennschärfe verliert, wenn situative Einflüsse in die Betrachtung einbezogen werden. Dabei ist hinsichtlich der Kundenzufriedenheit eine Indifferenz- bzw. Toleranzzone zu berücksichtigen. Damit ist derjenige Bereich gemeint, innerhalb dessen die negative Nichtbestätigung der Kundenerwartungen noch toleriert wird, es also noch nicht zu Unzufriedenheit kommt. Bei hohem Involvement fällt dieser Bereich relativ klein aus, die Zufriedenheit des Kunden reagiert schon bei kleinen Abweichungen sensibel. ${ }^{110}$ Daraus kann gefolgert werden, daß insbesondere in Situationen mit hohem emotionalen Potential die affektive Komponente an Bedeutung gewinnt, Kundenzufriedenheit ansonsten ähnlich wie Dienstleistungsqualität als vornehmlich kognitiver Prozeß interpretiert werden kann. ${ }^{111}$ Hohes Involvement fördert damit das bewußte und intensive Erleben von Zufriedenheit und damit die Verhaltensrelevanz von Kundenzufriedenheit. ${ }^{112}$

In der Literatur zum Dienstleistungsmarketing findet sich eine Vielzahl konzeptioneller Ansätze zur Bestimmung zentraler Dimensionen, die dem Konstrukt der Dienstleistungsqualität zugrunde liegen. An dieser Stelle soll jedoch auf eine detaillierte Auseinandersetzung mit diesen Modellen und Dimensionenkatalogen verzichtet werden, da dies bereits an anderer Stelle ausführlich erfolgt ist. ${ }^{113}$

Zu einem Überblick vgl. Nader, G., Zufriedenheit mit Finanzdienstleistungen: Erfolgswirksamkeit, Messung, Modellierung, a.a.O., S. 22.

Vgl. Nader, G., Zufriedenheit mit Finanzdienstleistungen: Erfolgswirksamkeit, Messung, Modellierung, a.a.O., S. 23.

$110 \mathrm{Vgl}$. Johnston, R., The Zone of Tolerance: Exploring the Relationship Between Service Transactions and Satisfaction with the Overall Service, a.a.O., S. $47 \mathrm{ff}$; Miller, J.A., Studying Satisfaction, Modifying Models, Elicting Expectations, Posing Problems, and Making Meaningful Measurements, in: Conceptualization and Measurement of Consumer Satisfaction and Dissatisfaction, Hunt, H.K. (Hrsg.), Cambridge 1977, S. 79 f.

111 Vgl. Dabholkar, P.A., Customer Satisfaction and Service Quality: Two Constructs or One?, in: AMA, Summer 1993, S. $12 \mathrm{ff}$.

112 Vgl. Day, R.L., Toward a Process Model of Consumer Satisfaction, in: Conceptualization and Measurement of Consumer Satisfaction and Dissatisfaction, Hunt, H.K. (Hrsg.), Cambridge, Mass. 1977, S. $169 \mathrm{ff}$.

Vgl. z.B. Haller, S., Beurteilung von Dienstleistungsqualităt: Dynamische Betrachtung des Qualitätsurteils im Weiterbildungsbereich, a.a.O., S. $65 \mathrm{ff}$;; Güthoff, J., Qualităt komplexer Dienstleistungen: Konzeption und empirische Analyse der Wahrnehmungsdimensionen, 
Exemplarisch soll hier nur auf das wohl bekannteste Konzept, den SERVQUALAnsatz, verwiesen werden. PARASURAMAN, ZEITHAML und BERRY ermittelten in Rahmen einer empirischen Studie die Qualitätsdimensionen Annehmlichkeit des tangiblen Umfeldes, Zuverlässigkeit, Reaktionsfähigkeit, Leistungskompetenz und Einfühlungsvermögen. ${ }^{114}$ Es ist offensichtlich, daß solche Dimensionen, in denen sich die einzelnen Eigenschaften einer Dienstleistung, wie z.B. die Höflichkeit des Personals, wiederfinden, sowohl auf die Kundenzufriedenheit als auch auf die wahrgenommene Dienstleistungsqualität Einfluß nehmen.

Im Gegensatz zur Dienstleistungsqualität werden der Kundenzufriedenheit jedoch weitere, über die spezifischen Dimensionen der Leistung hinausgehende Zufriedenheitsdimensionen zugeordnet. ${ }^{115}$ Diese stehen in keinem direktem Zusammenhang mit der Dienstleistung, können aber dennoch einen zentralen Einfluß auf den Prozeß der Zufriedenheitsbildung ausüben. So ist es denkbar, daß z.B. eine unbefriedigende Anreise oder Parkplatzsuche die Wahrnehmung einer Verkehrsdienstleistung nachhaltig beeinflussen, obwohl sie nicht unmittelbar die Qualität der eigentlichen Leistung, die Beförderung, berühren. Diese Zusammenhänge werden in Abbildung 7 graphisch veranschaulicht. Aufgrund der hohen Identität von Dienstleistungsqualität und Einstellungen beschränkt sich die Darstellung auf Differenzierungspotentiale von Kundenzufriedenheit und Dienstleistungsqualität.

a.a.O., S. 43 ff.; Scharitzer, D., Dienstleistungsqualităt - Kundenzufriedenheit, Wien 1994, S. $87 \mathrm{ff}$.

114 Vgl. Parasuraman, A., Zeithaml, A., Berry, L.L., SERVQUAL: A Multiple-Item Scale for Measuring Consumer Perceptions of Service Quality, a.a.O., S. $12 \mathrm{ff}$.

115 Vgl. Rust, R.T., Oliver, R.L., Service Quality: Insights and Managerial Implications from the Frontier, in: Service Quality: New Directions in Theory and Practice, Rust, R.T., Oliver, R.L. (Hrsg.), Thousand Oaks u.a. 1994, S. 6 und die dort angegebene Literatur. 




Abb. 7: Differenzierungspotentiale von Kundenzufriedenheit und Dienstleistungsqualität

Angesichts der konzeptionellen Unterschiede der Konstrukte wird im folgenden untersucht, in welchem Verhältnis Kundenzufriedenheit und Dienstleistungsqualität, insbesondere vor dem Hintergrund einer zeitbezogenen Entwicklung des Zufriedenheitsurteils, zueinander stehen.

\subsection{Analyse der kausalen Beziehungen der Konstrukte}

In der Literatur finden sich z.T. widersprüchliche Aussagen über den Kausalzusammenhang von Kundenzufriedenheit und Dienstleistungsqualität. Während Zufriedenheitsforscher die Qualität als Einflußfaktor der Zufriedenheit betrachten, nehmen Qualitätsforscher häufig die umgekehrte Sichtweise ein. ${ }^{116}$ Die Ursachen

116 Zu einem Überblick vgl. Esch, F.-R., Billen, P., Ansätze zum Zufriedenheitsmanagement: Das Zufriedenheitsportfolio, in: Kundennähe realisieren, Tomczak, T., Belz, Ch. (Hrsg.), St. Gallen 1994, S. 411. 
für diesen Unterschied sind primär darauf zurückzuführen, daß die Diskussion auf unterschiedlichen Ebenen geführt wird. Einige Autoren argumentieren in Abhängigkeit davon, ob eine transaktionale oder globale Sichtweise der Konstrukte eingenommen wird. Eine andere Gruppe von Forschern dagegen leitet die Kausalzusammenhänge aus den den Konstrukten zugeordneten Einflußfaktoren („antecedents“) ab.

Die erste Gruppe von Autoren ist der Meinung, daß sich Qualitätsurteile aus der Summe der bisherigen (zufriedenheitsrelevanten) Erfahrungen mit einem Anbieter ergeben. ${ }^{117}$ Hierin spiegelt sich die transaktionale Sichtweise der Kundenzufriedenheit und die Behandlung der Dienstleistungsqualität als einstellungsähnliches, vergleichsweise stabiles Globalurteil wieder. Qualität wird demzufolge als das übergeordnete Konstrukt gesehen und daher die Zufriedenheit als eine ihrer Einflußgrößen. HALLER hält folglich die Annahme, daß ein stabiles Konstrukt in einem instabilen mündet, für paradox. ${ }^{118}$

Eine andere Meinung wird dagegen von denjenigen Autoren vertreten, die anhand der den Konstrukten zugemessenen Einflußfaktoren argumentieren. Hinsichtlich der Zahl der Einflußfaktoren wird grundsätzlich die Meinung vertreten, daß "quality has fewer conceptual antecedents." ${ }^{119}$ Es wird daher der Schluß gezogen, daß die Dienstleistungsqualität in die Kundenzufriedenheit einfließt, da diese mehr (insbesondere affektive) Einflußfaktoren und die Qualität selbst enthält. Dementsprechend wird unterstellt, daß die Dienstleistungsqualität die Kundenzufriedenheit beeinflußt und diese daher das umfassendere Konstrukt darstellt. ${ }^{120}$

Beide Ausprägungen der hier postulierten Kausalzusammenhänge sind theoretisch vertretbar und konnten in verschiedenen Studien empirisch bestätigt werden. ${ }^{121}$ Die Ausführungen zeigen, daß beide Sichtweisen ihre Berechtigung haben

Vgl. z.B. Bitner, M.J., Evaluating Service Encounters: The Effects of Physical Surroundings and Employee Responses, in: JoM, Vol. 54, April 1990, S. 80; Oliver, R.L., A Conceptual Model of Service Quality and Service Satisfaction: Compatible Goals, Different Concepts, in: Advances in Service Marketing and Management: Research and Practice, Swart, T.A., Bowen, D.E., Brown, St.W. (Hrsg.), 2. Aufl., Greenwich, London 1993, S. 77.

118 Vgl. Haller, S., Beurteilung von Dienstleistungsqualităt: Dynamische Betrachtung des Qualitătsurteils im Weiterbildungsbereich, a.a.O., S. 48.

119 Rust, R.T., Oliver, R.L., Service Quality: Insights and Managerial Implications from the Frontier, a.a.O., S. 6. Vgl. hierzu auch die Ausführungen in Kap. B 2.2 dieser Arbeit. Vgl. Oliver, R.L., A Conceptual Model of Service Quality and Service Satisfaction: Compatible Goals, Different Concepts, a.a.O., S. 78.

121 Vgl. Bolton, R.N., Drew, J.H., A Multistage Model of Customers' Assessments of Service Quality and Value, in: JoCR, Vol. 17, March 1991, S. 375 ff.; Cronin, J.J., Taylor, St.A., Mea-

(Fortsetzung der Fußnote auf der nächsten Seite) 
und nicht in Konkurrenz zueinander gesehen werden sollten. Vielmehr ist eine integrierte Betrachtung vorzunehmen. So ist Kundenzufriedenheit der Dienstleistungsqualität in dem Sinne überzuordnen, daß die wahrgenommene Qualität eine (zentrale) Einflußgröße der Kundenzufriedenheit darstellt. Kundenzufriedenheit wiederum nimmt als das zeitlich weniger stabile (transaktionale) Konstrukt Einfluß auf zukünftige (globale) Qualitätsbeurteilungen. Diese Zusammenhänge sind in Abbildung 8 dargestellt. ${ }^{122}$

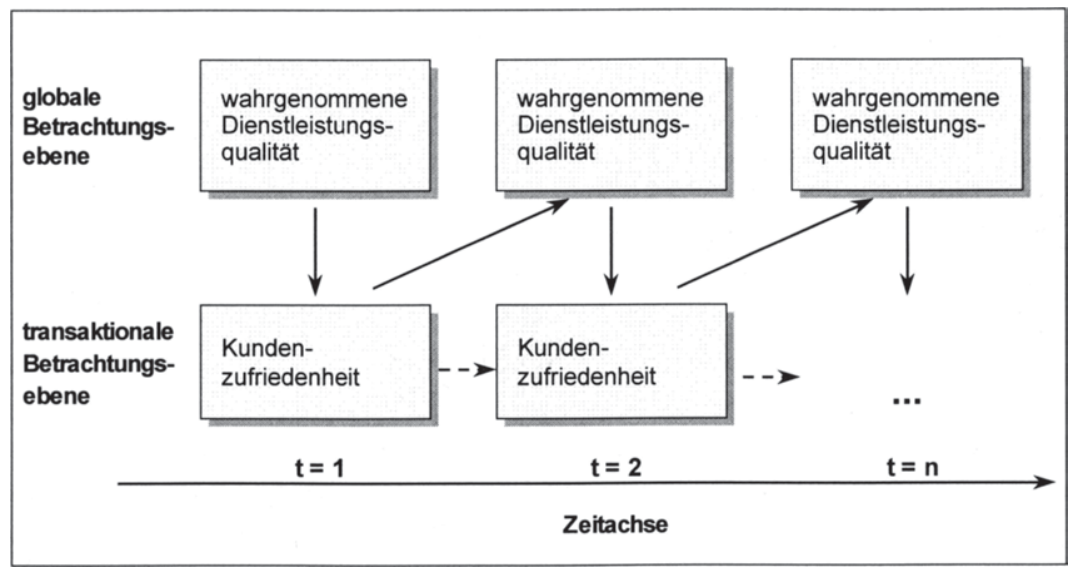

Abb. 8: Kausalzusammenhang von Kundenzufriedenheit und Dienstleistungsqualität

Es wird deutlich, daß nur in $t=1$, also zum Zeitpunkt eines erstmaligen Kontaktes mit einem Anbieter, eine eindeutige Kausalbeziehung im Sinne von „Qualität beeinflußt Kundenzufriedenheit" vorliegt. ${ }^{123}$ Ebenso beeinflußt die transaktionale Kundenzufriedenheit aus $t=1$ die Kundenzufriedenheit in $t=2 .{ }^{124}$ Sowohl eine einzelne Transaktion als auch die Summe aller bisherigen Erfahrungen mit dem Anbieter nehmen demnach Einfluß auf die wahrgenommene (globale) Dienst-

suring Service Quality: A Reexamination and Extension, in: JoM, Vol. 56, July 1992, S. 64 ff.; Parasuraman, A., Zeithaml, V.A., Berry, L.L., Reassessment of Expectations as a Comparison Standard in Measuring Service Quality: Implications for Further Research, a.a.O., S. $112 \mathrm{ff}$.

Zu einer empirischen Bestătigung dieser Zusammenhänge vgl. Taylor, St.A., Baker, Th., An Assessment of the Relationship Between Service Quality and Customer Satisfaction in the Formation of Consumers' Purchase Intention, a.a.O., S. $167 \mathrm{ff}$. 
leistungsqualität und die (transaktionale) Kundenzufriedenheit. Damit ist insgesamt festzuhalten, daß Kundenzufriedenheit und Dienstleistungsqualität zwei unterschiedliche, wenn auch verwandte, Konstrukte darstellen.

\subsection{Erklärungsansätze der Kundenzufriedenheit}

Im folgenden soll der Begriff der Kundenzufriedenheit mit Verkehrsdienstleistungen operationalisiert werden. Zu diesem Zweck werden zunächst zentrale Theorien und wesentliche Modellierungsrahmen der Kundenzufriedenheit in ihren Grundzügen vorgestellt.

\subsection{Grundlegende Theorien und Modellansätze}

Kundenzufriedenheit wird überwiegend als das Ergebnis eines komplexen psychischen Prozesses definiert. Vor allem in jüngerer Zeit wird die Zufriedenheit auch als die emotionale Reaktion auf diesen kognitiv geprägten Vergleichsprozeß bezeichnet. ${ }^{125}$ In diesem Zusammenhang wird jedoch davon ausgegangen, daß das Ergebnis des Vergleichsprozesses nicht zwingend mit der ursprünglichen Diskrepanz zwischen den Erwartungen, oder allgemeiner, dem Anspruchsniveau an eine Leistung (Soll-Komponente) und der wahrgenommenen Leistung (IstKomponente) übereinstimmt, sondern daß auch direkte Effekte von diesen Komponenten auf die Zufriedenheit ausgehen. ${ }^{126}$ Demzufolge wird die These aufgestellt, daß Folgereaktionen bzw. Rückkopplungseffekte denkbar sind, die zu einer nachträglichen Anpassung des Anspruchsniveaus und/oder der wahrgenommenen Ist-Leistung führen und damit einen Einfluß auf die Kundenzufriedenheit nehmen. Zur Erklärung dieser Wahrnehmungsprozesse werden unterschiedliche Theorien herangezogen. ${ }^{127}$

Nach der Assimilationstheorie erfolgt eine Korrektur der Wahrnehmung, um die Diskrepanz zu der Erwartung zu minimieren und somit kognitive Dissonanzen zu vermeiden. ${ }^{128}$ Die Kontrasttheorie stellt den Gegensatz zur Assimilationstheorie

Vgl. Homburg, Ch., Rudolph, B., Theoretische Perspektiven zur Kundenzufriedenheit, a.a.O., S. 31 und die dort angegebene Literatur.

Vgl. z.B. Bolton, R.N., Drew, J.H., A Multistage Model of Customers' Assessments of Service Quality and Value, a.a.O., S. $376 \mathrm{ff}$.

Vgl. Anderson, R.E., Consumer Dissatisfaction: The Effect of Disconfirmed Expectancy on Perceived Product Performance, in: JoMR, Vol. 10, February, 1973, S. $38 \mathrm{ff}$.

Nach der Theorie der kognitiven Dissonanz erstreben Individuen den Abbau psychischer Spannungen (Dissonanzen), um das innere Gleichgewicht wieder herzustellen. Vgl. Festinger, L., A Theory of Cognitive Dissonance, Stanford 1957, S. 3. 
dar. Hier wird davon ausgegangen, daß aufgrund der wahrgenommenen Diskrepanz die Leistung noch ungünstiger beurteilt wird und damit eine Vergrößerung der Diskrepanz erfolgt. Die Assimilations-Kontrast-Theorie vereint die Annahmen dieser beiden Theorien. Hier wird davon ausgegangen, daß die Beurteilung in Abhängigkeit von der Größe der wahrgenommenen Diskrepanz zwischen Anspruchsniveau und wahrgenommener Leistung stattfindet. Bei einer nur geringen Abweichung wird die Leistung noch assimiliert; wird dagegen der individuelle Annahmebereich in positiver oder negativer Hinsicht überschritten, tritt der Kontrasteffekt ein. ${ }^{129}$ Nach der Theorie der generellen Verneinung schließlich führt jede Form einer Abweichung von den Erwartungen, ob positiv oder negativ, zu einer ablehnenden Beurteilung. Eine Beurteilung dieser Ansätze erweist sich vor dem Hintergrund empirischer Untersuchungen und damit einhergehender unterschiedlicher Ergebnisse als problematisch. ${ }^{130}$ So kommt z.B. ANDERSON zu dem Ergebnis, daß die Anwendbarkeit der Ansätze insbesondere von der untersuchten Produktkategorie abhängt. ${ }^{131}$

Die unterschiedlichen Theorien werden vor allem im Zusammenhang mit dem "Confirmation/Disconfirmation-Paradigm"132 diskutiert, das die größte Verbreitung in der konzeptionellen und empirischen Zufriedenheitsforschung erfahren hat. Das Disconfirmation-Modell basiert auf zwei Prozessen, der Bildung eines Vergleichsstandards vor der Inanspruchnahme einer Leistung und der Bestätigung (Confirmation) bzw. Nichtbestätigung (Disconfirmation) des Vergleichsstandards durch die wahrgenommene Leistung. Dem Prozeß der (Nicht-) Bestätigung kommt dabei die Rolle einer vermittelnden Variablen zwischen den Konstruktkomponenten und der eigentlichen Zufriedenheit zu. Toleranzzone auf diesem Theorieansatz beruht.

130 heitsmessung als Informationsgrundlage des Hersteller- und Handelsmarketing am Beispiel der Automobilwirtschaft, a.a.O., S. 34.

131

132 $\mathrm{Vgl}$. Anderson, R.E., Consumer Dissatisfaction: The Effect of Disconfirmed Expectancy on Perceived Product Performance, a.a.O., S. 43.

Vgl. Oliver, R.L., A Cognitive Model of the Antecedents and Consequences of Satisfaction Decisions, in: JoMR, Vol. 17, November 1980, S. $460 \mathrm{ff}$. 
Weitere Ansätze, die auch im Kontext von (Verkehrs-)Dienstleistungen Berücksichtigung finden, stellen der Equity-Ansatz und die Attributionstheorie dar. ${ }^{133}$ Ebenso wie das Disconfirmation-Modell zählt auch das Equity-Modell zu der Gruppe der sogenannten Gleichgewichtsmodelle. ${ }^{134}$ Dieses Modell, dem in jüngerer Zeit im Zusammenhang mit der Zufriedenheitsforschung verstärkt Aufmerksamkeit gewidmet wird, beruht ebenfalls auf einem Vergleichsprozeß. Dessen Gegenstand bildet jedoch nicht eine bestimmte Leistung, sondern eine Austauschsituation. Hier wird unterstellt, daß ein Individuum eine distributive Gerechtigkeit erwartet und ein Kunde dementsprechend das eigene Input/Output-Verhältnis mit dem anderer Personen und/oder des Dienstleisters vergleicht. ${ }^{135}$ Die Wahrnehmung von Ungerechtigkeit innerhalb dieser Einsatz/Ergebnis-Verhältnisse hat einen negativen Einfluß auf die Kundenzufriedenheit. So ist es denkbar, daß z.B. im Falle einer Verkehrsdienstleistung von zwei Personen der gleiche Fahrpreis entrichtet worden ist, aber nur eine der beiden Personen einen Sitzplatz erhält oder eine ausführliche Beratung genießt. Dies kann Unzufriedenheit bei der anderen Person auslösen. ${ }^{136}$

In der neueren Forschungsliteratur wird darüber hinaus auch die Attributionstheorie ${ }^{137}$ zur Erklärung der Kundenzufriedenheit herangezogen. Attributionen stellen von Kunden wahrgenommene Ursachen für das eigene Verhalten und das Verhalten anderer dar. Attributionstheoretische Ansätze zielen auf kognitive Prozesse ab, auf deren Basis ein Kunde den Ort der Kausalität (intern, d.h. beim Kunden, oder extern), die Kontrollierbarkeit (durch den Anbieter beeinflußbar oder nicht) und die Stabilität (dauernd oder vorübergehend) von positiven und nega-

133 Einen umfassenden Überblick über die verschiedenen Ansătze und empirische Befunde liefern Agrawal, M., You haven't Seen All: Hypothesis for Extending Research on the Benefits of Customer Satisfaction, in: Marketing Today and for the 21st Century, Proceedings of the 24th Annual Conference of the European Marketing Academy, Bergadaa, M. (Hrsg.), Cergy 1995, S. $2 \mathrm{ff}$; Erevelles, S., Leavitt, C., A Comparison of Current Models of Consumer Satisfaction/Dissatisfaction, in: JoCS/D\&CB, Vol. 5, 1992, S. $104 \mathrm{ff}$.

Wăhrend Gleichgewichtsmodelle ausschließlich auf den Erkenntnissen der Zufriedenheitsforschung basieren, werden im Rahmen von sog. Integrationsmodellen auch Erkenntnisse über das individuelle Beschwerdeverhalten herangezogen. Vgl. Bruhn, M., Marketing und Konsumentenzufriedenheit, a.a.O., S. 302 f., der als weitere Gleichgewichtsmodelle das Normative-Deficit-Modell sowie das Composite-Modell nennt.

135 Vgl. Homburg, Ch., Rudolph, B., Theoretische Perspektiven zur Kundenzufriedenheit, a.a.O., S. 33 .

136 In neueren Ansătzen der Zufriedenheitsforschung findet der „Equity“-Gedanke gelegentlich auch im Rahmen des Disconfirmation-Paradigma Berücksichtigung. Hier wird vorgeschlagen, "Equity" als Soll-Komponente in das Modell zu integrieren. Zu unterschiedlichen Vergleichsstandards vgl. die Ausführungen in Kap. B 2.22 dieser Arbeit.

Vgl. Trommsdorff, V., Konsumentenverhalten, a.a.O., S. $261 \mathrm{ff}$. 
tiven Vorfällen bzw. Erlebnissen bei der Inanspruchnahme einer Dienstleistung abwägt. ${ }^{138}$

Empirische Untersuchungen der Zufriedenheit mit Flugdienstleistungen ergaben z.B., daß insbesondere Fälle, in denen die Verantwortung für den schlechten Verlauf einer Dienstleistung auf Seiten des Anbieters vermutet wurde, zu deutlicher Unzufriedenheit der Kunden führten. Passagiere, die davon ausgingen, daß die entsprechenden Fluggesellschaften einen Einfluß auf die Verspätung ihrer Flüge nehmen können, erwiesen sich als signifikant unzufriedener als solche Fluggäste, die die Verspätung als unvermeidbar einschätzten. ${ }^{139}$ Eine mögliche Folge von Attributionen besteht in einer Änderung des Erwartungsniveaus (Erhöhung oder Senkung) im Hinblick auf zukünftige Episoden oder Transaktionen.

Aufgrund seiner weiten Verbreitung und empirischen Bewährung bildet das Disconfirmation-Modell den gedanklichen Rahmen der weiteren Ausführungen. Daher werden das Modell und seine Komponenten im folgenden näher dargestellt.

\subsection{Analyse der Konstruktkomponenten und deren Einflußfaktoren im Disconfirmation-Paradigma}

Da das Zusammenspiel der Soll- und Ist-Komponente, die (Dis-)Confirmation, bereits in den Ausführungen zu den Theorien der Kundenzufriedenheit behandelt wurde, gilt es nun, sich näher mit dem konkreten Inhalt dieser Variablen des Zufriedenheitsprozesses zu beschäftigen. „Satisfaction is always judged in relation to a standard. " ${ }^{140}$ Wenngleich diese Aussage in der Literatur generell akzeptiert wird, können die Soll-Komponente bzw. der Vergleichsstandard im Disconfirmation-Modell durch sehr unterschiedliche Größen wie z.B. Erwartungen, Normen oder Wünsche auf der theoretischen Sprachebene operationalisiert wer-

Vgl. Oliver, R.L., DeSarbo, W.S., Response Determinants in Satisfaction Judgements, in: JoCR, Vol. 14, March 1988, S. 496.

139 Vgl. Bitner, M.J., Evaluating Service Encounters: The Effects of Physical Surroundings and Employee Responses, a.a.O., S. $72 \mathrm{ff}$; Taylor, Sh., Waiting for Service: The Relationship Between Delays and Evaluation of Services, in: JoM, Vol. 58, April 1994, S. $61 \mathrm{ff}$.

140 Olander, F., Consumer Satisfaction - A Sceptic's View, in: Conceptualization and Measurement of Consumer Satisfaction and Dissatisfaction, Hunt, H.K. (Hrsg.), Cambridge 1977, S. 412.
} 
den. ${ }^{141}$ So werden, wie in den bisherigen Ausführungen bereits implizit unterstellt, üblicherweise Erwartungen als Vergleichsmaßstab herangezogen (ExpectationDisconfirmation), die grundsätzlich als Ansichten und Meinungen über eine Dienstleistung oder über ein Produkt zu einem Zeitpunkt vor deren Inanspruchnahme interpretiert werden können. ${ }^{142}$ Darüber hinaus besteht weitgehend Einigkeit darin, daß Erwartungen durch eine Vielzahl von Bestimmungsfaktoren beeinflußt werden. Solche Faktoren stellen vor allem bisherige Konsumerfahrungen, die Kommunikation in der persönlichen Umgebung (mit Freunden, Verwandten, Kollegen) und das Wissen um Alternativen dar. Des weiteren sind Determinanten zu nennen, die unter dem direkten Einfluß des Anbieters stehen. In diesem Zusammenhang sind insbesondere der Preis sowie die Kommunikation des Leistungsversprechens im Rahmen der Werbung zu erwähnen. ${ }^{143}$

Im Hinblick auf den konkreten Inhalt bzw. die Beschaffenheit von Erwartungen wird in der Literatur eine Vielzahl von Konzepten zur Operationalisierung diskutiert. ${ }^{144}$ Am häufigsten werden „predictive expectations" genannt, in denen sich die auf Erfahrungen beruhenden angenommenen Wahrscheinlichkeiten einer bestimmten Ausprägung eines Merkmals oder Ereignisses als eine Art Durchschnittswert widerspiegeln ${ }^{145}$ und Erwartungen somit durch einen probalistischen Charakter geprägt sind. Darüber hinaus finden sich Ansätze, die sich an Idealvorstellungen oder als Gegenstück an Minimalanforderungen orientieren und damit dem Vergleichsmaßstab einen normativen Charakter verleihen. ${ }^{146}$ Ebenso

141 Vgl. Day, R.L., The Next Step: Commonly Accepted Constructs for Satisfaction Research, a.a.O., S. $113 \mathrm{ff}$.

Vgl. Haller, S., Beurteilung von Dienstleistungsqualität: Dynamische Betrachtung des Qualitătsurteils im Weiterbildungsbereich, a.a.O., S. 32; Boulding, W., Kalra, A., Staelin, R., Zeithaml, V.A., A Dynamic Process Model of Service Quality: From Expectations to Behavioral Intentions, in: JoMR, Vol. 30, February, 1993, S. 9.

Vgl. Burmann, Ch., Konsumentenzufriedenheit als Determinante der Marken- und Händlerloyalität, a.a.O., S. 250; Schütze, R., Kundenzufriedenheit: After-Sales-Marketing auf industriellen Märkten, a.a.O., S. $154 \mathrm{ff}$.

144

Zu einem Überblick vgl. Schütze, R., Kundenzufriedenheit: After-Sales-Marketing auf industriellen Märkten, a.a.O., S. 154 ff.; Liljander, V., Strandvik, T., Different Comparison Standards as Determinants of Service Quality, in: Dienstleistungsmarketing: Eine Bestandsaufnahme, Thelen, E.M., Mariamhof, G.B. (Hrsg.), Frankfurt am Main 1993, S. $131 \mathrm{ff}$.

Vgl. Hentschel, B., Dienstleistungsqualităt aus Kundensicht: Vom merkmals- zum ereignisorientierten Ansatz, a.a.O., S. 124.

146 Einige Autoren sehen hier eine Abgrenzungsmöglichkeit zwischen den Erwartungen, auf denen ein Zufriedenheitsurteil im Gegensatz zu einem Qualitatsurteil beruht. So werden die predictive expectations bzw. "would-expectations" der Zufriedenheit von sog. "shouldexpectations" der Dienstleistungsqualităt, die die Idealvorstellungen über eine Dienstleistung enthalten, abgegrenzt. Dieser Ansatz stieß jedoch auf Kritik, da sich auch in der Zufriedenheitsliteratur normative Standards finden. So beinhalten die sog. "experience based norms" auch die Erfahrungen, die mit anderen u. U. "besseren" Dienstleistern gemacht wurden. Dar-

(Fortsetzung der Fußnote auf der nächsten Seite) 
werden u.a. Erwartungen auch im Zusammenhang mit der erörterten Equity-Theorie im Sinne einer Abwägung von Kosten und Nutzen bei der Erwartungsbildung diskutiert. Da in der wissenschaftlichen Diskussion kein Konsens darüber besteht, welche Operationalisierungsform der Erwartungskomponente zu wählen ist, wird zuweilen in der Literatur die These aufgestellt, daß durch die Vielfalt an Erwartungskonzepten unterschiedliche Zufriedenheitskonstrukte analysiert und gemessen werden. ${ }^{147}$ Im Zuge dieser Diskussion wird in jüngerer Zeit insbesondere der Frage nachgegangen, inwieweit es überhaupt sinnvoll ist, davon auszugehen, daß ein Kunde nur einen Vergleichsmaßstab zur Bildung seines Zufriedenheitsurteils heranzieht und inwieweit eine explizite Operationalisierung von Erwartungen auf der empirischen Sprachebene erforderlich ist.

Grundsätzlich kann davon ausgegangen werden, daß jede der angesprochenen Erwartungsarten in der Realität anzutreffen ist und somit die Vergleichsstandards von Konsumenten für eine Dienstleistung interindividuell verschieden sind und in Abhängigkeit von der konkreten Konsumsituation und, z.B. beeinflußt durch neue Erfahrungen, auch im Zeitablauf intraindividuell divergieren. Des weiteren ist nicht auszuschließen, daß mehrere Standards gleichzeitig oder sequentiell existieren und die Zufriedenheit beeinflussen. So konnten TSE und WILTON im Rahmen einer empirischen Untersuchung den Nachweis erbringen, daß unterschiedliche Vergleichsniveaus (Equity, Erwartungen, Ideale) sowohl simultan als auch sequentiell von Kunden zur Beurteilung der Zufriedenheit herangezogen werden. ${ }^{148}$ Ebenso ist es durchaus denkbar, daß Kundenerwartungen nicht als Punktausprägungen auf einem Kontinuum, sondern vielmehr als Bandbreiten ausgeprägt sind, was Rückschlüsse auf die Existenz der bereits mehrfach angesprochenen Toleranzzone erlauben würde. Deren Größe wiederum kann in Abhängigkeit vom Involvement des Kunden und dem von ihm wahrgenommenen Risiko variieren.

über hinaus wird inzwischen auch im Rahmen der „should“-Komponente der Dienstleistungsqualităt zwischen dem sog. "desired service" und einem „adequate service" unterschieden und somit die "should"-Komponente in ihrer Ausprägung als Ideal-Erwartung relativiert. Vgl. zu dieser Diskussion insbes. Cronin, J.J., Taylor, St.A., Measuring Service Quality: A Reexamination and Extension, a.a.O., S. 56 ff. sowie Zeithaml, V.A., Berry, L.L., Parasuraman, A., The Nature and Determinants of Customer Expectations of Service, in: Managing Services Marketing: Text and Readings, Bateson, J.E.G (Hrsg.), 3. Aufl., Fort Worth u.a. 1995, S. 47 f.

Vgl. Kaas, K.P., Runow, H., Wie befriedigend sind die Ergebnisse der Forschung zur Verbraucherzufriedenheit?, a.a.O., S. 452.

148 Vgl. Tse, D.K., Wilton, P.C., Models of Consumer Satisfaction Formation: An Extension, a.a.O., S. $206 \mathrm{ff}$. 
Die Frage nach der Notwendigkeit einer expliziten Messung von Erwartungen gewinnt insbesondere dann an Bedeutung, wenn unterstellt wird, daß Zufriedenheit unmittelbar aus der Differenz von Soll- und Ist-Komponente resultiert. ${ }^{149}$ In der neueren Forschungsliteratur hat sich jedoch die Auffassung durchgesetzt, daß Zufriedenheit aus einer sich anschließenden Bewertung der wahrgenommenen Diskrepanz zwischen Soll und Ist entsteht. ${ }^{150}$ Dann ist jedoch unmittelbar der Frage nachzugehen, inwieweit die verschiedenen Komponenten des Zufriedenheitskonstruktes selbst auch einen direkten Einfluß auf die Zufriedenheit nehmen und inwieweit insbesondere eine separate Erfassung der Sollkomponente zu einer Verbesserung der Erklärung von Kundenzufriedenheit beiträgt. ${ }^{151} \mathrm{Im}$ Rahmen der Darstellung der theoretischen Grundlagen der Zufriedenheitsforschung wurde bereits darauf hingewiesen, daß sowohl von den Erwartungen als auch von der wahrgenommenen Leistung ein direkter Einfluß auf die Zufriedenheit ausgeht. ${ }^{152}$ Hier sei wieder beispielhaft auf die Untersuchung von TSE und WILTON hingewiesen, in der allein die wahrgenommene Leistung ca. $65 \%$ der Varianz der zusätzlich direkt erhobenen Gesamtzufriedenheit erklären konnte. ${ }^{153}$

Schütze bezeichnet diese Operationalisierungsform als die klassische Sichtweise von Kundenzufriedenheit. Vgl. Schütze, R., Kundenzufriedenheit: After-Sales-Marketing auf industriellen Märkten, a.a.O., S. 167.

Vgl. Dichtl, E., Schneider, W., Kundenzufriedenheit im Zeitalter des Beziehungsmanagement, a.a.O., S. 7; Cadotte, E.R., Woodruff, R.B., Jenkins, R.L., Expectations and Norms in Models of Consumer Satisfaction, in: JoMR, Vol. 24, August 1987, S. 307; Schütze, R., Kundenzufriedenheit: After-Sales-Marketing auf industriellen Märkten, a.a.O., S. 190 und 263.

Hieran wird mitunter ein weiterer Unterschied zum Konstrukt der Dienstleistungsqualităt ausgemacht, da diese ex definitione unmittelbar aus der wahrgenommen Differenz von Soll und Ist resultiert. Vgl. Esch, F.-R., Billen, P., Ansătze zum Zufriedenheitsmanagement: Das Zufriedenheitsportfolio, a.a.O., S. 411; Meyer, A., Ertl, R., Nationale Barometer zur Messung von Kundenzufriedenheit: Ein Vergleich zwischen dem "Deutschen Kundenbarometer - Qualität und Zufriedenheit" und dem "American Customer Satisfaction Index (ASCI)", a.a.O., S. 209.

Zu möglichen Ursachen eines unterschiedlichen Einflusses dieser Komponenten (neben dem Einfluß der Diskonfirmation) auf die Kundenzufriedenheit vgl. Gierl, H., Sipple, H., Zufriedenheit mit dem Kundendienst, in: JdAV, Jg. 39, H. 3, 1993, S. 244; McClure, N., The Role of Expectations, Ambiguity of Information, and Initial Impressions in Consumer Satisfaction Assessments: A Process Oriented Approach, Lubbock 1995, S. 23 ff.

$153 \mathrm{Vgl}$. Tse, D.K., Wilton, P.C., Models of Consumer Satisfaction Formation: An Extension, a.a.O., S. $206 \mathrm{ff}$. Zu weiteren Untersuchungen dieser Art vgl. Churchill, G.A., Surprenant, C., An Investigation into the Determinants of Customer Satisfaction, in: JoMR, Vol. 19, November 1982, S. $493 \mathrm{ff}$.; Liljander, V., Strandvik, T., The Relationship between Service Quality, Satisfaction and Intentions, Working Paper No. 243, Swedish School of Economics and Business Administration, Helsinki 1992, S. 13; Oliver, R.L., DeSarbo, W.S., Response Determinants in Satisfaction Judgements, a.a.O., S. $497 \mathrm{ff}$. 
Während die Diskussion der Soll-Komponente in der wissenschaftlichen Literatur breiten Raum einnimmt, wird der Operationalisierung der Ist-Komponente vergleichsweise wenig Aufmerksamkeit gewidmet. Grundsätzlich kann zwischen objektiver und subjektiver Leistung unterschieden werden. ${ }^{154}$ Da aber die Kundensicht für die empfundene Zufriedenheit ausschlaggebend ist und die "tatsächliche" (objektive) Leistung durch die oben aufgezeigten Wahrnehmungseffekte beeinflußt wird, liegt in aller Regel die vom Kunden (subjektiv) wahrgenommene Leistung Zufriedenheitsmodellen zugrunde. Als Bestimmungsfaktoren der wahrgenommenen Leistung werden regelmäßig Einstellungen und insbesondere die Qualität der Dienstleistung genannt. ${ }^{155}$ Darüber hinaus sind vor allem, wie bereits mehrfach angeklungen ist, situative Faktoren wichtige Determinanten der wahrgenommenen Leistung. In Abbildung 9 sind die bisherigen Erkenntnisse im Überblick dargestellt.

Unter Berücksichtigung der bisherigen Ausführungen liegt die Schlußfolgerung nahe, daß für die Operationalisierung der Kundenzufriedenheit auf empirischer Sprachebene auf eine getrennte Erfassung der Soll- und Ist-Komponente verzichtet und die Kundenzufriedenheit vielmehr direkt gemessen werden kann. ${ }^{156}$ „Insgesamt erscheint somit die direkte Erhebung ... der indirekten Messung ... überlegen, weil die Anwendbarkeits- und Operationalisierungsprobleme hinsichtlich der Idealvorstellungen und Erwartungen vermieden werden ... . Der möglicherweise geringere Informationswert der direkten Messung wird hierdurch überkompensiert. ${ }^{\text {157 }}$

154

Vgl. Homburg, Ch., Rudolph, B., Theoretische Perspektiven zur Kundenzufriedenheit, a.a.O., S. 39.

Bezüglich des unterstellten Einflusses von Einstellungen und insbesondere von der wahrgenommenen Dienstleistungsqualităt auf die Kundenzufriedenheit vgl. die Ausführungen in Kap. B 2.12 dieser Arbeit.

Vgl. Meyer, A., Ertl, R., Nationale Barometer zur Messung von Kundenzufriedenheit: Ein Vergleich zwischen dem "Deutschen Kundenbarometer - Qualităt und Zufriedenheit" und dem "American Customer Satisfaction Index (ASCI)", a.a.O., S. 212; Korte, Ch., Customer Satisfaction Measurement: Kundenzufriedenheitsmessung als Informationsgrundlage des Hersteller- und Handelsmarketing am Beispiel der Automobilwirtschaft, a.a.O., S. 40.

Hentschel, B., Dienstleistungsqualităt aus Kundensicht: Vom merkmals- zum ereignisorientierten Ansatz, a.a.O., S. 124 f. Inwieweit dieser Ansicht zuzustimmen ist, wird im Rahmen einer ausführlichen Auseinandersetzung mit alternativen Meßansătzen in Kap. B 3 dieser Arbeit überprüft. 


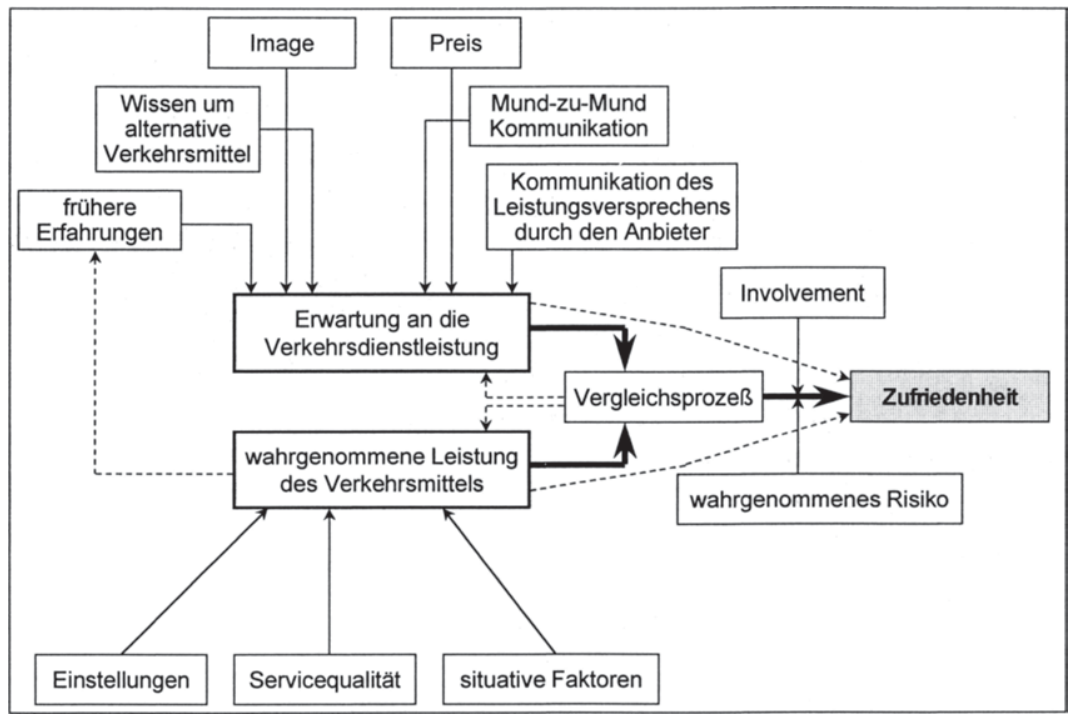

\section{Abb. 9: Erklärungsmodell der Kundenzufriedenheit}

(Quelle: In Anlehnung an Burmann, Ch., Konsumentenzufriedenheit als Determinante der Marken- und Händlerloyalität, a.a.O., S. 250)

In den bisherigen Ausführungen blieb die Frage nach dem letzten Element des Zufriedenheitsprozesses, dem Objekt der Kundenzufriedenheit, unbeantwortet. Die Sichtweise des Objektes der Zufriedenheit, des Konsumerlebnisses, ist jedoch für eine prozeßorientierte bzw. dynamische Auffassung der Kundenzufriedenheit von zentraler Bedeutung. Daher werden die verschiedenen in der Literatur diskutierten Erscheinungsformen der Kundenzufriedenheit sowie deren theoretische Grundlagen im folgenden differenziert dargestellt. 


\subsection{Erscheinungsformen der Kundenzufriedenheit}

Eine inhaltliche Konkretisierung der Konsumerlebniskomponente erfordert die Festlegung sowohl einer sachlichen als auch einer zeitlichen Dimension der Kundenzufriedenheit. Die Unterscheidung verschiedener sachlicher Bezugs- bzw. Aggregationsebenen der Kundenzufriedenheit erfolgt vornehmlich im Rahmen einer statischen Sichtweise. Fokus der Betrachtung ist der momentane Zufriedenheitszustand eines Kunden (Querschnittsanalyse). Im Rahmen einer prozeßorientierten und damit dynamischen Sichtweise von Kundenzufriedenheit sind dagegen die zeitlich aufeinander folgenden Konsumphasen bzw. Konsumakte Objekte der Zufriedenheit (Längsschnittanalyse).

\subsection{Psychologische Grundlagen der Wahrnehmung von Kunden- zufriedenheit}

Die Auseinandersetzung mit dem Konstrukt der Kundenzufriedenheit erfolgt in der wissenschaftlichen Literatur vornehmlich auf der grundsätzlichen Annahme einer merkmalsorientierten Wahrnehmung von Kundenzufriedenheit, die damit einer kognitiven Abbildung im menschlichen Gedächtnis unterliegt. ${ }^{158}$ Es wird davon ausgegangen, daß sich Erwartungen und Wahrnehmungen der Kunden auf einzelne Merkmale einer Leistung beziehen und ein Kunde dementsprechend differenzierte Merkmalszufriedenheiten empfindet. Aufbauend auf der jeweils unterstellten kognitiven Algebra (additives Modell, konjunktes Modell, diskonjunktes Modell) eines Individuums setzt sich dann die Gesamtzufriedenheit eines Kunden aus diesen (verdichteten) Teilzufriedenheiten zusammen. ${ }^{159}$ Dabei wird implizit unterstellt, daß die Wahrnehmung zufriedenheitsrelevanter Aspekte zeitunabhängig bzw. simultan erfolgt (statische Sichtweise), ${ }^{160}$ nicht zuletzt deshalb, weil die merkmalsorientierte Sichtweise ursprünglich für Sachgüter entwickelt wurde, also für Angebote, die sich durch ein vergleichbar hohes Maß an Sucheigenschaften auszeichnen. Dieser Ansatz wurde in der Vergangenheit häufig dennoch auch auf Dienstleistungen übertragen. Dementsprechend wird auch hier davon ausgegan-

diesem Problemkreis vgl. Kroeber-Riel, W., Konsumentenverhalten, 5. Aufl., München 1992, S. $311 \mathrm{ff}$. und 406 f.; Haller, S., Beurteilung von Dienstleistungsqualität: Dynamische Betrachtung des Qualitätsurteils im Weiterbildungsbereich, a.a.O., S. 27 ff.; Korte, Ch., Customer Satisfaction Measurement: Kundenzufriedenheitsmessung als Informationsgrundlage des Hersteller- und Handelsmarketing am Beispiel der Automobilwirtschaft, a.a.O., S. 35 ff. sowie die Ausführungen in Kap. B 3.221 dieser Arbeit.

$160 \mathrm{Vgl}$. McClure, N., The Role of Expectations, Ambiguity of Information, and Initial Impressions in Consumer Satisfaction Assessments: A Process Oriented Approach, a.a.O., S. 2. 
gen, daß sich das Zufriedenheitsurteil aus mehr oder weniger abstrakten und im Prinzip von der spezifischen Dienstleistung unabhängigen Dimensionen zusammensetzt. ${ }^{161}$

Dienstleistungen zeichnen sich aber dadurch aus, daß zu ihrer Beurteilung insbesondere Erfahrungseigenschaften herangezogen werden müssen, also Merkmale, in denen sich (auch) der Prozeßcharakter einer Dienstleistung widerspiegelt (z.B. Wartezeit beim Ticketerwerb). ${ }^{162}$ Wenngleich prozeßbezogene Merkmale in den verschiedenen entwickelten und z.T. auch empirisch fundierten dienstleistungsspezifischen Dimensionenkatalogen, wie z.B. dem bereits erwähnten Ansatz von PARASURAMAN et al., enthalten sind, so tragen diese Ansätze dem Episodencharakter und der Heterogenität von Dienstleistungen nicht ausreichend Rechnung. ${ }^{163}$ „Bei Dienstleistungen mit zeitlicher Ausdehnung des Dienstleister-KundeKontakts kann davon ausgegangen werden, daß sich die Qualitätsmerkmale entlang der Interaktionssequenz verteilen bzw. jeder einzelne „Kontaktpunkt“ ... potentiell qualitätsrelevant ist. "164

Vor diesem Hintergrund wird die rein merkmalsorientierte Sichtweise in jüngeren Forschungsarbeiten zum Dienstleistungsmarketing um eine ereignis- bzw. prozeßorientierte Sichtweise ergänzt. ${ }^{165}$ Eine theoretische Grundlage eines damit dynamischen Ansatzes besteht in dem Konzept der episodischen Informationsverarbeitung. ${ }^{166}$ Hier wird davon ausgegangen, daß das Zufriedenheitsurteil nicht auf dem Zusammenspiel zeitunabhängiger übergreifender Dimensionen beruht, sondern aus Einzelurteilen entsteht, die sich auf konkrete Episoden

161 Vgl. Güthoff, J., Qualität komplexer Dienstleistungen: Konzeption und empirische Analyse der Wahrnehmungsdimensionen, a.a.O., S. 78.

162 Vgl. hierzu die Ausführungen in Kap. A 2 dieser Arbeit.

163 Vgl. z.B. Güthoff, J., Qualität komplexer Dienstleistungen: Konzeption und empirische Analyse der Wahrnehmungsdimensionen, a.a.O., S. 78 und 88.

164 Hentschel, B., Dienstleistungsqualität aus Kundensicht: Vom merkmals- zum ereignisorientierten Ansatz, a.a.O., S. 73.

165 Vgl. Nader, G., Evaluation der Bankdienstleistungsqualităt mit Hilfe von Problementdeckungsverfahren, in: Der Markt, Jg. 32, H. 3, 1993, S. 152 ff.; Hentschel, B., Dienstleistungsqualităt aus Kundensicht: Vom merkmals- zum ereignisorientierten Ansatz, a.a.O., S. $155 \mathrm{ff}$. Durch die Hervorhebung spezifischer Ereignisse bzw. konkreter Prozeßabschnitte soll darüber hinaus der affektiven Komponente der Kundenzufriedenheit Rechnung getragen werden. Dieser Gedanke spiegelt sich auch in der zunehmenden Beschätigung mit der Attributionstheorie wider, die, wie gezeigt, auch auf bestimmte (positive oder negative) Erlebnisse und damit verbundene Emotionen wie Freude oder Ärger im Rahmen des Dienstleistungsprozesses abzielt.

166 Vgl. Hentschel, B., Dienstleistungsqualität aus Kundensicht: Vom merkmals- zum ereignisorientierten Ansatz, a.a.O., S. $158 \mathrm{ff}$ 
einer Dienstleistung beziehen. Dies bedeutet jedoch nicht, daß die in übergreifenden Dimensionen enthaltenen Kriterien, wie z.B. die Freundlichkeit des Personals, nicht auch im Kontext des ereignisorientierten Ansatzes Berücksichtigung finden, sondern daß diese vielmehr in den einzelnen Episoden unabhängig voneinander angewendet werden, wie am Beispiel einer Bahnreise und hier an den Abschnitten Schalterkontakt und Besuch des Zugrestaurants verdeutlicht werden kann. Im merkmalsorientierten Ansatz wird davon ausgegangen, daß die Freundlichkeit des Schalterbeamten sowie die des Servicepersonals zu einer Dimension Freundlichkeit verdichtet wird und als solche in das Zufriedenheitsurteil einfließt. Beim ereignisorientierten Ansatz dagegen erfolgt die Zufriedenheitsbildung durch eine Beurteilung der mit dem Schalterkontakt und dem Restaurantbesuch jeweils verbundenen Zufriedenheit. Die Zufriedenheit resultiert damit aus der Zufriedenheit mit den einzelnen Kontaktpunkten bzw. Episoden und den jeweils damit einhergehenden konkreten Merkmalen, wie z.B. der in den verschiedenen Abschnitten enthaltenen Zufriedenheit mit dem jeweiligen Bahnmitarbeiter.

In seinen Ausführungen zum Prinzip der episodischen Informationsverarbeitung unterscheidet HENTSCHEL in Anlehnung an TULVING zwischen der episodischen und der semantischen Informationsverarbeitung. ${ }^{167}$ Die Basis der episodischen Informationsverarbeitung stellen konkrete Ereignisse und Episoden dar, die als solche im Gedächtnis abgelegt werden. Den Inhalt der semantischen Informationsverarbeitung stellen dagegen kontextungebundene Begriffe und Vorstellungen (z.B. das Image eines bestimmten Verkehrsdienstleisters) dar. Diese Gedächtnissysteme sind allerdings nicht unabhängig voneinander. Vielmehr ist es durchaus denkbar, daß die episodischen Zufriedenheitsinformationen im Zeitablauf in das semantische Gedächtnis eingeordnet werden. Für eine erste Speicherung zufriedenheitsrelevanter Informationen im episodischen Gedächtnis sprechen die für viele Dienstleistungen typische Immaterialität und der hohe Interaktionsgrad zwischen Nachfrager und Anbieter. Nicht konkrete Eigenschaften bzw. Dimensionen, sondern vielmehr individuell erlebte Ereignissequenzen und von anderen Nachfragern erzählte Erlebnisse werden aufgenommen und verar-

Vgl. zum folgenden Hentschel, B., Dienstleistungsqualităt aus Kundensicht: Vom merkmalszum ereignisorientierten Ansatz, a.a.O., S. $158 \mathrm{ff}$; Tulving, E., How many Memory Systems are there?, in: American Psychologist, Vol. 40, No. 4, 1985, S. $385 \mathrm{ff}$. 
beitet und sind nur als solche abrufbar. ${ }^{168}$ HENTSCHEL vergleicht diese Art der Speicherung mit dem Eintrag von Informationen in ein Tagebuch mit konkreten zeitlichen und örtlichen Koordinaten. ${ }^{169}$ Im Rahmen der Erinnerung kann es im Zeitablauf zu Änderungen und Verzerrungen des Inhaltes solcher Informationen und zu einer Aufhebung der raumzeitlichen Bezüge kommen. Die Informationen werden in bereits vorhandene kognitive Strukturen des semantischen Gedächtnis eingeordnet und unterliegen damit weniger intensiven Vergessensprozessen. Der Inhalt des semantischen Systems besteht in dem grundsätzlichen Wissen eines Individuums über eine Dienstleistung. Dieses kann z.B. in eine globale Zufriedenheit bzw. Einstellung gegenüber einer Dienstleistung ohne konkreten Situationsbezug münden. Hier wird auch der Zusammenhang zwischen den beiden Gedächtnissystemen deutlich. Während das semantische Gedächtnis aus episodischen Informationen entsteht, kann dieses wiederum einen Einfluß auf die Strukturierung von episodischen Informationen ausüben. Wenngleich im Zeitablauf eine Einbettung episodischer Informationen in das semantische System erfolgt, ist dennoch die Kenntnis der Episodenzufriedenheiten von großer Bedeutung, da diese Einfluß auf die semantische Strukturen nehmen und sich somit als unmittelbar urteils- und handlungsrelevant erweisen können. ${ }^{170}$

Während die Erkenntnisse über das semantische System von Individuen damit mit dem rein merkmalsorientierten Ansatz der Zufriedenheitsforschung korrespondieren, kann das Konzept der episodischen Informationsverarbeitung als die psychologische Fundierung des ereignis- bzw. prozeßorientierten Ansatzes angesehen werden. Die Nähe des Merkmalsansatzes zum semantischen System wird besonders deutlich, wenn zu dessen Operationalisierung das aus der Psychologie bekannte hierarchische Modell herangezogen wird. ${ }^{171}$ Dieses basiert auf der Annahme, daß zentrale Eigenschaften bzw. Dimensionen z.B. von Dienstleistungen auf der höchsten Stufe und konkrete Merkmale auf tiefer liegenden Ebenen einer Wissenshierarchie repräsentiert werden.

Vgl. hierzu die Ausführungen in Kap. B 1.21 dieser Arbeit zu den Informationsquellen, die von Dienstleistungsnachfragern bevorzugt herangezogen werden.

Vgl. Hentschel, B., Dienstleistungsqualităt aus Kundensicht: Vom merkmals- zum ereignisorientierten Ansatz, a.a.O., S. 159.

$170 \mathrm{Vgl}$. McClure, N., The Role of Expectations, Ambiguity of Information, and Initial Impressions in Consumer Satisfaction Assessments: A Process Oriented Approach, a.a.O., S. 4; Hentschel, B., Dienstleistungsqualităt aus Kundensicht: Vom merkmals- zum ereignisorientierten Ansatz, a.a.O., S. 151 und 163.

171 Vgl. Hentschel, B., Dienstleistungsqualităt aus Kundensicht: Vom merkmals- zum ereignisorientierten Ansatz, a.a.O., S. 162 f. und die dort angegebene Literatur. 
Ähnlich wie das Konzept der episodischen Informationsverarbeitung stellt auch das „Service Script Concept" des Dienstleistungsmarketing auf den prozeduralen Charakter von Dienstleistungen ab. Hier wird davon ausgegangen, daß zufriedenheitsrelevante Informationen in Form von kognitiven Skripten strukturiert und gespeichert werden. ${ }^{172}$ Ein Skript stellt eine zusammenhängende Sequenz von Ereignissen bzw. Episoden dar, die ein Nachfrager bei der Inanspruchnahme einer Dienstleistung erwartet. Das Service Skript beinhaltet sowohl Informationen über das eigene Rollenverständnis eines Nachfragers als auch über dessen Erwartungen bezüglich des Verhaltens anderer (weiterer Nachfrager / Verhalten des Dienstleistungsanbieters). Es reflektiert die von einem Individuum erlernte oder angenommene Konzeption einer prototypischen Dienstleistungserfahrung. Hier sei z.B. die Untersuchung von BOWER, BLACK und TURNER erwähnt, die sich mit wahrgenommenen Ereignisabfolgen im Kontext von Restaurantbesuchen beschäftigt. ${ }^{173}$ Dabei ist zu beachten, daß Skripte sowohl auf Nachfrager- als auch auf Anbieterseite z.B. in Abhängigkeit von den Erfahrungen mit der Dienstleistung interindividuell verschieden sein können. Ebenso kann davon ausgegangen werden, daß die Skripte von Nachfragern nicht mit den Skripten der Mitarbeiter des Dienstleistungsanbieters übereinstimmen müssen. Abweichungen von den in den Skripten enthaltenen Erwartungen an die einzelnen Episoden einer Dienstleistung können Ursachen von Unzufriedenheit sein. ${ }^{174}$ Beispielhaft sei hier an die u.U. sehr unterschiedlichen Erwartungen von Konsumenten an das Verhalten des Personals beim Kleidungskauf gedacht. Während z.B. beim Kauf eines hochwertigen Anzugs die sofortige und intensive Beratung durch das Verkaufspersonal erwünscht sein kann, ist es ebenso denkbar, daß sich ein Nachfrager "ungestört" umsehen und seine Wahl alleine treffen möchte. Auch die auf Skripten basierende Sichtweise der Informationsverarbeitung und -speicherung legt somit eine an Episoden orientierte Auffassung von Kundenzufriedenheit im Dienstleistungskontext nahe.

172 Vgl. zum folgenden Solomon, M.R., Surprenant, C., Czepiel, J.A., Gutman, E.G., A Role Theory Perspective on Dyadic Interactions: The Service Encounter, a.a.O., S. $105 \mathrm{ff}$.; Smith, R.A., Houston, M.J., Script-Based Evaluations of Satisfaction with Services, a.a.O., S. $59 \mathrm{ff}$.

Vgl. Bower, G.H., Black, J.B., Turner, T.J., Scripts in Memory for Text, in: Cognitive Psychology, Vol. 11, No. 2, 1979, S. $177 \mathrm{ff}$.

$174 \mathrm{Vgl}$. McClure, N., The Role of Expectations, Ambiguity of Information, and Initial Impressions in Consumer Satisfaction Assessments: A Process Oriented Approach, a.a.O., S. 43. 
Im folgenden werden die in der Literatur anzutreffenden Forschungsansätze der statischen und insbesondere der dynamischen Sichtweise der Kundenzufriedenheit erörtert.

\subsection{Statischer Ansatz}

Eine erste Differenzierung unterschiedlicher Zufriedenheitsebenen im Rahmen einer statischen Betrachtung nimmt RENOUX vor, der zwischen einer Makro- und einer Mikroebene der Kundenzufriedenheit unterscheidet. ${ }^{175}$ Die Ausführungen zum Inhalt der Makrozufriedenheit sind stark durch die Gedanken der Konsumerismusbewegung der sechziger und siebziger Jahre geprägt. So zielt die Analyse der Makrozufriedenheit auf die allgemeine Versorgungszufriedenheit mit Gütern und Dienstleistungen ab. Sie stellt die Zufriedenheit mit dem gesamten Wirtschaftssystem oder bestimmten Branchen dar. ${ }^{176}$ Die Mikrozufriedenheit dagegen beinhaltet die Zufriedenheit mit konkreten Unternehmensleistungen. Eine Erweiterung erfährt dieser Ansatz durch MEFFERT und BRUHN, die eine Unterteilung der Mikrozufriedenheit vornehmen. ${ }^{177}$ Sie unterscheiden im Rahmen einer Hierarchie der Kundenzufriedenheit zwischen der Zufriedenheit mit einer Unternehmung, mit einer Produktgruppe bzw. einem bestimmten Dienstleistungsbereich sowie mit spezifischen Produkten oder Dienstleistungen. Dabei wird die Zufriedenheit mit einzelnen Produkt- bzw. Serviceeigenschaften auf der niedrigsten Hierarchieebene angesiedelt. In Abbildung 10 ist eine solche Zufriedenheitshierarchie beispielhaft für den Bereich von Verkehrsdienstleistungen dargestellt. Im Rahmen der statischen Ansätze wird davon ausgegangen, daß die verschiedenen Ebenen in einem interdependenten Verhältnis zueinander stehen und von Kunden differenziert wahrgenommen werden. ${ }^{178}$

Vgl. Renoux, Y., Consumer Dissatisfaction and Public Policy, in: Public Policy and Marketing Practices, Allvine, F.C. (Hrsg.), Chicago 1973, S. $55 \mathrm{ff}$.

Die Zufriedenheit mit einer Branche wird mitunter auch als Mesozufriedenheit bezeichnet. Vgl. Meyer, A., Ertl, R., Nationale Barometer zur Messung von Kundenzufriedenheit: Ein Vergleich zwischen dem "Deutschen Kundenbarometer - Qualităt und Zufriedenheit" und dem "American Customer Satisfaction Index (ASCI)", a.a.O., S. 207.

Vgl. Meffert, H., Bruhn, M., Beschwerdeverhalten und Zufriedenheit von Konsumenten, a.a.O., S. 598.

Vgl. Hansen, U., Schoenheit, I., Verbraucherzufriedenheit und Beschwerden - Strategische Herausforderungen für Unternehmen und Verbraucherorganisationen, in: Verbraucherzufriedenheit und Beschwerdeverhalten, Hansen, U., Schoenheit, I. (Hrsg.), Frankfurt am Main 1987, S. 17; Gierl, H., Sipple, H., Zufriedenheit mit dem Kundendienst, a.a.O., S. 245. 




Abb. 10: Hierarchie der Kundenzufriedenheit

(Quelle: In Anlehnung an Meffert, H., Bruhn, M., Beschwerdeverhalten und Zufriedenheit von Konsumenten, a.a.O., S. 598)

Die inhaltliche Nähe zu den Ausführungen zur merkmalsorientierten Sichtweise der Kundenzufriedenheit auf der einen Seite und der dargestellten Unterscheidung zwischen einer transaktionalen versus globalen (bzw. beziehungsorientierten) Betrachtung der Kundenzufriedenheit auf der anderen Seite ist hier offenkundig. So spiegelt sich in der Zufriedenheit mit einem Unternehmen die globale Sichtweise wider, in der sich die kumulierten Erfahrungen mit einem Unternehmen ausdrücken. Die transaktionale Betrachtung von Zufriedenheit weist wiederum enge Bezüge zu der Zufriedenheit auf Produkt- bzw. Dienstleistungsebene auf. Durch die explizite Betonung der Zufriedenheit auf der Merkmalsebene wird darüber hinaus die Verbindung zur merkmalsorientierten Auffassung von Kundenzufriedenheit und deren multiattributivem Charakter deutlich.

Dagegen wird dem Prozeßcharakter insbesondere von Dienstleistungen im Rahmen dieser Ansätze nicht Rechnung getragen. ,... this potential sequential and interactive dynamic component of $C S / D$ has been neglected in the $C S / D$ litera- 
ture."179 Aus diesem Grund sind in jüngerer Zeit zunehmend Bemühungen erkennbar, den Besonderheiten des Dienstleistungskonsums im Rahmen dynamischer Ansätze Rechnung zu tragen.

\subsection{Dynamischer Ansatz}

Einen ersten Schritt in Richtung einer dynamischen Analyse der Kundenzufriedenheit machte ebenfalls RENOUX, der im Rahmen der von inm formulierten Ebene der Mikrozufriedenheit drei idealtypische Kauf- bzw. Konsumphasen unterscheidet. ${ }^{180}$ Die "Shopping Satisfaction" beschreibt die Zufriedenheit eines Kunden mit den notwendigen Aktivitäten, die mit der Auswahl einer bestimmten Einkaufsstätte bzw. eines Anbieters verbunden sind (Vorkaufzufriedenheit). Die "Buying Satisfaction" umfaßt die Zufriedenheit mit den gesamten Erfahrungen, die beim eigentlichen Kauf gemacht werden (Kaufzufriedenheit). Die "Consuming Satisfaction" schließlich beinhaltet die Zufriedenheit mit der eigentlichen Nutzung (und anschließender Entsorgung) des Produktes als auch hiermit verbundener Serviceleistungen, wie z.B. dem Kundendienst (Nachkaufzufriedenheit). In dieser Aufteilung wird die inhaltliche Nähe zu den Ausführungen zur prozeßorientierten Sichtweise von Kundenzufriedenheit deutlich. Diese Betrachtung von Kundenzufriedenheit korrespondiert überdies mit der bereits vorgenommenen Unterscheidung in eine Vor-Reise-, eine Reise- und eine Nach-Reisezufriedenheit bei der Inanspruchnahme einer spezifischen Verkehrsdienstleistung. ${ }^{181}$ Des weiteren kann im Sinne des Beziehungsmarketing auf einer höheren Aggregationsstufe eine Betrachtung der Zufriedenheit mit zeitlich aufeinander folgenden Kauf- bzw. Konsumakten, die bei einem bestimmten Anbieter getätigt werden, stattfinden. Ebenso ist eine Analyse der Zufriedenheit auf der Makroebene denkbar. Hier würde die Zufriedenheit mit dem gesamten Wirtschaftssystem bzw. einer bestimmten Branche im Zeitablauf erfolgen. Damit wird deutlich, daß die statischen Ansätze nicht im Gegensatz zu den dynamischen Ansätzen stehen, sondern Zufriedenheit vielmehr aus einem anderen Blickwinkel beleuchten. Dieser Zusammenhang ist in Abbildung 11 dargestellt.

\footnotetext{
179 McClure, N., The Role of Expectations, Ambiguity of Information, and Initial Impressions in Consumer Satisfaction Assessments: A Process Oriented Approach, a.a.O., S. 42. 




Abb. 11: Integrierte Betrachtung der Erscheinungsformen von Kundenzufriedenheit

Die Felder I und II kennzeichnen die klassische statische Sichtweise der Kundenzufriedenheit auf unterschiedlichen Aggregationsebenen. Die Ausführungen zu den Komponenten des Disconfirmation-Modells haben jedoch gezeigt, daß Erwartungen und Wahrnehmungen keine statischen Größen darstellen, sondern sich im Zeitablauf verändern können. Damit erhält aber auch die Zufriedenheit selbst einen dynamischen Charakter. Die Analyse der Dynamik von Kundenzufriedenheit auf der höchsten Aggregationsstufe (Makroebene) stellen dabei die Felder III und V dar. Daneben kann in Anlehnung an die aufgestellte Prozeßhierarchie der Kundenzufriedenheit ${ }^{122}$ zwischen einer beziehungsspezifischen und einer transaktionsspezifischen Zufriedenheitsdynamik unterschieden werden (Mikroebene). Während erstere die Entwicklung der Zufriedenheit über mehrere Transaktionen beschreibt (Feld IV), besteht der Inhalt von Feld VI in der Analyse der Kundenzufriedenheit über die einzelnen Phasen einer spezifischen Transaktion bzw. eines Ausschnittes hieraus. In diesem Bereich liegt auch der Schwerpunkt der vorliegenden Untersuchung, also auf einer Analyse der Dynamik der Kundenzufriedenheit mit einer konkreten Transaktion im Verkehrsdienstleistungsbereich und der damit einhergehenden Wirkungseffekte. 


\subsection{Wirkungseffekte der Zufriedenheitsdynamik}

Während traditionelle Anwendungen des Disconfirmation-Modells Zufriedenheit als reines Nachkaufphänomen betrachten, ist für eine prozeßorientierte Untersuchung der Entwicklung der Zufriedenheit im Zeitablauf eine Dynamisierung des Modells erforderlich. ${ }^{183}$ Dies ist in Abbildung 12 beispielhaft für eine transaktionale Drei-Phasen-Betrachtung einer Verkehrsdienstleistung dargestellt. ${ }^{184}$

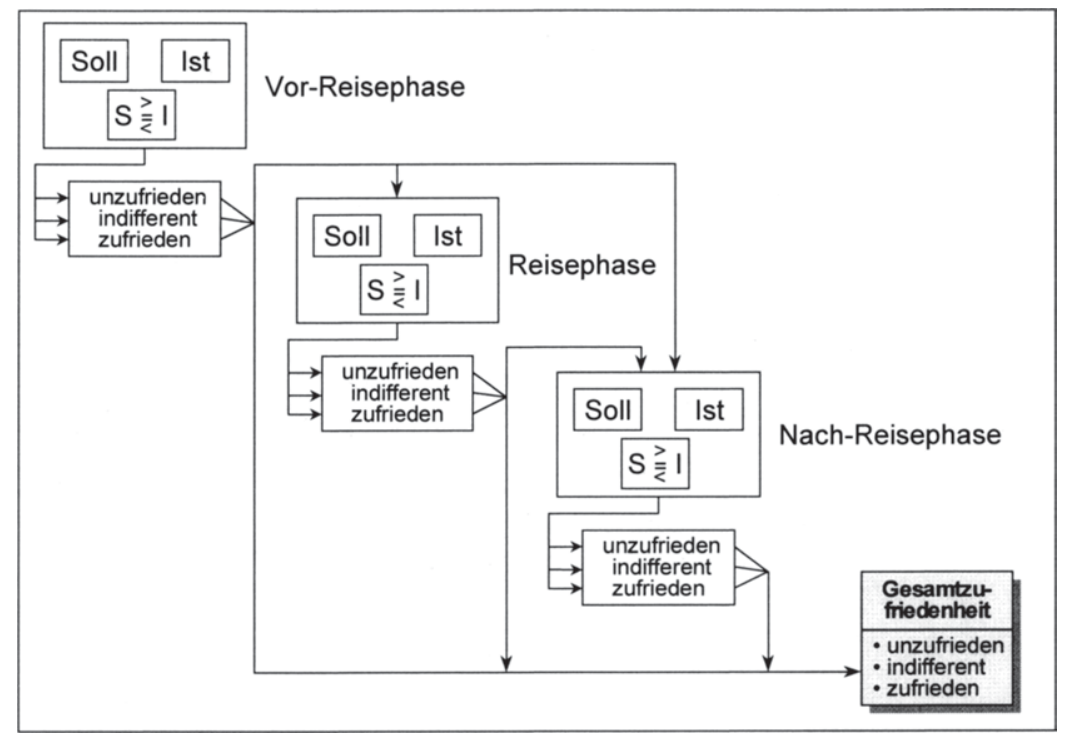

Abb. 12: Dynamisches Disconfirmation-Modell

(Quelle: In Anlehnung an Walker, J.L., Service Encounter Satisfaction:

Conceptualized, a.a.O., S. 8)

Grundsätzlich besteht in der wissenschaftlichen Literatur Einigkeit darin, daß die Zufriedenheit mit einer Transaktion bzw. einer einzelnen Phase einen Einfluß auf die Zufriedenheit mit einer späteren Transaktion bzw. Phase ausübt. Ebenso wird generell davon ausgegangen, daß sich die Gesamtzufriedenheit sowohl auf transaktionaler als auch globaler Ebene aus der Zufriedenheit mit den einzelnen

183 Vgl. Walker, J.L., Service Encounter Satisfaction: Conceptualized, a.a.O., S. 7 f.

184 Für eine Betrachtung der Entwicklung der Kundenzufriedenheit über mehrere Transaktionen sind analoge Überlegungen anzustellen. 
Konsumphasen bzw. Konsumakten zusammensetzt. ${ }^{185}$ Dagegen werden mögliche Wirkungsrichtungen (positiver versus negativer Einfluß) als auch die spezifische Stärke des Einflusses einzelner Phasen bzw. Transaktionen sehr unterschiedlich beurteilt. „We know very little about how satisfaction judgements evolve during the process." ${ }^{186}$

Die Zufriedenheitsforschung geht von der Annahme aus, daß ein Kunde die Erwartungen an eine Dienstleistung schon vor deren Inanspruchnahme gebildet hat. In Anlehnung an den Script-Ansatz bedeutet dies eine Festlegung von Erwartungen für jede Phase einer Dienstleistung vor dem Erleben der gesamten Transaktion. Es stellt sich jedoch die Frage, inwieweit eine Abweichung von der Erwartung an eine frühe Phase im Dienstleistungsprozeß einen Einfluß auf die Erwartung bzw. Wahrnehmungskomponente einer darauf folgenden Phase hat. Hier sind mehrere Fälle vorstellbar (vgl. zu einer vereinfachten Darstellung Abbildung 13). ${ }^{187}$

So ist es zunächst denkbar, daß bei einer negativen Abweichung in der ersten Phase die Erwartung an eine zweite Phase unverändert bleibt, die Wahrnehmung der zweiten Phase aber durch den Ausstrahlungseffekt (sog. Halo-Effekt) der ersten Phase negativ beeinflußt wird (Fall 1). Die Zufriedenheit mit der zweiten Phase fällt schlechter aus als ohne das negative Vorerlebnis. ${ }^{188}$

Ebenso liegt es aber auch im Bereich des Möglichen, daß die in der ersten Phase empfundene Unzufriedenheit zu einer Revision der Erwartung an den nächsten Abschnitt der Dienstleistung führt, dagegen die Wahrnehmung der zweiten Phase unbeeinflußt bleibt. Hier sind wiederum zwei Varianten denkbar. So kann die Unzufriedenheit mit der ersten Phase zu einer Senkung der Erwartung an die zweite Episode führen (Fall 2a). Dies sei an einem Beispiel verdeutlicht. Muß ein

Vgl. Stauss, B., Seidel, W., Prozessuale Zufriedenheitsermittlung und Zufriedenheitsdynamik bei Dienstleistungen, a.a.O., S. 192.

186 Danaher, P.J., Mattson, J., Customer Satisfaction during the Service Delivery Process, a.a.O., S. 6.

$187 \mathrm{Vgl}$. McClure, N., The Role of Expectations, Ambiguity of Information, and Initial Impressions in Consumer Satisfaction Assessments: A Process Oriented Approach, a.a.O., S. 46 ff.; von Lingen, Th., Zufriedenheitsmanagement, a.a.O., S. 8.

$188 \mathrm{Vgl}$. Johnston, R., The Zone of Tolerance: Exploring the Relationship Between Service Transactions and Satisfaction with the Overall Service, a.a.O., S. 48. 




\section{Abb. 13: Richtungseffekte der Kundenzufriedenheit}

Gast in einem Restaurant sehr lange auf die Bedienung warten, kann dies Skepsis hervorrufen und die Erwartungen an die Qualität des Menüs negativ beeinflussen. Schmeckt aber das Essen (wider Erwarten) gut, führt dies zu einer höheren Zufriedenheit mit dieser Phase als ohne das negative Vorerlebnis. GRÖRROOS 
umschreibt dieses Phänomen als "Learning-Paradox". ${ }^{189}$ Lernprozesse führen dazu, daß sich die Zufriedenheit entgegengesetzt zum erbrachten Leistungsniveau entwickelt. Bei der zweiten Variante dagegen erhöht der Kunde seine Erwartungen an die nächste Phase aufgrund seiner negativen Erfahrungen mit der vorhergegangenen (Fall 2b). Hier sei wieder an den Gast gedacht, der lange auf die Bedienung warten mußte und jetzt eine Erhöhung seiner Erwartungen an das Essen durch die lange Wartezeit gerechtfertigt sieht. In diesem Fall würde sich die Zufriedenheit mit der zweiten Phase (trotz gleichbleibender Qualität des Essens) verschlechtern.

Schließlich ist es auch vorstellbar, daß die Unzufriedenheit in der ersten Phase zu einer Veränderung der Erwartungs- und Wahrnehmungskomponente der sich anschließenden Phase führt (Fall 3). ${ }^{190}$ In Abhängigkeit davon, ob und in welchem Ausmaß sich diese beide Größen (eventuell auch voneinander abweichend) verändern, sind hier verschiedenste Zufriedenheitsreaktionen denkbar. ${ }^{191}$

Ebenso wie über die möglichen Richtungseffekte sind in der Literatur auch unterschiedliche Aussagen bezüglich der relativen Bedeutung einzelner Phasen bzw. Transaktionen für die Bildung des gesamten Zufriedenheitsurteils anzutreffen. So wird beispielsweise auf der transaktionalen Ebene die These aufgestellt, daß die jeweils letzte Phase einer Dienstleistung den stärksten Einfluß auf den aktuellen Stand der Transaktionszufriedenheit ausübt. ${ }^{192}$ In gleicher Weise werden z.T. kontroverse Überlegungen auf der globalen Ebene (bzw. Beziehungsebene) angestellt. Einerseits wird Z.B. die Ansicht vertreten, daß insbesondere die Zufriedenheiten mit den ersten Transaktionen das Ausmaß der Globalzufriedenheit und das künftige Verhalten eines Kunden gegenüber einem Anbieter bestimmen, dagegen spätere (und auch vereinzelt negative) einen vergleichsweise geringeren Einfluß ausüben. ${ }^{193}$ Andererseits ist aber auch die Meinung anzutreffen, daß negative Transaktionserlebnisse (unabhängig von ihrem zeitlichen

189 Vgl. Grönroos, Ch., Toward a Third Phase in Service Quality Research: Challenges and Future Directions, a.a.O., S. 55.

190 Vgl. Stauss, B., Seidel, W., Prozessuale Zufriedenheitsermittlung und Zufriedenheitsdynamik bei Dienstleistungen, a.a.O., S. 193.

191 Die Darstellung der potentiellen Wirkungseffekte beruht jeweils auf der Annahme einer empfundenen Unzufriedenheit in der ersten Phase. Stellt sich in dieser Phase Zufriedenheit ein, ist der jeweils umgekehrte Effekt in den einzelnen Fällen denkbar.

192 Vgl. Danaher, P.J., Mattson, J., Cumulative Encounter Satisfaction in the Hotel Conference Process, a.a.O., S. 74.

193 Vgl. Bitner, M.J., Hubbert, A.R., Encounter Satisfaction Versus Overall Satisfaction Versus Quality: The Customer's Voice, a.a.O., S. 77. 
Erscheinen) einen stärkeren Einfluß ausüben als positive. ${ }^{194}$ Schließlich wird darüber hinaus ein Reihenfolge- bzw. Positionseffekt für möglich gehalten, dergestalt, daß insbesondere die ersten als auch letzten und damit jüngsten Kontakte die Zufriedenheit mit einem Anbieter und damit das Kundenverhalten definieren. ${ }^{195}$ In Anlehnung an die Theorien der Gedächtnisforschung wären hier also Primat- und Rezenzeffekte denkbar, ${ }^{196}$ die zu einer unterschiedlichen Gewichtung bestimmter Transaktionszufriedenheiten führen würden. Diese Überlegungen können wiederum in analoger Weise auf der transaktionalen Ebene angestellt werden, was eine unterschiedliche Gewichtung einzelner Phasenzufriedenheiten innerhalb einer Transaktion implizieren würde.

STAUSS und SEIDEL stellen zu Recht fest, daß viele dieser Annahmen „häufig nicht mehr als (zum Teil sich widersprechende) Plausibilitätsüberlegungen“ darsteilen. ${ }^{197}$ Es stellt sich daher die Frage, inwieweit die postulierten Zusammenhänge einer empirischen Überprüfung standhalten. Im folgenden werden dazu die zentralen Ergebnisse verschiedener Untersuchungen sowohl auf der globalen als auch auf der transaktionalen Ebene vorgestellt.

\subsection{Dynamisch-konsumaktbezogene Betrachtung}

Die Auseinandersetzung mit Modellen und empirischen Analysen auf der Ebene von Konsumakten bedingt eine Konkretisierung des untersuchten Konstruktes. Der bisherigen Argumentation folgend, soll hier nicht zwischen einer globalen Zufriedenheit und einer globalen wahrgenommenen Dienstleistungsqualität unterschieden werden. Wenn entsprechend hier der Einfluß einzelner Kauf- bzw. Konsumakte auf die kundenseitige Gesamtbeurteilung eines Dienstleisters untersucht wird, soll Kundenzufriedenheit ebenso wie Dienstleistungsqualität als ein einstellungsähnliches, vergleichsweise stabiles Konstrukt verstanden werden. ${ }^{198}$

Vgl. Johnston, R., The Zone of Tolerance: Exploring the Relationship Between Service Transactions and Satisfaction with the Overall Service, a.a.O., S. 54; Homburg, Ch., Rudolph, B., Theoretische Perspektiven zur Kundenzufriedenheit, a.a.O., S. 41.

Vgl. Parasuraman, A., Zeithaml, V.A., Berry, L.L., Reassessment of Expectations as a Comparison Standard in Measuring Service Quality: Implications for Further Research, a.a.O., S. 122.

196 Vgl. Trommsdorff, V., Konsumentenverhalten, a.a.O., S. 267.

197 Stauss, B., Seidel, W., Prozessuale Zufriedenheitsermittlung und Zufriedenheitsdynamik bei Dienstleistungen, a.a.O., S. 192.

198 Vgl. hierzu die Ausführungen in Kap. B 2.1 dieser Arbeit. 




Abb. 14: Prozeßmodell der Dienstleistungsqualität nach Boulding et al. (Quelle: Boulding, W. et al., A Dynamic Process Model of Service Quality: From Expectations to Behavioral Intentions, a.a.O., S. 12)

In der jüngeren Zeit ist von mehreren Forschern die Notwendigkeit erkannt worden, die dynamische Komponente von Dienstleistungen in die existierenden Modelle der Dienstleistungsqualität bzw. Kundenzufriedenheit zu integrieren. ${ }^{199}$

BOULDING ET AL. formulieren vor diesem Hintergrund ein dynamisches Prozeßmodell der Dienstleistungsqualität, das auf folgenden Annahmen basiert (vgl. Abbildung 14). ${ }^{200}$

Zu einer Übersicht dynamischer Ansätze der Dienstleistungsqualităt vgl. Grönroos, Ch., Toward a Third Phase in Service Quality Research: Challenges and Future Directions, a.a.O. S. 60. Zu Untersuchungen im Konsumgüterbereich vgl. LaBarbera, P.A., Mazursky, D., A Longitudinal Assessment of Consumer Satisfaction/Dissatisfaction: The Dynamic Aspect of the Cognitive Process, in: JoMR, Vol. 20, November 1983, S. $393 \mathrm{ff}$.

200 Vgl. hierzu und im folgenden Boulding, W., Kalra, A., Staelin, R., Zeithaml, V.A., A Dynamic Process Model of Service Quality: From Expectations to Behavioral Intentions, a.a.O., S. 7 ff.; Meffert, H., Bruhn, M., Dienstleistungsmarketing: Grundlagen, Konzepte, Methoden; mit Fallbeispielen, a.a.O., S. $240 \mathrm{ff}$. 
Die Autoren gehen davon aus, daß ein Kunde im Zeitablauf seine Wahrnehmungen in Abhängigkeit von seinen (früheren) Erwartungen bezüglich der Dienstleistungsqualität eines Anbieters ändert. Aus diesen Veränderungen resultieren dann wiederum unterschiedliche Verhaltensabsichten gegenüber dem Anbieter. Zur Operationalisierung der Dienstleistungsqualität werden dabei zwei Arten von Erwartungen unterschieden, die ein Kunde seiner Beurteilung zugrunde legt. ${ }^{201}$ „Would“-Erwartungen zielen auf die für wahrscheinlich gehaltenen Ausprägungen der Dienstleistungsqualität $a b$, während sich in den „Should“-Erwartungen die Vorstellungen eines Kunden bezüglich eines als angemessen betrachteten Qualitätsniveaus widerspiegeln. Die ursprünglich gebildeten Erwartungen bezüglich der verschiedenen Eigenschaften der Dienstleistung ${ }^{202}$ sowie weitere im Zeitablauf aufgenommene Informationen, die als eine Art Korrekturfaktor der Erwartungen wirken (z.B. Kommunikation mit anderen Kunden des Dienstleisters), ${ }^{203}$ und die tatsächlich gelieferte Leistung führen dann zu einer kumuliert wahrgenommenen Leistung bzw. Qualität der einzelnen Dimensionen der Dienstleistung. Diese werden wiederum aggregiert und ergeben eine individuell unterschiedlich wahrgenommene Gesamtqualität des Dienstleisters, aus der dann eine bestimmte Verhaltensabsicht resultiert. Die empirische Überprüfung des Modells im Rahmen von Labor- und Feldstudien ${ }^{204}$ ergab, daß, je stärker die "Would“Erwartungen ausgeprägt sind, die (wahrgenommene) Dienstleistungsqualität umso besser beurteilt wird. Je höher dagegen die „Should“-Erwartungen ausgebildet sind, desto negativer wird die Dienstleistungsqualität eingestuft. Es zeigt sich also, daß die vor der Inanspruchnahme einer Dienstleistung aufgestellten Erwartungen von entscheidender Bedeutung für die Beurteilung eines Dienstleisters sind. Dabei ist insbesondere beachtenswert, daß die Resultate die Annahme eines dynamischen Prozesses bestätigten. So konnte nachgewiesen werden, daß die verschiedenen Komponenten im Zeitablauf einem dynamischen Veränderungsprozeß unterworfen sind und einen entsprechenden Einfluß auf die Wahrnehmung einer Dienstleistung ausüben.

Vgl. hierzu die Ausführungen in Kap. B 2.22 dieser Arbeit.

Hier dienen die von Parasuraman et al. entwickelten Dimensionen der Dienstleistungsqualitat als Maßstab. Vgl. hierzu die Ausführungen in Kap. B 2.11 dieser Arbeit.

Im Rahmen der empirischen Untersuchungen wurde auf die Messung solcher Einflußgrößen verzichtet.

204

Der Gegenstand des Laborexperimentes bestand in einer Abfolge simulierter Hotelaufenthalte, Inhalt der Feldstudie war die wahrgenommene Qualităt von universitären Ausbildungsmaßnahmen. 
Das Modell gewährt damit einen interessanten Einblick in die Entwicklungsprozesse der Erwartungsbildung und Wahrnehmung von Dienstleistungen und hieraus resultierender Verhaltensabsichten. Auch ergeben sich erste Implikationen für das Management von Dienstleistungsunternehmen im Hinblick auf eine gezielte Beeinflussung der verschiedenen Erwartungsarten. Kritisch ist jedoch anzumerken, daß keine konkreten Umsetzungsmaßnahmen bezüglich einzelner Dimensionen der Dienstleistungen abgeleitet werden. Darüber hinaus deuten die separate Erhebung der verschiedenen Erwartungsarten und die Erfassung der verschiedenen (dynamischen) Komponenten im Rahmen der Feldstudie zu einem Zeitpunkt auf meßtechnische Schwächen hin. ${ }^{205}$

Eine etwas andere Perspektive wird in dem Modell der Beziehungsqualität von LILJANDER und STRANDVIK eingenommen. ${ }^{206}$ Die Autoren greifen explizit auf die Konstrukte Kundenzufriedenheit und Dienstleistungsqualität zurück. Auch hier wird unterstellt, daß eine als positiv wahrgenommene Dienstleistungsqualität und Kundenzufriedenheit einen vorteilhaften Einfluß auf die Bindung eines Kunden an ein Unternehmen ausüben. Das Qualitätsurteil wird von der Kundenzufriedenheit abgegrenzt, indem der Dienstleistungsqualität eine „outsider perspective" zugeordnet wird, während Kundenzufriedenheit aus einer "insider perspective" resultiert. Ein Zufriedenheitsurteil entsteht somit nur im konkreten Dienstleistungskontakt, während ein Qualitätsurteil auch ohne Inanspruchnahme der Dienstleistung gebildet werden kann. In Anlehnung an die Unterscheidung zwischen Transaktions- und Globalperspektive der Kundenzufriedenheit wird zwischen der „Episoden"- und der „Beziehungsebene“ differenziert. Während eine Episode dem dieser Arbeit zugrunde gelegten Transaktionsbegriff entspricht, besteht eine Beziehung aus mindestens zwei Episoden. Aufbauend auf dem DisconfirmationModell wird mit dem sog. „Value“-Konstrukt eine zwischen der Dienstleistungsqualität und der Kundenzufriedenheit vermittelnde Variable eingeführt, die einem Qualitäts-Opfer-Abgleich entspricht und die auf beiden Kontaktebenen wirkt (Beziehungs- bzw. Episodenwert). Wie Abbildung $15 \mathrm{zu}$ entnehmen ist, wird darüber hinaus die Existenz einer Toleranzzone angenommen.

205 206

Vgl. Danaher, P.J., Mattson, J., Customer Satisfaction during the Service Delivery Process, a.a.O., S. 6.

Vgl. hierzu und im folgenden Strandvik, T., Liljander, V., A Comparison of Episode Performance and Relationship Performance for a Discrete Service, a.a.O., S. $111 \mathrm{ff}$. Liljander, V., Introducing Deserved Service and Equity into Service Quality Models, a.a.O., S. $141 \mathrm{ff}$;; Meffert, H., Bruhn, M., Dienstleistungsmarketing: Grundlagen, Konzepte, Methoden; mit Fallbeispielen, a.a.O., S. $243 \mathrm{ff}$. 


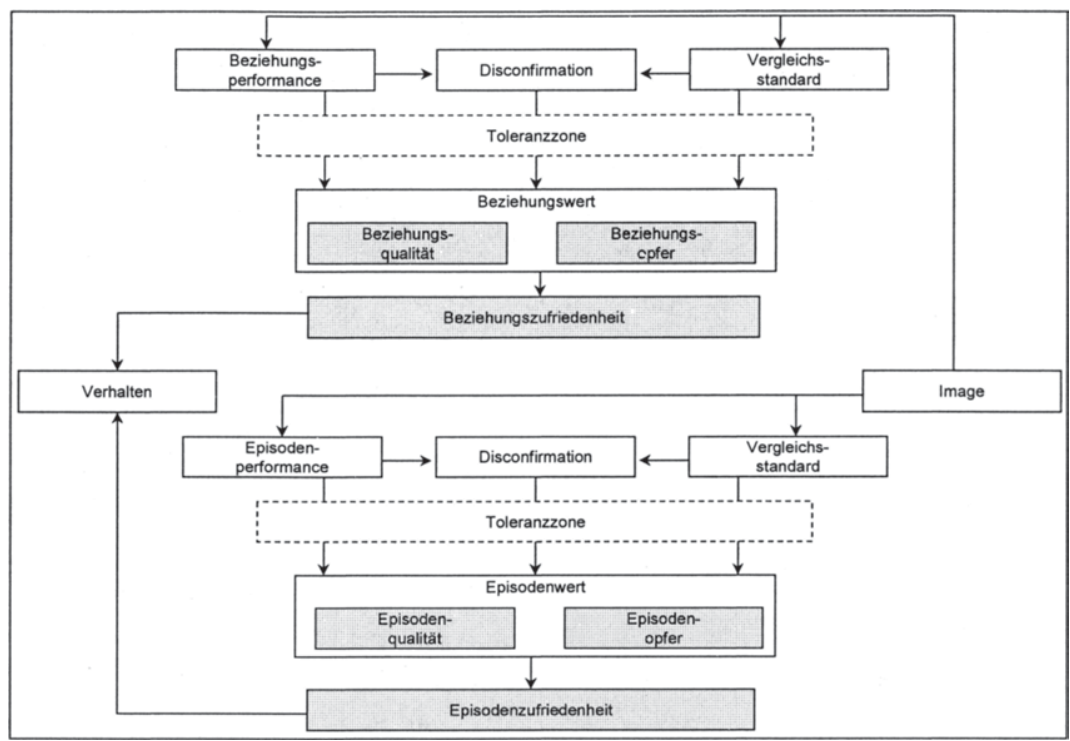

Abb. 15: Modell der Beziehungsqualität nach Strandvik / Liljander (Quelle: Strandvik, T., Liljander, V., A Comparison of Episode Performance and Relationship Performance for a Discrete Service, a.a.O., S. 122)

Besondere Bedeutung im Hinblick auf das zukünftige Verhalten des Kunden gegenüber dem Anbieter wird der Beziehungszufriedenheit beigemessen. So wird angenommen, daß nicht die einzelnen (positiven oder negativen) Transaktionszufriedenheiten an sich das Kundenverhalten determinieren, sondern daß erst eine durch diese im Zeitablauf bewirkte Änderung der Beziehungszufriedenheit einen nachhaltigen Einfluß auf das Kundenverhalten ausübt. Die empirische Überprüfung des Modells erfolgte im Kontext von Friseurdienstleistungen. Dabei stand nicht die Aggregation von Episodenzufriedenheiten zu einer Beziehungszufriedenheit im Vordergrund, sondern die Frage, inwieweit sich die untersuchte Episodenzufriedenheit von der gleichzeitig gemessenen Beziehungszufriedenheit unterscheidet und welche dieser Größen einen höheren Beitrag zur Erklärung des Kundenverhaltens leistet. Die Befragten bekundeten durchgehend eine höhere Zufriedenheit mit der Episode im Vergleich zur gesamten Beziehung. Eine mögliche Erklärung liegt in der Berücksichtigung weniger zufriedenstellender Kontakte in der Vergangenheit im Rahmen der Bildung des Gesamturteils. Die Beziehungszufriedenheit wiederum leistete einen höheren Beitrag zur Erklärung des bekundeten Wiedernutzungs- und Weiterempfehlungsverhalten der Kunden. Dies 
erlaubt allerdings nicht die Schlußfolgerung, auf eine Untersuchung von Episodenzufriedenheiten verzichten zu können. Vielmehr ist es von entscheidender Bedeutung zu erkennen, daß erst die Summe einzelner Erfahrungen die Beziehungszufriedenheit bestimmt. Das Modell leistet damit einen ersten Einblick in diesen Entwicklungsprozeß. ${ }^{207}$

Abschließend sei an dieser Stelle auf die Studie von BOLTON und DREW hingewiesen. ${ }^{208}$ Auch hier wird davon ausgegangen, daß sich die Gesamtzufriedenheit zu einem bestimmten Zeitpunkt aus der aktuellen Leistung und den Erfahrungen früherer Prozesse ergibt. Besonderes Augenmerk wurde hier dem zeitlich unterschiedlichen Einfluß von Serviceänderungen auf die wahrgenommene Dienstleistungsqualität gewidmet. Im Rahmen ihrer empirischen Längsschnittanalyse in der Telekommunikationsbranche stellten die Autoren fest, daß vier Wochen nach der vorgenommenen Serviceverbesserung (Reduktion des Hintergrundrauschens beim Telefonieren) die aktuell wahrgenommene globale Qualität stärker durch die Zufriedenheit mit der Serviceverbesserung und weniger durch die früheren Einstellungen gegenüber dem Anbieter geprägt wurde. Sechs Monate später konnte jedoch der gegenläufige Trend festgestellt werden. Hätte die Messung zu nur einem Zeitpunkt stattgefunden, wären diese Effekte nicht erkennbar gewesen. Damit zeigt sich auch in den Ergebnissen dieser Studie die Unterschiedlichkeit in der Zufriedenheit mit einzelnen Episoden und der kumulierten Wahrnehmung der Qualität eines Anbieters. Interessanterweise wurde im Rahmen der empirischen Untersuchung auf eine explizite Messung der Erwartungskomponente mit der Begründung verzichtet, daß diese bereits implizit in der erhobenen wahrgenommenen Ist-Leistung enthalten ist. Bemerkenswert an dieser Studie ist insbesondere der nachgewiesene Effekt einer im Zeitablauf nachlassenden Wirkung einzelner Episodenerlebnisse. In Abhängigkeit vom gewählten Zeitpunkt wurde die Qualitätsbeurteilung des Anbieters durch sehr unterschiedliche Faktoren dominiert.

Auch hier ist anzumerken, daß die Messung der verschiedenen Konstruktelemente nur zu einem Zeitpunkt stattfand. Wird allerdings berücksichtigt, daß mit der Beziehungszufriedenheit ein einstellungsăhnliches und damit relativ stabiles Konstrukt untersucht wird, ist die Notwendigkeit einer kontinuierlichen Messung der Zufriedenheit auf beiden Ebenen offenkundig. Service Changes on Customer Attitudes, in: JoM, Vol. 55, January, 1991, S. $1 \mathrm{ff}$. 


\subsection{Dynamisch-konsumphasenbezogene Betrachtung}

Während die Notwendigkeit der Untersuchung der Zufriedenheitsdynamik auf der Ebene von Konsumakten durch die in jüngerer Zeit zu beobachtende Popularität des Beziehungsmarketing eine Bestätigung erfährt, bilden phasenorientierte Untersuchungen auf der Transaktionsebene eher die Ausnahme. Wenngleich in der Fachliteratur bereits zu Beginn der achtziger Jahre die Bedeutung einer konsumphasenbezogenen Analyse der Kundenzufriedenheit betont wurde, ${ }^{209}$ beschränkte sich die Forschung vornehmlich auf konzeptionelle Ansätze.$^{210} \mathrm{Empi}$ rische Untersuchungen sind erst in jüngerer Zeit anzutreffen, wobei diese sich schwerpunktmäßig mit der Analyse des Prozeßcharakters von Dienstleistungen auseinandersetzen. ${ }^{211}$

Eine derartige Untersuchung wurde von WOODSIDE ET AL. durchgeführt. ${ }^{212}$ Die Zielsetzung dieser Studie bestand in der Analyse des Einflusses wahrgenommener Dienstleistungsqualität und Kundenzufriedenheit auf das künftige Kundenverhalten. Die aktuell wahrgenommene Dienstleistungsqualität wird dabei als die zentrale Bestimmungsgröße der Kundenzufriedenheit angesehen. Aus einer transaktionsbezogenen Perspektive heraus wurde eine empirische Untersuchung bei Krankenhauspatienten, die eine zweitägige Behandlung erfuhren, durchgeführt. Den Gedanken des Service-Script-Konzeptes aufgreifend, fand eine Zerlegung des Kundenprozesses in die sechs Episoden Aufnahme, Kontakte mit dem Krankenhauspersonal, Verpflegung, Zimmeraufenthalt sowie technische Dienstleistungen (z.B. Röntgen) und Entlassung statt, die durch insgesamt 18 Qualitätsmerkmale operationalisiert wurden. Die der empirischen Untersuchung zugrunde liegenden Daten entstammen einer telefonischen Befragung von Patienten, deren letzter Klinikaufenthalt nicht länger als 30 Tage zurücklag. Während die Qualitätsurteile anhand der wahrgenommenen Ist-Leistung erhoben wur-

Vgl. Day, R.L., Toward a Process Model of Consumer Satisfaction, a.a.O., S. $175 \mathrm{ff}$.; Oliver, R.L., Measurement and Evaluation of Satisfaction Processes in Retail Settings, a.a.O., S. $31 \mathrm{ff}$. Vgl. Ferriday, J.J., Simintiras, A.C., Customer Satisfaction: Present Knowledge and Some Directions for Future Research, in: Marketing: Its Dynamics and Challenges, Proceedings of the 23rd Annual Conference of the European Marketing Academy, Bloemer, J., Lemmink, J., Kasper, H. (Hrsg.), Maastricht 1994, S. 227 f.

Des weiteren ist auf einzelne Untersuchungen in der Automobilbranche hinzuweisen. Vgl. hierzu Burmann, Ch., Konsumentenzufriedenheit als Determinante der Marken- und Händlerloyalität, a.a.O., S. $250 \mathrm{ff}$;; Korte, Ch., Customer Satisfaction Measurement: Kundenzufriedenheitsmessung als Informationsgrundlage des Hersteller- und Handelsmarketing am Beispiel der Automobilwirtschaft, a.a.O., S. $167 \mathrm{ff}$.

212 Vgl. hierzu und im folgenden Woodside, A.G., Frey, L.L., Daly, R.T., Linking Service Quality, Customer Satisfaction, and Behavioral Intention, a.a.O., S. $5 \mathrm{ff}$. 
den, erfolgte die Erhebung der Zufriedenheitsurteile auf der Basis einer direkten Erfragung der empfundenen Zufriedenheit. Zur Auswertung der Daten wurden vorwiegend korrelations- und regressionsanalytische Verfahren eingesetzt.

Die Resultate bestätigten die Vermutung, daß die Episodenzufriedenheiten positiv mit der Transaktionszufriedenheit korrelieren. Ebenso konnte ermittelt werden, daß der Einfluß der einzelnen Episoden auf die Gesamtzufriedenheit unterschiedlich hoch ist. Des weiteren wurden Ausstrahlungseffekte zwischen den einzelnen Merkmalen einer Episode festgestellt. Überraschenderweise konnte dagegen nicht der Nachweis für einen Ausstrahlungseffekt zwischen den einzelnen Episoden erbracht werden. Dieser Umstand ist auf verschiedene Ursachen zurückzuführen. Wenngleich die Autoren selbst auf die Messung der verschiedenen Variablen zu nur einem Zeitpunkt hinweisen, ist in diesem Zusammenhang ein weiteres Problem erkennbar. So wird die Zufriedenheit mit einer Transaktion gemessen, die zum Zeitpunkt der Befragung bis zu 30 Tage zurücklag. Vergessenseffekte und eine nachträgliche Anpassung des Zufriedenheitsurteils sind daher nicht auszuschließen. So können z.B. in der Zwischenzeit Erfahrungen mit anderen medizinischen Einrichtungen gemacht worden sein. Ebenso sind Tendenzen zu einer Reduktion empfundener Dissonanzen denkbar. Darüber hinaus stellen die sechs Episoden eine nur grobe Beschreibung des Kundenpfades dar. Es kann vor allem keine eindeutige (zeitliche) Reihenfolge ausgemacht werden. Zudem ist z.B. die Episode "Verpflegung" ein Ereignis, das auch innerhalb eines nur zwei Tage dauernden Klinikaufenthaltes mehrfach vorkommt. Eine nachträgliche Beurteilung ergibt einen Durchschnittswert, der Einfluß auf eine andere Episode ist nicht zwingend nachweisbar. Dem originären Episodencharakter der Dienstleistung wird somit nur bedingt Rechnung getragen.

$\mathrm{Da} \beta$ eine Beziehung zwischen verschiedenen Episoden besteht, konnte dagegen von GüTHOFF nachgewiesen werden. ${ }^{213}$ Aufbauend auf dem Prinzip der episodischen Informationsverarbeitung entwickelte die Autorin ein sog. Teilleistungsmodell der Dienstleistungsqualität, dem ein zufriedenheitsorientiertes Verständnis von Dienstleistungsqualität zugrunde liegt. Bei diesem Modell wird davon ausgegangen, daß sich bei Dienstleistungen, die sich insbesondere durch eine hohe Zahl heterogener Teilleistungen auszeichnen, die Qualitätsbeurteilung aus einer differenzierten Wahrnehmung dieser einzelnen Abschnitte zusammensetzt. Im

213 Vgl. Güthoff, J., Qualităt komplexer Dienstleistungen: Konzeption und empirische Analyse der Wahrnehmungsdimensionen, a.a.O., S. $120 \mathrm{ff}$. 
Rahmen der empirischen Untersuchung der wahrgenommenen Qualität von Hoteldienstleistungen wurde der Aufenthalt in einem Vier-Sterne-Hotel in die vier sog. Teilleistungen Zimmer, Reservierung, Empfang und Buffet zerlegt. Die Messung der Dienstleistungsqualität erfolgte im Rahmen einer mündlichen Befragung und auf der Basis einer direkten Erfragung der Zufriedenheit mit verschiedenen Merkmalen der einzelnen Leistungsabschnitte. Wenngleich das primäre Ziel der Untersuchung nicht in einer Analyse des sequentiellen Einflusses von Episoden auf nachgelagerte Phasen bestand und auch hier nicht von einer eindeutigen zeitlichen Abfolge der verschiedenen Episoden ausgegangen werden kann, zeigen sich dennoch signifikante korrelative Beziehungen zwischen den identifizierten Teilleistungen im Rahmen der durchgeführten konfirmatorischen Faktorenanalyse. Es unterbleibt jedoch eine Untersuchung des Einflusses der einzelnen Teilleistungen bzw. Episoden auf die Gesamtzufriedenheit.

Eine solche Analyse erfolgt dagegen in den Studien von DANAHER und MATTSON. ${ }^{214}$ In beiden Untersuchungen bestand die zentrale Zielsetzung in einer Analyse der Zufriedenheitsdynamik und der Entwicklung des Zufriedenheitsurteils im Zeitablauf. Ebenso wie GüTHOFF befassen sich die Autoren in ihrer ersten Studie mit der Zufriedenheit von Hotelgästen, während die Zufriedenheit von Konferenzteilnehmern den Gegenstand der zweiten Studie darstellt. Im Rahmen ihrer empirischen Untersuchung der Zufriedenheit mit Hoteldienstleistungen zerlegten die Autoren den Kundenprozeß in die fünf Episoden Check-In, Zimmeraufenthalt, Restaurantbesuch, Frühstück und Check-Out. Befragt wurden ausschließlich Hotelgäste, die nur eine Übernachtung gebucht hatten, um sicherzustellen, daß jede der genannten Episoden genau einmal erlebt wurde. Im Gegensatz zu den bisher genannten Studien wurden die Gäste jedoch nicht erst nach Abschluß der gesamten Transaktion, sondern jeweils im direkten Anschluß an eine erlebte Episode nach ihrer aktuell empfundenen Zufriedenheit mit der Transaktion befragt, wobei sich die Frage nach dem Check-Out auf die Zufriedenheit mit dem gesamten Hotelaufenthalt bezog. Darüber hinaus wurde jeweils die Zufriedenheit mit unterschiedlichen Qualitätsmerkmalen der verschiedenen Episoden erhoben.

Vgl. Danaher, P.J., Mattson, J., Customer Satisfaction during the Service Delivery Process, a.a.O., S. 6 ff. Danaher, P.J., Mattson, J., Cumulative Encounter Satisfaction in the Hotel Conference Process, a.a.O., S. $70 \mathrm{ff}$. 
Eine ähnliche Vorgehensweise wählten die Autoren im Rahmen ihrer Untersuchung der Zufriedenheit von Konferenzbesuchern (Studie 2). Hier bestand der Kundenprozeß aus den vier untersuchten Episoden Ankunft, Kaffepause, Mittagessen und Aufenthalt im Konferenzraum. Befragt wurden hier Teilnehmer einer eintägigen Konferenz, die in einem Tagungshotel ausgerichtet wurde.

Die Ergebnisse beider Studien bestätigten die zwei zentralen Annahmen der Autoren über die Zufriedenheitsdynamik. So konnte zum einen festgestellt werden, daß die aktuelle Transaktionszufriedenheit im Zeitablauf nicht konstant bleibt, sondern vielmehr durch die Zufriedenheit mit den einzelnen Episoden beeinflußt wird und zu den verschiedenen Meßzeitpunkten unterschiedlich hoch ausfällt. Zum anderen bestätigte sich die Vermutung eines vergleichsweise stärkeren Einflusses der jeweils letzten Episode auf die aktuelle Transaktionszufriedenheit im Rahmen faktorenanalytischer Untersuchungen. Zur Überprüfung des Einflusses der einzelnen Merkmale auf den jeweiligen Stand der Transaktionszufriedenheit bzw. der Bedeutung der einzelnen Episoden im Hinblick auf die Gesamtzufriedenheit wurden regressionsanalytische Verfahren eingesetzt. Auch hier konnte sowohl ein unterschiedlicher Einfluß der einzelnen Merkmale als auch der verschiedenen Episoden ermittelt werden. In diesem Zusammenhang ist erwähnenswert, daß die Zufriedenheit mit dem Restaurant keinen signifikanten Einfluß auf die Gesamtzufriedenheit mit dem Hotelaufenthalt ausübt, obwohl die aktuelle Transaktionszufriedenheit nach dieser Episode am niedrigsten ausfiel (Studie 1). Die Autoren führen dieses Ergebnis auf den Umstand zurück, daß der Restaurantaufenthalt von den Gästen als eine eigenständige Dienstleistung und nicht als Bestandteil der Hoteldienstleistung wahrgenommen wird, da dieser nicht in dem Leistungspaket der Gäste enthalten war. Dagegen war der Restaurantaufenthalt Bestandteil des Leistungspaketes der Konferenzteilnehmer, deren Zufriedenheit innerhalb der zweiten Studie untersucht wurde. Ebenso wie in der ersten Studie fiel das Zufriedenheitsurteil über den Restaurantbesuch am niedrigsten aus. Interessanterweise hatte hier jedoch die Zufriedenheit mit dem Mittagessen, die Vermutung der Autoren bestätigend, einen signifikanten Einfluß auf die Gesamtzufriedenheit der Konferenzteilnehmer. Bemerkenswert an den Studien von DANAHER und MATTSON ist das Vorgehen, Zufriedenheit im direkten Anschluß an eine Dienstleistungsepisode im Rahmen eines "On-Process"-Ansatzes zu unterschiedlichen Zeitpunkten zu messen. STAUSS weist jedoch zu Recht darauf 
hin, daß „die dabei zu bewältigenden methodischen Probleme noch nicht vollständig geklärt sind."215

Eine explizite Untersuchung der Dynamik von Erwartungen und der wahrgenommen Leistung auf der Transaktionsebene unternimmt MCCLURE. ${ }^{216}$ Basierend auf dem Disconfirmation-Modell untersucht die Autorin im Rahmen von Laborstudien mit Studenten die Dynamik der Konstruktkomponenten anhand unterschiedlicher Szenarien für den Besuch eines Freizeitparks als auch für einen Restaurantbesuch. Um die Resultate beider Untersuchungen vergleichbar zu halten, wurden für beide Dienstleistungsarten die Episoden Wartezeit, Umfang der erhaltenen Leistungen und Zahlungsvorgang ausgewählt. Die mit Hilfe regressionsanalytischer Verfahren ermittelten Ergebnisse bestätigten die Vermutung, daß die Befragten ihre ursprünglichen Erwartungen an eine Dienstleistung während der Transaktion im Zeitablauf erneuern und revidieren. Darüber hinaus konnte nachgewiesen werden, daß solche Veränderungen einen Einfluß auf die Erwartungen und Wahrnehmungen späterer Episoden und dementsprechend auf die Zufriedenheit mit diesen Episoden ausüben. Bemerkenswert sind insbesondere die nachgewiesenen Richtungseffekte der Zufriedenheitsdynamik. So stellte sich heraus, daß eine positive Diskonfirmation der Erwartungen an eine frühe Phase des Dienstleistungsprozesses zu einer Senkung der Erwartungen an eine spätere Episode führt und daher die Zufriedenheit mit folgenden Episoden größer ausfällt als ohne dieses Vorerlebnis. Im Falle einer Nichtbestätigung der Erwartungen an eine frühe Phase der Dienstleistung stellte sich der gegenläufige Effekt ein. Ebenso gelang der Autorin der Nachweis für die Existenz von Rezenzeffekten, während Primateffekte nicht ausgemacht werden konnten. So wurde festgestellt, daß das positive Erleben der letzten Episode einen starken Einfluß auf die Zufriedenheit ausübt, während eine negative Wahrnehmung der letzten Phase einen vergleichsweise geringeren, aber dennoch signifikanten Einfluß auf die Transaktionszufriedenheit nimmt. Wenngleich die Ergebnisse dieser Untersuchung vor dem Hintergrund der verwendeten Szenariotechnik als auch unter Berücksichtigung der z.T. sehr kleinen Fallzahlen lediglich unter Vorbehalt zu

Stauss, B., Kundenprozeßorientiertes Qualitătsmanagement im Dienstleistungsbereich, a.a.O., S. 45. Eine Diskussion der Vor- und Nachteile einer solchen Vorgehensweise wird in den folgenden Abschnitten dieser Arbeit vorgenommen.

216 Vgl. McClure, N., The Role of Expectations, Ambiguity of Information, and Initial Impressions in Consumer Satisfaction Assessments: A Process Oriented Approach, a.a.O., S. 80 ff. 
interpretieren sind, ${ }^{217}$ bestätigt auch diese Studie die Notwendigkeit einer prozessorientierten Analyse der Kundenzufriedenheit.

Die dargestellten empirischen Studien vermitteln einen interessanten Einblick in verschiedene Aspekte der Dynamik von Kundenzufriedenheit sowohl auf transaktionaler als auch auf globaler Ebene. So werden die unterstellten Einflüsse einzelner Transaktionen bzw. Episoden untereinander als auch auf die Beziehungsbzw. Transaktionszufriedenheit theoretisch hergeleitet und zumindest teilweise empirisch bestätigt. Demgegenüber unterbleibt nahezu in allen Untersuchungen eine nähere Analyse von Bestimmungsfaktoren, die zu einer unterschiedlichen Ausbildung des Zufriedenheitsurteils führen können. Es ist aber durchaus denkbar, daß z.B. in Abhängigkeit vom Geschlecht, dem Involvement oder auch dem spezifischen $A n l a ß$ der Dienstleistungsnutzung die Zufriedenheit mit einzelnen Episoden und deren Einfluß auf das Gesamturteil unterschiedlich ausfällt. Die Frage, inwieweit solche Faktoren das Zufriedenheitsurteil und die damit einhergehende Zufriedenheitsdynamik beeinflussen, bleibt in der Regel unbeantwortet. Ebenso ist kritisch anzumerken, daß in den Studien häufig nur eine grobe Unterteilung des Kundenprozesses vorgenommen und ein eindeutig sequentieller Verlauf der verschiedenen Episoden nur bedingt sichergestellt wird. Darüber hinaus beruhen die Ergebnisse teilweise auf Laborexperimenten sowie auf Stichproben mit sehr geringer Fallzahl. Die nicht einheitliche Operationalisierung der Konstruktkomponenten der Kundenzufriedenheit sowohl auf der theoretischen als auch auf der empirischen Sprachebene erschwert überdies die Vergleichbarkeit der Ergebnisse. ${ }^{218}$

\subsection{Würdigung der Erklärungsansätze und Erscheinungsformen der Kundenzufriedenheit}

Die bisherigen Ausführungen haben gezeigt, daß bei einer Analyse der Kundenzufriedenheit im Dienstleistungsbereich eine Vielzahl von Aspekten zu berücksichtigen ist. Dabei sind insbesondere unterschiedliche Interpretationsformen sowohl der Soll- als auch der Konsumerlebniskomponente anzutreffen. Eine allgemeingültige Empfehlung für den Einsatz einer bestimmten Ausprägungsform

\footnotetext{
217 So beruhten einzelne Analyseschritte der Untersuchung nur auf den Angaben von ca. 20 Befragten.

218 Auf die mit den eingesetzten Auswertungstechniken verbundenen Probleme wird in Teil C dieser Arbeit differenziert eingegangen.
} 
bzw. für eine Kombination verschiedener Ausgestaltungsvarianten erscheint nicht zweckmäßig. Vielmehr gilt es, den Erklärungsbeitrag der verschiedenen Ansätze vor dem Hintergrund der spezifischen Untersuchungssituation und dem verfolgten Untersuchungsziel kritisch zu würdigen.

Von den verschiedenen Theorien und Modellansätzen hat das DisconfirmationModell, welches auch den gedanklichen Rahmen der weiteren Ausführungen darstellt, die größte Verbreitung in der Literatur erfahren. In diesem Zusammenhang ist jedoch anzumerken, daß das Modell in seiner ursprünglichen Form den Prozeßcharakter von Dienstleistungen nicht ausreichend berücksichtigt. Es ist aber gerade der Episodencharakter von Dienstleistungen, der eine Dynamisierung des Disconfirmation-Modells zwingend erforderlich macht. Mit dem Konzept der episodischen Informationsverarbeitung bzw. dem Service-Script-Ansatz verfügt eine dynamische Sichtweise der Kundenzufriedenheit über ein solides psychologisches Fundament für eine prozeßorientierte Operationalisierung auf der theoretischen Sprachebene.

Hiermit eng verbunden ist jedoch die Operationalisierung der Kundenzufriedenheit auf der empirischen Sprachebene. Wird eine dynamische Analyse der Kundenzufriedenheit angestrebt, ist eine genaue Analyse des Kundenprozesses und eine entsprechend differenzierte Messung notwendig. Hieraus resultiert die Forderung nach einer prozeßbegleitenden Messung von Kundenzufriedenheit, also einer Messung zu unterschiedlichen Zeitpunkten während des Dienstleistungsprozesses. ${ }^{219}$ Eine solche On-Process-Messung, die die verschiedenen Konsumprozeßphasen der Dienstleistungsnutzung berücksichtigt, stellt nicht nur spezifische Anforderungen an das Meßinstrumentarium, sondern birgt auch einige besondere Problembereiche in sich.

So wird gelegentlich die Vermutung geäußert, daß erst die Frage nach Zufriedenheit einen Beurteilungsprozeß auslöst. ${ }^{220}$ Ist dies der Fall, wird dieses Problem bei On-Process-Ansätzen besonders virulent. Da ein Proband bereits vor der Untersuchung mit der Befragungsmethodik vertraut gemacht werden muß, sind die Generierung einer künstlichen Befragungssituation und ein damit einhergehender

Vgl. Voss, C.A., Where should Service Quality be measured? Conclusions from a Process Based Model, a.a.O., S. 29; Danaher, P.J., Mattson, J., Customer Satisfaction during the Service Delivery Process, a.a.O., S. 15. braucherzufriedenheit?, a.a.O., 1984, S. 453. 
"unnatürlicher" Aktivierungsgrad des Befragten nicht auszuschließen. Findet dagegen eine ex post-Messung (Off-Process-Ansatz) statt, ist zu berücksichtigen, daß ein dynamisches Phänomen in einer grundsätzlich statischen Weise erfaßt wird. ${ }^{221}$ Der Ausgestaltung des Meßinstrumentariums und dem Zeitpunkt der Messung kommt damit eine zentrale Bedeutung zu. Im folgenden wird daher der Frage nachgegangen, welche Verfahren und Meßmethoden grundsätzlich geeignet sind, Kundenzufriedenheit phasen- bzw. prozeßorientiert zu erfassen und welche spezifischen Vor- und Nachteile mit dem Einsatz dieser Verfahren verbunden sind.

\section{Ansätze zur prozeßorientierten Strukturanalyse und Messung von Kundenzufriedenheit im Dienstleistungsbereich}

Voraussetzung einer prozeßorientierten Messung der Kundenzufriedenheit ist die Ermittlung des vom Kunden während der Nutzung der Dienstleistung erlebten Prozesses. Zu diesem Zweck ist eine Reihe von Instrumenten entwickelt worden, die im folgenden näher dargestellt und im Hinblick auf ihre Eignung zur Abbildung des Kundenprozesses diskutiert werden. Hierauf aufbauend erfolgt dann eine Darstellung und Bewertung unterschiedlicher Meßverfahren zur Erfassung der Kundenzufriedenheit aus einer prozeßorientierten Sichtweise.

\subsection{Strukturanalyse und Visualisierung der Prozeßkomponente von Dienstleistungen}

Informationen über den vom Kunden typischerweise erlebten Dienstleistungsprozeß können z.B. auf Basis von Beobachtungen, Einzelinterviews oder Gruppendiskussionen mit den Konsumenten der Dienstleistung gewonnen werden. ${ }^{222}$ Zur Umsetzung der aus solchen Erhebungen gewonnenen Informationen in eine systematische Darstellung des Dienstleistungsprozesses lassen sich unterschiedliche Verfahren einsetzen. Diese Verfahren teilen dabei die Gemeinsamkeit, Varianten von Ablaufdiagrammen oder auch sog. Flow Charts darzustellen.

Vgl. Gronroos, Ch., Toward a Third Phase in Service Quality Research: Challenges and Future Directions, a.a.O., S. 60.

Vgl. Bateson, J.E.G., Managing Services Marketing: Text and Readings, a.a.O., S. 214; Botschen, G., Bstieler, L., Woodside, A.G., Sequence-Oriented Problem Identification within Service Encounters, in: Manuskript, Januar 1993, S. 13. 
SHOSTACK entwickelte Mitte der achtziger Jahre mit dem Instrument des Blueprinting ein erstes Verfahren zur Planung und Kontrolle von Dienstleistungen, dessen zentraler Gegenstand die systematische Erfassung und Darstellung des Dienstleistungsproduktions- und nutzungsprozesses ist. ${ }^{223}$ Das Verfahren stellt eine Weiterentwicklung der aus dem Einzelhandelsbereich bekannten Kundenlaufstudien dar, welche bereits wichtige Hinweise über die räumlichen und zeitlichen Nutzungsprofilverläufe von Dienstleistungskunden bereitstellen können, jedoch primär durch einen empirischen (quantitativen) Charakter geprägt sind. ${ }^{224}$ Das Ziel der Blueprinting Methode besteht dagegen eher in einer (möglichst) vollständigen und systematischen Erfassung der Kundenkontaktsituation und der hiermit verbundenen Prozesse. Zu diesem Zweck erfolgt eine Zerlegung des Dienstleistungsprozesses in Teilphasen, die wiederum weitere Teilprozesse beinhalten können. Die ermittelten Prozeßphasen und die hiermit verbundenen Schnittstellen werden in einem graphischen Ablaufdiagramm visualisiert. Eine "Line of Visibility" markiert in einem solchem Diagramm den für den Kunden sichtbaren Bereich der Dienstleistungserstellung und damit den Ausschnitt des Produktionsprozesses, den er seinem Zufriedenheitsurteil zugrunde legt. In dieser grundlegenden Vorgehensweise wird die inhaitliche Nähe zum dargestellten "Service Script Konzept“ deutlich, das ebenfalls auf eine Abfolge erlebter Dienstleistungssequenzen bei der Bildung des Zufriedenheitsurteils abstellt.

Es liegt im Ermessen des Forschers, wie detailliert ein solcher Blueprint im konkreten Anwendungsfall ausgestaltet wird. Skeptiker bezweifeln die Möglichkeit, einen Dienstleistungsprozeß in all seinen Schattierungen abbilden zu können. ${ }^{225}$ Dieses Argument wird jedoch der Idee des Blueprinting nicht gerecht. Vielmehr gilt es, sich vor dem Hintergrund der Zielsetzung einer Untersuchung auf einen bestimmten Differenzierungsgrad bei der Darstellung der einzelnen Episoden der Dienstleistung zu beschränken. Soll die mit einer vollständigen Transaktion verbundene Kundenzufriedenheit ermittelt werden, empfiehlt es sich, zunächst ein

Vgl. Shostack, G.L., Designing Services that Deliver, in: HBR, Vol. 62, January/February, 1984, S. 133 ff.; Shostack, G.L., How to Design a Service, in: EJoM, Vol. 18, No. 1, 1984, S. 54 ff.; Shostack, G.L., Service Positioning through Structural Change, in: JoM, Vol. 51, January, 1987, S. $35 \mathrm{ff}$.

Zu der Eignung von Kundenlauf-Beobachtungen bzw. Passagierstromanalysen im Kontext von Verkehrsdienstleistungen vgl. Birkelbach, R., Qualitätsmanagement in Dienstleistungscentern: Konzeption und typenspezifische Ausgestaltung unter besonderer Berücksichtigung von Verkehrsflughäfen, a.a.O., S. $91 \mathrm{f}$.

Zu einer Aufzählung entsprechender Autoren vgl. Westerbarkey, P., Methoden zur Messung und Beeinflussung der Dienstleistungsqualităt: Feedback- und Anreizsysteme in Beherbergungsunternehmen, a.a.O., S. 50. 
globales Blueprint für den gesamten Prozeß zu entwerfen und dieses dann sukzessiv dem Untersuchungszweck gemäß in seine Bestandteile zu zerlegen. Sind einzelne Ausschnitte des Dienstleistungsprozesses von besonderem Interesse, kann eine entsprechend vertiefte Darstellung der Dienstleistungselemente vorgenommen werden. Der Untersuchungszweck determiniert damit die Detailgenauigkeit der Darstellung. ${ }^{226}$

In diesem Zusammenhang ist jedoch anzumerken, daß die primäre Zielsetzung von SHOSTACK in einer Analyse und Visualisierung des Unternehmensprozesses besteht. Die Analyse des Dienstleistungsprozesses erfolgt vornehmlich aus der Unternehmensperspektive und hat in erster Linie den Zweck, produktionsbedingte Ursachen für Leistungsmängel offenzulegen. ${ }^{227}$ Das Verfahren des Blueprinting erscheint daher für die interne Problementdeckung besonders geeignet, da es eine instrumentelle Hilfe darstellt, den komplexen Charakter von Dienstleistungen zu strukturieren und für interne Planungszwecke in Form graphischer Darstellungen zu kommunizieren.

Einen Perspektivenwechsel erfuhr das Konzept des Blueprinting durch KINGMANBRUNDAGE und GUMMESSON. In dem von diesen Autoren entwickelten Instrument des Service Mapping ist der Kundenprozeß Ausgangspunkt der Überlegungen. ${ }^{228}$ Zentraler Bestandteil einer solchen "Dienstleistungskarte“ ist der Kundenpfad, der die chronologische Abfolge der Interaktionen des Kunden mit dem Dienstleistungsangebot umfaßt und horizontal in einem entsprechenden Abflußdiagramm abgetragen wird. Auch hier trennt eine „Line of Visibility“ den für den Kunden sichtbaren Teil des Dienstleistungserstellungsprozesses von dem für inn unsichtbaren Teil. Die hierbei entstehenden Bereiche des Front-Office („Onstage Actions“) und Back-Office („Backstage“) werden im Rahmen des Service Mapping um weitere vertikale Schichten ergänzt, um die Beziehungen zum System der

Vgl. Stauss, B., Kundenprozeßorientiertes Qualitătsmanagement im Dienstleistungsbereich, a.a.O., S. 32 .

227 Vgl. Stauss, B., Seidel, W., Prozessuale Zufriedenheitsermittlung und Zufriedenheitsdynamik bei Dienstleistungen, a.a.O., S. 189; Bateson, J.E.G., Managing Services Marketing: Text and Readings, a.a.O., S. 214.

228 Vgl. Kingman-Brundage, J., The ABC's of Service System Blueprinting, in: Designing a Winning Service Strategy, Bitner, M.J., Crosby, L.A. (Hrsg.), Chicago 1989, S. 30 ff.; KingmanBrundage, J., Service Mapping: Gaining a Concrete Perspective on Service System Design, in: QUiS 3: Quality in Services Conference - Proceedings, Scheuing, E.E. et al. (Hrsg.), New York 1994, S. 235 ff.; Gummesson, E., Kingman-Brundage, J., Service Design and Quality: Applying Service Blueprinting and Service Mapping to Railroad Services, a.a.O., S. $105 \mathrm{ff}$. 




Abb. 16: Vereinfachte Service Map am Beispiel einer Flugreise

Leistungserstellung zu verdeutlichen. In Abbildung 16 ist ein Ausschnitt einer solchen Service Map für eine Flugreise dargestellt. Diese wurde bewußt einfach gehalten, um die Übersichtlichkeit der dargestellten Prozesse möglichst wenig zu beeinträchtigen. ${ }^{229}$

Die "Line of External Interaction“ unterscheidet in einem solchen Ablaufdiagramm die allein vom Dienstleistungskunden durchgeführten Tätigkeiten von den Interaktionen, die mit dem Kundenkontaktpersonal stattfinden. Dagegen trennt die "Line of Internal Interaction" die unterschiedlichen Interaktionen von Kundenkontakt- und Back-Office-Personal und Geschäftsleitung. Darüber hinaus besteht

Neben den dargestellten Dienstleistungselementen sind auch die eingezeichneten Verbindungslinien von besonderer Bedeutung. Aus innen lassen sich wertvolle Hinweise auf etwaige Schnittstellenprobleme gewinnen. Dabei gilt es zu beachten, daß verschiedene Prozeßelemente optional durchlaufen werden. Eine genaue Analyse des Kundenpfades im konkreten Anwendungsfall kann zu einer weitaus komplexeren Darstellung des mit der Nutzung einer Dienstleistung verbundenen Prozesses als in der obigen Abbildung führen. So stellt Shostack im Hinblick auf ein Blueprint für den Scheckverkehr im Privatkundengeschäft von Kreditinstituten fest, daß "It is a large and complex system that in fully-detailed diagrammatic form would occupy more than one wall of a very large room." Shostack, G.L., Understanding Services Through Blueprinting, in: Advances in Service Marketing and Management: Research and Practice, Swartz, T.A., Bowen, D.E., Brown, St.W. (Hrsg.), Greenwich, London 1992, S. 80 . 
schließlich die Möglichkeit, eine „Line of Implementation“ zu berücksichtigen, die zwischen Planungs- und Organisationstätigkeiten der Unternehmensleitung und operativen Tätigkeiten unterstützender Funktionsbereiche differenziert. Die Autoren betonen mit einer solchen Sichtweise des Dienstleistungsprozesses die hohe Interdependenz einer Vielzahl sehr unterschiedlicher Prozesse, aus deren Zusammenspiel sich die Qualität einer Dienstleistung ergibt.

Eine Erweiterung erfährt das Konzept des Service Mapping durch BITNER. ${ }^{230}$ Die Wichtigkeit des physischen Umfeldes bei der Wahrnehmung einer Dienstleistung betonend, ergänzt die Autorin die „Service Map" um Angaben über Aspekte des physischen Umfeldes, mit denen der Dienstleistungskunde während des Nutzungsprozesses an den verschiedenen Kontaktpunkten in Berührung kommt. Diese werden oberhalb des Kundenpfades in das Ablaufdiagramm entsprechend eingetragen (vgl. Abbildung 16). Wie in den bisherigen Ausführungen deutlich wurde, erscheint es gerade im Kontext von Verkehrsdienstleistungen angezeigt, auch die vor und nach dem direkten Kontakt mit dem Verkehrsdienstleister durch den Kunden erbrachten Teilprozesse (z.B. Informationsbeschaffung, An- und Abreise zur bzw. von der Transportlokation etc.) in einer solchen Darstellung zu berücksichtigen. Nur so können alle etwaig zufriedenheitsrelevanten Aspekte des Nutzungsprozesses adäquat erfaßt werden.

Blueprints bzw. Service Maps sind ein geeignetes Instrument zur Visualisierung des Prozeßcharakters von Dienstleistungen. So hat sich ihr Einsatz bei der Erfassung, Planung, Steuerung und Kontrolle z.T. sehr unterschiedlicher Dienstleistungen wie Tankstellen, Speditionen, Hotels oder Krankenhäuser bewährt. ${ }^{231}$ Auch im Kontext von Verkehrsdienstleistungen finden diese Verfahren inzwischen vereinzelt Anwendung. ${ }^{232}$ Über die Funktion der Ermittlung und Visualisierung von

230 Vgl. Bitner, M.J., Managing the Evidence of Service, in: The Service Quality Handbook, Scheuing, E.E., Christopher, W.F. (Hrsg.), New York u.a. 1993, S. $358 \mathrm{ff}$.

231 Vgl. Stauss, B., "Augenblicke der Wahrheit" in der Dienstleistungserstellung, a.a.O., S. 386; Mangold, W.G., Babakus, E., Service Quality. The Front-Stage vs. the Back-Stage Perspective, in: JoSM, Vol. 5, No. 4, 1991, S. 59 ff. Westerbarkey, P., Methoden zur Messung und Beeinflussung der Dienstleistungsqualitat: Feedback- und Anreizsysteme in Beherbergungsunternehmen, a.a.O., S. 49 ff. Zu einer differenzierten Darstellung der Einsatzmöglichkeiten der Blueprinting Technik im Finanzdienstleistungsbereich vgl. Schmitz, G., Qualitătsmanagement im Privatkundengeschaft von Banken: Konzeption und aufbauorganisatorische Verankerung, a.a.O., S. $118 \mathrm{ff}$.

232 Vgl. Birkelbach, R., Qualitătsmanagement in Dienstleistungscentern: Konzeption und typenspezifische Ausgestaltung unter besonderer Berücksichtigung von Verkehrsflughäfen, a.a.O., S. 93 f.; Laakmann, K., Value-Added Services als Profilierungsinstrument im Wettbewerb: Analyse, Generierung und Bewertung, a.a.O., S. 143 ff.; Gummesson, E., Kingman-Brundage,

(Fortsetzung der Fußnote auf der náchsten Seite) 
Kontaktpunkten hinaus stellen Blueprints bzw. Service Maps die Basis für den Einsatz bestimmter Meßmethoden der Kundenzufriedenheit dar. Dabei gilt es zu beachten, daß mit dem Einsatz dieser Techniken zeit- und kostenintensive Erhebungen verbunden sind. ${ }^{233}$

\subsection{Verfahren zur Messung der Kundenzufriedenheit}

Im Rahmen der Marktforschung ist grundsätzlich zwischen dem Rückgriff auf sekundärstatistische Verfahren und die der Primärerhebung zu unterscheiden. ${ }^{234}$ Wenngleich die Sekundärforschung wichtige Informationen insbesondere für einen Vergleich mit anderen Verkehrsdienstleistern bietet, ist für eine prozeßorientierte Analyse der Kundenzufriedenheit eine unternehmensindividuelle Erfassung und Messung durch Primärforschung unerläßlich. In der wissenschaftlichen Literatur finden sich unterschiedliche Vorschläge zur Systematisierung von primärforscherischen Verfahren zur Messung der Kundenzufriedenheit. ${ }^{235}$ Grundsätzlich lassen sich nachfrager-, anbieter- und mitarbeiterorientierte Meßverfahren unterscheiden. ${ }^{236}$ Diese Unterteilung orientiert sich an dem „Objekt" der Messung. Konzentrieren sich anbieter- und mitarbeiterorientierte Ansätze auf die Erfassung der Kundenzufriedenheit aus Unternehmenssicht, steht bei nachfragerorientierten Verfahren die Kundenperspektive im Mittelpunkt. Des weiteren können die Verfahren in „objektive“ und „subjektive" Ansätze eingeteilt werden. Objektive Verfahren stellen auf eine intersubjektiv überprüfbare Zufriedenheitsbewertung ab. Es wird davon ausgegangen, daß eine Zufriedenheitsanalyse mit Hilfe neutraler dritter Personen oder anhand objektiver Indikatoren (z.B. Beschaffenheit bestimmter tangibler Bestandteile der Dienstleistung) vorgenommen werden kann.

J., Service Design and Quality: Applying Service Blueprinting and Service Mapping to Railroad Services, a.a.O., S. $105 \mathrm{ff}$.

233 Vgl. Laakmann, K., Value-Added Services als Profilierungsinstrument im Wettbewerb: Analyse, Generierung und Bewertung, a.a.O., S. 144.

234 Vgl. Meffert, H., Marketingforschung und Käuferverhalten, a.a.O., S. $195 \mathrm{ff}$. Als Basis einer Sekundärforschung kann z.B. das seit 1992 jährlich durchgeführte Deutsche Kundenbarometer dienen, das eine branchenübergreifende Studie zur Messung der Kundenzufriedenheit mit über 30 Branchen in Deutschland darstellt. Vgl. Meyer, A., Dornach, F., Das Deutsche Kundenbarometer: Qualität und Zufriedenheit, a.a.O., S. $161 \mathrm{ff}$.

$235 \mathrm{Vgl}$. Meffert, H., Bruhn, M., Beschwerdeverhalten und Zufriedenheit von Konsumenten, a.a.O., S. 600; Schmitz, G., Qualitătsmanagement im Privatkundengeschätt von Banken: Konzeption und aufbauorganisatorische Verankerung, a.a.O., S. 255; Bruhn, M., Hennig, K., Selektion und Strukturierung von Qualitătsmerkmalen - auf dem Weg zu einem umfassenden Qualitätsmanagement für Kreditinstitute (Teil 1), a.a.O., S. 219; Schütze, R., Kundenzufriedenheit: After-Sales-Marketing auf industriellen Märkten, a.a.O., S. 184 und die dort angegebenen Literaturquellen.

236 Vgl. Meffert, H., Bruhn, M., Dienstleistungsmarketing: Grundlagen, Konzepte, Methoden; mit Fallbeispielen, a.a.O., S. 205. 
Dagegen stützen sich subjektive Verfahren auf die interindividuell unterschiedlichen Wahrnehmungen und Bedürfnisse der beurteilenden Person. ${ }^{237}$

Da eine Messung der Kundenzufriedenheit insbesondere aus Kundensicht zu erfolgen hat, konzentriert sich die folgende Darstellung auf solche Verfahren, die eine nachfragerorientierte Perspektive einnehmen. ${ }^{238}$ Dazu wurden diejenigen Verfahren ausgewählt, die sich in ihrer grundsätzlichen Konzeption für eine phasenorientierte Messung der Kundenzufriedenheit eignen. Wie in Abbildung 17 dargestellt, kann innerhalb der subjektiven Meßansätze eine Unterteilung in merkmals- und ereignisgestützte Verfahren vorgenommen werden. ${ }^{239}$ Während im Rahmen merkmalsgestützter Verfahren eine Analyse der Zufriedenheit mit Eigenschaften einer Dienstleistung vorgenommen wird, stehen bei ereignisgestützten Ansätzen einzelne Erlebnisse bei der Inanspruchnahme einer Dienstleistung im Vordergrund.

Im Rahmen einer Eignungsdiskussion dieser Instrumente ist es erforderlich, einheitliche Bewertungskriterien festzulegen. In der Literatur werden vornehmlich als generelle Bewertungskriterien die Relevanz, Eindeutigkeit, Vollständigkeit, Aktualität, Steuerbarkeit und Kostenwirtschaftlichkeit eines Meßansatzes herangezogen. ${ }^{240}$ Die Eignung der Verfahren muß jedoch vor dem Hintergrund ihres Anwendungsbereiches erörtert werden. Daher sind die bereits genannten Kriterien um jene Anforderungen zu ergänzen, die sich aus einer prozeßorientierten Sichtweise der Kundenzufriedenheit mit Verkehrsdienstleistungen ergeben. Dabei ist es besonders wichtig, daß ein Meßansatz über die Möglichkeit verfügt, den Episoden- bzw. Schnittstellencharakter einer Dienstleistung auf unterschiedlichen Aggregationsebenen zu erfassen. Ebenso ist zu überprüfen, ob mit Hilfe des

237 Vgl. Bruhn, M., Hennig, K., Selektion und Strukturierung von Qualitätsmerkmalen - auf dem Weg zu einem umfassenden Qualitätsmanagement für Kreditinstitute (Teil 1), a.a.O., S. 220. $\mathrm{Zu}$ einer umfassenden Darstellung von anbieter- und mitarbeiterorientierten Meßverfahren vgl. Meffert, H., Bruhn, M., Dienstleistungsmarketing: Grundlagen, Konzepte, Methoden; mit Fallbeispielen, a.a.O., S. $225 \mathrm{ff}$.

239 Vgl. Stauss, B., Service-Qualität als strategischer Erfolgsfaktor, in: Erfolg durch Service-Qualität, Stauss, B. (Hrsg.), München 1991, S. 17.

240 Vgl. Stauss, B., Hentschel, B., Verfahren der Problementdeckung und -analyse im Qualitătsmanagement von Dienstleistungsunternehmen, in: JdAV, Jg. 36, H. 3, 1990, S. 235; Meyer, A., Hüttinger, S., Möglichkeiten und Grenzen der Implementierung eines Total Quality Managements in Kreditinstituten: Konzeptionelle Vorschläge und empirische Analyse, in: JdAV, Jg. 42, H. 3, 1996, S. 227. 


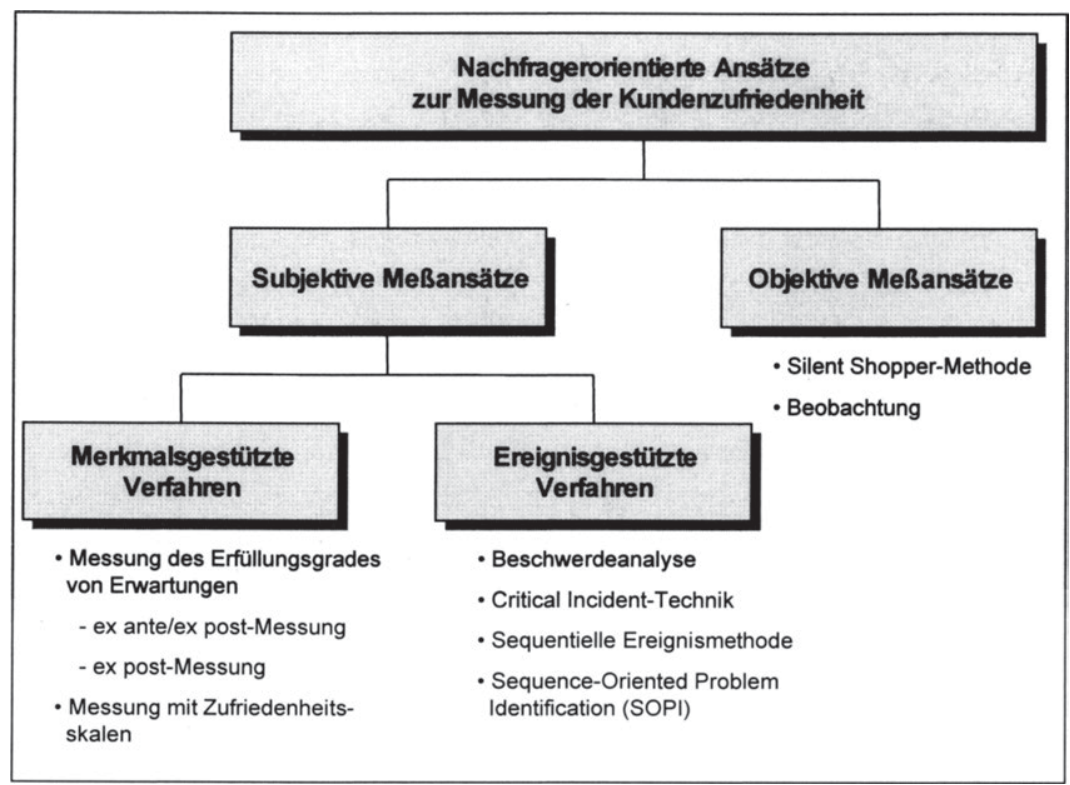

\section{Abb. 17: Ansätze zur Messung der Kundenzufriedenheit}

Meßansatzes Rückschlüsse auf die Relevanz einzelner Phasen für das gesamte Zufriedenheitsempfinden sowie auf Effekte der Zufriedenheitsdynamik gezogen werden können. Ist ein Ansatz primär auf eine statische Analyse der Kundenzufriedenheit ausgerichtet, gilt es zu untersuchen, inwieweit Modifikationsmöglichkeiten im Hinblick auf eine „Dynamisierung“ des Verfahrens bestehen.

\subsection{Objektive Meßansätze}

Instrumente, die nicht das subjektive Empfinden einzelner Kunden zu ermitteln versuchen, sondern durch eine dritte Person oder durch objektive Zufriedenheitsindikatoren eine intersubjektiv nachprüfbare Zufriedenheitsbewertung vornehmen, stellen insbesondere die Silent Shopper-Methode sowie das Verfahren der Beobachtung dar. 
"Silent" bzw. „Mystery Shopper" sind Beobachter und Testpersonen, die als Dienstleistungskunde auftreten, um durch die Simulation einer realen Situation Hinweise auf wesentliche Mängel bei der Dienstleistungserstellung zu erhalten. ${ }^{241}$ Auf diese Weise sollen die subjektiven Empfindungen der Kunden im Hinblick auf die Erfahrungen mit dem Dienstleistungsprozeß auf einer möglichst objektiven Ebene erfaßt werden. Als Vorteil dieser auch als Testkauf bezeichneten Vorgehensweise ist anzuführen, daß nicht nur ein Überblick über das Leistungsvermögen der eigenen Unternehmung, sondern durch anonym durchgeführte Testkäufe auch ein Konkurrenzvergleich ermöglicht wird. Eine solche vergleichende „aktive“ Beobachtung kann im Verkehrsdienstleistungsbereich z.B. von Mitarbeitern auf Dienstreisen durchgeführt werden.

Als kritisch erweist sich jedoch der Umstand, daß die evaluierten Leistungsmerkmale zumeist nicht nach der Relevanz aus Kundensicht geordnet sind und ein solches Vorgehen daher eher für unternehmensinterne Vergleichsstudien geeignet ist. ${ }^{242}$ Für eine prozeßorientierte Analyse der Kundenzufriedenheit ist somit sicherzustellen, daß die Beobachtung auf einer differenzierten Analyse des Kundenpfades beruht. STAUSS empfiehlt in diesem Zusammenhang den Einsatz geschulter Sozialforscher als Silent Shopper. ${ }^{243}$ Dennoch stellt sich auch hier die Frage, insbesondere im Hinblick auf die Vollständigkeit und Relevanz der erhaltenen Informationen, inwieweit Testkäufer in der Lage sind, die Wahrnehmungen und Empfindungen „realer" Kunden nachzuvollziehen. Darüber hinaus ist der zeitaufwendige Einsatz spezialisierter Testpersonen mit vergleichsweise hohen Kosten verbunden. ${ }^{244}$

Zielsetzung des Meßverfahrens der Beobachtung ist das Erfassen offensichtlicher Mängel im Dienstleistungserstellungsprozeß und hieraus resultierender Kundenreaktionen. Hier kann zum einen mit Hilfe technischer Einrichtungen das kontaktpunktbezogene Verhalten von Dienstleistungskunden beobachtet werden.

241 Vgl. Bruhn, M., Hennig, K., Selektion und Strukturierung von Qualitătsmerkmalen - auf dem Weg zu einem umfassenden Qualitătsmanagement für Kreditinstitute (Teil 1), a.a.O., S. $220 f$.

242 Vgl. Westerbarkey, P., Methoden zur Messung und Beeinflussung der Dienstleistungsqualităt: Feedback- und Anreizsysteme in Beherbergungsunternehmen, a.a.O., S. 92.

Vgl. Stauss, B., Kundenprozeßorientiertes Qualitătsmanagement im Dienstleistungsbereich, a.a.O., S. 44. Auf der Grundlage einer differenzierten Rollen- und Problemvorgabe können Silent Shopper zentrale Faktoren der Kontaktsituation, des Personalverhaltens und der Kundensituation (z.B. Zahl und Verhalten anderer Kunden) realitătsnah erkunden und in standardisierten Protokollen festhalten.

244 Vgl. Schmitz, G., Qualitătsmanagement im Privatkundengeschaft von Banken: Konzeption und aufbauorganisatorische Verankerung, a.a.O., S. 267. 
MEFFERT und BIRKELBACH berichten in diesem Zusammenhang von dem Einsatz von Videoaufzeichnungen am Frankfurter Flughafen, anhand derer Orientierungsprobleme von Flughafenbesuchern identifiziert werden konnten. ${ }^{245}$ Auf diese Weise können wichtige Hinweise bezüglich der Gestaltung des physischen Umfeldes gewonnen werden. Eine weitere Möglichkeit besteht in dem Einsatz geschulter Sozialforscher, die jedoch nicht als Testkunde auftreten, sondern als „passive“ Beobachter agieren und das Interaktionsverhalten von Kunden und Mitarbeitern an unterschiedlichen Kontaktpunkten analysieren. In Abhängigkeit von der Art der Kundenkontaktsituation (z.B. Beratung beim Ticketerwerb) ist ein solches Vorgehen nur eingeschränkt ohne das Wissen der Beteiligten möglich. Dies hat unter Umständen Beobachtungseffekte zur Folge. Ein weiteres zentrales Problem besteht in der Tatsache, daß das Verfahren der Beobachtung vor allem auf die äußeren Aspekte des Dienstleistungserlebens abzielt und daher die mit der Inanspruchnahme der Dienstleistung verbundenen emotionalen Reaktionen von Kunden nur sehr eingeschränkt erkennbar sind. Soll der gesamte Kundenpfad analysiert werden, wirkt sich zudem wie beim Silent Shopper-Ansatz der mit dem Einsatz des Verfahrens verbundene hohe Zeit- und Kostenaufwand negativ auf die Anwendbarkeit dieser Meßmethode aus.

Insgesamt erweisen sich die objektiven Ansätze zur Messung der Kundenzufriedenheit zwar als grundsätzlich geeignet, wichtige Anhaltspunkte über den prozessualen Charakter von Dienstleistungen und die Bildung der Zufriedenheit mit Verkehrsdienstleistungen liefern zu können. Aufgrund der genannten Nachteile der Verfahren sind diese jedoch zwingend um solche Verfahren zu ergänzen, die eine Bewertung der erbrachten Leistung aus Sicht „wirklicher" Kunden garantieren. tionelle Grundlagen und typenspezifische Ausgestaltung, a.a.O., S. 221. 


\subsection{Subjektive Meßansätze}

\subsection{Merkmalsgestützte Verfahren}

Die am häufigsten anzutreffende Variante merkmalsorientierter Meßansätze stellen die sog. multiattributiven Kundenbefragungen dar, die in sehr unterschiedlichen Anwendungsvarianten vorkommen. ${ }^{246}$ Diese Verfahren beruhen auf der Annahme, daß sich die Gesamtzufriedenheit eines Kunden aus einer Vielzahl individuell bewerteter Merkmale der Dienstleistung zusammensetzt (kompositioneller Ansatz). ${ }^{247}$ Die Zufriedenheit mit einzelnen Merkmalen der Dienstleistung wird anhand vorstrukturierter Fragebögen auf der Basis von Ratingskalen erhoben. Die Gesamtzufriedenheit kann dann mittels unterschiedlicher Aggregationsalgorithmen aus den Einzelzufriedenheiten berechnet oder ebenfalls auf einer Ratingskala erhoben werden. Da die verschiedenen Anwendungsvarianten und grundsätzlichen Problembereiche des Multiattributansatzes bereits an anderer Stelle ausführlich behandelt wurden, ${ }^{248}$ sollen hier insbesondere die Aspekte dargestellt werden, die bei einer prozeßorientierten Messung der Kundenzufriedenheit von besonderer Relevanz sind.

Innerhalb der Gruppe kompositioneller Multiattributverfahren kann zwischen indirekten und direkten Verfahren unterschieden werden. Während bei indirekten Verfahren die Erwartungskomponente vor und die Wahrnehmungskomponente nach dem Konsumerlebnis erfaßt werden (ex ante/ex post-Messung), wird bei

246 Zu weiteren, bislang weniger häufig eingesetzten Verfahren, wie z.B. der Penalty-RewardAnalyse vgl. Haller, S., Beurteilung von Dienstleistungsqualităt: Dynamische Betrachtung des Qualitătsurteils im Weiterbildungsbereich, a.a.O., S. $108 \mathrm{ff}$.; Meffert, H., Bruhn, M., Dienstleistungsmarketing: Grundlagen, Konzepte, Methoden; mit Fallbeispielen, a.a.O., S. $213 \mathrm{ff}$.

247 Vgl. Hentschel, B., Multiattributive Messung von Dienstleistungsqualität, in: Dienstleistungsqualităt: Konzepte, Methoden, Erfahrungen, Bruhn, M., Stauss, B. (Hrsg.), 2. Aufl., Wiesbaden 1995, S. 355.

Dieser kompositionellen Sichtweise des Multiattributansatzes stehen Verfahren gegenüber, die aus einer dekompositionellen Perspektive die Zufriedenheit von Kunden ermitteln. Hier wird anhand globaler Zufriedenheitsurteile eine Rangreihe verschiedener Leistungspakete mit unterschiedlichen Merkmalsausprägungen gebildet. Hierauf aufbauend wird dann mit Hilfe unterschiedlicher Auswertungsmethoden, wie z.B. der Conjoint-Analyse, die Zufriedenheit mit einzelnen Merkmalen statistisch ermittelt. Zu der Diskussion der Eignung der verschiedenen Ansätze vgl. Hentschel, B., Dienstleistungsqualität aus Kundensicht: Vom merkmals- zum ereignisorientierten Ansatz, a.a.O., S. 113. Zur Anwendung der Conjoint-Analyse im Rahmen von Zufriedenheitsanalysen vgl. Sebastian, K.-H., Paffrath, R., Lauszus, D., Runneboon, T., Messung von Kundenzufriedenheit bei industriellen Dienstleistungen, in: Kundenzufriedenheit: Konzepte, Methoden, Erfahrungen, Simon, H., Homburg, Ch. (Hrsg.), Wiesbaden 1995, S. $357 \mathrm{ff}$

248 Vgl. Peterson, R.A., Wilson, W.R., Measuring Customer Satisfaction: Fact and Artifact, a.a.O., S. $61 \mathrm{ff}$; Hentschel, B., Dienstleistungsqualität aus Kundensicht: Vom merkmals- zum ereignisorientierten Ansatz, a.a.O., S. $111 \mathrm{ff}$; Andritzky, K., Die Operationalisierbarkeit von Theorien zum Konsumentenverhalten, a.a.O., S. $56 \mathrm{ff}$. 
direkten Ansätzen nur die nachträgliche Diskrepanz zwischen diesen beiden Größen ermittelt (ex post-Messung des Erfüllungsgrades von Erwartungen) bzw. unmittelbar nach dem Ergebnis des Abwägungsprozesses - der Kundenzufriedenheit - gefragt.

Neben der bereits erörterten schwierigen Aufgabe der inhaltlichen Festlegung der Erwartungskomponente ${ }^{249}$ sind mit einer separaten Erhebung weitere Validitätsprobleme verbunden. Die zweimalige Verwendung der gleichen Meßskala birgt die Gefahr eines konsistenten Antwortverhaltens der Befragten. Die als Zufriedenheit interpretierte Differenz zwischen den ermittelten Größen kann damit deutlich von der tatsächlich empfundenen Zufriedenheit abweichen. ${ }^{250}$ Die bei dieser Variante notwendige zweifache Befragung ist zudem mit Durchführungsproblemen behaftet, da den Kunden eine erhöhte Kooperationsbereitschaft abverlangt wird. Darüber hinaus kann die ex ante-Erhebung von Erwartungen zu einer „unnatürlichen“ Aktivierung des Befragten sowie der Formulierung überhöhter Anforderungen (sog. Anspruchsinflation) und entsprechend verzerrter Wahrnehmung führen. ${ }^{251}$ Die Zweckmäßigkeit einer Differenzbildung ist aber auch bezüglich der Auswertungslogik zu bezweifeln, als daß in Einzelfällen irreführende Interpretationen die Folge sein können. ${ }^{252}$ Darüber hinaus wurde bereits darauf hingewiesen, daß Erwartungen einem dynamischen Veränderungsprozeß während des Dienstleistungskonsums unterliegen und der Sinn einer separaten Messung von Erwartungen damit grundsätzlich fragwürdig erscheint.

Diesen Aspekten kann dadurch Rechnung getragen werden, daß eine ex postMessung des Erfüllungsgrades von Erwartungen durchgeführt wird. Hier wird der Kunde im Anschluß an den Dienstleistungsprozeß gefragt, inwieweit die Erfahrungen mit der Dienstleistung den ursprünglichen Erwartungen entsprechen. Die Möglichkeit veränderter Erwartungen wird damit berücksichtigt. Der bewertende Vergleich beruht nicht auf einer durch den Forscher durchgeführten Dif-

Vgl. Homburg, Ch., Rudolph, B., Theoretische Perspektiven zur Kundenzufriedenheit, a.a.O., S. 44.

251 Vgl. Haller, S., Beurteilung von Dienstleistungsqualität: Dynamische Betrachtung des Qualitätsurteils im Weiterbildungsbereich, a.a.O., S. 97. Diesen Problemen kann zumindest teilweise dadurch begegnet werden, daß sowohl die Erwartungs- als auch die Wahrnehmungskomponente im Anschluß an den Dienstleistungskonsum getrennt abgefragt werden. Dies stellt jedoch hohe Anforderungen an die Urteilsbereitschaft und -făhigkeit der Kunden, nachträglich die Inanspruchnahme der Dienstleistung in die Einzelkomponenten zu zerlegen.

Vgl. Hentschel, B., Dienstleistungsqualităt aus Kundensicht: Vom merkmals- zum ereignisorientierten Ansatz, a.a.O., S. 140. 
ferenzbildung, sondern findet auf der intrapersonellen Ebene statt. Von dem bekundeten Erfüllungsgrad wird dann direkt auf die Zufriedenheit geschlossen. Damit wird aber auch bei dieser Vorgehensweise unterstellt, daß aus der Bestätigung bzw. Nicht-Bestätigung von Erwartungen unmittelbar Zufriedenheit resultiert. Die affektive Reaktion auf den kognitiv geprägten Vergleichsprozeß wird dagegen nicht berücksichtigt. ${ }^{253}$

Der Prozeßcharakter von Dienstleistungen und die damit verbundene Entstehung des Zufriedenheitsurteils während des Dienstleistungskonsums führen zu einer Verstärkung der aufgezeigten Probleme. GRÖROOS faßt die mit einer Messung der Erfüllungsgrades von Erwartungen verbundenen Schwierigkeiten anschaulich in drei Punkten zusammen: ${ }^{254}$

- Die Messungen von Erwartungen im Anschluß an den Dienstleistungskonsum (ex post-Messung) erlaubt keine Rückschlüsse auf die Erwartungen, die ein Kunde a priori an die Dienstleistung gebildet hat. Die ermittelte Größe ist vielmehr bereits durch die Erfahrungen mit dem Prozeß geprägt.

- Da sich aber die Erwartungen an eine Dienstleistung im Laufe des Nutzungsprozesses kontinuierlich ändern, erscheint auch die Messung von Erwartungen vor der Inanspruchnahme der Dienstleistung wenig zweckmäßig (ex ante-Messung). Die so erhobenen Standards entsprechen nicht dem Vergleichsmaßstab, mit denen der Kunde seine Wahrnehmungen im Kundenprozeß vergleicht.

- Eine Messung von Erwartungen ist grundsätzlich zu hinterfragen, da in den Wahrnehmungen verarbeitete Erwartungen bereits enthalten sind. Demzufolge führt eine separate Messung der Erwartungen zu einer doppelten Erhebung der gleichen Komponente.

Die Ergebnisse der in den vorhergehenden Kapiteln vorgestellten Studien bestätigen die grundsätzliche Richtigkeit der beiden erstgenannten Aussagen. ${ }^{255}$ Daher ist es nicht verwunderlich, daß die Anwendung der beschriebenen Methoden zur Messung der Kundenzufriedenheit mit Dienstleistungen einer zunehmenden Kritik

Vgl. hierzu die Ausführungen in Kap. B 2.22 dieser Arbeit.

Vgl. Gronroos, Ch., Toward a Third Phase in Service Quality Research: Challenges and Future Directions, a.a.O., S. 56.

255 Insbesondere die in Kap. B 2.333 vorgestellte Studie von MCCLURE zeigt jedoch auch erste Ansatzpunkte zu der Überwindung dieser Problembereiche im Rahmen einer prozeßorientierten Messung der Kundenzufriedenheit auf. So ist insbesondere bei Ersterhebungen die Kenntnis der Erwartungen von Kunden an eine Dienstleistung von zentraler Bedeutung, da sie wichtige Anhaltspunkte bezüglich der grundsätzlichen Ausgestaltung einer Dienstleistung liefern. Vgl. Zeithaml, V.A., Berry, L.L., Parasuraman, A., The Behavioral Consequences of Service Quality, a.a.O., S. 40. 
ausgesetzt ist und Empfehlungen für eine direkte Messung der Kundenzufriedenheit ausgesprochen werden. ${ }^{256}$

Unabhängig von der gewählten Meßvariante sind bei der Konstruktion eines Fragebogens zur Analyse der Kundenzufriedenheit mit Dienstleistungen einige weitere wichtige Aspekte zu beachten. So stellt sich die Frage, ob neben dem als Differenz ermittelten bzw. direkt erhobenen Zufriedenheitsurteil noch das den einzelnen Merkmalen subjektiv beigemessene Gewicht erhoben werden soll. Der Ausgangspunkt einer solchen Bedeutungsgewichtung ist die Annahme, daß nicht jedes Teilmerkmal für jeden Kunden in jeder Situation gleich wichtig ist. ${ }^{257}$ Während Einkomponentenansätze auf eine explizite Erhebung des Bedeutungsgewichtes verzichten, erfolgt im Rahmen von Zweikomponentenansätzen neben der Messung des Zufriedenheitsurteils eine Ermittlung der Gewichtungskomponente auf Ratingskalen. ${ }^{258}$ Ähnlich wie bei einer separaten Erhebung von Erwartungen existiert auch hier die Gefahr der Anspruchsinflation. ${ }^{259}$ Um dieser entgegenzuwirken, besteht z.B. die Möglichkeit, die Befragten zu bitten, eine vorgegebene Punktsumme auf die festgelegten Merkmale zu verteilen und damit

256 Vgl. Homburg, Ch., Werner, H., Ein Meßsystem für Kundenzufriedenheit, in: asw, Jg. 39, H. 11, 1996, S. 94; Meyer, A., Ertl, R., Nationale Barometer zur Messung von Kundenzufriedenheit: Ein Vergleich zwischen dem "Deutschen Kundenbarometer - Qualităt und Zufriedenheit" und dem "American Customer Satisfaction Index (ASCI)", a.a.O., S. 212; Korte, Ch., Customer Satisfaction Measurement: Kundenzufriedenheitsmessung als Informationsgrundlage des Hersteller- und Handelsmarketing am Beispiel der Automobilwirtschaft, a.a.O., S. 38, 40 und die dort angegebene Literatur.

$257 \mathrm{Zu}$ einer ausführlichen Diskussion der Gewichtungsproblematik vgl. Scharitzer, D., Dienstleistungsqualităt - Kundenzufriedenheit, a.a.O., S. 144 ff.; Schütze, R., Kundenzufriedenheit: After-Sales-Marketing auf industriellen Märkten, a.a.O., S. $175 \mathrm{ff}$.

258 Die Verknüpfung der Urteils- und Bedeutungskomponente kann in additiver oder multiplikativer Weise erfolgen. Dabei zeigen empirische Untersuchungen keine einheitlichen Ergebnisse hinsichtlich eines höheren Erklärungsbeitrages der Resultate durch die Venwendung erhobener Gewichte. Vgl. zu einem Überblick Korte, Ch., Customer Satisfaction Measurement: Kundenzufriedenheitsmessung als Informationsgrundlage des Hersteller- und Handelsmarketing am Beispiel der Automobilwirtschaft, a.a.O., S. 38.

Trommsdorff hält es für grundsătzlich „schädlich, die (immer fehlerbehafteten) Ratings miteinander zu multiplizieren, so daß die Fehler zum Quadrat in das Ergebnis eingehen." Trommsdorff, V., Konsumentenverhalten, a.a.O., S. 141.

259 Vgl. Dichtl, E., Müller, St., Anspruchsinflation und Nivellierungstendenzen als meßtechnische Probleme in der Absatzforschung, in: Marketing ZFP, Jg. 8, H. 4, 1986, S. 233 ff. Mit einem solchem Effekt ist um so mehr zu rechnen, wenn bei der Auswahl von Items nur solche berücksichtigt werden, die sich im Rahmen explorativer Vorstudien als besonders wichtig erwiesen und dementsprechend Eingang in den Fragebogen gefunden haben. Ebenso stellt sich die Frage, ob durch die explizite Erhebung von Wichtigkeiten auf Ratingskalen der unterschiedlichen Bedeutung von Merkmalen Rechnung getragen wird. So kann ein Flugreisender bei der Frage nach der Wichtigkeit der Bequemlichkeit des Sitzplatzes und der Sicherheit des Flugzeuges in beiden Fällen auf der vorgegeben Skala den gleichen (hohen) Wert ankreuzen. Es ist aber fraglich, ob dies dem Dringlichkeitsempfinden realiter entspricht. 
Präferenzen zu setzen (Konstant-Summen-Verfahren). ${ }^{260}$ Dieses Verfahren eignet sich jedoch nur für die Messung einer geringen Anzahl von Merkmalen. Hierin konstatiert sich überdies ein weiteres Problem merkmalsorientierter Verfahren.

So hat ein Forscher eine Entscheidung über die Anzahl der zu messenden Merkmale zu treffen. Dem Wunsch nach einer möglichst detaillierten und vollständigen Messung stehen Reaktanz- und Ermüdungserscheinungen seitens der Befragten gegenüber, die durch eine separate Messung von Erwartungen und/oder der Erhebung von Bedeutungsgewichten weiter verstärkt werden. Daher erfolgt im Rahmen von Einkomponentenansätzen eine implizite Gewichtung der Merkmale. Mit Hilfe statistischer Verfahren kann die Bedeutung der Zufriedenheit mit einzelnen Merkmalen bzw. Merkmalskonstrukten (z.B. der Zufriedenheit mit einer bestimmten Phase des Dienstleistungsprozesses) im Zuge der Auswertung der Befragungsergebnisse ermittelt und so ihr Einfluß auf die Gesamtzufriedenheit offengelegt werden. ${ }^{261}$ Es liegen zahlreiche Befragungs- und Testmethoden vor, die der Generierung und Selektion potentieller Kriterien dienen. Um sicherzustellen, daß hierbei die Merkmale erfaßt werden, die von den Befragten üblicherweise zur Beurteilung des betreffenden Objektes herangezogen werden, empfehlen sich hier solche Verfahren, die die Konsumenten bei der Ermittlung der Kriterien mit einbeziehen. ${ }^{262}$ Es ist unmittelbar einsichtig, daß für die Erfassung der Kundenzufriedenheit mit Verkehrsdienstleistungen bei der Auswahl geeigneter Indikatoren der explizite Bezug zu der untersuchten Verkehrsdienstleistung herzustellen ist.

Ist die Entscheidung über die zu berücksichtigenden Merkmale getroffen, besteht der nächste Schritt in der Festlegung der Skalierungstechnik. Wie bereits ausgeführt, werden im Marketing hierzu häufig Rating-Skalen eingesetzt, bei denen

260 Vgl. Schütze, R., Kundenzufriedenheit: After-Sales-Marketing auf industriellen Märkten, a.a.O., S. 176. Zu weiteren Verfahren vgl. Schmidt, R., Marktorientierte Konzeptfindung für langlebige' Gebrauchsgüter: Messung und QFD-gestützte Umsetzung von Kundenanforderungen und Kundenurteilen, a.a.O., S. $156 \mathrm{ff}$.

261 In diesem Zusammenhang wird insbesondere der Einsatz von Verfahren wie der Regressionsund Kausalanalyse empfohlen. Vgl. Homburg, Ch., Rudolph, B., Werner, H., Messung und Management von Kundenzufriedenheit in Industriegüterunternehmen, in: Kundenzufriedenheit: Konzepte, Methoden, Erfahrungen, Simon, H., Homburg, Ch. (Hrsg.), Wiesbaden 1995, S. 322; Wittink, D.R., Bayer, L.R., The Measurement Imperative, in: Customer Satisfaction Measurement: A Management Information System for Total Quality, Marr, S.L., Crosby, A. (Hrsg.), Chicago 1993, S. 17.

Zu einem umfassenden Überblick über Methoden zur Erhebung und Selektion von Indikatoren $\mathrm{vgl}$. Schmidt, R., Marktorientierte Konzeptfindung für langlebige Gebrauchsgüter: Messung und QFD-gestützte Umsetzung von Kundenanforderungen und Kundenurteilen, a.a.O., S. $127 \mathrm{ff}$. 
der Befragte seine Position bezüglich eines Merkmals auf einer mehrstufigen Skala angeben soll. Solche ordinalen ${ }^{263}$ Skalen sind streng genommen bei vielen statistischen Auswertungsverfahren nicht verwendbar, die ein metrisches Skalenniveau voraussetzen. ${ }^{264}$ Der Einsatz solcher Skalen rechtfertigt sich durch die Annahme, daß die Befragten die Abstände zwischen den einzelnen Abschnitten als gleich groß - semantisch äquidistant - empfinden und so ein höheres Skalenniveau angenommen werden kann. ${ }^{265}$

Ein weiteres Problem der prozeßorientierten Messung von Kundenzufriedenheit ergibt sich bezüglich der Aggregation der Einzelzufriedenheiten. Durch die Identifikation von Prozeßphasen und ihre hierarchische Zuordnung treten mehrfache Aggregationsstufen auf. ${ }^{266}$ Hier stellt sich verstärkt die Frage, welcher kognitiven Algebra der Prozeß der Zufriedenheitsbildung unterliegt. In diesem Zusammenhang werden verschiedene Modellansätze diskutiert. Bei nichtkompensatorischen Modellen wird einerseits davon ausgegangen, daß die einzelnen Merkmale ein bestimmtes minimal tolerierbares Niveau aufweisen müssen. Ist dies bereits bei nur einem Merkmal nicht der Fall, fällt die Beurteilung der Dienstleistung negativ aus und der Kunde ist unzufrieden (Konjunktionsansatz). Andererseits wird ebenso die These vertreten, daß bereits die Zufriedenheit mit einem subjektiv als wichtig empfundenen Merkmal zu einer positiven Gesamtzufriedenheit führt (Disjunktionsansatz). ${ }^{267}$ Kompensatorische Modelle beruhen

$263 \mathrm{Zu}$ den verschiedenen Skalenniveaus und ihren statistischen Eigenschaften vgl. z.B. Bleymüller, J., Gehlert, G., Gülicher, H., Statistik für Wirtschaftswissenschaftler, 10. Aufl., München 1996, S. 3 f.

Die konkrete Ausgestaltung von Rating-Skalen liegt dabei weitgehend im Ermessen des Forschers und führte daher zu der Entwicklung einer Vielzahl von unterschiedlichen Anwendungsund Gestaltungsoptionen.

265 Vgl. Berekoven, L., Eckert, W., Ellenrieder, P., Marktforschung: methodische Grundlagen und praktische Anwendung, a.a.O., S. 71; Andritzky, K., Vier Hypothesen bei der Anwendung der Rating-Methode in der Wirtschafts- und Sozialpsychologie, in: JdAV, Jg. 22, 1976, S. 292. In der Literatur findet sich eine Vielzahl unterschiedlicher Varianten von Ratingskalen, die sich hinsichtlich des Skalenaufbaus, der Skalenbreite sowie des Skalenniveaus und der Berücksichtigung von Ausweichrubriken („keine Antwort") unterscheiden. So hat Hausknecht mehr als dreißig Varianten in einer Übersicht zusammengestellt. Vgl. Hausknecht, D.R., Measurement Scales in Consumer Satisfaction/Dissatisfaction, in: JoCS/D\&CB, Vol. 3, 1990, $\mathrm{S}$. $1 \mathrm{ff}$. Ebenso finden sich Beiträge, die sich speziell mit der Frage der optimalen Anzahl von Kategorien einer Ratingskala beschäftigen. Prinzipiell werden Empfehlungen für die Verwendung einer ungeraden Anzahl von drei bis neun Kategorien ausgesprochen. Vgl. Cox, E., The Optimal Number of Response Alternatives for a Scale: A Review, in: JoMR, Vol. 17, November 1980, S. $407 \mathrm{ff}$.

Vgl. Stauss, B., Seidel, W., Prozessuale Zufriedenheitsermittlung und Zufriedenheitsdynamik bei Dienstleistungen, a.a.O., S. 199.

$267 \mathrm{Zu}$ einer Darstellung dieser Modelle vgl. Kroeber-Riel, W., Konsumentenverhalten, a.a.O., S. $406 \mathrm{f}$. 
dagegen auf der Annahme, daß die schlechte Bewertung eines Merkmals durch die positive Wahrnehmung eines anderen Merkmals aufgefangen werden kann. Hier wird also die Ansicht vertreten, daß sich die Teilzufriedenheiten durch eine linear-additive Aggregation zu einer Gesamtzufriedenheit zusammensetzen. Diese Auffassung hat in wissenschaftlichen Untersuchungen die größte Verbreitung erfahren. ${ }^{268}$

Insgesamt ist festzustellen, daß Multiattributansätze in ihrer herkömmlichen Anwendung den Prozeßcharakter des Dienstleistungskonsums kaum berücksichtigen, da lediglich die Zufriedenheit mit einer Vielzahl von Merkmalen und deren Einfluß auf die Gesamtzufriedenheit gemessen wird. Wenn eine Zuordnung von Merkmalen zu bestimmten Teilleistungen vorgenommen wird, erfolgt dies zumeist nicht auf der Basis einer Analyse des Kundenpfades. Im folgenden werden daher Ansatzpunkte aufgezeigt, die bei einer prozeßorientierten Messung der Kundenzufriedenheit mit einer Dienstleistungstransaktion auf der Basis merkmalsgestützter Verfahren zu beachten sind: ${ }^{269}$

Aus der oben erarbeiteten Notwendigkeit einer differenzierten Analyse des Kundenpfades sowie der Ermittlung der Teilphasen kann unmittelbar gefolgert werden, daß sich die Auswahl der zu messenden Merkmale an den identifizierten Kontaktpunkten zu orientieren hat (z.B. „Wie zufrieden waren Sie mit der Freundlichkeit des Schalterpersonals im Bahnhof?"). Zur Ermittlung des Einflusses dieser Merkmalszufriedenheiten auf das Zufriedenheitsurteil mit der jeweiligen Episode, ist entsprechend die Zufriedenheit mit den verschiedenen Teilabschnitten festzustellen („Wie zufrieden waren Sie mit Ihrer Vor-Reise-Phase?"). ${ }^{270}$ Darüber hinaus ist die Zufriedenheit mit der gesamten Transaktion zu erheben („Wie zufrieden waren Sie insgesamt mit dieser Bahnreise?"), um Rückschlüsse über den Einfluß der einzelnen Phasen auf die Gesamtzufriedenheit ziehen zu können. ${ }^{271}$ Im Idealfall durchläuft ein Kunde jede der angegeben Episoden nur einmal,

Vgl. Dichtl, E., Peter, S., Kundenzufriedenheit und Kundenbindung in der Automobilindustrie: Ergebnisse einer empirischen Untersuchung, a.a.O., S. 19.

Vgl. hierzu und im folgenden Stauss, B., Seidel, W., Prozessuale Zufriedenheitsermittlung und Zufriedenheitsdynamik bei Dienstleistungen, a.a.O., S. $197 \mathrm{f}$.

270 Um die Verständlichkeit der Frage nach der Episodenzufriedenheit zu erhőhen, sollte der Fragebogen eine kurze Beschreibung der einzelnen Teilphasen beinhalten.

271 Sollen auch Erkenntnisse über die Zufriedenheit mit den bisherigen Erfahrungen eines Kunden mit einem Dienstleister gewonnen werden, ist ebenso eine Frage nach der Beziehungszufriedenheit zu stellen ("Wie zufrieden sind Sie generell mit der Bahn, wenn Sie alle Ihre bisherigen Erfahrungen mit der Bahn berücksichtigen?"). 
da nur dann Rückschlüsse auf die Zufriedenheitsdynamik gezogen werden können. Kommt ein Kunde dagegen mit einzelnen Episoden mehrfach in Berührung, besteht die Gefahr einer Durchschnittsbildung im Antwortverhalten und damit einer „Verwässerung" der Ergebnisse.

Werden die Fragen nach der Gesamt- bzw. Episodenzufriedenheit im Anschluß an die kontaktpunktbezogenen Merkmalsfragen gestellt, führt dies zu einer stark kognitiv geprägten Replikation des Dienstleistungserlebnisses und einer Durchschnittsbildung im Antwortverhalten. Soll der affektiven Komponente der Kundenzufriedenheit Rechnung getragen werden, bietet sich eine Voranstellung der Fragen nach der Gesamt- bzw. Episodenzufriedenheit an. „Questions about overall satisfaction are asked before questions about individual performance attributes. This follows the accepted rule that general questions should precede specific questions." ${ }^{272}$

Unbeantwortet ist bei einem solchen Vorgehen jedoch die Frage nach dem Zeitpunkt der Messung. Neben den bereits angesprochenen Problemen einer prozeßbegleitenden Messung ${ }^{273}$ sprechen auch finanzielle und erhebungstechnische Aspekte gegen ein solches methodisches Vorgehen. Es erscheint vielmehr, insbesondere bei der Zielsetzung einer regelmäßigen und repräsentativen Messung der Kundenzufriedenheit, zweckmäßig, im direkten Anschluß an den Dienstleistungsprozeß die Befragung durchzuführen. ${ }^{274}$

Dies würde zudem auch einen Vorwurf entkräften, der zuweilen im Zusammenhang mit Zufriedenheitsmessungen erhoben wird. ${ }^{275}$ Dieser besteht darin, daß häufig zwischen dem Zeitpunkt der Befragung und dem zu analysierenden Konsumerlebnis unterschiedlich lange Zeiträume liegen, in denen neue Erfahrungen mit der Dienstleistung gemacht werden und weitere Informationen über das Angebot vorliegen können. Die Rekonstruktion des konkreten Dienstleistungserlebnisses ist damit vielfältigen Faktoren ausgesetzt und erschwert so die Ana-

Dutka, A.F., AMA Handbook for Customer Satisfaction: Research, Planning, and Implementation, Chicago 1994, S. 73. Zu einer gegenteiligen Ansicht vgl. Gierl, H., Sipple, H., Zufriedenheit mit dem Kundendienst, a.a.O., S. 251.

$273 \mathrm{Vgl}$. die Ausführungen in Kap. B 2.4 dieser Arbeit.

274 Vgl. Stauss, B., Seidel, W., Prozessuale Zufriedenheitsermittlung und Zufriedenheitsdynamik bei Dienstleistungen, a.a.O., S. $197 \mathrm{f}$.

275 Vgl. Kaas, K.P., Runow, H., Wie befriedigend sind die Ergebnisse der Forschung zur Verbraucherzufriedenheit?, a.a.O., S. $452 \mathrm{ff}$. 
lyse des Konstruktes, da auch Dissonanzreduktionseffekte nicht ausgeschlossen werden können. ${ }^{276}$

Auch in der Praxis von Verkehrsdienstleistungsunternehmen kommen häufig Multiattributverfahren in unterschiedlichen Varianten zum Einsatz. ${ }^{277}$ Jedoch zeigen nur wenige Untersuchungen Ansatzpunkte für ein prozeßorientiertes Verständnis von Kundenzufriedenheit auf. Ein Beispiel für einen Meßansatz, in dem sich die Idee einer Kundenpfadanalyse widerspiegelt, stellt der „Thermograaf" der niederländischen Eisenbahnen AG dar. ${ }^{278}$ Dabei werden Kundenurteile zu insgesamt achtzig Merkmalen einer Bahnreise erhoben. Die aus über vierhundert Aspekten einer Bahnreise ausgewählten Merkmale orientieren sich an den Episoden Zugang (Kontaktaufnahme, Reservierung, Bewegung im Bahnhof), Transport sowie Verlassen des Verkehrsmittels und des Bahnhofs. Wenngleich diesem Ansatz der Phasenablauf einer Bahnreise zugrunde liegt, wird er den Anforderungen einer prozeßorientierten Messung der Kundenzufriedenheit nur ansatzweise gerecht. So erfolgt zwar eine an Kontaktpunkten orientierte Messung, eine Erhebung der Episoden- bzw. Gesamtzufriedenheit jedoch unterbleibt. Darüber hinaus wird mit dem Instrument eher der Vergleich der Wahrnehmungen der einzelnen Merkmale und deren Zusammenhänge im langfristigen Zeitablauf auf der globalen Ebene bezweckt und weniger die Analyse der Zufriedenheitsdynamik innerhalb einer konkreten Transaktion.

Angesichts der angedeuteten vielfältigen Ausgestaltungs- und Interpretationsmöglichkeiten merkmalsorientierter Befragungsmethoden ist eine generelle Beurteilung ihrer Aussagefähigkeit für eine prozeßorientierte Analyse der Kundenzufriedenheit nur eingeschränkt möglich. Ihre große Akzeptanz innerhalb der Marketingforschung belegt, daß auch Verkehrsdienstleistungsunternehmen mittels

Zu diesem Problemkreis vgl. Peterson, R.A., Wilson, W.R., Measuring Customer Satisfaction: Fact and Artifact, a.a.O., S. 66.

277 Vgl. Klein, H., Management von Kundenzufriedenheit bei der Deutschen Lufthansa AG, a.a.O., S. 379 ff.; Gohlke, R., Wie sichert die Bundesbahn ihre Dienstleistungsqualität?, in: Qualität Die Herausforderung: Erfahrungen, Perspektiven, Lisson, A. (Hrsg.), Berlin u.a. 1987, S. 236 f.; Garre, K.-H., Müller, J., Das Qualitätssicherungssystem Personenverkehr der DB, in: DBB, Jg. 67, H. 9, 1991, S. 850 ff;; Deutsche Lufthansa AG (Hrsg.), Servicequalität - Unsere Kunden halten uns den Spiegel vor: Customer Service Index, in: Lufthanseat, Jg. 42, H. 642 vom 16. Februar 1996, S. 1, 6 f. Zu weiteren von der Lufthansa eingesetzten Verfahren vgl. Schörcher, U., Buchholz, R., Qualitätsmanagement in der Luftfahrt, in: Handbuch Qualitätsmanagement, Masing, W. (Hrsg.), 3. Aufl., München, Wien 1994, S. $841 \mathrm{ff}$.

278 Vgl. Hertel, G., Rasemann, A., In der Kundenklasse reisen, in: QZ, Jg. 38, H. 6, 1993, S. 367 ff.; Forschungsgemeinschaft Qualitätssicherung e.V. (FQS) (Hrsg.), Qualitätssicherung in Dienstleistungsprozessen: theoretische Grundlagen für die strategische Planung von Qualitătszielen im Dienstleistungsbereich; Abschlußbericht, Berlin u.a. 1995, S. $140 \mathrm{ff}$. 
ihres Einsatzes wichtige Erkenntnisse über Zufriedenheit von Kunden gewinnen können. In ihrer grundsätzlichen Ausrichtung eignen sie sich jedoch nicht für eine phasenorientierte Messung der Kundenzufriedenheit. Die aufgezeigten Modifikationsmöglichkeiten sind gleichwohl ein Indiz der Adaptionsfähigkeit für diese spezielle Zielsetzung. Dennoch ist auch bei einer solchen Anpassung des Instrumentariums nur die Möglichkeit gegeben, eine begrenzte Menge an Merkmalen einer Beurteilung zu unterziehen. So ist die Eignung merkmalsorientierter Verfahren insbesondere bezüglich der Vollständigkeit, Eindeutigkeit und Aktualität vor dem Hintergrund der konkreten Anwendungssituation differenziert zu beurteilen. Sollen repräsentative Umfrageergebnisse erzielt werden, sind die Verfahren mit entsprechenden Kosten verbunden. Aus diesen Gründen gilt es, zur Entdeckung neuer, bislang unbekannter Ursachen von (Un-)Zufriedenheit und zur Konkretisierung der aus merkmalsorientierten Verfahren erhaltenen Informationen in Ergänzung ereignisorientierte Methoden heranzuziehen.

\subsection{Ereignisgestützte Verfahren}

Die Beschwerdeanalyse zählt zu den „klassischen" ereignisorientierten Verfahren der Zufriedenheitsforschung. Ihr Gegenstand besteht in der systematischen Auswertung und Analyse der in Beschwerden enthaltenen Informationen. Beschwerden stellen in diesem Zusammenhang Artikulationen von Unzufriedenheit dar, die in Abhängigkeit von verschiedenen Einflußfaktoren bei dem Verursacher des Beschwerdegrundes vorgebracht werden. ${ }^{279} \mathrm{Da}$ Kunden durch das aktive Vorbringen von Beschwerden bewußt den materiellen und zeitlichen Aufwand der Beschwerdeführung auf sich nehmen, ist die Relevanz und Eindeutigkeit der Informationen dabei in besonderem Maße gegeben. Auch ist von einer hohen Aktualität der Beschwerdeinformationen auszugehen, da sich Kunden erfahrungsgemäß schon kurze Zeit nach dem Problemeintritt beschweren. Der spezifische Charakter von Dienstleistungen hat jedoch zur Folge, daß sich Kunden im Vergleich zum Konsumgüterbereich weniger häufig beschweren ${ }^{280}$ und damit hinter einer einzelnen Beschwerde eine Vielzahl von „Unvoiced Complaints"

279 Zu dieser Definition und den Grundlagen der Beschwerdemessung vgl. Meffert, H., Bruhn, M., Beschwerdeverhalten und Zufriedenheit von Konsumenten, a.a.O., S. $597 \mathrm{ff}$.; Bruhn, M., Konsumentenzufriedenheit und Beschwerden: Erklärungsansätze und Ergebnisse einer empirischen Untersuchung in ausgewählten Konsumbereichen, a.a.O., S. 1 ff.; Stauss, B., Seidel, W., Beschwerdemanagement: Fehler vermeiden, Leistung verbessern, Kunden binden, München, Wien 1996, S. $1 \mathrm{ff}$.

$280 \mathrm{Zu}$ den Determinanten der Beschwerdewahrscheinlichkeit vgl. Stauss, B., Beschwerdepolitik als Instrument des Dienstleistungsmarketing, in: JdAV, Jg. 35, H. 1, 1989, S. $43 \mathrm{ff}$. 
zu vermuten ist. ${ }^{281}$ Ein wesentlicher Grund besteht darin, daß es Kunden aufgrund der mehr oder minder starken Beteiligung am Dienstleistungserstellungsprozeß häufig schwerfällt, die Ursache der Unzufriedenheit eindeutig dem Verantwortungsbereich des Anbieters zuzurechnen. Der Erfolg einer Beschwerde wird dementsprechend niedrig eingeschätzt. Dies hat zur Konsequenz, daß die Beschwerde zurückgehalten wird. ${ }^{282}$ Aus einer niedrigen Beschwerdequote kann daher nicht auf die Zufriedenheit von Kunden geschlossen werden. Die Repräsentativität und Vollständigkeit von vorgetragenen Beschwerden ist damit als problematisch anzusehen, während das Instrument aus Kostenüberlegungen heraus als vorteilhaft zu beurteilen ist, da das Unternehmen die Informationen auf Initiative des sich beschwerenden Kunden erhält.

Da Beschwerden in der Regel ausführliche Informationen über den Gegenstand der Beschwerdeursache beinhalten, besteht grundsätzlich die Möglichkeit, diese unterschiedlichen Kontaktpunkten, Episoden oder Transaktionen zuzuordnen und damit prozessual zu systematisieren und auszuwerten. Geschieht dies auf der Basis eines Blueprints bzw. auf einer Service Map, können wertvolle Hinweise auf Probleme im Dienstleistungserstellungsprozeß generiert werden. Da Beschwerden mitunter auch die Beschreibung von Prozeßverläufen beinhalten, besteht überdies die Möglichkeit, Erkenntnisse über die Dynamik der Zufriedenheitsbildung zu gewinnen. ${ }^{283}$

Ähnliches gilt für die Critical Incident-Technik, die der Erfassung außergewöhnlicher Kundenerlebnisse während des Dienstleistungskonsums dient. ${ }^{284}$ Während

Vgl. Stauss, B., Hentschel, B., Verfahren der Problementdeckung und -analyse im Qualitătsmanagement von Dienstleistungsunternehmen, a.a.O., S. 239.

282

So stellt Breuer für die Deutsche Lufthansa $A G$ fest, daß sich nur ca. $4 \%$ der unzufriedenen Kunden tatsächlich beschweren. Vgl. Breuer, H., Qualitătsmanagement in einem Dienstleistungsunternehmen, in: Erfolg durch Service-Qualităt, Stauss, B. (Hrsg.), München 1991, S. 217.

283 Vgl. Stauss, B., Seidel, W., Prozessuale Zufriedenheitsermittlung und Zufriedenheitsdynamik bei Dienstleistungen, a.a.O., S. 201.

284 Dieses Verfahren wurde bereits 1954 von Flanagan mit dem Ziel der Identifikation von Kriterien eines effektiven Arbeitsverhaltens entwickelt. Vgl. Flanagan, J.C., The Critical Incident Technique, in: Psychological Bulletin, Vol. 51, July 1954, S. $327 \mathrm{ff}$.

Zu einer ausführlichen Darstellung der Methode und ihren Einsatzmöglichkeiten im Dienstleistungsbereich vgl. Stauss, B., Der Einsatz der "Critical Incident Technique" im Dienstleistungsmarketing, in: Kundennähe realisieren, Tomczak, T., Belz, Ch. (Hrsg.), St. Gallen 1994, S. 233 ff.; Bitner, M.J., Booms, B.H., Tetreault, M.S., The Service Encounter: Diagnosing Favorable and Unfavorable Incidents, in: JoM, Vol. 54, January, 1990, S. 71 ff.; Nyquist, J.D., Bitner, M.J., Booms, B.H., Identifying Communication Difficulties in the Service Encounter: A Critical Incident Approach, in: The Service Encounter: Managing Employee/Customer 
Beschwerden eine Form „kundeninitiierter Kommunikation" darstellen, werden bei der Methode der Kritischen Ereignisse Kundenerlebnisse detailliert im Rahmen mündlicher Interviews anhand standardisierter offener Fragen erhoben. Kritische Ereignisse werden in diesem Zusammenhang als „specific interactions between customers and service firm employees that are especially satisfying or especially dissatisfying" definiert. ${ }^{285}$ STAUSS erweitert diese Definition auf alle personalbezogenen und nichtpersonalbezogenen Vorfälle im Rahmen des Interaktionsprozesses zwischen Dienstleister und Kunde, die vom Nachfrager als besonders positiv oder negativ wahrgenommen werden. ${ }^{286}$ Diese Definition ist insbesondere für Verkehrsdienstleistungen geeignet, da sie eine Reihe nicht personalbezogener Transport-, Informations- und Aufenthaltsprozesse enthalten, denen ebenfalls Potentiale für kritische Erlebnisse innewohnen. ${ }^{287}$ Im Vergleich zur Beschwerdeanalyse werden damit zwar ebenfalls nicht alle Aspekte eines Dienstleistungsprozesses abgedeckt, jedoch auch positive Ereignisse explizit berücksichtigt.

Die Interpretation der Befragungsergebnisse erfolgt im Rahmen eines mehrstufigen Auswertungsverfahrens anhand festzulegender Kodierungs- und Klassifikationsregeln. Neben der Bildung von Problemkategorien werden zur Ableitung von Handlungsmaßnahmen die Häufigkeiten der kategorisierten Ereignisse ermittelt. ${ }^{288}$ Wie bei der Beschwerdeanalyse ist diesen Richtlinien für eine prozeßorientierte Analyse der Kundenzufriedenheit ein am Kundenprozeß orientierter Bezugsrahmen zugrundezulegen. Grundsätzlich sind die mit Hilfe dieses Verfahrens ermittelten Informationen handlungsrelevant, eindeutig und ihrem Informationsgehalt nach sehr konkret. Dementsprechend besteht die Möglichkeit, kontaktpunkt-, episoden- und transaktionsspezifisch Ansatzpunkte über zusatznutzenstiftende Dienstleistungseigenschaften (sog. Werterhöhungsqualitäten) als auch über Mindesterwartungen gegenüber bestimmten Merkmalen des Angebotes

Interaction in Service Businesses, Czepiel, J.A., Solomon, M.R., Surprenant, C.F. (Hrsg.), Lexington 1985, S. $195 \mathrm{ff}$.

285 Bitner, M.J., Booms, B.H., Tetreault, M.S., The Service Encounter: Diagnosing Favorable and Unfavorable Incidents, a.a.O., S. 73.

Vgl. Birkelbach, R., Qualitătsmanagement in Dienstleistungscentern: Konzeption und typenspezifische Ausgestaltung unter besonderer Berücksichtigung von Verkehrsflughäfen, a.a.O., S. 79 .

288 Zu einem Einsatz dieses Verfahrens im Verkehrsdienstleistungsbereich vgl. Edvardsson, B., Service Breakdowns: A Study of Critical Incidents in an Airline, in: IJoSIM, Vol. 3, No. 4, 1992, S. $17 \mathrm{ff.;}$ Bitner, M.J., Booms, B.H., Tetreault, M.S., The Service Encounter: Diagnosing Favorable and Unfavorable Incidents, a.a.O., S. $74 \mathrm{ff}$. 
zu erhalten (sog. Minimumqualitäten). ${ }^{289}$ Da sich kritische Ereignisse in Frequenz sowie Intensität unterscheiden und insbesondere aus Kundensicht von unterschiedlicher Bedeutung sein können, ist es sinnvoll, in einem nächsten Schritt Prioritäten der Problembeseitigung für das Kundenzufriedenheitsmanagement zu ermitteln. Zur genaueren Ermittlung der Dringlichkeit der Problembehebung bietet sich der Einsatz hierzu konzipierter Verfahren wie die Problem-Detecting-Methode bzw. die Frequenz-Relevanz-Analyse für Probleme (FRAP) an. ${ }^{290}$

Bei den bisher geschilderten Verfahren erfolgt eine prozeßorientierte Analyse erst im Anschluß an den Datensammlungsprozeß. Ein Verfahren, das bereits bei der Messung der Kundenzufriedenheit den Prozeßcharakter der Dienstleistungskonsums explizit berücksichtigt, stellt die Sequentielle Ereignismethode dar. ${ }^{291}$ Dieses in der Praxis noch weitgehend unerprobte Meßinstrument beruht auf den bereits dargestellten Methoden des Blueprinting bzw. Service Mapping. Auf der Basis einer solchen bildlichen Darstellung des Dienstleistungsprozesses werden Kunden gebeten, den von innen erlebten Nutzungsprozeß noch einmal gedanklich zu durchlaufen und zu jeder Kontaktsituation die wahrgenommen Erlebnisse zu schildern. Im Gegensatz zur Critical Incident-Technik sollen hier nicht nur kritische, sondern sämtliche wahrgenommen Ereignisse des Dienstleistungskonsums erfaßt werden. Durch die gestützte Art der Befragung besteht die Möglichkeit, (nahezu) alle Kontaktpunkte des Dienstleistungsprozesses einer Betrachtung zu unterziehen. ${ }^{292}$ Damit können auch solche Prozeßbestandteile erfaßt werden, die sich nicht im Handlungsbereich des Verkehrsdienstleisters befinden, sehr wohl aber Einfluß auf die Kundenzufriedenheit nehmen können (Anreise, Parkplatzsuche, Zollkontrolle etc.). ${ }^{293}$

289 Vgl. Stauss, B., Der Einsatz der "Critical Incident Technique" im Dienstleistungsmarketing, a.a.O., S. 243.

Zu einer ausführlichen Darstellung dieser Verfahren vgl. Brandt, D.R., Reffett, K.L., Focusing on Customer Problems to Improve Service Quality, in: Designing a Winning Service Strategy, Bitner, M.J., Crosby, L.A. (Hrsg.), Chicago 1989, S. 93 ff.; Stauss, B., Hentschel, B., Verfahren der Problementdeckung und -analyse im Qualitätsmanagement von Dienstleistungsunternehmen, a.a.O., S. $247 \mathrm{ff}$.

291 Vgl. Stauss, B., Hentschel, B., Verfahren der Problementdeckung und -analyse im Qualitătsmanagement von Dienstleistungsunternehmen, a.a.O., S. $244 \mathrm{ff}$.

292 Eine ähnliche Untersuchungsmethode stellt das Verfahren des "Story Telling" dar. Im Gegensatz zur Critical Incident-Technik und Sequentiellen Ereignismethode werden hier jedoch ohne Bezugnahme auf konkrete Fragestellungen die Befragten gebeten, ihre Erfahrungen mit einem Dienstleister zu schildern. Vgl. Scharitzer, D., Dienstleistungsqualität - Kundenzufriedenheit, Wien 1994, S. 137.

293 Im Anschluß an die Datenerhebung erfolgt ebenfalls eine Inhaltsanalyse, bei der die Äußerungen selektiert und zuvor gebildeten Ereigniskategorien zugeordnet werden. Während die

(Fortsetzung der Fußnote auf der nächsten Seite) 
Mit dem Meßverfahren der Sequence-Oriented Problem Identification stellen BOTSCHEN/BSTIELERMOODSIDE ein Befragungsvariante vor, die die Vorgehensweisen der Critical Incident-Technik und der Sequentiellen Ereignismethode miteinander vereint. ${ }^{294}$ Erwartungsgemäß zeigen die Ergebnisse der durchgeführten empirischen Untersuchung im Bereich beruflicher Fortbildungsmaßnahmen, daß die beiden Verfahren unterschiedliche Schwächen und Stärken einer Dienstleistung zu Tage fördern. Der kombinierte Einsatz der Verfahren gewährleistet damit eine Abbildung des Kontaktpunkterlebens innerhalb und außerhalb der Indifferenzzone.

Um jedoch im Rahmen dieser Ansätze nähere Informationen über den Zufriedenheitsgrad und seine Dynamik zu erhalten, sind Modifikationen notwendig. STAUSS und SEIDEL präsentieren in diesem Zusammenhang eine interessante integrierte Applikation der verschiedenen bisher beschriebenen Verfahren am Beispiel von Clubreisen. ${ }^{295}$ Zur Ermittlung des Kundenpfades wurde eine explorative Voruntersuchung durchgeführt und eine Visualisierung des Ergebnisses mit Hilfe einer Service Map vorgenommen. Im Rahmen der dann vor Ort durchgeführten Hauptuntersuchung erfolgte die Sammlung von Ereignissen auf Basis dieses Kundenpfades. Dabei wurde die Betrachtung auf den Reiseabschnitt „Empfang der Gäste" eingegrenzt (vgl. Abbildung 18).

Im Anschluß an die Schilderung der einzelnen Ereignisse wurden die Urlauber gebeten, die Erlebnisse als positiv oder negativ einzustufen und ihre Relevanz auf einer vorgegeben Skala zu beurteilen. Auf diese Weise bestand die Möglichkeit, kritische von üblichen Ereignissen zu trennen und episodenbezogen auszuwerten. Ebenso konnten auf diese Art Aussagen über die Häufigkeit und Relevanz der Erlebnisse getroffen und damit die Ergebnisse einer Frequenz-Relevanz-Analyse zugänglich gemacht werden. Ein besonderer Vorteil bestand darin, daß die FRAPAnalyse in ungestützter Weise erfolgen konnte, da keine Problemkategorien vorgegeben werden mußten, sondern vieimehr von den Befragten selbst genannt

Methode der Kritischen Ereignisse auf Kundenerlebnisse abzielt, die außerhalb der „Zone of Tolerance" liegen, besteht die Zielsetzung dieses Verfahren insbesondere in einer detaillierten Analyse des vom Kunden üblicherweise wahrgenommenen Nutzungsprozesses.

Vgl. Botschen, G., Bstieler, L., Woodside, A.G., Sequence-Oriented Problem Identification within Service Encounters, a.a.O., S. 2 ff.

$295 \mathrm{Vgl}$. hierzu und im folgenden Stauss, B., Weinlich, B., Die Sequentielle Ereignismethode - ein Instrument der prozeßorientierten Messung von Dienstleistungsqualität, a.a.O., S. $50 \mathrm{ff}$. 


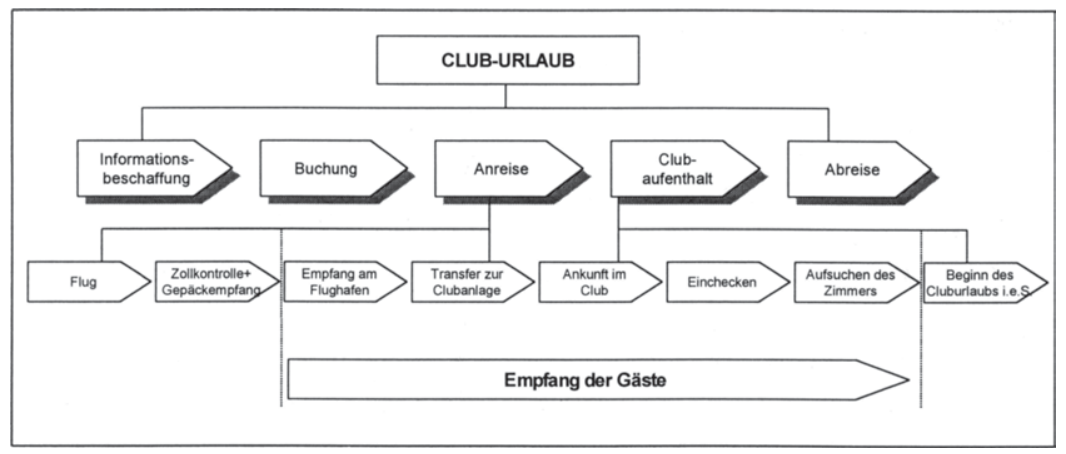

Abb. 18: Ausschnitt aus dem Kundenpfad einer Cluburlaubsreise

(Quelle: Stauss, B., Weinlich, B., Die Sequentielle Ereignismethode - ein Instrument der prozeßorientierten Messung von Dienstleistungsqualität, a.a.O., S. 53)

wurden. ${ }^{296}$ Darüber hinaus wurden die Befragten gebeten, auf einer Ratingskala Angaben über die Zufriedenheit mit dem gesamten Teilabschnitt „Empfang der Gäste" zu machen. ${ }^{297}$ Damit konnten Rückschlüsse im Hinblick auf den Einfluß kritischer Ereignisse auf die gesamte Zufriedenheit mit diesem Teilabschnitt der Urlaubsreise gezogen werden. Die Ergebnisse der Untersuchung bestätigen die episodenbezogene Wahrnehmung der Dienstleistung. Überdies konnte der Nachweis erbracht werden, daß nicht nur kritische, sondern auch übliche Erlebnisse einen Einfluß auf die Episodenzufriedenheit ausüben. Doch zeigten sich auch Schwächen des gewählten Untersuchungsdesigns. So fiel es den Befragten schwer, die Problemrelevanz auf der vorgegeben 5er-Skala zu bewerten. Darüber hinaus ist die zeitintensive Erhebung von Daten mit einer komplexen, nicht-standardisierten Form der Auswertung verbunden und das beschriebene Meßinstrument daher als sehr aufwendig zu bezeichnen. Dieser Umstand kommt auch darin zum Ausdruck, daß nur ein Ausschnitt aus dem Kundenpfad untersucht wurde. Ein großer Vorteil des Verfahrens besteht in der

Da durch die beschriebene Vorgehensweise auch positive Erlebnisse erhoben werden, besteht ebenso die Möglichkeit, eine entsprechende Auswertung im Hinblick auf als besonders positiv empfundene Ereignisse durchzuführen. Wienczierz bezeichnet ein entsprechendes Vorgehen als "Frequenz-Relevanz-Analyse für positive und negative Ereignisse (FRAPNE). Vgl. Wienczierz, zitiert in Stauss, B., Weinlich, B., Die Sequentielle Ereignismethode - ein Instrument der prozeßorientierten Messung von Dienstleistungsqualităt, a.a.O., S. 52. Eine solche Untersuchung wurde jedoch in der oben beschrieben Studie nicht durchgeführt.

297 Dagegen wurde auf eine Erhebung von Kontaktpunkt- und Episodenzufriedenheiten innerhalb des untersuchten Abschnittes des Kundenpfades verzichtet. 
Gewinnung einer Vielzahl konkreter Einzelinformationen. Zudem konnten die Autoren durch die kombinierte Anwendung der verschiedenen Verfahren wichtige Ansatzpunkte für eine prozeßorientierte Messung der Kundenzufriedenheit aufzeigen.

Im Rahmen einer generellen Beurteilung der ereignisorientierten Meßverfahren ist festzustellen, daß diese für eine regelmäßige und repräsentative Ermittlung der Kundenzufriedenheit kaum geeignet sind. Sie sind daher nicht als Substitut, sondern vielmehr als wichtige Ergänzung merkmalsorientierter Verfahren anzusehen. Ähnlich wie merkmalsorientierte Methoden liefern ereignisorientierte Methoden nur bedingt Ansatzpunkte für eine prozeßbegleitende Messung, da auch innerhalb dieser Verfahren ein dynamisches Phänomen in einer grundsätzlich statisch ausgerichteten Weise untersucht wird. Dennoch bieten die Verfahren bei entsprechender Anwendung grundsätzlich die Möglichkeit einer nach Kontaktpunkten, Episoden und Transaktionen differenzierten Analyse der Kundenzufriedenheit. Zudem können bis zu einem gewissen Grad Erkenntnisse über die Dynamik der Kundenzufriedenheit im Zeitablauf gewonnen werden. Gleichwohl ist bei ihrem üblichen Einsatz nur bedingt gesichert, daß sich die erhobenen Informationen auf das letzte Konsumerlebnis beziehen und wirklich auf den eigenen Erfahrungen des Befragten beruhen. Dem Zeitpunkt der Befragung kommt damit wie bei den merkmalsorientierten Verfahren eine große Bedeutung zu. ${ }^{298}$ Mögliche Interviewereinflüsse sowie der hohe Zeit- und Kostenaufwand stehen dem Einsatz dieser Verfahren ebenfalls nachteilig gegenüber.

\subsection{Würdigung der Meßansätze zur Kundenzufriedenheit}

Die bisherigen Ausführen zeigen, daß letztlich allen dargestellten Verfahren spezifische Vor- und Nachteile innewohnen und mit innen teilweise unterschiedliche Aspekte der Kundenzufriedenheit gemessen werden. Wie aus Abbildung 19 deutlich wird, ist ein einzelnes Verfahren nicht in der Lage, die phasenorientierte Entwicklung der Kundenzufriedenheit mit Verkehrsdienstleistungen in sämtlichen Facetten vollständig zu erfassen. ${ }^{299}$

Vgl. Haller, S., Beurteilung von Dienstleistungsqualität: Dynamische Betrachtung des Qualitătsurteils im Weiterbildungsbereich, a.a.O., S. 125.

299 Die Bewertung basiert auf der vorangegangenen Diskussion der verschiedenen Methoden sowie auf den in diesem Zusammenhang zitierten Literaturquellen. Darüber hinaus sind in die Beurteilung die Ergebnisse von Expertengesprächen eingeflossen, die der Autor mit Vertretern der Verkehrsdienstleistungsbranche, insbesondere der Deutschen Bahn AG, geführt hat. 


\begin{tabular}{|c|c|c|c|c|c|c|c|c|c|c|c|}
\hline \multirow{2}{*}{\multicolumn{4}{|c|}{$\begin{array}{l}\text { Legende: } \\
++ \text { : besonders geeignet } \\
+\quad \text { : geeignet } \\
0 \text { : bedingt geeignet } \\
-\quad: \text { weniger geeignet }\end{array}$}} & \multicolumn{6}{|c|}{ Generelle Bewertungskriterien } & \multicolumn{2}{|c|}{ 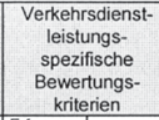 } \\
\hline & & & & $\begin{array}{l}\text { Relevanz } \\
\text { der erfabs } \\
\text { en Kriterien }\end{array}$ & $\begin{array}{l}\text { Eindeutig- } \\
\text { keit der Er- } \\
\text { gebnisse }\end{array}$ & $\begin{array}{l}\text { Aktualitat } \\
\text { der Beur- } \\
\text { teillungen }\end{array}$ & $\begin{array}{l}\text { Vollstandig- } \\
\text { keit der er- } \\
\text { fasten Kri- } \\
\text { terien }\end{array}$ & $\begin{array}{l}\text { Kosten- } \\
\text { wirtschati- } \\
\text { lichkeit }\end{array}$ & $\begin{array}{l}\text { Steuerbar- } \\
\text { keit (Maß- } \\
\text { nahmen } \\
\text { ableitbar?) }\end{array}$ & \multirow{2}{*}{\begin{tabular}{|c}
$\begin{array}{c}\text { Errassung } \\
\text { des E piso- } \\
\text { den- bzw. } \\
\text { Schnititse- } \\
\text { lencharak- } \\
\text { ters }\end{array}$ \\
0 \\
\end{tabular}} & \multirow{2}{*}{$\begin{array}{c}\begin{array}{c}\text { Hinweis- } \\
\text { farhighetit } \\
\text { aut Zutrie } \\
\text { denheits- } \\
\text { dynamik }\end{array} \\
-\end{array}$} \\
\hline \multirow{7}{*}{ 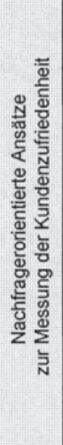 } & \multirow{2}{*}{\multicolumn{2}{|c|}{ 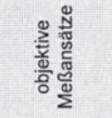 }} & $\begin{array}{l}\text { Silent Shopper- } \\
\text { Methode }\end{array}$ & 0 & 0 & + & O & - & O & & \\
\hline & & & Beobachtung & 0 & $\mathrm{O}$ & + & - & - & 0 & 0 & - \\
\hline & \multirow{5}{*}{  } & 旁费 & $\begin{array}{l}\text { Multiattribut- } \\
\text { verfahrent" }\end{array}$ & + & + & + & 0 & + & + & + & + \\
\hline & & \multirow{4}{*}{ 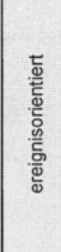 } & $\begin{array}{l}\text { Beschwerde- } \\
\text { analyse }\end{array}$ & ++ & ++ & ++ & - & ++ & ++ & O & O \\
\hline & & & $\begin{array}{l}\text { Critical Incident- } \\
\text { Technik }\end{array}$ & ++ & ++ & O & - & - & ++ & $\mathrm{O}$ & O \\
\hline & & & $\begin{array}{l}\text { Sequentielle } \\
\text { Ereignis- } \\
\text { methode. }\end{array}$ & ++ & ++ & $\mathrm{O}$ & $\mathrm{O}$ & - & ++ & ++ & + \\
\hline & & & $\begin{array}{l}\text { Sequence-Oriented } \\
\text { Problem } \\
\text { Identification }\end{array}$ & ++ & ++ & $\mathrm{O}$ & + & - & ++ & ++ & 0 \\
\hline
\end{tabular}

* Beachte aufgrund der Ausgestaltungsvielfalt differenzierte Argumentation im Textteil Abb. 19: Vergleichende Bewertung der Ansätze zur Messung der Kunden-
zufriedenheit

Es empfiehlt sich daher, die Verfahren in einer integrierten Weise einzusetzen. Dabei bietet sich ein stufenweise gestaltetes Methodenmix an. Zunächst gilt es, anhand der Verfahren des Blueprinting bzw. Service Mapping den Kundenpfad einer eingehenden Untersuchung zu unterziehen. Daran anschließend erfolgt sinnvollerweise die Identifikation von zentralen Zufriedenheitsindikatoren mit Hilfe der objektiven und ereignisorientierten Verfahren zur Messung der Kundenzufriedenheit. Zur regelmäßigen Überprüfung der ermittelten Indikatoren und deren Verdichtung bietet sich der Einsatz merkmalsorientierter Verfahren an. Deren konkrete Ausgestaltung ist dabei in hohem Maße von der jeweiligen Zielsetzung einer Zufriedenheitsuntersuchung abhängig.

Basierend auf den bisherigen Ausführungen erfolgt nun die Entwicklung eines Ansatzes zur Operationalisierung und Erklärung der Kundenzufriedenheit mit Verkehrsdienstleistungen am Beispiel des innerdeutschen schienengebundenen Personenfernverkehrs. 


\section{Phasenorientierter Ansatz zur Erfassung und Erklärung der Kunden- zufriedenheit mit Verkehrsdienstleistungen}

Die Basis der Untersuchung der Kundenzufriedenheit mit Verkehrsdienstleistungen stellt auf der theoretischen Sprachebene das Disconfirmation-Modell der Kundenzufriedenheit dar. Aufgrund der bei einer traditionellen Anwendung dieses Modells aufgezeigten Defizite zur Abbildung des Prozeßcharakters von Dienstleistungen erfährt dieser Modellansatz in den folgenden Ausführungen die zur Erfassung der Kundenzufriedenheit mit Verkehrsdienstleistungen notwendigen Anpassungen und Erweiterungen.

Zur Erklärung der Kundenzufriedenheit mit Verkehrsdienstleistungen werden zentrale personenbezogene und reise- bzw. verkehrsdienstleistungsspezifische Bestimmungsfaktoren einer Analyse unterzogen.

\subsection{Erfassung der Kundenzufriedenheit mit Verkehrsdienstleistungen}

\subsection{Strukturanalyse und Visualisierung der untersuchten Verkehrs- dienstleistung}

Aufbauend auf den bisherigen Ausführungen gilt es zunächst, den Kundenprozeß umfassend zu strukturieren und zu visualisieren. Dies geschieht in der vorliegenden Untersuchung am Beispiel des innerdeutschen schienengebundenen Personenfernverkehrs. Im Mittelpunkt der Untersuchung steht die prozeßorientierte Analyse der Kundenzufriedenheit auf der Transaktionsebene. Unter einer Dienstleistungstransaktion soll in diesem Zusammenhang das aus Sicht des Kunden spezifische und vollständige Erleben einer einzelnen Bahnreise verstanden werden.

Zur Strukturierung des kundenseitigen Nutzungsprozesses wurde eine aus drei Ebenen bestehende Prozeßhierarchie entwickelt, die das Ergebnis umfassender explorativer Voruntersuchungen sowie einer detaillierten Auswertung bahneigener Studien zu verschiedenen Aspekten der Kundenzufriedenheit und die Erkenntnisse aus Expertengesprächen darstellt. ${ }^{300}$ Wie Abbildung $20 \mathrm{zu}$ entnehmen ist, wurden auf der Episodenebene vier Teilprozesse des Kundenpfades identifiziert. zahlreiche Studien der Deutschen Bahn AG zurückgreifen, die wichtige Hinweise für eine prozeßorientierte Analyse der Kundenzufriedenheit lieferten. Das zur Verfügung stehende Datenmaterial entstammt dabei unterschiedlichen Erhebungs- und Auswertungstechniken. Einem Großteil der Studien gingen explorative Vorarbeiten in Form von Gruppendiskussionen (Fortsetzung der Fußnote auf der năchsten Seite) 


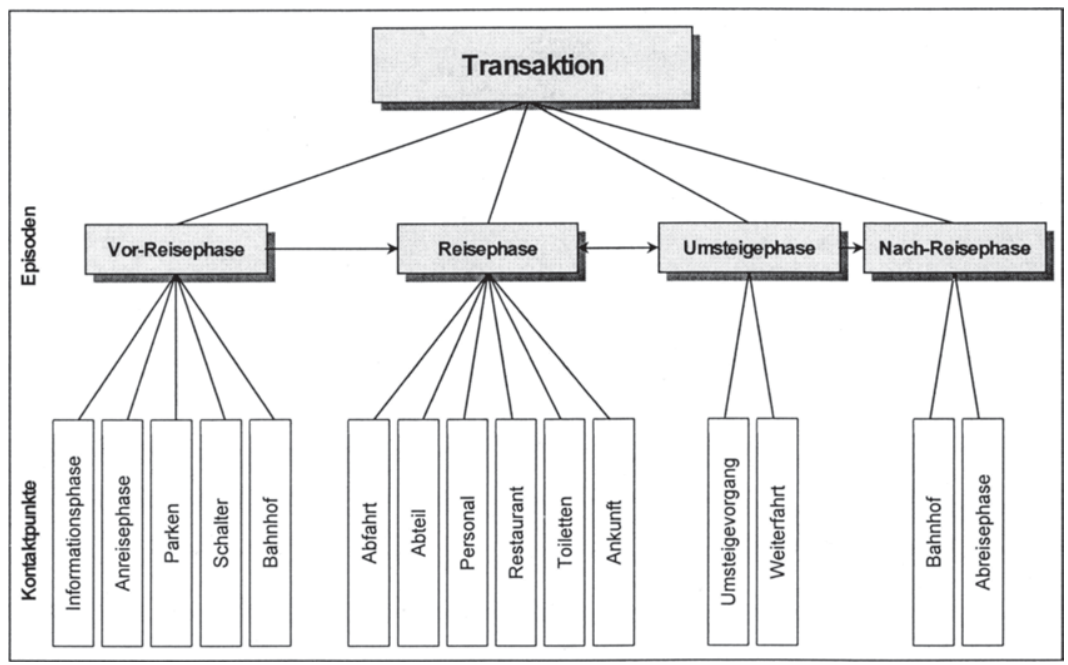

Abb. 20: Hierarchie des Kundenprozesses bei schienengebundenen Verkehrsdienstleistungen

Der erste Teilprozeß, die Vor-Reisephase, umfaßt alle Aspekte einer Bahnreise von der Vorbereitung bis zur Abfahrt des Zuges am Startbahnhof. Die Reisephase beinhaltet als zweiter Teilprozeß den Aufenthalt im Zug einschließlich der Ankunft am Zielbahnhof. Die Umsteigephase wiederum zielt auf die mit dem Wechsel eines Zuges verbundenen Tatbestände ab. Die Nach-Reisephase schließlich stellt den Teil einer Bahnreise dar, der das Verlassen des Zuges am Zielbahnhof bis zur Ankunft des Kunden am endgültigen Zielort der Reise beschreibt. Während ein Kunde die Vor-Reisephase sowie die Reisephase und Nach-Reisephase bei der erfolgreichen Inanspruchnahme einer Verkehrsdienstleistung grundsätzlich immer durchläuft, wird die Umsteigephase in der Regel nur im Falle der Nichtexistenz einer Direktverbindung wahrgenommen. ${ }^{301}$ Ist ein Umsteigevorgang Bestandteil einer Bahnreise, kommt dem internen Schnittstellenmanagement eine besondere Bedeutung zu.

und Tiefeninterviews voraus. Innerhalb der verschiedenen Studien wurden sowohl merkmalsals auch ereignisgestützte Verfahren eingesetzt. einer "schnelleren" Verbindung zu realisieren. 
Innerhalb der Vor-Reisephase werden auf der Kontaktpunktebene fünf Prozeßabschnitte unterschieden. Die Informationsphase (z.B. telefonische Kontaktaufnahme mit dem Reisezentrum) als auch die Anreise zum Bahnhof sowie der hiermit unter Umständen verbundene Parkvorgang verkörpern Teilabschnitte der Vor-Reisephase, die der eigentlichen Kernleistung, der „Beförderung“, vorgelagert sind und häufig durch den Kunden selbst erbracht werden. Ein erster direkter Kontakt mit dem Personal des Dienstleisters findet am Fahrkartenschalter bzw. im Reisezentrum des Startbahnhofes statt. Der Bahnhof stellt mit seinen unterschiedlichen Ausstattungs- und Einrichtungskomponenten einen weiteren zentralen Kontaktpunkt dar. Hier sind beispielhaft die Ausstattung mit Fahrplänen, der Wetterschutz am Bahnsteig oder die verschiedenen im Bahnhof angesiedelten Ladenlokale, aber auch die nicht-tangible „Bahnhofsatmosphäre“ zu nennen.

Wie bei den weiteren Episoden kann auch hier nicht davon ausgegangen werden, daß alle Bahnkunden die verschiedenen Kontaktpunkte vollständig und in der beschriebenen Reihenfolge durchlaufen. So kann beispielsweise die Anreise mit dem öffentlichen Nahverkehr erfolgen. Dies hat zur Folge, daß der Parkvorgang entfält. Ebenso ist es möglich, daß der Fahrschein bereits im Vorfeld in einem Reisebüro erworben worden wurde; der Schalterkontakt ist somit nicht zwingend Bestandteil des Kundenpfades. Die Darstellung der Vor-Reisephase dokumentiert dennoch anschaulich, daß bei der Auswahl von Indikatoren der Kundenzufriedenheit mit einer Bahnreise sowohl Elemente der räumlichen und zeitlichen Kontaktebene als auch der funktionalen sowie sozialen Kontaktebene Berücksichtigung finden müssen. ${ }^{302}$

Die Reisephase beinhaltet in der gewählten Unterteilung sechs Kontaktpunkte. Während die Abfahrt am Startbahnhof und die Ankunft am Zielbahnhof Komponenten der zeitlichen Ebene des Kundenkontaktes darstellen, sind die Interaktionen mit dem Zugbegleitpersonal Bestandteil der sozialen Kontaktebene. Der Aufenthalt im Zugabteil (bzw. Großraumwagen) sowie der etwaige Besuch des Zugrestaurants spiegeln wichtige Elemente der räumlichen Kontaktebene und bedingt durch die etwaig stattfindenden Interaktionen mit anderen Reisenden auch der sozialen Kontaktebene wider. Dagegen umfaßt das Aufsuchen der Zugtoiletten in erster Linie Aspekte der räumlichen Kontaktebene.

Vgl. die Ausführungen in Kap. B 1.21 dieser Arbeit. 
Innerhalb der Umsteigephase werden zwei zentrale Teilabschnitte unterschieden. Der Umsteigevorgang umschreibt den physischen Wechsel des Zuges und die hiermit einhergehenden Aktivitäten des Kunden. Der Teilprozeß „Weiterfahrt" wiederum ist der zeitlichen Kontaktebene der Dienstleistung zuzuordnen und zielt auf die mit dem Zugwechsel verbundene Wartezeit sowie die Pünktlichkeit des Anschlußzuges ab.

Zu einer Strukturierung der Nach-Reisephase schließlich wird diese ebenfalls in zwei Subprozesse unterteilt. Dies ist zum einen der Aufenthalt im Zielbahnhof, der ähnliche Komponenten wie der gleichnamige Kontaktpunkt der Vor-Reisephase zum Inhalt hat. Den zweiten Kontaktpunkt stellt die Abfahrt vom Zielbahnhof und damit die eigentliche Anreise zum endgültigen Zielort der Reise dar.

Damit basiert das der Untersuchung der Kundenzufriedenheit mit Verkehrsdienstleistungen zugrunde liegende Modell auf der Unterscheidung von vier Episoden und fünfzehn Kontaktpunkten einer Bahnreise, die es im folgenden näher zu charakterisieren gilt. Darüber hinaus werden die Beziehungszusammenhänge innerhalb und zwischen den verschiedenen Prozeßebenen konkretisiert.

\subsection{Ebenen und Indikatoren der Kundenzufriedenheit mit Verkehrs- dienstleistungen}

Die identifizierten Ebenen des bahnbezogenen Kundenprozesses ermöglichen eine intra- sowie eine interphasenspezifische Analyse der Kundenzufriedenheit. Während die intraphasenspezifische Betrachtung eine Analyse der einzelnen Episoden zum Gegenstand hat, beschäftigt sich die interphasenspezifische Untersuchung mit der Erforschung der Zusammenhänge zwischen diesen Abschnitten einer Bahnreise. Darüber hinaus wird der Frage nachgegangen, welchen Einfluß die Zufriedenheit mit einer einzelnen Bahnreise auf die globale Zufriedenheit eines Bahnreisenden mit dem Unternehmen sowie dessen zukünftiges Verhalten gegenüber dem Anbieter hat. Auf diese Weise sollen Anhaltspunkte für das Marketing eines Verkehrsdienstleisters gewonnen werden. 


\subsection{Struktur und Dynamik der Transaktionszufriedenheit}

Die Basis für die Ableitung eines Hypothesengerüstes der Kundenzufriedenheit mit Verkehrsdienstleistungen bilden die in der Forschung diskutierten Strukturund Wirkungseffekte der Zufriedenheitsdynamik. ${ }^{303}$ Dabei wird auf die Theorie der episodischen Informationsverarbeitung bzw. auf den Service-Script-Ansatz sowie auf die in diesem Zusammenhang dargestellten empirischen Forschungsarbeiten zurückgegriffen. ${ }^{304}$ Aufgrund des aufgezeigten Prozeßcharakters von Verkehrsdienstleistungen und der damit einhergehenden prozeßorientierten Entwicklung des Zufriedenheitsurteils soll, basierend auf dem vorgestellten Kundenpfad, für die interphasenspezifische Untersuchung die folgende Basishypothese formuliert werden: $:^{305}$

\section{Hyp Trans}

Die Kundenzufriedenheit mit einer Bahnreise (Transaktionsebene) setzt sich aus drei signifikant voneinander verschiedenen Episodenzufriedenheiten zusammen: ${ }^{306}$

- der Vor-Reisezufriedenheit

- der Reisezufriedenheit

- der Nach-Reisezufriedenheit

Ist eine solche Struktur der Transaktionszufriedenheit nachweisbar, stellt sich unmittelbar die Frage nach den zwischen diesen Phasen bestehenden Wirkungsbeziehungen. In Anlehnung an die Erkenntnisse der vorgestellten empirischen Studien wird folgender Tendenzhypothese nachgegangen:

Hyp D Episod

Die Zufriedenheit mit einer zeitlich vorgelagerten Episode übt einen positiven Einfluß auf die Zufriedenheit mit darauf folgenden Episoden aus.

303

304

Im folgenden wird zwischen Basis-, Tendenz- und Intensitätshypothesen unterschieden. Basishypothesen dienen einer ersten Orientierung im Untersuchungsverlauf. Eine Formulierung von Tendenzhypothesen erfolgt, wenn konkrete Wirkungsrichtungen zwischen den untersuchten Variablen abgeleitet werden können. Intensitätshypothesen werden aufgestellt, wenn über eine vermutete Wirkungsrichtung hinaus Annahmen über die relative Stärke einer Wirkungsbeziehung gemacht werden. Vgl. Kirchgeorg, M., Ökologieorientiertes Unternehmensverhalten: Typologien und Erklärungsansätze auf empirischer Basis, Wiesbaden 1990, S. 50; Schanz, G., Zwei Arten von Empirismus, in: ZfbF, Jg. 27, 1975, S. 325; Korte, Ch., Customer Satisfaction Measurement: Kundenzufriedenheitsmessung als Informationsgrundlage des Hersteller- und Handelsmarketing am Beispiel der Automobilwirtschaft, a.a.O., S. 163.

Die Zufriedenheit mit der Umsteigephase wird als ein integraler Bestandteil der Reisezufriedenheit angesehen. Vgl. hierzu die Ausführungen in Kap. C 2.31 dieser Arbeit. 
Auf der Transaktionsebene ist es für das Marketing eines Verkehrsdienstleisters von zentraler Bedeutung, den Einfluß der einzeinen Reiseabschnitte auf die Gesamtzufriedenheit zu kennen, um so etwaige Schwachpunkte aufdecken und diesen gezielt entgegenwirken zu können. Da die Reisephase, also die Beförderung von $A$ nach $B$, die Kerndienstleistung eines Verkehrsunternehmens darstellt, ist zu vermuten, daß diese mit der größten Intensität auf die Gesamtzufriedenheit wirkt:

Hyp I Trans 1 Die Zufriedenheit mit der Reisephase übt den stärksten Einfluß auf die Transaktionszufriedenheit aus.

Die Nach-Reisephase wiederum ist durch eine vergleichsweise geringe Interaktionsintensität des Verkehrsdienstleisters mit dem Reisenden geprägt, da sie primär vom Kunden selbst erbrachte Teilprozesse beinhaltet, deren positiver oder negativer Verlauf der Bahnreisende nicht unmittelbar dem Verantwortungsbereich des Unternehmens zuordnet. Diese Annahme beruht auf dem Sachverhalt, daß mit dem Erwerb der Fahrkarte grundsätzlich kein Anspruch auf eine Weiterbeförderung des Fahrgastes vom Zielbahnhof zum endgültigen Ziel der Reise verbunden ist. In bezug auf den Einfluß dieser Episode ist daher folgende Hypothese zu formulieren:

Hyp I Trans 2

Die Zufriedenheit mit der Nach-Reisephase übt den gering sten Einfluß auf die Transaktionszufriedenheit aus.

Aus einer konsumaktbezogenen Perspektive ist es vor dem Hintergrund der hohen Bedeutung der Kundenbindung für den langfristigen Unternehmenserfolg ${ }^{307}$ von besonderem Interesse, den Einfluß der Transaktionszufriedenheit auf die globale Zufriedenheit mit dem Verkehrsdienstleister (Beziehungszufriedenheit) und auf das künftige Kundenverhalten zu ermitteln. Aus den vorausgegangenen Ausführungen läßt sich dabei schließen, daß die Zufriedenheit mit einer spezifischen Transaktion in einem signifikant positiven Verhältnis zu der Zufriedenheit auf der Beziehungsebene und dem weiteren Verhalten eines Kunden gegenüber dem Anbieter steht: ${ }^{308}$

\footnotetext{
307 Vgl. hierzu die Ausführungen in Kap. A 1 und B 2.332 dieser Arbeit.

308 Vgl. Strandvik, T., Liljander, V., A Comparison of Episode Performance and Relationship Performance for a Discrete Service, a.a.O., S. $120 \mathrm{ff}$.
} 
Hyp D $B Z$

Die Zufriedenheit mit der Transaktion hat einen positiven Einfluß auf die Beziehungszufriedenheit.

Hyp D Loyal

Die Zufriedenheit mit der Transaktion übt einen positiven Einfluß auf die Kundenloyalität aus.

\subsection{Struktur und Dynamik der Episodenzufriedenheiten}

Analog zu den Überlegungen auf der Transaktionsebene sind für eine intraphasenspezifische Betrachtung Struktur und Dynamik der Kundenzufriedenheit auf der Episodenebene einer eingehenden Untersuchung zu unterziehen. Auf diese Weise sollen Erkenntnisse über die Zusammensetzung der Episodenzufriedenheiten und die ihnen zugrunde liegenden Wirkungszusammenhänge gewonnen werden. In Anlehnung an die bisherigen Ausführungen werden daher die folgenden Hypothesen formuliert:

Hyp Episod Die Kundenzufriedenheit mit einer Episode einer Bahnreise setzt sich aus signifikant voneinander verschiedenen Kontaktpunktzufriedenheiten zusammen:

Hyp Episod 1 Die Zufriedenheit mit der Vor-Reisephase beinhaltet die Zufriedenheit mit fünf Kontaktpunkten:

- der Informationsphase

- der Anreisephase

- dem Parkvorgang

- dem Schalterkontakt

- dem Aufenthalt am Startbahnhof

Hyp Episod 2 Die Zufriedenheit mit der Reisephase ergibt sich aus der Zufriedenheit mit sechs Kontaktpunkten:

- der Abfahrt vom Startbahnhof

- dem Abteilaufenthalt

- dem Personalkontakt

- dem Besuch des Zugrestaurants/-bistros

- den sanitären Anlagen im Zug

- der Ankunft am Zielbahnhof

Hyp Episod 3 Die Zufriedenheit mit der Umsteigephase setzt sich aus der Zufriedenheit mit zwei Kontaktpunkten zusammen:

- dem Umsteigevorgang

- der Weiterfahrt 
Hyp Episod 4 Die Zufriedenheit mit der Nach-Reisephase ist durch die Zufriedenheit mit zwei Kontaktpunkten gekennzeichnet:

- dem Aufenthalt am Zielbahnhof

- der Abreise zum endgültigen Ziel der Reise

Da die verschiedenen Kontaktpunkte einer Episode zeitlich eng aufeinanderfolgende Erlebnisse darstellen, kann nicht davon ausgegangen werden, daß diese isoliert voneinander wahrgenommen werden. Vielmehr liegt die Vermutung nahe, daß auch die Kontaktpunktzufriedenheiten in einem engen Verhältnis zueinander stehen:

Hyp D KP

Die Zufriedenheit mit einem zeitlich vorgelagerten Kontaktpunkt übt einen positiven Einfluß auf die Zufriedenheit mit darauf folgenden Kontaktpunkten aus.

Auf der Episodenebene ist die Kenntnis der Einflußstärke einzelner Kontaktpunktzufriedenheiten auf die jeweilige Episodenzufriedenheit von hoher Bedeutung, da insbesondere hier konkrete Anhaltspunkte für Handlungsempfehlungen im Hinblick auf das Zufriedenheitsmanagement eines Verkehrsdienstleisters abgeleitet werden können.

Der erste Reiseabschnitt, die Vor-Reisephase, ist durch eine hohe Interaktion der Fahrgäste mit dem Kundenkontaktpersonal des Verkehrsdienstleisters geprägt (z.B. Informationsphase und Fahrkartenerwerb). Daher liegt die Vermutung nahe, daß insbesondere die durch das Personal des Unternehmens zur Verfügung gestellten Informationen und die in diesem Zusammenhang wahrgenommenen Merkmale der Kompetenz und Freundlichkeit der Bediensteten einen zentralen Einfluß auf die Zufriedenheit mit der Vor-Reisephase nehmen. ${ }^{309}$ So konnte auch KLAUS im Rahmen einer empirischen Untersuchung im Verkehrsdienstleistungsbereich die besondere Rolle der persönlichen Interaktionen in der Vor-Reisephase nachweisen. ${ }^{310}$ In diesem Zusammenhang soll die folgende Hypothese einer Untersuchung unterzogen werden:

309 Zur Bedeutung dieser Komponenten einer Dienstleistung vgl. z.B. Güthoff, J., Qualităt komplexer Dienstleistungen: Konzeption und empirische Analyse der Wahrnehmungsdimensionen, a.a.O., S. 33; Stauss, B., Kundenprozeßorientiertes Qualitătsmanagement im Dienstleistungsbereich, a.a.O., S. 32 f.

310 Vgl. Klaus, P.G., Face-to-Face Service Encounters: The Issue of Quality in the Mass-Delivery of Services by Public Enterprises, Boston 1983, S. 32 ff.; Klaus, P.G., Service-Qualităt im offentlichen Personenverkehr: Ein retrograder Ansatz zur Analyse und Verănderung, in: ZőgU, Bd. 7, H. 1, 1984, S. $57 \mathrm{ff}$. 
Hyp I Episod 1

Innerhalb der Vor-Reisephase übt die Zufriedenheit mit personenbezogenen Kontaktpunkten den stärksten Einfluß auf die Episodenzufriedenheit aus.

Für das Management eines Verkehrsdienstleisters ist es zur Ableitung von Handlungsmaßnahmen von entscheidender Bedeutung zu erkennen, welche Bestandteile der angebotenen Kerndienstleistung den größten Einfluß auf die Zufriedenheit ausüben. Ein zentraler Maßstab, an dem die Qualität von Verkehrsdienstleistungsunternehmen aus Kundensicht gemessen wird, ist die Pünktlichkeit der Flugzeuge bzw. Züge. So konnte in einer Reihe von Studien die pünktliche Ankunft von Reisenden an der Zieldestination als ein zentraler Einflußfaktor der Kundenwahrnehmung identifiziert werden. ${ }^{311}$ Insbesondere in diesem Bereich ist der deutsche Schienenpersonenfernverkehr in der jüngeren Zeit deutlicher Kritik ausgesetzt, wenngleich die Bahn in der Pünktlichkeit ihrer Züge einen zentralen Systemvorteil sieht und diesen auch als Leistungsversprechen kommuniziert. ${ }^{312}$ Vor diesem Hintergrund soll daher folgender Hypothese nachgegangen werden:

Hyp I Episod 2a

Innerhalb der Reisephase beeinflußt die Zufriedenheit mit der Pünktlichkeit am Zielbahnhof die Episodenzufriedenheit am stärksten.

Während bei Fluggesellschaften Mahlzeiten und Getränke „on Board“ in der Regel Bestandteile des vom Kunden erworbenen Leistungspaketes darstellen, erhält ein Reisender in den Linienzügen der Deutschen Bahn AG durch den Kauf des Fahrscheins keinen Anspruch auf derartige Verpflegungsleistungen. Es besteht für den Kunden jedoch die Möglichkeit, das Zugrestaurant bzw. -bistro aufzusuchen, um hier gegen Entgelt entsprechende Leistungen zu erwerben. Aufgrund des engen zeitlichen Verbundes mit den anderen Kontaktpunkten der Reisephase ist davon auszugehen, daß der Aufenthalt im Zugrestaurant in einem signifikanten Wahrnehmungsverbund mit den weiteren Abschnitten der Reisephase steht. Dennoch liegt die Vermutung nahe, daß der Einfluß dieses Kontaktpunktes auf die gesamte Zufriedenheit mit der Reisephase vergleichs-

311 Vgl. Ungefug, H.-G., Bahnservice - KKV gesucht, in: WiWo, Jg. 49, H. 22, 1995, S. 85; Taylor, Sh., Waiting for Service: The Relationship Between Delays and Evaluation of Services, a.a.O., S. 56 ff.; Taylor, Sh., Claxton, J.D., Delays and the Dynamics of Service Evaluations, in: JAMS, Vol. 22, No. 3, 1994, S. $254 \mathrm{ff}$.

312 Vgl. Klein, H., InterCityExpress: mehr als nur ein neuer Zug, in: DBB, Jg. 67, H. 5, 1991, S. 499. 
weise niedrig ausfällt, da die Verpflegung nicht in dem Leistungspaket "Bahnreise“ enthalten ist. ${ }^{313}$ Daher wird die folgende Hypothese formuliert:

$\begin{array}{ll}\text { Hyp I Episod 2b } & \text { Innerhalb der Reisephase übt die Zufriedenheit mit dem } \\ & \text { Zugrestaurant/-bistro einen nur geringen Einfluß auf die } \\ & \text { Zufriedenheit mit dieser Episode aus. }\end{array}$

Fahrgäste, die während ihrer Bahnreise in einen anderen Zug umsteigen müssen, sind darauf angewiesen, auf einen reibungslosen Verlauf dieser "internen Schnittstelle" einer Bahnreise zu vertrauen. Ist die Ursache für das Versäumen des Anschlußzuges eindeutig dem Verantwortungsbereich des Verkehrsdienstleisters zuzuordnen, wird sich möglicherweise eine hohe Unzufriedenheit des Reisenden einstellen. ${ }^{314}$ So kann davon ausgegangen werden, daß insbesondere die Zufriedenheit mit dem Umsteigen durch den hiermit verbundenen Zeitaufwand bestimmt wird:

Hyp I Episod 3

Innerhalb der Umsteigephase übt die Zufriedenheit mit den zeitlichen Komponenten der Weiterfahrt den stärksten Einfluß auf die Zufriedenheit mit der Episode aus.

Der oben aufgezeigte vergleichsweise „autonome“ Charakter der Nach-Reisephase hat zur Folge, daß selbst erbrachte Teilprozesse für den Kunden in der Vordergrund rücken. So hat ein Bahnreisender grundsätzlich eigenständig sein Weiterkommen vom Zielbahnhof zu regeln und auch zu finanzieren, da mit dem Verlassen des Zuges der Anspruch auf die durch den Fahrscheinkauf erworbenen Leistungen des Verkehrsdienstleisters endet. ${ }^{315}$ Es ist daher zu vermuten, daß der Verlauf der Nach-Reisephase durch die Zufriedenheit mit den für die Weiterfahrt notwendigen Aktivitäten und dem hiermit verbundenen Zeitaufwand dominiert wird:

313 Vgl. Danaher, P.J., Mattson, J., Cumulative Encounter Satisfaction in the Hotel Conference Process, a.a.O., S. 76 sowie die Ausführungen in Kap. B 2.333 dieser Arbeit.

$314 \mathrm{Vgl}$. hierzu die Ausführungen zur Attributionstheorie der Kundenzufriedenheit in Kap. B 2.21 dieser Arbeit sowie Chebat, J.-Ch. et al., Impact of Waiting Attribution and Consumers' Mood on Perceived Quality, in: JoBR, Vol. 28, No. 3, 1995, S. $191 \mathrm{ff}$.

315 Eine Ausnahme bildet der seit November 1996 von der Deutschen Bahn AG angebotene „BahnTaxi"-Service. Hier wird der Kunde zu einem bestimmten Pauschalpreis mit einem Sammeltaxi vom Bahnhof zum Zielort seiner Reise (bzw. vom Startort seiner Reise zum Bahnhof) gebracht. Vgl. Henß, R., Sammeltaxi im Zug, in: Die Zeit, Nr. 45 vom 1.11.1996, S. 71; Deutsche Bahn AG (Hrsg.), BahnTaxi, Broschüre, Frankfurt am Main 1997. 
Hyp I Episod 4

Innerhalb der Nach-Reisephase ist die Zufriedenheit mit der Abreise zum endgültigen Zielort die dominierende Teilzufriedenheit dieser Episode.

\subsection{Bestimmungsfaktoren der Kundenzufriedenheit mit Verkehrsdienst- leistungen}

Für die Erklärung der Kundenzufriedenheit bietet sich grundsätzlich eine Unterscheidung in personenbezogene und reise- bzw. verkehrsdienstleistungsspezifische Bestimmungsfaktoren an. Zu den personalen Bestimmungsfaktoren zählen soziodemographische sowie psychographische Faktoren, bei deren Betrachtung mögliche Unterschiede in der Kundenzufriedenheit auf der Episodenund Transaktionsebene sowie etwaige Abweichungen in der Zufriedenheitsdynamik im Mittelpunkt der Untersuchung stehen. Die zweite Gruppe von Bestimmungsfaktoren befaßt sich mit der spezifischen Reisesituation von Bahnreisenden. Hier werden vor allem solche Bestimmungsfaktoren einer Analyse unterzogen, die einen potentiellen Einfluß auf die Zufriedenheit mit der Kerndienstleistung, der Beförderung von $A$ nach $B$, ausüben.

\subsection{Personenbezogene Bestimmungsfaktoren}

In der wissenschaftlichen Literatur liegen vergleichsweise nur wenige gesicherte Erkenntnisse über zentrale personenbezogene Bestimmungsfaktoren der Kundenzufriedenheit mit schienengebundenen Verkehrsdienstleistungen vor. Daher sollen im folgenden auch solche Einflußfaktoren einer Untersuchung unterzogen werden, denen allgemein im Marketing eine nur bedingte Aussagekraft zugestanden wird. In diesem Zusammenhang sind insbesondere soziodemographische Merkmale zu nennen. ${ }^{316}$ Eine Untersuchung dieser Merkmale ist insbesondere im Hinblick darauf von Interesse, daß diese in der Verkehrsdienstleistungspraxis häufig zur Bildung von Zielsegmenten herangezogen werden. ${ }^{317}$ Soziodemographische Bestimmungsfaktoren lassen sich in demographische und sozioökonomische Merkmale unterscheiden. Zu den demographischen Merkmalen zählen z.B. Geschlecht und Alter von Konsumenten. Der Kriteriengruppe

Vgl. Meffert, H., Perrey, J., Nutzensegmentierung im Verkehrsdienstleistungsbereich - theoretische Grundlagen und empirische Erkenntnisse am Beispiel des Schienenpersonenverkehrs, in: Tourismus Journal, Jg. 1, H. 1, 1997, S. $13 \mathrm{ff}$.

317 Vgl. Firner, H., Köster, J., Das Wachstum des Personenverkehrsmarktes, in: DBB, Jg. 65, H. 12,1989, S. $1037 \mathrm{ff}$. 
der sozioökonomischen Merkmale werden Bestimmungsfaktoren wie Beruf sowie Einkommen und Pkw-Besitz zugeordnet.

Im Verkehrsdienstleistungsbereich wird oft auf das Alter der Reisenden als Merkmal zur Gestaltung des Leistungsangebotes abgestellt. ${ }^{318}$ Dies geschieht vor dem Hintergrund der Erkenntnis, daß in Abhängigkeit vom Alter auch die verschiedenen Leistungsdimensionen einer Bahnreise eine unterschiedliche Bedeutung für den Reisenden haben. ${ }^{319}$ Im folgenden wird vermutet, daß mit zunehmender Anzahl von Lebensjahren die Zufriedenheit mit der Vor- und der Nach-Reisephase von besonderer Bedeutung ist, da diese Episoden zahlreiche vom Kunden selbst durchzuführende Teilprozesse (z.B. Gepäcktransport) enthalten, die ihm eine vergleichsweise hohe Selbständigkeit und Mobilität abverlangen: ${ }^{320}$

Hyp Dem 1

Das Alter der Reisenden beeinflußt die Zufriedenheit mit den einzelnen Episoden sowie die Zufriedenheit mit der gesamten Bahnreise.

Hyp Dem 2

Mit steigendem Alter der Reisenden nimmt der Einfluß der Vor- und der Nach-Reisephase auf die Transaktionszufriedenheit zu.

Neben dem Alter ist zusätzlich ein Einfluß des Geschlechts auf die Zufriedenheit mit Verkehrsdienstleistungen zu erwarten. Der bei Männern deutlich höhere Motorisierungsgrad ${ }^{321}$ legt die Vermutung nahe, daß eine Bahnfahrt von männlichen Reisenden kritischer als von Frauen beurteilt wird:

Hyp Dem 3

Das Geschlecht der Reisenden beeinflußt die Zufriedenheit mit den einzelnen Episoden sowie die Zufriedenheit mit der gesamten Bahnreise.

Hierbei ist z.B. an die speziellen Angebote für Junioren (bis 26 Jahre) und Senioren (ab 60 Jahre) zu denken. Vgl. Firner, H., Köster, J., Das Wachstum des Personenverkehrsmarktes, a.a.O., S. 1038 .

319 Vgl. Bretthauer, I., Deutsche Bahn AG: Von der Behörde zum marktorientierten Touristikanbieter, a.a.O., S. $250 \mathrm{f}$.

Vgl. Neuhaus, H., Ziele des Personenverkehrs, in: ETR, Jg. 43, H. 1/2, 1994, S. 41; Keuchel, St., Wirkungsanalyse von Maßnahmen zur Beeinflussung des Verkehrsmittelwahlverhaltens, Gottingen 1994, S. 37 f.

321 Vgl. Deutsche Shell AG (Hrsg.), Grenzen der Motorisierung in Sicht, Hamburg 1989, S. $14 \mathrm{f}$. Ein weiterer Grund für die höhere psychische Distanz von Männern gegenüber Bahnreisen liegt möglicherweise in der Bedeutung eines Pkws als Statussymbol, wăhrend das Reisen mit der Bahn einen solchen Nimbus nicht innehat. 
Ebenso ist anzunehmen, daß im Bereich der sozioökonomischen Bestimmungsfaktoren das verfügbare Einkommen und der Besitz eines Pkws eine deutliche Wirkung auf das an eine Bahnreise gestellte Anspruchsniveau und damit auf die Kundenzufriedenheit haben. Gerade der Pkw-Besitz ermöglicht einem Reisenden den Vergleich der Bahn mit einem grundsätzlich „mobileren" und "flexibleren" Verkehrsmittel. ${ }^{322}$ Es ist daher eine prinzipiell kritischere Beurteilung der Verkehrsdienstleistung „Bahnreise“ durch diese Personengruppe zu erwarten:

Hyp ökon 1

Das Einkommen der Reisenden übt einen signifikanten Einfluß auf die Zufriedenheit mit einer Bahnreise und mit ihren einzelnen Episoden aus.

Hyp Ökon 2

Die dauerhafte Verfügbarkeit eines Pkws hat einen negativen Einfluß auf die Zufriedenheit mit einer Bahnreise und mit ihren einzelnen Episoden.

Der Besitz eines Pkws eröffnet einem Nachfrager von Verkehrsdienstleistungen eine weitere Alternative bei der Wahl des Verkehrsmittels für eine bestimmte Reise. Ist der Pkw für eine Reise in Erwägung gezogen worden, wird aber die Bahn aufgrund situativer Umstände genutzt, kann vermutet werden, daß auch die Größe des Evoked Set einen Einfluß auf die Zufriedenheit mit einer Bahnreise ausübt: ${ }^{323}$

Hyp vm

Die Erwägung eines alternativen Verkehrsmittels beeinflußt die Zufriedenheit mit einer Bahnreise und mit ihren einzelnen Episoden.

Aufgrund ihrer vergleichsweise hohen Kaufverhaltensrelevanz sind zur Erklärung der Kundenzufriedenheit mit Verkehrsdienstleistungen des weiteren psychographische Merkmale einer Untersuchung zu unterziehen. ${ }^{324}$ Den bisherigen

Vgl. Schmidt, F.K., Das Verkehrsangebot „Bahn“, a.a.O., S. 160.

324

Die in diesem Zusammenhang durchzuführenden Analysen beschränken sich dabei auf die Untersuchung des Einflusses psychographischer Bestimmungsfaktoren auf die transaktionale Kundenzufriedenheit. Eine nach den verschiedenen Episoden einer Bahnreise differenzierte Betrachtung wird dagegen nicht vorgenommen, da die einbezogenen psychographischen Konstrukte in einer phasenübergreifenden Weise operationalisiert wurden. Eine phasenspezifische Analyse des Einflusses psychographischer Variablen hätte hingegen eine dezidierte Ausgestaltung dieser facettenreichen Konstrukte erfordert. Eine derartige Operationalisierung wurde in der vorliegenden Untersuchung nicht vorgenommen, da dies nicht der Zielsetzung dieser Arbeit entsprach und nicht zuletzt aus forschungsökonomischen Gründen nicht sinnvoll erschien. 
Ausführungen folgend wird davon ausgegangen, daß ein als hoch empfundenes Kaufrisiko einen negativen Einfluß auf die Kundenzufriedenheit ausübt. ${ }^{325}$ Ebenso ist anzunehmen, daß ein höheres Involvement der Reisenden das bewußte und intensive Erleben von Zufriedenheit fördert: ${ }^{326}$

Hyp Psycho 1

Das von Kunden wahrgenommene Kaufrisiko übt einen negativen Einfluß auf die Zufriedenheit mit einer Bahnreise aus.

Hyp Psycho 2

Das Involvement der Reisenden gegenüber der Bahn hat einen positiven Einfluß auf die Zufriedenheit mit einer Bahnreise.

Neben dem wahrgenommenen Kaufrisiko sowie dem Involvement werden vor allem Einstellungen als Bestimmungsfaktoren der Kundenzufriedenheit angesehen. ${ }^{327}$ "Die Bahn leidet an einem Mangel an sozialer Akzeptanz". ${ }^{328}$ Diese Aussage wird durch die Ergebnisse einer aktuellen verkehrsmittelübergreifenden Studie bestätigt, in der der Bahn auch einige Jahre nach ihrer Privatisierung insbesondere im Vergleich zur Deutschen Lufthansa AG ein schlechtes Image attestiert wird. ${ }^{329}$ Daher soll überprüft werden, inwieweit auch die grundsätzliche Einstellung gegenüber der Deutschen Bahn AG die Zufriedenheit mit einer konkreten Bahnreise beeinflußt. GRONROOS bezeichnet in diesem Zusammenhang das Image eines Unternehmens als eine Art "Wahrnehmungsfilter", der den Grad der Zufriedenheit mit der Transaktion mitbestimmt: ${ }^{300}$

Vgl. hierzu und im folgenden die Ausführungen in Kap. B 1.21 und B 2.22 dieser Arbeit.

Zu einer entsprechenden Untersuchung im Automobilbereich vgl. Richins, M.L., Bloch, P.H., Post-Purchase Product Satisfaction: Incorporating the Effects of Involvement and Time, in: JoBR, Vol. 23, No. 2, 1991, S. $145 \mathrm{ff}$.

Vgl. hierzu die Ausführungen in Kap. B 2.1 sowie B 2.2 dieser Arbeit.

Klein, H., Deutsche Bundesbahn: Die Entwicklung zu einem europäischen Dienstleistungsund Verkehrskonzern, in: Touristik-Marketing: das Marketing der Tourismus-Organisationen, Verkehrsträger, Reiseveranstalter und Reisebüros, Roth, P., Schrand, A. (Hrsg.), München 1992, S. 288.

Vgl. Antrecht, R., Claßen, W., Friese, U., Wem die Deutschen trauen, a.a.O., S. 38 ff.

Der Begriff Image wird der in der Literatur gängigen Konvention folgend in diesem Zusammenhang als überwiegend deckungsgleich mit dem Begriff der Einstellungen angesehen. Vgl. hierzu Meffert, H., Marketingforschung und Käuferverhalten, a.a.O., S. 55; Trommsdorff, V., Konsumentenverhalten, a.a.O., S. 155.

Vgl. Grönroos, Ch., Toward a Third Phase in Service Quality Research: Challenges and Future Directions, a.a.O., S. 52 f. 
Hyp Psycho 3

Die Zufriedenheit mit einer Bahnreise wird durch eine positive Einstellung der Reisenden gegenüber der Bahn vorteilhaft beeinflußt.

\subsection{Reisebezogene Bestimmungsfaktoren}

Zusätzlich zu den personenbezogenen Bestimmungsfaktoren sind reise- bzw. verkehrsdienstleistungsspezifische Determinanten zu berücksichtigen. So kann eine Reihe von Einflußfaktoren identifiziert werden, die für die spezifische Reisesituation und die damit verbundene Zufriedenheit eines Nachfragers von Verkehrsdienstleistungen von Bedeutung sein können. In diesem Kontext stellt der Reiseanlaß ein zentrales Kriterium zur Unterscheidung von Konsumentengruppen im Verkehrsdienstleistungsbereich dar.

Bei den Reiseanlässen wird in der Unternehmenspraxis üblicherweise zwischen Privat- und Geschäftsreisen und damit nach der „zahlenden Stelle" unterschieden. ${ }^{331}$ Diesen Reiseanlässen liegen unterschiedlichen Motive für die Inanspruchnahme einer Verkehrsdienstleistung zugrunde. Für Geschäftsreisende stellt eine Bahnfahrt in erster Linie einen integrierten Bestandteil des Arbeitstages dar. Sie ist daher Mittel zum Zweck und dient als "Doppelnutzung der Zeit". ${ }^{332}$ Privatreisende verbinden mit einer Bahnreise dagegen z.B. den Beginn eines Urlaubs, so daß der Erholungswert und Erlebnischarakter einer Bahnreise in den Vordergrund der Kundenwahrnehmung rücken. ${ }^{333}$ Es wird daher vermutet:

Hyp VDL 1

Der Reiseanlaß hat eine signifikante Wirkung auf die Zufriedenheit mit den einzelnen Episoden sowie die Zufriedenheit mit der gesamten Bahnreise.

Vgl. Schörcher, U., Marketing im Luftverkehr, a.a.O., S. 133 ff.; Pompl, W., Luftverkehr: Eine okonomische Einführung, a.a.O., S. 64 und 85 ff. Innerhalb der Gruppe der Privatreisenden ist eine weitere Differenzierung Z.B. in touristische Reisen und Verwandten- und Bekanntenbesuche denkbar. Innerhalb des Reiseanlasses "Geschäftsreise" kann wiederum zwischen den Dienstreisen von Beamten und Beschäftigten des offentlichen Dienstes und der Reisetätigkeit von Angestellten im privatwirtschaftlichen Bereich unterschieden werden. Vgl. Merckens, R., Analyse des Verkehrsmittelwahlverhaltens von Geschäftsreisenden, Bundesminister für Verkehr (Hrsg.), Bonn 1984, S. 2.

Stauss, B., Dienstleister und die vierte Dimension, a.a.O., S. 85. So nutzen Geschäftsreisende eine Bahnreise häufig nicht nur zur Anreise zu einem Geschäftstermin, sondern auch zu dessen Vorbereitung.

333 Aufgrund der verschiedenartigen Motive des Reiseantritts ist auch davon auszugehen, daß die "Zeitwahrnehmung" bzw. "Zeitsensibilitatt" dieser Kundensegmente (z.B. in bezug auf die Dauer der Fahrt oder die Pünktlichkeit am Zielbahnhof) unterschiedlich ausgeprägt sind. Vgl. Pompl, W., Luftverkehr: Eine ökonomische Einführung, a.a.O., S. 88. 
Im Rahmen einer Betrachtung des schienengebundenen Verkehrsmittels "Bahn“ können darüber hinaus weitere Faktoren identifiziert werden, die einen potentiellen Einfluß insbesondere auf die Zufriedenheit mit der Reisephase ausüben und damit auch die Transaktionszufriedenheit beeinflussen. Hier sind vor allem die unterschiedlichen Zugarten (InterCityExpress, InterCity bzw. EuroCity, InterRegio), die genutzte Reise- bzw. Beförderungsklasse ${ }^{334}$ sowie der Abteiltyp (Großraumwagen versus „klassische“ Abteilwagen) zu nennen, die sich hinsichtlich ihrer Ausstattung nachhaltig voneinander unterscheiden: ${ }^{335}$ \begin{tabular}{|ll}
\hline Hyp VDL 2 & Die genutzte Zugart übt einen signifikanten Einfluß auf die \\
& $\begin{array}{l}\text { Zufriedenheit mit der Reisephase und die Zufriedenheit mit } \\
\text { der gesamten Bahnreise aus. }\end{array}$
\end{tabular}

Hyp VDL 3

Die Reiseklasse nimmt Einfluß auf die Zufriedenheit mit der Reisephase und die Zufriedenheit mit der gesamten Bahnreise.

Hyp VDL 4

Die Zufriedenheit mit der Reisephase und mit der gesamten Bahnreise wird durch den Abteiltyp beeinflußt.

Im Tagesablauf sind deutliche Schwankungen in der Nachfrage nach Verkehrsdienstleistungen festzustellen. Zeiten hoher Nachfrage werden von einem großen Personenaufkommen bzw. Kundenandrang am Bahnhof und insbesondere in den Zügen begleitet (sog. „Crowding"-Phänomen). ${ }^{336}$ Es ist zu enwarten, daß die hohe physische Nähe zu den Mitreisenden und die hiermit verbundenen Begleit-

334 Während im Schienenpersonenverkehr üblicherweise zwischen der „ersten“ und "zweiten“ Klasse unterschieden wird, differenzieren Fluggesellschaften im innerdeutschen Flugverkehr gängigerweise zwischen der "Business"- und „Economy"-Klasse. Zum letzteren vgl. Laakmann, K., Value-Added Services als Profilierungsinstrument im Wettbewerb: Analyse, Generierung und Bewertung, a.a.O., S. $211 \mathrm{ff}$.

Zu der Ausstattung der verschiedenen Zugvarianten und ihren Einsatzfeldern vgl. Giese, D., Regionalluftverkehr und Eisenbahn als Wettbewerber im deutschen und grenzüberschreitenden Verkehr, Berlin 1993, S. 148 ff.; Garre, K.-H., InterRegio: Ein neues Leistungsangebot der Deutschen Bundesbahn im Schienenpersonenfernverkehr, a.a.O., S. 775 ff.; Rahn, T., Der Hochgeschwindigkeitszug InterCityExpress der DB, in: DBB, Jg. 67, H. 5, 1991, S. 537 ff.; Klein, H., InterCityExpress: mehr als nur ein neuer Zug, a.a.O., S. $497 \mathrm{ff}$.

Da der Schwerpunkt dieser Arbeit auf dem (Linien-)Personenfernverkehr der Deutschen Bahn liegt, kann hier auf eine Erörterung des Nahverkehrs sowie eine Darstellung weiterer Spezialverkehrsarten wie z.B. des Autoreise- oder des Hotel- bzw. Nachtreiseverkehrs verzichtet werden. Vgl. hierzu Neuhaus, H., Ziele des Personenverkehrs, a.a.O., S. 42 ff.; Klein, H., Personenverkehr, in: DDB, Jg. 69, H. 1, 1993, S. 34. Deutsche Bahn AG (Hrsg.), Service \& Züge, Broschüre, Frankfurt am Main 1997, S. $58 \mathrm{ff}$.

Vgl. Bateson, J.E.G., Hui, M.K., Crowding in the Service Environment, in: Creativity in Services Marketing: What's New, What Works, What's Developing, Venkatesan, M., Schmalensee, D.M., Marshall, C. (Hrsg.), Chicago 1986, S. $85 \mathrm{ff}$. 
erscheinungen wie Wartezeiten und empfundene Enge die Zufriedenheit mit der Fahrt im Zug beeinflussen:

Hyp VDL 5 Eine hohe Auslastung der Züge übt einen negativen Einfluß auf die Zufriedenheit mit der Reisephase und die Zufriedenheit mit der gesamten Bahnreise aus.

Hierauf aufbauend kann angenommen werden, daß die Zufriedenheit mit der Reisephase durch eine vorgenommene Reservierung eines Sitzplatzes positiv beeinflußt wird. Es wird daher folgende Hypothese formuliert:

Hyp VDL 6 Die Sitzplatzreservierung hat einen positiven Einfluß auf die Zufriedenheit mit der Reisephase und die Zufriedenheit mit der gesamten Bahnreise.

Die dargestellten Beziehungszusammenhänge des Kundenprozesses und die erörterten Bestimmungsfaktoren haben unterschiedliche Wirkungen auf die Zufriedenheit mit einer Bahnreise. Im empirischen Teil dieser Arbeit sollen diese Einflüsse weiter konkretisiert werden.

\section{Bezugsrahmen der empirischen Untersuchung}

Aufbauend auf den Überlegungen zu Struktur und Dynamik der Kundenzufriedenheit mit Verkehrsdienstleistungen sowie den thematisierten Bestimmungsfaktoren und den in diesem Zusammenhang formulierten Untersuchungshypothesen läßt sich der in Abbildung 21 dargestellte Bezugsrahmen der empirischen Untersuchung ableiten.

Im Mittelpunkt der Betrachtung steht die Validierung eines operationalen Meßinstrumentes zur Erfassung des prozessualen Charakters der Kundenzufriedenheit mit Verkehrsdienstleistungen sowie die Identifikation von zentralen Bestimmungsfaktoren zur Erklärung der Kundenzufriedenheit. Zu diesem Zweck werden die im Rahmen der konzeptionellen Überlegungen erarbeiteten Wirkungsbeziehungen und die daraus abgeleiteten Untersuchungshypothesen in den Erklärungsansatz einbezogen. 




Abb. 21: Bezugsrahmen der empirischen Untersuchung der Kundenzufriedenheit mit Verkehrsdienstleistungen

Die Erfassung der Kundenzufriedenheit mit Verkehrsdienstleistungen bedingt die Festlegung geeigneter Indikatoren zur Operationalisierung der Kundenzufriedenheit auf den verschiedenen Prozeßebenen. Daher orientiert sich die Auswahl der zu messenden Merkmale an den identifizierten Kontaktpunkten einer Bahnreise. Bei der Auswahl von Kriterien ist zunächst eine Reduktion aller hypothetisch denkbaren Merkmale auf die aus Konsumentensicht relevanten Merkmale erforderlich. Wie bereits erörtert sollen die Indikatoren auch solche Kontaktpunkte abbilden, die vom Verkehrsdienstleister nicht direkt kontrolliert werden können. Zur Vermeidung von Verzerrungseffekten und Doppelzählungen ist darüber hinaus der Unabhängigkeitsprämisse der Merkmale weitestgehend Rechnung zu tragen. Der Merkmalskatalog der empirischen Untersuchung beruht analog zu der Ermittlung des Kundenpfades auf der Auswertung explorativer Kundeninterviews und bahneigener Untersuchungen sowie den Ergebnissen von Expertengesprächen und wurde gemäß den Anforderungen an einen Operationa- 
lisierungsansatz der Kundenzufriedenheit mit Verkehrsdienstleistungen zusammengestellt. ${ }^{337}$

Auf Grundlage der vorgestellten Kundenprozeßhierarchie werden zunächst die einzelnen Episoden und die hierin befindlichen Kontaktpunkte einer Analyse unterzogen (Intraphasen-Betrachtung). Auf diesen Erkenntnissen aufbauend erfolgt dann die interphasenspezifische Untersuchung der Kundenzufriedenheit mit einer Bahnreise. Hinsichtlich der thematisierten Bestimmungsfaktoren sind soziodemographische sowie psychographische und insbesondere reise- bzw. verkehrsdienstleistungsspezifische Faktoren berücksichtigt worden.

Der Messung der Kundenzufriedenheit auf der empirischen Sprachebene liegt ein direkter Einkomponenten-Ansatz aus dem Bereich merkmalsorientierter Verfahren zugrunde. ${ }^{338}$ Damit wird auf eine separate Erhebung der Soll- und IstKomponente verzichtet und so u.a. der Tatsache Rechnung getragen, daß erst aus der nachträglichen Bewertung des Soll-Ist-Vergleiches ein Zufriedenheitsurteil resultiert. Aufgrund der nicht erwiesenen grundsätzlichen Vorteilhaftigkeit einer expliziten Erhebung von Merkmalsgewichten auf Ratingskalen findet die Ermittlung der Bedeutungsgewichte anhand statistischer Auswertungsverfahren statt. Diese wird um die Erhebung kritischer Ereignisse auf der Episodenebene ergänzt. $^{339}$

Da sich in der wissenschaftlichen Diskussion hinsichtlich der Verdichtung von Einzelmerkmalen bisher kein konsensfähiger Modellansatz durchsetzen konnte, ${ }^{340}$ wird im Rahmen der vorliegenden Untersuchung auf die Formulierung einer Gewichtungs- bzw. Integrationsregel zur Errechnung der Episodenzufriedenheiten bzw. der Transaktionszufriedenheit verzichtet. Statt dessen erfolgt eine explizite Messung der Zufriedenheit sowohl mit den verschiedenen Episoden als auch mit der gesamten Bahnreise (Transaktionszufriedenheit). ${ }^{341}$

\footnotetext{
337 Vgl. hierzu die Ausführungen in Kap. B 1 dieser Arbeit.

$338 \mathrm{Vgl}$. hierzu und im folgenden die Ausführungen in Kap. B 3.221 dieser Arbeit.

339 Eine detaillierte Darstellung der zugrunde gelegten Meß- und Auswertungsmethodik erfolgt in Kap. C 1 dieser Arbeit.

$340 \mathrm{Vgl}$. hierzu die Ausführungen in Kap. B 3.221 dieser Arbeit.

341 Dieser Vorgehensweise liegt die implizite Annahme zugrunde, daß die Episodenzufriedenheiten bzw. die Transaktionszufriedenheit als valide zu betrachten sind. Vgl. hierzu Dichtl, E., Peter, S., Kundenzufriedenheit und Kundenbindung in der Automobilindustrie: Ergebnisse einer empirischen Untersuchung, a.a.O., S. 19.
} 


\section{Empirische Analyse zur phasenorientierten Erfassung und Erklärung der Zufriedenheit mit Verkehrsdienstleistungen}

Die Überprüfung der Hypothesen zur Kundenzufriedenheit mit Verkehrsdienstleistungen erfolgt auf der Basis einer von der Forschungsstelle Bahnmarketing in der Wissenschaftlichen Gesellschaft für Marketing und Unternehmensführung e.V. im Sommer 1996 durchgeführten Befragung von Fernreisenden der Deutschen Bahn AG.

Zunächst werden die Datenbasis und ihre Erhebungsform sowie die zur Überprüfung des Hypothesensystems herangezogenen Methoden der statistischen Datenauswertung näher dargestellt. Die empirische Untersuchung gliedert sich in drei Abschnitte, die intraphasenspezifische Analyse der Kundenzufriedenheit mit Verkehrsdienstleistungen, die episodenübergreifende Untersuchung der Kundenzufriedenheit (interphasenspezifische Betrachtung) und die Analyse des Einflusses ausgewählter Bestimmungsfaktoren auf die Kundenzufriedenheit mit Verkehrsdienstleistungen.

\section{Design der Untersuchung}

\subsection{Wahl der Stichprobe}

Der empirischen Überprüfung des Bezugsrahmens liegt eine in Zusammenarbeit mit der Deutschen Bahn AG durchgeführte schriftliche Befragung von Reisenden mit einer Fahrtlänge von über $100 \mathrm{~km}$ zugrunde. ${ }^{1}$ Um dem Ziel einer prozeßorientierten Analyse der Kundenzufriedenheit zu entsprechen, wurde ein auf dem Bezugsrahmen der Untersuchung basierender Fragebogen entwickelt. Der detaillierte Fragebogen sowie die Frageformulierungen wurden auf der Basis zufriedenheitsorientierter Studien der Deutschen Bahn AG sowie aufgrund der Erkenntnisse aus Expertengesprächen konzipiert. Nach der Durchführung von Pretestinterviews und einer hierauf aufbauenden Anpassung des Befragungsdesigns erfolgte die Ausgabe der Fragebögen im August 1996 auf ausgewählten innerdeutschen Strecken in den verschiedenen Zugarten des Fernverkehrs der Deutschen Bahn AG sowie in ausgewählten Reisebüros in Münster. Dabei wurden die Befragten gebeten, den Fragebogen in direktem Anschluß an ihre aktuelle

Die Vorgabe einer Mindestreiselänge von $100 \mathrm{~km}$ diente zum einen der eindeutigen Abgrenzung Bahnfernreisender von Teilnehmern des schienengebundenen Nahverkehrs. Vgl. zur Definition des Schienenpersonenfernverkehrs die Ausführungen in Kap. B 1.22 dieser Arbeit. Zum anderen konnte auf diese Weise das kundenseitige Erleben eines Großteils der zu untersuchenden Kontaktpunkte sichergestellt werden. 
Bahnreise auszufüllen und an die Forschungsstelle Bahnmarketing zurückzusenden. Von den zurückgesandten Fragebögen erwiesen sich 603 als auswertbar. $^{2}$ Die Zusammensetzung der Stichprobe kann Abbildung 22 entnommen werden.

\begin{tabular}{|l|c|c|c|c|}
\cline { 2 - 4 } \multicolumn{1}{l|}{} & InterCityExpress & $\begin{array}{c}\text { InterCityl } \\
\text { EuroCity }\end{array}$ & InterRegio & Gesamt \\
\hline Stichprobenanteil: & $45 \%$ & $38 \%$ & $17 \%$ & $\mathrm{n}=603$ \\
\hline $\begin{array}{l}\text { Reiseanlaß: } \\
\text { • geschäftlich } \\
\text { •privat (inkl. Fern- } \\
\text { pendler) }\end{array}$ & $25 \%$ & $32 \%$ & $15 \%$ & $26 \%$ \\
\hline $\begin{array}{l}\text { Reiseklasse: } \\
\text {-1. Klasse }\end{array}$ & $75 \%$ & $68 \%$ & $85 \%$ & $74 \%$ \\
-2. Klasse & $29 \%$ & $26 \%$ & $13 \%$ & $25 \%$ \\
\hline $\begin{array}{l}\text { Geschlecht: } \\
\text { - weiblich } \\
\text { • männlich }\end{array}$ & $71 \%$ & $74 \%$ & $87 \%$ & $75 \%$ \\
\hline $\begin{array}{l}\text { Alter: } \\
\text { - bis 26 Jahre } \\
\text {-27-59 Jahre } \\
\text {-60 Jahre und älter }\end{array}$ & $16 \%$ & $46 \%$ & $43 \%$ & $44 \%$ \\
\hline
\end{tabular}

\section{Abb. 22: Zusammensetzung der Stichprobe}

Der Schwerpunkt der Befragung lag auf standardisierten Fragen. Zur Ermittlung der Kundenzufriedenheit dienten für die vier Episoden einer Bahnreise vorgegebene Kriterienkataloge. Die Zufriedenheitsurteile wurden anhand einer fünfstufigen Ratingskala erfaßt, die in einem Kontinuum von "sehr zufrieden" bis "gar nicht zufrieden“ die Einschätzung der Befragten mißt. ${ }^{3}$ Darüber hinaus wurden die Reisenden in offenen Fragen um eine nach den verschiedenen Episoden differenzierte Schilderung von positiven und negativen kritischen Ereignissen gebeten. Die weiteren Befragungsinhalte basieren auf den Bestimmungsfaktoren

2 Die Nettorücklaufquote betrug deutlich über $50 \%$ und ist damit als sehr hoch zu bezeichnen. Vgl. Meffert, H., Marketingforschung und Käuferverhalten, a.a.O., S. 202. Die durchschnittliche Rücksendedauer lag unter drei Tagen, so daß von einem aktuellen Zufriedenheitsurteil der Befragten ausgegangen werden kann.

3 Darüber hinaus wurde den Befragten die Moglichkeit gegeben, auf eine Antwortkategorie ${ }_{n}$ trifft nicht zu/kein Urteil" auszuweichen. Da im Fragebogen explizit um eine Beurteilung der aktuellen Bahnreise gebeten wurde, sollte auf diese Weise gewăhrleistet werden, daß die Befragten nur die im Rahmen dieser konkreten Bahnreise erlebten Kontaktpunkte beurteilen. 
der Kundenzufriedenheit mit Verkehrsdienstleistungen und der bekundeten Loyalität gegenüber der Verkehrsdienstleistung „Bahnreise“. Dabei wurden folgende Fragenkomplexe erfaßt:

- Spezifische Reisesituation der Befragten,

- Verhaltensabsichten gegenüber der Deutschen Bahn AG,

- Einstellungen, wahrgenommenes Risiko und Involvement gegenüber einer Bahnreise,

- Allgemeines Reiseverhalten der letzten 12 Monate sowie

- soziodemographische Merkmale der Befragten.

Die Einbindung der einzelnen Variablengruppen in das Befragungsdesign, die konkreten Fragenformulierungen und die eingesetzten Ratingskalen sind dem Fragebogen im Anhang II dieser Arbeit zu entnehmen.

\subsection{Methoden der statistischen Auswertung}

Mit dem Ziel, die Struktur und die Dynamik der Kundenzufriedenheit mit Verkehrsdienstleistungen zu untersuchen, verbinden sich hohe Anforderungen an die Leistungsfähigkeit der zu diesen Zwecken einzusetzenden Verfahren. So kommen einerseits nur solche Analysetechniken in Betracht, die es ermöglichen, das Beziehungsgeflecht einer Vielzahl von möglichen abhängigen und unabhängigen Variablen gleichzeitig zu untersuchen. Zum anderen sollten diese Verfahren den Forscher in die Lage versetzen, zwischen beobachtbaren und nicht-beobachtbaren (latenten) Variablen und damit zwischen den zu untersuchenden Konstrukten und ihrer Operationalisierung unterscheiden zu können. ${ }^{4}$ Für die dieser Arbeit zugrunde liegenden Fragestellungen sind daher insbesondere Verfahren der Dependenzanalyse in Erwägung zu ziehen. ${ }^{5}$

4 Vgl. Büschken, J., Multipersonale Kaufentscheidungen - Empirische Analyse zur Operationalisierung von Einflußbeziehungen im Buying Center, Wiesbaden 1994, S. 133 und $141 \mathrm{f}$.; Korte, Ch., Customer Satisfaction Measurement: Kundenzufriedenheitsmessung als Informationsgrundlage des Hersteller- und Handelsmarketing am Beispiel der Automobilwirtschaft, a.a.O., S. $174 \mathrm{f}$.

5 Während der Dependenzanalyse die Annahme eines Kausalzusammenhanges zwischen unabhängigen Variablen und von ihnen abhängigen Variablen zugrunde liegt, ist die Analyse wechselseitiger Beziehungen zwischen Variablengruppen Gegenstand der Interdependenzanalyse. Zu den Verfahren der Dependenzanalyse zählen insbesondere die Varianz-, Regressions- und Kausalanalyse. Verfahren der Interdependenzanalyse stellen z.B. die Korrelations-, Cluster- und Faktorenanalyse dar. Vgl. z.B. Berekoven, L., Eckert, W., Ellenrieder, P., Marktforschung: methodische Grundlagen und praktische Anwendung, a.a.O., S. $210 \mathrm{ff}$.

Andreas Siefke and Universität Münster - 978-3-631-75103-9 
Die Beziehungsstruktur zwischen den einzelnen Kontaktpunktzufriedenheiten auf der einen Seite und deren Einfluß auf die entsprechenden Episodenzufriedenheiten auf der anderen Seite sowie die Zusammenhänge dieser Art auf der Transaktionsebene lassen eine ausschließliche Untersuchung der verschiedenen Konstellationen mit Hilfe einfacher multivariater Verfahren wie z.B. der Regressionsanalyse nur eingeschränkt zu. So besteht eine zentrale Voraussetzung der Regressionsanalyse in der Forderung nach der statistischen Unabhängigkeit der zur Erklärung eines Konstruktes (endogene Variable) herangezogenen (exogenen) Variablen. ${ }^{6}$ Dies würde z.B. bedeuten, daß in die Analyse der Episodenzufriedenheiten statistisch voneinander unabhängige Kontaktpunktzufriedenheiten einfließen würden. Die bisherigen Ausführungen zu dem Konstrukt der Kundenzufriedenheit auf der theoretischen Sprachebene beruhen jedoch auf der zentralen Annahme eines signifikanten Zusammenhangs zwischen diesen Teilzufriedenheiten. Daher ist es notwendig, auf Ansätze zurückzugreifen, die die mögliche Existenz solcher Beziehungen explizit berücksichtigen. In der Literatur werden derartige Analysetechniken als multivariate Verfahren der „zweiten Generation" bezeichnet und in jüngeren Arbeiten der Marketingforschung zunehmend berücksichtigt. ${ }^{7}$ Zu diesen Ansätzen zählen die Verfahren der Kausalanalyse. ${ }^{8}$

Die grundsätzliche Zielsetzung der Kausalanalyse besteht in der Untersuchung der Beziehungen zwischen latenten Variablen. Solche nicht-beobachtbare Variable stellen z.B. die Kontaktpunkt- und Episodenzufriedenheiten oder die Transaktionszufriedenheit einer Bahnreise dar. Zur Analyse der aus theoretischen Vorüberlegungen abgeleiteten Beziehungsstrukturen erfolgt die Formulierung eines sog. Kausalmodells häufig in Form eines graphisches Pfaddiagrammes, das dann in ein lineares Gleichungssystem umgesetzt und damit einer mathematischen Analyse zugänglich gemacht wird. ${ }^{9}$ Neben dem Vorteil einer simultanen Analyse mehrerer latenter Variablen ist durch die explizite Trennung der Konstrukte und

Vgl. Nader, G., Zufriedenheit mit Finanzdienstleistungen: Erfolgswirksamkeit, Messung, Modellierung, a.a.O., S. 55. Zu den Problemen eines ausschließlichen Einsatzes der Regressionsanalyse zur Analyse von Zusammenhangsstrukturen vgl. Homburg, Ch., Die Kausalanalyse: Eine Einführung, in: WiSt, Jg.21, H. 10, 1992, S. 499 f.; Wöllenstein, St., Betriebstypenprofilierung in vertraglichen Vertriebssystemen: Eine Analyse von Einflußfaktoren und Erfolgswirkungen auf der Grundlage eines Vertragshändlersystems im Automobilhandel, Frankfurt am Main u.a. 1996, S. 272 f.

7 Vgl. Homburg, Ch., Giering, A., Konzeptualisierung und Operationalisierung komplexer Konstrukte, a.a.O., S. 8.

8 Einen Überblick über Einsatzfelder der Kausalanalyse in der Kaufverhaltensforschung liefert Büschken, J., Multipersonale Kaufentscheidungen - Empirische Analyse zur Operationalisierung von Einflußbeziehungen im Buying Center, a.a.O., S. $132 \mathrm{f}$.

$9 \quad$ Vgl. Balderjahn, I., Das umweltbewußte Konsumentenverhalten - Eine empirische Studie, Berlin 1986, S. 72. 
der zu ihrer Operationalisierung herangezogenen Indikatoren die Möglichkeit gegeben, Meßfehler dieser Indikatorvariablen in die Analyse einzubeziehen.

Daher wurde im Rahmen der vorliegenden Untersuchung die Kausal- oder auch Strukturgleichungsanalyse als ein geeigneter Ansatz zur Spezifizierung der Beziehungen zwischen den verschiedenen Zufriedenheitskonstrukten der Kundenprozeßhierarchie eingesetzt. ${ }^{10}$ Dies gilt analog für ausgewählte Bestimmungsfaktoren der Kundenzufriedenheit sowie die bekundete Verhaltensabsicht gegenüber der Deutschen Bahn AG, deren jeweilige Zusammenhangsstruktur mit dem Zufriedenheitskonstrukt anhand des Verfahrens der Kausalanalyse untersucht werden kann. Als hypothesenprüfendes statistisches Verfahren kommt in der vorliegenden Arbeit der EQS-Ansatz (Equations based Structural Program) von BENTLER zum Einsatz. ${ }^{11}$

Aufgrund der inzwischen häufigen Venwendung der Kausalanalyse in der empirischen Kaufverhaltensforschung kann auf eine Darstellung des Verfahrens verzichtet werden, da dies bereits an anderer Stelle in ausführlicher Weise erfolgt ist. $^{12}$ Zur Schätzung der Modellparameter und deren Gütebeurteilung werden in

In der wissenschaftlichen Literatur hat sich der Begriff der "Kausalanalyse" durchgesetzt, wenngleich diese Bezeichnung und damit die Annahme kausaler Beziehungen aus wissenschaftstheoretischer Sicht nicht unproblematisch ist. Zum sog. Kausalitätsproblem vgl. Büschken, J., Multipersonale Kaufentscheidungen - Empirische Analyse zur Operationalisierung von Einflußbeziehungen im Buying Center, a.a.O., S. 141; Hildebrandt, L., Trommsdorff, V., Konfirmatorische Analysen in der empirischen Forschung, in: Innovative Marktforschung, Forschungsgruppe Konsum und Verhalten (Hrsg.), Würzburg, Wien 1983, S. 139 f.

11 Vgl. Bentler, P.M., Theory and Implementation of EQS. A Structural Equations Program, Los Angeles 1985. Bentler, P.M., EQS Structural Equations Program Manual, Multivariate Software Inc., Encino 1995. Dem Verfasser stand für die Datenanalyse die windows-gestützte Programmversion EQS 5.3 zur Verfügung, die sich gegenüber dem in der Marktforschung häufig eingesetzten LISREL-Ansatz der Kausalanalyse durch flexiblere Modellannahmen und eine deutlich höhere Bedienungsfreundlichkeit auszeichnet. So beinhaltet die eingesetzte Programmversion u.a. eine graphische Benutzeroberfläche („Diagrammer"), die der Erstellung graphischer Pfaddiagramme dient. Diese werden dann durch das Programm in die entsprechende Befehlssyntax umgesetzt und die Modellparameter sowie Anpassungsmaße berechnet. Methodisch stellen der LISREL-Ansatz und der EQS-Ansatz eng verwandte Verfahren dar. So ist der LISREL-Ansatz als ein Sonderfall des EQS-Modells anzusehen. Zu einem Vergleich dieser Verfahren vgl. Homburg, Ch., Sütterlin, St., Kausalmodelle in der Marktforschung: EQS als Alternative zu LISREL?, in: Marketing ZFP, Jg.12, H. 3, 1990, S. $181 \mathrm{ff}$.

Des weiteren ist in diesem Zusammenhang der PLS-Ansatz (Partial Least Square) von Wold zu nennen. Während der LISREL-Ansatz sowie der EQS-Ansatz auf einer Analyse von Kovarianzstrukturen beruhen, basiert der PLS-Ansatz auf einer Kleinste-Quadrate-Schătzung. Zum letzteren vgl. Wold, H., Soft Modeling: The Basic Design and Some Extensions, in: Systems under Indirect Observation: Causality, Structure, Prediction, Jöreskog, K.G., Wold, H. (Hrsg.), 2. Aufl., Amsterdam 1982, S. 1 ff.; Lohmüller, G.B., Path models with Latent Variables and Partial Least Squares (PLS) Estimation, Würzburg 1984.

Vgl. z.B. Hildebrandt, L., Konfirmatorische Analysen von Modellen des Konsumentenverhaltens, Berlin 1983, Homburg, Ch., Die Kausalanalyse: Eine Einführung, a.a.O., S. 499 ff.; Backhaus, K. et al., Multivariate Analysemethoden - Eine anwendungsorientierte Einführung, 8. 
der Literatur zahlreiche Konzepte diskutiert, die im Hinblick auf ihre Eignung für die Durchführung und Bewertung eines Kausalmodells unterschiedlich eingeschätzt werden. ${ }^{13} \mathrm{Da}$ die auszuwertende Datendatei nicht normalverteilte Variablen enthält, wurde im Rahmen dieser Arbeit von den in EQS implementierten Schätzverfahren der AGLS-Ansatz ausgewählt, da diese Methode gegenüber anderen Verfahren auf keiner Verteilungsannahme beruht. ${ }^{14}$ In Abhängigkeit vom verwendeten Schätzalgorithmus stehen in EQS verschiedene Kriterien für die Beurteilung der Parameterschätzungen und der Anpassungsgüte von der modelltheoretischen und der vorliegenden empirischen Kovarianzmatrix zur Verfügung, auf die im Kontext der spezifizierten Kausalmodelle zur Analyse der Kundenzufriedenheit mit Verkehrsdienstleistungen näher eingegangen wird. ${ }^{15}$

Unter Berücksichtigung des jeweiligen Skalenniveaus wurde darüber hinaus das Softwarepaket SPSS zur Durchführung von Mittelwertvergleichstests sowie weiterer multivariater Verfahren (z.B. Korrelationsanalyse) im Rahmen der statistischen Datenauswertung eingesetzt. ${ }^{16}$

\section{Phasenbezogene Analyse der Zufriedenheit mit Verkehrsdienst- leistungen}

Die folgenden Abschnitte dienen der Zielsetzung, ein operationales Instrument zur Messung der Kundenzufriedenheit mit Verkehrsdienstleistungen empirisch zu überprüfen. Den Ausgangspunkt bilden die auf der Basis des identifizierten Kundenpfades erhobenen Merkmale einer Bahnreise. Dazu erfolgt zunächst die Überprüfung zentraler Operationalisierungskriterien. ${ }^{17}$

Aufl., Berlin u.a. 1996, S. 322 ff.; Fritz, W., Marktorientierte Unternehmensführung und Unternehmenserfolg: Grundlagen und Ergebnisse einer empirischen Untersuchung, Stuttgart 1992, S. $115 \mathrm{ff}$.

Vgl. Homburg, Ch., Baumgartner, H., Beurteilung von Kausalmodellen: Bestandsaufnahme und Anwendungsempfehlungen, in: Marketing ZFP, Jg.17, H. 3, 1995, S. 162 ff.

14 AGLS steht für Arbitrary Generalized Least Squares. Zu den Anwendungsvoraussetzungen verschiedener Schatzverfahren vgl. Homburg, Ch., Sütterlin, St., Kausalmodelle in der Marktforschung: EQS als Alternative zu LISREL?, a.a.O., S. 186 f.; Balderjahn, I., Das umweltbewußte Konsumentenverhalten - Eine empirische Studie, a.a.O., S. 94 ff.; Backhaus, K. et al., Multivariate Analysemethoden - Eine anwendungsorientierte Einführung, a.a.O., S. 423.

$15 \mathrm{Zu}$ einer allgemeinen Darstellung derartiger Kriterien vgl. Homburg, Ch., Giering, A., Konzeptualisierung und Operationalisierung komplexer Konstrukte, a.a.O., S. 8 ff.; Hildebrandt, L., Kausalanalytische Validierung in der Marketingforschung, a.a.O., S. $41 \mathrm{ff}$.

Zum Einsatz kam dabei die Programmversion SPSS (Superior Performing Software Systems) für Windows Version 6.13.

17 Vgl. hierzu die Ausführungen in Kap. B 1 dieser Arbeit. Im Zentrum der Analyse steht die Überprüfung der Operationalisierungsanforderungen auf der empirischen Sprachebene, da

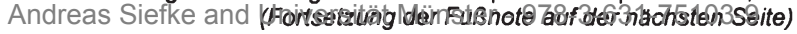




\section{1 Überprüfung zentraler Operationalisierungsanforderungen}

Die Anforderung der Zweckmäßigkeit des Zufriedenheitskonstruktes als Ausgangspunkt der Untersuchung läßt sich aus der aktuellen Literatur und der Vielzahl empirischer Studien ableiten. Unter Berücksichtigung der diskutierten konzeptionellen Differenzierungspotentiale und der in dieser Arbeit eingenommen transaktionsbezogenen Perspektive ist auch das Kriterium der Eindeutigkeit für den Zufriedenheitsbegriff als erfült anzusehen. Der multiattributive Charakter des Zufriedenheitskonstruktes macht die Bestimmung relevanter Indikatoren erforderlich. Angesichts der mehrfachen Evaluation des Fragebogens hinsichtlich der inhaltlichen und semantischen Gestaltung kann davon ausgegangen werden, daß die vorliegende Befragung auch der Anforderung der Adäquanz gerecht wird.

Die Objektivität der Konstrukterfassung ist aufgrund der Erhebung des Datenmaterials in Form einer standardisierten Befragung gewährleistet. ${ }^{18}$ Im Hinblick auf die Validität des gewählten Meßverfahrens gilt es zu prüfen, inwieweit die Meßergebnisse den zu analysierenden Sachverhalt unter Berücksichtigung inhaltlicher und konstruktspezifischer Aspekte tatsächlich wiedergeben. Ebenso ist sicherzustellen, daß sich die Messung der Kundenzufriedenheit mit Verkehrsdienstleistungen als reliabel bzw. zuverlässig und damit als reproduzierbar erweist.

Die Zuverlässigkeitsprüfung der verwendeten Skala erfolgte anhand des Reliabilitätskoeffizienten Cronbach's Alpha sowie der Split-Half-Methode. ${ }^{19}$ Die Überprüfung ergab für die zugrunde gelegten zufriedenheitsorientierten Itembatterien Werte des Reliabilitätskoeffizienten zwischen 0,7876 und 0,9064 für die verschiedenen Episoden einer Bahnreise. Bei der Split-Half-Methode lagen die entsprechende Werte des Spearman-Brown-Koeffizienten zwischen 0,6145 und $0,8785 .^{20}$ Es kann daher von einer hinreichenden Reliabilität der Skala ausgegangen werden. Da eine gegebene Reliabilität lediglich eine notwendige, aber

eine ausführliche Untersuchung der Anforderungen auf der theoretischen Sprachebene insoweit vernachlässigt werden kann, als daß der zugrunde gelegte modelltheoretische Ansatz auf dem in der Wissenschaft akzeptierten Modell des Disconfirmation-Paradigmia aufbaut. Vgl. hierzu die Ausführungen in Kap. B 2.2 dieser Arbeit.

Vgl. Berekoven, L., Eckert, W., Ellenrieder, P., Marktforschung: methodische Grundlagen und praktische Anwendung, a.a.O., S. 85.

Vgl. Hildebrandt, L., Kausalanalytische Validierung in der Marketingforschung, a.a.O., S. $41 \mathrm{f}$. Zur Berechnung der Zuverlässigkeitskoeffizienten vgl. Brosius, G., SPSS/PC+ Advanced Statistics and Tables, Hamburg, New York 1989, S. $261 \mathrm{ff}$.

20

Die genannten Koeffizienten können wie der ebenfalls herangezogene Guttmann-Koeffizient, dessen Werte in einem Bereich von 0, 5978 und 0,8776 lagen, Ausprägungen zwischen 0 und 1 annehmen. Bei einem Maß von 1 liegt kein Meßfehler vor.

Andreas Siefke and Universität Münster - 978-3-631-75103-9 
nicht hinreichende Bedingung für die Validität eines Meßverfahrens darstellt, verbleibt die Notwendigkeit einer Überprüfung der inhaltlichen Genauigkeit der Merkmalserfassung.

In Anlehnung an HILDEBRANDT kann zwischen drei Aspekten der Validität von Konstrukten unterschieden werden. ${ }^{21}$ Die Inhaltsvalidität bezeichnet den Grad, zu dem Variablen eines Meßmodells inhaltlich und semantisch das untersuchte Konstrukt abbilden. Zu ihrer Überprüfung werden häufig Expertenurteile herangezogen (Face-Validität), ${ }^{22}$ um durch geeignete Itembatterien alle Facetten des interessierenden Konstruktes zu erfassen. Wie den vorherigen Ausführungen zu entnehmen ist, wurde dieser Forderung in der vorliegenden Untersuchung Rechnung getragen. ${ }^{23}$ Es ist daher von einer ausreichenden inhaltlichen Validität des vorliegenden Meßinstrumentariums auszugehen.

Die Überprüfung der Kriteriumsvalidität eines Meßverfahrens erfolgt in der Regel derart, daß ein Prüfkriterium herangezogen wird, das mit dem zu messenden Konstrukt in einer theoretisch engen Beziehung steht und mit dem Meßinstrument des Konstruktes in hohem Maße korreliert. Häufig werden hierzu direkt erfaßte Globalurteile als Außenkriterium genutzt. ${ }^{24}$ Zur Überprüfung der Kriteriumsvalidität der Meßinstrumente für die vier Episoden einer Bahnreise wurden die Zufriedenheitsindikatoren mit den zusätzlich erhobenen Episodenzufriedenheiten korreliert. Die ermittelten Korrelationskoeffizienten lassen auf eine befriedigende Kriteriumsvalidität der verwendeten Meßinstrumente schließen. ${ }^{25}$

Vgl. Hildebrandt, L., Kausalanalytische Validierung in der Marketingforschung, a.a.O., S. $42 \mathrm{ff}$.

Vgl. ebenda.

Vgl. hierzu die Ausführungen in Kap. C 1.1 dieser Arbeit.

Vgl. z.B. Dichtl, E., Peter, S., Kundenzufriedenheit und Kundenbindung in der Automobilindustrie: Ergebnisse einer empirischen Untersuchung, a.a.O., S. 19 f.; Büker, B., Qualitätsbeurteilung investiver Dienstleistungen: Operationalisierungsansätze an einem Beispiel zentraler EDV-Dienste, a.a.O., S. 113. Diese Vorgehensweise unterstellt implizit eine hinreichende Validität globaler Zufriedenheitsurteile. Deren Validităt kann wiederum z.B. anhand des Außenkriteriums „Wiedernutzungsabsicht" überprüft werden. Bei dieser Form der Untersuchung tritt jedoch das Problem des „unendlichen Regresses" auf, dem üblicherweise dadurch begegnet wird, das Außenkriterium als valide anzusehen. Vgl. Nieschlag, R., Dichtl, E., Horschgen, H., Marketing, 17. Aufl., Berlin 1994, S. 723.

Die niedrigste ermittelte Korrelation betrug 0,38. Zur Überprüfung der Kriteriumsvalidität der Episodenzufriedenheiten wurden diese mit der erhobenen Transaktionszufriedenheit und diese schließlich mit der Wiedernutzungsabsicht korreliert. Auch hier ergaben sich zufriedenstellende Werte. 
Die Konstruktvalidität schließlich umfaßt die Eignung eines Meßansatzes, ein nicht beobachtbares theoretisches Konstrukt wie die Kundenzufriedenheit mit Verkehrsdienstleistungen weitestgehend zu erfassen und Erklärungsansätze liefern zu können. Die Konstruktvalidität wird als das anspruchsvollste und gleichzeitig genaueste Kriterium der Validität angesehen. ${ }^{26}$ In der Literatur wird eine Vielzahl an Verfahren beschrieben, die der Ermittlung von Orientierungswerten zur Beurteilung der Validität eines Konstruktes dienen. ${ }^{27}$ Im Rahmen der vorliegenden Untersuchung wird die Konstruktvalidität auf der einen Seite anhand der Gütemaße der untersuchten Kausalmodelle überprüft. Die Konstruktvalidität geht jedoch über die reine Gültigkeit durchgeführter Tests hinaus. Vielmehr dient der Nachweis der Konstruktvalidität auch dem Versuch, eine dahinterstehende Theorie zu validieren. Daher werden auf der anderen Seite die aufgestellten theoriegestützten Hypothesen einem Falsifikationsversuch unterzogen. Können die postulierten Beziehungszusammenhänge in den folgenden Kapiteln bestätigt werden, ist auch die nomologische Validität, die den wichtigsten Teilaspekt der Konstruktvalidität verkörpert, als erfüllt anzusehen. ${ }^{28}$

\subsection{Intraphasenspezifische Analyse der Kundenzufriedenheit}

Gemäß der erarbeiteten Kundenprozeßhierarchie erfolgt in den nächsten Abschnitten zunächst eine Analyse der Kundenzufriedenheit mit Verkehrsdienstleistungen auf der Kontaktpunkt- bzw. Episodenebene. Der Überprüfung der Struktur und Dynamik der Episodenzufriedenheiten schließt sich dann die phasenübergreifende Untersuchung der Kundenzufriedenheit an.

\subsection{Analyse der Vor-Reisezufriedenheit}

Für die Messung der Zufriedenheit mit den fünf Kontaktpunkten der Vor-Reisephase wurde das Urteil über siebzehn Meßindikatoren erhoben. Wie das Mittelwertprofil aller Befragten in Abbildung 23 zeigt, sind hier deutliche Unterschiede in den Merkmalsausprägungen festzustellen. So liegt im Rahmen der Informationsphase die Zufriedenheit mit der telefonischen Erreichbarkeit des Reise-

Vgl. Peter, P.J., Construct Validity: A Review of Basic Issues and Marketing Practices, in: JoMR, Vol. 18, May 1981, S. 133.

27 Vgl. z.B. Hilke, R., Grundlagen normorientierter und kriteriumorientierter Tests, Bern 1980, S. $47 \mathrm{f}$.

Zum Begriff der nomologischen Validităt vgl. Homburg, Ch., Giering, A., Konzeptualisierung und Operationalisierung komplexer Konstrukte, a.a.O., S. 7 f. und die dort angegebene Literatur. 


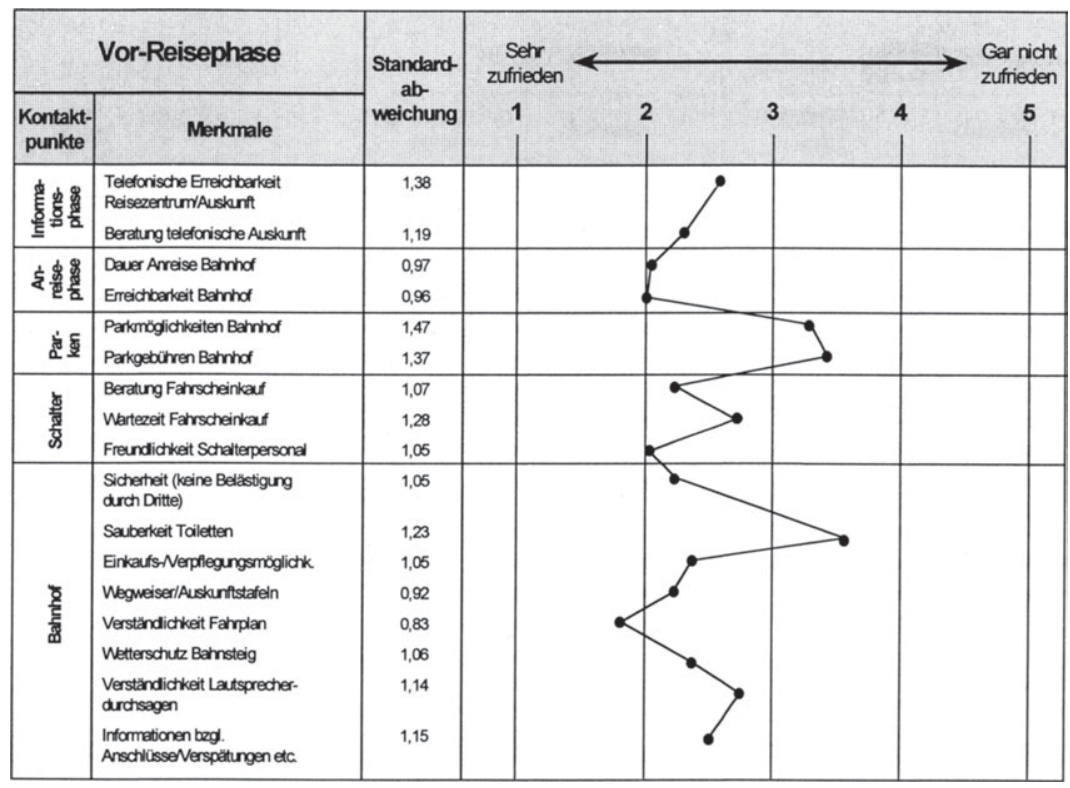

\section{Abb. 23: Zufriedenheitsprofil der Vor-Reisephase}

zentrums auf einem nur mittleren Niveau. Auch die Zufriedenheit mit der hier erfahrenen Beratung ist nur leicht besser ausgeprägt. ${ }^{29}$ Als durchaus zufrieden enweisen sich die Reisenden mit den Merkmalen der Anreisephase. ${ }^{30}$ Dagegen zeigen sich die Befragten bezüglich des Kontaktpunktes "Parken" als relativ unzufrieden. ${ }^{31}$ Die Analyse der Zufriedenheit mit dem Kontaktpunkt „Schalter" ergibt wiederum ein differenziertes Bild. Während die Freundlichkeit des Schalterpersonals und die hiermit verbundene Beratung der Kunden vergleichsweise positiv beurteilt werden, fält die Zufriedenheit mit der Wartezeit des Fahrscheinerwerbs deutlich niedriger aus. Auch die Zufriedenheit mit dem Bahnhof ist durch einen heterogenen Profilverlauf gekennzeichnet. Besonders

Hier ergibt sich bereits auf der Merkmalsebene ein erster Hinweis auf die Dynamik der Kundenzufriedenheit. So bestätigt die hohe Korrelation $(r=0,618)$ der beiden Merkmale den deutlichen Zusammenhang aufeinanderfolgender Teilaspekte einer Bahnreise.

Bei diesen Items gaben $73 \%$ bzw. $76 \%$ der Befragten an, daß sie mit diesen Merkmalen ihrer Bahnreise zufrieden bzw. sehr zufrieden waren. Auch die vergleichsweise niedrigen Standardabweichungen zeigen eine hohe Homogenităt im Antwortverhalten.

31 Die hohen Standardabweichungen dieser Merkmale sind ein Indiz für die starke Streuung der Einzelurteile. Es ist daher in den folgenden Abschnitten zu prufen, inwieweit die (Un-)Zufriedenheit mit diesem Kontaktpunkt einen signifikanten Einfluß auf die Zufriedenheit mit der VorReisephase ausübt. 
auffällig ist die hohe Zufriedenheit mit der Verständlichkeit des Fahrplans. Demgegenüber können die Verständlichkeit der Lautsprecherdurchsagen und insbesondere die Bahnhofstoiletten als Quellen der Unzufriedenheit mit dem Bahnhof identifiziert werden.

Die Unterschiede in den Standardabweichungen der Merkmale der Vor-Reisephase lassen auf unterschiedlich stark ausgeprägte Variationsmuster der Einzelzufriedenheiten schließen. Inwieweit sich dieser Umstand auch in dem Erleben kritischer Erlebnisse widerspiegelt, soll im folgenden nachgegangen werden.

Neben der merkmalsgestützten Abfrage der Kundenzufriedenheit enthielt der Fragebogen zwei offene Fragen, in denen die Reisenden gebeten wurden, besonders positive bzw. negative Ereignisse getrennt nach den unterschiedlichen Episoden ihrer Reise zu beschreiben. ${ }^{32}$ Im Rahmen der Vor-Reisephase wurden insgesamt 272 positive und 241 negative Erlebnisse genannt und geschildert (vgl. Abbildung 24). Dieses Ergebnis ist ein eindeutiger Beleg dafür, daß Reisende Ereignisse episodenbezogen speichern. ${ }^{33}$ Die Anzahl der erinnerten Vorfälle ist dabei insbesondere vor dem Hintergrund zu würdigen, daß es sich bei der VorReisephase nicht um die eigentliche Kerndienstleistung einer Bahnreise handelt.

Über $50 \%$ der positiven Erlebnisse entfallen auf den Kontaktpunkt „Schalter", die insbesondere die kompetente Beratung beim Fahrscheinerwerb betreffen (23,9\%). Hierbei ist jedoch zu beachten, daß dieser Kontaktpunkt durch einen hybriden Charakter geprägt ist. So werden auch $33,6 \%$ der erlebten negativen Ereignisse im Zusammenhang mit diesen Kontaktpunkt genannt. Umgekehrt stellt sich das Verhältnis von positiven zu negativen Nennungen für den Bahnhof dar. Mit 46,5\% werden hier die meisten negativen Erlebnisse geschildert. Insbesondere die Drängelei am Bahnsteig, die unzureichende Sauberkeit des Bahnhofs und das (negative) "Bahnhofsmilieu“ werden als Mängel betont. Dem stehen lediglich $17,6 \%$ der positiven Ereignisse gegenüber, von denen das zufriedenstellende Angebot an Einkaufsstätten den Großteil (7,7\%) ausmacht.

Die Zuordnung und Verdichtung der Ereignisse orientierten sich dabei an den Kontaktpunkten des Kundenpfades einer Bahnreise. Die Kataloge der gewonnenen Gruppierungen wurden zwei weiteren Kodierern vorgelegt und von diesen unabhängig voneinander einer Validitätsprüfung unterzogen. Vgl. hierzu Haller, S., Beurteilung von Dienstleistungsqualität: Dynamische Betrachtung des Qualitätsurteils im Weiterbildungsbereich, a.a.O., S. 170; Hentschel, B., Dienstleistungsqualität aus Kundensicht: Vom merkmals- zum ereignisorientierten Ansatz, a.a.O., S. 237 f.; Stauss, B., Hentschel, B., Verfahren der Problementdeckung und -analyse im Qualitätsmanagement von Dienstleistungsunternehmen, a.a.O., S. 242.

33 Vgl. Stauss, B., Weinlich, B., Die Sequentielle Ereignismethode - ein Instrument der prozeßorientierten Messung von Dienstleistungsqualität, a.a.O., S. 54.

Andreas Siefke and Universität Münster - 978-3-631-75103-9 


\begin{tabular}{|c|c|c|c|c|c|c|c|}
\hline \multicolumn{8}{|c|}{ Vor-Reisephase } \\
\hline \multicolumn{4}{|c|}{$\begin{array}{l}\text { Positive Ereignisse } \\
(n=272 \text { bzw. } 53,0 \%)\end{array}$} & \multicolumn{4}{|c|}{$\begin{array}{l}\text { Negative Ereignisse } \\
(n=241 \text { bzw. } 47,0 \%)\end{array}$} \\
\hline \multirow{2}{*}{$\begin{array}{l}\text { Kontakt- } \\
\text { punkte }\end{array}$} & \multicolumn{3}{|c|}{ Häufigkeiten } & \multirow{2}{*}{$\begin{array}{l}\text { Kontakt- } \\
\text { punkte }\end{array}$} & \multicolumn{3}{|c|}{ Häufigkeiten } \\
\hline & absolut & relativ & Summe & & absolut & relativ & Summe \\
\hline $\begin{array}{l}\text { Informationsphase: } \\
\text { - Beratung im Reisebüro } \\
\text { - Beratung durch telef. } \\
\text { Auskunft } \\
\text { - elektron. Kursbuch } \\
\text { - Gepäckservice }\end{array}$ & $\begin{array}{r}12 \\
8 \\
4 \\
5\end{array}$ & $\begin{array}{l}4,4 \% \\
2,9 \% \\
1,5 \% \\
1,8 \% \\
\end{array}$ & $\begin{array}{c}29 \\
\text { bzw. } \\
10,7 \%\end{array}$ & $\begin{array}{l}\text { Informationsphase: } \\
\text { - Beratung im Reisebüro } \\
\text { - telef. Auskunft besetzt }\end{array}$ & $\begin{array}{l}4 \\
3\end{array}$ & $\begin{array}{l}1,7 \% \\
1,2 \%\end{array}$ & $\begin{array}{c}7 \\
\text { bzw. } \\
2,9 \%\end{array}$ \\
\hline $\begin{array}{l}\text { Anreisephase: } \\
\text { - zentrale Lage Bahnhof } \\
\text { - guter OPNV-Anschluß }\end{array}$ & $\begin{array}{l}19 \\
10\end{array}$ & $\begin{array}{l}7,0 \% \\
3,6 \%\end{array}$ & $\begin{array}{c}2,9 \\
\text { bzw. } \\
10,7 \%\end{array}$ & $\begin{array}{l}\text { Anreisephase: } \\
\text { - ungünstige Lage Bhf. } \\
\text { - Schlechter OPNV- } \\
\text { Anschluß }\end{array}$ & $\begin{array}{r}14 \\
9\end{array}$ & $\begin{array}{l}5,8 \% \\
3,7 \%\end{array}$ & $\begin{array}{c}23 \\
\text { bzw. } \\
9,5 \%\end{array}$ \\
\hline $\begin{array}{l}\text { Parken: } \\
\text { - ausreichendes Angebot } \\
\text { (gut gelegen) }\end{array}$ & 18 & $6,6 \%$ & $\begin{array}{c}18 \\
\text { bzw. } \\
6.6 \%\end{array}$ & $\begin{array}{l}\text { Parken: } \\
\text { - kein ausreichendes } \\
\text { Angebot }\end{array}$ & 18 & $7,5 \%$ & $\begin{array}{c}18 \\
\text { bzw. } \\
7.5 \%\end{array}$ \\
\hline $\begin{array}{l}\text { Schalter: } \\
\text { - Freundlichkeit } \\
\text { - gute Verbindung } \\
\cdot \text { (Preis)Beratung } \\
\text { - Reservierung } \\
\text { - unkomplizierter Fahr- } \\
\text { kartenerwerb (z.B. } \\
\text { Express-Schalter) } \\
\text { - Reiseunterlagen (PC- } \\
\text { Ausdruck) } \\
\end{array}$ & $\begin{array}{l}20 \\
19 \\
65 \\
22 \\
18\end{array}$ & $\begin{array}{r}7,4 \% \\
7,0 \% \\
23,9 \% \\
8,1 \% \\
6,6 \% \\
\\
1,5 \%\end{array}$ & $\begin{array}{c}148 \\
\text { bzw. } \\
54,4 \%\end{array}$ & $\begin{array}{l}\text { Schalter: } \\
\text { - Unfreundlichkeit } \\
\text { - schlechte Verbindung } \\
\text { - (Preis)Beratung } \\
\text { - Reservierung nicht } \\
\text { möglich bzw. falsch } \\
\text { - „Drängelei“/Enge } \\
\text { - zu hohe Preise } \\
\text { - Wartezeit }\end{array}$ & $\begin{array}{r}7 \\
7 \\
15 \\
8 \\
\\
6 \\
9 \\
29\end{array}$ & $\begin{array}{r}2,9 \% \\
2,9 \% \\
6,2 \% \\
3,3 \% \\
\\
2,5 \% \\
3,7 \% \\
12,0 \%\end{array}$ & $\begin{array}{c}81 \\
\text { bzw. } \\
33,6 \%\end{array}$ \\
\hline $\begin{array}{l}\text { Bahnhof: } \\
\text { - Auswahl Einkaufs- } \\
\text { stätten } \\
\text { - Angebot Kofferkulis } \\
\text { - Angebot Gepăckband } \\
\text { - gute Fahrplan- } \\
\text { gestaltung } \\
\text { - Wagenstandsanzeiger } \\
\text { - Sauberkeit/Übersicht- } \\
\text { lichkeit }\end{array}$ & $\begin{array}{l}21 \\
4 \\
2 \\
7 \\
\\
5 \\
9\end{array}$ & $\begin{array}{l}7,7 \% \\
1,5 \% \\
0,7 \% \\
2,6 \% \\
1,8 \% \\
3,3 \%\end{array}$ & $\begin{array}{c}48 \\
\text { bzw. } \\
17,6 \%\end{array}$ & $\begin{array}{l}\text { Bahnhof: } \\
\text { - Gedränge auf } \\
\text { Bahnsteig } \\
\text { - schlechte } \\
\text { Verständlichkeit der } \\
\text { Durchsagen } \\
\text { - keine Kofferkulis } \\
\text { - kein bzw. defektes } \\
\text { Gepäckband } \\
\text { - mangelnde } \\
\text { Beschilderung } \\
\text { - „Milieu“/Atmosphäre } \\
\text { - mangelnde Sauberkeit }\end{array}$ & $\begin{array}{r}6 \\
14 \\
9 \\
24 \\
23\end{array}$ & $\begin{array}{r}10,4 \% \\
4,6 \% \\
\\
2,5 \% \\
5,8 \% \\
3,7 \% \\
\\
9,9 \% \\
9,5 \%\end{array}$ & $\begin{array}{c}112 \\
\text { bzw. } \\
46,5 \%\end{array}$ \\
\hline & & $100 \%$ & $100 \%$ & & & $100 \%$ & $100 \%$ \\
\hline
\end{tabular}

\section{Abb. 24: Kritische Ereignisse der Vor-Reisephase}


Auffällig ist die insgesamt deutlich geringe Anzahl an Nennungen für die Teilaspekte der Bahnreise, die dem ersten direkten Kontakt mit dem Dienstleister vorgelagert sind. Nahezu ausgeglichen verhält sich dabei die Anzahl positiver und negativer Erlebnisse mit den Kontaktpunkten "Anreisephase" (10,7\% bzw. 9,5\%) und „Parken“ (6,6\% bzw. 7,5\%), wobei insbesondere die Lage des Bahnhofs eine zentrale Ursache für (Un-)Zufriedenheit mit der Anreisephase darstellt. Die (Un-) Zufriedenheit mit der Informationsphase wird auf der einen Seite durch die Erreichbarkeit der telefonischen Auskunft bestimmt. Auf der anderen Seite sind auch Erfahrungen, die beim Fahrkartenerwerb in einem Reisebüro gemacht werden, zu beachten. ${ }^{34}$

Insgesamt ist festzustellen, daß die Frage nach kritischen Ereignissen die Ergebnisse der merkmalsgestützten Befragung zumindest in der Tendenz bestätigen. Als Ausnahme ist jedoch der Parkvorgang zu nennen. Unter Berücksichtigung der Ergebnisse der merkmalsgestützten Befragung wäre hier ein größerer Anteil an Nennungen zu erwarten. ${ }^{35}$

Die bisher vorgenommene Betrachtung diente in erster Linie der Identifikation potentieller Quellen der (Un-)Zufriedenheit mit den einzelnen Kontaktpunkten der Vor-Reisephase; die Bedeutung dieser Kontaktpunkte im Hinblick auf die Episodenzufriedenheit und ihr Zusammenspiel untereinander wurde bis hierher noch nicht untersucht. Zur Ermittlung dieser Beziehungszusammenhänge dient das in Abbildung 25 dargestellte Kausalmodell. ${ }^{36}$

35 Diese Erkenntnis steht im Einklang mit vergleichbaren Untersuchungen, bei denen sich ebenfalls ein differenziertes Bild hinsichtlich der Resultate der beiden Befragungsvarianten ergibt. Vgl. Stauss, B., Hentschel, B., Messung von Kundenzufriedenheit - Merkmals- oder ereignisorientierte Beurteilung von Dienstleistungsqualităt, in: M\&M, Jg.36, H. 3, 1992, S. $117 \mathrm{ff}$., Haller, S., Beurteilung von Dienstleistungsqualităt: Dynamische Betrachtung des Qualitătsurteils im Weiterbildungsbereich, a.a.O., S. 173. Ein möglicher Grund hierfür ist darin zusehen, daß die genannten Vorfälle außerhalb der Indifferenzzone der Befragten liegen. Die Frage nach kritischen Ereignissen komplettiert damit die Erkenntnisse einer merkmalsgestützten Befragung. Vgl. hierzu die Ausführungen in Kap. B 3.3 dieser Arbeit.

Zur Interpretation der Beziehungsstrukturen wird im Rahmen der vorliegenden Arbeit jeweils die standardisierte Lösung herangezogen, da diese die Vergleichbarkeit der einzelnen Parameterschătzungen gewährleistet. Vgl. hierzu u.a. Bentler, P.M., EQS Structural Equations Program Manual, a.a.O., S. 98. 


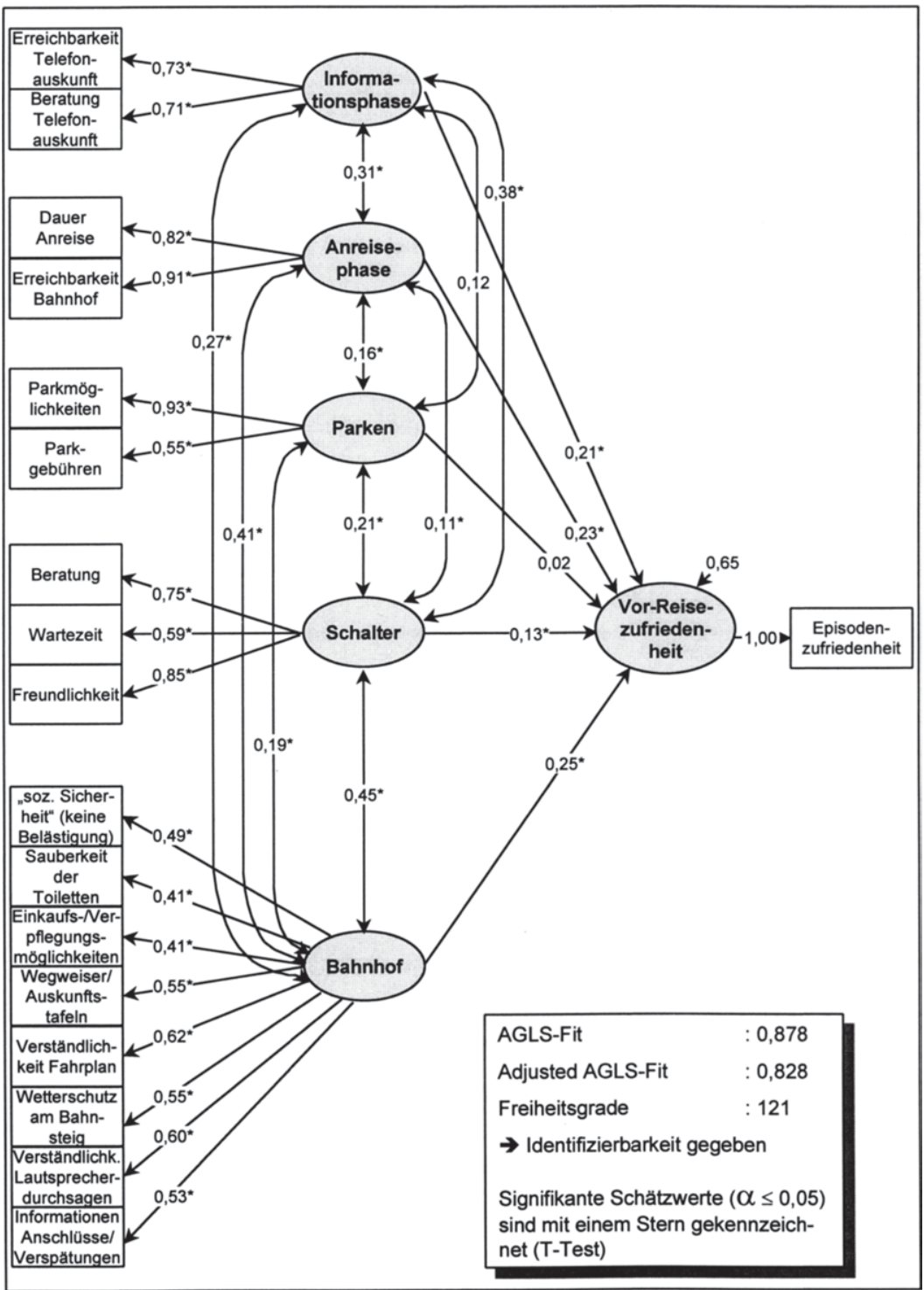

Abb. 25: Kausalmodell zur Struktur und Dynamik der Vor-Reisezufriedenheit 
Zunächst gilt es, die Identifizierbarkeit des spezifizierten Modells zu überprüfen. Eine notwendige, jedoch nicht hinreichende Bedingung identifizierbarer Modelle besteht in der Forderung nach einer positiven Anzahl an Freiheitsgraden. ${ }^{37}$ Für das Modell in Abbildung 25 ist diese Bedingung mit 121 Freiheitsgraden als erfüllt anzusehen. Ebenso ist festzuhalten, daß das Modell aufgrund der ermittelten Anpassungsmaße nicht abgelehnt und damit auf eine hinreichende Konstruktvalidität geschlossen werden kann. ${ }^{38}$ Demnach wird zumindest vorläufig von der Existenz der in Hypothese Hyp Episod 1 angenommenen Struktur der Vor-Reisezufriedenheit ausgegangen.

Die Schätzwerte in den Teilstrukturen des Modells können als zufriedenstellend beurteilt werden. So weisen die Meßindikatoren durchgehend reliable Werte (Faktorladungen > 0,4) auf und stellen damit akzeptable Operationalisierungen der Kontaktpunktzufriedenheiten dar. ${ }^{39}$ Die Höhe dieser Korrelationen gibt überdies einen Hinweis auf die Bedeutung einzelner Variablen bei der inhaltlichen Interpretation der Teilkonstrukte. Dabei zeigt sich beispielsweise, daß die Zufrie-

Vgl. hierzu und zur Berechnung von Freiheitsgraden: Backhaus, K. et al., Multivariate Analysemethoden - Eine anwendungsorientierte Einführung, a.a.O., S. $377 \mathrm{f}$.

38 Das hier verwendete Programmpaket EQS bietet eine Reihe unterschiedlicher globaler Anpassungsmaße. Im Rahmen der vorliegenden Untersuchung wurden die mit dem verwendeten Schätzalgorithmus korrespondierenden Fit-Maße AGLS-Fit und Adjusted AGLS-Fit, das zusätzlich die Freiheitsgrade des untersuchten Modells berücksichtigt, zur Beurteilung der globalen Anpassung des spezifizierten Modells an die erhobenen Daten herangezogen. Diese Maße sind inhaltlich mit den in LISREL-Anwendungen üblichenweise verwendeten Fit-Maßen GFI bzw. AGFI vergleichbar, die in EQS ebenfalls bereitgestellt werden. Vgl. Bentler, P.M., EQS Structural Equations Program Manual, a.a.O., S. 94.

Bezüglich solcher Gütekriterien ist jedoch festzustellen, daß bislang eindeutige kritische Werte zur Beurteilung eines Kausalmodells fehlen. Methodologisch gesehen bedeutet dies die Nichtverfügbarkeit eindeutiger Falsifikationskriterien für Kausalmodelle. In der Forschungspraxis werden daher üblicherweise Faustregeln oder Erfahrungswerte herangezogen. Als Konvention werden für diese Maße Mindestwerte von 0,9 sowie für das Fit-Maß RMR, das sowohl in LISREL als auch in EQS verfügbar ist, ein Höchstwert von 0,1 als akzeptabel angesehen. Vgl. z.B. Fritz, W., Marktorientierte Unternehmensführung und Unternehmenserfolg: Grundlagen und Ergebnisse einer empirischen Untersuchung, a.a.O., S. 126. Büschken stellt im Zusammenhang mit der Diskussion von Grenzwerten in Kovarianzstrukturanalysen richtiger Weise fest: „Jeder Grenzwert hat letztendlich willkürlichen Charakter und ist stets mit den Informationswünschen des Anwenders abzuwägen." Büschken, J., Multipersonale Kaufentscheidungen - Empirische Analyse zur Operationalisierung von Einflußbeziehungen im Buying Center, a.a.O., S. 181.

Dabei ist anzumerken, daß die für das vorliegende Modell ferner durchgeführte Untersuchung der Maße GFI $(0,941)$, AGFI $(0,917)$ und RMR $(0,072)$ ebenfalls den Schluß auf eine gute Modellanpassung erlaubt.

39 Zur Beurteilung werden üblicherweise die quadrierten Korrelationskoeffizienten für jede beobachtete Variable und die latenten Variablen berechnet. Diese können Werte zwischen 0 und 1 annehmen. Auch hier existieren keine verbindlichen Richtwerte. In der Literatur werden in Abhängigkeit vom Stichprobenumfang unterschiedlich hohe Anforderungen an die Indikatorreliabilitäten gestellt. Für die vorliegende Untersuchung mit einem Stichprobenumfang von 603 Fällen stellen Koeffizienten größer 0,2 bzw. Faktorladungen größer 0,4 als akzeptabel zu wertende Reliabilităten der Meßvariablen dar. Vgl. Balderjahn, I., Das umweltbewußte Konsumentenverhalten - Eine empirische Studie, a.a.O., S. 117.

Andreas Siefke and Universität Münster - 978-3-631-75103-9 
denheit mit dem Kontaktpunkt „Parken“ im besonderen Maße durch die Zufriedenheit mit dem Angebot an Parkmöglichkeiten repräsentiert wird. Im Rahmen des Schalterkontaktes erweist sich die Zufriedenheit mit der Freundlichkeit des Personals als zuverlässigster Indikator. Die Faktorladungen des Kontaktpunktes "Bahnhof" sind als vergleichsweise homogen zu bezeichnen, wobei die Verständlichkeit des Fahrplans sowie der Lautsprecherdurchsagen und damit personalunabhängige Informationsaspekte einen tendenziell höheren Beitrag zur Erklärung der Varianz der Zufriedenheit mit diesem Kontaktpunkt leisten.

Die zwischen den Kontaktpunkten bestehenden Interdependenzen werden durch Doppelpfeile repräsentiert. Wird der Frage nachgegangen, inwieweit sich die Kontaktpunktzufriedenheiten auf der einen Seite nachweisbar voneinander unterscheiden (Diskriminanzvalidität), auf der anderen Seite aber in einem erkennbaren Verhältnis zueinander stehen (Konvergenzvalidität), ${ }^{40}$ sind unter Berücksichtigung der Höhe der Korrelationen zwischen den Kontaktpunkten diese beiden Aspekte als grundsätzlich erfüllt anzusehen. ${ }^{41}$ So ergeben sich mit lediglich einer Ausnahme statistisch signifikante Werte, die durchgehend positiv ausgeprägt sind, in ihrer Größe jedoch durchaus unterschiedlich ausfallen. ${ }^{42}$

Die stärkste Interdependenz ist hier zwischen den Kontaktpunkten „Bahnhof“ und „Schalter“ zu beobachten $(r=0,45)$, die angesichts des „physischen Verbundes“ dieser Teilaspekte der Vor-Reisephase verständlich ist. Eine ähnliche Beziehung besteht zwischen der Anreisephase und dem Bahnhof $(r=0,41)$. Ein günstiger Verlauf der Anreise beeinflußt damit die Zufriedenheit mit dem Kontaktpunkt „Bahnhof". ${ }^{43}$ Ein vergleichbarer Einfluß geht von der Informationsphase auf den

Zu den Begriffen der Konvergenz- und Diskriminanzvalidităt vgl. Homburg, Ch., Giering, A., Konzeptualisierung und Operationalisierung komplexer Konstrukte, a.a.O., S. 7.

41 Bei praktischen Anwendungen der Kausalanalyse kann die Forderung nach Diskriminanzvalidität als erfüllt angesehen werden, wenn jeweils zwei latente Variablen zu einem Wert kleiner 1 miteinander korrelieren. Vgl. Bagozzi, R.P., Causal Modelling: General Method for Developing and Testing Theories in Consumer Research, in: Advances in Consumer Research, Monroe, K.B. (Hrsg.), Vol. 8, Ann Arbor 1981, S. 197; Hildebrandt, L., Kausalanalytische Validierung in der Marketingforschung, a.a.O., S. 47. Zu weiteren Kriterien vgl. Korte, Ch., Customer Satisfaction Measurement: Kundenzufriedenheitsmessung als Informationsgrundlage des Hersteller- und Handelsmarketing am Beispiel der Automobilwirtschaft, a.a.O., S. $184 \mathrm{f}$.

42 So ist kein signifikanter Zusammenhang zwischen den Kontaktpunkten "Informationsphase" und "Parken" festzustellen. Da mit Ausnahme der Kontaktpunkte "Schalter" und "Bahnhof" ein sequentieller Ablauf der einzelnen Teilabschnitte der Vor-Reisephase unterstellt werden kann, sollen die Korrelationen im folgenden für zeitlich aufeinander folgende Abschnitte hinsichtlich der Zufriedenheitsdynamik auch kausal interpretiert werden.

Interessanterweise ist die Beziehung zwischen der Anreisephase und dem Schalterkontakt deutlich niedriger ausgeprägt $(r=0,11)$. Der Einfluß der Anreisephase bezieht sich damit primär auf die Erreichbarkeit des Bahnhofs an sich.

Andreas Siefke and Universität Münster - 978-3-631-75103-9 
Schalterkontakt aus $(r=0,38)$. Eine als positiv wahrgenommene telefonische Beratung erweist sich damit als vorteilhaft für die Beurteilung des Fahrkartenerwerbs. Insgesamt ist damit die Hypothese Hyp D KP für die Vor-Reisephase als bestätigt anzusehen. ${ }^{44}$

Die Parameter auf den Verbindungslinien zwischen den Kontaktpunkten und der Vor-Reisezufriedenheit stellen die sog. Pfadkoeffizienten dar. Diese sind ein Maß für die Stärke des Einflusses des jeweiligen Kontaktpunktes auf die gesamte Zufriedenheit mit dieser Episode. ${ }^{45}$ Entgegen den konzeptionellen Vorüberlegungen wird die Vor-Reisezufriedenheit nicht eindeutig durch die personenbezogenen Kontaktpunkte "Informationsphase" und "Schalter" determiniert. So fällt der Einfluß des Schalterkontaktes relativ niedrig aus $(0,13)$. Dagegen üben der Bahnhof, die Anreise- und die Informationsphase (als personenbezogener Kontaktpunkt) einen vergleichsweise starken und in der Höhe ähnlichen Einfluß auf die Episodenzufriedenheit aus. In der Bedeutung des Bahnhofs für die Bildung des Zufriedenheitsurteils wird die Rolle des physischen Umfeldes und damit die "Visitenkartenfunktion" dieses Kontaktpunktes besonders deutlich. ${ }^{46}$ Trotz der bekundeten Unzufriedenheit mit dem Abschnitt „Parken“ hat dieser Kontaktpunkt interessanterweise keinen nachweisbaren Einfluß auf die Vor-Reisezufriedenheit. ${ }^{47}$ Hypothese Hyp Episod 1 über die Struktur der Vor-Reisezufriedenheit kann daher nur unter Berücksichtigung dieser Einschränkung endgültig angenommen werden, da mit Ausnahme des Kontaktpunktes „Parken" alle weiteren Abschnitte dieser Episode einer Bahnreise einen signifikanten Einfluß auf die Vor-Reise-

Gleichwohl ist im Hinblick auf die Konvergenzvalidität festzustellen, daß sich einige Korrelationen ungeachtet ihrer statistischen Signifikanz, auch im Vergleich zu den weiteren Modellen dieser Arbeit, auf einem relativ niedrigen Niveau bewegen. So stellt insbesondere der Kontaktpunkt "Parken" einen vergleichsweise eigenständigen Abschnitt der Vor-Reisephase dar. Vgl. hierzu auch die Ausführungen in Kap. D 1 dieser Arbeit.

45 Diese latente Variable ist aufgrund ihrer eindimensionalen Messung mit ihrem Meßindikator identisch. Eine Ladung von 1 und eine Residualgröße von 0 unterstellen, daß die latente Variable vollständig durch den einzelnen Meßindikator (und damit ohne Meßfehler) repräsentiert werden kann. Für die Analyse der weiteren Episoden einer Bahnreise wurde analog vorgegangen. Alternativ ist auch die Möglichkeit gegeben, Werte kleiner 1 vorzugeben und/oder Meßfehler zuzulassen. Vgl. Backhaus, K. et al., Multivariate Analysemethoden - Eine anwendungsorientierte Einführung, a.a.O., S. $373 \mathrm{ff}$. Der ebenfalls auf die Vor-Reisezufriedenheit gerichtete freistehende Pfeil kennzeichnet die durch das Modell nicht erklärte Varianz der VorReisezufriedenheit.

Vgl. Klein, H., Bahnhöfe - Visitenkarten der Bahn, in: DBB, Jg.65, H. 9, 1989, S. 707 ff.

47 In der Literatur werden Pfadkoeffizienten < 0,1 aufgrund ihrer geringen Größe lediglich für Tendenzaussagen herangezogen. Vgl. Monhemius, K.Ch., Umweltbewußtes Kaufverhalten von Konsumenten: ein Beitrag zur Operationalisierung, Erklärung und Typologie des Verhaltens in der Kaufsituation, Frankfurt am Main u.a. 1993, S. 186.

Das obige Ergebnis ist auch auf den Umstand zurückzuführen, daß nur ca. $37 \%$ der Befragten einen Pkw für die Anreise zum Startbahnhof genutzt haben und dieser Kontaktpunkt daher von nur gut einem Drittel der Befragten erlebt wurde. 
zufriedenheit ausüben. Insgesamt zeigt sich also, daß die Zufriedenheit mit der Vor-Reisephase auch durch den Bahnhof und den Verlauf der von den Kunden selbst erbrachten Teilleistungen geprägt wird. Unter Berücksichtigung dieser Ergebnisse ist daher die Hypothese Hyp I Episod 1 über die Dominanz personenbezogener Kontaktpunkte bei der Bildung der Episodenzufriedenheit der VorReisephase abzulehnen.

Im Zusammenhang mit der Beurteilung der Anpassungsgüte eines Modells bezüglich dieser Kausalbeziehungen zwischen den latenten unabhängigen Variablen (den Kontaktpunktzufriedenheiten) und der latenten abhängigen Variable (der Vor-Reisezufriedenheit) werden in der Literatur sehr unterschiedliche Mindestwerte gefordert. ${ }^{48}$ In dem vorliegenden Modell werden $35 \%$ der Varianz der Vor-Reisezufriedenheit durch die Kontaktpunktzufriedenheiten erklärt, so daß unter Berücksichtigung der bisherigen Ausführungen von einer hinreichenden nomologischen Validität des Modells ausgegangen werden kann. ${ }^{49}$

Im folgenden wird die Reisephase und damit die Kerndienstleistung des Leistungsangebotes „Bahnreise“ einer ausführlichen Betrachtung unterzogen. Da das Umsteigen in einen Anschlußzug für Reisende ohne Direktverbindung einen integralen Bestandteil der Reisephase darstellt, erfolgt daran anschließend eine detaillierte Analyse der Zufriedenheit mit der Umsteigephase.

So findet sich auch für diese Große kein verbindlicher Richtwert. Dieses Problem wird zusătzlich durch den Umstand verstärkt, daß der Aussagewert des häufig als Beurteilungsgröße herangezogenen sog. TCD-Wertes in neueren Publikationen stark kritisiert wird. Vgl. Homburg, Ch., Giering, A., Konzeptualisierung und Operationalisierung komplexer Konstrukte, a.a.O., S. 10 und 22; Homburg, Ch., Baumgartner, $\mathrm{H}$., Beurteilung von Kausalmodellen: Bestandsaufnahme und Anwendungsempfehlungen, a.a.O., S. $170 \mathrm{ff}$. und die dort jeweils angegebene Literatur.

Homburg und Baumgartner argumentieren in diesem Zusammenhang: „Es erscheint uns allerdings nicht immer sinnvoll, hier einen Mindestwert zu fordern. Eine solche Forderung wäre nur sinnvoll, wenn das substanzwissenschaftliche Erkenntnisziel der Untersuchung darin besteht, die jeweiligen endogenen latenten Variablen möglichst vollständig zu erklären. Dann könnte man beispielsweise eine quadrierte multiple Korrelation von mindestens 0,4 fordern. Geht es dem Anwender der Kausalanalyse aber lediglich um die Prüfung bestimmter vermuteter Beziehungen zwischen den latenten Variablen, so sollte er die quadrierten multiplen Korrelationen zwar zur Kenntnis nehmen, hier aber keine Mindestanforderungen vorgeben." Homburg, Ch., Baumgartner, H., Beurteilung von Kausalmodellen: Bestandsaufnahme und Anwendungsempfehlungen, a.a.O., S. 172. Mit einem Wert von 0,35 für den multiplen Korrelationskoeffizienten der Vor-Reisezufriedenheit wird auch die strengere Richtlinie von Homburg und Baumgartner (Mindestwert 0,4) im hiesigen Modell nahezu erfüllt. Vgl. hierzu auch die Ausführungen in Kap. D 1 dieser Arbeit. 


\subsection{Analyse der Reisezufriedenheit}

Zur Messung der Zufriedenheit mit den sechs Kontaktpunkten der Reisephase wurde das Urteil über achtzehn Meßindikatoren erhoben. Wie bereits in der VorReisephase können auch in der Reisephase deutliche Unterschiede in der Zufriedenheit mit den einzelnen Merkmalen bei einer Betrachtung über alle Befragten festgestellt werden (vgl. Abbildung 26).



\section{Abb. 26: Zufriedenheitsprofil der Reisephase}

Entgegen der häufig in Verbindung mit einer Bahnreise geäußerten Kritik an der unzureichenden Pünktlichkeit der Züge erweist sich die Zufriedenheit der Reisenden mit der Abfahrt und Ankunft als überraschend hoch. Einen ähnlich positiven Wert erzielt die "soziale" Sicherheit im Zug. Hiermit ist der Umstand angesprochen, nicht durch Dritte (z.B. betrunkene Fahrgäste oder Fußballfans) belästigt worden zu sein. ${ }^{50}$ Auch das Zugpersonal wird insgesamt sehr positiv beurteilt. Dagegen fällt die Zufriedenheit mit den Kontaktpunkten „Zugrestaurant“

50 Dieses Merkmal wurde dem Kontaktpunkt Personal zugeordnet, da sich im Rahmen der explorativen Vorstudien zeigte, daß Reisende in hohem Maße die Gewährleistung der „sozialen" Sicherheit dem Verantwortungsbereich des Zugbegleitpersonals zuweisen.

Andreas Siefke and Universität Münster - 978-3-631-75103-9 
bzw. "Toiletten" deutlich niedriger aus. Insbesondere in bezug auf das Preis-1 Leistungsverhältnis der angebotenen Speisen und Getränke bekunden die Befragten eine hohe Unzufriedenheit. Die Merkmalszufriedenheiten des Kontaktpunktes „Abteil" weisen einen relativ heterogenen Verlauf auf. Während sich die Reisenden mit der Sauberkeit des Abteils und der Verständlichkeit der Lautsprecherdurchsagen vergleichsweise zufrieden zeigen, können in diesem Kontaktpunkt insbesondere die Beinfreiheit am Sitzplatz sowie die Luft- bzw. Temperaturverhältnisse als Quellen der Unzufriedenheit ausgemacht werden. Aber auch die Möglichkeit, ungestört arbeiten, lesen bzw. schlafen zu können, ist aus Sicht der Befragten nur eingeschränkt gegeben. Zur Gewinnung zusätzlicher Informationen wurde auch für die Reisephase eine Auswertung der in diesem Zusammenhang von den Befragten genannten kritischen Ereignisse vorgenommen.

Die Auswertung ergab eine im Vergleich zur Vor-Reisephase deutlich höhere Anzahl an Nennungen. Dies kann bereits als ein erster Hinweise auf die hohe Bedeutung dieser Episode für die Zufriedenheit mit einer Bahnreise gewertet werden. So wurden von den Befragten 556 positive und 457 negative Erlebnisse geschildert, deren Zusammenfassung in Abbildung 27 wiedergegeben ist.

Auch hier können einige Ergebnisse der merkmalsgestützten Befragung in ihrer Tendenz bestätigt werden. So entfallen $12,9 \%$ bzw. $7,2 \%$ der positiven Nennungen auf die pünktliche Abfahrt bzw. Ankunft der Züge. Diesen stehen mit 7,0\% bzw. 5,9\% tendenziell geringe Anteile der diesbezüglichen negativen Äußerungen gegenüber. Der weitaus größte Anteil der positiven Erlebnisse entfällt auf den Kontaktpunkt "Abteil“ $(39,8 \%)$. Hier werden in erster Linie der Sitz- und Abteilkomfort $(9,2 \%)^{51}$ sowie die Möglichkeit, ungestört arbeiten oder schlafen zu können $(6,1 \%)$, hervorgehoben. Diesen als positiv empfundenen Erlebnissen steht mit $61,0 \%$ jedoch auch der mit Abstand größte Anteil der negativen Nennungen gegenüber. Hier fallen insbesondere der relativ hohe Anteil an Äußerungen über die Klimatisierung der Züge auf (12,0\%). Ebenso wird die Überfüllung der Züge bzw. das damit verbundene mangelnde (Sitz-)Platzangebot vergleichsweise häufig kritisiert (15,3\%). In diesem Zusammenhang erfährt auch der Sitz- bzw. Abteilkomfort Kritik (10,9\%), die sich insbesondere in einer mangelhaften Beinfreiheit niederschlägt. 


\begin{tabular}{|c|c|c|c|c|c|c|c|}
\hline \multicolumn{8}{|c|}{ Reisephase } \\
\hline \multicolumn{4}{|c|}{$\begin{array}{l}\text { Positive Ereignisse } \\
(\mathrm{n}=556 \text { bzw. } 54,9 \%)\end{array}$} & \multicolumn{4}{|c|}{$\begin{array}{l}\text { Negative Ereignisse } \\
(\mathrm{n}=457 \text { bzw. } 45,1 \%)\end{array}$} \\
\hline \multirow{2}{*}{$\begin{array}{l}\text { Kontakt- } \\
\text { punkte }\end{array}$} & \multicolumn{3}{|c|}{ Häufigkeiten } & \multirow{2}{*}{$\begin{array}{c}\text { Kontakt- } \\
\text { punkte }\end{array}$} & \multicolumn{3}{|c|}{ Häufigkeiten } \\
\hline & absolut & relativ & Summe & & absolut & relativ & Summe \\
\hline $\begin{array}{l}\text { Abfahrt: } \\
\text { - Pünktlichkeit }\end{array}$ & 72 & $12,9 \%$ & \begin{tabular}{|c|}
72 \\
bzw. \\
$12,9 \%$
\end{tabular} & $\begin{array}{l}\text { Abfahrt: } \\
\text { - Verspätungen }\end{array}$ & 32 & $7,0 \%$ & $\begin{array}{l}32 \\
\text { bzw. } \\
7.0 \%\end{array}$ \\
\hline $\begin{array}{l}\text { Abteil: } \\
\text { - Minibar } \\
\text { - Komfort des Abteils/ } \\
\text { des Sitzes } \\
\text { - Bequemlichkeit } \\
\text { - Durchsagen } \\
\text { - Sitzplatzangebot } \\
\text { - Klimatisierung } \\
\text { - Sauberkeit/neue Züge } \\
\text { - Ruhe/Arbeitsmöglichk. } \\
\text { - Leeres Abteil } \\
\text { - Bewegungsfreiheit (im } \\
\text { Zug) } \\
\text { - Gepäckverstauung } \\
\text { - Zeitung/Video } \\
\text { - nette Mitreisende } \\
\text { - Telefon/lnformations- } \\
\text { tafeln }\end{array}$ & $\begin{array}{r}7 \\
51 \\
28 \\
10 \\
5 \\
16 \\
25 \\
34 \\
20 \\
6\end{array}$ & $\begin{array}{l}1,3 \% \\
9,2 \% \\
5,0 \% \\
1,8 \% \\
0,9 \% \\
2,9 \% \\
4,5 \% \\
6,1 \% \\
3,6 \% \\
1,1 \% \\
\\
0,5 \% \\
1,1 \% \\
0,9 \% \\
0,9 \%\end{array}$ & $\begin{array}{c}221 \\
\text { bzw. } \\
39,8 \%\end{array}$ & \begin{tabular}{|l|} 
Abteil: \\
- volle/fehlende Müll- \\
eimer/Aschenbecher \\
- Komfort des Abteils/ \\
des Sitzes \\
- Durchsagen (Verständ- \\
lichkeit) \\
- Überfüllung \\
- Klimatisierung \\
- Belästigung durch \\
Zigarettenrauch \\
- Sauberkeitalte Züge \\
- Fahrtgeräusche \\
- fehlende Laptop- \\
Anschlüsse \\
- kein Platz für Kinderl \\
keine Wickelmöglichk. \\
- Sitzplatz-Nummern \\
unübersichtlich \\
- mangelnder Stauraum \\
für Gepäck
\end{tabular} & $\begin{array}{r}12 \\
50 \\
13 \\
\\
70 \\
55 \\
16 \\
21 \\
12 \\
3 \\
8 \\
4 \\
15\end{array}$ & $\begin{array}{r}2,6 \% \\
10,9 \% \\
2,8 \% \\
15,3 \% \\
12,0 \% \\
3,5 \% \\
4,6 \% \\
2,6 \% \\
0,7 \% \\
1,8 \% \\
0,9 \% \\
3,3 \%\end{array}$ & $\begin{array}{c}279 \\
\text { bzw. } \\
61,0 \%\end{array}$ \\
\hline $\begin{array}{l}\text { Personal: } \\
\text { - Am-Platz-Service } \\
\text { - Kompetenz } \\
\text { - Freundlichkeit }\end{array}$ & $\begin{array}{l}39 \\
29 \\
96\end{array}$ & $\begin{array}{r}7,0 \% \\
5,2 \% \\
17,3 \%\end{array}$ & $\begin{array}{c}164 \\
\text { bzw. } \\
29,4 \%\end{array}$ & $\begin{array}{l}\text { Personal: } \\
\text { - Am-Platz-Service } \\
\text { - Unfreundlichkeit } \\
\text { - Häufige Fahrschein- } \\
\text { kontrollen } \\
\end{array}$ & $\begin{array}{r}2 \\
15 \\
9\end{array}$ & $\begin{array}{l}0,4 \% \\
3,3 \% \\
2,0 \%\end{array}$ & $\begin{array}{c}26 \\
\text { bzw. } \\
5,7 \%\end{array}$ \\
\hline $\begin{array}{l}\text { Restaurant: } \\
\text { - Preis-/Leistungs- } \\
\text { Verhältnis } \\
\text { - Servicepersonal }\end{array}$ & $\begin{array}{r}22 \\
7\end{array}$ & $\begin{array}{l}4,0 \% \\
1,3 \%\end{array}$ & $\begin{array}{c}29 \\
\text { bzw. } \\
5,3 \%\end{array}$ & $\begin{array}{l}\text { Restaurant: } \\
\text { - Preis-/Leistungs- } \\
\text { Verhältnis } \\
\text { - Servicepersonal } \\
\text { - Atmosphäre } \\
\end{array}$ & $\begin{array}{l}43 \\
4 \\
6 \\
\end{array}$ & $\begin{array}{l}9,4 \% \\
0,9 \% \\
1,3 \% \\
\end{array}$ & $\begin{array}{c}53 \\
\text { bzw. } \\
11,6 \%\end{array}$ \\
\hline $\begin{array}{l}\text { Toiletten: } \\
\text { - Sauberkeit }\end{array}$ & 2 & $0,4 \%$ & $\begin{array}{c}2 \\
\text { bzw. } \\
0.4 \%\end{array}$ & \begin{tabular}{|l|} 
Toiletten: \\
- mangelnde Sauberkeit \\
\end{tabular} & 25 & $5,5 \%$ & $\begin{array}{c}25 \\
\text { bzw. } \\
5.5 \%\end{array}$ \\
\hline $\begin{array}{l}\text { Ankunft: } \\
\text { - Schnelligkeit } \\
\text { - Pünktlichkeit }\end{array}$ & $\begin{array}{l}28 \\
40\end{array}$ & $\begin{array}{l}5,0 \% \\
7,2 \%\end{array}$ & $\begin{array}{c}68 \\
\text { bzw. } \\
12,2 \%\end{array}$ & $\begin{array}{l}\text { Ankunft: } \\
\text { - zu lange Fahrtdauer } \\
\text { - Verspătung } \\
\text { - (unplanmäßige) } \\
\text { Zwischenstopps }\end{array}$ & $\begin{array}{r}8 \\
27 \\
7\end{array}$ & $\begin{array}{l}1,8 \% \\
5,9 \% \\
1,5 \%\end{array}$ & $\begin{array}{c}42 \\
\text { bzw. } \\
9,2 \%\end{array}$ \\
\hline & & $100 \%$ & $100 \%$ & & & $100 \%$ & $100 \%$ \\
\hline
\end{tabular}


Negative Erlebnisse mit dem Personal bilden eher die Ausnahme. So beruhen nur 5,7\% der negativen Ereignisse auf schlechten Erfahrungen mit dem Zugbegleitpersonal. Vergleichsweise häufig wird das Personal dagegen lobend erwähnt $(29,4 \%)$. So macht bereits allein der (ausschließlich) in der ersten Klasse angebotene "Am-Platz-Service" $7,0 \%$ der positiven Erlebnisse aus. ${ }^{52}$ Die Aussagen bezüglich des Zugrestaurants bestätigen die Ergebnisse der merkmalsgestützten Befragung. So überwiegen hier die negativen Nennungen insbesondere in bezug auf das Preis-/Leistungsverhältnis der angebotenen Speisen und Getränke eindeutig $(9,4 \%$ zu 4,0\%). Auch die Sauberkeit der Zugtoiletten ist für Bahnreisende eher ein Anlaß zum Ärgernis. So steht einem Anteil von $5,5 \%$ diesbezüglicher negativer Nennungen mit $0,4 \%$ ein ausgesprochen geringer Teil der positiven Äußerungen gegenüber.

Die kontaktpunktorientierte Auswertung der merkmals- und ereignisgestützten Befragung konnte unterschiedliche Quellen der (Un-)Zufriedenheit mit der Reisephase offenlegen. Im folgenden ist zu untersuchen, inwieweit sich die Zufriedenheiten mit den verschiedenen Abschnitten der Reisephase gegenseitig beeinflussen und welchen Beitrag sie zur Erklärung der Zufriedenheit mit der gesamten Episode leisten. Abbildung 28 zeigt das zur Analyse der Struktur und Dynamik der Reisezufriedenheit spezifizierte Kausalmodell.

Auch für dieses identifizierbare Modell ist festzuhalten, daß aufgrund der ermittelten globalen Gütekriterien eine insgesamt zufriedenstellende Konstruktvalidität angenommen werden kann. ${ }^{53}$ Die Meßindikatoren der Kontaktpunktzufriedenheiten weisen ohne Ausnahme reliable Werte aus, so daß auch für dieses Modell akzeptable Operationalisierungen der verschiedenen Kontaktpunktzufriedenheiten festzuhalten sind. Als ein besonders zuverlässiger Indikator der Zufriedenheit mit dem Abteil stellt sich der Sitzkomfort heraus $(r=0,83)$. Die Zufriedenheit mit dem Personal wird in besonderem Maße durch die hier erfahrene Freundlichkeit und Fachkompetenz repräsentiert. Während das Angebot an Speisen und Getränken für die Zufriedenheit mit dem Zugrestaurant eine hohe Bedeutung einnimmt, wird die Zufriedenheit mit dem Ende der Reisephase, der Ankunft am Zielbahnhof, in besonderer Weise durch das Urteil über die Pünktlichkeit der Züge beeinflußt. am Sitzplatz serviert zu bekommen. Vgl. Deutsche Bahn AG (Hrsg.), Service \& Züge, Broschüre, a.a.O., S. 31. 


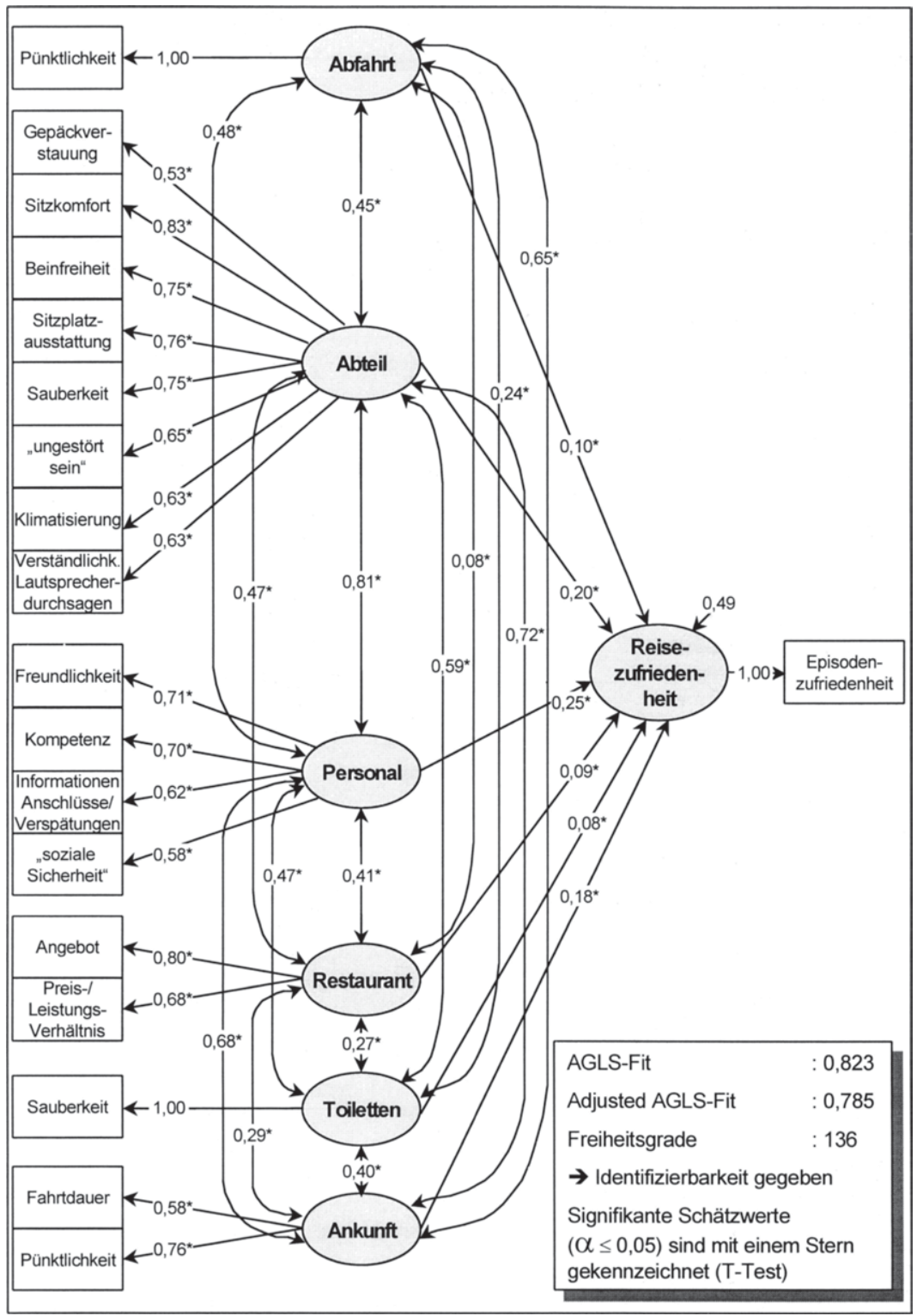

Abb. 28: Kausalmodell zur Struktur und Dynamik der Reisezufriedenheit 
Die Korrelationen zwischen den einzelnen Kontaktpunkten sind ohne Ausnahme positiv und durchgehend von statistischer Signifikanz. Im Vergleich zur Vor-Reisephase fallen diese Werte teilweise deutlich höher aus. ${ }^{54}$ So ist insbesondere zwischen den Kontaktpunkten „Abteil“ und „Personal“ ein sehr hoher Zusammenhang zu beobachten $(r=0,81)$. Diese zwar inhaltlich eindeutig zu differenzierenden Kontaktpunkte stellen damit auf der Wahrnehmungsseite eng verwandte Teilaspekte der Reisephase dar. Dies liegt zum einen darin begründet, daß der Kontakt mit dem Personal in erster Linie bei der Fahrkartenkontrolle am Sitzplatz des Reisenden und damit innerhalb des Kontaktpunktes „Abteil" stattfindet. Auf der anderen Seite zeichnet sich das Personal aber auch für den Inhalt und die Verständlichkeit der per Lautsprecher erteilten Informationen sowie für die Gewährleistung der bereits angesprochenen „sozialen“ Sicherheit der Reisenden während des Abteilaufenthaltes verantwortlich. Die enge Verbindung zwischen diesen Kontaktpunkten wird damit nachvollziehbar.

Der Beginn der Reisephase, die Abfahrt, hat einen erkennbaren Einfluß auf zeitlich darauf folgende Kontaktpunkte. Insbesondere die vergleichsweise hohe Beziehung zum Kontaktpunkt „Ankunft“ ist hier auffällig ( $r=0,65)$. Offensichtlich assoziiert ein Reisender mit einer (un-)pünktlichen Abfahrt auch eine (un-)pünktliche Ankunft des Zuges. Dies und die weiteren Korrelationen wie z.B. mit dem Abteil $(r=0,45)$ oder dem Personal $(r=0,48)$ unterstreichen die Bedeutung des Anfangs der Reisephase für die Zufriedenheit mit anderen Kontaktpunkten.

Die Untersuchung der Zusammenhänge zwischen den Kontaktpunkten zeigt darüber hinaus, daß die Zufriedenheit mit der Ankunft der Züge am Zielbahnhof durch weitere zeitlich vorgelagerte Kontaktpunkte beeinflußt wird. Bemerkenswert erscheint hier insbesondere die Stärke der Beziehung zu den Kontaktpunkten „Abteil" $(r=0,72)$ und „Personal“ $(r=0,68)$. Diese Zusammenhänge erklären sich aus der Beeinflußbarkeit der Zeitwahrnehmung von Dienstleistungskunden durch Maßnahmen des Verkehrsdienstleisters. ${ }^{55}$ Die ansprechende Gestaltung des Abteils sowie die Sicherstellung verständlicher und konkreter Informationen per Lautsprecher bzw. „vor Ort“ durch das Personal üben im Vergleich zu dem Unterlassen solcher Maßnahmen eine positive Wirkung auf die Einschätzung der Dauer

Da nicht davon ausgegangen werden kann, daß die Kontaktpunkte "Abteil", "Personal", "Restaurant" und "Toiletten" von den Reisenden in einer identischen Reihenfolge durchlaufen "wurden, werden die Korrelationen nur jeweils im Hinblick auf die Kontaktpunkte "Abfahrt" und "Ankunft" in kausaler Weise interpretiert; hier besteht ein eindeutig sequentieller Zusammenhang. 
der Transaktionszeit aus. So konnte empirisch nachgewiesen werden, daß die Befriedigung des Bedürfnisses an Informationen über Gründe und die Dauer von etwaigen Wartezeiten bzw. Verspätungen die Zufriedenheit von Verkehrsdienstleistungskunden meßbar steigert. ${ }^{56}$ Unterbleiben dagegen solche Informationen, wird das Gefühl der Unsicherheit und des "Zeitverlustes“ verstärkt und die subjektive Zeitwahrnehmung der Kunden negativ beeinflußt. Insbesondere in Fällen, in denen die Verantwortlichkeit für eine Verzögerung im Reiseverlauf eindeutig dem Verantwortungsbereich des Verkehrsdienstleisters zuzuordnen ist, kann durch eine proaktive Informationspolitik solchen kundenseitigen Irritationen erfolgreich begegnet werden.

Schließlich ist auf die Beziehung der Kontaktpunkte "Abteil“ und "Zugtoiletten" hinzuweisen $(r=0,59)$. Hiernach zeichnet sich die zufriedenstellende Wahrnehmung der Ausstattung des Abteils zu einem gewissen Grad für die Zufriedenheit mit den Zugtoiletten (und umgekehrt) verantwortlich. Ähnliches gilt in leicht abgeschwächter Form auch für den Zusammenhang zwischen den Kontaktpunkten „Abteil" und "Zugrestaurant" $(r=0,47)$. Interessanterweise fallen jedoch die weiteren Korrelationen des Kontaktpunktes „Zugrestaurant“ mit den übrigen Abschnitten der Reisephase vergleichsweise niedrig aus. Wie im folgenden noch näher zu erörtern ist, liegt die Vermutung nahe, daß dieser Kontaktpunkt vergleichsweise eigenständig und damit relativ unabhängig von den weiteren Kontaktpunkten wahrgenommen wird. Insgesamt bestätigen die im Modell zu beobachtenden Korrelationen die Annahme der Dynamik der Kundenzufriedenheit in der Reisephase, so daß vor dem Hintergrund der empirischen Befunde die Hypothese Hyp D KP auch für diese Episode einer Bahnreise nicht abgelehnt werden kann. Der insgesamt im Vergleich zur Vor-Reisephase größere Anteil an höheren Korrelationen ist auch auf den in dieser Phase stärker ausgeprägten physischen und zeitlichen Verbund der einzelnen Kontaktpunkte zurückzuführen.

Die empirische Analyse des Einflusses der einzelnen Kontaktpunkte auf die Zufriedenheit mit der gesamten Reisephase führt zu der Erkenntnis, daß entgegen Hypothese Hyp I Episod 2a die Zufriedenheit mit der Ankunft nicht den stärksten Einfluß auf die Episodenzufriedenheit ausübt $(0,18)$. Ein ebenfalls nur geringer Effekt geht von dem Kontaktpunkt „Abfahrt" aus $(0,10)$. Somit läßt sich die Information, and Initial Impressions in Consumer Satisfaction Assessments: A Process Oriented Approach, a.a.O., S. $63 \mathrm{ff}$; Haynes, P.J., Hating to Wait: Managing the Final Service Encounter, in: JoSM, Vol. 4, No. 4, 1990, S. $20 \mathrm{ff}$. 
Pünktlichkeit der Züge bei der Abfahrt und bei der Ankunft in Anlehnung an die Terminologie von HERZBERG in erster Linie als ein „Hygienefaktor" charakterisieren. ${ }^{57}$ Hiermit ist der Umstand angesprochen, daß bestimmte Merkmale einer Dienstleistung erst bei Nichterfüllung einer bestimmten Mindestnorm einen starken Effekt auf die (Un-)Zufriedenheit eines Dienstleistungskunden ausüben. Während die (Über-)Erfüllung dieser Norm einen vergleichsweise schwachen zufriedenheitsstiftenden Charakter innehat, ist bei Untererfüllung eine spürbare Verschlechterung der Kundenzufriedenheit die Folge. ${ }^{58}$ Angesichts des hohen Anteils mit der Pünktlichkeit der Züge (sehr) zufriedener Kunden in der vorliegenden Stichprobe kann von einer solchen Wirkungsweise der Pünktlichkeit auf die Zufriedenheit mit der Reisephase ausgegangen werden. ${ }^{59}$ Hyp I Episod 2a ist damit zu verwerfen.

Ein ebenfalls schwacher Einfluß auf die Episodenzufriedenheit ist dem Kontaktpunkt „Zugtoiletten" zu attestieren $(0,08)$. Diese können im doppelten Sinne als "Hygienefaktor" interpretiert werden. Während der einwandfreie Zustand der sanitären Anlagen als Selbstverständlichkeit vorausgesetzt wird und damit nicht zwingend zufriedenheitsfördernd wirkt, führt eine Nichterfüllung dieser Norm zu starker Unzufriedenheit. In Anbetracht der vergleichsweise wenigen diesbezüglich unzufriedenen Reisenden wird der schwache Einfluß dieses Kontaktpunktes auf die Zufriedenheit mit der Reisephase verständlich. ${ }^{60}$ Als "Motivator" erweist sich dagegen die Zufriedenheit der Befragten mit dem Zugbegleitpersonal, da diese den vergleichsweise höchsten Einfluß auf die Reisezufriedenheit ausübt $(0,25)$. Eine (Über-)Erfüllung der Erwartungen der Kunden an das Zugbegleitpersonal beeinflußt damit die Zufriedenheit mit der Reisephase relativ stark. ${ }^{61}$

57 Im Rahmen der von ihm aufgestellten Zwei-Faktoren-Theorie zur Arbeitszufriedenheit unterscheidet Herzberg zwischen sog. Hygienefaktoren und Motivatoren. Vgl. Herzberg, F., Work and Nature of Men, Cleveland, Ohio 1966; Herzberg, F., The Motivation-Hygiene Concept and Problems of Manpower, in: Personal Administration, Jg.27, 1964, S. 3 ff. sowie zu einer kritischen Würdigung dieser Theorie: Schütze, R., Kundenzufriedenheit: After-Sales-Marketing auf industriellen Markten, a.a.O., S. $141 \mathrm{ff}$.

$\mathrm{Zu}$ einer derartigen Interpretation von Hygienefaktoren im Dienstleistungsbereich vgl. Eckert, St., Rentabilitătssteigerung durch Kundenbindung, a.a.O., S. 99 ff.

Über $\mathbf{8 7 \%}$ der Befragten zeigten sich als (sehr) zufrieden mit der Pünktlichkeit ihres Zuges bei der Abfahrt am Startbahnhof. Ca. $80 \%$ der Reisenden gaben an, mit der Pünktlichkeit ihres Zuges bei der Ankunft am Zielbahnhof (sehr) zufrieden gewesen zu sein.

60 Von den Befragten, die die sanitären Anlagen der Züge aufgesucht haben, zeigten sich ca. $26 \%$ weniger bis gar nicht zufrieden mit dem Zustand der Zugtoiletten.

61

Insgesamt bekundeten ca. $80 \%$ der Reisenden eine (hohe) Zufriedenheit mit der Freundlichkeit des Personals sowie über $78 \%$ in bezug auf die fachliche Kompetenz der Zugbegleiter. $73 \%$ zeigten sich (sehr) zufrieden mit den wahrend der Fahrt erhaltenen Informationen und

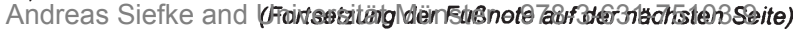


Einen Nachweis für ihre Gültigkeit erfährt die Hypothese Hyp I Episod 2b zum Einfluß des Zugrestaurants auf die Episodenzufriedenheit. Nach dem Ergebnis der empirischen Untersuchung ist dieser Einfluß als nur gering zu bezeichnen $(0,09)$. Da die Verpflegung nicht in dem Leistungspaket der Fahrgäste enthalten ist, kann es als bestätigt angesehen werden, daß Bahnreisende den Restaurantaufenthalt als eine eigenständige Dienstleistung und nicht als einen integrierten Bestandteil der Verkehrsdienstleistung „Bahnreise“ wahrnehmen. ${ }^{62}$ Der Aufenthalt im Zugabteil ist dagegen eine wichtige Komponente des vom Kunden erworbenen Leistungspaketes, deren Bedeutung sich in dem relativ hohen Pfadkoeffizienten widerspiegelt $(0,20)$.

Unter Berücksichtigung dieser Ergebnisse kann damit von der grundsätzlichen Gültigkeit der in Hypothese Hyp Episod 2 formulierten Struktur der Reisezufriedenheit ausgegangen werden. Insgesamt werden durch die erörterten kausalen Zusammenhänge ca. $51 \%$ der Varianz der Reisezufriedenheit erklärt. Angesichts der lediglich eindimensionalen Operationalisierung der Episodenzufriedenheit kann dem Modell damit eine ausreichende Stärke der Kausalbeziehungen zuerkannt werden, die im Vergleich zur Vor-Reisezufriedenheit deutlich höher ausfällt.

\subsection{Analyse der Umsteigezufriedenheit}

Die Betrachtung der im Zusammenhang mit der Messung der Kontaktpunktzufriedenheiten der Umsteigephase erhobenen fünf Dienstleistungsmerkmale führt zu der Erkenntnis, daß sich die Zufriedenheit mit dem eigentlichen Umsteigevorgang auf einem nur mittleren Niveau bewegt (vgl. Abbildung 29). ${ }^{63}$ Dagegen fällt die Zufriedenheit mit der pünktlichen Abfahrt der Anschlußzüge im Kontaktpunkt „Weiterfahrt" mit einem Mittelwert von 1,8 verhältnismäßig positiv aus. Als relativ hoch erweist sich hier die Standardabweichung des Merkmals "Anschluß an andere Züge" $(1,29)$, dessen Mittelwert den Ausprägungen der Indikatoren des Umsteigevorganges ungefähr entspricht. Die Streuung dieses

wiederum knapp 90\% der Befragten gaben an, mit der „sozialen“ Sicherheit im Zug (sehr) zufrieden gewesen zu sein. 


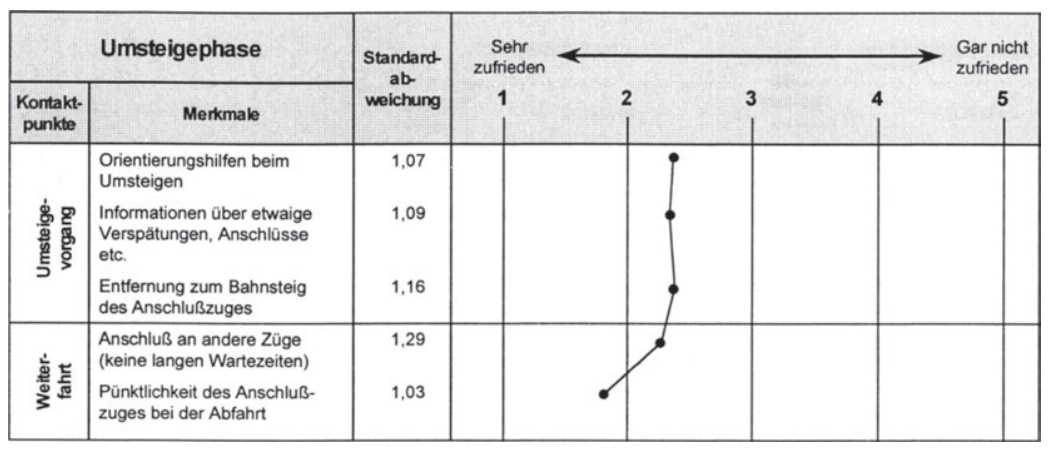

Abb. 29: Zufriedenheitsprofil der Umsteigephase

Merkmals kann als ein Indiz für ein vergleichsweise heterogenes Antwortverhalten der Befragten in bezug auf die Zufriedenheit mit diesem Aspekt des Kontaktpunktes „Weiterfahrt" gedeutet werden. Im folgenden wird daher der Frage nachgegangen, inwieweit sich diese Streuung auch in den bekundeten negativen und positiven Erlebnissen mit dieser Phase der Bahnreise widerspiegelt.

Abbildung 30 vermittelt einen Überblick über die kritischen Ereignisse der Umsteigephase. Dabei entfallen 142 Nennungen auf vorteilhafte Erlebnisse mit dieser Episode, während die entsprechende Anzahl an negativen Momenten 126 beträgt. ${ }^{64}$ Es zeigt sich, daß die (Un-)Zufriedenheit mit dem Anschluß an den nächsten Zug auf durchaus unterschiedlichen Ursachen beruhen kann. Zunächst soll mit dem Kontaktpunkt „Weiterfahrt" die zeitliche Komponente dieser Episode einer Bahnreise näher beleuchtet werden.

Hier wird die subjektive Zeitwahrnehmung von Kunden besonders deutlich. Während in $19 \%$ der negativen Äußerungen ein zu langer Aufenthalt am Umsteigebahnhof und der damit einhergehende Zeitverlust bemängelt werden, entfallen $33,1 \%$ der positiven Ereignisse auf eine diesbezüglich vorteilhafte Wahrnehmung der mit einem Zugwechsel verbundenen Wartezeit. Weitere 21,4\% der als negativ empfundenen Erlebnisse betreffen eine als zu kurz und damit hektisch empfundene Zeit, die den Reisenden auch bei planmäßiger Ankunft bzw. Abfahrt beider

64 Innerhalb eines absoluten Vergleichs der Anzahl an Nennungen von positiven und negativen Erlebnissen zu den verschiedenen Episoden einer Bahnreise gilt es zu berücksichtigen, daß ca. ein Drittel der Befragten nicht in einen Anschlußzug umgestiegen sind und daher keine diesbezüglichen Ereignisse geschildert haben.

Andreas Siefke and Universität Münster - 978-3-631-75103-9 


\begin{tabular}{|c|c|c|c|c|c|c|c|}
\hline \multicolumn{8}{|c|}{ Umsteigephase } \\
\hline \multicolumn{4}{|c|}{$\begin{array}{l}\text { Positive Ereignisse } \\
\text { ( } n=142 \text { bzw. } 53,0 \% \text { ) }\end{array}$} & \multicolumn{4}{|c|}{$\begin{array}{l}\text { Negative Ereignisse } \\
\text { (n=126 bzw. 47,0\%) }\end{array}$} \\
\hline \multirow{2}{*}{$\begin{array}{l}\text { Kontakt- } \\
\text { punkte }\end{array}$} & \multicolumn{3}{|c|}{ Häufigkeiten } & \multirow{2}{*}{$\begin{array}{l}\text { Kontakt- } \\
\text { punkte }\end{array}$} & \multicolumn{3}{|c|}{ Häufigkeiten } \\
\hline & absolut & relativ & Summe & & absolut & relativ & Summe \\
\hline $\begin{array}{l}\text { Umsteigevorgang: } \\
\text { - Wegweiser/ } \\
\text { Informationen } \\
\text { - Durchsagen gut } \\
\text { verständlich } \\
\text { - Anschlußzug auf } \\
\text { gegenüberliegendem } \\
\text { Gleis }\end{array}$ & $\begin{array}{l}9 \\
9\end{array}$ & $\begin{array}{r}6,3 \% \\
6,3 \% \\
28,2 \%\end{array}$ & $\begin{array}{c}58 \\
\text { bzw. } \\
40,8 \%\end{array}$ & $\begin{array}{l}\text { Umsteigevorgang: } \\
\text { - keine Wegweiser/ } \\
\text { Informationen } \\
\text { - unfreundliches } \\
\text { Personal auf } \\
\text { Bahnsteig } \\
\text { - keine Gepäckbänder/ } \\
\text { Kofferkulis } \\
\text { - weite Wege zum } \\
\text { Anschlußzug } \\
\text { - schlecht verständliche } \\
\text { Durchsagen }\end{array}$ & $\begin{array}{l}12 \\
17 \\
3\end{array}$ & $\begin{array}{r}12,7 \% \\
2,4 \% \\
9,5 \% \\
13,5 \% \\
2,4 \%\end{array}$ & $\begin{array}{c}51 \\
\text { bzw. } \\
40,5 \%\end{array}$ \\
\hline $\begin{array}{l}\text { Weiterfahrt } \\
\text { - Anschlußzug wartet } \\
\text { - ausreichende } \\
\text { Umsteigezeit } \\
\text { - guter Anschluß/ } \\
\text { wenig Wartezeit } \\
\text { - pünktliche Weiterfahrt }\end{array}$ & $\begin{array}{r}8 \\
10 \\
47\end{array}$ & $\begin{array}{r}5,6 \% \\
7,0 \% \\
33,1 \% \\
13,4 \%\end{array}$ & $\begin{array}{c}84 \\
\text { bzw. } \\
59,2 \%\end{array}$ & $\begin{array}{l}\text { Weiterfahrt: } \\
\text { - Anschluß wegen } \\
\text { Verspätung des } \\
\text { ersten Zuges verpaßt } \\
\text { - zu kurze Umsteigezeit } \\
\text { - schlechter Anschluß/ } \\
\text { zuviel Wartezeit } \\
\text { - Unpünktliche } \\
\text { Weiterfahrt }\end{array}$ & $\begin{array}{r}27 \\
24 \\
2\end{array}$ & $\begin{array}{r}17,5 \% \\
21,4 \% \\
19,0 \% \\
1,6 \%\end{array}$ & $\begin{array}{c}75 \\
\text { bzw. } \\
60,5 \%\end{array}$ \\
\hline & & $100 \%$ & $100 \%$ & & & $100 \%$ & $100 \%$ \\
\hline
\end{tabular}

\section{Abb. 30: Kritische Ereignisse der Umsteigephase}

Züge für den Zugwechsel zur Verfügung stand. Diesen stehen lediglich $7,0 \%$ der diesbezüglichen positiven Nennungen gegenüber. $17,5 \%$ der als nachteilig wahrgenommenen Erlebnisse beruhen auf dem Umstand, daß die Verspätung des ersten Zuges das Verpassen des Anschlußzuges zur Folge hatte. In diesem Zusammenhang wurde von den Befragten insbesondere kritisiert, daß die Folgezüge auch bei nur geringfügiger Verspätung nicht auf die ankommenden Züge gewartet haben. Dagegen wurde in 5,6\% der positiven Ereignisse das Warten der Anschlußzüge explizit gelobt. 
Besonders positiv blieb den Bahnreisenden im Kontaktpunkt "Umsteigevorgang" die Tatsache in Erinnerung, am gleichen Bahnsteig auf der gegenüberliegenden Seite in den Anschlußzug umsteigen zu können $(28,2 \%$ der positiven Nennungen). Dagegen entfallen $13,5 \%$ der als kritisch empfundenen Ereignisse auf den genau gegenteiligen Sachverhalt. Demzufolge wird hier auch das mangelnde Angebot an Gepäckbändern und Kofferkulis bzw. deren Funktionsuntüchtigkeit vergleichsweise häufig kritisiert ( $9,5 \%$ der negativen Nennungen). In diesem Zusammenhang sind ebenso die als unzureichend empfundene Versorgung mit Informationen durch das Bahnpersonal als auch das Fehlen von Orientierungshilfen in Form von Wegweisern zu nennen (12,7\%).

Über die Bedeutung der verschiedenen Kontaktpunkte für die Zufriedenheit mit dieser Episode einer Bahnreise gibt das folgende Kausalmodell Aufschluß. Es erweist sich als identifizierbar, und die in Abbildung 31 angegebenen globalen Gütekriterien bescheinigen dem spezifizierten Modell eine insgesamt gelungene Anpassung an die empirische Datenstruktur. ${ }^{65}$ Auch die Reliabilität der Meßindikatoren der beiden Kontaktpunkte ist durchgängig positiv zu beurteilen. Während die Orientierungshilfen am Umsteigebahnhof den zuverlässigsten Indikator der Zufriedenheit mit dem eigentlichen Umsteigevorgang darstellen, wird die Zufriedenheit mit der Weiterfahrt am stärksten durch das Merkmal „Anschluß an andere Züge" wiedergegeben.

Die vergleichsweise hohe Korrelation zwischen den beiden Kontaktpunkten $(r=0,65)$ ist ein sicherer Nachweis für den engen Wahrnehmungsverbund zwischen den Abschnitten des Umsteigevorganges. Die Erteilung eindeutiger sowie verständlicher Informationen in Verbindung mit einer ausreichenden Ausstattung an Orientierungshilfen und die Gewährleistung möglichst kurzer physischer Distanzen zum Anschlußzug beeinflussen die Zufriedenheit mit der eigentlichen Weiterfahrt des Anschlußzuges in eindeutiger Weise. Die Hypothese Hyp $D_{K P}$ über den positiven Einfluß der Zufriedenheit mit einem zeitlich vorgelagerten Kontaktpunkt auf die Zufriedenheit mit einem darauf folgenden Kontaktpunkt kann somit auf der Basis der empirischen Ergebnisse für die Umsteigephase nicht abgelehnt werden. 


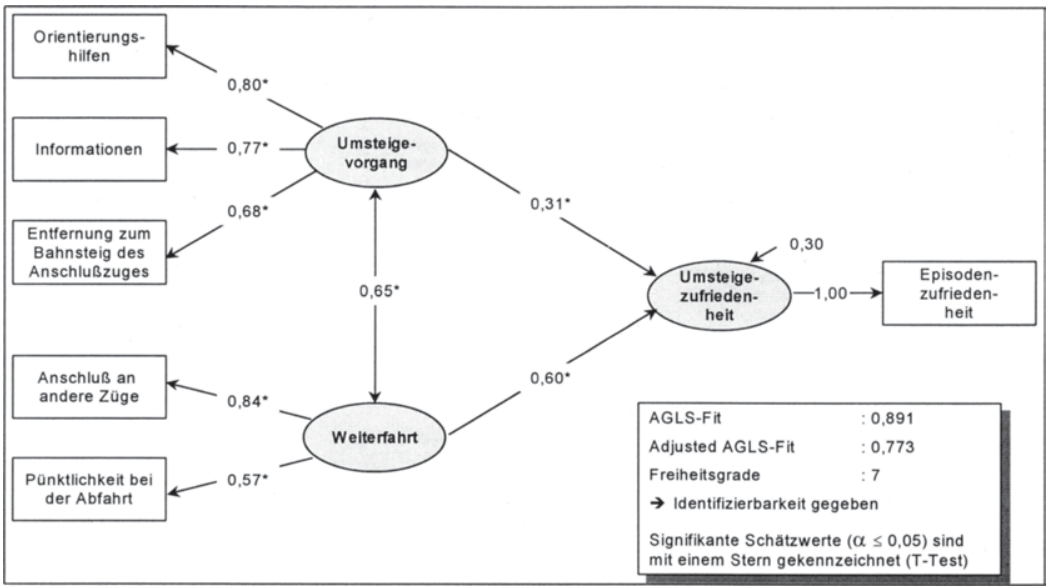

Abb. 31: Kausalmodell zur Struktur und Dynamik der Umsteigezufriedenheit

Die Überprüfung des Einflusses der beiden Kontaktpunktzufriedenheiten auf die Zufriedenheit mit der gesamten Umsteigephase zeigt, daß die zeitliche Komponente dieser Episode, die Weiterfahrt, mit einem Pfadkoeffizienten von 0,60 einen besonders starken Einfluß auf die Episodenzufriedenheit ausübt. ${ }^{66}$ Ebenso geht eine eindeutige Wirkung von der Zufriedenheit mit dem physischen Umsteigevorgang aus, der jedoch mit einem Koeffizienten von 0,31 vergleichsweise niedriger ausfällt, so daß auf der Basis dieser Ergebnisse Hypothese Hyp I Episod 3 als bestätigt anzusehen ist.

Unter Berücksichtigung der empirischen Ergebnisse kann die Hypothese Hyp Episod 3 über die Struktur der Umsteigezufriedenheit als bestätigt angesehen werden. Mit einem Anteil von $70 \%$ erklärter Varianz der Episodenzufriedenheit erzielt dieses Modell von den bisher betrachteten Kausalmodellen den höchsten Wert. Unter Berücksichtigung der bisherigen Ausführungen kann damit in Verbindung mit diesem Wert die Forderung nach nomologischer Validität als gut erfüllt angesehen werden.

66 Hildebrandt bezeichnet Pfadkoeffizienten mit Werten über 0,55 als "die Ursache". Vgl. Hildebrandt, L., Konfirmatorische Analysen von Modellen des Konsumentenverhaltens, a.a.O., S. 34 . 


\subsection{Analyse der Nach-Reisezufriedenheit}

Der Analyse der Zufriedenheit mit der letzten Episode einer Bahnreise, der NachReisephase, liegt die Messung der Zufriedenheit mit sieben Indikatoren zugrunde, deren Zuordnung zu den verschiedenen Kontaktpunkten der Nach-Reisephase Abbildung 32 zu entnehmen ist.

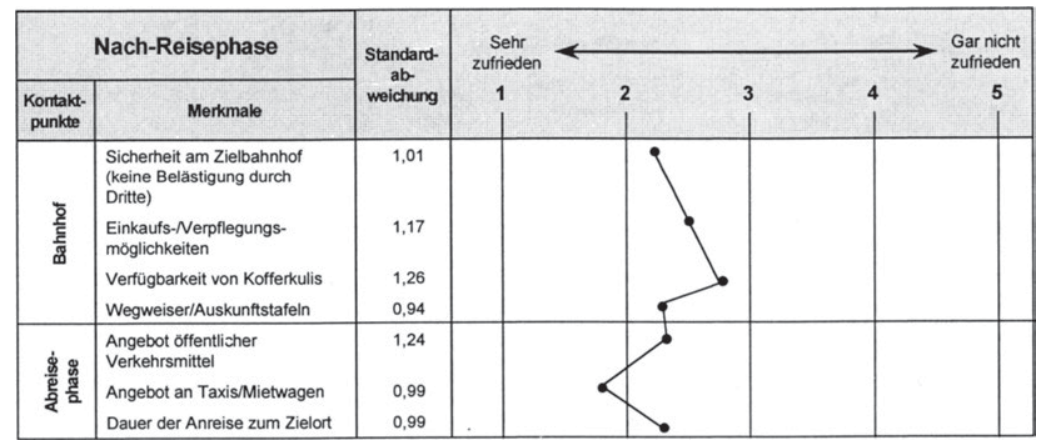

\section{Abb. 32: Zufriedenheitsprofil der Nach-Reisephase}

Hier zeigt sich, daß von den Indikatoren des Kontaktpunktes „Bahnhof" die Verfügbarkeit von Kofferkulis vergleichsweise negativ beurteilt wird. Auch die Zufriedenheit mit den Einkaufs- und Verpflegungsmöglichkeiten am Zielbahnhof bewegt sich auf einem nur mittleren Niveau. Bei einer Beurteilung dieser Merkmalsausprägungen gilt es jedoch zu berücksichtigen, daß sich die Bahnhöfe der Deutschen Bahn in ihrer Größe und Ausstattung nachhaltig unterscheiden. ${ }^{67}$ Innerhalb der Abreisephase wird das Angebot an Taxis/Mietwagen vergleichsweise positiv beurteilt. Dagegen zeigen sich die Befragten mit dem Angebot der öffentlichen Verkehrsmittel nur durchschnittlich zufrieden. Zudem ist für dieses Merkmal eine relativ hohe Standardabweichung zu beobachten, so daß hier ein gewisses (Un-)Zufriedenheitspotential der Nach-Reisezufriedenheit zu vermuten ist.

Die Bahn unterscheidet fünf Kategorien von Bahnhöfen. Diese reichen von „sehr großen Bahnhöfen" mit einer Nutzerfrequenz von über 25 Mio. Reisenden pro Jahr, wie z.B. dem Münchner Hauptbahnhof, bis hin zu "Kleinstbahnhöfen" mit einer Nutzerfrequenz von 0,5 Mio. Reisenden pro Jahr. Zu einer ausführlichen Darstellung vgl. Glöckner, G., Jäger, W., Bahnhof 2000: Erlebniswelt mit Gleisanschluß, in: DBB, Jg. 65, H. 9, 1989, S. 709 ff.; Bahn Extra (Hrsg.), Lexikon Deutsche Bahn, München 1996, S. 26 ff.

$\mathrm{Zu}$ einer differenzierten Analyse des "Dienstleistungscenters Bahnhof" vgl. Birkelbach, R., Qualitătsmanagement in Dienstleistungscentern: Konzeption und typenspezifische Ausgestaltung unter besonderer Berücksichtigung von Verkehrsflughäfen, a.a.O., S. 179 ff.

Andreas Siefke and Universität Munster - 978-3-631-75103-9 
Eine Auswertung der für diese Phase einer Bahnreise genannten kritischen Ereignisse für die Abreisephase bestätigt diese Vermutung. So werden $20,0 \%$ der als nachteilig empfundenen Erlebnisse in Zusammenhang mit dem Angebot des öffentlichen Nahverkehrs genannt. Ebenso entfallen aber auch knapp 33\% der positiven Erlebnisse auf diesen Aspekt der Nach-Reisephase (vgl. Abbildung 33). ${ }^{68}$ Interessanterweise betreffen $11,0 \%$ der positiven Nennungen den Umstand, daß die Reisenden von Angehörigen am Zielbahnhof (überwiegend mit dem Pkw) abgeholt wurden. Darin spiegelt sich u.a. der Wunsch nach einer möglichst problemlosen Anreise zum Zielort wider. ${ }^{69}$ Gerade solche Bahnreisende sind aber darauf angewiesen, daß die abholende Person einen Parkplatz am Zielbahnhof findet. Das mangelnde Parkplatzangebot und die hier anfallenden Parkgebühren werden jedoch in ca. $16 \%$ der negativen Erlebnisse in Verbindung mit dem Kontaktpunkt „Bahnhof" kritisiert. Mit jeweils 15,0\% der als nachteilig wahrgenommenen Ereignisse werden ebenso die Sauberkeit des Bahnhofs bzw. der Bahnhofstoiletten und die unzureichende „soziale" Sicherheit am Bahnhof bemängelt. Als weitere Kritikpunkte werden von den Befragten bei den negativen Ereignissen fehlende bzw. defekte Gepäckbänder $(9,2 \%)$ und eine als nicht ausreichend empfundene Ausstattung der Bahnhöfe mit Orientierungshilfen (10,8\%) vergleichsweise häufig genannt. Als vorteilhaft werden, wie bereits in der Vor-Reisephase, die Einkaufs- und Verpflegungsmöglichkeiten an den Bahnhöfen empfunden, auf die $11,0 \%$ der positiven Erlebnisse entfallen.

Insgesamt werden in Zusammenhang mit der Nach-Reisephase 73 positive und 120 negative Ereignisse von den Befragten geschildert. Im Vergleich zu den anderen Episoden einer Bahnreise sind diese Werte als niedrig zu bezeichnen. Inwieweit dies bereits als ein Indikator für die relativ geringe Bedeutung der NachReisephase für die Gesamtzufriedenheit gedeutet werden kann, soll in den nächsten Kapiteln untersucht werden. Bevor jedoch eine solche episodenübergreifende Perspektive eingenommen wird, werden zunächst die Beziehungszusammenhänge der Nach-Reisezufriedenheit näher betrachtet. zum Zielort ihrer Reise.

$\mathrm{Zu}$ einer differenzierten Analyse derartiger unentgeltlicher Serviceleistungen vgl. Häberli, V., Greuter, B., Serviceleistungen - ein zu Unrecht vernachlässigter Verkehrszweck, in: IVW, Jg.48, H. 10, 1996, S. $20 \mathrm{ff}$. 


\begin{tabular}{|c|c|c|c|c|c|c|c|}
\hline \multicolumn{8}{|c|}{ Nach-Reisephase } \\
\hline \multicolumn{4}{|c|}{$\begin{array}{l}\text { Positive Ereignisse } \\
\text { ( } n=73 \text { bzw. } 37,8 \% \text { ) }\end{array}$} & \multicolumn{4}{|c|}{$\begin{array}{l}\text { Negative Ereignisse } \\
\text { ( } n=120 \text { bzw. } 62,2 \% \text { ) }\end{array}$} \\
\hline \multirow{2}{*}{$\begin{array}{l}\text { Kontakt- } \\
\text { punkte }\end{array}$} & \multicolumn{3}{|c|}{ Häufigkeiten } & \multirow{2}{*}{$\begin{array}{c}\text { Kontakt- } \\
\text { punkte }\end{array}$} & \multicolumn{3}{|c|}{ Häufigkeiten } \\
\hline & absolut & relativ & Summe & & absolut & relativ & Summe \\
\hline $\begin{array}{l}\text { Bahnhof: } \\
\text { - zentrale Lage } \\
\text { - Gepäckbänder } \\
\text { - Kofferkulis } \\
\text { - Wegweiser/Informa- } \\
\text { tionstafeln } \\
\text { - Verpflegungs-/ Ein- } \\
\text { kaufsmőglichkeiten } \\
\text { - Parkplatzangebot }\end{array}$ & $\begin{array}{l}5 \\
3 \\
6 \\
6 \\
8 \\
5\end{array}$ & $\begin{array}{r}6,8 \% \\
4,1 \% \\
8,2 \% \\
8,2 \% \\
11,0 \% \\
6,8 \%\end{array}$ & $\begin{array}{c}33 \\
\text { bzw. } \\
45,2 \%\end{array}$ & $\begin{array}{l}\text { Bahnhof: } \\
\text { - ungünstige Lage } \\
\text { - fehlende/defekte } \\
\text { Gepäckbänder } \\
\text { - fehlende/defekte } \\
\text { Kofferkulis } \\
\text { - unzureichende } \\
\text { Orientierungshilfen } \\
\text { - schlechte Einkaufs- } \\
\text { möglichkeiten } \\
\text { - Sauberkeit (insb. } \\
\text { Toiletten) } \\
\text { - „soziale Sicherheit“ } \\
\text { am Bahnhof } \\
\text { - Lage und Gebühren } \\
\text { der Parkplătze } \\
\text { - zu wenig Telefone }\end{array}$ & $\begin{array}{r}2 \\
11 \\
8 \\
13 \\
13 \\
2 \\
18 \\
18 \\
19 \\
3\end{array}$ & $\begin{array}{r}1,7 \% \\
9,2 \% \\
6,7 \% \\
10,8 \% \\
1,7 \% \\
15,0 \% \\
15,0 \% \\
15,8 \% \\
2,5 \%\end{array}$ & $\begin{array}{c}94 \\
\text { bzw. } \\
78,3 \%\end{array}$ \\
\hline $\begin{array}{l}\text { Abreisephase: } \\
\text { - gute OPNV-An- } \\
\text { bindung/Nahverkehr } \\
\text { der Deutschen Bahn } \\
\text { - Erreichbarkeit der } \\
\text { Zielorte vom Bahnhof } \\
\text { - Angebot an Taxis } \\
\text { - Abholung durch } \\
\text { Angehörige }\end{array}$ & \begin{tabular}{l|l}
3 & \\
5 & \\
8 &
\end{tabular} & $\begin{array}{r}32,9 \% \\
4,1 \% \\
6,8 \% \\
11,0 \%\end{array}$ & $\begin{array}{c}40 \\
\text { bzw. } \\
54,8 \%\end{array}$ & $\begin{array}{l}\text { Abreisephase: } \\
\text { - schlechte OPNV-An- } \\
\text { bindung/Nahverkehr } \\
\text { der DB } \\
\text { - unzureichendes } \\
\text { Angebot an Taxis }\end{array}$ & 24 & $1,7 \%$ & $\begin{array}{c}26 \\
\text { bzw. } \\
21,7 \%\end{array}$ \\
\hline & & $100 \%$ & $100 \%$ & & & $100 \%$ & $100 \%$ \\
\hline
\end{tabular}

\section{Abb. 33: Kritische Ereignisse der Nach-Reisephase}

Das zur Überprüfung der Beziehungsstrukturen der Nach-Reisephase spezifizierte und identifizierbare Kausalmodell ist in Abbildung 34 dargestellt. Die ermittelten Gütemaße bestätigen eine insgesamt zufriedenstellende Modellanpassung. So liegen der AGLS-Wert sowie der Adjusted AGLS-Wert in einem ähnlichen Be- 


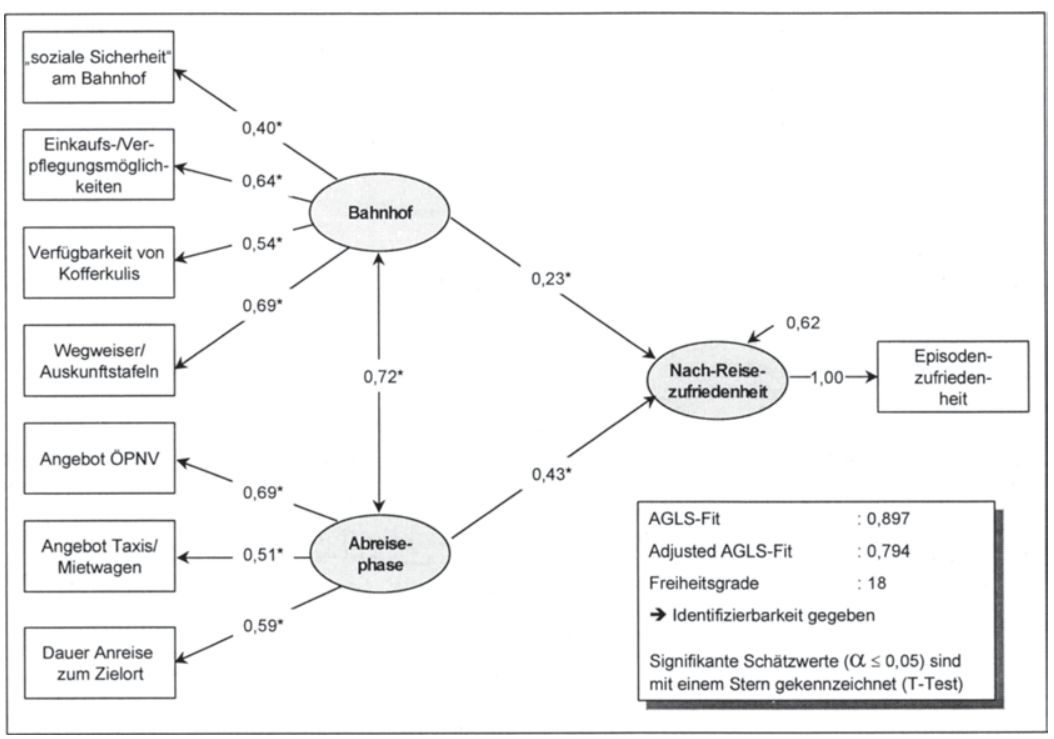

Abb. 34: Kausalmodell zur Struktur und Dynamik der Nach-Reisezufriedenheit

reich wie für die vorhergehenden Modelle und weisen damit auf eine akzeptable Anpassung der theoretischen Modellstruktur an die empirische Datenstruktur hin. ${ }^{70}$

Für die Meßindikatoren liegen unter Berücksichtigung der Stichprobengröße reliable Werte vor. Dabei zeigt sich jedoch, daß sich das Merkmal „soziale Sicherheit" nur mit einem vergleichsweise hohen Informationsverlust als Indikator des Kontaktpunktes „Bahnhof” verwenden läßt. Als zuverlässigste Indikatoren dieses Kontaktpunktes erweisen sich die Ausstattung des Bahnhofs mit Orientierungshilfen sowie die im Bahnhof befindlichen Einkaufs- und Verpflegungsmöglichkeiten. Die Zufriedenheit mit der Abreisephase wird wiederum am stärksten durch die Zufriedenheit mit dem Angebot der öffentlichen Verkehrsmittel repräsentiert.

Die Analyse der Beziehung zwischen den beiden Kontaktpunkten zeigt mit einem Korrelationskoeffizienten von 0,72 einen deutlichen Zusammenhang. Die Zufriedenheit mit dem Bahnhof ist damit zu einem nicht unerheblichen Grad für die Zufriedenheit mit der Abreisephase verantwortlich. Da der Kontaktpunkt „Bahnhof“

70 Für diese Modell betragen die LISREL-Gütekriterien: GFI=0,979, AGFI=0,938, RMR=0,069. Auch auf Basis dieser Werte ist die Modellanpassung als gelungen zu bezeichnen Andreas Siefke and Universität Münster - 978-3-631-75103-9 
der Abreisephase zeitlich vorgelagert ist, kann Hypothese Hyp D KP auch für die Nach-Reisephase als bestätigt angesehen werden.

Während der Bahnhof in der Vor-Reisephase den vergleichsweise stärksten Einfluß auf die Episodenzufriedenheit ausübt, steht der Zielbahnhof in der NachReisephase in seiner Bedeutung (Pfadkoeffizient=0,23) eindeutig hinter dem Kontaktpunkt „Abreise“ zurück (Pfadkoeffizient=0,43). Hierin wird der relativ autonome Charakter der letzten Episode einer Bahnreise deutlich. Wenngleich die Zufriedenheit mit dem Zielbahnhof einen eindeutigen und nicht zu vernachlässigenden Einfluß auf die Episodenzufriedenheit ausübt, nimmt der reibungslose Verlauf der Anreise zum letztendlichen Zielort der Reise für die Befragten die dominierende Rolle bei der Bildung des Zufriedenheitsurteils über die NachReisephase ein. Hypothese Hyp I Episod 4 wird damit auf der Basis der empirischen Ergebnisse angenommen. Ebenso kann von der Existenz der in Hypothese Hyp Episod 4 formulierten Struktur der Nach-Reisezufriedenheit ausgegangen werden.

Insgesamt werden durch die erörterten kausalen Zusammenhänge ca. 38\% der Varianz der Nach-Reisezufriedenheit erklärt. Dieser Anteil entspricht damit ungefähr dem Wert der erklärten Varianz der Vor-Reisezufriedenheit, ist jedoch erkennbar niedriger als die entsprechenden Werte der Reise- und Umsteigezufriedenheit. $^{71}$

\subsection{Interphasenspezifische Analyse der Kundenzufriedenheit}

Nachdem in den bisherigen Ausführungen die Strukturen und Beziehungszusammenhänge der Kundenzufriedenheit innerhalb der einzelnen Episoden einer Bahnreise einer ausführlichen Untersuchung unterzogen wurden, werden in den folgenden Abschnitten die Zusammenhänge zwischen den verschiedenen Abschnitten im Hinblick auf die gesamte Zufriedenheit mit einer Bahnreise und deren Auswirkungen auf die Beziehungszufriedenheit und das zukünftige Verhalten der Reisenden gegenüber dem Verkehrsdienstleistungsunternehmen näher betrachtet. 


\subsection{Struktur und Dynamik der Transaktionszufriedenheit}

Aufgrund der Komplexität der Kundenprozeßhierarchie und der Vielzahl von Zufriedenheitsindikatoren einer Bahnreise werden im folgenden die Beziehungszusammenhänge zwischen den einzelnen Episoden auf der Basis der zusätzlich erfaßten Episodenzufriedenheiten untersucht. Da diese im Rahmen der intraphasenspezifischen Kausalmodelle als hinreichend geeignete Indikatoren der Konstruktvalidität erkannt wurden, ist ein solches Vorgehen als zieladäquat anzusehen. $^{72}$

Die Operationalisierung der Transaktionszufriedenheit erfolgt anhand von zwei Indikatoren. So wurden die Befragten auf der einen Seite gebeten, ihre Zufriedenheit mit der gesamten Bahnreise unter Einbeziehung der erlebten Episoden anzugeben. Darüber hinaus wurde auf der anderen Seite die Zufriedenheit mit dem Preis-iLeistungsverhältnis der Bahnreise erhoben. Mit dieser Fragestellung wird dem mit dem Erwerb einer Fahrkarte verbundenen finanziellen Opfer stärker entsprochen. $^{73}$

Wenngleich sich zweifellos auch in der globalen Frage nach der Kundenzufriedenheit der Preis der Verkehrsdienstleistung als impliziter Vergleichsmaßstab widerspiegelt, ist aufgrund der Frageformulierung davon auszugehen, daß dieses Urteil durch eine stärker affektive, ereignisorientierte Wahrnehmung der Bahnreise geprägt ist und daß insoweit der emotionalen Komponente des Zufriedenheitskonstruktes in einem größeren Umfang Rechnung getragen wird. Die explizite Frage nach der Einschätzung des Preis-/Leistungsverhältnisses zielt

72 Zu einer ähnlichen Vorgehensweise vgl. Woodside, A.G., Frey, L.L., Daly, R.T., Linking Service Quality, Customer Satisfaction, and Behavioral Intention, a.a.O., S. 9 ff.; Danaher, P.J., Mattson, J., Customer Satisfaction during the Service Delivery Process, a.a.O., S. $10 \mathrm{ff}$.; Danaher, P.J., Mattson, J., Cumulative Encounter Satisfaction in the Hotel Conference Process, a.a.O., S. 73 ff. und ferner Nader, G., Zufriedenheit mit Finanzdienstleistungen: Erfolgswirksamkeit, Messung, Modellierung, a.a.O., S $86 \mathrm{ff}$.

Alternativ besteht die Möglichkeit einer Indexbildung über die einzelnen Merkmale des jeweiligen Kontaktpunktes einer Episode. Aufgrund des hiermit unvermeidlich verbundenen Informationsverlustes, der mit einer Durchschnittsbildung einhergeht, wurde im Rahmen der vorliegenden Untersuchung auf ein solches Vorgehen verzichtet und die dargestellte Variante, nicht zuletzt aufgrund der geringeren Modellkomplexität, bevorzugt.

Kundenzufriedenheit stellt in diesem Sinne ein als günstig beurteiltes Preis-I Leistungsverhältnis der wahrgenommenen Transaktion dar und kann damit auch als "Okonomische" Kundenzufriedenheit bezeichnet werden. Zu den in ein Zufriedenheitsurteil eingehenden "Give"- und "Get"-Komponenten vgl. Haller, S., Beurteilung von Dienstleistungsqualität: Dynamische Betrachtung des Qualitătsurteils im Weiterbildungsbereich, a.a.O., S. 47 f. sowie die Diskussion des Service Value-Konzeptes von Liljander und Strandvik: Liljander, V., Strandvik, T., Estimating Zones of Tolerance in Perceived Service Quality and Perceived Service Value, in: IJoSIM, Vol. 4, No. 2, 1993, S. $14 \mathrm{ff}$.; vgl. hierzu auch die Ausführungen in Kap. B 2.332 sowie die Definition der Kundenzufriedenheit nach Howard/Sheth in Kap. A 3 dieser Arbeit. 
dagegen eher auf das kognitive Element der Kundenzufriedenheit und eine somit "objektivere" Beurteilung ab. Insofern ist zu vermuten, daß auf diese Weise der hohen Komplexität des Konstruktes Kundenzufriedenheit mit einer Verkehrsdienstleistung im Vergleich zu einer eindimensionalen Operationalisierung besser entsprochen wird. Messen jedoch beide Indikatoren entgegen diesen Ausführungen die gleichen Facetten der Kundenzufriedenheit, ist eine hohe Korrelation zwischen den beiden Zufriedenheitsmaßen zu vermuten. In der vorliegenden Stichprobe beträgt dieser Koeffizient 0,40 . Es kann damit von inhaltich verbundenen, aber dennoch unterschiedlichen Operationalisierungen der Gesamtzufriedenheit mit einer Bahnreise ausgegangen werden. Daher finden beide Ansätze in der vorliegenden Untersuchung Berücksichtigung. Das sich aus diesen Merkmalen ergebende Mittelwertprofil der verschiedenen Komponenten des Zufriedenheitskonstruktes ist in Abbildung 35 wiedergegeben.

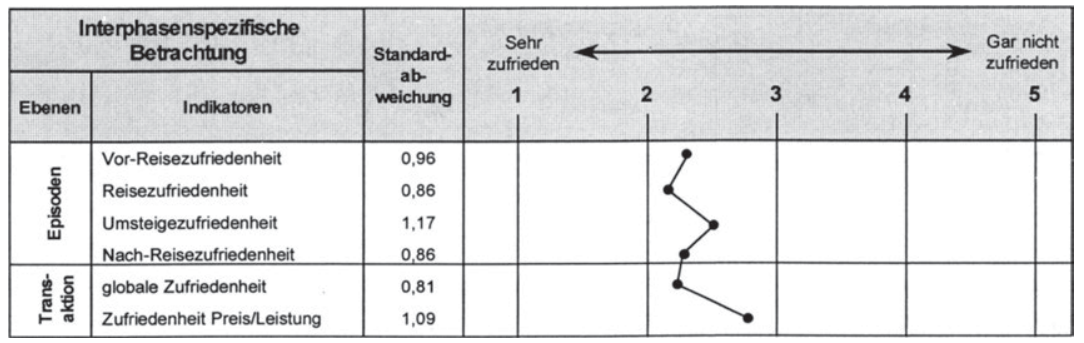

Abb. 35: Profil der Kundenzufriedenheit auf der Episoden- und Transaktionsebene

Hier zeigt sich auf der Episodenebene, daß die Zufriedenheit mit dem Umsteigevorgang im Vergleich zu den anderen Abschnitten einer Bahnreise niedriger ausfällt. Die relativ hohe Standardabweichung dieses Indikators kann als ein Indiz für den vergleichsweise starken Einfluß der in dieser Phase einer Bahnreise erlebten positiven und negativen Ereignisse gewertet werden. ${ }^{74}$ Auf der Transaktionsebene ist der Unterschied zwischen der globalen episodenübergreifenden Zufriedenheit und der Zufriedenheit mit dem Preis-/Leistungsverhältnis offenkundig. So ist unter expliziter Einbeziehung des Preises die Zufriedenheit mit einer Bahnreise deutlich niedriger ausgeprägt. Dies ist auch darauf zurückzuführen, daß die Bahn generell als ein „teures" Verkehrsmittel betrachtet wird. Ein möglicher Grund hierfür besteht 


\begin{tabular}{|c|c|c|c|c|c|c|}
\cline { 2 - 7 } \multicolumn{1}{c|}{} & \multicolumn{2}{c|}{ Gesamt } & \multicolumn{2}{c|}{$\begin{array}{c}\text { Positive } \\
\text { Ereignisse } \\
(52,5 \%)\end{array}$} & \multicolumn{2}{c|}{$\begin{array}{c}\text { Negative } \\
\text { Ereignisse } \\
(47,5 \%)\end{array}$} \\
\hline $\begin{array}{c}\text { Vor-Reise- } \\
\text { phase }\end{array}$ & 513 & $25,8 \%$ & 272 & $26,1 \%$ & 241 & $25,5 \%$ \\
\hline Reisephase & 1013 & $51,0 \%$ & 556 & $53,3 \%$ & 457 & $48,4 \%$ \\
\hline $\begin{array}{c}\text { Umsteige- } \\
\text { phase }\end{array}$ & 268 & $13,5 \%$ & 142 & $13,6 \%$ & 126 & $13,4 \%$ \\
\hline $\begin{array}{c}\text { Nach-Reise- } \\
\text { phase }\end{array}$ & 193 & $9,7 \%$ & 73 & $7,0 \%$ & 120 & $12,7 \%$ \\
\hline \hline Summe & 1987 & $100 \%$ & 1043 & $100 \%$ & 944 & $100 \%$ \\
\hline
\end{tabular}

\section{Abb. 36: Episodenübergreifende Betrachtung der kritischen Ereignisse}

darin, daß Reisende häufig den Pkw als Vergleichsmaßstab zugrunde legen und hierbei in einem überwiegenden Maße die sogenannten "out-of-pocket"-Kosten zur Kalkulation heranziehen. Während die Kosten einer Bahnfahrt in Form des Fahrpreises klar definiert sind, neigen Reisende bei der Kalkulation einer PkwFahrt dazu, ausschließlich die Benzinkosten anstatt der Betriebs- und Kapitalkosten zu berücksichtigen. ${ }^{75}$

Bei einer episodenübergreifenden Betrachtung der kritischen Ereignisse ist festzustellen, daß die weit überwiegende Zahl der Nennungen in Zusammenhang mit der Reisephase erfolgt. So entfällt ca. die Hälfte aller Ereignisse auf diesen Reiseabschnitt (vgl. Abbildung 36). Dagegen werden nur ca. 10\% der Ereignisse in Verbindung mit der Nach-Reisephase geschildert.

Vgl. Institut für Marketing, Non User-Analyse bei der Deutschen Bahn AG, unveröffentlichte Studie im Auftrag der Deutschen Bahn AG, Münster 1996; Jänsch, E., Wiese, J., Der Stellenwert des Komforts in der Systemplanung "Hochgeschwindigkeitsverkehr" (HGV), in: DBB, Jg. 64, H. 9, 1988, S. 806; Wilken, D., Kriterien der Verkehrsmittelwahl im Personenfernverkehr und ihre Bedeutung in Verkehrsnachfragemodellen, a.a.O., S. 6

Andreas Siefke and Universität Münster - 978-3-631-75103-9 


\begin{tabular}{|c|c|c|c|c|c|}
\hline & & \multicolumn{2}{|c|}{ (sehr) zufrieden 1} & \multicolumn{2}{|c|}{ weniger zufrieden ${ }^{2}$} \\
\hline & & $\begin{array}{l}\text { Positives } \\
\text { Ereignis }\end{array}$ & $\begin{array}{c}\text { Negatives } \\
\text { Ereignis }\end{array}$ & $\begin{array}{l}\text { Positives } \\
\text { Ereignis }\end{array}$ & $\begin{array}{c}\text { Negatives } \\
\text { Ereignis }\end{array}$ \\
\hline \multirow{2}{*}{ 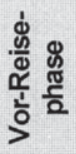 } & Anzahl & 202 & 99 & 118 & 157 \\
\hline & Quotient & \multicolumn{2}{|c|}{2,04} & \multicolumn{2}{|c|}{0,75} \\
\hline \multirow{2}{*}{$\begin{array}{l}\dot{\Phi} \\
\frac{\Phi}{\omega} \\
\frac{\pi}{\square} \\
\frac{\pi}{2}\end{array}$} & Anzahl & 275 & 174 & 143 & 161 \\
\hline & Quotient & \multicolumn{2}{|c|}{1,58} & \multicolumn{2}{|c|}{0,89} \\
\hline \multirow{2}{*}{ 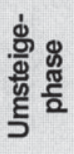 } & Anzahl & 117 & 44 & 71 & 127 \\
\hline & Quotient & \multicolumn{2}{|c|}{2,66} & \multicolumn{2}{|c|}{0,56} \\
\hline \multirow{2}{*}{ 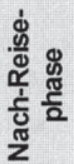 } & Anzahl & 99 & 49 & 68 & 107 \\
\hline & Quotient & \multicolumn{2}{|c|}{2,02} & \multicolumn{2}{|c|}{0,63} \\
\hline
\end{tabular}

$\begin{array}{lll}\text { 1: Skalenwerte } 1 \text { und } 2 \quad \text { 2: Skalenwerte }>2 & 2\end{array}$

\section{Abb. 37: Zusammenhang von kritischen Ereignissen und der Zufriedenheit auf der Episodenebene}

Zur Analyse des Einflusses dieser Ereignisse auf die Zufriedenheit mit einer Episode wurden die Fahrgäste in zwei Gruppen aufgeteilt. Die erste Gruppe umfaßt jeweils die Reisenden, die sich mit einer Episode als zufrieden oder sehr zufrieden gezeigt haben. Die andere Gruppe besteht aus den Fahrgästen mit einer jeweils schlechteren Bewertung. ${ }^{76}$ In Abbildung 37 ist dabei für jede Episode die Anzahl derjenigen Fahrgäste angegeben, die mindestens ein negatives und/oder positives Ereignis für die einzelnen Abschnitte einer Bahnreise genannt haben. 
Es zeigt sich, daß in den jeweils mit einer Episode zufriedenen Gruppen die Anzahl an Personen mit (mindestens) einem positiven Erlebnis eindeutig überwiegt. Das genau umgekehrte Verhältnis ergibt sich dagegen bei einer Betrachtung der entsprechenden negativer wertenden Gruppen. Wie auch in den ermittelten Quotienten zwischen den positiven und negativen Ereignissen deutlich wird, dokumentieren diese Ergebnisse einen gut erkennbaren Zusammenhang zwischen dem Dienstleistungserleben und der Episodenzufriedenheit.

Inwieweit diese bisher rein quantitativen Betrachtungen der kritischen Ereignisse bereits als Hinweis auf die Bedeutung dieser Episoden bei der Bildung des gesamten Zufriedenheitsurteils interpretiert werden können, soll im folgenden näher untersucht werden. $\mathrm{Zu}$ diesem Zweck wurde das in Abbildung 38 dargestellte Kausalmodell zur Analyse der Beziehungsstrukturen zwischen den Episoden einer Bahnreise spezifiziert. ${ }^{77}$ In diesem Zusammenhang ist auf eine Berücksichtigung der Umsteigephase als einer eigenständigen Episode verzichtet worden, da sie einen integralen Bestandteil der Reisephase darstellt. ${ }^{78}$ Das Modell erfültt die notwendige Bedingung der Identifizierbarkeit, und die ermittelten Gütemaße kennzeichnen eine guten Modellfit. ${ }^{79}$

Interessanterweise erweist sich die Frage nach der Beurteilung des Preis-I Leistungsverhältnisses als der schwächere Indikator der Transaktionszufriedenheit. Dies steht in Einklang mit den Ergebnissen anderer Forschungsarbeiten, in denen ebenfalls ein vergleichsweise geringer Einfluß der Preiskomponente in

77 Das vorliegenden Modell ist als ein Sonderfall des allgemeinen Modells der Kausalanalyse zu bezeichnen. Da in diesem Modell ausschließlich kausale Beziehungen zwischen den Teilkonstrukten spezifiziert wurden, handelt es sich um ein Meßmodell von latenten endogenen Variablen (konfirmatorische Faktorenanalyse). Vgl. hierzu Backhaus, K. et al., Multivariate Analysemethoden - Eine anwendungsorientierte Einführung, a.a.O., S. 408; Bentler, P.M., EQS Structural Equations Program Manual, a.a.O., S. $26 \mathrm{ff}$.

Für eine explizite Berücksichtigung der Umsteigephase ist die Notwendigkeit der Messung der Reisezufriedenheit vor und nach dem Umsteigevorgang gegeben. Dies würde die zweifache Messung von inhaltlich sich überlappenden Episoden einer Bahnreise bedeuten. Aus erhebungsstechnischen Gründen und zur Vermeidung der Überforderung der Befragten wurde auf ein derartige Anlage des Befragungsdesigns verzichtet.

79 Die entsprechenden LISREL-Kriterien dieses Modells lauten: $G F I=0,982, A G F I=0,908$ RMR=0,067. Zur Sicherstellung der Identifizierbarkeit des Modells werden für die einzelnen Indikatoren der Episodenzufriedenheiten Ladungen von 1 und entsprechende Residualgroßßen von 0 unterstellt. Zu einer ähnlichen Vorgehensweise vgl. Kirchgeorg, M., Okologieorientiertes Unternehmensverhalten: Typologien und Erklärungsansätze auf empirischer Basis, a.a.O., S. 225 . 


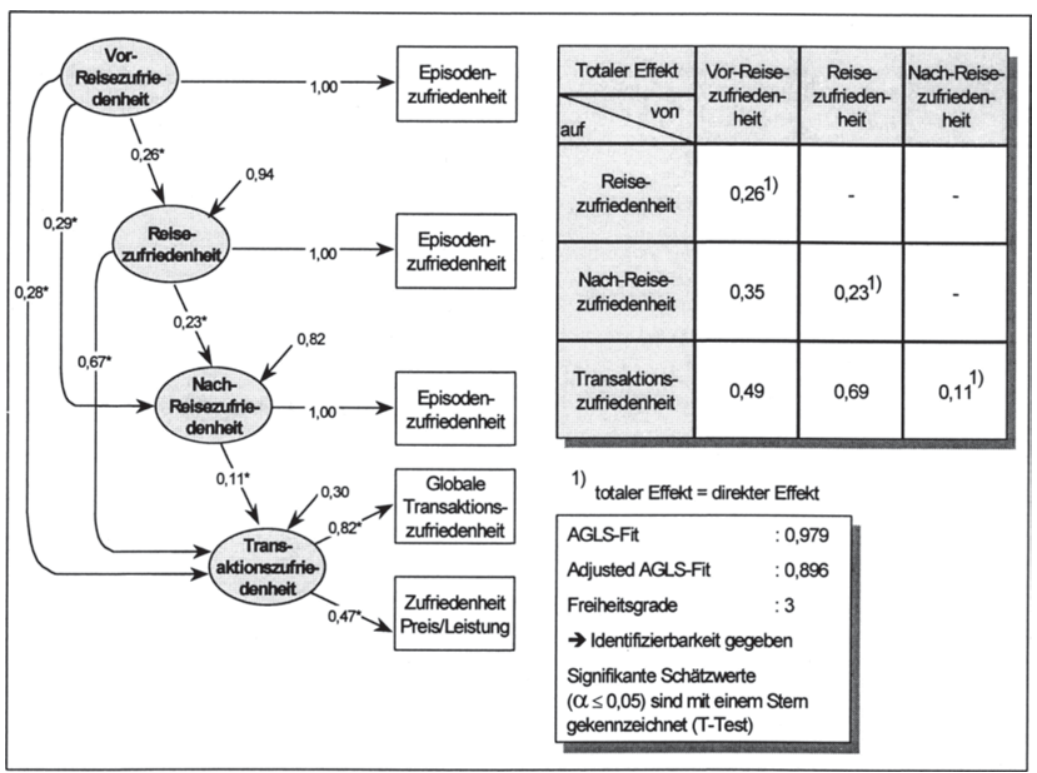

Abb. 38: Konfirmatorische Faktorenanalyse zur Struktur und Dynamik der Transaktionszufriedenheit

bezug auf die Gesamtbewertung einer Dienstleistung ermittelt wurde.$^{80}$ Die global formulierte Gesamtzufriedenheit mit der Bahnreise stellt mit einer Faktorladung von 0,82 einen sehr zuverlässigen Indikator der Transaktionszufriedenheit dar.

Zur Überprüfung der in Hypothese Hyp D Episod aufgestellten Vermutung eines positiven Einflusses der Zufriedenheit mit einer zeitlich vorgelagerten Episode auf die Beurteilung nachfolgender Episoden sollen die ermittelten Pfadkoeffizienten einer genaueren Analyse unterzogen werden. Zunächst ist hier festzuhalten, daß von allen Episoden ein direkter positiver Einfluß auf zeitlich nachgelagerte Episoden ausgeht. Hypothese Hyp $D$ Episod ist damit zu bestätigen. Jedoch ist der Erklärungsbeitrag zeitlich vorgelagerter Episoden für die Zufriedenheit nachfolgender Episoden über alle Befragte als relativ gering einzustufen. So werden lediglich $6 \%$ der Varianz der Reisezufriedenheit und $18 \%$ der Varianz der NachReisezufriedenheit durch die davor erlebten Reiseabschnitte erklärt. Diese 
Ergebnisse stellen damit auf der einen Seite einen eindeutigen Nachweis für die Dynamik der Kundenzufriedenheit mit Verkehrsdienstleistungen auf der Episodenebene dar. Auf der anderen Seite ist aber ebenso eine vergleichsweise eigenständige Wahrnehmung der einzelnen Abschnitte einer Bahnreise festzustellen.

Den eindeutig stärksten direkten Einfluß übt die Reisezufriedenheit auf die Transaktionszufriedenheit aus $(0,67)$. Die sehr hohe Bedeutung eines zufriedenstellenden Verlaufs der Kerndienstleistung einer Bahnreise, der Beförderung, ist hier unverkennbar. Hyp I Trans 1 kann damit auf der Basis der empirischen Ergebnisse angenommen werden. Gleichwohl üben auch die der Reisephase vor- und nachgelagerten Episoden einen eindeutigen Einfluß auf die Transaktionszufriedenheit aus. Hieran wird deutlich, daß sich ein Verkehrsdienstleistungsunternehmen keinesfalls auf die Optimierung des eigentlichen Kerns der Dienstleistung beschränken darf, sondern auch den Erfahrungen, die der Kunde im Vorfeld und nach dem eigentlichen Dienstleistungskonsum macht, eine besondere Beachtung zukommen lassen muß. Dies ist nicht zuletzt aufgrund des Einflusses der Vor-Reisephase auf die Reisezufriedenheit als notwendig zu erachten. In diesem Zusammenhang kann festgestellt werden, daß der Einfluß der Zufriedenheit mit der Nach-Reisephase relativ niedrig ausfällt $(0,11)$. Hypothese Hyp I Trans 2 über den vergleichsweise „autonomen“ Charakter der NachReisephase im Hinblick auf die Entstehung der Transaktionszufriedenheit ist damit anzunehmen. So scheint ein Bahnreisender mit dem Verlassen des Zielbahnhofs die Verkehrsdienstleistung "Bahnreise" als (weitgehend) beendet anzusehen, wie dies bereits in der intraphasenspezifischen Analyse der Nach-Reisezufriedenheit zum Ausdruck kam. ${ }^{81}$

Neben den bisher erläuterten direkten Einflüssen sind darüber hinaus die totalen Effekte der einzelnen Abschnitte einer Bahnreise von Interesse, da eine Episodenzufriedenheit über die danach folgenden Episoden indirekt auf die weiteren Phasen und damit auch auf die gesamte Zufriedenheit mit der Bahnreise Wirkungen ausübt. ${ }^{82}$ Wie Abbildung $38 \mathrm{zu}$ entnehmen ist, erfährt die Vor-Reise-

Vgl. hierzu die Ausführungen in Kap. C 2.24 dieser Arbeit.

Indirekte Effekte werden durch die Multiplikation und anschließende Addition der entsprechenden Koeffizienten ermittelt. Aus der Addition der direkten und indirekten Einflüsse ergeben sich die totalen Effekte, die von der EQS-Software berechnet werden und dem Output-Protokoll des Programms zu entnehmen sind. Vgl. Bentler, P.M., EQS Structural Equations Program Manual, a.a.O., S. 97. Bestehen keine indirekten Wirkungen, entsprechen die totalen Effekte den direkten Effekten. Zu einer ausführlichen Darstellung der Berechnung derartiger Effekte vgl. Backhaus, K. et al., Multivariate Analysemethoden - Eine anwendungsorientierte Einführung, a.a.O., S. $389 \mathrm{ff}$. 
zufriedenheit bei einer Integration der indirekten Effekte einen merklichen Bedeutungszuwachs. Auch wenn die Reisezufriedenheit weiterhin als die dominierende Episode einer Bahnreise anzusehen ist, wird auf diese Weise nochmals die Notwendigkeit der Berücksichtigung insbesondere der Episode deutlich, die der Kerndienstleistung zeitlich vorgelagert ist.

Insgesamt werden durch die dargestellten Beziehungszusammenhänge $70 \%$ der Varianz der Transaktionszufriedenheit erklärt. Auf der Basis der bisherigen Ausführungen und vor dem Hintergrund dieses Ergebnisses kann schließlich auch Hypothese Hyp Trans über die Struktur der Transaktionszufriedenheit als grundsätzlich bestätigt angesehen und dem Modell eine zufriedenstellende nomologische Validität bescheinigt werden.

\subsection{Einfluß auf die Beziehungszufriedenheit}

Nachdem im vorhergegangenen Abschnitt die transaktionsspezifische Zufriedenheitsdynamik Gegenstand der Untersuchung war, soll im folgenden die Forschungsperspektive auf die höchste Ebene der Hierarchie von Dienstleistungsprozessen, die Beziehungsebene, ausgedehnt werden. ${ }^{83}$ Der Begriff der Beziehungszufriedenheit beinhaltet die transaktionsübergreifende Zufriedenheit mit dem Verkehrsdienstleister Bahn und umfaßt die Summe aller bisher erlebten Transaktionen. Im Hinblick auf die beziehungsspezifische Zufriedenheitsdynamik ist zu hinterfragen, welchen Einfluß die Zufriedenheit mit einer konkreten Transaktion auf den aktuellen Stand der Beziehungszufriedenheit ausübt. ${ }^{84} \mathrm{Zu}$ diesem Zweck wurden die Reisenden gebeten, ihre Zufriedenheit mit der Bahn unter Berücksichtigung aller bisherigen Erfahrungen mit der Bahn anzugeben.

Mit einem Durchschnitt von 2,53 über alle Befragten bewegt sich die Beziehungszufriedenheit mit der Bahn auf einem nur mäßigen Niveau und fällt damit in einem nicht unerheblichen Ausmaß niedriger als die episodenübergreifende Transaktionszufriedenheit (Mittelwert 2,19) aus. ${ }^{85}$ Offensichtlich spiegeln sich in der Beziehungszufriedenheit auch weniger zufriedenstellende Kontakte der Vergangenheit wider. Ebenso konnte in Zufriedenheitsstudien nachgewiesen werden,

Vgl. hierzu die Ausführungen in Kap. A 2 und B 2.332 dieser Arbeit.

Da die Analyse der Zufriedenheit mit einer spezifischen Transaktion im Mittelpunkt dieser Arbeit steht, ist hier nicht die Möglichkeit gegeben, den Einfluß auf zeitlich nachgelagerte bzw. von zeitlich vorgelagerten Transaktionen zu untersuchen.

Diese Ergebnis steht in Einklang mit den Ergebnissen der empirischen Untersuchung von Strandvik/Liljander. Vgl. hierzu die Ausführungen in Kap. B 2.332 dieser Arbeit.

Andreas Siefke and Universität Münster - 978-3-631-75103-9 
daß Kundenzufriedenheit im Zeitablauf abnimmt. Ein mögliche Ursache wird darin gesehen, daß sich Kunden eher an negative als an positive Transaktionen erinnern und demzufolge die Beziehungszufriedenheit geringer einschätzen. ${ }^{86}$

Für die Analyse des Einflusses der Transaktionszufriedenheit auf die Beziehungszufriedenheit wird die im vorhergegangenen Kapitel vorgestellte konfirmatorische Faktorenanalyse zur Struktur und Dynamik der Transaktionszufriedenheit aufgegriffen und um das Konstrukt der Beziehungszufriedenheit ergänzt (vgl. Abbildung 39).

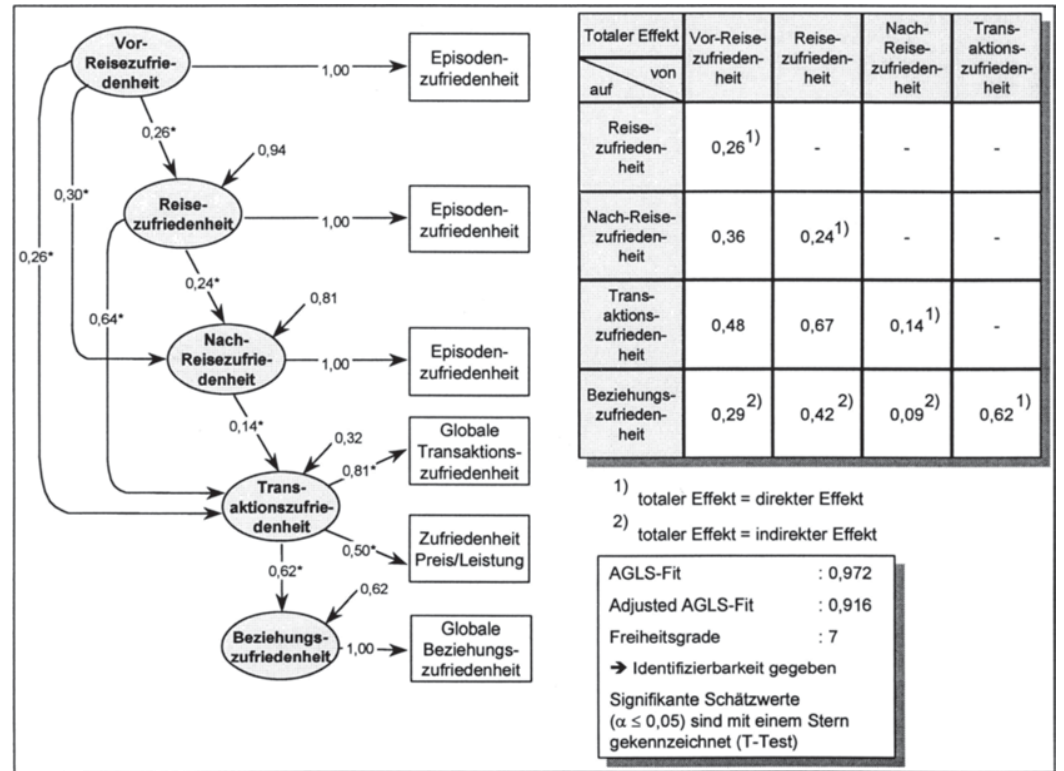

Abb. 39: Konfirmatorische Faktorenanalyse zur Beziehungszufriedenheit

Auch dieses Modell ist identifizierbar und weist eine gute Anpassung an die empirische Datenstruktur auf (AGLS=0,972, Adjusted AGLS=0,916) ${ }^{87}$ Hier ist zunächst ein starker direkter Einfluß der Transaktionszufriedenheit auf die Beziehungs-

86 Vgl. Folkes, V., How Consumers Predict Service Quality: What Do They Expect?, in: Service Quality: New Directions in Theory and Practice Rust, R.T., Oliver, R.L. (Hrsg.), Thousand Oaks u.a. 1994, S. $108 \mathrm{ff}$.

87 Die entsprechenden LISREL-Gütemaße betragen: GFI=0,983, AGFI=0,945, RMR=0,06 und führen ebenfalls zu einer positiven Beurteilung des Modells. 
zufriedenheit zu erkennen $(0,62)$. Damit kann Hypothese Hyp $\mathrm{Bz}$ über den positiven Einfluß der Transaktionszufriedenheit auf die Zufriedenheit mit der Beziehung zum Verkehrsdienstleistungsunternehmen Bahn unterstützt werden. Die ebenfalls in Abbildung 39 angegebenen totalen Effekte der einzelnen Konstrukte bestätigen überdies jedoch auch die Bedeutung einzelner Episoden im Hinblick auf die Bildung des Urteils über die Beziehung zum Unternehmen. So geht auch von der Vor-Reise- und insbesondere von der Reisephase ein deutlicher (totaler) Effekt auf die Beziehungszufriedenheit aus. Dagegen kann der Einfluß der Zufriedenheit mit der Nach-Reisephase auf die Beziehungszufriedenheit vernachlässigt werden $(0,09)$.

Die im Modell dargestellten Kausalbeziehungen vermögen ca. 38\% der Varianz der Zufriedenheit auf der Beziehungsebene zu erklären. Unter Berücksichtigung der Tatsache, daß der Großteil der Befragten über vergleichsweise „junge" Erfahrungen mit Bahnreisen verfügt, ${ }^{88}$ ist dieser Wert als hoch anzusehen, da im allgemeinen davon ausgegangen wird, daß das Urteil über die Beziehungszufriedenheit auf der Summe aller Erfahrungen beruht. Eine mögliche Erklärung für den hier ermittelten Wert besteht darin, daß ein Rezenzeffekt vorliegt. ${ }^{89}$ Dies würde bedeuten, daß insbesondere der letzte und damit jüngste Kontakt mit dem Verkehrsdienstleister die Beziehungszufriedenheit in entscheidender Weise bestimmt.

In diesem Zusammenhang ist allerdings anzumerken, daß die Messung der Transaktions- und Beziehungszufriedenheit zum gleichen Zeitpunkt stattfand. Da jedoch, den bisherigen Ausführungen folgend, die Beziehungszufriedenheit als ein vergleichsweise stabiles Konstrukt anzusehen ist, kann erst eine als Zeitreihenanalyse angelegte Messung genauen Aufschluß über den Einfluß einzelner Transaktionen auf die Beziehungszufriedenheit geben. ${ }^{90}$ Dennoch ist das vorliegende Ergebnis auf der einen Seite ein Beleg für die Unterschiedlichkeit beider Konstrukte. Auf der anderen Seite ist deutlich erkennbar, daß die Beziehungszufriedenheit in einem nicht unerheblichen Ausmaß durch das aktuelle Dienstleistungserleben beeinflußt wird. Reisen über $100 \mathrm{~km}$ genutzt zu haben.

89 Vgl. hierzu die Ausführungen in Kap. B 2.331 dieser Arbeit sowie Parasuraman, A., Zeithaml, V.A., Berry, L.L., Reassessment of Expectations as a Comparison Standard in Measuring Service Quality: Implications for Further Research, a.a.O., S. 122.

90 Vgl. Alpert, M.I., Golden, L.L., Transportation Attitudes over Time: A Longitudinal Approach, in: Advances in Consumer Research, Hunt, H.K. (Hrsg.), Vol. 5, 1978, S. $194 \mathrm{ff}$. 
Insgesamt bleibt zu vermuten, daß das einmalige positive Erleben einer Dienstleistungstransaktion die Beziehungszufriedenheit nur in einem befristeten Zeitraum vorteilhaft stimuliert. ${ }^{91}$ So ist davon auszugehen, daß erst das regelmäßige positive Erleben einer Bahnreise einen nachhaltigen Einfluß auf die Beziehungszufriedenheit ausübt und damit eine dauerhaft (positive) Änderung der Beziehung zum Verkehrsdienstleister Bahn bewirkt. ${ }^{92}$

\subsection{Einfluß auf die Kundenloyalität}

In der Literatur wird der häufig postulierten prognostischen Relevanz der Kundenzufriedenheit für die Loyalität von Kunden nicht immer uneingeschränkt zugestimmt. ${ }^{93} \mathrm{Da}$, wie gezeigt, das Verkehrsmittelwahlverhalten durch eine Vielzahl von Komponenten geprägt wird, stellt sich die Frage nach der Bedeutung der Kundenzufriedenheit für die Kundenbindung hier mit besonderem Nachdruck.

Die Kundenloyalität wurde in der vorliegenden Untersuchung einerseits als die Absicht, bei der nächsten Reise wieder mit der Bahn zu fahren, operationalisiert. Darüber hinaus wurde die Empfehlungsloyalität, also die Bereitschaft, die Bahn an Freunde, Bekannte oder Kollegen weiter zu empfehlen, als Indikator der Kundenloyalität herangezogen. ${ }^{94}$ Auch das auf der Basis dieser Indikatoren spezifizierte Kausalmodell zur Loyalität von Bahnreisenden baut auf der konfirmatorischen Faktorenanalyse zur Untersuchung der Transaktionszufriedenheit auf. ${ }^{95}$ Wie Abbildung $40 \mathrm{zu}$ entnehmen ist, kann diesem identifizierbaren Modell mit

Vgl. Bolton, R.N., Drew, J.H., A Longitudinal Analysis of the Impact of Service Changes on Customer Attitudes, a.a.O., S. $1 \mathrm{ff}$.

Vgl. Bitner, M.J., Hubbert, A.R., Encounter Satisfaction Versus Overall Satisfaction Versus Quality: The Customer's Voice, a.a.O., S. 77.

93 Vgl. Stauss, B., Neuhaus, P., Das Qualitative Zufriedenheitsmodell (QZM), a.a.O., S. 2 ff.; Dichtl, E., Peter, S., Kundenzufriedenheit und Kundenbindung in der Automobilindustrie: Ergebnisse einer empirischen Untersuchung, a.a.O., S. $23 \mathrm{ff}$.

Die Begriffe Loyalität, Markentreue und Wiederkauf(-absicht) werden in der Literatur häufig synonym behandelt. Vgl. hierzu Homburg, Ch., Rudolph, B., Theoretische Perspektiven zur Kundenzufriedenheit, a.a.O., S. 46. Auch in der vorliegenden Arbeit wird keine diesbezügliche konzeptionelle Differenzierung vorgenommen.

$94 \mathrm{Zu}$ einem ähnlichen Vorgehen vgl. Dichtl, E., Peter, S., Kundenzufriedenheit und Kundenbindung in der Automobilindustrie: Ergebnisse einer empirischen Untersuchung, a.a.O., S. 26; Strandvik, T., Liljander, V., A Comparison of Episode Performance and Relationship Performance for a Discrete Service, a.a.O., S. $130 \mathrm{f}$.

95 Um den spezifischen Einfluß der aktuellen Transaktion auf die Kundenloyalität zu ermitteln, wurden die Reisenden gebeten, die Fragen nach der Loyalităt vor dem Hintergrund der aktuellen Bahnreise zu beantworten. Aus diesem Grund wird die Beziehungszufriedenheit nicht in das Modell miteinbezogen.

Andreas Siefke and Universität Münster - 978-3-631-75103-9 


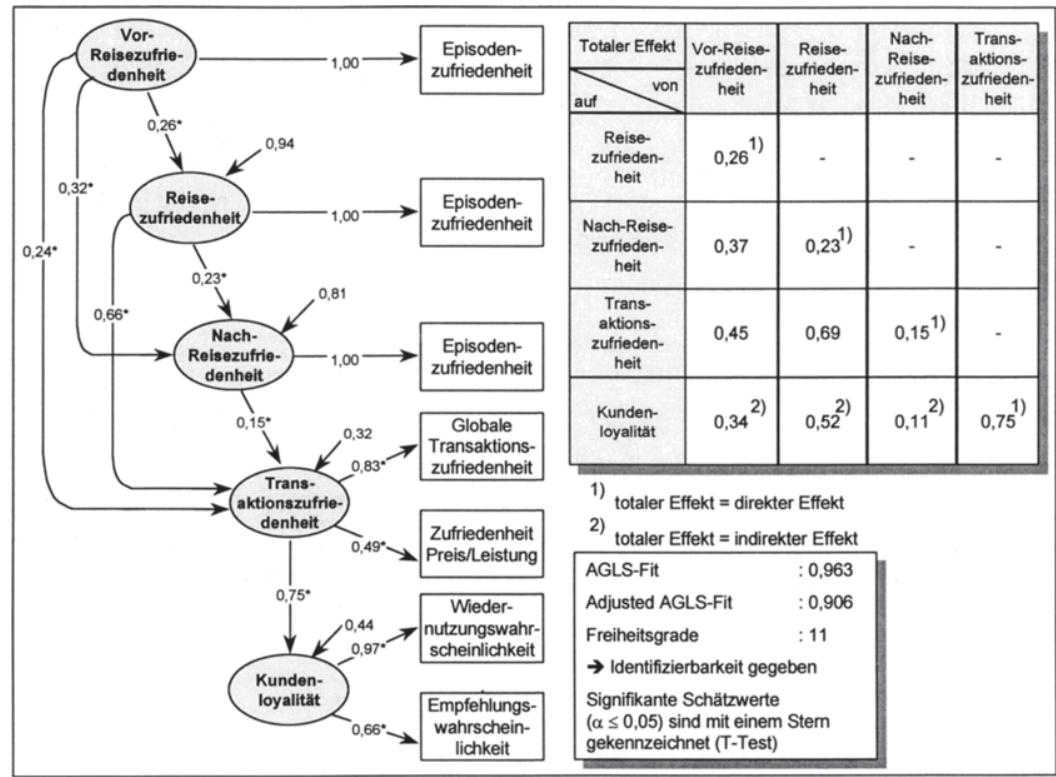

Abb. 40: Konfirmatorische Faktorenanalyse zur Kundenloyalität

einem AGLS-Fit von 0,963 eine gute Anpassungsgüte attestiert werden (Adjusted AGLS-Fit=0,906). ${ }^{96}$ Dabei zeigt sich die Wiedernutzungsabsicht als der zuverlässigere Indikator des theoretischen Konstruktes Kundenloyalität.

Mit einem Pfadkoeffizienten von 0,75 ist der Transaktionszufriedenheit eine sehr starke Wirkung auf die Kundenloyalität zuzusprechen. Die hohe Bedeutung der Kundenzufriedenheit für die Kundenbindung ist damit unverkennbar. Die weitere Betrachtung der totalen Effekte führt zu dem Ergebnis, daß von den einzelnen Episoden einer Bahnreise und dabei insbesondere von der Reisezufriedenheit ein nachhaltiger Effekt auf die Kundenloyalität ausgeht $(0,52)$. Auch hier wird die zentrale Bedeutung eines zufriedenstellenden Ablaufs der Kerndienstleistung deutlich, wenngleich der Einfluß der Vor-Reisephase nicht vernachlässigt werden darf. Mit einem totalen Effekt von 0,34 hat auch die Zufriedenheit mit Kundenprozeßelementen, die der eigentlichen Beförderung vorgelagert sind, einen erkennbaren Einfluß auf die zukünftige Verkehrsmittelwahl. Insgesamt werden

96 Für diese Modell betragen die LISREL-Gütekriterien: GFI=0,984, AGFI=0,959, RMR=0,059. Auch diese Werte deuten auf eine gute Modellanpassung hin. 
durch das Modell 56\% der Varianz der Kundenloyalität erklärt. Daher ist Hypothese Hyp D Loyal anzunehmen und die Kundenzufriedenheit als eine wichtige Determinante der Kundenloyalität anzusehen.

Wie bereits für die Analyse der Beziehungszufriedenheit angemerkt wurde, gilt es jedoch auch bei der hier vorgenommenen Untersuchung der Kundenloyalität zu beachten, daß die Kundenzufriedenheit eine vergleichsweise zeitlich instabile Größe darstellt, die nach Beendigung der Transaktion einem gewissen Abnahmeprozeß unterliegt. Da zudem hier nicht das tatsächliche, sondern das bekundete Verhalten der Kunden kurz nach Erleben der Transaktion abgefragt wurde, ist es durchaus denkbar, daß das geäußerte Verhalten von dem tatsächlichen Verhalten in der Folgezeit abweicht. ${ }^{97}$ Verschiedene Faktoren können zu einem von der Wiedernutzungsabsicht divergierenden Verkehrsmittelwahlverhalten führen. Der Einfluß solcher Faktoren fällt dabei umso geringer aus, je kürzer der Zeitraum zwischen der Messung der Verhaltensabsicht und der tatsächlichen Nutzung ist.

In der Literatur werden unterschiedliche Faktoren diskutiert, die neben der Kundenzufriedenheit die Kundenloyalität beeinflussen und eine mögliche Erklärung für die durch das vorliegende Modell nicht erfaßte Varianz der Kundenloyalität in Höhe von ca. $44 \%$ darstellen. In diesem Zusammenhang ist insbesondere die Existenz möglicher Wechselbarrieren von Bedeutung. ${ }^{98}$ Dabei spielt vor allem der Besitz eines Pkws eine entscheidende Rolle für die Verkehrsmittelwahl. ${ }^{99}$ Der Besitz eines Pkws eröffnet einem potentiellen Bahnkunden eine grundsätzlich ständig zur Verfügung stehende und flexibel einsetzbare Alternative, so daß die Wechselbarrieren für einen Bahnreisenden vergleichsweise gering ausfallen. Die Attraktivität von Konkurrenzangeboten, wie z.B. die in jüngerer Zeit im innerdeutschen Flugverkehr offerierten Niedrigpreisangebote, oder der grundsätzliche Wunsch nach Abwechslung stellen weitere mögliche Einflußfaktoren dar.

Auch ist der Einfluß situativer Faktoren nicht auszuschließen. Wetterbedingt kann "unfreiwillige Loyalität" die Folge sein. ${ }^{100}$ In diesem Fall binden nicht vertragliche Verpflichtungen oder eine hohe Kundenzufriedenheit einen Nachfrager an das Feedback- und Anreizsysteme in Beherbergungsunternehmen, a.a.O., S. 62. der Automobilindustrie: Ergebnisse einer empirischen Untersuchung, a.a.O., S. 27. leistungen: Erfolgswirksamkeit, Messung, Modellierung, a.a.O., S. $101 \mathrm{f}$. 
Unternehmen, sondern rein situative Komponenten. Ebenso ist es denkbar, daß ein Arbeitnehmer den Reisevorschriften des Unternehmens folgend die Bahn nutzt, private Reisen trotz hoher Zufriedenheit dagegen mit dem Pkw unternimmt, um am Zielort der Reise über eine höhere Mobilität zu verfügen.

Insgesamt ist davon auszugehen, daß nur ausgesprochen zufriedene Kunden ein höheres Maß an Loyalität gegenüber der Bahn zeigen. Für die Überprüfung dieser Vermutung wurde die Transaktionszufriedenheit der Befragten dem bekundeten Wiedernutzungsverhalten gegenübergestellt (vgl. Abbildung 41).

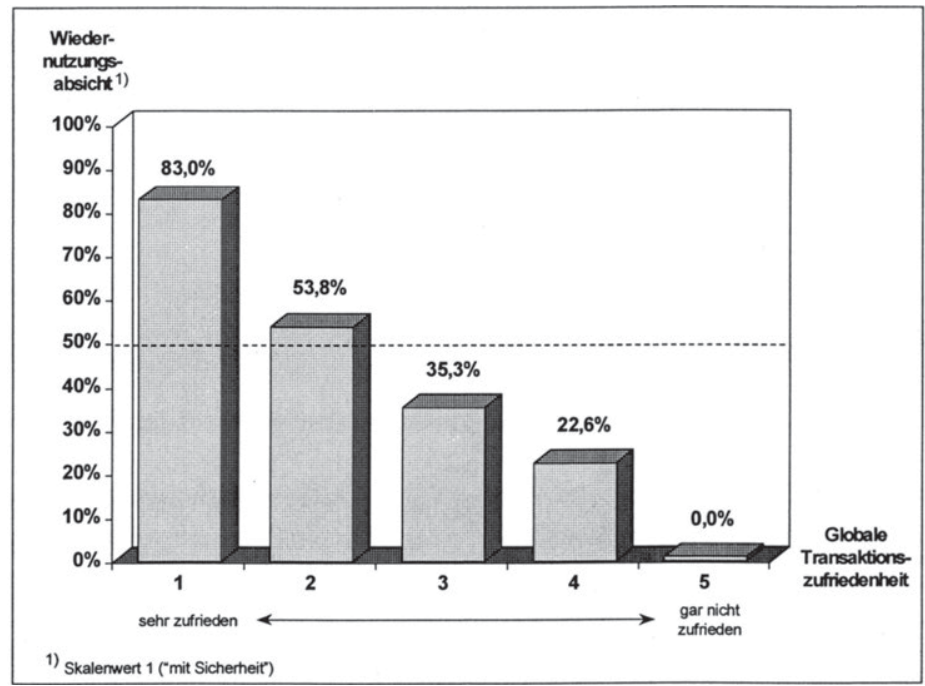

Abb. 41: Kundenloyalität in Abhängigkeit vom Niveau der Transaktionszufriedenheit

Hier zeigt sich, daß das (bekundete) Wiedernutzungsverhalten in hohem Maße von der Transaktionszufriedenheit beeinflußt wird. Darüber hinaus ist Abbildung $41 \mathrm{zu}$ entnehmen, daß die Kundenloyalität eine ausgesprochen sensible Größe darstellt. Nimmt die Zufriedenheit vom Optimalwert 1 nur um eine Einheit ab, sind sich bereits nur noch gut die Hälfte der Befragten sicher, die Bahn ein erneutes Mal zu nutzen. Sinkt die Zufriedenheit um einen weiteren Punkt, bekunden sogar nur noch ca. $35 \%$ der Bahnreisenden, bei ihrer nächsten Reise mit Sicherheit wieder mit der Bahn fahren zu wollen. Auf der einen Seite wird damit deutlich, daß der Gesamtheit der Bahnkunden angesichts ihrer augenscheinlich geringen Wechselbarrieren ein hohes Fluktuationspotential inhärent ist. Auf der anderen 
Seite sind aber auch die Chancen für eine höhere Kundenbindung offenkundig, da bereits eine leichte Erhöhung des Zufriedenheitsniveaus zu einer deutlich höheren Loyalitätsrate führt. Damit kommt dem Zufriedenheitsmanagement eine zentrale Stellung für die Kundenbindung und die Sicherung des langfristigen Unternehmenserfolges zu.

Zusammenfassend ist festzuhalten, daß hohe Zufriedenheit ein sehr wichtiger, aber kein absoluter Garant für die Wiedernutzung der Bahn ist, da die Verkehrsmittelwahl von einer Vielzahl von Komponenten abhängt. Ungeachtet dieser Einschränkung bleibt jedoch aufgrund der empirischen Untersuchungsergebnisse festzuhalten, daß die Zufriedenheit einen nachhaltigen Einfluß auf die Wiedernutzung des Verkehrsmittels Bahn ausübt. Somit läßt eine (sehr) zufriedenstellende Bahnreise das Verkehrsmittel Bahn (wieder bzw. weiterhin) zu einer echten Alternative für einen Reisenden bei seiner nächsten Verkehrsmittelwahl werden, womit das Fundament für eine langfristige Aufnahme der Bahn in das "Evoked Set" eines Nachfragers von Verkehrsdienstleistungen geschaffen wird. ${ }^{101}$

Inwieweit die oben angesprochenen Einflußfaktoren die Zufriedenheit mit einer Bahnreise und damit auch das zukünftige Verhalten der Kunden gegenüber dem Verkehrsmittel Bahn beeinflussen, ist Gegenstand der folgenden Ausführungen.

\subsection{Analyse der Bestimmungsfaktoren der Zufriedenheit mit Verkehrs- dienstleistungen}

Die Analyse der Bestimmungsfaktoren der Kundenzufriedenheit mit Bahnreisen ist in zwei Abschnitte aufgeteilt. Dabei erfolgt zunächst die Untersuchung ausgewählter personenbezogener Determinanten, der sich eine Betrachtung zentraler reisebzw. verkehrsdienstleistungsspezifischer Einflußfaktoren anschließt.

\subsection{Personenbezogene Bestimmungsfaktoren}

Für die Untersuchung der Wirkung personenbezogener Einflußfaktoren auf die Kundenzufriedenheit mit Verkehrsdienstleistungen wird zwischen soziodemographischen und psychographischen Bestimmungsfaktoren unterschieden. Im Mittelpunkt der Ausführungen stehen die Unterschiede in der Kundenzufriedenheit auf der Episoden- und Transaktionsebene sowie etwaige Abweichungen in der Zufriedenheitsdynamik. 


\subsection{Soziodemographische Bestimmungsfaktoren}

Zunächst werden die Ausprägungen der Kundenzufriedenheit differenziert nach den zentralen demographischen Merkmalen Geschlecht und Alter analysiert. Die entsprechenden Ergebnisse auf der Episoden- und Transaktionsebene sind in Abbildung 42 dargestellt.

Eine erste Betrachtung der Mittelwertunterschiede ${ }^{102}$ über alle Altersgruppen ( $F$ Test) führt zu dem Ergebnis, daß mit Ausnahme der Umsteigephase signifikante Unterschiede in der Zufriedenheit mit den einzelnen Episoden als auch der gesamten Bahnreise in Abhängigkeit vom Alter der Befragten festzustellen sind. Interessanterweise fält das Zufriedenheitsurteil der Reisenden, die 60 Jahre und älter sind, durchgehend positiver aus. Augenscheinlich stehen ältere Menschen einer Bahnreise weniger kritisch gegenüber. ${ }^{103}$ Dies zeigt sich auch in den zwischen den einzelnen Altersgruppen bestehenden Merkmalsunterschieden. So unterscheiden sich die Zufriedenheiten der älteren Reisenden mit Ausnahme der Umsteigephase signifikant positiv von denen der beiden anderen Altersgruppen. Während sich die jüngeren Bahnfahrer (bis 26 Jahre) von den Reisenden der mittleren Altersgruppe hinsichtlich ihrer Vor-Reise-, Nach-Reise- und globalen Transaktionszufriedenheit nicht nachweislich voneinander abheben, ergeben sich im Hinblick auf die weiteren Episoden sowie insbesondere auf das Preis-/ Leistungsverhältnis erkennbar niedrigere Zufriedenheitsurteile dieser Altersgruppe. Demnach wird eine Bahnreise von jüngeren Reisenden tendenziell kritischer beurteilt. Das relativ schlechte Urteil über das Preis-/Leistungsverhältnis der Bahnfahrt ist vermutlich auf das dieser Gruppe zur Verfügung stehende vergleichsweise geringere Einkommen zurückzuführen. ${ }^{104}$ Ebenso ist es denkbar, daß diese Altersgruppe ein grundsätzlich höheres Anspruchsniveau an eine

Zur Untersuchung von Merkmalsunterschieden zwischen den verschiedenen Teilstichproben wurden als gruppenübergreifendes, globales Verfahren der F-Test sowie für Einzelunterschiede zwischen zwei Mittelwerten im Mehr-Gruppenfall der Scheffee'-Test und im ZweiGruppenfall der T-Test eingesetzt. Zu den verschiedenen Verfahren vgl. Bauer, F., Datenanalyse mit SPSS, a.a.O., S. $58 \mathrm{ff}$.

Dies wird durch die Ergebnisse der offenen Frage nach positiven Erlebnissen bestätigt. Hier wurde von 40 hauptsächlich älteren Reisenden der hohe Erholungswert einer Bahnreise explizit hervorgehoben. Diese Angaben sind nicht Bestandteil der bisherigen Auswertung kritischer Ereignisse, da sie nicht einer spezifischen Episode einer Bahnreise zugeordnet werden können, sondern vielmehr für die gesamte Reise Gültigkeit haben.

So verfügen lediglich ca. $23 \%$ der dieser Altersgruppe angehörenden Personen über ein monatliches Haushaltsnettoeinkommen von über 2.500 DM. 


\begin{tabular}{|c|c|c|c|c|c|c|c|c|c|c|c|}
\hline \multicolumn{2}{|c|}{$\begin{array}{l}\text { Interphasen- } \\
\text { betrachtung }\end{array}$} & \multicolumn{10}{|c|}{ Demographische Bestimmungsfaktoren } \\
\hline \multirow{3}{*}{ Ebenen } & \multirow{3}{*}{ Indikatoren } & \multicolumn{7}{|c|}{ Alter } & \multicolumn{3}{|c|}{ Geschlecht } \\
\hline & & \multirow{2}{*}{$\begin{array}{r}\text { Bis } 26 \mathrm{~J} . \\
\square .1 \\
\end{array}$} & \multirow{2}{*}{$\begin{array}{l}27 \text { bis } \\
59 \mathrm{~J} . \\
2\end{array}$} & \multirow{2}{*}{$\begin{array}{r}\begin{array}{c}60 \mathrm{JJ} \\
\text { und älter } \\
3\end{array} \\
\end{array}$} & \multicolumn{3}{|c|}{ Scheffeé-Test } & \multirow{2}{*}{ F-Test } & \multirow{2}{*}{ weibl. } & \multirow{2}{*}{ männl. } & \multirow{2}{*}{ T-Test } \\
\hline & & & & & $1 / 2$ & $1 / 3$ & $2 / 3$ & & & & \\
\hline \multirow{4}{*}{$\begin{array}{l}\frac{5}{9} \\
\frac{8}{8} \\
\frac{.0}{2} \\
\text { யி }\end{array}$} & $\begin{array}{l}\text { Vor-Reise- } \\
\text { zufriedenheit }\end{array}$ & 2,30 & 2,32 & 1,95 & n.s. & $\star \star$ & $\star \star$ & $\star \star$ & 2,18 & 2,34 & $\star$ \\
\hline & $\begin{array}{c}\text { Reise- } \\
\text { zufriedenheit }\end{array}$ & 2,35 & 2,17 & 1,70 & * & $\star \star$ & $\star \star$ & $\star \star$ & 2,17 & 2,12 & n.s. \\
\hline & $\begin{array}{l}\text { Umsteige- } \\
\text { zufriedenheit }\end{array}$ & 2,77 & 2,40 & 2,47 & * & n.s. & n.s. & n.s. & 2,44 & 2,59 & n.s. \\
\hline & $\begin{array}{l}\text { Nach-Reise- } \\
\text { zufriedenheit }\end{array}$ & 2,30 & 2,25 & 1,81 & n.s. & $\star \star$ & $\star \star$ & $\star \star$ & 2,04 & 2,33 & $\star \star$ \\
\hline \multirow{2}{*}{ 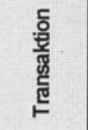 } & $\begin{array}{c}\text { Globale } \\
\text { Zufriedenheit }\end{array}$ & 2,25 & 2,21 & 1,98 & n.s. & $\star \star$ & $\star \star$ & $\star \star$ & 2,19 & 2,18 & n.s. \\
\hline & $\begin{array}{l}\text { Zufriedenheit } \\
\text { Preis/Leistung }\end{array}$ & 3,00 & 2,76 & 2,23 & $\star \star$ & $\star \star$ & $\star \star$ & $\star \star$ & 2,79 & 2,68 & n.s. \\
\hline
\end{tabular}

Signifikanzniveau: $\quad \alpha<0,05=\star \star \quad / \alpha<0,1=\star \quad /$ nicht signifikant $=$ n.s.

\section{Abb. 42: Einfluß demographischer Bestimmungsfaktoren auf die Kundenzufriedenheit}

Bahnreise stellt. ${ }^{105}$ Insgesamt ist damit festzuhalten, daß das Alter der Reisenden einen nachweisbaren Einfluß auf die Zufriedenheit sowohl mit einzelnen Episoden einer Bahnreise als auch mit der gesamten Transaktion ausübt. Auf der Basis der empirischen Ergebnisse ist damit Hypothese Hyp Dem 1 mit Ausnahme der Umsteigephase als bestätigt anzusehen.

In einem nächsten Schritt soll der Frage nachgegangen werden, ob in Abhängigkeit vom Alter der Reisenden sowohl zwischen den einzelnen Episoden einer Bahnreise als auch in Hinblick auf die gesamte Zufriedenheit unterschiedlich starke Wirkungen festgestellt werden können. Zu diesem Zweck wurden für die verschiedenen Altersgruppen separate konfirmatorische Faktorenanalysen durchgeführt, deren Ergebnisse in Abbildung 43 dargestellt sind. ${ }^{106}$ Anspruchshaltung gegenüber Produkten und Dienstleistungen auszumachen. Vgl. Lanz, I., Die Masse steigt aus der Massengesellschaft aus, in: FWW, H. 24, 1996, S. 34. zur Analyse der Struktur und Dynamik der Transaktionszufriedenheit verwendet. Die Güte- 


\begin{tabular}{|c|c|c|c|c|c|c|c|c|c|c|}
\hline & & \multicolumn{3}{|c|}{ bis 26 Jahre $(20 \%)$} & \multicolumn{3}{|c|}{27 - 59 Jahre $(65 \%)$} & \multicolumn{3}{|c|}{60 Jahre und älter $(15 \%)$} \\
\hline & auf & $\begin{array}{c}\begin{array}{c}\text { Voi- } \\
\text { Reise- } \\
\text { zufrieden- } \\
\text { heit }\end{array} \\
\end{array}$ & $\begin{array}{c}\text { Reise- } \\
\text { zufrieden- } \\
\text { heit }\end{array}$ & $\begin{array}{c}\begin{array}{c}\text { Nach- } \\
\text { Reise- } \\
\text { zufrieden- } \\
\text { heit }\end{array} \\
\end{array}$ & \begin{tabular}{|c|}
$\begin{array}{c}\text { Vor- } \\
\text { Reise- } \\
\text { zufrieden- } \\
\text { heit }\end{array}$ \\
\end{tabular} & $\begin{array}{c}\begin{array}{c}\text { Reise- } \\
\text { zufrieden- } \\
\text { heit }\end{array}\end{array}$ & $\begin{array}{c}\begin{array}{c}\text { Nach- } \\
\text { Reise- } \\
\text { zufrieden- } \\
\text { heit }\end{array} \\
\end{array}$ & $\begin{array}{c}\text { Vor- } \\
\text { Reise- } \\
\text { zufrieden- } \\
\text { heit }\end{array}$ & $\begin{array}{c}\text { Reise- } \\
\text { zufrieden- } \\
\text { heit }\end{array}$ & $\begin{array}{c}\begin{array}{c}\text { Nach- } \\
\text { Reise- } \\
\text { zufrieden- } \\
\text { heit }\end{array} \\
\end{array}$ \\
\hline \multirow{3}{*}{ 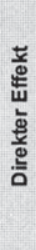 } & $\begin{array}{c}\text { Reise- } \\
\text { zufriedenheit }\end{array}$ & 0,10 & - & - & 0,38 & - & - & 0,11 & - & - \\
\hline & $\begin{array}{l}\text { Nach-Reise- } \\
\text { zufriedenheit }\end{array}$ & 0,36 & 0,32 & - & 0,22 & 0,22 & - & 0,46 & 0,17 & - \\
\hline & $\begin{array}{l}\text { Transaktions- } \\
\text { zufriedenheit }\end{array}$ & 0,12 & 0,60 & 0,06 & 0,29 & 0,69 & 0,11 & 0,20 & 0,48 & 0,15 \\
\hline \multirow{3}{*}{ 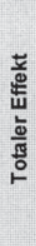 } & $\begin{array}{c}\text { Reise- } \\
\text { zufriedenheit }\end{array}$ & $0,10^{1)}$ & - & - & $0,38^{1)}$ & - & - & $0,11^{1)}$ & - & - \\
\hline & $\begin{array}{l}\text { Nach-Reise- } \\
\text { zufriedenheit }\end{array}$ & 0,39 & $0,32^{1)}$ & - & 0,30 & $0,22^{1)}$ & - & 0,48 & $0,17^{1)}$ & - \\
\hline & $\begin{array}{l}\text { Transaktions- } \\
\text { zufriedenheit }\end{array}$ & 0,20 & 0,62 & $0,06^{1)}$ & 0,59 & 0,71 & $0,11^{1)}$ & 0,32 & 0,51 & $0,15^{1)}$ \\
\hline
\end{tabular}

1) ${ }_{\text {totaler Effekt }}=$ direkter Effekt

Abb. 43: Beziehungszusammenhänge der Kundenzufriedenheit in Abhängigkeit vom Alter

Eine nähere Betrachtung der hier angegebenen direkten Effekte der Kausalanalysen zeigt, daß die Dynamik der Kundenzufriedenheit auf der Episodenebene in den Gruppen der jüngeren und älteren Reisenden vergleichbar ausgeprägt ist. ${ }^{107}$ Im Gegensatz zu diesen Altersklassen ist für die Personen der mittleren Alterskategorie ein deutlich höherer Einfluß der Vor-Reisezufriedenheit auf die Zufriedenheit mit der Reisephase und der Transaktion auszumachen (Koeffizienten 0,38 bzw. 0,29). Eine mögliche Erklärung ist darin zu finden, daß die mittlere Altersgruppe den vergleichsweise höchsten Anteil an Familienreisen ausweist. Besonders für diese Reisenden ist ein tendenziell höherer Planungsund Koordinationsaufwand in der Vor-Reisephase zu erwarten, was mit einem stärkeren Einfluß der Zufriedenheit mit dieser Episode auf die Reise- und Transaktionszufriedenheit einhergeht. Eine weitere Ursache hierfür liegt in dem

beurteilung dieser und der weiteren in Kap. C 2.4 dieser Arbeit behandelten Modelle führte in allen Fällen auch unter Berücksichtigung der jeweiligen Stichprobengrößen zu insgesamt zufriedenstellenden Werten. Zu einer Übersicht vgl. die im Anhang I dokumentierten Abbildungen $A 1$ und $A 2$.

So werden in diesen Altersklassen die Varianzen der Reise- und Nach-Reisezufriedenheit zu vergleichbaren Anteilen erklärt. Vgl. hierzu die im Anhang I dokumentierte Abbildung A1. 
Umstand begründet, daß die Geschäftsreisen zu nahezu $90 \%$ von dieser Altersgruppe getätigt werden und damit der problemlose Start in den Arbeitstag eine besondere Stellung einnimmt. ${ }^{108}$ Darüber hinaus ist bei einer Betrachtung aller Altersklassen eine mit steigendem Alter der Befragten leicht zunehmende Bedeutung der Nach-Reisephase für die Bildung des Zufriedenheitsurteils über die gesamte Bahnreise zu konstatieren, was möglicherweise auf die geringere körperliche Mobilität älterer Personen zurückzuführen ist.

Der hohe Einfluß der Vor-Reisephase auf die Gesamtzufriedenheit in der mittleren Altersgruppe wird bei einer Betrachtung der totalen Effekte nochmals unterstrichen (Koeffizient 0,59). Bemerkenswert ist darüber hinaus der relativ geringe Einfluß der Vor- und der Nach-Reisephase auf die Gesamtzufriedenheit der Gruppe der jüngeren Bahnreisenden. Augenscheinlich basiert das globale transaktionsbezogene Zufriedenheitsurteil dieser Altersklasse in erster Linie auf der Zufriedenheit mit der Reisephase, während die weiteren Episoden einen nur geringen Stellenwert einnehmen. Denkbare Ursachen sind die vergleichsweise hohe Mobilität jüngerer Menschen sowie die damit verbundene flexiblere Gestaltung und die von der eigentlichen Kerndienstleistung weitgehend losgelöste Wahrnehmung dieser Abschnitte einer Bahnreise. Insgesamt kann Hypothese Hyp Dem 2 über den mit zunehmendem Alter der Reisenden steigenden Einfluß der Vor- und der Nach-Reisephase auf die Transaktionszufriedenheit hinsichtlich der Nach-Reisephase angenommenen werden. In bezug auf die Vor-Reisephase kann der vermutete Zusammenhang lediglich für die ersten beiden Altersgruppen bestätigt werden.

Die Untersuchung von Merkmalsunterschieden in der Kundenzufriedenheit differenziert nach dem Geschlecht der Befragten führt schließlich zu der Erkenntnis, daß die Episoden, die vor und nach der Kerndienstleistung erlebt werden, von Frauen positiver beurteilt werden (vgl. Abbildung 42). Demnach stehen Männer diesen Abschnitten einer Bahnreise tendenziell kritischer gegenüber. Dagegen können keine nennenswerten Unterschiede hinsichtlich der Beurteilung der Kerndienstleistung, also der Beförderung, als auch nicht in bezug auf die Transaktions- 


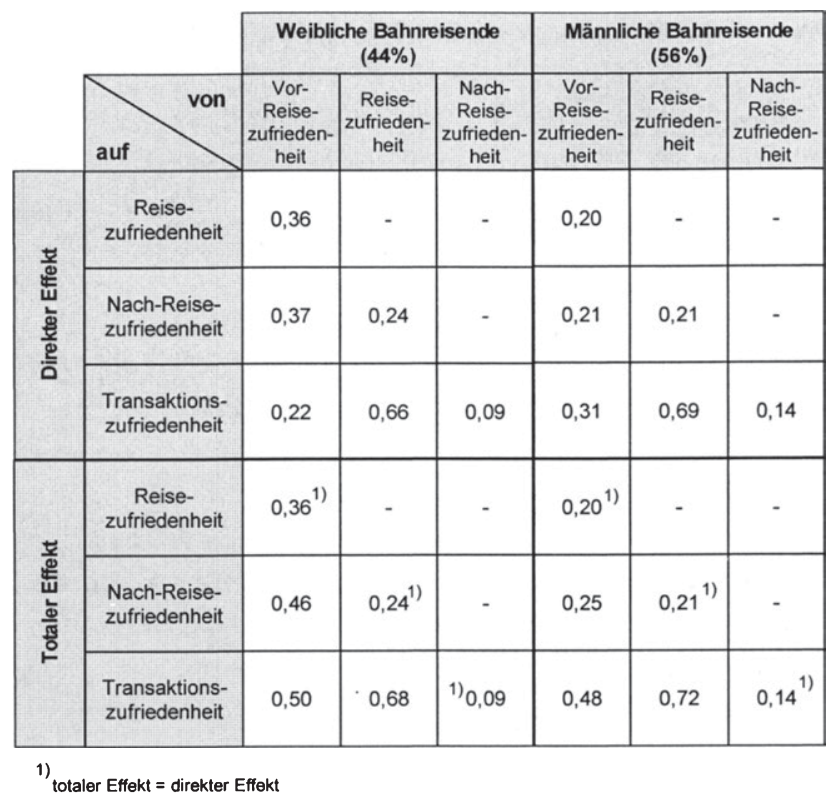

\section{Abb. 44: Beziehungszusammenhänge der Kundenzufriedenheit in Abhängigkeit vom Geschlecht}

zufriedenheit ausgemacht werden. Inwieweit dies den Schluß erlaubt, daß von den verschiedenen Episoden unterschiedliche Wirkungen auf die Transaktionszufriedenheit dieser Kundensegmente ausgehen, die zu einer Angleichung der Zufriedenheitsurteile auf der Transaktionsebene führen, soll auf der Basis der in Abbildung 44 dargestellten Ergebnisse der entsprechenden konfirmatorischen Faktorenanalysen für diese beiden Kundengruppen untersucht werden.

Hier ist zunächst bei der Analyse der direkten Effekte eine höhere Dynamik der Kundenzufriedenheit auf der Episodenebene für die weiblichen Befragten festzustellen. $^{109}$ Dagegen werden die verschiedenen Episoden von den männlichen Reisenden, die dabei der Reisephase ein leicht höheres Gewicht bei der Bildung der Gesamtzufriedenheit zumessen, vergleichsweise eigenständiger wahrgenommen. Dieser Zusammenhang wird bei einer Betrachtung der totalen Effekte nachhaltig bestätigt. Während die direkten Effekte für die männlichen

Dies wird auch bei einer Betrachtung der in Abbildung A1 im Anhang I angegebenen Varianzerklärungsanteile für die Reise- und Nach-Reisezufriedenheit deutlich, die bei den weiblichen Befragten erkennbar höher ausfallen. 
Befragten noch einen deutlich höheren Einfluß der Vor-Reisezufriedenheit auf die Transaktionszufriedenheit ausweisen $(0,31$ zu 0,22 bei den weiblichen Personen), führt die bei den weiblichen Fahrgästen stärker ausgeprägte Dynamik der Kundenzufriedenheit zu einer Nivellierung dieser Effekte. Dabei ist sogar ein etwas höherer totaler Effekt der Vor-Reisezufriedenheit auf die Transaktionszufriedenheit bei den weiblichen Reisenden festzustellen $(0,50$ zu 0,48 bei den männlichen Personen).

Darüber hinaus ist ein etwas schwächerer Einfluß der Nach-Reisephase hinsichtlich der Entstehung der Transaktionszufriedenheit bei den weiblichen Reisenden zu erkennen. Da festgestellt wurde, daß sich beide Kundensegmente hinsichtlich ihrer Gesamtzufriedenheit mit der Bahnreise nicht signifikant voneinander unterscheiden, ist zu vermuten, daß der tendenziell unterschiedliche Einfluß der verschiedenen Episoden zu einer Angleichung der Kundenurteile der beiden Segmente führt. Insgesamt ist Hypothese Hyp Dem 3 über den Einfluß des Geschlechts auf die Kundenzufriedenheit im Hinblick auf die Zufriedenheit mit einzelnen Episoden anzunehmen, dagegen aber in bezug auf die Gesamtzufriedenheit abzulehnen.

Eine Analyse des Einflusses sozioökonomischer Faktoren auf die Kundenzufriedenheit zeigt, daß die globale transaktionsbezogene Zufriedenheit nicht nachhaltig durch das Netto-Haushaltseinkommen der Befragten beeinflußt wird (vgl. Abbildung 45). Dagegen ist ein signifikanter Unterschied in der Beurteilung des Preis-/Leistungsverhältnisses festzustellen, wenngleich zwischen den Befragten mit mittlerem und höherem Einkommen keine nennenswerten Differenzen vorliegen. Erwartungsgemäß erweisen sich Personen mit einem geringeren Einkommen (bis DM 2.500) als weniger zufrieden. Auf der Episodenebene werden die Vor- und Reisephase ebenfalls durch die Reisenden, die dieser Einkommensgruppe angehören, vergleichsweise schlechter beurteilt. Dies schlägt sich jedoch kaum in der transaktionsbezogenen Zufriedenheit nieder. Die unterschiedliche Beurteilung des Preis-/Leistungsverhältnisses dagegen ist vermutlich darauf zurückzuführen, daß die Befragten bei ihrer Urteilsbildung insbesondere auf die Vor- und Reisephase abstellen, da diese den (erweiterten) Kern der in Anspruch genommenen und bezahlten Dienstleistung darstellen. Im Rahmen der kausalanalytischen Überprüfung der Struktur und Dynamik der Kundenzufriedenheit in Abhängigkeit vom Einkommen konnten keine deutlichen Unterschiede bzw. eindeutigen Trends festgestellt werden, so daß die episodenbezogene Wahrnehmung der Kundenzufriedenheit als vom Einkommen 


\begin{tabular}{|c|c|c|c|c|c|c|c|c|c|c|c|c|c|c|}
\hline \multicolumn{2}{|c|}{$\begin{array}{l}\text { Interphasen- } \\
\text { betrachtung }\end{array}$} & \multicolumn{13}{|c|}{ Sozioökonomische Bestimmungsfaktoren } \\
\hline \multirow{3}{*}{ Ebenen } & \multirow{3}{*}{ Indikatoren } & \multicolumn{7}{|c|}{ Haushaltseinkommen (Netto) } & \multicolumn{3}{|c|}{$\begin{array}{l}\text { PKW (dauerhaft) } \\
\text { verfügbar }\end{array}$} & \multicolumn{3}{|c|}{$\begin{array}{l}\text { Alternatives Ver- } \\
\text { kehrsmittel erwogen }\end{array}$} \\
\hline & & \multirow{2}{*}{$\begin{array}{c}\text { bis } \\
2.500 \\
\mathrm{DM}\end{array}$} & \multirow{2}{*}{$\begin{array}{r}2.501 \\
-5.000 \\
\mathrm{DM} \\
\end{array}$} & \multirow{2}{*}{\begin{tabular}{c|c} 
aber \\
5.000 \\
$\mathrm{DM}$ \\
\end{tabular}} & \multicolumn{3}{|c|}{ Scheffeé-Test } & \multirow{2}{*}{ F-Test } & \multirow{2}{*}{ Ja } & \multirow{2}{*}{ Nein } & \multirow{2}{*}{ T-Test } & \multirow{2}{*}{$\mathrm{Ja}$} & \multirow{2}{*}{ Nein } & \multirow{2}{*}{ T-Tes: } \\
\hline & & & & & $1 / 2$ & $1 / 3$ & $2 / 3$ & & & & & & & \\
\hline \multirow{4}{*}{$\begin{array}{l}\frac{5}{5} \\
\frac{8}{8} \\
\text { 훔 }\end{array}$} & $\begin{array}{l}\text { Vor-Reise- } \\
\text { zufriedenheit }\end{array}$ & 2,41 & 2,15 & 2,27 & $\star \star$ & n.s. & n.s. & * & 2,30 & 2,10 & * & 2,41 & 2,21 & $\star \star$ \\
\hline & $\begin{array}{l}\text { Reise- } \\
\text { zufriedenheit }\end{array}$ & 2,30 & 2,06 & 2,14 & $\star \star$ & n.s. & n.s. & * & 2,17 & 1,98 & $\star$ & 2,37 & 2,06 & $\star \star$ \\
\hline & $\begin{array}{l}\text { Umsteige- } \\
\text { zufriedenheit }\end{array}$ & 2,63 & 2,54 & 2,46 & n.s. & n.s. & n.s. & n.s. & 2,51 & 2,65 & n.s. & 2,84 & 2,40 & $\star \star$ \\
\hline & $\begin{array}{l}\text { Nach-Reise- } \\
\text { zufriedenheit }\end{array}$ & 2,31 & 2,13 & 2,20 & n.s. & n.s. & n.s. & n.s. & 2,23 & 2,10 & n.s. & 2,35 & 2,15 & $\star \star$ \\
\hline \multirow{2}{*}{ 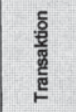 } & $\begin{array}{c}\text { Globale } \\
\text { Zufriedenheit }\end{array}$ & 2,28 & 2,14 & 2,16 & n.s. & n.s. & n.s. & n.s. & 2,22 & 1,96 & $\star \star$ & 2,39 & 2,09 & $\star \star$ \\
\hline & $\begin{array}{l}\text { Zufriedenheit } \\
\text { Preis/Leistung }\end{array}$ & 2,92 & 2,71 & 2,61 & * & $\star \star$ & n.s. & $\star \star$ & 2,74 & 2,73 & n.s. & 3,00 & 2,62 & $\star \star$ \\
\hline
\end{tabular}

Signifikanzniveau: $\quad \alpha<0,05=\star$ / $\alpha<0,1=\star I$ nicht signifikant $=$ n.s.

Abb. 45: Einfluß sozioökonomischer Bestimmungsfaktoren auf die Kundenzufriedenheit

der Befragten weitgehend unabhängig angesehen werden kann. ${ }^{110}$ Hypothese Hyp Okon 1 ist damit insgesamt abzulehnen. Bei differenzierter Betrachtung kann jedoch eine Beeinflussung einzelner Episodenzufriedenheiten sowie der Zufriedenheit mit dem Preis-/Leistungsverhältnis im Ansatz bestätigt werden.

Die Verfügbarkeit eines Pkws stellt ein sozioökonomisches Merkmal dar, welches einen Reisenden in die Lage versetzt, die Bahn mit einem grundsätzlich dauerhaft zur Verfügung stehenden und vermeintlich „preiswerteren“ Verkehrsmittel zu vergleichen. ${ }^{111}$ Inwieweit die Verfügbarkeit eines Pkws die Zufriedenheit mit einer Bahnreise beeinflußt, soll daher im folgenden untersucht werden. Die in Abbildung 45 angegebenen Mittelwerte führen zu dem interessanten Ergebnis, daß die Beurteilung des Preis-/Leistungsverhältnisses durch diesen Bestimmungsfaktor nicht nachhaltig beeinflußt wird. Dagegen ist die Transaktionszufriedenheit der Befragten, die ständig über einen Pkw verfügen, signifikant

110 Vgl. hierzu die Abbildungen A2 und A3 im Anhang I. Auf eine entsprechende Untersuchung des soziookonomischen Merkmals Beruf wird an dieser Stelle verzichtet, da diesem Bestimmungsfaktor nur dann eine hőhere Verhaltensrelevanz zugesprochen wird, wenn die Nachfrage nach einem Produkt oder einer Dienstleistung in einem engen Zusammenhang mit der beruflichen Tătigkeit steht. Vgl. Frömbling, S., Zielgruppenmarketing im Fremdenverkehr von Regionen: Ein Beitrag zur Marktsegmentierung auf der Grundlage von Werten, Motiven und Einstellungen, Frankfurt am Main 1993, S. 74. Daher erfolgt in Kap. C 2.42 die Untersuchung etwaiger Unterschiede in der Kundenzufriedenheit in Abhängigkeit vom Reiseanlaß, in der zwischen Geschăfts- bzw. Dienstreisen und Reisen aus privaten Anlässen differenziert wird. 
niedriger ausgeprägt. Anscheinend kommt hier eine vergleichsweise höhere psychische Distanz von Pkw-Fahrern gegenüber der Reiseform „Bahnfahren“ zum Ausdruck. Dies ist vermutlich auch die Ursache für die niedrigere Vor-Reise- und Reisezufriedenheit dieses Kundensegmentes. Insgesamt ist damit Hypothese Hyp Ökon 2 über den Einfluß der Pkw-Verfügbarkeit auf die Zufriedenheit mit einer Bahnreise als zumindest teilweise bestätigt anzusehen.

Diese Tendenzen erfahren eine Verstärkung, wenn von den Befragten neben der Bahn ein alternatives Verkehrsmittel für ihre Reise in Erwägung gezogen wurde (vgl. Abbildung 45). In der vorliegenden Stichprobe sind dies ca. $30 \%$ der Befragten, die vornehmlich den Pkw als Alternative angesehen haben. ${ }^{112}$ Diese Personen bekunden sowohl auf der Episoden- als auch auf der Transaktionsebene durchgehend signifikant niedrigere Zufriedenheiten. Insbesondere der vergleichsweise hohe Unterschied in der Zufriedenheit mit der Umsteigephase ist hier auffällig. Offensichtlich werden mit der Nutzung alternativer Verkehrsmittel Vorteile z.B. hinsichtlich der grundsätzlichen Bequemlichkeit oder einer höheren Mobilität am Zielort verbunden, die zu einer Beeinträchtigung der Zufriedenheit mit einer Bahnreise und mit ihren einzelnen Abschnitten führen. Damit kann Hypothese Hyp vм über den Einfluß der Erwägung eines alternativen Verkehrsmittels auf die Zufriedenheit mit einer Bahnreise auf der Basis der empirischen Ergebnisse angenommen werden.

In Abbildung 46 sind die mit den beschriebenen Differenzen einhergehenden Unterschiede in den Beziehungszusammenhängen der Kundenzufriedenheit für die Bestimmungsfaktoren „Pkw-Verfügbarkeit“ und „Erwägung eines alternativen Verkehrsmittels" dargestellt.

112 Ca. $80 \%$ der Befragten, die über die Nutzung eines anderen Verkehrsmittel nachgedacht haben, nannten dabei den Pkw, weitere $19 \%$ das Flugzeug als Alternative.

Andreas Siefke and Universität Münster - 978-3-631-75103-9 


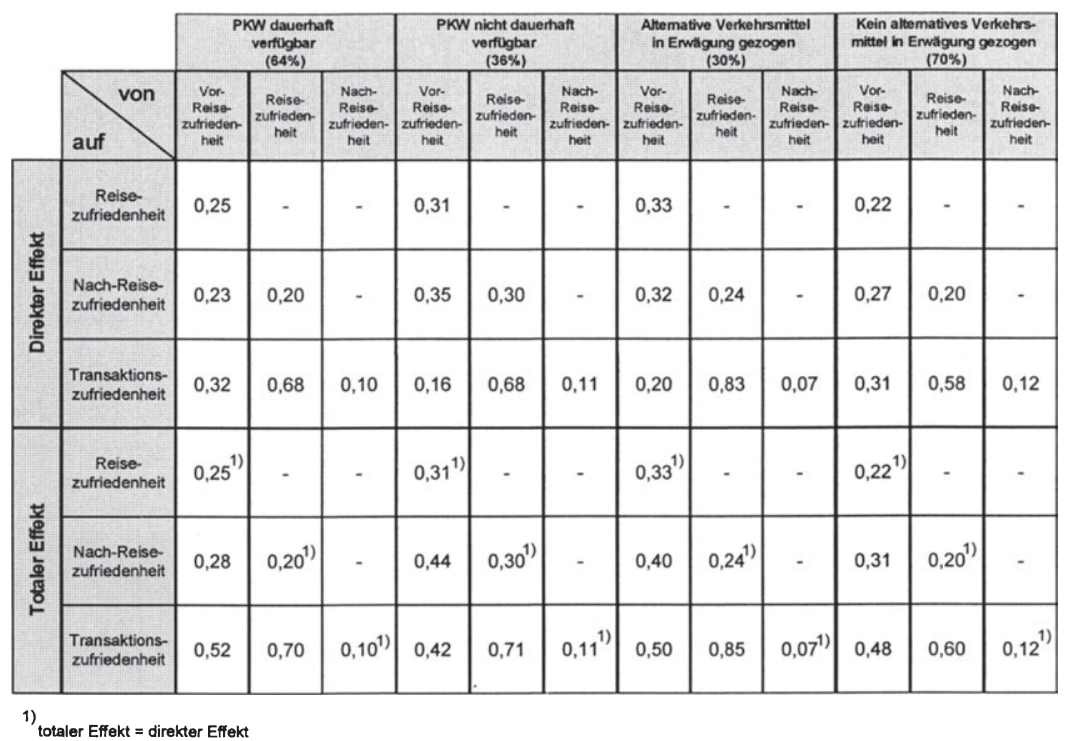

\section{Abb. 46: Beziehungszusammenhänge der Kundenzufriedenheit in Abhängigkeit von sozioökonomischen Bestimmungsfaktoren}

In bezug auf den Bestimmungsfaktor „Pkw-Verfügbarkeit" ist bei einer Betrachtung der direkten Effekte zunächst eine leicht höhere Zufriedenheitsdynamik auf der Episodenebene für diejenigen Reisenden festzustellen, die nicht über einen $P k w$ verfügen. Demnach stehen die einzelnen Episoden hier in einem insgesamt stärkeren Wahrnehmungsverbund. ${ }^{113}$ Dabei messen die "Pkw-Besitzer" der VorReisezufriedenheit eine höhere Bedeutung bei der Bildung der Zufriedenheit mit der Transaktion zu. Dies ist auch bei einer Analyse der totalen Effekte zu erkennen. ${ }^{114} \mathrm{Da}$ die „Pkw-Besitzer" mit dieser Phase weniger zufrieden sind, wird auch die geringere Transaktionszufriedenheit dieser Gruppe von Reisenden nachvollziehbar. Offenkundig nimmt der zufriedenstellende Verlauf der Vor-Reisephase für diesen Kundenkreis eine wichtige Rolle ein. Diejenigen Personen dagegen, die nicht über einen Pkw verfügen, messen dieser Phase eine geringere Bedeutung bei. Angesichts der Tatsache, daß dieser Gruppe nicht dauerhaft ein Pkw zur freien Verfügung steht, sind diese Personen zur Befriedigung ihrer Mobilitäts-

Vgl. hierzu auch die entsprechenden Varianzerklärungsanteile für die einzelnen Episodenzufriedenheiten in der Abbildung A1 im Anhang I dieser Arbeit.

114 Wăhrend der totale Effekt der Vor-Reisephase bei den „Pkw-Besitzern 0,52 betrăgt, liegt der entsprechende Wert bei den Personen, die nicht dauerhaft über einen Pkw verfügen, bei 0,42.

Andreas Siefke and Universität Münster - 978-3-631-75103-9 
bedürfnisse in erster Linie auf das Angebot von Verkehrsdienstleistungsunternehmen angewiesen. Es liegt daher nahe zu vermuten, daß diese Personengruppe grundsätzlich mit der Nutzung von Verkehrsdienstleistungen vertrauter ist bzw. über eine größere Routine verfügt, so daß der Einfluß der Vor-Reisephase auf die Transaktionszufriedenheit entsprechend geringer ausfällt, da zudem für diese Personen die (dauerhafte) Vergleichsmöglichkeit mit einer Pkw-Nutzung entfällt.

Auch die Erwägung eines alternativen Verkehrsmittels führt zu unterschiedlichen Ausprägungen in den Beziehungsstrukturen der Kundenzufriedenheit. Hier sind insbesondere Unterschiede in der Wichtigkeit der Reisephase für die Bildung der Transaktionszufriedenheit zu erkennen. Die Zufriedenheit mit der Reisephase übt einen deutlich höheren direkten $(0,83)$ als auch totalen Effekt $(0,85)$ auf die Gesamtzufriedenheit derjenigen Personen aus, die für ihre Reise auch die Nutzung eines alternativen Verkehrsmittels in Betracht gezogen haben und damit in ihrer Verkehrsmittelwahl nicht vollständig sicher waren. ${ }^{115}$ Eine mögliche Erklärung für dieses Ergebnis ist darin zu sehen, daß die Personengruppe der „Unentschlossenen“ deshalb der Kerndienstleistung eine zentrale Bedeutung beimißt, da deren (nicht) erfolgreicher Verlauf eindeutig dem Verantwortungsbereich des Unternehmens zugeordnet werden kann. Die mit dieser Episode einer Bahnreise empfundene Zufriedenheit dient somit als zentraler Gradmesser für die Richtigkeit der Verkehrsmittelwahl.

Die Analyse personenbezogener Bestimmungsfaktoren der Kundenzufriedenheit wird im folgenden um die Untersuchung zentraler psychographischer Einflußgrößen erweitert, denen in der Literatur eine besondere Bedeutung bei der Bildung des Zufriedenheitsurteil zuerkannt wird. ${ }^{116}$

\subsection{Psychographische Bestimmungsfaktoren}

Bei den im Rahmen der vorliegenden Arbeit berücksichtigten psychographischen Einflußfaktoren handelt es sich zum einen um das Einstellungskonstrukt, das die grundsätzliche Haltung der Befragten gegenüber der Deutschen Bahn AG abbildet. Zum anderen werden das in Verbindung mit Bahnreisen wahrgenommene Risiko und das Involvement der Befragten in die Analyse einbezogen. Ebenso wie

\footnotetext{
115 Die entsprechen Werte der Personen, die ausschließlich die Bahn für ihre Reise in Erwägung gezogen haben, betragen lediglich 0,58 bzw. 0,60.

116 Vgl. hierzu die Ausführungen in Kap. B 2.1 und B 2.2 dieser Arbeit.
} 
bei der Kundenzufriedenheit handelt es sich auch bei diesen Größen um Variable des Käuferverhaltens, die sich einer direkten Beobachtung entziehen und daher einer Operationalisierung bedürfen.

Zur Erfassung der Einstellungen der Reisenden gegenüber dem Unternehmen Deutsche Bahn AG wurden die Befragten in der vorliegenden Untersuchung gebeten, den Grad ihrer Zustimmung $\mathrm{zu}$ den in Abbildung 47 dargestellten Statements anzugeben. ${ }^{117}$

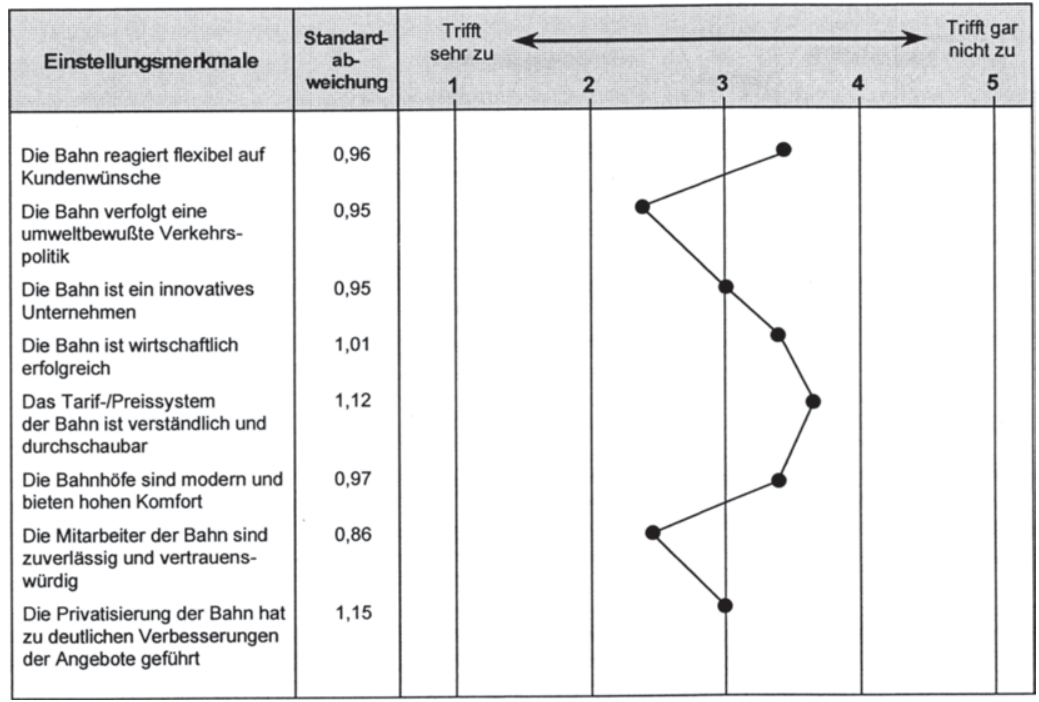

\section{Abb. 47: Einstellungen gegenüber dem Verkehrsdienstleister Deutsche Bahn AG}

Die grundsätzliche Einschätzung der Befragten deutet auf Defizite der Bahn aus Kundensicht hin. So stimmen unter Berücksichtigung der Häufigkeitsverteilung der Skalenwerte lediglich ca. 15\% der Befragten der Aussage in hohem Maße zu, daß die Bahn als Unternehmen flexibel auf Kundenwünsche reagiert und damit eine

Die Urteile wurden anhand einer fünfstufigen Ratingskala erfaßt, die in einem Kontinuum von "trifft sehr $\mathrm{zu}^{\prime \prime}$ bis "trifft gar nicht $\mathrm{zu}^{4}$ die Einschätzung der Befragten mißt. Die Überprüfung dieser auch zur Messung der weiteren Konstrukte verwendeten Ratingskala läßt auf eine hinreichende Reliabilităt der Meßskala schließen. So ergab die Zuverlässigkeitsprüfung anhand Cronbach's Alpha Reliabilitätskoeffizienten zwischen 0,6336 und 0,7839 für die drei Konstrukte. Bei der Split-Half-Methode lagen die entsprechende Werte des Spearman-BrownKoeffizienten zwischen 0,6012 und 0,762. Zur Abgrenzung der Kundenzufriedenheit vom Einstellungskonstrukt vgl. die Ausführungen in Kap. B 2.1 dieser Arbeit. 
ausreichende Kundenorientierung zeigt (Skalenwerte 1 und 2). Dagegen können fast $45 \%$ der Reisenden ein solches Verhalten der Bahn nicht erkennen (Skalenwerte 4 und 5). Ebenso wird die Bahn von über $40 \%$ der Befragten als wirtschaftlich nicht erfolgreich eingeschätzt. Weitere $40 \%$ sind hier in ihrem Urteil indifferent (Skalenwert 3). Auch die in jüngerer Zeit von der Bahn stark forcierten Bemühungen um eine Verbesserung des Serviceniveaus der Bahnhöfe ${ }^{118}$ konnten bislang keine nachhaltige Wirkung auf das grundsätzliche Kundenurteil nach sich ziehen. ${ }^{119}$ So halten nur ca. $18 \%$ der Befragten die Bahnhöfe für modern und komfortabel. Die schlechteste Beurteilung erfährt jedoch das Tarif- bzw. Preissystem der Bahn. Ca. $60 \%$ der Befragten halten dieses für unverständlich und nicht durchschaubar, während lediglich $20 \%$ der Reisenden dieses nicht bzw. kaum als kritikwürdig ansehen.

Auch die Einschätzung der Innovationskraft ist als nur mäßig zu bezeichnen. So zeigen sich $42 \%$ der Reisenden in diesem Punkt indifferent, während weitere $28 \%$ der Bahn diese Fähigkeit (nahezu) gänzlich absprechen. Ebenso sind ca. 35\% der Befragten unentschlossen, der Bahn nach ihrer Privatisierung eine Verbesserung ihrer Angebote zu bescheinigen. Die vergleichsweise hohe Standardabweichung dieses Merkmals deutet jedoch darauf hin, daß zumindest ein Teil der Befragten hier einen positiven Trend erkennt. So sehen immerhin ca. $34 \%$ der Befragten eine Verbesserung des Angebotes seit der Umwandlung des Unternehmens in eine Aktiengesellschaft. Durchaus positiv beurteilen die Befragten die von der Bahn verfolgte Verkehrspolitik. Nahezu 62\% der Befragten attestieren der Bahn ein umweltbewußtes verkehrspolitisches Handeln. Auch die Mitarbeiter der Bahn erfahren eine grundsätzlich positive Beurteilung. So halten lediglich ca. $10 \%$ der Reisenden die Bediensteten der Bahn für nicht zuverlässig und vertrauenswürdig, während ca. $60 \%$ das Personal der Bahn positiv beurteilen. Insgesamt ist jedoch eine eher kritische Grundhaltung der Befragten gegenüber dem Unternehmen zu konstatieren.

Bevor im Rahmen einer kausalanalytischen Untersuchung der Einfluß der Einstellungen der Befragten gegenüber der Deutschen Bahn AG auf die Zufriedenheit mit der erlebten Bahnreise überprüft wird, werden zunächst die weiteren in

118 Vgl. Bahn Extra (Hrsg.), Lexikon Deutsche Bahn, a.a.O., S. $26 \mathrm{ff}$.

119 Auch im folgenden werden die zusammengefaßten Häufigkeiten der Skalenwerte 1 und 2 als eine (hohe) Zustimmung zu dem jeweiligen Item gedeutet. Als indifferent werden die Personen bezeichnet, die der jeweiligen Aussage mit dem mittleren Skalenwert (3) zustimmen. Die zusammengefaßten Häufigkeiten der Skalenwerte 4 und 5 werden wiederum als Zeichen einer (starken) Ablehnung des entsprechenden Indikators interpretiert.

Andreas Siefke and Universität Münster - 978-3-631-75103-9 
der vorliegenden Arbeit berücksichtigten psychographischen Bestimmungsfaktoren näher vorgestellt.

Das Involvement kennzeichnet das Interesse an bzw. die Wichtigkeit von z.B. Produkten oder Dienstleistungen im Wertesystem von Menschen. ${ }^{120}$ Das Involvementkonstrukt wird in dieser Arbeit in zwei verschiedenen Facetten erfaßt. So wird auf der einen Seite der Erlebnischarakter einer Bahnreise berücksichtigt. Dieser auch als hedonistisch ${ }^{121}$ bezeichnete Aspekt des Involvements zielt auf die emotionale Komponente des Konstruktes ab und umfaßt den Spaß und die eigene Belohnung, die mit dem Kauf bzw. dem Konsum eines Produktes oder einer Dienstleistung verbunden sind. ${ }^{122}$ Andererseits wird das Interesse, das der Dienstleistung "Bahnreise" entgegengebracht wird, erfaßt und der Bedeutung, die ein Konsument der Dienstleistung beimißt, Rechnung getragen. Diese hier als Dienstleistungsartinvolvement ${ }^{123}$ bezeichnete Dimension des Involvements beinhaltet den Grad der gedanklichen Auseinandersetzung eines Konsumenten mit einer Dienstleistung und ist somit auf die kognitive Ausprägung des Konstruktes ausgerichtet. ${ }^{124}$

Eine Analyse des in Abbildung 48 dargestellten Mittelwertprofils der Involvementindikatoren führt zu dem Ergebnis, daß das Reisen mit der Bahn mit einem vergleichsweise positiven Erlebniswert verbunden wird. So sehen knapp $54 \%$ der Befragten in einer Bahnreise ein schönes Erlebnis. ${ }^{125}$ Das Dienstleistungsartinvolvement ist dagegen tendenziell schwächer ausgeprägt. Während noch ca. die Hälfte der Befragten angibt, die Bahn für die Anreise zu wichtigen Terminen häufig zu nutzen, zeigen sich nur noch knapp $37 \%$ der Reisenden mit den Angeboten und Preisen der Bahn gut bzw. sehr gut vertraut. Mit einem Anteil von ca. $23 \%$ ist der Personenkreis, der sich regelmäßig über die Angebote der Bahn informiert, nochmals deutlich kleiner (Skalenwerte 1 und 2).

Vgl. Diller, H., Kundenbindung als Marketingziel, a.a.O., S. 87 sowie die Ausführungen in Kap. B 1.21 dieser Arbeit.

Vgl. Laurent, G., Kapferer, J.-N., Measuring Consumer Involvement Profiles, in: JoMR, Vol. 22, February 1985, S. 43.

Vgl. Diller, H., Kundenbindung als Marketingziel, a.a.O., S. 88, Esch, F.-R., Billen, P., Ansätze zum Zufriedenheitsmanagement: Das Zufriedenheitsportfolio, a.a.O., S. 412 f.

Vgl. Watzlik, S., Die Bedeutung von Involvement und kognitiven Strukturen für das Marketing von Dienstleistungen am Beispiel von Finanzdienstleistungen, in: Dienstleistungsmarketing: Konzeptionen und Anwendungen, Kleinaltenkamp, M. (Hrsg.), Wiesbaden 1995, S. 89 ff.

Zu weiteren Involvementdeterminanten vgl. Trommsdorff, V., Konsumentenverhalten, a.a.O., S. 54 f.; Monhemius, K.Ch., Umweltbewußtes Kaufverhalten von Konsumenten: ein Beitrag zur Operationalisierung, Erklärung und Typologie des Verhaltens in der Kaufsituation, a.a.O., S. 113.

Zusammengefaßte Häufigkeiten der Skalenwerte 1 und 2 


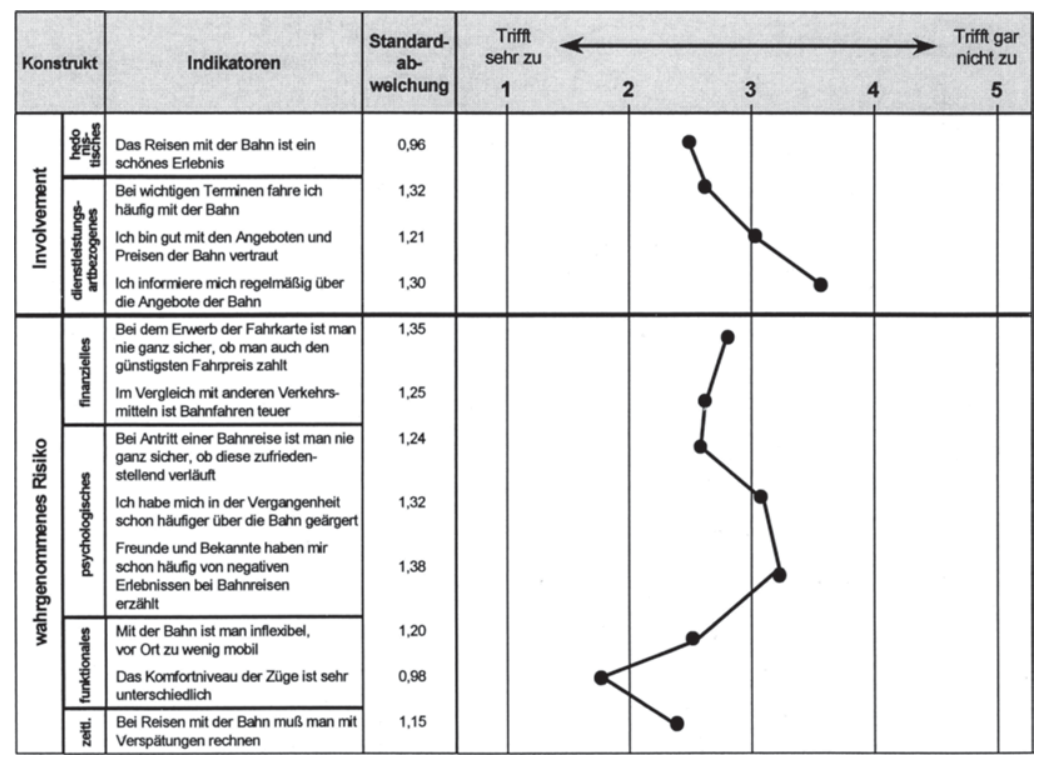

Abb. 48: Ausprägungen des wahrgenommenen Risikos und des Involvement von Bahnreisenden

In der Marketingliteratur wird unter dem wahrgenommenen Kaufrisiko die Nichtvorhersehbarkeit als nachteilig empfundener Folgen des Verhaltens verstanden. Wie Abbildung 48 zu entnehmen ist, werden zur Erfassung des Risikokonstruktes im folgenden vier Teilrisiken unterschieden. ${ }^{126}$ Das finanzielle Risiko beinhaltet die Gefahr monetärer Einbußen, die aus einem Fehlkauf resultieren können. Das psychische Kaufrisiko entsteht zum einen aus der globalen Unsicherheit über den zufriedenstellenden Verlauf einer Bahnreise. Dieses kann zum anderen durch eigene schlechte Erfahrungen und durch negative Berichte Dritter eine Verstärkung erfahren. ${ }^{127}$ Die spezifischen Besonderheiten von Dienstleistungen wiederum haben zur Folge, daß im Gegensatz zum Konsumgüterbereich der Umtausch bzw. die Rückgabe der Leistung in der Regel nicht möglich und dementsprechend auch ein funktionales Risiko zu berücksichtigen ist. Im Kontext von Verkehrsdienst-

Vgl. hierzu Katz, R., Informationsquellen der Konsumenten: Eine Analyse der Divergenzen zwischen der Beurteilung und Nutzung, Wiesbaden 1983, S. 79 sowie die Ausführungen in Kap. B 1.21 dieser Arbeit.

127 Zur Bedeutung des Einflusses von Mund-zu-Mund-Kommunikation für die Ausprägung des psychischen Risikos vgl. File, K.M., Judd, B.B., Prince, R.A., Interactive Marketing: The Influence of Participation on Positive Word-of-Mouth and Referrals, in: JoSM, Vol. 6, No. 4, 1992, S. $6 \mathrm{f}$. 
leistungen ist schließlich ein zeitliches Risiko zu berücksichtigen, das die Unsicherheit über etwaige Verspätungen beinhaltet.

Wie in Abbildung $48 \mathrm{zu}$ erkennen ist, sind die verschiedenen Teilrisiken in der vorliegenden Untersuchung in ihren Mittelwerten durchaus unterschiedlich stark ausgebildet. Eine vergleichsweise hohe Ausprägung ist für das funktionale Risiko festzuhalten. So stimmen ca. $78 \%$ der Befragten in hohen Maße der Aussage zu, daß das Komfortniveau der Züge sehr unterschiedlich ausfällt (Skalenwerte 1 und 2). Eine mangelnde Flexibilität am Zielort bekunden weitere $54 \%$ der Reisenden. Auch das von den Befragten wahrgenommene zeitliche Risiko ist relativ hoch. So empfinden immerhin knapp 57\% der Reisenden eine (sehr) starke Unsicherheit im Hinblick auf die Pünktlichkeit der Züge. Während im Rahmen des psychischen Risikos noch über $50 \%$ der Befragten die grundsätzliche Sicherheit eines zufriedenstellenden Verlaufs (in hohem Maße) bezweifeln, sind die Anteile derjenigen, die selbst negative Erfahrungen mit der Bahn gemacht haben bzw. von negativen Erlebnissen Dritter wissen, mit jeweils ca. 35\% der Befragten vergleichsweise gering. Jedoch darf nicht übersehen werden, daß damit jeder dritte Reisende eigene negative Erlebnisse im Zusammenhang mit Bahnreisen bekundet und/oder von solchen Erfahrungen Dritter schon häufiger gehört hat. Schließlich darf das finanzielle Risiko trotz der nur durchschnittlichen Mittelwerte der beiden Meßindikatoren in seiner Bedeutung nicht unterschätzt werden. So empfinden ca. $46 \%$ der Befragten das Reisen mit der Bahn im Vergleich zu anderen Verkehrsmitteln als teuer. ${ }^{128}$ Weitere $45 \%$ bezweifeln darüber hinaus, daß mit dem Kauf der Fahrkarte auch grundsätzlich das günstigste Angebot erworben wird. ${ }^{129}$

Sowohl bei den Involvement- als auch bei den Risikoindikatoren sind vergleichsweise hohe Standardabweichungen festzustellen. Diese können als Hinweis auf interindividuelle Unterschiede in den Ausprägungen der einzelnen Konstrukte und ihrer Bestandteile angesehen werden. Demnach fält das Involvement der

Interessanterweise wird diese Ansicht auch von ca. der Halfte der Befragten vertreten, die über eine BahnCard verfügt und die damit einen um 50\% reduzierten Fahrpreis bezahit. In der vorliegenden Untersuchung macht der Anteil an BahnCard-Besitzern ca. 48\% der Gesamtstichprobe aus.

Daß diese Einschătzung nicht unberechtigt ist, zeigt eine Untersuchung der Stiftung Warentest, aus der hervorgeht, daß es in Abhängigkeit vom Engagement und der Sachkenntnis des Schalterpersonals durchaus vorkommt, daß preislich z.T. deutlich voneinander abweichende Tickets für identische Reiserouten ausgestellt werden. Vgl. Stiftung Warentest (Hrsg.), Service der Bahn - Zügig informiert, zuviel kassiert, a.a.O., S. $80 \mathrm{ff}$.

Ein weiteres Teilrisiko stellt das physische Kaufrisiko dar, das von weniger als $8 \%$ der Befragten in Zusammenhang mit einer Bahnreise als hoch empfunden wird. Da mehr als $78 \%$ der Reisenden in der Bahn ein ausgesprochen sicheres Verkehrsmittel sehen, wird auf eine weitergehende Erorterung dieses Teilrisikos im folgenden verzichtet. 
Befragten gegenüber der Bahn unterschiedlich stark aus, und auch das Kaufrisiko wird in eher divergierenden Intensitätsstufen wahrgenommen.

Zur Untersuchung des Einflusses dieser psychographischen Bestimmungsfaktoren auf die Zufriedenheit mit der erlebten Bahnreise wurde auf der Basis der vorgestellten Meßindikatoren das in Abbildung 49 dargestellte Kausalmodell spezifiziert. Das Modell erweist sich als identifizierbar und die ermittelten globalen EQS-Gütekriterien ( $A G L S=0,854$, Adjusted $A G L S=0,819$ ) bestätigen eine insgesamt zufriedenstellende Modellanpassung. ${ }^{130}$

Die Meßindikatoren der einzelnen Konstrukte weisen unter Berücksichtigung des Stichprobenumfangs reliable Werte aus, so daß für dieses Modell akzeptable Operationalisierungen der verschiedenen psychographischen Bestimmungsfaktoren festzuhalten sind. Als zuverlässigster Indikator der Einstellungen erweist sich die Einschätzung der Innovationskraft des Unternehmens $(r=0,77)$. Das Involvement der Befragten wird in besonderem Maße durch ihr Informationsverhalten und den Erlebnisgehalt einer Bahnreise repräsentiert. Im Rahmen des wahrgenommenen Risikos nehmen schließlich vor allem die Indikatoren des psychischen Risikos und hier insbesondere die eigenen negativen Erfahrungen der Reisenden eine besondere Stellung ein.

Die Höhe der Korrelationen zwischen den einzelnen psychographischen Bestimmungsfaktoren deuten darauf hin, daß die einzelnen Konstrukte in einem relativ stark interdependenten Verhältnis zueinander stehen. ${ }^{131}$ Die vergleichsweise hohe positive Korrelation $(0,64)$ zwischen dem Einstellungskonstrukt und dem Involvement der Befragten weist darauf hin, daß eine positive Einstellung gegenüber der Bahn AG mit einer intensiveren Erlebniskomponente einer Bahnreise und einem größeren Interesse für die Angebote des Unternehmens einhergeht. In ähnlicher Höhe, jedoch in negativer Weise, sind die Einstellungen der Befragten mit dem Kaufrisiko korreliert $(-0,65)$. Demnach hat eine positive Grundhaltung gegenüber der Bahn einen risikoreduzierenden Charakter. Andererseits

Die entsprechenden LISREL-Maße betragen $\mathrm{GFI}=0,882, \mathrm{AGFI}=0,854$ und RMR $=0,141$ und können daher in ihrer Höhe nicht vollständig befriedigen. Da mit dem Modell jedoch ca. $50 \%$ der Varianz der Kundenzufriedenheit erfaßt werden, kann von einer hinreichend genauen Modellanpassung ausgegangen werden.

131 Da es sich hierbei um korrelative Beziehungen handelt, ist die Formulierung gerichteter Aussagen lediglich auf der Basis theoretischer Überlegungen, jedoch nicht aufgrund der im Modell spezifizierten Beziehungen möglich.

Andreas Siefke and Universität Münster - 978-3-631-75103-9 


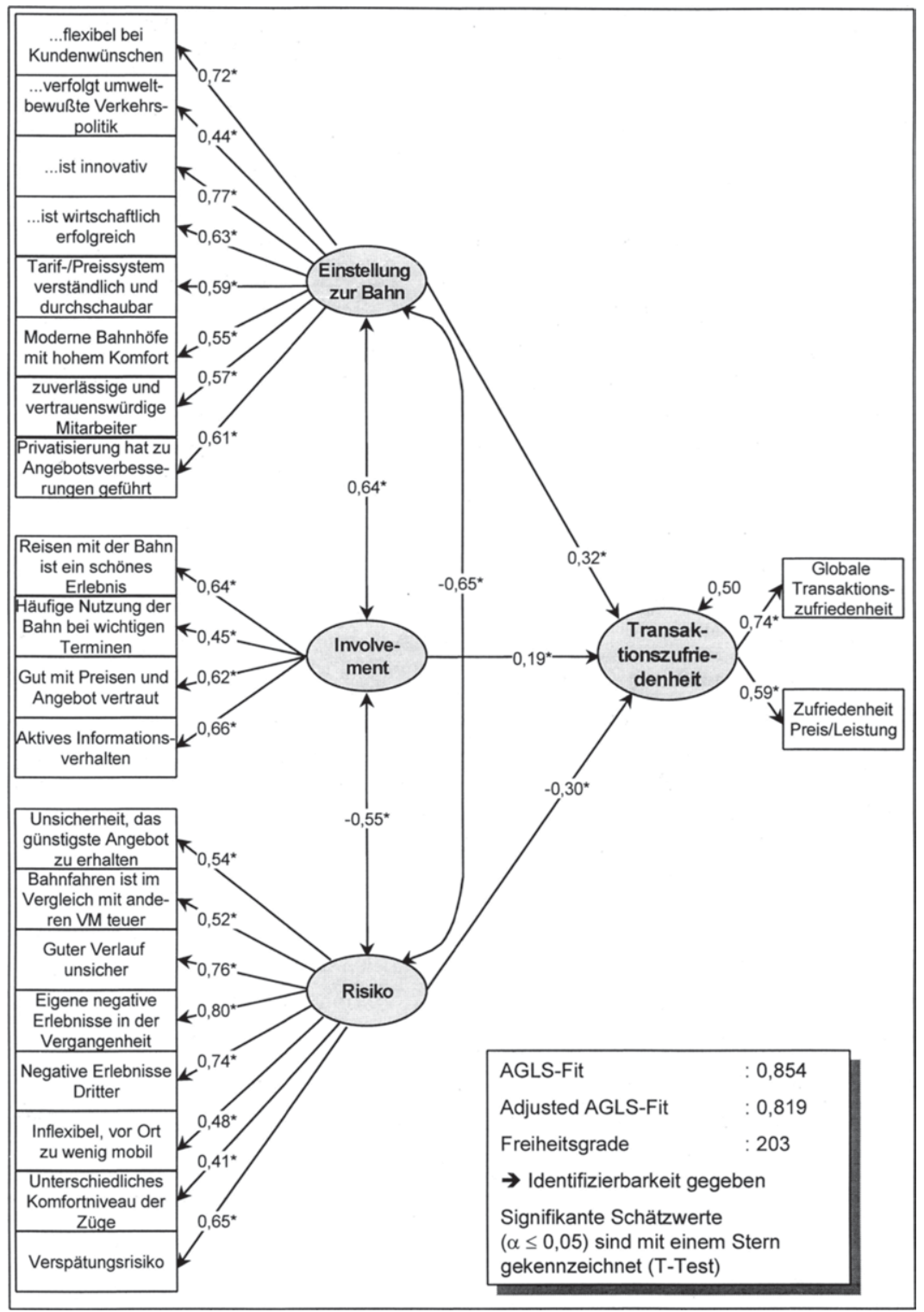

Abb. 49: Kausalmodell zum Einfluß psychographischer Bestimmungsfaktoren auf die Kundenzufriedenheit 
ist ein größeres wahrgenommenes Risiko mit einer Verschlechterung der Einstellungen verbunden. Auch der Bestimmungsfaktor Involvement korreliert in negativer Weise mit dem Risikokonstrukt $(-0,55)$. Das Kaufrisiko wird demzufolge umso weniger intensiv empfunden, desto positiver der Erlebnisgehalt einer Bahnreise eingeschätzt wird und desto vertrauter die Befragten mit der Dienstleistung Bahnreise sind. Andererseits führt auf der Basis dieses Ergebnisses ein als hoch empfundenes Risiko nicht zu einer stärkeren Auseinandersetzung mit der Dienstleistung. Danach sind Reisende, die ein höheres Kaufrisiko empfinden, nicht dazu bereit, dieses durch entsprechende Aktivitäten zu reduzieren. ${ }^{132}$

Über die Einflußstärke der einzelnen Bestimmungsfaktoren auf die Kundenzufriedenheit geben die in Abbildung 49 angegebenen Pfadkoeffizienten Aufschluß. Hier ist zunächst ein relativ starker positiver Effekt des Einstellungskonstruktes auf die Kundenzufriedenheit festzustellen $(0,32)$. Nach diesem Ergebnis wird die Zufriedenheit mit einer Bahnreise von einer positiven Einstellung gegenüber dem Unternehmen Bahn in vorteilhafter Weise beeinflußt. Damit kann Hyp Psycho 3 als bestätigt angesehen werden. Mit einem Pfadkoeffizienten von 0,19 geht ein ebenfalls positiver Effekt vom Involvementkonstrukt auf die Kundenzufriedenheit aus. Damit kann auch Hypothese Hyp Psycho 2 über den positiven Einfluß des Involvements auf die Zufriedenheit mit einer Bahnreise auf der Basis der vorliegenden Untersuchung nicht verworfen werden. Folglich fördert ein hohes Involvement das bewußte und intensive Erleben von Zufriedenheit.

Schließlich ist ein vergleichsweise starker negativer Einfluß des wahrgenommenen Risikos auf die Kundenzufriedenheit erkennbar $(-0,30)$. Die Nichtabschätzbarkeit vermeintlich nachteiliger Konsequenzen der Dienstleistungsnutzung übt damit eine negative Wirkung auf die Zufriedenheit aus. Auf der Basis dieses Ergebnisses ist somit auch Hyp Psycho 1 über den negativen Zusammenhang von Kaufrisiko und Zufriedenheit anzunehmen.

Nach der Betrachtung soziodemographischer und psychographischer Determinanten der Kundenzufriedenheit sollen nun Bestimmungsfaktoren untersucht werden, die sich aus der spezifischen Reisesituation eines Bahnkunden ergeben. 


\subsection{Reisebezogene Bestimmungsfaktoren}

Aus der Vielzahl denkbarer reise- bzw. verkehrsdienstleistungsspezifischer Determinanten der Kundenzufriedenheit mit einer Bahnreise ${ }^{133}$ werden im folgenden solche Einflußgrößen einer Analyse unterzogen, die von Verkehrsdienstleistungsunternehmen häufig als zentrale Stellgrößen für die Differenzierung und Profilierung des Angebotes im Markt angesehen werden. Auch bei der Deutschen Bahn AG erfolgt die Kundenansprache in erster Linie aus einer angebotsbezogenen Perspektive. Dabei werden z.B. Kriterien wie der Reiseanlaß oder die Reiseklasse zur Aufteilung der Konsumenten in Teilsegmente verwendet. Der Frage, inwieweit sich derartige Faktoren auch im Zufriedenheitsurteil der Befragten niederschlagen, wird im folgenden nachgegangen.

Das Motiv für den Antritt einer Bahnreise kann vielfältiger Natur sein und mit einer unterschiedlichen Wahrnehmung und Gewichtung der verschiedenen Bestandteile der Dienstleistung einhergehen. ${ }^{134}$ In der Verkehrsdienstleistungspraxis wird in der Regel zwischen Reisen aus privatem Anlaß und dienstlich bzw. geschäftlich motivierten Reisen unterschieden. Eine solche Einteilung liegt auch den in Abbildung 50 dargestellten Beziehungszusammenhängen der Kundenzufriedenheit in Abhängigkeit vom Reiseanlaß zugrunde. ${ }^{135}$

Eine Betrachtung der Mittelwertunterschiede im linken Teil der Abbildung 50 führt zu dem Ergebnis, daß sich die Geschäftsreisenden, die etwa ein Viertel der gesamten Stichprobe ausmachen, mit dem Verlauf der Vor- und NachReisephase weniger zufrieden zeigen. Dagegen ist bei diesen Personen eine höhere Zufriedenheit mit dem Preis-/Leistungsverhältnis festzustellen. Dies ist sicherlich auf den Umstand zurückzuführen, daß die Aufwendungen für Geschäfts- bzw. Dienstreisen in der Regel durch den Arbeitgeber getragen werden und somit die Sensibilität gegenüber dem Verhältnis von Preis und

Vgl. hierzu die Ausführungen in Kap. B 1.22 dieser Arbeit.

135

Vgl. hierzu und im folgenden die Ausführungen in Kap. B 4.22 dieser Arbeit.

In der vorliegenden Untersuchung wurden (Kurz-)Urlaubsreisen und sonstige Privatreisen (z.B. Verwandten-/Bekanntenbesuche) zum Reiseanlaß „Privatreise“ zusammengefaßt. Eine Einbeziehung von Fernpendlerfahrten wurde dagegen nicht vorgenommen, da sich diese aufgrund signifikanter Unterschiede in den Wahrnehmungs- und Nutzenstrukturen von Pendlern keinem der oben angeführten Reiseanlässe eindeutig zuordnen lassen. Vgl. Institut für Marketing, User-Analyse bei der Deutschen Bahn AG, unveroffentlichte Studie im Auftrag der Deutschen Bahn AG, Münster 1995; Institut für Marketing, Non User-Analyse bei der Deutschen Bahn AG, unveroffentlichte Studie im Auftrag der Deutschen Bahn AG, Münster 1996. Auf eine eigenständige Berücksichtigung dieses Reiseanlasses wurde aufgrund der geringen Fallzahl $(n=63)$ verzichtet. 


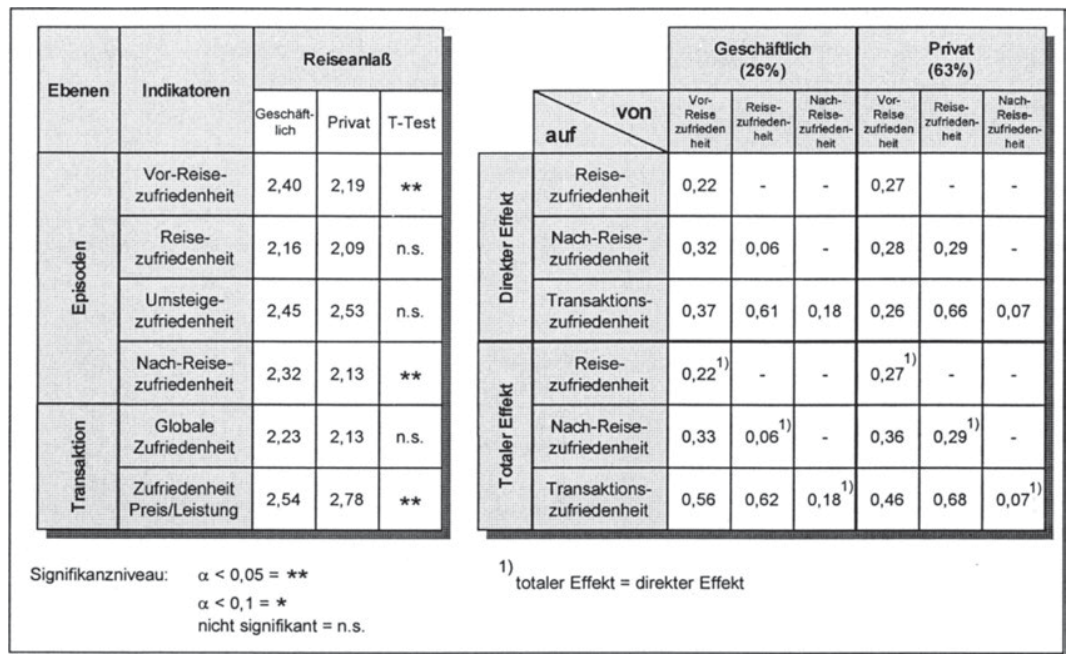

\section{Abb. 50: Beziehungszusammenhänge der Kundenzufriedenheit in Abhängigkeit vom Reiseanlaß}

Leistung nicht so stark in den Vordergrund tritt. In bezug auf die transaktionsbezogene Zufriedenheit sowie die Zufriedenheit mit der Reise- und Umsteigephase unterscheiden sich die beiden Gruppen dagegen nicht nachhaltig voneinander.

Die Überprüfung der im rechten Teil der Abbildung 50 dargestellten Effekte der kausalanalytischen Untersuchung der Kundenzufriedenheit zeigt eine höhere Dynamik auf der Episodenebene für die Gruppe der Privatreisenden (ca. 63\% der Befragten). ${ }^{136}$ Diese ist insbesondere in bezug auf den Einfluß der Reisezufriedenheit auf die Zufriedenheit mit der Nach-Reisephase festzustellen (direkter Effekt=0,29). Bei den totalen Effekten ist für die Geschäftsreisenden ein vergleichsweise etwas schwächerer Einfluß der Reisephase hinsichtlich der Entstehung der Transaktionszufriedenheit zu erkennen, während diese Fahrgäste der Vor- und der Nach-Reisezufriedenheit eine relativ höhere Bedeutung beimessen als die Privatreisenden. Hier liegt die Vermutung nahe, daß für Reisende, die aus beruflichen Gründen unterwegs sind, der reibungslose Ablauf aller Phasen wichtig

$136 \mathrm{Vgl}$. hierzu die entsprechenden Varianzerklärungsanteile der Reise- und Nach-Reisezufriedenheit in Abbildung A2 im Anhang I dieser Arbeit.

Andreas Siefke and Universität Münster - 978-3-631-75103-9 
ist, da die Reise einen integrierten Bestandteil der Arbeitstages darstellt und dabei insbesondere die Vor-Reisephase für einen „erfolgreichen“ Start in den Arbeitstag an Bedeutung gewinnt. Der für die Privatreisenden im Vergleich beider Gruppen tendenziell höhere Einfluß der Reisephase auf die Gesamtzufriedenheit liegt möglicherweise darin begründet, daß diese Fahrgäste dem „Fahrerlebnis“ einen größeren Stellenwert einräumen. ${ }^{137} \mathrm{Da}$ festgestellt wurde, daß sich die Gruppen hinsichtlich ihrer transaktionsbezogenen Zufriedenheit mit der Bahnreise kaum voneinander unterscheiden, ist anzunehmen, daß der unterschiedliche Einfluß der einzelnen Abschnitte einer Bahnreise eine Angleichung der Kundenurteile der beiden Kundengruppen zur Folge hat. Hypothese Hyp VDL 1 über den Einfluß des Reiseanlasses auf die Kundenzufriedenheit kann somit im Hinblick auf die Zufriedenheit mit einzelnen Episoden und das Urteil über das Preis/Leistungsverhältnis angenommen werden, ist aber in bezug auf die transaktionale Gesamtzufriedenheit als nicht bestätigt anzusehen.

Die bisher vorgenommenen Analysen haben gezeigt, daß die Zufriedenheit mit der Reisephase auf der Episodenebene die zentrale Einflußgröße der Zufriedenheit mit einer Bahnreise darstellt. Im folgenden werden daher Bestimmungsfaktoren untersucht, die vor allem die Zufriedenheit mit dem Aufenthalt im Zug und hier insbesondere die Wahrnehmung des Kontaktpunktes "Abteil" beeinflussen und vom Verkehrsdienstleister konkret ausgestaltet werden können. $\mathrm{Zu}$ diesen Einflußgrößen gehört der Zugtyp, mit dem eine Bahnreise unternommen wird. ${ }^{138}$ Wie Abbildung $51 \mathrm{zu}$ entnehmen ist, können hier signifikante Unterschiede auf den verschiedenen Ebenen der Kundenzufriedenheit festgestellt werden. ${ }^{139}$

So ist zunächst auf der Transaktionsebene die globale Zufriedenheit der ICE-Reisenden am höchsten ausgeprägt. Dagegen sind hinsichtlich der Beurteilung des Preis-/Leistungsverhältnisses zwischen den Nutzern der verschiedenen Zugarten keine signifikanten Unterschiede zu erkennen. Auf der Ebene der Reisephase sind wiederum die ICE-Nutzer im Vergleich zu den Reisenden der beiden anderen

137 Dies zeigt sich auch in dem Involvementindikator "Bahnfahren ist ein schönes Erlebnis", der bei dieser Gruppe mit einem Mittelwert von 2,4 im Vergleich zu einem Mittelwert von 2,7 bei den Geschäftsreisenden signifikant stärker ausgeprägt ist. 


\begin{tabular}{|c|c|c|c|c|c|c|c|c|c|}
\hline \multirow{3}{*}{\multicolumn{2}{|c|}{ Ebene }} & \multirow{3}{*}{ Merkmale } & \multicolumn{7}{|c|}{ Zugtyp } \\
\hline & & & \multirow{2}{*}{ ICE } & \multirow{2}{*}{$\stackrel{\mathrm{IR}}{2}$} & \multirow{2}{*}{ IC/EC } & \multicolumn{3}{|c|}{ Scheffeé-Test } & \multirow{2}{*}{ F-Test } \\
\hline & & & & & & $1 / 2$ & $1 / 3$ & $2 / 3$ & \\
\hline \multirow{2}{*}{\multicolumn{2}{|c|}{ Transaktion }} & Globale Zufriedenheit & 2,01 & 2,27 & 2,36 & $\star \star \star$ & $\star \star$ & n.s. & $\star \star$ \\
\hline & & Zufriedenheit Preis/Leistung & 2,63 & 2,85 & 2,79 & n.s. & n.s. & n.s. & n.s. \\
\hline \multicolumn{2}{|c|}{ Episode } & Reisezufriedenheit & 1,90 & 2,28 & 2,38 & $\star \star$ & $\star \star$ & n.s. & $\star \star$ \\
\hline \multirow{18}{*}{ 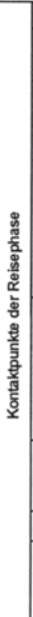 } & Abfahrt & Pünktlichkeit Abfahrt & 1,38 & 1,63 & 1,82 & $\star *$ & $\star \star *$ & n.s. & $\star \star$ \\
\hline & \multirow{8}{*}{ 體 } & Möglichkeit zur Gepăckverstauung & 2,32 & 2,47 & 2,72 & n.s. & $\star \star$ & $\star$ & $\star \star$ \\
\hline & & Komfort des Sitzes & 2,12 & 2,27 & 2,63 & n.s. & $\star \star$ & $\star \star$ & $\star *$ \\
\hline & & Beinfreiheit am Sitzplatz & 2,32 & 2,72 & 2,80 & $\star \star$ & $\star \star$ & n.s. & $\star \star$ \\
\hline & & $\begin{array}{l}\text { Ausstattung des Sitzplatzes } \\
\text { (z. B. Arbeitstisch, Ablage) }\end{array}$ & 2,05 & 2,45 & 2,83 & $\star \star$ & $\star \star$ & $\star \star$ & $* \star$ \\
\hline & & Sauberkeit des Abteils/Wagens & 1,78 & 2,24 & 2,45 & $\star \star$ & $\star \star$ & $\star \star$ & $\star \star$ \\
\hline & & $\begin{array}{l}\text { Möglichkeit, ungestört } \\
\text { arbeiten/schlafen/esen zu können }\end{array}$ & 2,48 & 2,63 & 2.73 & n.s. & $\star \star$ & n.s. & * \\
\hline & & Luft-rTemperaturverhältnisse & 2,42 & 3,22 & 2,82 & $\star \star$ & $\star \star$ & $\star \star$ & $\star \star$ \\
\hline & & $\begin{array}{l}\text { Verständlichkeit der } \\
\text { Lautsprecherdurchsagen }\end{array}$ & 1,86 & 2,43 & 2,48 & $\star \star$ & $\star \star$ & n.s. & $\star \star$ \\
\hline & \multirow{4}{*}{  } & Freundlichkeit des Zugpersonals & 1,76 & 1,90 & 2,02 & n.s. & $\star \star$ & n.s. & $\star \star$ \\
\hline & & Fachikompetenz des Zugpersonals & 1,85 & 2,02 & 2,03 & n.s. & $\star$ & n.s. & n.s. \\
\hline & & $\begin{array}{l}\text { Informationen über etwaige } \\
\text { Anschlüsse, Verspätungen etc. }\end{array}$ & 1,87 & 2,30 & 2,32 & $\star \star$ & $\star \star$ & n.s. & $\star \star$ \\
\hline & & $\begin{array}{l}\text { Sicherheit im Zug (keine Belastigung } \\
\text { durch Dritte) }\end{array}$ & 1,58 & 1,85 & 1,88 & $\star \star$ & $\star \star$ & n.s. & $\star \star$ \\
\hline & \multirow{2}{*}{ 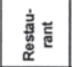 } & Angebot im Speisewagen/Zugbistro & 2,62 & 3,31 & 2,84 & $\star \star$ & n.s. & $\star \star$ & $\star \star$ \\
\hline & & $\begin{array}{l}\text { Preis-/Leistungsverhalitnis der } \\
\text { Speisen und Getrănke }\end{array}$ & 3.57 & 3,98 & 3,84 & $\star \star$ & $\star \star$ & n.s. & $\star \star$ \\
\hline & Toiletten & Sauberkeit der Zugtoiletten & 2,20 & 3,21 & 3,20 & $\star \star$ & $\star \star$ & n.s. & $\star \star$ \\
\hline & \multirow{2}{*}{$\frac{\text { E }}{\frac{E}{3}}$} & Fahrtdauer & 1,74 & 2,45 & 2,48 & $\star \star *$ & $\star \star$ & n.s. & $\star \star$ \\
\hline & & $\begin{array}{l}\text { Pünktlichkeit des Zuges am } \\
\text { Zielbahnhof }\end{array}$ & 1,66 & 1,78 & 2,23 & n.s. & $\star \star$ & $\star \star$ & $\star \star$ \\
\hline
\end{tabular}

Signifikanzniveau:

$\alpha<0,05=\star \star$

$\alpha<0,1 \quad=\star$

nicht signifikant $=$ n.s.

Abb. 51: Differenzen in der Kundenzufriedenheit in Abhängigkeit vom Zugtyp

Zugarten zufriedener. Dieser Trend ist auch bei einer Vielzahl der Meßindikatoren der einzelnen Kontaktpunkte auszumachen. So ist die Zufriedenheit der ICEFahrer mit Ausnahme der Beurteilung des Angebotes im Speisewagen bei allen Indikatoren signifikant höher als bei den IC/EC-Reisenden. Ebenso findet sich kein Merkmal, mit dem InterRegio-Reisende deutlich zufriedener sind als Personen, die den ICE nutzen. Bemerkenswert erscheint in diesem Zusammenhang der Umstand, daß der seitens der Bahn leistungsmäßig und preislich unter den InterCity- bzw. EuroCity eingestufte Zugtyp InterRegio ${ }^{140}$ in einigen Merkmalen (z.B. Sitzkomfort, Möglichkeiten der Gepäckverstauung) besser als die IC/ECZüge beurteilt wird. Andererseits darf nicht übersehen werden, daß IR-Reisende z.B. in bezug auf die Luft- und Temperaturverhältnisse oder das Angebot im Speisewagen/Zugbistro die vergleichsweise höchste Unzufriedenheit bekunden.

140 Vgl. hierzu Bretthauer, I., Deutsche Bahn AG: Von der Behorde zum marktorientierten Touristikanbieter, a.a.O., S. 250. 
Insgesamt ist festzuhalten, daß nicht nur Eigenschaften des materiellen Umfeldes, sondern auch intangible Aspekte der Reisephase wie z.B. die Pünktlichkeit der Züge bei Start und Ankunft oder die "soziale" Sicherheit im Zug in Abhängigkeit vom genutzten Zugtyp differenziert wahrgenommen werden. Da nicht davon ausgegangen werden kann, daß die zufriedenstellende Erfüllung dieser Merkmale grundsätzlich durch einen bestimmten Zugtyp mit höherer Sicherheit gewährleistet wird, ist insbesondere dem auch als "Flaggschiff der Bahn" bezeichneten InterCityExpress ${ }^{141}$ die Funktion eines „Wahrnehmungsfilters“ zuzusprechen, der neben der Zufriedenheit mit den Aspekten des materiellen Umfeldes auch die Zufriedenheit mit immateriellen Komponenten der Dienstleistung vorteilhaft beeinflußt. Hypothese Hyp VDL 2 über den Einfluß des Zugtyps auf die Zufriedenheit mit der Reisephase als auch auf die transaktionsbezogene Kundenzufriedenheit ist damit auf der Basis der empirischen Ergebnisse als bestätigt anzusehen.

Zur Überprüfung der Annahme, daß auch die genutzte Reiseklasse einen Einfluß auf die Kundenzufriedenheit ausübt, werden die in Abbildung 52 dargestellten Mittelwertprofile herangezogen. ${ }^{142}$ Hier kann zunächst festgestellt werden, daß die Reisenden der ersten Klasse mit allen Indikatoren, für die ein signifikanter Mittelwertunterschied zwischen den beiden Beförderungsklassen ermittelt wurde, zufriedener sind als Reisende der zweiten Klasse. So ist auch die Zufriedenheit mit dem Preis-/Leistungsverhältnis bei den Reisenden der ersten Klasse signifikant höher, wenngleich für diese Klasse ein (deutlich) höherer Fahrpreis zu entrichten ist. Ebenso ist die globale Kundenzufriedenheit als auch die Zufriedenheit mit der Reisephase bei dieser Kundengruppe stärker ausgeprägt. Auch auf der Kontaktpunktebene zeigen sich die Reisenden der ersten Klasse in allen Merkmalen mit signifikant unterschiedlichen Mittelwerten zufriedener. Hier können einige besonders starke Differenzen in den Mittelwerten des Kontaktpunktes „Abteil“ ausgemacht werden. Erwartungsgemäß werden der Sitzkomfort und die Beinfreiheit deutlich positiver beurteilt. Ferner ist die Möglichkeit, während der Fahrt ungestört arbeiten, lesen oder schlafen zu können, durch ein besseres Zufriedenheitsurteil gekennzeichnet, wobei dies vermutlich auch darauf zurück-

141 Rahn, T., Der Hochgeschwindigkeitszug InterCityExpress der DB, a.a.O., S. 537.

$14225 \%$ der Befragten reisten in der ersten Klasse, wahrend mit $75 \%$ der Großteil der Reisenden in der zweiten Klasse fuhr. 


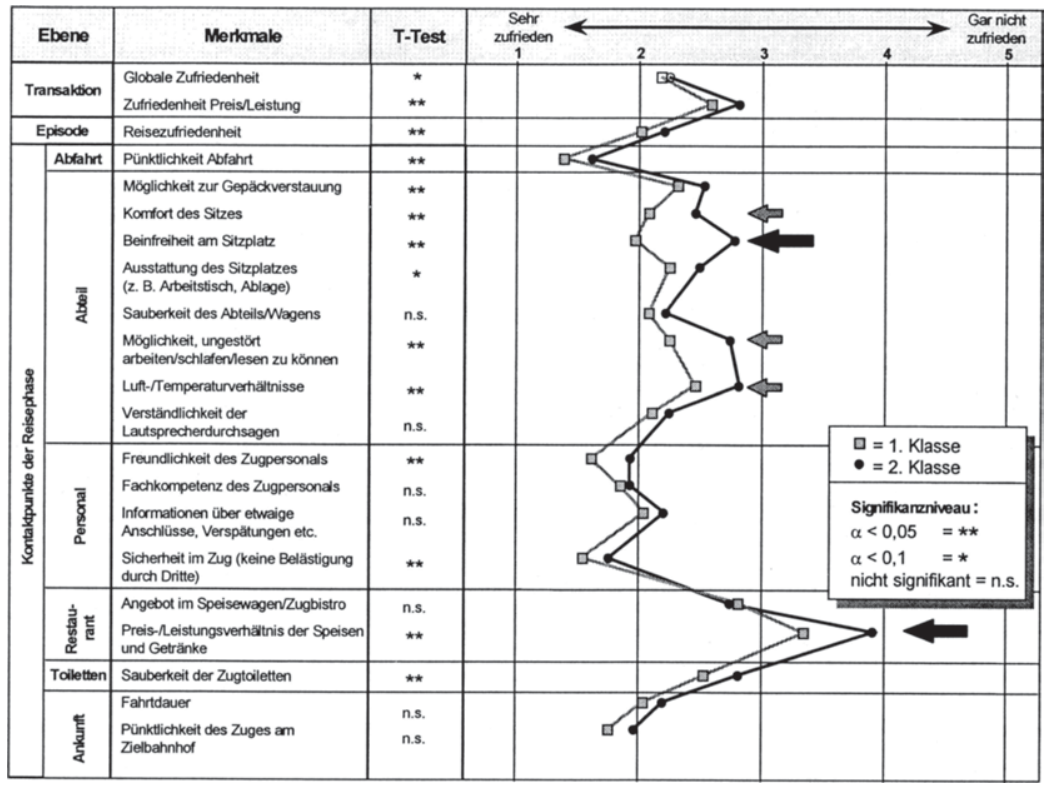

\section{Abb. 52: Zufriedenheitsprofile in Abhängigkeit von der Reiseklasse}

zuführen ist, daß in den Wagen der ersten Klasse im Durchschnitt deutlich weniger Sitzplätze belegt waren. ${ }^{143}$

Interessanterweise werden aber auch weitere immaterielle Merkmale der Reisephase als zufriedenstellender wahrgenommen, die von der Reiseklasse grundsätzlich unabhängig sind. Dies gilt z.B. für die Luft- und Temperaturverhältnisse im Zug oder für die Freundlichkeit des Zugpersonals. Ob letzteres auf ein tatsächlich freundlicheres Verhalten des Zugbegleitpersonals oder aber lediglich auf eine kundenseitig differenzierte Wahrnehmung zurückzuführen ist, kann an dieser Stelle nicht abschließend beantwortet werden. Eine mögliche Erklärung für diese Ergebnis besteht jedoch darin, daß der Am-Platz-Service, der nur in der ersten Wagenklasse angeboten wird, die Zufriedenheit mit der Freundlichkeit des Personals positiv beeinflußt. ${ }^{144}$ Der deutliche Unterschied in der Zufriedenheit mit dem

143 Während ca. $70 \%$ der Reisenden der zweiten Klasse angaben, daß bei ihrer Fahrt $3 / 4$ und mehr aller Sitzplätze belegt waren, beträgt dieser Wert in der vorliegenden Stichprobe für die erste Klasse lediglich ca. $34 \%$.

$144 \mathrm{Vgl}$. hierzu die Auswertungen der kritischen Ereignisse der Reisephase in Kap. C 2.22 dieser Arbeit. 
Preis-/Leistungsverhältnis der im Speisewagen bzw. Zugbistro angebotenen Speisen und Getränke schließlich liegt sicherlich auch darin begründet, daß die Fahrgäste der ersten Klasse über ein deutlich höheres Einkommen verfügen. ${ }^{145}$ Zusammenfassend bleibt festzuhalten, daß von der genutzten Reiseklasse erkennbare Wirkungen auf die Zufriedenheit mit der Reisephase als auch mit der gesamten Bahnreise ausgehen und dementsprechend Hypothese Hyp VDL 3 anzunehmen ist.

Eine interessante Ergänzung dieser Analyse besteht in der Untersuchung potentieller Unterschiede in der Kundenzufriedenheit in Abhängigkeit vom Abteiltyp, da in beiden Beförderungsklassen die Möglichkeit besteht, in Abteilen "klassischer" Konzeption als auch in Großßraumabteilen mit Reihenbestuhlung und Sitzgruppen zu reisen. ${ }^{146}$ In Abbildung 53 sind die entsprechenden Mittelwertprofile für die beiden Abteiltypen abgetragen. ${ }^{147}$ Hier ist zu erkennen, daß die Nutzer von Großraumwagen sowohl auf der Transaktionsebene als auch auf der Ebene der Reisephase eine höhere Zufriedenheit bekunden.

Auf der Kontaktpunktebene ist auffällig, daß auch von den Reisenden, die ihre Fahrt in einem Großraumwagen unternommen haben, ähnlich wie von den Fahrgästen der ersten Klasse, die Beinfreiheit am Sitzplatz positiver beurteilt wird. Da ca. $50 \%$ aller Befragten in einem Großraumabteil der zweiten Klasse reisten, ist dieses Ergebnis nur zu einem gewissen Teil auf die genutzte Reiseklasse zurückzuführen. Bemerkenswert erscheint in diesem Zusammenhang, daß die Sauberkeit der Abteile von Fahrgästen, die in einem Großraumabteil gereist sind, signifikant besser eingeschätzt wird; ein Ergebnis, das bei der Analyse nach der Reiseklasse nicht festgestellt werden konnte. Deutliche Unterschiede ergeben sich des weiteren in der Wahrnehmung der Möglichkeiten der Gepäckverstauung sowie in bezug auf die Ausstattung des Sitzplatzes. Neben diesen einrichtungsbezogenen Merkmalen werden ebenso immaterielle Aspekte wie z.B. die Fahrtdauer und die Pünktlichkeit der Züge von Reisenden dieses Abteiltyps positiver beurteilt. Wenngleich dies keinen Schluß auf die eigentliche Fahrtlänge und

145 So verfügen ca. $65 \%$ der Reisenden der ersten Klasse über ein monatliches Nettohaushaltseinkommen von über 5.000 DM. Der entsprechende Anteil der Fahrgäste in der zweiten Klasse liegt dagegen bei ca. $29 \%$.

146 Vgl. Rahn, T., Der Hochgeschwindigkeitszug InterCityExpress der DB, a.a.O., S. 540.

147 Knapp 65\% der Reisenden fuhren in einem Großraumabteil, wăhrend mit ca. 35\% ein gutes Drittel der Fahrgäste in einem klassischen Abteil reisten.

Andreas Siefke and Universität Münster - 978-3-631-75103-9 


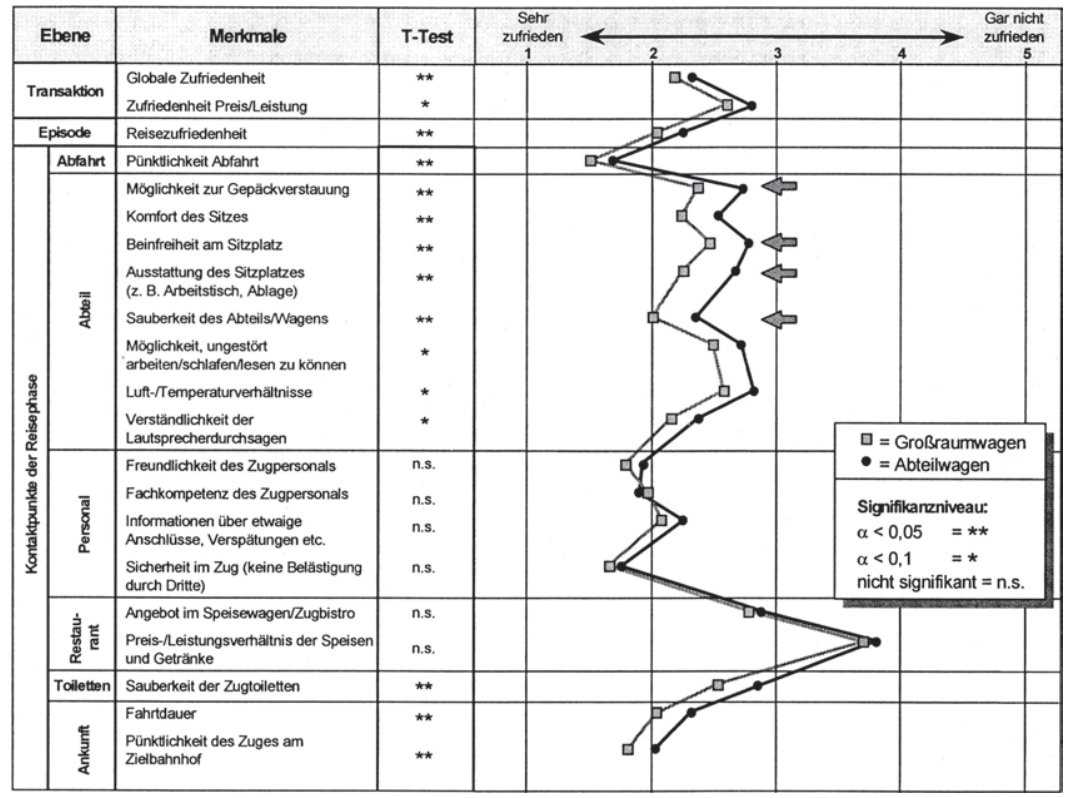

Abb. 53: Zufriedenheitsprofile in Abhängigkeit vom Abteiltyp

Pünktlichkeit der Züge zuläßt, bleibt dennoch zu vermuten, daß auch die Zeitwahrnehmung der Fahrgäste durch die Ausgestaltung des physischen Umfeldes der Reisephase beeinflußt wird. ${ }^{148}$ Insgesamt ist damit Hypothese Hyp VDL 4 über den Einfluß des Abteiltyps auf die Kundenzufriedenheit mit einer Bahnreise anzunehmen.

Während die Bestimmungsfaktoren Zugart, Reiseklasse und Abteiltyp in zumindest kurzfristiger Hinsicht als weitgehend konstante Größen angesehen werden können, sind in bezug auf den Auslastungsgrad der Züge deutliche zeitliche Schwankungen im Tagesablauf zu erkennen. So werden insbesondere bei Eintagesreisen die sog. „Tagesrandverbindungen“ am Morgen und am Abend bevorzugt in Anspruch genommen. ${ }^{149}$ Das damit verbundene entsprechend hohe Personenaufkommen am Bahnhof und in den Zügen führt möglicherweise dazu, daß

Vgl. Stauss, B., Dienstleister und die vierte Dimension, a.a.O., S. 87 f. und ferner Bitner, M.J., Evaluating Service Encounters: The Effects of Physical Surroundings and Employee Responses, in: JoM, Vol. 54, April 1990, S. 69 ff. 
von den Fahrgästen eine größere Hektik und Unruhe wahrgenommen wird, die sich nachteilig auf die Kundenzufriedenheit auswirken können. Da bereits in der Analyse der kritischen Ereignisse der Reisephase deutlich wurde, daß die Überfüllung der Züge bzw. das damit verbundene mangelnde (Sitz-)Platzangebot vergleichsweise häufig kritisiert wurden, ${ }^{150}$ ist zu vermuten, daß auch die Zufriedenheit mit weiteren Merkmalen der Reisephase durch den Auslastungsgrad der Züge beeinflußt wird. Die in Abbildung 54 wiedergegeben Mittelwertprofile bestätigen diese Annahme. So bekunden die Befragten, die in weniger stark besetzten Zügen gereist sind, ${ }^{151}$ eine höhere globale Transaktionszufriedenheit als die Reisenden, die in Zügen mit einem relativ hohen Sitzladefaktor ${ }^{152}$ unterwegs gewesen sind. Während in der Zufriedenheit mit dem Preis-/Leistungsverhältnis kein Unterschied zwischen diesen Personengruppen festgestellt werden kann, sind die Reisenden in den „volleren“ Zügen mit der Reisephase und damit mit der Fahrt im Zug signifikant unzufriedener.

Bedingt durch die höhere physische Nähe zu den Mitreisenden zeigen sich diese Befragten auf der Kontaktpunktebene der Reisephase insbesondere mit der Beinfreiheit am Sitzplatz sowie mit den Möglichkeiten zur Gepäckunterbringung als deutlich unzufriedener. Damit einher geht eine höhere Unzufriedenheit in bezug auf die Möglichkeit, ungestört die Reise zu verbringen. Auch die Luft- und Temperaturverhältnisse in diesen Zügen werden von diesen Fahrgästen deutlich schlechter beurteilt. Darüber hinaus ist bei diesen Befragten sowohl die Zufriedenheit mit der Freundlichkeit und Kompetenz des Zugbegleitpersonals als auch mit der Pünktlichkeit der Züge schwächer ausgeprägt. Wenngleich nicht gänzlich ausgeschlossen werden kann, daß das Personal in Zügen mit einem sehr hohen Sitzladefaktor bedingt durch den damit einhergehenden stärkeren Streß in der Tendenz weniger freundlich ist und diese Züge durch größere Verspätungen gekennzeichnet sind, ist zu vermuten, daß bereits der Umstand, in einem Zug mit einer hohen Auslastung zu reisen, die Wahrnehmung dieser Aspekte der Reisephase nachteilig beeinflußt. Insgesamt kann damit die Hypothese Hyp vDL 5 über den Einfluß des Auslastungsgrades auf die Kundenzufriedenheit mit Bahnreisen als bestätigt angesehen werden.

Vgl. hierzu die Ausführungen in Kap. C 2.22 dieser Arbeit.

151 Hierunter werden die Reisenden zusammengefaßt, in deren Zügen über die Hälfte aller Sitzplätze des genutzten Abteils belegt waren. $\mathrm{Ca}$. $60 \%$ der Befragten gaben an, mit einem solchen Zug gefahren zu sein. Die verbleibenden ca. $40 \%$ der Fahrgäste entfallen auf Züge, in denen entsprechend weniger Sitzplätze besetzt waren.

Zum Begriff des Sitzladefaktors vgl. Pompl, W., Luftverkehr: Eine okonomische Einführung, a.a.O., S. $33 \mathrm{ff}$. 




\section{Abb. 54: Zufriedenheitsprofile in Abhängigkeit vom Auslastungsgrad der Züge}

Es liegt nahe anzunehmen, daß die Kundenzufriedenheit auch durch den Umstand beeinflußt wird, ob von den Fahrgästen eine Sitzplatzreservierung vorgenommen wurde, da diese einem Reisenden bereits im Vorfeld einer Reise den Anspruch auf einen Sitzplatz in einem auch stark besetzten Zug garantiert. Die in Abbildung 55 dargestellten Mittelwertunterschiede sind ein Beleg für die grundsätzliche Richtigkeit dieser Vermutung. So sind Personen mit einer Sitzplatzreservierung sowohl auf der Transaktions- als auch auf der Ebene der Reisephase signifikant zufriedener als Reisende ohne Platzreservierung. ${ }^{153}$

Insbesondere der vergleichsweise hohe Unterschied in der Zufriedenheit mit dem Preis-/Leistungsverhältnis der beiden Kundengruppen, die bei Reisenden ohne Platzreservierung deutlich niedriger ausfällt, ist hier auffällig. Da der Sitzplatz einen zentralen Bestandteil der Kerndienstleistung darstellt, wirkt sich offenbar der Umstand, daß mit dem Kauf des Bahntickets im Gegensatz z.B. zu Flugdienstplatzreservierung Gebrauch gemacht. Ca. $47 \%$ der Reisenden verfügten dagegen nicht über eine Platzreservierung. Hierin sind ca. $5 \%$ aller Fahrgäste enthalten, denen laut Auskunft/Schalterpersonal keine Sitzplatzreservierung für ihre Reise ausgestellt werden konnte.

Andreas Siefke and Universitat Münster - 978-3-631-75103-9 


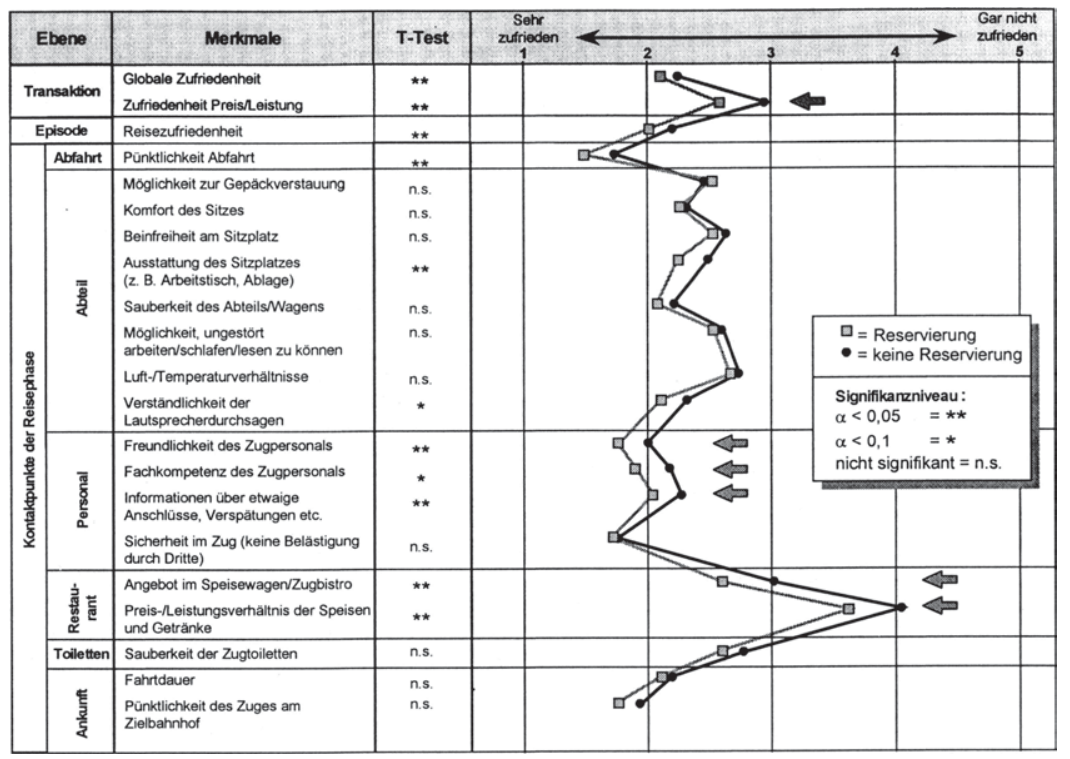

Abb. 55: Zufriedenheitsprofile in Abhängigkeit von der Inanspruchnahme einer Sitzplatzreservierung

leistungen nicht gleichzeitig der garantierte Anspruch auf eine Sitzmöglichkeit erworben wird, negativ auf das Kundenurteil aus. Dies spiegelt sich auch auf der Kontaktpunktebene wider; hier zeigen sich die Fahrgäste mit einer Platzreservierung in einigen Merkmalen und hier insbesondere mit den Kontaktpunkten Personal und Restaurant insgesamt zufriedener. Darüber hinaus ist für kein Merkmal eine vergleichsweise höhere Unzufriedenheit dieser Personengruppe festzustellen.

Diese Ergebnisse sind alles in allem darauf zurückzuführen, daß die Sitzplatzgarantie einem Reisenden die Suche nach einem freien Platz erspart und er damit dem Verlauf der Reisephase gelassener entgegen sehen kann. Dagegen laufen Personen ohne Sitzplatzreservierung besonders in stark frequentierten Zügen Gefahr, nicht den erwünschten Sitzplatz (z.B. am Fenster oder in einem Nichtraucherabteil) oder gar keinen freien Platz zu erhalten bzw. diesen, z.B. bei Aufsuchen des Zugrestaurants, wieder zu verlieren. Unter Berücksichtigung der von diesen Reisenden bekundeten größeren Unzufriedenheit mit der Reisephase wird auch die geringere Transaktionszufriedenheit dieses Personenkreises nachvollziehbar. Insgesamt ist somit die Hypothese Hyp vDL 6 über den Einfluß der Sitzplatzreservierung auf dieckundenzufriedenheit miẗ̈Bahnreisenoanzunghmen03-9 


\section{Zusammenfassung und Implikationen}

\section{Zusammenfassung und Würdigung der Untersuchungsergebnisse}

Der Ausgangspunkt der vorliegenden Arbeit war der wenig befriedigende Forschungsstand zur prozeßorientierten Analyse der Kundenzufriedenheit im Dienstleistungsbereich im allgemeinen sowie im Verkehrsdienstleistungsbereich im besonderen. So wird sowohl in der Wissenschaft als auch in der Unternehmenspraxis die Zufriedenheit von Kunden mit Produkten oder Dienstleistungen vornehmlich als ein reines Nachkaufphänomen behandelt. Diese Sichtweise greift jedoch insbesondere im Dienstleistungsbereich zu kurz. Vielmehr hat der Prozeßcharakter des Dienstleistungskonsums zur Folge, daß Kundenzufriedenheit aus der Beurteilung zeitlich aufeinanderfolgender Teilerlebnisse resultiert. Damit entsteht die Zufriedenheit von Kunden nicht erst im Anschluß an den Dienstleistungskonsum, sondern auch bereits während des Dienstleistungserlebens. Angesichts der nachweisbaren Erkenntnisdefizite in der wissenschaftlichen Diskussion und in Anbetracht des hohen Stellenwertes, den die Kundenzufriedenheit in der Verkehrsdienstleistungspraxis einnimmt, bestand die generelle Zielsetzung der Arbeit darin, eine theoretische Fundierung und umfassende empirische Analyse dieses Untersuchungskomplexes zu leisten. Als empirisches Beispiel dienten die Angebote des schienengebundenen Personenfernverkehrs der Deutschen Bahn AG.

Im Rahmen einer definitorischen Konkretisierung des Zufriedenheitsbegriffes war es in diesem Zusammenhang zunächst erforderlich, zwischen verschiedenen Ausprägungsformen der Kundenzufriedenheit mit Dienstleistungen zu differenzieren. Aus einer konsumaktübergreifenden Perspektive heraus wurde dabei die Zufriedenheit eines Kunden in bezug auf alle bisherigen Erfahrungen mit dem Anbieter von der Zufriedenheit mit einer konkreten Dienstleistungstransaktion abgegrenzt. Letztere wiederum beinhaltet die Zufriedenheit mit den verschiedenen Episoden einer einzelnen Dienstleistung. Diese Episodenzufriedenheiten resultieren ihrerseits aus der Zufriedenheit mit den einzelnen Kontaktpunkten einer Dienstleistungsepisode.

Vor diesem Hintergrund stand die Fragestellung im Mittelpunkt, wie aus einer prozeßbezogenen Perspektive die Kundenzufriedenheit mit personenbezogenen Verkehrsdienstleistungen $\mathrm{zu}$ operationalisieren ist und welche Bestimmungsfaktoren die kundenseitige Wahrnehmung der in Anspruch genommenen Dienstleistung beeinflussen. Dabei sollte insbesondere der Episodencharakter des 
Dienstleistungskonsums und die damit einhergehende Dynamik der Zufriedenheitsbildung in expliziter Weise Berücksichtigung finden.

Um den dargestellten Zielsetzungen gerecht zu werden, wurden in einem ersten Schritt die Anforderungen an einen Operationalisierungsansatz zur Erfassung und Erklärung der Kundenzufriedenheit mit Verkehrsdienstleistungen erörtert. Neben allgemeinen Anforderungen standen dabei generelle dienstleistungsspezifische sowie verkehrsdienstleistungsbezogene Besonderheiten im Mittelpunkt. Hier zeigte sich, daß aus dem immateriellen Gutscharakter von Dienstleistungen und den zur Leistungserstellung notwendigen Fähigkeiten des Anbieters ein im Vergleich zum Konsumgüterbereich höheres wahrgenommenes Kaufrisiko resultiert, das wiederum mit Veränderungen im Involvement der Kunden einhergehen und damit die Informationsaufnahme und -verarbeitung eines Verkehrsdienstleistungskunden in entscheidender Weise beeinflussen kann. Des weiteren wurde deutlich, daß die zur Dienstleistungserstellung grundsätzlich notwendige Integration eines externen Faktors in unterschiedlicher Intensität auf einer räumlichen, zeitlichen, funktionalen sowie sozialen Ebene des Kundenkontaktes stattfindet und damit in vielfältiger Weise auf die Kundenwahrnehmung einwirken kann. So erfordert z.B. die Beförderung eines Fahrgastes zumindest dessen physische und zeitliche Präsenz.

In diesem Zusammenhang wurden Verkehrsdienstleistungen in der Weise definiert, daß unter diese neben der eigentlichen Beförderungsleistung auch die mit der Beförderung verbundenen ergänzenden Leistungsprozesse und die hierzu erforderlichen Potentiale subsumiert werden. Dieses Verständnis von Verkehrsdienstleistungen führte dazu, auch die der eigentlichen Kerndienstleistung vorund nachgelagerten Teilprozesse in die Betrachtung einzubeziehen. Aus einer solchen Sichtweise wird der einer Verkehrsdienstleistung inhärente Schnittstellenund Prozeßcharakter deutlich, der die Berücksichtigung des Zusammenspiels einer Vielzahl von Teilprozessen der von einem Kunden erlebten Reisekette zwingend erforderlich macht, um Erkenntnisse über den Entstehungsprozeß der Kundenzufriedenheit gewinnen zu können. Während die Reisekette in erster Linie die unternehmensseitig erbrachten Teilprozesse umfaßt, beinhaltet der Kundenprozeß darüber hinaus auch die vom Kunden vor, während und nach einer Reise selbst erstellten Teilprozesse, deren Verlauf wiederum die Kundenzufriedenheit in entscheidender Weise prägen und damit auch die zukünftige Verkehrsmittelwahl und -beurteilung beeinflussen kann. Die verschiedenen Determinanten der aus Kundensicht wahrgenommenen Verkehrswertigkeit und die Beachtung der spezifischen Reisesituation von Verkehrsdienstleistungskunden wurden in diesem Andreas Siefke and Universität Münster - 978-3-631-75103-9 
Zusammenhang als weitere zentrale Voraussetzungen für einen Operationalisierungsansatz der Kundenzufriedenheit mit Verkehrsdienstleistungen identifiziert.

Die erarbeiteten Anforderungen an einen Operationalisierungsansatz der Kundenzufriedenheit bildeten das Fundament für die sich anschließende Diskussion der in der Literatur erörterten Erklärungsansätze der Kundenzufriedenheit. Da bisher in der Wissenschaft kein Konsens über das konzeptionelle Grundverständnis von Kundenzufriedenheit erzielt werden konnte und darüber hinaus auch die Abgrenzung der Kundenzufriedenheit von dem Begriff der Dienstleistungsqualität sowie dem Einstellungskonstrukt in der wissenschaftlichen Diskussion nicht unstrittig ist, wurden zunächst konzeptionelle Differenzierungspotentiale dieser Konstrukte einer Analyse unterzogen. Hier zeigte sich, daß vor allem eine starke inhaltliche Nähe zwischen der Dienstleistungsqualität und dem Konstrukt der Einstellungen festzustellen ist, während insbesondere die „klassische" Sichtweise der Kundenzufriedenheit als ein auf eine spezifische Transaktion gerichtetes Konstrukt eine Abgrenzung der Kundenzufriedenheit von den beiden anderen Begriffen ermöglicht. Eine solche Sichtweise der Kundenzufriedenheit erleichterte überdies die Analyse der kausalen Beziehungen zwischen den verschiedenen Konstrukten, aus der hervor ging, daß die Kundenzufriedenheit nicht in einem konkurrierenden, sondern vielmehr in einem sich ergänzenden Verhältnis zu den beiden anderen Konstrukten steht.

Auf einem vergleichsweise abstrakten Niveau besteht in der Literatur weitestgehend Einigkeit darin, daß unter Kundenzufriedenheit das Ergebnis eines psychischen Soll-Ist-Vergleiches über ein Konsumerlebnis zu verstehen ist. Die Erörterung grundlegender Theorien und Modellansätze der Kundenzufriedenheit zeigte jedoch, daß sehr unterschiedliche Interpretationsformen insbesondere hinsichtlich der konkreten Operationalisierung der Soll- als auch der Konsumerlebniskomponente anzutreffen sind. In diesem Kontext wurde deutlich, daß vor allem die Notwendigkeit einer expliziten Operationalisierung der Soll-Komponente auf der empirischen Sprachebene in jüngeren Zeit zunehmend in Frage gestellt und einer direkten Messung der Vorzug eingeräumt wird. Des weiteren konnte festgestellt werden, daß das Konsumerlebniselement der Kundenzufriedenheit in zwei verschiedenen Erscheinungsformen (zeitliche Dimension) und auf unterschiedlichen Aggregationsebenen (sachliche Dimension) diskutiert und interpretiert wird. Während die statische Sichtweise die Kundenzufriedenheit im Sinne einer Momentaufnahme behandelt, spiegelt sich in der dynamischen Sichtweise ein konsumakt- bzw, konsumphasenbezogenes und damit prozeßorientierAndreas Siefke and Universität Munster - 978-3-631-75103-9 
tes Verständnis der Kundenzufriedenheit wider. Darüber hinaus gilt es zu unterscheiden, ob die Mikro- oder die Makroebene der Kundenzufriedenheit Gegenstand des Interesses ist.

Die Diskussion dieser Sachverhalte erfolgt in der Literatur vornehmlich im Zusammenhang mit dem Disconfirmation-Modell der Kundenzufriedenheit, das die höchste Verbreitung in der Zufriedenheitsforschung erfahren hat. Über die Problematik der Operationalisierung der Soll-Komponente hinaus konnte im Rahmen der weiteren Ausführungen aufgezeigt werden, daß das Modell in seiner ursprünglichen Form den Prozeßcharakter von Dienstleistungen nur unzureichend berücksichtigt. Der Episodencharakter von (Verkehrs-)Dienstleistungen auf der einen Seite sowie die damit einhergehende prozeßbezogene Entstehung des Zufriedenheitsurteils auf der anderen Seite machen jedoch eine Dynamisierung des Disconfirmation-Modells zwingend erforderlich. Dabei wurde in der Erörterung der psychologischen Grundlagen des Entstehungsprozesses von Kundenzufriedenheit deutlich, daß für eine prozeßorientierte Operationalisierung der Kundenzufriedenheit auf der theoretischen Sprachebene mit dem Konzept der episodischen Informationsverarbeitung bzw. dem Service-Script-Ansatz eine fundierte theoretische Basis zur Verfügung steht.

Die in diesem Zusammenhang in der Wissenschaft diskutierten Wirkungseffekte einer im Zeitablauf zu beobachtenden Dynamik der Kundenzufriedenheit spiegeln sich auch in den Ergebnissen der wenigen in diesem Bereich anzutreffenden empirischen Forschungsarbeiten wider, zu deren Analyse zwischen einer konsumakt- sowie einer konsumphasenbezogenen Betrachtungsweise unterschieden wurde. Diese Studien erwiesen sich einerseits als ein eindeutiger Beleg für die Sinnhaftigkeit einer am Kundenprozeß orientierten Analyse der Kundenzufriedenheit und der Existenz dynamischer Effekte der Kundenzufriedenheit, offenbarten auf der anderen Seite aber auch Schwächen im Hinblick auf die Strukturierung des Kundenprozesses sowie hinsichtlich der Berücksichtigung von Bestimmungsfaktoren, die die Kundenzufriedenheit beeinflussen.

Für eine prozeßorientierte Analyse der Kundenzufriedenheit ist neben der Operationalisierung des Konstruktes auf der theoretischen Sprachebene auch dessen Zugänglichkeit auf der empirischen Sprachebene sicherzustellen. Eine zentrale Voraussetzung hierfür besteht in der Ermittlung des vom Kunden wahrgenommenen Dienstleistungsprozesses. Die Darstellung der zu diesem Zweck in der Wissenschaft entwickelten Instrumente verdeutlichte, daß die Konzepte des 
Blueprinting bzw. des Service Mapping in besonderer Weise dazu geeignet sind, den Kundenpfad zu strukturieren und zu visualisieren.

Im Rahmen der sich anschließenden vergleichenden Diskussion von Verfahren zur Messung der Kundenzufriedenheit aus Nachfragersicht konnte kein für das vorliegende Untersuchungsziel allein ausreichend geeignetes Verfahren identifiziert werden. So ergaben sich anhand des zur Bewertung der verschiedenen Meßansätze aufgestellten Kriteriensets sowohl für die analysierten objektiven und als auch für die subjektiven Meßverfahren spezifische Vor- und Nachteile, die den alleinigen Einsatz eines Verfahrens nicht rechtfertigen. Da sich keines der Verfahren hinsichtlich aller Bewertungskriterien als dominant erwies, wurden Empfehlungen hinsichtlich eines kombinierten Einsatzes der verschiedenen Meßansätze abgeleitet.

Aufbauend auf diesen Ausführungen erfolgte die Formulierung eines phasenorientierten Ansatzes zur Erfassung und Erklärung der Kundenzufriedenheit mit Verkehrsdienstleistungen. Da die Analyse der Kundenzufriedenheit mit einer spezifischen Transaktion im Mittelpunkt der Arbeit stand, wurden zunächst die Strukturierung und Visualisierung der zu untersuchenden Verkehrsdienstleistung "Bahnfernreise“ vorgenommen, die zu einer Unterscheidung von insgesamt fünfzehn Kontaktpunkten führten. Diese wiederum bildeten das Fundament zur Ableitung der vier Dienstleistungsepisoden „Vor-Reisephase", „Reisephase", "Umsteigephase" und „Nach-Reisephase".

Der ermittelte idealtypische Kundenpfad stellte die Basis für die Ableitung eines Hypothesengerüstes der Kundenzufriedenheit mit Verkehrsdienstleistungen dar. Zur Formulierung von Untersuchungshypothesen wurde auf die in der Forschung diskutierten Struktur- und Wirkungseffekte der Zufriedenheitsdynamik sowie auf die in diesem Zusammenhang erörterten theoretischen und empirischen Forschungsarbeiten zurückgegriffen. Für die Analyse von Bestimmungsfaktoren der Kundenzufriedenheit wurden eine Unterscheidung zwischen personenbezogenen und reise- bzw. verkehrsdienstleistungsspezifischen Determinanten vorgenommen sowie entsprechende Untersuchungshypothesen abgeleitet. Aufbauend auf den Überlegungen zu der Struktur und der Dynamik der Kundenzufriedenheit sowie den identifizierten Bestimmungsfaktoren konnte ein Bezugsrahmen aufgestellt werden, der gleichzeitig die Struktur der sich anschließenden empirischen Untersuchung vorgab. Der neueren Forschungsliteratur folgend wurde im Rahmen des gewählten Meßansatzes auf eine explizite Operationalisierung der Soll- und der Ist-Komponente der Kundenzufriedenheit verzichtet und ein direkter Ein- 
komponenten-Ansatz zur Erfassung der Kundenzufriedenheit auf der Basis einer fünfstufigen Zufriedenheitsskala gewählt, der eine Ergänzung durch die Erhebung kritischer Ereignisse auf der Episodenebene erfuhr.

Die zusammenfassende Würdigung der empirischen Ergebnisse setzt an der Zielsetzung einer modelltheoretischen Validierung und Erklärung der Kundenzufriedenheit mit Verkehrsdienstleistungen an. Die Eignung des entwickelten Meßinstrumentes zur Erfassung der Kundenzufriedenheit mit Verkehrsdienstleistungen konnte durch eine Überprüfung am Beispiel des innerdeutschen Schienenpersonenfernverkehrs bestätigt werden. Dabei ist zunächst festzuhalten, daß sich alle Kausalmodelle empirisch bewährt und dabei z.T. zur Präzisierung der aufgestellten Untersuchungshypothesen über die Struktur und die Dynamik der Kundenzufriedenheit beigetragen haben ( $v g l$. Abbildung 56).

Als erstes zentrales Ergebnis der empirischen Untersuchung ist hervorzuheben, daß sowohl auf der Kontaktpunkt- als auch auf der Episodenebene eindeutige Effekte der Dynamik der Kundenzufriedenheit beobachtet werden konnten. Die Resultate dieser Analysen sind als ein klares Indiz für die prozeßbezogene Wahrnehmung und Entstehung der Kundenzufriedenheit mit Verkehrsdienstleistungen zu werten. In Kombination mit den Ergebnissen der Fragen nach kritischen Ereignissen konnte der Informationsgehalt der Ausführungen weiter gesteigert werden.

Die differenzierte intraphasenspezifische Analyse der Kundenzufriedenheit offenbarte in der Vor-Reisephase die besondere Bedeutung der Bahnhöfe als physischer Ausgangspunkt der Kerndienstleistung „Bahnreise“, während der Einfluß personenbezogener Kontaktpunkte auf die Episodenzufriedenheit vergleichsweise niedrig ausfiel. In diesem Zusammenhang zeigte sich überdies, daß der Parkvorgang keinen nachhaltigen Einfluß auf die Vor-Reisezufriedenheit ausübt. Innerhalb dieser Episode einer Bahnreise wurden zum Teil relativ geringe Korrelationen zwischen den einzelnen Kontaktpunkten festgestellt, die als ein Indiz für die vergleichsweise eigenständige Wahrnehmung bzw. bedingte Unabhängigkeit der einzelnen Abschnitte der Vor-Reisephase angesehen werden können und damit die theoretischen Ausführungen zum Prozeßcharakter der Kundenzufriedenheit zumindest für diese Episode einer Bahnreise zu einem gewissen Grad relativieren. 


\begin{tabular}{|c|c|c|}
\hline Hypothese & Inhalt & Befund \\
\hline Hyp Trans & $\begin{array}{l}\text { Die Kundenzufriedenheit mit einer Bahnreise (Transaktionsebene) setzt } \\
\text { sich aus drei signifikant voneinander verschiedenen } \\
\text { Episodenzufriedenheiten zusammen: der Vor-Reisezufriedenheit, der } \\
\text { Reisezufriedenheit sowie der Nach-Reisezufriedenheit. }\end{array}$ & bestätigt \\
\hline Hyp D Episod & $\begin{array}{l}\text { Die Zufriedenheit mit einer zeitlich vorgelagerten Episode übt einen } \\
\text { positiven Einfluß auf die Zufriedenheit mit darauf folgenden Episoden aus. }\end{array}$ & bestătigt \\
\hline Hyp I Trans 1 & $\begin{array}{l}\text { Die Zufriedenheit mit der Reisephase übt den stärksten Einfluß auf die } \\
\text { Transaktionszufriedenheit aus. }\end{array}$ & bestätigt \\
\hline Hyp I Trans 2 & $\begin{array}{l}\text { Die Zufriedenheit mit der Nach-Reisephase übt den geringsten Einfluß auf } \\
\text { die Transaktionszufriedenheit aus. }\end{array}$ & bestätigt \\
\hline Hyp D $\mathrm{Bz}$ & $\begin{array}{l}\text { Die Zufriedenheit mit der Transaktion hat einen positiven Einfluß auf die } \\
\text { Beziehungszufriedenheit. }\end{array}$ & bestätigt \\
\hline Hyp D Loyal & $\begin{array}{l}\text { Die Zufriedenheit mit der Transaktion übt einen positiven Einfluß auf die } \\
\text { Kundenloyalität aus. }\end{array}$ & bestätigt \\
\hline Hyp Episod & $\begin{array}{l}\text { Die Kundenzufriedenheit mit einer Episode einer Bahnreise setzt sich aus } \\
\text { signifikant voneinander verschiedenen Kontaktpunktzufriedenheiten } \\
\text { zusammen. }\end{array}$ & $\begin{array}{l}\text { für alle } \\
\text { Episoden } \\
\text { bestätigt }\end{array}$ \\
\hline Hyp Episod 1 & $\begin{array}{l}\text { Die Zufriedenheit mit der Vor-Reisephase beinhaltet die Zufriedenheit mit } \\
\text { fünf Kontaktpunkten: der Informationsphase, der Anreisephase, dem } \\
\text { Parkvorgang, dem Schalterkontakt sowie dem Aufenthalt am Startbahnhof. }\end{array}$ & $\begin{array}{l}\text { eingeschränkt } \\
\text { bestätigt }\end{array}$ \\
\hline Hyp Episod 2 & $\begin{array}{l}\text { Die Zufriedenheit mit der Reisephase ergibt sich aus der Zufriedenheit mit } \\
\text { sechs Kontaktpunkten: der Abfahrt vom Startbahnhof, dem Abteilaufenthalt, } \\
\text { dem Personalkontakt, dem Besuch des Zugrestaurants/-bistros, den } \\
\text { sanitären Anlagen im Zug sowie der Ankunft am Zielbahnhof. }\end{array}$ & bestätigt \\
\hline Hyp Episod 3 & $\begin{array}{l}\text { Die Zufriedenheit mit der Umsteigephase setzt sich aus der Zufriedenheit } \\
\text { mit zwei Kontaktpunkten zusammen: dem Umsteigevorgang sowie der } \\
\text { Weiterfahrt. }\end{array}$ & bestätigt \\
\hline Hyp Episod 4 & $\begin{array}{l}\text { Die Zufriedenheit mit der Nach-Reisephase ist durch die Zufriedenheit mit } \\
\text { zwei Kontaktpunkten gekennzeichnet: dem Aufenthalt am Zielbahnhof } \\
\text { sowie der Abreise zum endgültigen Ziel der Reise. }\end{array}$ & bestätigt \\
\hline Hyp D $\mathrm{kP}$ & $\begin{array}{l}\text { Die Zufriedenheit mit einem zeitlich vorgelagerten Kontaktpunkt übt einen } \\
\text { positiven Einfluß auf die Zufriedenheit mit darauf folgenden Kontaktpunkten } \\
\text { aus. }\end{array}$ & $\begin{array}{l}\text { für alle } \\
\text { Episoden } \\
\text { bestätigt }\end{array}$ \\
\hline Hyp I Episod 1 & $\begin{array}{l}\text { Innerhalb der Vor-Reisephase übt } \text { die Zufriedenheit mit } \\
\text { personenbezogenen Kontaktpunkten den stärksten Einfluß auf die } \\
\text { Episodenzufriedenheit aus. }\end{array}$ & $\begin{array}{c}\text { nicht } \\
\text { bestätigt }\end{array}$ \\
\hline Hyp I Episod 2a & $\begin{array}{l}\text { Innerhalb der Reisephase beeinflußt die Zufriedenheit mit der Pünktlichkeit } \\
\text { am Zielbahnhof die Episodenzufriedenheit am stärksten. }\end{array}$ & $\begin{array}{c}\text { nicht } \\
\text { bestätigt }\end{array}$ \\
\hline Hyp I Episod 2b & $\begin{array}{l}\text { Innerhalb der Reisephase übt die Zufriedenheit mit dem Zugrestaurant/- } \\
\text { bistro einen nur geringen Einfluß auf die Zufriedenheit mit dieser Episode } \\
\text { aus. }\end{array}$ & bestätigt \\
\hline Hyp I Episod 3 & $\begin{array}{l}\text { Innerhalb der Umsteigephase übt die Zufriedenheit mit den zeitlichen } \\
\text { Komponenten der Weiterfahrt den stärksten Einfluß auf die Zufriedenheit } \\
\text { mit der Episode aus. }\end{array}$ & bestätigt \\
\hline Hyp I Episod 4 & $\begin{array}{l}\text { Innerhalb der Nach-Reisephase ist die Zufriedenheit mit der Abreise zum } \\
\text { endgültigen Zielort die dominierende Teilzufriedenheit dieser Episode. }\end{array}$ & bestätigt \\
\hline
\end{tabular}

\section{Abb. 56: Zusammenfassende Übersicht zur Prüfung der Untersuchungs- hypothesen zur Struktur und Dynamik der Kundenzufriedenheit mit Verkehrsdienstleistungen}

Andreas Siefke and Universität Münster - 978-3-631-75103-9

Downloaded from PubFactory at 01/11/2019 08:18:16AM 
Eine solche Einschränkung erfahren hingegen die Ergebnisse der weiteren Abschnitte einer Bahnreise nicht. So waren in der Reisephase vergleichsweise hohe Korrelationen zwischen verschiedenen Kontaktpunkten dieser Episode festzustellen, die den Wahrnehmungsverbund der Teilerlebnisse dieser Phase einer Bahnreise nachhaltig bestätigen. Im Hinblick auf den Einfluß der einzelnen Kontaktpunktzufriedenheiten auf die Zufriedenheit mit der Episode konnte die Zufriedenheit mit der Pünktlichkeit der Züge als ein „Hygienefaktor" der Kundenzufriedenheit identifiziert werden, während sich die Zufriedenheit mit dem Personal, die den relativ höchsten Einfluß auf die Episodenzufriedenheit ausübt, als „Motivator" der Kundenzufriedenheit herausstellte. Des weiteren wurde deutlich, daß der Einfluß der nicht im Fahrpreis enthaltenen Dienstleistung „Verpflegung im Zugrestaurant" keinen nennenswerten Einfluß auf die Reisezufriedenheit ausübt.

Die besondere Bedeutung zeitlicher Komponenten für die Zufriedenheit mit der Dienstleistung zeigte sich in der Analyse der Umsteigephase. Neben dem Wunsch nach einem auf der zeitlichen Ebene reibungslosen Verlauf der mit dem Umsteigen in einen Anschlußzug verbundenen Teilprozesse ist in den Ergebnissen darüber hinaus zu erkennen, daß das interne Schnittstellenmanagement der Bahn hier in besonderer Weise gefordert ist, die Kunden mit entsprechenden Informationen und Orientierungshilfen zu versorgen.

Die Analyse der Zufriedenheit mit der Nach-Reisephase führte zu dem Ergebnis, daß insbesondere die Zufriedenheit mit dem Verlauf der Weiterreise zum endgültigen Zielort der Reise die Episodenzufriedenheit in entscheidender Weise beeinflußt. Wenngleich auch von der Zufriedenheit mit dem Zielbahnhof eine vergleichsweise starke Wirkung auf die Nach-Reisezufriedenheit ausgeht, wird in den Ergebnissen deutlich, daß ein Reisender mit dem Verlassen des Zielbahnhofs die Verkehrsdienstleistung "Bahnreise" als weitgehend abgeschlossen ansieht und damit die vom Kunden selbst erstellten Teilprozesse an Bedeutung gewinnen.

In der interphasenspezifischen Analyse der Kundenzufriedenheit war zunächst die sehr hohe Bedeutung der Reisephase für die Bildung des Zufriedenheitsurteils über die gesamte Bahnreise auffällig, in der die zentrale Stellung der Kerndienstleistung für das phasenübergreifende Zufriedenheitsurteil klar zum Ausdruck kommt. Dies spiegelte sich auch in der quantitativen Betrachtung der für die verschiedenen Episoden genannten kritischen Ereignisse wider, von denen allein ca. 50\% auf diesen Abschnitt einer Bahnreise entfielen. Da jedoch auch von den weiteren Episoden und hier insbesondere von der Vor-Reisephase ein erkennbarer Einfluß auf die Transaktionszufriedenheit ausgeht, wird die Notwen- 
digkeit eines umfassenden Verständnisses der Dienstleistung „Bahnreise“ deutlich. Allerdings konnte ein nur vergleichsweise geringer Einfluß der Nach-Reisezufriedenheit auf die gesamte Zufriedenheit mit einer Bahnreise ermittelt werden, der auf den eher „autonomen“ Charakter dieser Phase einer Bahnreise hindeutet. So wird augenscheinlich der Verlauf dieses Abschnittes einer Bahnreise nicht unmittelbar dem Verantwortungsbereich des Verkehrsdienstleisters zugeordnet.

Hinsichtlich der Dynamik der Kundenzufriedenheit auf der Episodenebene konnte ein positiver Einfluß der Zufriedenheit mit einer vorgelagerten Episode auf die Zufriedenheit mit einer zeitlich nachfolgenden Episode eindeutig nachgewiesen werden. Gleichwohl dürfen diese Einflußstrukturen in ihrer Stärke nicht überinterpretiert werden, da die Ergebnisse, trotz der erkennbaren Dynamik zwischen den verschiedenen Episoden, auf eine vergleichsweise eigenständige VVahrnehmung der verschiedenen Phasen auf der Episodenebene hindeuten.

Die Analyse des Einflusses der aktuellen Transaktionszufriedenheit auf die Zufriedenheit mit allen bisherigen Erfahrungen und damit auf die Zufriedenheit auf der Beziehungsebene ergab, daß hier die Existenz eines Rezenzeffektes zu vermuten ist. So beeinflußt die Zufriedenheit mit der aktuellen Transaktion die Beziehungszufriedenheit in einem nicht unerheblichen Ausmaß.

Ebenso wurde in den Ausführungen die zentrale Stellung der Kundenzufriedenheit im Hinblick auf das künftige Verhalten der Kunden gegenüber dem Verkehrsdienstleister Deutsche Bahn AG deutlich. Während eine (sehr) hohe Zufriedenheit zu einer vergleichsweise starken (bekundeten) Kundenloyalität führt, ist bereits bei einer nur leichten Abnahme des Zufriedenheitsurteils eine deutliche Vergrößerung des Abwanderungspotentials zu beobachten, dessen mögliche Zunahme überdies durch die nur geringen Wechselbarrieren von Bahnkunden begünstigt wird.

Die Analyse des Einflusses personen- und reisebezogener Bestimmungsfaktoren auf die Kundenzufriedenheit mit einer Bahnreise bildete den Gegenstand der weiteren Ausführungen. Wie aus Abbildung 57 hervorgeht, ist die Aussagekraft der verschiedenen Einflußfaktorengruppen differenziert zu beurteilen. 


\begin{tabular}{|c|c|c|}
\hline Hypothese & Inhalt & Befund \\
\hline Hyp $_{\text {Dem } 1}$ & $\begin{array}{l}\text { Das Alter der Reisenden beeinflußt die Zufriedenheit mit den einzelnen } \\
\text { Episoden sowie die Zufriedenheit mit der gesamten Bahnreise. }\end{array}$ & $\begin{array}{c}\text { eingeschränkt } \\
\text { bestätigt }\end{array}$ \\
\hline Hyp Dem 2 & $\begin{array}{l}\text { Mit steigendem Alter der Reisenden nimmt der Einfluß der Vor- und der } \\
\text { Nach-Reisephase auf die Transaktionszufriedenheit zu. }\end{array}$ & $\begin{array}{l}\text { eingeschränkt } \\
\text { bestätigt }\end{array}$ \\
\hline Hyp Dem 3 & $\begin{array}{l}\text { Das Geschlecht der Reisenden beeinflußt die Zufriedenheit mit den } \\
\text { einzelnen Episoden sowie die Zufriedenheit mit der gesamten Bahnreise. }\end{array}$ & $\begin{array}{l}\text { eingeschränkt } \\
\text { bestätigt }\end{array}$ \\
\hline Hyp okon 1 & $\begin{array}{l}\text { Das Einkommen der Reisenden übt einen signifikanten Einfluß auf die } \\
\text { Zufriedenheit mit einer Bahnreise und mit ihren einzelnen Episoden aus. }\end{array}$ & $\begin{array}{l}\text { eingeschränkt } \\
\text { bestätigt }\end{array}$ \\
\hline Hyp okon 2 & $\begin{array}{l}\text { Die dauerhafte Verfügbarkeit eines Pkws hat einen negativen Einfluß auf } \\
\text { die Zufriedenheit mit einer Bahnreise und mit ihren einzelnen Episoden. }\end{array}$ & $\begin{array}{l}\text { eingeschränkt } \\
\text { bestätigt }\end{array}$ \\
\hline Hyp vм $_{\text {vi }}$ & $\begin{array}{l}\text { Die Erwăgung eines alternativen Verkehrsmittels beeinflußt die } \\
\text { Zufriedenheit mit einer Bahnreise und mit ihren einzelnen Episoden. }\end{array}$ & bestätigt \\
\hline Hyp Psycho 1 & $\begin{array}{l}\text { Das von Kunden wahrgenommene Kaufrisiko übt einen negativen Einfluß } \\
\text { auf die Zufriedenheit mit einer Bahnreise aus. }\end{array}$ & bestätigt \\
\hline Hyp Psycho 2 & $\begin{array}{l}\text { Das Involvement der Reisenden gegenüber der Bahn hat einen positiven } \\
\text { Einfluß auf die Zufriedenheit mit einer Bahnreise. }\end{array}$ & bestätigt \\
\hline Hyp Psycho 3 & $\begin{array}{l}\text { Die Zufriedenheit mit einer Bahnreise wird durch eine positive Einstellung } \\
\text { der Reisenden gegenüber der Bahn vorteilhaft beeinflußt. }\end{array}$ & bestätigt \\
\hline Hyp vol 1 & $\begin{array}{l}\text { Der Reiseanlaß hat eine signifikante Wirkung auf die Zufriedenheit mit den } \\
\text { einzelnen Episoden sowie die Zufriedenheit mit der gesamten Bahnreise. }\end{array}$ & $\begin{array}{c}\text { eingeschränkt } \\
\text { bestătigt }\end{array}$ \\
\hline Hyp $_{\text {voL } 2}$ & $\begin{array}{l}\text { Die genutzte Zugart übt einen signifikanten Einfluß auf die Zufriedenheit mit } \\
\text { der Reisephase und die Zufriedenheit mit der gesamten Bahnreise aus. }\end{array}$ & bestătigt \\
\hline Hyp voL $3_{3}$ & $\begin{array}{l}\text { Die Reiseklasse nimmt Einfluß auf die Zufriedenheit mit der Reisephase } \\
\text { und die Zufriedenheit mit der gesamten Bahnreise. }\end{array}$ & bestätigt \\
\hline$H_{\text {ypp }}$ vo 4 & $\begin{array}{l}\text { Die Zufriedenheit mit der Reisephase und mit der gesamten Bahnreise wird } \\
\text { durch den Abteiltyp beeinflußt. }\end{array}$ & bestätigt \\
\hline Hyp vol 5 & $\begin{array}{l}\text { Eine hohe Auslastung der Züge übt einen negativen Einfluß auf die } \\
\text { Zufriedenheit mit der Reisephase und die Zufriedenheit mit der gesamten } \\
\text { Bahnreise aus. }\end{array}$ & bestätigt \\
\hline Hyp vol 6 & $\begin{array}{l}\text { Die Sitzplatzreservierung hat einen positiven Einfluß auf die Zufriedenheit } \\
\text { mit der Reisephase und die Zufriedenheit mit der gesamten Bahnreise. }\end{array}$ & bestätigt \\
\hline
\end{tabular}

\section{Abb. 57: Zusammenfassende Übersicht zur Prüfung der Untersuchungs- hypothesen zum Einfluß personen- und reisebezogener Bestimmungsfaktoren auf die Kundenzufrìedenheit}

Danach kann der Forschungsliteratur im Hinblick auf die (eingeschränkte) Erklärungskraft soziodemographischer Einflußfaktoren im Grundsatz zugestimmt werden, da sich die vermutete Wirkung dieser Größen auf die Wahrnehmung der in Anspruch genommenen Verkehrsdienstleistung und ihrer Elemente nur teilweise bestätigte. So zeigte sich bei der Analyse demographischer Bestimmungsfaktoren, daß das Alter der Reisenden sowohl die Zufriedenheit mit einzelnen Episoden der Bahnreise als auch die Zufriedenheit mit der gesamten Transaktion 
beeinflußt. Auch konnte eine leichte Bedeutungszunahme der Nach-Reisephase im Hinblick auf die Bildung des Gesamturteils mit zunehmendem Alter der Befragten festgestellt werden; ein Trend, der in dieser Form jedoch für die VorReisephase nicht ermittelt wurde. Bei der Analyse des Bestimmungsfaktors Geschlecht ergaben sich keine signifikanten Unterschiede in der transaktionalen Kundenzufriedenheit, wobei jedoch durchaus Unterschiede in der Dynamik und in den Einflußstrukturen der Kundenzufriedenheit aufgedeckt werden konnten. Ähnliches trifft für die analysierten sozioökonomischen Merkmale Einkommen und Pkw-Verfügbarkeit zu. Auch hier konnten die vermuteten Wirkungsannahmen nicht vollständig bestätigt werden. Dagegen hat die Erwägung eines alternativen Verkehrsmittels eine vergleichsweise starke und eindeutig interpretierbare negative Wirkung auf die Kundenzufriedenheit mit einer Bahnreise, die sich zudem in einer unterschiedlichen Dynamik auf der Episodenebene äußert. Insgesamt ist den hier betrachteten demographischen und sozioökonomischen Merkmalen im Vergleich zu den anderen Einflußfaktoren aber eine eher untergeordnete Bedeutung als Determinanten der Kundenzufriedenheit mit Verkehrsdienstleistungen zu bescheinigen.

Dagegen sind die Ergebnisse hinsichtlich der Wirkung psychographischer Bestimmungsfaktoren auf die Kundenzufriedenheit als ein eindeutiger Beleg für deren vergleichsweise hohe Kaufverhaltensrelevanz zu bewerten. So konnte sowohl für die Einstellungen der Bahnreisenden als auch für das Involvement, das einer Bahnreise von den Befragten entgegengebracht wird, eine positive Wirkung auf die Ausprägung der Kundenzufriedenheit mit einer Bahnreise ermittelt werden. Ebenso gelang der Nachweis, daß von dem wahrgenommenen Kaufrisiko eine eindeutig negative Wirkung auf die Transaktionszufriedenheit ausgeht.

Auch die Aussagekraft reisebezogener Einflußfaktoren zur Erklärung der Kundenzufriedenheit mit Verkehrsdienstleistungen ist insgesamt positiv zu beurteilen. Während die Analyse des Bestimmungsfaktors Reiseanlaß die vermuteten Zusammenhänge zwar nicht vollständig belegen konnte, erwiesen sich dagegen die weiteren untersuchten Faktoren als wichtige Determinanten der Zufriedenheit mit der Reisephase sowie der Transaktionszufriedenheit eines Bahnreisenden.

Die Würdigung der empirischen Untersuchungsergebnisse setzt auch eine kritische Auseinandersetzung mit der methodischen Vorgehensweise der Arbeit voraus. So soll im Hinblick auf die intraphasenspezifische Analyse der Kundenzufriedenheit nicht ignoriert werden, daß in der Vor- bzw. Nach-Reisephase lediglich 35\% bzw. 38\% der Varianz der Zufriedenheit mit diesen Episoden durch die 
verschiedenen Kontaktpunkte abgebildet werden. Eine denkbare Erklärung besteht darin, daß die diesen Phasen kundenseitig beigemessene Bedeutung im Hinblick auf die Bildung des Gesamturteils vergleichsweise niedrig ausfällt und damit ein eher inkonsistentes Antwortverhalten der Befragten die Folge ist. Ein weiterer Grund liegt möglicherweise in der nur eindimensionalen Operationalisierung der Episodenzufriedenheiten. Insofern offenbart sich in diesem Punkt eine vorstellbare Schwäche des Befragungsdesigns der zugrunde gelegten Erhebung, die aber die grundsätzliche Gültigkeit der identifizierten Struktur und Dynamik der Zufriedenheit mit diesen Phasen nicht in Frage stellt. Des weiteren ist zu erwähnen, daß aus forschungsökonomischen Gründen keine explizite Berücksichtigung der Umsteigephase als eigenständiger Episode im Rahmen der Analyse der Struktur und Dynamik der Transaktionszufriedenheit stattgefunden hat. Schließlich ist auch auf die zeitpunktbezogene Messung der Kundenzufriedenheit und der (bekundeten) Loyalität gegenüber der Verkehrsdienstleistung hinzuweisen, die damit der grundsätzlichen Kritik einer primär statischen Erfassung dynamischer Phänomene unterliegt, wobei, wie gezeigt, insbesondere die mit einer „Echtzeitmessung" der Kundenzufriedenheit verbundenen Probleme als nicht unerheblich einzustufen sind.

Demgegenüber liegt eine Stärke der vorliegenden Untersuchung in der umfangreichen Stichprobe von 603 Bahnreisenden. Damit konnte sichergestellt werden, daß die ermittelten Ergebnisse auf einer hinreichenden Datenbasis beruhen. Dabei gilt es jedoch zu beachten, daß die Untersuchung für eine durch spezifische Eigenarten geprägte Verkehrsdienstleistung durchgeführt wurde. Aus einer gebotenen kritischen Distanz können die ermittelten Ergebnisse jedoch im Prinzip auch auf weitere Verkehrsdienstleistungen, z.B. aus dem Flugbereich, übertragen werden.

Insgesamt ist damit festzustellen, daß die empirischen Befunde der vorliegenden Arbeit unbeschadet der aufgeführten Einschränkungen zahlreiche statistisch abgesicherte Aufschlüsse über den prozessualen Charakter sowie über die Struktur und die Dynamik der Kundenzufriedenheit mit Verkehrsdienstleistungen liefern. 


\section{Implikationen für das Management von Verkehrsdienstleistungs- unternehmen}

Der zunehmende Wettbewerbsdruck auf der Angebotsseite sowie ein deutlich gestiegenes Anspruchsniveau an die Qualität von Dienstleistungen auf der Nachfragerseite führen dazu, daß auch Verkehrsdienstleister in besonderer Weise gefordert sind, ihr Leistungsangebot im Wettbewerbsumfeld zu profilieren und die Zufriedenheit ihrer Kunden sicherzustellen.

Die empirischen Ergebnisse der vorliegenden Untersuchung belegen, daß die prozeßorientierte Analyse der Kundenzufriedenheit zentrale Hinweise zur Sicherung des langfristigen Unternehmenserfolges geben und damit wichtige Aufschlüsse für die Ausgestaltung des Zufriedenheitsmanagements von Verkehrsdienstleistungsunternehmen im allgemeinen und der Deutschen Bahn AG im besonderen liefern kann. In diesem Zusammenhang lassen sich vor allem folgende Implikationen für das Marketing der Deutschen Bahn AG ableiten:

- Die vorliegenden Resultate dokumentieren, daß eine Vielzahl von Aspekten entlang der gesamten Reisekette die Zufriedenheit mit einer Bahnreise prägt, wobei insbesondere der Reisephase und ihren einzelnen Kontaktpunkten eine zentrale Stellung bei der Bildung des Zufriedenheitsurteils zukommt. Im Vergleich zu Fluggesellschaften beziehen sich die Zufriedenheitsurteile eines Bahnreisenden zum Großteil auf Dienstleistungen, die von der Bahn erbracht werden. Durch die (bisherige) alleinige Nutzung der Bahnhöfe verfügt die Bahn über einen im Vergleich zu anderen Verkehrsdienstleistern sehr großen autonomen Gestaltungsbereich. So sind die externen Leistungsverflechtungen mit anderen Dienstleistungsunternehmen beispielsweise in Relation zu Fluggesellschaften weniger stark ausgeprägt. Der damit vergleichsweise hohe Autonomiegrad des bahnspezifischen Zufriedenheitsmanagements ermöglicht grundsätzlich eine optimale Ausgestaltung und Integration der von der Bahn selbst erstellten Teilprozesse in den Kundenprozeß. Diesem Vorteil steht jedoch der Umstand entgegen, daß es einem Reisenden leichter fält, die Verantwortlichkeit für einen ungünstigen Verlauf eines Teilabschnittes seiner Reise, wie z.B. das Verpassen des Anschlußzuges, dem Unternehmen Deutsche Bahn AG zuzuweisen.

- Insgesamt verdeutlichen die Untersuchungsergebnisse, daß dem Schnittstellenmanagement eines Verkehrsdienstleisters große Bedeutung zukommt. Neben den externen sind dabei insbesondere die internen Schnittstellen derart auszugestalten, daß der reibungslose und zufriedenstellende Verlauf der gesamten Reisekette sichergestellt wird. Im Sinne einer auf den Kundenprozeß ausgerichteten Gesamtbetrachtung gilt es in diesem Zusammenhang zu berücksichtigen, daß auch die der eigentlichen Kerndienstleistung vor- und nachgelagerten „Augenblicke der Wahrheit" ebenfalls „Augenblicke der (Un-) 
Zufriedenheit" darstellen und das Gesamturteil eines Kunden in entscheidender Weise beeinflussen können.

- Trotz der eindeutig zu erkennenden Dynamik der Kundenzufriedenheit werden die einzelnen Episoden einer Bahnreise von den Fahrgästen vergleichsweise eigenständig wahrgenommen. Dies eröffnet dem Unternehmen die Möglichkeit, ein Zufriedenheitsmanagement einzurichten, dessen Struktur sich an den verschiedenen Reiseabschnitten orientiert. Konkret könnte dies durch die Einrichtung von selbständigen Organisationseinheiten für die Vor-, Reise- und Nach-Reisephase realisiert werden. Da die Gesamtzufriedenheit mit einer Bahnreise jedoch auf der Wahrnehmung aller Episoden beruht, müssen diese Einheiten, auch unter Wirtschaftlichkeitsgesichtspunkten, eng miteinander vernetzt sein. Nicht die isolierte, sondern nur die integrierte Betrachtung der verschiedenen Reiseabschnitte wird dem Prozeßcharakter des Dienstleistungskonsums in ausreichender Weise gerecht.

- Vor dem Hintergrund der nachgewiesenen Dynamik der Kundenzufriedenheit kommt einer unternehmensinternen Kommunikation der ermittelten Daten eine nachhaltige Bedeutung zu. Im Sinne eines Markt-Feedback-Konzeptes gilt es, den Mitarbeitern und insbesondere dem Kundenkontaktpersonal bewußt zu machen, daß die prozeßorientierte Entstehung der Kundenzufriedenheit zur Folge hat, daß ein negatives Erlebnis in einer Phase einer Bahnreise auch die Wahrnehmung darauffolgender Teilprozesse und damit die Gesamtzufriedenheit in entscheidender Weise prägen kann. Das bereichsübergreifende Verständnis, ein wichtiger und zufriedenheitswirksamer Bestandteil in einer komplexen und im Verbund wahrgenommenen Dienstleistung zu sein, ist ein zentraler Garant für die Schaffung zufriedener Kunden.

- Eine wichtige Funktion der Kundenzufriedenheit ist in ihrer Eignung als Kontrollund Steuerungsgröße zu sehen. Die hierzu erforderliche regelmäßige Messung der Kundenzufriedenheit kann dabei in sinnvoller Weise gleichzeitig, bei entsprechender Akzeptanz auf der Mitarbeiterseite, als eine Bezugsgröße für die Entwicklung eines Mitarbeiter-Incentive-Systems genutzt werden. Die phasenorientierte Messung der Kundenzufriedenheit erleichtert dabei, neben dem Kundenkontaktpersonal auch die Mitarbeiter hinter der "Line of Visibility" in ein solches System zu integrieren.

- Des weiteren lassen sich die Ergebnisse der Zufriedenheitsmessung im Sinne eines Frühwarnsystem nutzen, indem neue oder sich im Zeitablauf verändernde Kundenanforderungen an die verschiedenen Abschnitte einer Bahnreise sowie die damit einhergehenden Veränderungen in der Struktur und Dynamik der Kundenzufriedenheit rechtzeitig erkannt werden. Diese Ergeb- 
nisse können wichtige Hinweise bei der Planung und Weiterentwicklung des Dienstleistungserstellungsprozesses liefern. ${ }^{1}$

- Die Umsetzung der aus Kundenzufriedenheitsmessungen gewonnenen Erkenntnisse in konkrete Handlungsmaßnahmen ist in hohem Maße von deren Realisierbarkeit abhängig. Dabei sind gleichermaßen technische wie ökonomische Faktoren zu berücksichtigen. Die prozeßorientierte Messung der Kundenzufriedenheit ermöglicht hierbei eine Hierarchisierung der die Kundenzufriedenheit konstituierenden Elemente und eine damit einhergehende Priorisierung einzelner Verbesserungsmaßnahmen. So läßt erst die zufriedenstellende Erfüllung der zentralen Hygienefaktoren aller Phasen einer Bahnreise, wie z.B. der Pünktlichkeit der Züge, die Einführung additiver Sekundärdienstleistungen im Sinne von Value-Added Services sinnvoll erscheinen. ${ }^{2}$

- Schließlich gilt es, die Eignung der Kundenzufriedenheit als BenchmarkingInstrument zu prüfen. Die Erhebung von Daten der Kunden konkurrierender Unternehmen ermöglicht einen Vergleich der eigenen Leistung mit dem Wettbewerbsumfeld. Eine diesbezüglich differenzierte Analyse kann über eine verkehrsmittelspezifische Betrachtung hinaus weitere potentielle Verbesserungsmöglichkeiten für die Ausgestaltung der einzelnen Episoden einer Bahnreise offenlegen. Meßmethodisch ist jedoch damit die sehr komplexe Aufgabe verbunden, die Vergleichbarkeit prozeßorientiert erhobener Daten sicherzustellen.

Insgesamt ist jedoch zu beachten, daß ein prozeßorientiertes Zufriedenheitsmanagement nur eine, wenn auch zentrale, Herausforderung an das Unternehmen Deutsche Bahn AG darstellt. So ist zu berücksichtigen, daß insbesondere im Verkehrsdienstleistungsbereich die Abstimmung des Kundenprozesses und der Unternehmensprozesse mit einer Vielzahl von Integrations- und Koordinationsaufgaben einhergeht. Dies hat eine deutliche Komplexitätserhöhung der zur Befriedigung der Mobilitätsbedürfnisse sowie der zur Sicherstellung einer hohen Kundenzufriedenheit notwendigen Tätigkeiten zur Folge, die die Deutsche Bahn $A G$ in Zukunft in verstärkter Weise zu bewältigen hat.

Vgl. Muffatto, M., Panizzolo, R., A Process-Based View for Customer Satisfaction, a.a.O., S. $155 \mathrm{ff}$.

2 Zum Begriff der Value-Added Services vgl. Laakmann, K., Value-Added Services als Profilierungsinstrument im Wettbewerb: Analyse, Generierung und Bewertung, a.a.O. S. $6 \mathrm{ff}$.

Andreas Siefke and Universität Münster - 978-3-631-75103-9 


\section{Implikationen für die Zufriedenheitsforschung im Dienstleistungs- bereich}

In Anbetracht der theoretischen Konzeption und der empirischen Untersuchungsergebnisse der vorliegenden Arbeit sowie der hieraus für die Unternehmenspraxis abgeleiteten Implikationen zeichnen sich für weiterführende konzeptionelle und empirische Forschungsarbeiten sowohl inhaltliche als auch methodische Ansatzpunkte ab:

- Ausgehend von dem vorliegenden Untersuchungsgegenstand "Bahnfernreise“ erscheint es wünschenswert, eine Vergleichbarkeit der für den Schienenpersonenverkehr erzielten Ergebnisse mit weiteren Verkehrsdienstleistungen herzustellen. Dabei sind insbesondere zwei Fragestellungen von Interesse. Zum einen ist zu analysieren, inwieweit sich auch bei anderen Verkehrsdienstleistungen (etwa im Luftverkehr) ähnliche Zufriedenheitsstrukturen nachweisen lassen. Zum anderen ist der Frage nachzugehen, ob sich die in der vorliegenden Untersuchung identifizierte Dynamik der Kundenzufriedenheit auch bei nicht schienengebundenen Verkehrsdienstleistungen wiederfinden läßt. Darüber hinaus besteht in .der Einbeziehung nicht verkehrsspezifischer Dienstleistungen eine interessante Ausweitung dieses Forschungsfeldes.

- Im Rahmen einer möglichen Replizierung der Untersuchung ist die Einbeziehung der Umsteigephase als einer eigenständigen Episode bei der Analyse der Struktur und der Dynamik der Transaktionszufriedenheit anzudenken. Ein derartiges Vorgehen impliziert jedoch die Messung der Zufriedenheit mit mindestens fünf Episoden und damit die Berücksichtigung von zumindest zwei Reisephasen (vor und nach dem Umsteigen in einen Anschlußzug). Darüber hinaus sollten die Möglichkeiten einer mehr als eindimensionalen Erfassung der einzelnen Episodenzufriedenheiten evaluiert werden.

- Ein durch die vorliegende Untersuchung nur flankierend behandelter Bereich stellt die Analyse des Einflusses psychographischer Bestimmungsfaktoren auf die Kundenzufriedenheit dar. Die in dieser Arbeit durchgeführte Betrachtung beschränkte sich auf die Untersuchung des Einflusses psychographischer Bestimmungsfaktoren auf die transaktionale Kundenzufriedenheit. Für eine phasenspezifische Analyse des Einflusses psychographischer Variablen sind in weiterführenden Forschungsarbeiten die Möglichkeiten einer prozeßorientierten Operationalisierung dieser Konstrukte zu prüfen.

- Wenngleich die Kundenzufriedenheit als eine zentrale Determinante der Kundenbindung identifiziert wurde, sollten im Rahmen vertiefender Forschungsbemühungen die Ergebnisse über die bekundete Wiedernutzungsabsicht mit Daten über das tatsächliche Kundenverhalten bzw. mit ökonomischen Erfolgsgrößen konfrontiert werden, um die Aussagekraft der Kundenzufriedenheit als Indikator der Kundenloyalität und des Unternehmenserfolges einer weiteren Prüfung unterziehen und mögliche Abweichungen näher ergründen zu können. 
- Eng damit verbunden ist die Frage nach dem „richtigen“ Zeitpunkt einer Messung der unterschiedlichen Konstrukte. Vor allem im Zusammenhang mit dem Zufriedenheitskonstrukt gilt es, sich auf der meßmethodischen Ebene in Zukunft mit den Möglichkeiten und Grenzen einer „on-process-Messung“ kritisch auseinanderzusetzen.

- Insbesondere im Hinblick auf die konsumakt- und damit transaktionsübergreifende Analyse der Struktur und Dynamik der Kundenzufriedenheit besteht in der Durchführung von Längsschnittuntersuchungen, z.B. in Form von Panelerhebungen, eine interessante Erweiterung der vorliegenden Arbeit. Speziell ist hier der Einfluß der Zufriedenheit mit zeitlich vorgelagerten Transaktionen auf die aktuelle Transaktionszufriedenheit zu untersuchen, um Erkenntnisse über die Bedeutung der in der Vergangenheit gemachten Erfahrungen für die aktuelle Kundenzufriedenheit zu gewinnen.

- Darüber hinaus sind sowohl Ansatzpunkte für die Umsetzung der aus Kundenzufriedenheitsmessungen gewonnenen Erkenntnisse in konkrete Handlungsmaßnahmen als auch die Möglichkeiten einer organisatorischen Verankerung des Zufriedenheitsmanagements im Rahmen zukünftiger Forschungsaktivitäten zu generieren. In diesem Zusammenhang sind insbesondere die notwendigen internen und externen Abstimmungsprozesse innerhalb der Reisekette zu analysieren.

- Schließlich stellen die Schaffung von Kundenzufriedenheit und deren Analyse stets Mittel zum Zweck eines übergreifenden ökonomischen Unternehmungserfolges dar. Ein zieladäquates Verhältnis von Kosten und Nutzen der Zufriedenheitsmessung ist dabei gleichermaßen wie die Ermittlung und Realisierung eines „optimalen" Zufriedenheitsniveaus anzustreben. Insbesondere vor dem Hintergrund des Prozeßcharakters der Kundenzufriedenheit sind hierin sowohl für die Wissenschaft als auch für die Unternehmenspraxis zentrale Herausforderungen angesprochen.

Insgesamt wird deutlich, daß trotz der bereits mannigfaltigen Bemühungen im Bereich der Erforschung der Kundenzufriedenheit weitere Fragestellungen durch die Wissenschaft zu beantworten sind, die angesichts des hohen Stellenwertes der Kundenzufriedenheit in der Unternehmenspraxis gegenwärtig und in der Zukunft von besonderer Relevanz sind. 
Andreas Siefke and Universität Münster - 978-3-631-75103-9

Downloaded from PubFactory at 01/11/2019 08:18:16AM

via free access 


\section{Anhang I:}

\section{Ergänzende Abbildungen}

\section{Abbildungsverzeichnis des Anhangs I}

Abb. A1: Ausgewählte Prüfkriterien der Kausalmodelle zur Untersuchung des Einflusses soziodemographischer Bestimmungsfaktoren auf die Kundenzufriedenheit

Abb. A2: Ausgewählte Prüfkriterien der Kausalmodelle zur Untersuchung des Einflusses soziodemographischer und reisebezogener Bestimmungsfaktoren auf die Kundenzufriedenheit.

Abb. A3: Beziehungszusammenhänge der Kundenzufriedenheit in Abhängigkeit vom Netto-Haushaltseinkommen. 


\begin{tabular}{|c|c|c|c|c|c|c|c|c|c|}
\hline \multirow{2}{*}{$\begin{array}{l}\begin{array}{c}\text { Bestimmungs- } \\
\text { faktor }\end{array} \\
\begin{array}{l}\text { Gute- Modell } \\
\text { kitterien }\end{array} \\
\end{array}$} & \multicolumn{3}{|c|}{ Alter } & \multicolumn{2}{|c|}{ Geschlecht } & \multicolumn{2}{|c|}{$\begin{array}{l}\text { PKW dauerhaft } \\
\text { verfügbar }\end{array}$} & \multicolumn{2}{|c|}{$\begin{array}{l}\text { Altematives Verkehrs } \\
\text { mittel erwogen }\end{array}$} \\
\hline & bis $26 \mathrm{~J}$. & $27-59 \mathrm{~J}$. & $\begin{array}{l}60 \mathrm{~J} \text {. und } \\
\text { âlter }\end{array}$ & weiblich & mănnlich & Ja & Nein & Ja & Nein \\
\hline AGLS & 0,958 & 0,977 & 0,993 & 0,977 & 0,981 & 0,984 & 0,960 & 0,973 & 0,976 \\
\hline Adjusted AGLS & 0,792 & 0,886 & 0,967 & 0,885 & 0,904 & 0,922 & 0,801 & 0,867 & 0,879 \\
\hline GFI & 0,966 & 0,974 & 0,987 & 0,973 & 0,985 & 0,986 & 0,958 & 0,978 & 0,976 \\
\hline AGFI & 0,830 & 0,870 & 0,934 & 0,864 & 0,925 & 0,932 & 0,792 & 0,891 & 0,878 \\
\hline RMR & 0,085 & 0,076 & 0,062 & 0,079 & 0,078 & 0,056 & 0,097 & 0,072 & 0,072 \\
\hline Freiheitsgrade & 3 & 3 & 3 & 3 & 3 & 3 & 3 & 3 & 3 \\
\hline $\begin{array}{c}\text { erklärte Varianz } \\
\text { Reise- } \\
\text { zufriedenheit }\end{array}$ & $1 \%$ & $14 \%$ & $2 \%$ & $14 \%$ & $4 \%$ & $6 \%$ & $10 \%$ & $12 \%$ & $6 \%$ \\
\hline $\begin{array}{l}\text { erklärte Varianz } \\
\text { Nach-Reise- } \\
\text { zufriedenheit }\end{array}$ & $23 \%$ & $14 \%$ & $26 \%$ & $26 \%$ & $12 \%$ & $12 \%$ & $28 \%$ & $21 \%$ & $14 \%$ \\
\hline $\begin{array}{l}\text { erklärte Varianz } \\
\text { Transaktions- } \\
\text { zufriedenheit }\end{array}$ & $41 \%$ & $80 \%$ & $38 \%$ & $65 \%$ & $75 \%$ & $74 \%$ & $65 \%$ & $89 \%$ & $59 \%$ \\
\hline $\begin{array}{l}\text { Faktorladung } \\
\text { globale Transak- } \\
\text { tionszufriedenheit }\end{array}$ & 0,95 & 0,80 & 0,91 & 0,87 & 0,78 & 0,79 & 0,85 & 0,80 & 0,83 \\
\hline $\begin{array}{l}\text { Faktorladung } \\
\text { Zufriedenheit } \\
\text { Preis/Leistung }\end{array}$ & 0,39 & 0,48 & 0,47 & 0,53 & 0,43 & 0,49 & 0,50 & 0,43 & 0,48 \\
\hline
\end{tabular}

Abb. A1: Ausgewählte Prüfkriterien der Kausalmodelle zur Untersuchung des Einflusses soziodemographischer Bestimmungsfaktoren auf die Kundenzufriedenheit

\begin{tabular}{|c|c|c|c|c|c|}
\hline $\begin{array}{c}\text { Bestimmungs- } \\
\text { faktor }\end{array}$ & \multicolumn{3}{|c|}{ Einkommen } & \multicolumn{2}{c|}{ Reiseanlaß } \\
\hline $\begin{array}{c}\text { Güte- Modell } \\
\text { kriterien }\end{array}$ & $\begin{array}{c}\text { bis } \\
2500 \mathrm{DM}\end{array}$ & $\begin{array}{c}2.501 \text { - } \\
5.000 \mathrm{DM}\end{array}$ & $\begin{array}{c}\text { Ober } \\
5.000 \mathrm{DM}\end{array}$ & $\begin{array}{c}\text { Geschäft- } \\
\text { lich }\end{array}$ & Privat \\
\hline AGLS & 0,980 & 0,990 & 0,963 & 0,947 & 0,990 \\
\hline Adjusted AGLS & 0,901 & 0,948 & 0,817 & 0,734 & 0,952 \\
\hline GFI & 0,978 & 0,994 & 0,958 & 0,934 & 0,991 \\
\hline AGFI & 0,892 & 0,969 & 0,792 & 0,772 & 0,956 \\
\hline RMR & 0,076 & 0,038 & 0,087 & 0,106 & 0,048 \\
\hline Freiheitsgrade & 3 & 3 & 3 & 3 & 3 \\
\hline $\begin{array}{c}\text { erklärte Varianz } \\
\text { Reise- } \\
\text { zufriedenheit }\end{array}$ & $10 \%$ & $2 \%$ & $14 \%$ & $4 \%$ & $8 \%$ \\
\hline $\begin{array}{c}\text { erklärte Varianz } \\
\text { Nach-Reise- } \\
\text { zufriedenheit }\end{array}$ & $28 \%$ & $18 \%$ & $10 \%$ & $12 \%$ & $21 \%$ \\
\hline $\begin{array}{c}\text { erklärte Varianz } \\
\text { Transaktions- } \\
\text { zufriedenheit }\end{array}$ & $74 \%$ & $66 \%$ & $70 \%$ & $72 \%$ & $65 \%$ \\
\hline $\begin{array}{c}\text { Faktorladung } \\
\text { globale Transak- } \\
\text { tionszufriedenheit }\end{array}$ & 0,85 & 0,82 & 0,78 & 0,79 & 0,86 \\
\hline $\begin{array}{c}\text { Faktorladung } \\
\text { Zufriedenheit } \\
\text { Preis/Leistung }\end{array}$ & 0,46 & 0,51 & 0,44 & 0,58 & 0,49 \\
\hline
\end{tabular}

Abb. A2: Ausgewählte Prüfkriterien der Kausalmodelle zur Untersuchung des Einflusses soziodemographischer und reisebezogener Bestimmungsfaktoren auf die Kundenzufriedenheit

Andreas Siefke and Universität Münster - 978-3-631-75103-9 


\begin{tabular}{|c|c|c|c|c|c|c|c|c|c|c|}
\hline & & \multicolumn{3}{|c|}{$\begin{array}{c}\text { Bis } 2.500 \mathrm{DM} \\
(28 \%)\end{array}$} & \multicolumn{3}{|c|}{$\begin{array}{c}2.501-5.000 \mathrm{DM} \\
(34 \%)\end{array}$} & \multicolumn{3}{|c|}{$\begin{array}{c}\text { Über } 5.000 \mathrm{DM} \\
(38 \%)\end{array}$} \\
\hline & auf & $\begin{array}{c}\text { Vor- } \\
\text { Reise- } \\
\text { zufrieden- } \\
\text { heit }\end{array}$ & $\begin{array}{c}\text { Reise- } \\
\text { zufrieden- } \\
\text { heit }\end{array}$ & $\begin{array}{c}\text { Nach- } \\
\text { Reise- } \\
\text { zufrieden- } \\
\text { heit }\end{array}$ &  & $\begin{array}{c}\text { Reise- } \\
\text { zufrieden- } \\
\text { heit }\end{array}$ & $\begin{array}{c}\begin{array}{c}\text { Nach- } \\
\text { Reise- } \\
\text { zufrieden- } \\
\text { heit }\end{array} \\
\end{array}$ & $\begin{array}{c}\text { Vor- } \\
\text { Reise- } \\
\text { zufrieden- } \\
\text { heit }\end{array}$ & $\begin{array}{c}\text { Reise- } \\
\text { zufrieden- } \\
\text { heit }\end{array}$ & $\begin{array}{c}\text { Nach- } \\
\text { Reise- } \\
\text { zufrieden- } \\
\text { heit }\end{array}$ \\
\hline \multirow{3}{*}{ 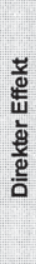 } & $\begin{array}{c}\text { Reise- } \\
\text { zufriedenheit }\end{array}$ & 0,31 & - & - & 0,14 & - & - & 0,36 & - & - \\
\hline & $\begin{array}{l}\text { Nach-Reise- } \\
\text { zufriedenheit }\end{array}$ & 0,37 & 0,27 & - & 0,34 & 0,18 & - & 0,15 & 0,21 & - \\
\hline & $\begin{array}{l}\text { Transaktions- } \\
\text { zufriedenheit }\end{array}$ & 0,19 & 0,72 & 0,12 & 0,30 & 0,64 & 0,15 & 0,32 & 0,64 & 0,06 \\
\hline \multirow{3}{*}{  } & $\begin{array}{c}\text { Reise- } \\
\text { zufriedenheit }\end{array}$ & $0,31^{1)}$ & - & - & $0,14^{1)}$ & - & - & $0,36^{1)}$ & - & - \\
\hline & $\begin{array}{l}\text { Nach-Reise- } \\
\text { zufriedenheit }\end{array}$ & 0,45 & $0,27^{1)}$ & - & 0,37 & $0,18^{1)}$ & - & 0,23 & $0,21^{1)}$ & - \\
\hline & $\begin{array}{l}\text { Transaktions- } \\
\text { zufriedenheit }\end{array}$ & 0,47 & 0,75 & $0,12^{1)}$ & 0,44 & 0,67 & $0,15^{1)}$ & 0,56 & 0,65 & $0,06^{1)}$ \\
\hline
\end{tabular}

\section{Abb. A3: Beziehungszusammenhänge der Kundenzufriedenheit in Abhängigkeit vom Netto-Haushaltseinkommen}


Andreas Siefke and Universität Münster - 978-3-631-75103-9

Downloaded from PubFactory at 01/11/2019 08:18:16AM

via free access 


\section{Anhang II:}

\section{Fragebogen der empirischen Untersuchung}


Westfälische Wilhelms-Universität Münster

Institut für Marketing

Direktor: Prof. Dr. Dr. h.c. H. Meffert

Forschungsprojekt

„Zufriedenheit mit der aktuellen Bahnreise“

Vom Interviewer auszufüllen:

\begin{tabular}{|l|l|l|l|l|l|l|l|}
\hline Interviewer & Zugtyp & Zug-Nr. & Klasse & Wagentyp & Datum & Uhrzeit & Strecke \\
\hline & & & & & & & \\
& & & & & & & \\
\hline
\end{tabular}


1. Wie lang war in etwa Ihre (einfache) Fahrtstrecke?

2. Wie haben Sie den Startbahnhof erreicht?

3. Wie sind Sie vom Zielbahnhof zum Ziel Ihrer Reise gekommen?

4. Was war der $\underline{\text { Anlaß }}$ für diese Reise?

5. Waren Sie alleine oder in Begleitung unterwegs?

6. Wie lange dauerte Ihre gesamte Reise von Tür zu Tür (einfacher Weg)?

7. Wie lange dauerte Ihre eigentliche Bahnreise vom Start- zum Zielbahnhof?

8. Wieviel hat der Fahrschein für diese Reise (ohne Zuschlăge) pro Person gekostet?

9. Haben Sie einen Sondertarif der Bahn für diese Reise genutzt?

10. Wo haben Sie sich über mögliche Zugverbindungen informiert?

11. Wo haben Sie Ihre Fahrkarte gekauft?

12. Hatten Sie für diese Reise auch andere Verkehrsmittel in Erwägung gezogen?

13. Wie viele größere Gepäckstücke/Koffer hatten Sie bei dieser Reise dabei?

14. Hatten Sie für Ihre Bahnreise einen Sitzplatz reserviert?

15. Wie oft sind Sie umgestiegen? (falls Hin- und Rückreise unterschiedlich, bitte für die Strecke mit den häufigeren Zugwechseln angeben)

16. Wie viele Plätze waren in Ihrem Abteil/Großraumwagen während Ihrer Bahnreise (durchschnittlich) besetzt? $\square$ weniger als $100 \mathrm{~km}$

$\square 100-200 \mathrm{~km}$

$\square 201-400 \mathrm{~km}$

$\square$ mehr als $600 \mathrm{~km}$

$\square$ Offentlicher Nahverkehr

zu Fuß

$\square$ Taxi

$\square$ Mietwagen

$\square$ Fahrrad

$\square$ mit PKW

Offentlicher Nahverkehr
$\square$ zu Fuß

$\square$ Taxi

$\square$ Fahrrad

$\square$ Mietwagen

$\square$ mit PKW

$\square$ Geschäts-/Dienstreise

$\square$ Fernpendler (Studium, Beruf etc.)

$\square$ Kurzurlaub; Wochenend-, Tagesausflug (bis 3 Übernachtungen)

$\square$ Urlaubsreise (4 Übernachtungen und mehr)

$\square$ sonstige Privatreise (z.B. Besuch von Verwandten/ Einkäufe etc.)

$\square$ Alleine $\square$ Mit Partner(in) $\quad \square$ Familie (mit Kindern)

$\square$ Mit Geschäftspartner / Kollege(in)

$\checkmark$ In einer Gruppe (3 Personen und mehr, nicht Familie)

ca. Stunden Minuten

ca. Stunden Minuten

DM $\square$ Einfache Fahrt $\quad \square$ Hin- und Rückfahrt

$\begin{array}{lll}\square \text { BahnCard } & \square \text { Großkundenabo } & \square \text { (Super-)Sparpreis } \\ \square \text { Netz-/Dauerkarte } & \square \text { Sonstiges } & \square \text { keinen }\end{array}$

$\square$ Reisezentrum/Bahnhof $\square$ Reisebüro $\square$ Fahrplanaushang $\square$ (elektronisches) Kursbuch $\square$ Telefonauskunft $\square$ Sonstiges

$\square$ Reisezentrum/Bahnhof $\square$ Reisebüro $\square$ Sonstiges

$\square$ Nein $\quad \square$ Ja, und zwar

$\square$ kein grő̉eres Gepăck $\square$ eins $\square$ zwei $\quad \square$ drei und mehr

$\square \mathrm{Ja} \quad \square$ Nein

$\square$ laut Auskunft/Schalterpersonal nicht mehr möglich gewesen

$\square$ gar nicht $\square$ einmal $\square$ mehr als einmal

$\square$ (nahezu) alle $\square$ etwa die Hälfte $\quad \square$ (fast) keiner

$\square$ etwa $3 / 4 \quad \square$ etwa $1 / 4$ 
Eine Bahnreise kann man in unterschiedliche Abschnitte aufteilen. Wir unterscheiden in den năchsten Fragen insgesamt vier Abschnitte. Diese Abschnitte lauten: "Vor der Bahnfahrt", "Wăhrend der Bahnfahrt", "Umsteigen" und "Nach der Bahnfahrt"!

Der Abschnitt „Vor der Bahnfahrt" beinhaltet dabei alle Aspekte Ihrer Bahnreise von der Vorbereitung der Reise bis zur Abfahrt Ihres Zuges am Startbahnhof. Unter dem Abschnitt "Während der Fahrt" sollen alle Aspekte, die die Fahrt im Zug einschließlich der Ankunft am Zielbahnhof betreffen, verstanden werden. Der Abschnitt "Umsteigen“ enthalt alle Aspekte, die sich auf das Umsteigen in einen anderen Zug beziehen. Bei dem Abschnitt "Nach der Bahnfahrt" handelt es sich um den Teil Ihrer Bahnreise, der das Verlassen Ihres Zuges am Zielbahnhof bis einschließlich Ihrer Ankunft an Ihrem endgaltigen Zielort umfaßt.

17a. Was hat Ihnen auf dieser Reise mit der Bahn besonders gut gefallen. Wofür würden Sie die Bahn loben? Denken Sie dabei bitte an die vier verschiedenen Reiseabschnitte!

- Vor der Bahnfahrt:

- Während der Bahnfahrt:

- Umsteigen:

- Nach der Bahnfahrt:

17b. Was hat Ihnen auf dieser Reise mit der Bahn nicht gefallen. Wofür würden Sie die Bahn tadeln? Denken Sie auch hier bitte an die vier verschiedenen Reiseabschnitte!

- Vor der Bahnfahrt:

- Wăhrend der Bahnfahrt:

- Umsteigen:

- Nach der Bahnfahrt:

18. Welche Bedeutung hat der gute, relbungslose Ablauf dleser Abschnitte einer Bahnrelse far Sle? Verteilen Sie bitte eine Gesamtsumme von 100 Punkten auf die Reiseabschnitte, wobei der for Sie wichtigste Abschnitt die hochste Punktzahl erhältl Geben Sie einem Abschnitt keinen Punkt, so ist dieser fur Sie vollig unwichtigl Falls Sie umgestlegen sind, beantworten Sie die Frage bitte unter a), falls Sie nicht umgestlegen sind, beantworten Sie die Frage bitte unter b)!

Beantworten Sle die Frage bltte far Ihre aktuelle Bahnreisel

\begin{tabular}{|c|c|c|c|}
\hline \multicolumn{2}{|c|}{ a) Bahnreise mit Umsteigen } & \multicolumn{2}{|c|}{ b) Bahnreise ohne Umsteigen } \\
\hline Reiseabschnitt & Punkte & Reiseabschnitt & Punkte \\
\hline Vor der Bahnfahrt: & & Vor der Bahnfahrt: & \\
\hline Während der Bahnfahrt: & & Während der Bahnfahrt: & \\
\hline Umsteigen: & & Nach der Bahnfahrt: & \\
\hline Nach der Bahnfahrt: & & Summe & 100 Punkte \\
\hline Summe & DO Punkte & & \\
\hline
\end{tabular}


19. Wir möchten Sie nun bitten, Ihre aktuelle Bahnreise hinsichtlich Ihrer Zufriedenheit zu beurteilen. Dabei bedeutet "1" sehr zufrieden und ${ }_{n}{ }^{4}$ gar nicht zufrieden. Mit den dazwischen liegenden Ziffern können Sie Ihr Urteil abstufen.

Wenn Sie die verschiedenen Reiseabschnitte bedenken: Wie zufrieden waren Sie alles in allem mit Ihrer Bahnreise?

\begin{tabular}{|c|c|c|c|}
\hline $\begin{array}{l}\text { sehr } \\
\text { frieden }\end{array}$ & & & \\
\hline $\begin{array}{l}1 \\
\square\end{array}$ & $\begin{array}{l}2 \\
\square\end{array}$ & י3 & $\begin{array}{l}4 \\
\square\end{array}$ \\
\hline
\end{tabular}

Wie zufrieden waren Sie mit dem Preis-/Leistungsverhältnis?

20. Wir möchten Ihnen nun einige Fragen bezüglich Ihrer aktuellen Bahnreise zum Reiseabschnitt „Vor der Bahnfahrt" stellen! Bitte beantworten Sie nur die Aspekte, mit denen Sie bei dieser Reise Erfahrungen gemacht haben!

Wie zufrieden waren Sie insgesamt mit dieser Phase Ihrer Reise, also dem Reiseabschnitt „Vor der Bahnfahrt"?

\section{Wie zufrieden waren Sie im einzelnen mit}

der telefonischen Erreichbarkeit des Reisezentrums bzw. der Auskunft

\begin{tabular}{ccccc}
$\begin{array}{c}\text { sehr } \\
\text { zufrieden } \\
1\end{array}$ & 2 & 3 & \multicolumn{3}{c}{$\begin{array}{c}\text { gar nicht } \\
\text { zufrieden }\end{array}$} \\
$\square$ & $\square$ & $\square$ & $\square$ & $\square$
\end{tabular}

der Beratung bei der telefonischen Auskunft

der Dauer Ihrer Anreise zum Bahnhof

der Erreichbarkeit des Bahnhofs

den Parkmöglichkeiten am Bahnhof

den Parkgebühren am Bahnhof

der Beratung beim Fahrscheinkauf

der Wartezeit beim Fahrscheinkauf

der Freundlichkeit des Schalterpersonals

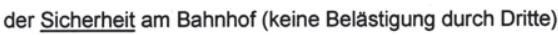



der Sauberkeit der Bahnhofstoiletten

den Einkaufs-Nerpflegungsmoglichkeiten im Bahnhof

den Wegweisern und Auskunftstafeln im Bahnhof

der Verständlichkeit des Fahrplans

dem Wetterschutz am Bahnsteig

der Verständlichkeit der Lautsprecherdurchsagen

den Informationen über etwaige Anschlüsse, Verspätungen etc.

$\begin{array}{llllll}\square & \square & \square & \square & \square & 0 \\ \square & \square & \square & \square & \square & 0 \\ \square & \square & \square & \square & \square & 0 \\ \square & \square & \square & \square & \square & 0 \\ \square & \square & \square & \square & \square & 0 \\ \square & \square & \square & \square & \square & 0 \\ \square & \square & \square & \square & \square & 0 \\ \square & \square & \square & \square & \square & 0 \\ \square & \square & \square & \square & \square & 0 \\ \square & \square & \square & \square & \square & 0 \\ \square & \square & \square & \square & \square & 0 \\ \square & \square & \square & \square & \square & 0 \\ \square & \square & \square & \square & \square & 0 \\ \square & \square & \square & \square & \square & 0 \\ \square & \square & \square & \square & \square & 0 \\ \square & \square & \square & \square & \square & 0\end{array}$


21. Es folgen nun einige Fragen zum Reiseabschnitt "Während der Bahnfahrt“ Ihrer aktuellen Bahnreise! Falls Sie während Ihrer Bahnreise den Zug gewechselt haben, möchten wir Sie bitten, die folgenden Fragen für den Zug zu beantworten, in dem Sie die meiste Zeit Ihrer Reise verbracht haben!

Wie zufrieden waren Sie insgesamt mit dieser Phase Ihrer

Reise, also dem Reiseabschnitt „Während der Bahnfahrt"?

\begin{tabular}{|c|c|c|c|c|c|}
\hline \multirow{2}{*}{$\begin{array}{c}\text { sehr } \\
\text { zufrieden } \\
1 \\
\square \\
\text { sehr } \\
\text { zufrieden } \\
1 \\
\square\end{array}$} & \multirow{2}{*}{$\begin{array}{l}2 \\
\square \\
2 \\
\square\end{array}$} & \multirow{2}{*}{$\begin{array}{l}3 \\
\square\end{array}$} & \multicolumn{2}{|c|}{$\begin{array}{cc}\begin{array}{c}\text { gar nicht } \\
\text { zufrieden }\end{array} \\
4 & 5 \\
4 & \square\end{array}$} & \multirow[b]{2}{*}{$\begin{array}{c}\text { trifft nicht } \\
\text { zu I } \\
\text { kein Urteil } \\
0\end{array}$} \\
\hline & & & $\begin{array}{l}9 \\
4^{2} \\
\square\end{array}$ & $\begin{array}{l}\text { ar nicht } \\
\text { dfrieden } \\
5 \\
\square\end{array}$ & \\
\hline 口 & ㅁ & $\square$ & ㅁ & 口 & 0 \\
\hline 口 & $\square$ & $\square$ & $\square$ & $\square$ & 0 \\
\hline$\square$ & $\square$ & $\square$ & $\square$ & $\square$ & O \\
\hline$\square$ & $\square$ & $\square$ & 口 & 口 & 0 \\
\hline$\square$ & $\square$ & $\square$ & $\square$ & $\square$ & 0 \\
\hline$\square$ & $\square$ & $\square$ & ㅁ & $\square$ & 0 \\
\hline 口 & $\square$ & $\square$ & 口 & $\square$ & 0 \\
\hline$\square$ & $\square$ & $\square$ & $\square$ & $\square$ & 0 \\
\hline$\square$ & 口 & 口 & 口 & ㅁ & 0 \\
\hline$\square$ & 口 & $\square$ & 口 & 口 & 0 \\
\hline$\square$ & $\square$ & $\square$ & 口 & $\square$ & 0 \\
\hline$\square$ & $\square$ & $\square$ & 口 & $\square$ & 0 \\
\hline$\square$ & $\square$ & $\square$ & $\square$ & 口 & 0 \\
\hline$\square$ & $\square$ & $\square$ & ㅁ & $\square$ & 0 \\
\hline$\square$ & 口 & $\square$ & 口 & 口 & 0 \\
\hline$\square$ & $\square$ & $\square$ & $\square$ & $\square$ & 0 \\
\hline 口 & 口 & 口 & 口 & 口 & 0 \\
\hline
\end{tabular}

\section{Wie zufrieden waren Sie im einzelnen mit}

der Pünktlichkeit Inres Zuges bei der Abfahrt am Startbahnhof

der Freundlichkeit des Zugpersonals

der Fachkompetenz des Zugpersonals

den Möglichkeiten zur Gepäck- und Garderobeverstauung

dem Komfort Ihres Sitzes

der Beinfreiheit an Ihrem Sitzplatz

der Ausstattung Ihres Sitzplatzes (z.B. Arbeitstisch, Ablage)

der Sauberkeit Ihres Abteils/Wagens

der Sauberkeit der Zugtoiletten

der Möglichkeit, ungestört arbeiten / lesen / schlafen zu können

dem Angebot im Speisewagen/Zugbistro

dem Preis-/Leistungsverhältnis der Speisen und Getränke

den Luft-/Temperaturverhältnissen im Zug

der Verständlichkeit der Lautsprecherdurchsagen

den Informationen über etwaige Anschlüsse, Verspătungen etc.

der Sicherheit im Zug (keine Belästigung durch Dritte)

der Fahrtdauer, die Ihr Zug für Ihre Reisestrecke benőtigte

der Pünktlichkeit Inres Zuges am Zielbahnhof

22. Falls Sie während Ihrer Bahnreise umsteigen mußten, möchten wir Sie bitten, die nachfolgenden Fragen zu beantworten. Wenn Sie mehr als einmal umgestiegen sind, beantworten Sie die Fragen bitte für den Umsteigevorgang, an den Sie sich am besten erinnern können. Ansonsten weiter mit Frage 23!

Wie zufrieden waren Sie insgesamt mit dieser Phase Ihrer

Reise, also dem Reiseabschnitt „Umsteigen"?

\section{Wie zufrieden waren Sie im einzelnen mit}

dem $\underline{A n s c h l u ß}$ an andere Züge (keine lange Wartezeit)

\begin{tabular}{ccccc}
$\begin{array}{c}\text { sehr } \\
\text { zufrieden }\end{array}$ & & \multicolumn{2}{c}{$\begin{array}{c}\text { gar nicht } \\
\text { zufrieden }\end{array}$} \\
1 & 2 & 3 & 4 & 5 \\
$\square$ & $\square$ & $\square$ & $\square$ & $\square$
\end{tabular}

den Orientierungshilfen beim Umsteigen

den Informationen über etwaige Verspätungen, Anschlüsse etc.

$\begin{array}{cccccc}\begin{array}{c}\text { sehr } \\ \text { zufrieden } \\ 1\end{array} & 2 & 3 & 4 & \begin{array}{c}\text { gar nicht } \\ \text { zufrieden }\end{array} & \begin{array}{c}\text { trifft nicht } \\ \text { zu I } \\ \text { kein Urteil } \\ \square\end{array} \\ \square & \square & \square & \square & 0 \\ \square & \square & \square & \square & \square & 0 \\ \square & \square & \square & \square & \square & 0 \\ \square & \square & \square & \square & \square & 0 \\ \square & \square & \square & \square & \square & 0\end{array}$

der Entfernung zum Bahnsteig des Anschlußzuges

der Pünktlichkeit Ihres Anschlußzuges bei der Abfahrt 
23. Es folgen nun einige Fragen zum Reiseabschnitt "Nach der Bahnfahrt"!

\begin{tabular}{|c|c|c|c|c|c|c|}
\hline $\begin{array}{l}\text { Wie zufrieden waren Sie insgesamt mit dieser Phase Ihrer } \\
\text { Reise, also dem Reiseabschnitt „Nach der Bahnfahrt"? }\end{array}$ & $\begin{array}{c}\text { sehr } \\
\text { zufrieden } \\
1 \\
\square\end{array}$ & 2 & 3 & $4^{9}$ & $\begin{array}{l}\text { ar nicht } \\
\text { ufrieden } \\
5 \\
\square\end{array}$ & \\
\hline $\begin{array}{l}\text { Wie zufrieden waren Sie im einzelnen mit } \\
\text { der Sicherheit am Zielbahnhof (keine Belästigung durch Dritte) }\end{array}$ & $\begin{array}{c}\text { sehr } \\
\text { zufrieden } \\
1 \\
\square\end{array}$ & 2 & $\begin{array}{l}3 \\
\square\end{array}$ & $4^{g}$ & $\begin{array}{l}\text { ar nicht } \\
\text { ufrieden } \\
5 \\
\square\end{array}$ & $\begin{array}{l}\text { trifft nicht } \\
\text { zu I } \\
\text { kein Urteil } \\
0\end{array}$ \\
\hline den Einkaufs-Nerpflegungsmöglichkeiten im Zielbahnhof & 口 & $\square$ & $\square$ & $\square$ & $\square$ & 0 \\
\hline der Verfügbarkeit von Kofferkulis & ㅁ & $\square$ & $\square$ & $\square$ & $\square$ & 0 \\
\hline den Wegweisern und Auskunftstafeln im Zielbahnhof & $\square$ & $\square$ & $\square$ & $\square$ & $\square$ & 0 \\
\hline dem Angebot der öffentlichen Verkehrsmittel am Zielbahnhof & $\square$ & $\square$ & $\square$ & $\square$ & $\square$ & 0 \\
\hline dem Angebot an Taxis /Mietwagen & $\square$ & $\square$ & $\square$ & $\square$ & $\square$ & 0 \\
\hline der Dauer Ihrer Anreise vom Zielbahnhof zum Zielort & $\square$ & $\square$ & $\square$ & $\square$ & $\square$ & O \\
\hline
\end{tabular}

24. Falls Sie sich auf Ihrer aktuellen Bahnreise aber die Bahn geărgert haben: Inwieweit treffen dann die folgenden Aussagen auf Ihre aktuelle Bahnreise zu? Ansonsten weiter mit Frage 25!

a) Die Aussage:

Ich habe mich mit Mitreisenden über meinen Årger unterhalten

$\begin{array}{ccccc}\begin{array}{c}\text { trifft } \\ \text { sehr zu }\end{array} & & & \begin{array}{c}\text { triff gar } \\ \text { nicht zu }\end{array} \\ 1 & 2 & 3 & 4 & 5 \\ \square & \square & \square & \square & \square \\ \square & \square & \square & \square & \square \\ \square & \square & \square & \square & \square\end{array}$

b) In welchem Reiseabschnitt haben Sie sich über die Bahn geärgert?
$\square$ "Vor der Bahnfahrt"
$\square$ „Während der Bahnfahrt"
口 „Umsteigen"
$\square$ „Nach der Bahnfahrt"

c) Schildern Sie bitte kurz, worüber Sie sich geärgert haben!

25. Wie würden Sie vor dem Hintergrund Ihrer aktuellen Bahnreise die folgenden Fragen beantworten?

\begin{tabular}{|c|c|c|c|c|c|}
\hline \multirow{2}{*}{$\begin{array}{l}\text { Würden Sie die Bahn an Freunde, Bekannte oder } \\
\text { Kollegen weiterempfehlen? }\end{array}$} & $\underset{1}{\text { mit }}$ & 2 & 3 & \multicolumn{2}{|c|}{$\begin{array}{c}\text { wahrscheinlich } \\
\text { nicht }\end{array}$} \\
\hline & $\square$ & $\square$ & 口 & $\square$ & $\square$ \\
\hline $\begin{array}{l}\text { Werden Sie bei Ihrer năchsten Reise dieser Art wieder } \\
\text { mit der Bahn fahren? }\end{array}$ & $\square$ & 口 & 口 & $\square$ & 口 \\
\hline $\begin{array}{l}\text { Würden Sie sich über negative Erlebnisse, wie z.B. eine deutliche } \\
\text { Verspätung Ihres Zuges (z.B. mehr als } 30 \text { Minuten), bei Mitarbeitern } \\
\text { der Bahn beschweren? }\end{array}$ & 口 & $\square$ & 口 & $\square$ & 口 \\
\hline $\begin{array}{l}\text { Würden Sie Freunden oder Bekannten von einem solchen } \\
\text { Erlebnis erzăhlen? }\end{array}$ & 口 & $\square$ & ㅁ & ㅁ & ㅁ \\
\hline
\end{tabular}

\begin{tabular}{|ccc|}
\hline 26. Wie häufig werden Sie die Bahn In Zukunft im Vergleich zu heute Ihrer Meinung nach nutzen? \\
\hline$\square$ deutlich mehr $\quad \square$ etwas mehr $\quad \square$ wie bisher $\quad \square$ etwas weniger $\square$ deutlich weniger \\
\hline
\end{tabular}


Es folgen nun einige allgemeine Fragen zum Unternehmen Bahn sowie zum Reisen mit der Bahn!

1. Wie zufrieden sind Sie generell mit der Bahn, wenn Sie alle Ihre bisherigen Erfahrungen mit der Bahn berücksichtigen?

\begin{tabular}{|c|c|c|c|c|}
\hline $\begin{array}{c}\text { sehr } \\
\text { zufrieden } \\
1 \\
\square\end{array}$ & 2 &  & $\stackrel{4}{\square}$ & $\begin{array}{c}\text { gar nich } \\
\text { zufrieder } \\
5 \\
\square\end{array}$ \\
\hline $\begin{array}{r}\text { sehr } \\
\text { gut } \\
1\end{array}$ & 2 & 3 & 4 & $\begin{array}{c}\text { nangel- } \\
\text { haft } \\
5\end{array}$ \\
\hline 口 & 口 & $\square$ & 口 & $\square$ \\
\hline
\end{tabular}

2a. Wenn Sie einmal, abgesehen von Ihrer letzten Bahnreise, ein allgemeines Gesamturteil über die Bahn im Vergleich zu anderen Verkehrsmitteln fällen müßten. Wie würden Sie die Bahn dann insgesamt beurteilen?

2b. Wenn Sie ein Urteil über den Fernverkehr der Bahn fällen müßten. Wie würde dann Ihr Urteil ausfallen?

2c. Wenn Sie ein Urteil über den Nahverkehr der Bahn fällen müßten. Wie würde dann Ihr Urteil ausfallen?

3. Im folgenden finden Sie einige Aussagen uber das Unternehmen Deutsche Bahn AG. Bitte geben Sie an, inwieweit diese aus Ihrer Sicht zutreffen!

\section{Die Aussage:}

Die Bahn reagiert flexibel auf Kundenwünsche

trifft sehr zu

$\square$
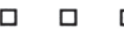

$\square \quad \square$

$\mathrm{O}$

0

Die Bahn verfolgt eine umweltbewußte Verkehrspolitik

Die Bahn ist ein innovatives Unternehmen

Die Bahn ist wirtschaftlich erfolgreich

Das Tarif-/Preissystem der Bahn ist verständlich und durchschaubar

Die Bahnhofe sind modern und bieten hohen Komfort

Die Mitarbeiter der Bahn sind zuverlässig und vertrauenswürdig

Die Privatisierung der Bahn hat zu deutlichen Verbesserungen der Angebote geführt

4. Im folgenden finden Sie einige Aussagen uber das Relsen mit der Bahn. Bitte geben Sie an, inwieweit diese aus Ihrer Sicht zutreffen!

\section{Die Aussage:}

Das Reisen mit der Bahn ist ein schönes Erlebnis

Bei wichtigen Terminen fahre ich häufig mit der Bahn

Ich bin gut mit den Angeboten und Preisen der Bahn vertraut

Ich informiere mich regelmäßig über die Angebote der Bahn

Im Vergleich mit anderen Verkehrsmitteln ist Bahnfahren teuer

$\begin{array}{ccccc}\begin{array}{c}\text { trifft } \\ \text { sehr zu } \\ 1\end{array} & 2 & 3 & 4 & \begin{array}{c}\text { trifft gar } \\ \text { nicht zu } \\ \square\end{array} \\ \square & \square & \square & \square \\ \square & \square & \square & \square & \square \\ \square & \square & \square & \square & \square \\ \square & \square & \square & \square & \square \\ \square & \square & \square & \square & \square\end{array}$


Fortsetzung von Frage 4

Die Aussage:

Bei Reisen mit der Bahn muß man mit Verspätungen rechnen

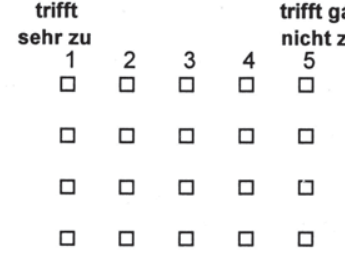

Das Komfortniveau der Züge ist sehr unterschiedlich

Bei Antritt einer Bahnreise ist man nie ganz sicher, ob diese zufriedenstellend verläuft

Freunde und Bekannte haben mir schon häufig von negativen Erlebnissen bei Bahnreisen erzählt

Bei dem Erwerb der Fahrkarte ist man nie ganz sicher, ob man auch den günstigsten Fahrpreis zahlt

Ich habe mich in der Vergangenheit schon häufiger über die Bahn geärgert

5a. Die folgenden Fragen betreffen Ihr allgemeines Reiseverhaltenl Wenn Sie an die letzten 12 Monate denken, wie würden Sie dann die folgenden Fragen beantworten? Bitte denken Sie dabei an Reisen uber 100 km Lănge (einfache Fahrt)!

Wie hăufig fuhren Sie privat längere Strecken

$\begin{array}{rrrrr}\text { gar } & 1-3 & 4-12 & 12-25 & \begin{array}{r}\text { über } \\ \text { nicht }\end{array} \\ \text { mal } & \text { mal } & \text { mal } & 25 \text { mal }\end{array}$

unabhängig vom Verkehrsmittel?

Wie hăufig fuhren Sie privat mit der Bahn längere Strecken?

Wie hăufig fuhren Sie geschăftlich längere Strecken

unabhängig vom Verkehrsmittel?

Wie häufig fuhren Sie geschäftlich mit der Bahn längere Strecken?

5b. Wie häufig benutzten Sie Züge im Nahverkehr (bis $100 \mathrm{~km}$ )

(z.B. S-Bahn, RegionalBahn oder Stadtexpress)?

$\begin{array}{lllll}\square & \square & \square & \square & \square \\ \square & \square & \square & \square & \square\end{array}$

$\square \quad \square \quad \square \quad \square \quad \square$

$\square \quad \square \quad \square \quad \square \quad \square$

Zur Ableitung geeigneter Maßnahmen zur Erhø̋ung der Qualităt von Bahnreisen mochten wir Ihnen abschlieBend noch einige Fragen zu Ihrer Person stellen! Selbstverständlich bleibt die Anonymitat und Vertraulichkeit Ihrer Angaben dabei gewahrt!

1. Steht Ihnen ein PKW zur Verfügung?

Immer $\square \quad$ Gelegentlich $\square \quad$ Nie $\square$

2. Welchen Wagentyp bevorzugen Sie bei Bahnreisen?

Großraumwagen $\square \quad$ Abteilwagen $\square$

3. Wie gro $B$ ist Ihr Wohnort (Einwohner)?

weniger als $20.000 \square \quad 20.000-100.000 \square \quad 100.001-250.000 \square \quad 250.001-500.000 \square \quad$ über $500.000 \square$ 
4. Wie groß ist die Entfernung von Ihrer Wohnung zum nächsten Bahnhof, von dem aus Fernzüge fahren (bitte in $\mathrm{km}$ antworten)? $\mathrm{km}$

5. Welche abgeschlossene Ausbildung haben Sie? (bitte nur eine Nennung)

Haupt- bzw. Realschulabschluß $\square$

Fach-/Abitur $\square$
Berufsausbildung/Lehre $\square$

Meister $\square$
Hochschulstudium $\square$

Promotion $\square$

6. Welchen Beruf üben Sie aus?

Arbeiter, Facharbeiter, Handwerker $\square$ Beamter / Angestellter $\square$ höherer Beamter / leitender Angestellter $\square$

Selbständiger / Freiberufler $\square$
In Berufsausbildung /Schule /Studium $\square$ Soldat / Zivildienstleistender $\square$ Rentner / Pensionăr $\square$ Hausfrau / Hausmann $\square$ Sonstiges $\square$

7. Wie alt sind Sie? Jahre

8. Welches Geschlecht haben Sie? Weiblich $\square \quad$ Männlich $\square$

9. In welche der folgenden Gruppen würden Sie das monatliche Nettoeinkommem Ihres Haushaltes einordnen? Bitte denken Sie dabei auch an zusätzliche Einkommen wie z.B. Kindergeld und Mieteinnahmen!

$\begin{array}{rrrr}\text { bis 1.500 DM } & 2.501-3.500 \mathrm{DM} \square & 5.001-7.500 \mathrm{DM} \square & \text { über } 10.000 \mathrm{DM} \square \\ 1.501-2.500 \mathrm{DM} \square & 3.501-5.000 \mathrm{DM} \square & 7.501-10.000 \mathrm{DM} \square & \end{array}$

\section{Vielen Dank für Ihre freundliche Unterstützung!}




\section{Literaturverzeichnis}

Agrawal, M., You haven't Seen All: Hypothesis for Extending Research on the Benefits of Customer Satisfaction, in: Marketing Today and for the 21st Century, Proceedings of the 24th Annual Conference of the European Marketing Academy, Bergadaa, M. (Hrsg.), Cergy 1995, S. 1-17.

Albrecht, K., At America's Service - How Corporations can Revolutionize the Way they Treat their Customers, Homewood 1988.

Albrecht, K., Zemke, R., Service-Strategien, Hamburg u.a. 1987.

Alpert, M.I., Golden, L.L., Transportation Attitudes over Time: A Longitudinal Approach, in: Advances in Consumer Research, Vol. 5, Hunt, H.K. (Hrsg.), 1978, S. 194-200.

Anderson, E.W., Fornell, C., Lehmann, D.R., Customer Satisfaction, Market Share, and Profitability: Findings from Sweden, in: JoM, Vol. 58, July 1994, S. 53-66.

Anderson, R.E., Consumer Dissatisfaction: The Effect of Disconfirmed Expectancy on Perceived Product Performance, in: JoMR, Vol. 10, February 1973, S. 38-44.

Andritzky, K., Die Operationalisierbarkeit von Theorien zum Konsumentenverhalten, Berlin 1976.

Andritzky, K., Vier Hypothesen bei der Anwendung der Rating-Methode in der Wirtschafts- und Sozialpsychologie, in: JdAV, Jg. 22, 1976, S. 291-306.

Antrecht, R., Claßen, W., Friese, U., Wem die Deutschen trauen, in: Capital, H. 4, 1996, S. 38-74.

Backhaus, K., Investitionsgütermarketing, 4. Aufl., München 1995.

Backhaus, K., Erichson, B., Plinke, W., Weiber, R., Multivariate Analysemethoden - Eine anwendungsorientierte Einführung, 8. Aufl., Berlin u.a. 1996.

Backhaus, K., Weiber, R., Entwicklung einer Marketing-Konzeption mit SPSS/PC+, Berlin u.a. 1989.

Bagozzi, R.P., Causal Modelling: General Method for Developing and Testing Theories in Consumer Research, in: Advances in Consumer Research, Vol. 8, Monroe, K.B. (Hrsg.), Ann Arbor 1981, S. 195-202.

Bagozzi, R.P., Fornell, C., Theoretical Concepts, Measurement, and Meaning, in: A Second Generation of Multivariate Analysis, Bd. 2, Fornell, C. (Hrsg.). New York 1982, S. 24-38. 
Bahn Extra (Hrsg.), Lexikon Deutsche Bahn, München 1996.

Balderjahn, I., Das umweltbewußte Konsumentenverhalten - Eine empirische Studie, Berlin 1986.

Bamberg, S., Zeit und Geld: Empirische Verhaltenserklärung mittels Restriktionen am Beispiel der Verkehrsmittelwahl, in: ZUMA-Nachrichten, Jg. 38, 20. Mai 1996, S. 7-32.

Bamberg, S., Bien, W., Angebot (des OV) nach Wunsch (des MIV-Nutzers), in: IVW, Jg. 47, H. 3, 1995, S. 108-115.

Bamberg, S., Schmidt, P., Verkehrsmittelwahl: Eine Anwendung der Theorie des geplanten Verhaltens, in: Zeitschrift für Sozialpsychologie, Jg. 24, 1993, S. 25-37.

Bänsch, A., Käuferverhalten, München, Wien 1983.

Bänsch, A., Variety Seeking: Marketingfolgerungen aus Überlegungen und Untersuchungen zum Abwechslungsbedürfnis von Konsumenten, in: JdAV, Jg. 41, H. 4, 1995, S. 342-365.

Bateson, J.E.G., Managing Services Marketing: Text and Readings, 3. Aufl., Fort Worth u.a. 1995.

Bateson, J.E.G., Hui, M.K., Crowding in the Service Environment, in: Creativity in Services Marketing: What's New, What Works, What's Developing, Venkatesan, M., Schmalensee, D.M., Marshall, C. (Hrsg.), Chicago 1986, S. 85-88.

Bauer, F., Datenanalyse mit SPSS, Berlin u.a. 1984.

Benkenstein, M., Dienstleistungsqualität: Ansätze zur Messung und Implikationen für die Steuerung, in: ZfB, Jg. 63, H. 11, 1993, S. 1095-1116.

Bentler, P.M., Theory and Implementation of EQS.A Structural Equations Program, Los Angeles 1985.

Bentler, P.M., EQS Structural Equations Program Manual, Multivariate Software Inc., Encino 1995.

Berekoven, L., Der Begriff "Dienstleistung" und seine Bedeutung für eine Analyse der Dienstleistungsbetriebe, in: JdAV, Jg. 12, H. 4, 1966, S. 314-326.

Berekoven, L., Eckert, W., Ellenrieder, P., Marktforschung: methodische Grundlagen und praktische Anwendung, 4. Aufl., Wiesbaden 1989.

Birkelbach, R., Qualitätsmanagement in Dienstleistungscentern: Konzeption und typenspezifische Ausgestaltung unter besonderer Berücksichtigung

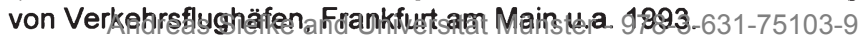


Bitner, M.J., Evaluating Service Encounters: The Effects of Physical Surroundings and Employee Responses, in: JoM, Vol. 54, April 1990, S. 69-82.

Bitner, M.J., Managing the Evidence of Service, in: The Service Quality Handbook, Scheuing, E.E., Christopher, W.F. (Hrsg.), New York u.a. 1993, S. 358-370.

Bitner, M.J., Booms, B.H., Tetreault, M.S., The Service Encounter: Diagnosing Favorable and Unfavorable Incidents, in: JoM, Vol. 54, January 1990, S. 71-84.

Bitner, M.J., Hubbert, A.R., Encounter Satisfaction Versus Overall Satisfaction Versus Quality: The Customer's Voice, in: Service Quality: New Directions in Theory and Practice, Rust, R.T., Oliver, R.L. (Hrsg.), Thousand Oaks u.a. 1994, S. 72-94.

Bleymüller, J., Gehlert, G., Gülicher, H., Statistik für Wirtschaftswissenschaftler, 10. Aufl., München 1996.

Böhm, W., Zur Motivation junger Auslandsreisender, in: Jahrbuch für Jugendreisen und Internationaler Jugendaustausch, Bonn 1962, S. 111-122.

Bolton, R.N., Drew, J.H., A Longitudinal Analysis of the Impact of Service Changes on Customer Attitudes, in: JoM, Vol. 55, January 1991, S. 1-9.

Bolton, R.N., Drew, J.H., A Multistage Model of Customers' Assessments of Service Quality and Value, in: JoCR, Vol. 17, March 1991, S. 375-384.

Botschen, G., Bstieler, L., Woodside, A.G., Sequence-Oriented Problem Identification within Service Encounters, Manuskript, Januar 1993.

Boulding, W., Kalra, A., Staelin, R., Zeithaml, V.A., A Dynamic Process Model of Service Quality: From Expectations to Behavioral Intentions, in: JoMR, Vol. 30, February 1993, S. 7-27.

Bower, G.H., Black, J.B., Turner, T.J., Scripts in Memory for Text, in: Cognitive Psychology, Vol. 11, No. 2, 1979, S. 177-220.

Brandt, D.R., Reffett, K.L., Focusing on Customer Problems to Improve Service Quality, in: Designing a Winning Service Strategy, Bitner, M.J., Crosby, L.A. (Hrsg.), Chicago 1989, S. 92-97.

Bretthauer, I., Deutsche Bahn AG: Von der Behörde zum marktorientierten Touristikanbieter, in: Touristik-Marketing: das Marketing der Tourismus-Organisationen, Verkehrsträger, Reiseveranstalter und Reisebüros, Roth, P., Schrand, A. (Hrsg.), 2. Aufl., München 1995, S. $243-253$. 
Breuer, H., Qualitätsmanagement in einem Dienstleistungsunternenmen, in: Erfolg durch Service-Qualität, Stauss, B. (Hrsg.), München 1991, S. $171-239$.

Brosius, G., SPSS/PC+ Advanced Statistics and Tables, Hamburg, New York 1989.

Bruhn, M., Konsumentenzufriedenheit und Beschwerden: Erklärungsansätze und Ergebnisse einer empirischen Untersuchung in ausgewählten Konsumbereichen, Frankfurt am Main 1982.

Bruhn, M., Marketing und Konsumentenzufriedenheit, in: WISU, Jg. 14, H. 6 , 1985, S. 300-307.

Bruhn, M., Qualitätsmanagement für Dienstleistungen: Grundlagen, Konzepte, Methoden, Berlin u.a. 1996.

Bruhn, M., Bunge, B., Beziehungsmarketing: Neuorientierung für Marketingwissenschaft und -praxis?, in: Marktorientierte Führung im Umbruch: Effizienz und Flexibilität als Herausforderungen des Marketing, Bruhn, M., Meffert, H., Wehrle, F. (Hrsg.), Stuttgart 1994, S. 41-84.

Bruhn, M., Hennig, K., Selektion und Strukturierung von Qualitätsmerkmalen - auf dem Weg zu einem umfassenden Qualitätsmanagement für Kreditinstitute (Teil 1), in: JdAV, Jg. 39, H. 3, 1993, S. 214-238.

Bruhn, M., Schneider, M., Fallstudie Deutsche Lufthansa AG, in: Dienstleistungsmarketing: Grundlagen, Konzepte, Methoden; mit Fallbeispielen, Meffert, H., Bruhn, M. (Hrsg.), 2. Aufl., Wiesbaden 1997, S. 601-637.

Büker, B., Qualitätsbeurteilung investiver Dienstleistungen: Operationalisierungsansätze an einem Beispiel zentraler EDV-Dienste, Frankfurt am Main u.a. 1991.

Burmann, Ch., Konsumentenzufriedenheit als Determinante der Marken- und Händlerloyalität, in: Marketing ZFP, Jg. 13, H. 4, 1991, S. 249-258.

Burmann, Ch., Touristik-Marketing, in: Lexikon der aktuellen Marketingbegriffe, Meffert, H. (Hrsg.), Wien 1994, S. 233-240.

Büschken, J., Multipersonale Kaufentscheidungen - Empirische Analyse zur Operationalisierung von Einflußbeziehungen im Buying Center, Wiesbaden 1994.

Cadotte, E.R., Woodruff, R.B., Jenkins, R.L., Expectations and Norms in Models of Consumer Satisfaction, in: JoMR, Vol. 24, August 1987, S. 305-314. 
Chebat, J.-Ch., Fliatrault, P., Gelinas-Chebat, C., Vaninsky, A., Impact of Waiting Attribution and Consumers Mood on Perceived Quality, in: JoBR, Vol. 28, No. 3, 1995, S. 191-196.

Churchill, G.A., A Paradigm for Developing Better Measures of Marketing Constructs, in: JoMR, Vol. 16, February 1979, S. 64-73.

Churchill, G.A., Surprenant, C., An Investigation into the Determinants of Customer Satisfaction, in: JoMR, Vol. 19, November 1982, S. 491-504.

Corsten, H., Betriebswirtschaftslehre der Dienstleistungsunternehmen, München 1988.

Cox, E., The Optimal Number of Response Alternatives for a Scale: A Review, in: JoMR, Vol. 17, November 1980, S. 407-422.

Cronin, J.J., Taylor, St.A., Measuring Service Quality: A Reexamination and Extension, in: JoM, Vol. 56, July 1992, S. 55-68.

Dabholkar, P.A., Customer Satisfaction and Service Quality: Two Constructs or One?, in: AMA, Summer 1993, S. 10-18.

Danaher, P.J., Mattson, J., Cumulative Encounter Satisfaction in the Hotel Conference Process, in: IJoSIM, Vol. 5, No. 4, 1994, S. 69-80.

Danaher, P.J., Mattson, J., Customer Satisfaction during the Service Delivery Process, in: EJoM, Vol. 28, No. 5, 1994, S. 5-16.

Das Deutsche Bundesrecht, Allgemeines Eisenbahngesetz (AEG), 730. Lieferung, 1995.

Day, R.L., Toward a Process Model of Consumer Satisfaction, in: Conceptualization and Measurement of Consumer Satisfaction and Dissatisfaction, Hunt, H.K. (Hrsg.), Cambridge, Mass. 1977, S. 153-183.

Day, R.L., The Next Step: Commonly Accepted Constructs for Satisfaction Research, in: International Fare in Consumer Satisfaction and Complaining Behavior, Day, R.L., Hunt, H.K. (Hrsg.), Bloomington 1983, S. 113-117.

Deutsche Bahn AG (Hrsg.), Service \& Züge, Broschüre, Frankfurt am Main 1997.

Deutsche Bahn AG (Hrsg.), BahnTaxi, Broschüre, Frankfurt am Main 1997.

Deutsche Lufthansa AG (Hrsg.), Servicequalität - Unsere Kunden halten uns den Spiegel vor: Customer Service Index, in: Lufthanseat, Jg. 42, H. 642 vom 16. Februar 1996, S. 1, 6-7.




Dichtl, E., Müller, St., Anspruchsinflation und Nivellierungstendenzen als meßtechnische Probleme in der Absatzforschung, in: Marketing ZFP, Jg. 8 , H. 4, 1986, S. 233-236.

Dichtl, E., Peter, S., Kundenzufriedenheit und Kundenbindung in der Automobilindustrie: Ergebnisse einer empirischen Untersuchung, in: Automobilmarktforschung: Nutzenorientierung von Pkw-Herstellern, Bauer, H.H., Dichtl, E., Herrmann, A. (Hrsg.), München 1996, S. $15-31$.

Dichtl, E., Schneider, W., Kundenzufriedenheit im Zeitalter des Beziehungs! management, in: Lean Management und Lean Marketing, Belz, C., Schögel, M., Kramer, M. (Hrsg.), St. Gallen 1994, S. 6-12.

Diederich, H., Verkehrsbetriebslehre, Wiesbaden 1977.

Diller, H., Kundenbindung als Marketingziel, in: Marketing ZFP, Jg. 18, H. 2, 1996, S. 81-94.

Dutka, A.F., AMA Handbook for Customer Satisfaction: Research, Planning, and Implementation, Chicago 1994.

Eckert, St., Rentabilitätssteigerung durch Kundenbindung, Hammer 1995.

Edvardsson, B., Service Breakdowns: A Study of Critical Incidents in an Airline, in: IJoSIM, Vol. 3, No. 4, 1992, S. 17-29.

Engelhardt, W.H., Kleinaltenkamp, M., Reckenfelderbäumer, M., Leistungsbündel als Absatzobjekte: Ein Ansatz zur Überwindung der Dichotomie von Sach- und Dienstleistungen, in: ZfbF, Jg. 45, H. 5, 1993, S. 395-426.

Erevelles, S., Leavitt, C., A Comparison of Current Models of Consumer Satisfaction/Dissatisfaction, in: JoCS/D\&CB, Vol. 5, 1992, S. 104-114.

Esch, F.-R., Billen, P., Ansätze zum Zufriedenheitsmanagement: Das Zufriedenheitsportfolio, in: Kundennähe realisieren, Tomczak, T., Belz, Ch. (Hrsg.), St. Gallen 1994, S. 407-424.

Ferriday, J.J., Simintiras, A.C., Customer Satisfaction: Present Knowledge and Some Directions for Future Research, in: Marketing: Its Dynamics and Challenges, Proceedings of the 23rd Annual Conference of the European Marketing Academy, Bloemer, J., Lemmink, J., Kasper, H. (Hrsg.), Maastricht 1994, S. 221-234.

Festinger, L., A Theory of Cognitive Dissonance, Stanford 1957.

File, K.M., Judd, B.B., Prince, R.A., Interactive Marketing: The Influence of Participation on Positive Word-of-Mouth and Referrals, in: JoSM, Vol. 6, No. 4, 1992, S. 5-14. 
Firner, H., Köster, J., Das Wachstum des Personenverkehrsmarktes, in: DBB, Jg. 65, H. 12, 1989, S. 1037-1039.

Fisk, R.P., Toward a Consumption/Evaluation Process Model for Services, in: Marketing of Services, Proceedings Series, AMA, Donnelly, J.H., George, W.R. (Hrsg.), Chicago 1981, S. 191-195.

Flanagan, J.C., The Critical Incident Technique, in: Psychological Bulletin, Vol. 51, July 1954, S. 327-357.

Folkes, V., How Consumers Predict Service Quality: What Do They Expect?, in: Service Quality: New Directions in Theory and Practice, Rust, R.T., Oliver, R.L. (Hrsg.), Thousand Oaks u.a. 1994, S. 108-122.

Forschungsgemeinschaft Qualitätssicherung e.V. (FQS) (Hrsg.), Qualitätssicherung in Dienstleistungsprozessen: theoretische Grundlagen für die strategische Planung von Qualitätszielen im Dienstleistungsbereich; Abschlußbericht, Berlin u.a. 1995.

Fourastié, J., Die große Hoffnung des zwanzigsten Jahrhunderts, Köln 1954.

Fritz, W., Marktorientierte Unternehmensführung und Unternehmenserfolg: Grundlagen und Ergebnisse einer empirischen Untersuchung, Stuttgart 1992.

Frömbling, S., Zielgruppenmarketing im Fremdenverkehr von Regionen: Ein Beitrag zur Marktsegmentierung auf der Grundlage von Werten, Motiven und Einstellungen, Frankfurt am Main 1993.

Garre, K.-H., InterRegio: Ein neues Leistungsangebot der Deutschen Bundesbahn im Schienenpersonenfernverkehr, in: DBB, Jg. 64, H. 9, 1988, S. $775-780$.

Garre, K.-H., Müller, J., Das Qualitätssicherungssystem Personenverkehr der DB, in: DBB, Jg. 67, H. 9, 1991, S. 849-857.

Garvin, D.A., What does "Product Quality" Really Mean?, in: SMR, Vol. 25, Fall 1984, S. 25-43.

Geise, W., Einstellung und Marktverhalten: Analyse der theoretisch-empirischen Bedeutung des Einstellungskonzepts im Marketing und Entwicklung eines alternativen Forschungsprogramms aus alltagstheoretischer Sicht, Thun, Frankfurt 1984.

Geva, A., Goldman, A., Changes in the Perception of a Service during its Consumption: A Case of Organised Tours, in: EJoM, Vol. 23, No. 12, 1989, S. 44-52.

Gierl, H., Sipple, H., Zufriedenheit mit dem Kundendienst, in: JdAV, Jg. 39, H. 3, 1993, S. 239-260. 
Giese, D., Regionalluftverkehr und Eisenbahn als Wettbewerber im deutschen und grenzüberschreitenden Verkehr, Berlin 1993.

Glöckner, G., Jäger, W., Bahnhof 2000: Erlebniswelt mit Gleisanschluß, in: DBB, Jg. 65, H. 9, 1989, S. 709-714.

Gohlke, R., Wie sichert die Bundesbahn ihre Dienstleistungsqualität?, in: Qualität Die Herausforderung: Erfahrungen, Perspektiven, Lisson, A. (Hrsg.), Berlin u.a. 1987, S. 235-246.

Grönroos, Ch., Service Management and Marketing: Managing the Moment of Truth in Service Competition, Lexington, Mass. 1990.

Grönroos, Ch., Toward a Third Phase in Service Quality Research: Challenges and Future Directions, in: Advances in Service Marketing and Management: Research and Practice, Swartz, T.A., Bowen, D.E., Brown, St.W. (Hrsg.), 2. Aufl., Greenwich, London 1993, S. 49-64.

Große, K.-H., Der außertarifliche Wettbewerb der Unternehmen des Personenund Güterverkehrs, in: Veröffentlichungen der Akademie für Wirtschaft und Politik Hamburg, Ortlieb, H.-D. (Hrsg.), Tübingen 1963.

Gummesson, E., Kingman-Brundage, J., Service Design and Quality: Applying Service Blueprinting and Service Mapping to Railroad Services, in: Quality Management in Services, Kunst, P., Lemmink, J. (Hrsg.), Assen 1992, S. 101-114.

Güthoff, J., Qualität komplexer Dienstleistungen: Konzeption und empirische Analyse der Wahrnehmungsdimensionen, Wiesbaden 1995.

Häberli, V., Greuter, B., Serviceleistungen - ein zu Unrecht vernachlässigter Verkehrszweck, in: IVW, Jg. 48, H. 10, 1996, S. 20-26.

Haist, F., Fromm, H., Qualität im Unternehmen: Prinzipien - Methoden Techniken, 2. Aufl., München, Wien 1991.

Haller, S., Beurteilung von Dienstleistungsqualität: Dynamische Betrachtung des Qualitätsurteils im Weiterbildungsbereich, Wiesbaden 1995.

Hansen, U., Schoenheit, I., Verbraucherzufriedenheit und Beschwerden - Strategische Herausforderungen für Unternehmen und Verbraucherorganisationen, in: Verbraucherzufriedenheit und Beschwerdeverhalten, Hansen, U., Schoenheit, I. (Hrsg.), Frankfurt am Main 1987, S. 11-27.

Hausknecht, D.R., Measurement Scales in Consumer Satisfaction/Dissatisfaction, in: JoCS/D\&CB, Vol. 3, 1990, S. 1-11. 
Haynes, P.J., Hating to Wait: Managing the Final Service Encounter, in: JoSM, Vol. 4, No. 4, 1990, S. 20-26.

Henß, R., Sammeltaxi im Zug, in: Die Zeit, Nr. 45 vom 1.11.1996, S. 71.

Hentschel, B., Dienstleistungsqualität aus Kundensicht: Vom merkmals- zum ereignisorientierten Ansatz, Wiesbaden 1992.

Hentschel, B., Multiattributive Messung von Dienstleistungsqualität, in: Dienstleistungsqualität: Konzepte, Methoden, Erfahrungen, Bruhn, M., Stauss, B. (Hrsg.), 2. Aufl., Wiesbaden 1995, S. 347-378.

Herrmann, A., Produktqualität, Kundenzufriedenheit und Unternehmensrentabilität: Eine branchenübergreifende Analyse, in: Wege des Marketing: Festschrift zum 60. Geburtstag von Erwin Dichtl, Bauer, H.H., Diller, H. (Hrsg.), Berlin 1995, S. 237-247.

Hertel, G., Rasemann, A., In der Kundenklasse reisen, in: QZ, Jg. 38, H. 6, 1993, S. 367-371.

Herzberg, F., The Motivation-Hygiene Concept and Problems of Manpower, in: Personal Administration, Jg. 27, 1964, S. 3-7.

Herzberg, F., Work and Nature of Men, Cleveland, Ohio 1966.

Hildebrandt, L., Konfirmatorische Analysen von Modellen des Konsumentenverhaltens, Berlin 1983.

Hildebrandt, L., Kausalanalytische Validierung in der Marketingforschung, in: Marketing ZFP, Jg. 6, H. 1, 1984, S. 41-51.

Hildebrandt, L., Trommsdorff, V., Konfirmatorische Analysen in der empirischen Forschung, in: Innovative Marktforschung, Forschungsgruppe Konsum und Verhalten (Hrsg.), Würzburg, Wien 1983, S. 139-160.

Hilke, R., Grundlagen normorientierter und kriteriumorientierter Tests, Bern 1980.

Hilke, W., Grundprobleme und Entwicklungstendenzen des DienstleistungsMarketing, in: Dienstleistungs-Marketing, Schriften zur Unternehmensführung, Bd. 35, Jacob, H. et al. (Hrsg.), Wiesbaden 1989, S. 6-44.

Hinterhuber, H.H., Handlbauer, G., Matzler, K., Kundenzufriedenheit durch Kernkompetenzen: eigene Potentiale erkennen - entwickeln - umsetzen, München, Wien 1997.

Hirschman, A.O., Abwanderung und Widerspruch - Reaktionen auf Leistungsabfall bei Unternehmungen, Organisationen und Staaten, Tübingen 1974. 
Holzmüller, H.H., Nentwich, A., Ansätze zur Entwicklung eines Marketing-Informationssystems (MAIS) im Bahnbetrieb, in: Der Markt, Jg. 29, H. 1, 1990, S. 16-26.

Homburg, Ch., Die Kausalanalyse: Eine Einführung, in: WiSt, Jg. 21, H. 10, 1992, S. 499-508.

Homburg, Ch., Baumgartner, H., Beurteilung von Kausalmodellen: Bestandsaufnahme und Anwendungsempfehlungen, in: Marketing ZFP, Jg. 17, H. 3, 1995, S. 162-176.

Homburg, Ch., Giering, A., Konzeptualisierung und Operationalisierung komplexer Konstrukte, in: Marketing ZFP, Jg. 18, H. 1, 1996, S. 5-24.

Homburg, Ch., Rudolph, B., Theoretische Perspektiven zur Kundenzufriedenheit, in: Kundenzufriedenheit: Konzepte, Methoden, Erfahrungen, Simon, H., Homburg, Ch. (Hrsg.), Wiesbaden 1995, S. 29-49.

Homburg, Ch., Rudolph, B., Werner, H., Messung und Management von Kundenzufriedenheit in Industriegüterunternehmen, in: Kundenzufriedenheit: Konzepte, Methoden, Erfahrungen, Simon, H., Homburg, Ch. (Hrsg.), Wiesbaden 1995, S. 313-340.

Homburg, Ch., Sütterlin, St., Kausalmodelle in der Marktforschung: EQS als Alternative zu LISREL?, in: Marketing ZFP, Jg. 12, H. 3, 1990, S. 181-192.

Homburg, Ch., Werner, H., Ein Meßsystem für Kundenzufriedenheit, in: asw, Jg. 39, H. 11, 1996, S. 92-100.

Howard, J.H., Sheth, J.N., The Theory of Buyer Behavior, New York 1969.

Inde, G.B., Transport, Verkehr, Logistik: gesamtwirtschaftliche Aspekte und einzelwirtschaftliche Handhabung, 2. Aufl., München 1991.

Illetschko, L.L., Transportbetriebswirtschaft im Grundriss, Wien 1957.

Illetschko, L.L., Betriebswirtschaftliche Probleme der Verkehrswirtschaft, Wiesbaden 1959.

Institut der deutschen Wirtschaft Köln (Hrsg.), EU-Verkehr - Freie Fahrt für die Eisenbahn, in: iwd Informationsdienst des Instituts der deutschen Wirtschaft, Nr. 41 vom 10. Oktober 1996, S. 7.

Institut für Marketing, User-Analyse bei der Deutschen Bahn AG, unveröffentlichte Studie im Auftrag der Deutschen Bahn AG, Münster 1995.

Institut für Marketing, Non User-Analyse bei der Deutschen Bahn AG, unveröffentlichte Studie im Auftrag der Deutschen Bahn AG, Münster 1996. 
Jacoby, J., Information Load and Decision Quality: Some Contest Issues, in: JoMR, Vol. 14, November 1977, S. 569-573.

Jänsch, E., Wiese, J., Der Stellenwert des Komforts in der Systemplanung "Hochgeschwindigkeitsverkehr" (HGV), in: DBB, Jg. 64, H. 9, 1988, S. 805-809.

Jarvis, L.P., Wilcox, J.B., Evoked Set Size - Some Theoretical and Empirical Evidence, in: Combined Proceedings Fall Conference of the AMA, No. 35, Greer, Th.V. (Hrsg.), Chicago 1973, S. 236-240.

Jeschke, K., Institutionalisierungsformen der Kundenorientierung in öffentlichen Unternehmen am Beispiel der Deutschen Bundesbahn, Lehr- und Forschungsberichte des Lehrstuhls Markt und Konsum der Universität Hannover, Nr. 12, Hannover 1988.

Jeschke, K., Nachkaufmarketing: Kundenzufriedenheit und Kundenbindung auf Konsumgütermärkten, Frankfurt am Main u.a. 1995.

Johnston, R., The Zone of Tolerance: Exploring the Relationship Between Service Transactions and Satisfaction with the Overall Service, in: IJOSIM, Vol. 6, No. 2, 1995, S. 46-61.

Kaas, K.P., Runow, H., Wie befriedigend sind die Ergebnisse der Forschung zur Verbraucherzufriedenheit?, in: DBW, Jg. 44, H. 3, 1984, S. 451-460.

Katz, R., Informationsquellen der Konsumenten: Eine Analyse der Divergenzen zwischen der Beurteilung und Nutzung, Wiesbaden 1983.

Kern, E., Der Interaktionsansatz im Investitionsgütermarketing: Eine konfirmatorische Analyse, Berlin 1990.

Keuchel, St., Wirkungsanalyse von Maßnahmen zur Beeinflussung des Verkehrsmittelwahlverhaltens, Göttingen 1994.

Kingman-Brundage, J., The ABC's of Service System Blueprinting, in: Designing a Winning Service Strategy, Bitner, M.J., Crosby, L.A. (Hrsg.), Chicago 1989 , S. 30-33.

Kingman-Brundage, J., Service Mapping: Gaining a Concrete Perspective on Service System Design, in: QUiS 3: Quality in Services Conference Proceedings, Scheuing, E.E., Edvardsson, B., Lascelles, D., Little, C.H. (Hrsg.), New York 1994, S. 235-246.

Kirchgeorg, M., Ökologieorientiertes Unternehmensverhalten: Typologien und Erklärungsansätze auf empirischer Basis, Wiesbaden 1990.

Kirsch, W., Kutschker, M., Das Marketing von Investitionsgütern - Theoretische und empirische Perspektiven, Wiesbaden 1978. 
Kirsch, W., Kutschker, M., Lutschewitz, H., Ansätze und Entwicklungstendenzen im Investitionsgütermarketing, 2. Aufl., Stuttgart 1980.

Klaus, P.G., Face-to-Face Service Encounters: The Issue of Quality in the Mass-Delivery of Services by Public Enterprises, Boston, Mass. 1983.

Klaus, P.G., Auf dem Weg zu einer Betriebswirtschaftslehre der Dienstleistungen: Der Interaktionsansatz, in: DBW, Jg. 44, H. 3, 1984, S. 467-475.

Klaus, P.G., Service-Qualität im öffentlichen Personenverkehr: Ein retrograder Ansatz zur Analyse und Veränderung, in: ZögU, Bd. 7, H. 1, 1984, S. 55-73.

Klein, H., Deutsche Bundesbahn - Dienstleistung als Marke, in: FAZ vom 26.5.1987, S. B 18.

Klein, H., Bahnhöfe - Visitenkarten der Bahn, in: DBB, Jg.65, H. 9, 1989, S. 707-709.

Klein, H., InterCityExpress: mehr als nur ein neuer Zug, in: DBB, Jg. 67, H. 5, 1991, S. 497-504.

Klein, H., Deutsche Bundesbahn: Die Entwicklung zu einem europäischen Dienstleistungs- und Verkehrskonzern, in: Touristik-Marketing: das Marketing der Tourismus-Organisationen, Verkehrsträger, Reiseveranstalter und Reisebüros, Roth, P., Schrand, A. (Hrsg.), München 1992, S. 287-296.

Klein, H., Personenverkehr, in: DDB, Jg. 69, H. 1, 1993, S. 32-40.

Klein, H., Management von Kundenzufriedenheit bei der Deutschen Lufthansa $A G$, in: Kundenzufriedenheit: Konzepte, Methoden, Erfahrungen, Simon, H., Homburg, Ch. (Hrsg.), Wiesbaden 1995, S. 368-385.

Klein, H., Qualitätsmanagement der Deutschen Lufthansa AG, in: Dienstleistungsqualität: Konzepte, Methoden, Erfahrungen, Bruhn, M., Stauss, B. (Hrsg.), 2. Aufl., Wiesbaden 1995, S. 477-493.

Knoblich, H., Oppermann, R., Dienstleistung: Ein Produkttyp - Eine Erfassung und Abgrenzung des Dienstleistungsbegriffes auf produkttypologischer Basis, in: Der Markt, Jg. 35, H. 1, 1996, S. 13-22.

Koelemeijer, K., Roest, H., Verhallen, T., An Integrative Framework of Perceived Service Quality and its Relations to Satisfaction/Dissatisfaction, Attitude and Repurchase Intention, in: Marketing for the New Europe: Dealing with Complexity, Chias, J., Sureda, J. (Hrsg.), Barcelona 1993, S. 683-699. 
Korte, Ch., Customer Satisfaction Measurement: Kundenzufriedenheitsmessung als Informationsgrundlage des Hersteller- und Handelsmarketing am Beispiel der Automobilwirtschaft, Frankfurt am Main u.a. 1995.

Kroeber-Riel, W., Konsumentenverhalten, 5. Aufl., München 1992.

Kube, Ch., Erfolgsfaktoren in Filialsystemen - Diagnose und Umsetzung im strategischen Controlling, Wiesbaden 1991.

Kuß, A., Valide Daten: Die Grundlage für aussagefähige Marktforschung, in: Marktforschung, Tomczak, T., Reinecke, S. (Hrsg.), St. Gallen 1994, S. $16-28$.

Laakmann, K., Mobilitätsmarketing, in: Lexikon der aktuellen Marketingbegriffe, Meffert, H. (Hrsg.), Wien 1994, S. 161-166.

Laakmann, K., Value-Added Services als Profilierungsinstrument im Wettbewerb: Analyse, Generierung und Bewertung, Frankfurt am Main 1995.

Laaser, C.-F., Die Bahnstrukturreform: Richtige Weichenstellung oder Fahrt aufs Abstellgleis?, Kieler Diskussionsbeiträge Nr. 239, Kiel 1994.

LaBarbera, P.A., Mazursky, D., A Longitudinal Assessment of Consumer Satisfaction/Dissatisfaction: The Dynamic Aspect of the Cognitive Process, in: JoMR, Vol. 20, November 1983, S. 393-404.

Lanz, I., Die Masse steigt aus der Massengesellschaft aus, in: FWW, H. 24, 1996, S. 32-35.

Laurent, G., Kapferer, J.-N., Measuring Consumer Involvement Profiles, in: JoMR, Vol. 22, February 1985, S. 41-53.

Lehmann, A., Dienstleistungsmanagement: Strategien und Ansatzpunkte zur Schaffung von Servicequalität, 2. Aufl., Stuttgart 1995.

Leonhardt-Weber, B., Die Entwicklung der Qualitätsmerkmale im Verkehr: Eine Analyse vor dem Hintergrund der technischen, wirtschaftlichen und gesellschaftlichen Entwicklung, München 1990.

Lewis, R.C., Booms, B.H., The Marketing Aspects of Service Quality, in: Emerging Perspectives on Services Marketing, Berry, L.L., Shostack, L., Upah, G. (Hrsg.), Chicago 1983, S. 99-104.

Liljander, V., Introducing Deserved Service and Equity into Service Quality Models, in: Dienstleistungsmarketing: Konzeptionen und Anwendungen, Kleinaltenkamp, M. (Hrsg.), Wiesbaden 1995, S. 141-169.

Liljander, V., Strandvik, T., The Relationship between Service Quality, Satisfaction and Intentions, Working Paper No. 243, Swedish School of Economics and Business Administration, Helsinki 1992. 
Liljander, V., Strandvik, T., Different Comparison Standards as Determinants of Service Quality, in: Dienstleistungsmarketing: Eine Bestandsaufnahme, Thelen, E.M., Mariamhof, G.B. (Hrsg.), Frankfurt am Main 1993, S. 127-150.

Liljander, V., Strandvik, T., Estimating Zones of Tolerance in Perceived Service Quality and Perceived Service Value, in: IJoSIM, Vol. 4, No. 2, 1993, S. 6-28.

Lingen, Th. von, Zufriedenheitsmanagement, in: P\&A, H. 1, 1994, S. 5-13.

Litzenroth, H., Dem Verbraucher auf der Spur - quantitative und qualitative Konsumtrends, in: JdAV, Jg. 41, H. 3, 1995, S. 213-305.

Lohmüller, G.B., Path models with Latent Variables and Partial Least Squares (PLS) Estimation, Würzburg 1984.

Lovelock, Ch.H., Classifying Services to Gain Strategic Marketing Insights, in: JoM, Vol. 47, Summer 1983, S. 9-20.

Lovelock, Ch.H., Developing Frameworks for Understanding Service Marketing, in: Services Marketing, Lovelock, Ch.H. (Hrsg.), 2. Aufl., Englewood Cliffs, N.J. 1991, S. 24-38.

Lovelock, Ch.H., Product Plus: How Product + Service $=$ Competitive Advantage, New York u.a. 1994.

Mangold, W.G., Babakus, E., Service Quality. The Front-Stage vs. the Back-Stage Perspective, in: JoSM, Vol. 5, No. 4, 1991, S. 59-70.

Mano, H., Oliver, R.L., Assessing the Dimensionality and Structure of the Consumption Experience: Evaluation, Feeling, and Satisfaction, in: JoCR, Vol. 20, December 1993, S. 451-466.

Marcati, A., Cecere, F., Marzocchi, G.L., Towards a Better Understanding of the Economics of Customer Satisfaction. An Analysis of Customer Satisfaction, Spending Levels and Spending Patterns, in: Marketing Today and for the 21st Century, Proceedings of the 24th Annual Conference of the European Marketing Academy, Bergadaa, M. (Hrsg.), Cergy 1995, S. 1871-1875.

McClure, N., The Role of Expectations, Ambiguity of Information, and Initial Impressions in Consumer Satisfaction Assessments: A Process Oriented Approach, Lubbock 1995.

McNeal, J.U., Consumer Satisfaction: The Measure of Marketing Effectiveness, in: MSU Business Topics, Summer 1969, S. 31-35.

Meffert, H., Marketing und Konsumerismus, in: ZfB, Jg. 45, H. 2, 1975, S. 69-90. 
Meffert, H., Die Beurteilung und Nutzung von Informationsquellen beim Kauf von Konsumgütern, in: Konsumentenverhalten und Information, Meffert, H., Steffenhagen, H., Freter, H. (Hrsg.), Wiesbaden 1979, S. 39-65.

Meffert, H., Marketing: Grundlagen der Absatzpolitik, 7. Aufl., Wiesbaden 1986.

Meffert, H., Euromarketing im Spannungsfeld zwischen nationalen Bedürfnissen und globalem Wettbewerb, in: Europa 1992 - Chancen und Risiken für das Marketing, Bruhn, M., Wehrle, F. (Hrsg.), 2. Aufl., Münster 1990, S. 13-18.

Meffert, H., Marketingforschung und Käuferverhalten, 2. Aufl., Wiesbaden 1992.

Meffert, H., Marktorientierte Führung von Dienstleistungsunternehmen - neuere Entwicklungen in Theorie und Praxis, in: DBW, Jg. 54, H. 4, 1994, S. 519-541.

Meffert, H., Kundenorientierung und Kundenzufriedenheit - Zwei Seiten einer Medaille?, Manuskript, Februar 1997.

Meffert, H., Birkelbach, R., Qualitätsmanagement in Dienstleistungszentren: Konzeptionelle Grundlagen und typenspezifische Ausgestaltung, in: Dienstleistungsqualität: Konzepte, Methoden, Erfahrungen, Bruhn, M., Stauss, B. (Hrsg.), 2. Aufl., Wiesbaden 1995, S. 207-237.

Meffert, H., Bruhn, M., Beschwerdeverhalten und Zufriedenheit von Konsumenten, in: DBW, Jg. 41, H. 4, 1981, S. 597-613.

Meffert, H., Bruhn, M., Dienstleistungsmarketing: Grundlagen, Konzepte, Methoden; mit Fallbeispielen, 2. Aufl., Wiesbaden 1997.

Meffert, H., Perrey, J., Nutzensegmentierung im Verkehrsdienstleistungsbereich theoretische Grundlagen und empirische Erkenntnisse am Beispiel des Schienenpersonenverkehrs, in: Tourismus Journal, Jg. 1, H. 1, 1997, S. 13-40.

Meffert, H., Siefke, A., Lean Marketing - mehr als ein Schlagwort?, Arbeitspapier Nr. 88 der Wissenschaftlichen Gesellschaft für Marketing und Unternehmensführung e.V., Meffert, H., Wagner, H., Backhaus, K. (Hrsg.), Münster 1994.

Meffert, H., Steffenhagen, H., Marketing-Prognosemodelle. Quantitative Grundlagen des Marketing, Stuttgart 1977.

Mengen, A., Konzeptgestaltung von Dienstleistungsprodukten: Eine ConjointAnalyse im Luftfrachtmarkt unter Berücksichtigung der Qualitätsunsicherheit beim Dienstleistungskauf, Stuttgart 1993.

Merckens, R., Analyse des Verkehrsmittelwahlverhaltens von Geschäftsreisenden, Bundesminister für Verkehr (Hrsg.), Bonn 1984.

Andreas Siefke and Universität Múnster - 978-3-631-75103-9 
Meyer, A., Dienstleistungsmarketing: Erkenntnisse und praktische Beispiele, 6. Aufl., München 1994.

Meyer, A., Dornach, F., Das Deutsche Kundenbarometer: Qualität und Zufriedenheit, in: Kundenzufriedenheit: Konzepte, Methoden, Erfahrungen, Simon, H., Homburg, Ch. (Hrsg.), Wiesbaden 1995, S. 161-178.

Meyer, A., Ertl, R., Nationale Barometer zur Messung von Kundenzufriedenheit: Ein Vergleich zwischen dem "Deutschen Kundenbarometer - Qualität und Zufriedenheit" und dem "American Customer Satisfaction Index $(\mathrm{ASCl})$ ", in: Grundsatzfragen und Herausforderungen des Dienstleistungsmarketing, Meyer, A. (Hrsg.), Wiesbaden 1996, S. 201-231.

Meyer, A., Hüttinger, S., Möglichkeiten und Grenzen der Implementierung eines Total Quality Managements in Kreditinstituten: Konzeptionelle Vorschläge und empirische Analyse, in: JdAV, Jg. 42, H. 3, 1996, S. 216-238.

Meyer, A., Mattmüller, R., Qualität von Dienstleistungen - Entwurf eines praxisorientierten Qualitätsmodells, in: Marketing ZFP, Jg. 9, H. 3, 1987 , S. $187-195$.

Meyer, A., Westerbarkey, P., Zufriedenheit von Hotelgästen - Entwurf eines selbstregulierenden Systems, in: Kundenzufriedenheit: Konzepte, Methoden, Erfahrungen, Simon, H., Homburg, Ch. (Hrsg.), Wiesbaden 1995, S. 387-402.

Miller, G.A., The Magical Number Seven, Plus or Minus Two: Some Limits on Our Capacity for Processing Information, in: Psychological Review, Vol. 63, March 1956, S. 81-97.

Miller, J.A., Studying Satisfaction, Modifying Models, Elicting Expectations, Posing Problems, and Making Meaningful Measurements, in: Conceptualization and Measurement of Consumer Satisfaction and Dissatisfaction, Hunt, H.K. (Hrsg.), Cambridge 1977, S. 72-91.

Monhemius, K.Ch., Umweltbewußtes Kaufverhalten von Konsumenten: ein Beitrag zur Operationalisierung, Erklärung und Typologie des Verhaltens in der Kaufsituation, Frankfurt am Main u.a. 1993.

Muffatto, M., Panizzolo, R., A Process-Based View for Customer Satisfaction, in: IJoQRM, Vol. 12, No. 9, 1995, S. 154-169.

Müller, W., Riesenbeck, H.-J., Wie aus zufriedenen auch anhängliche Kunden werden, in: HM, Jg. 13, H. 3, 1991, S. 67-79.

Nader, G., Evaluation der Bankdienstleistungsqualität mit Hilfe von Problementdeckungsverfahren, in: Der Markt, Jg. 32, H. 3, 1993, S. 152-162. 
Nader, G., Zufriedenheit mit Finanzdienstleistungen: Erfolgswirksamkeit, Messung, Modellierung, Wien, New York 1995.

Neuhaus, H., Ziele des Personenverkehrs, in: ETR, Jg. 43, H. 1/2, 1994, S. 41-44.

Nieschlag, R., Dichtl, E., Hörschgen, H., Marketing, 17. Aufl., Berlin 1994.

Norling, P., Service Design - A Frame of Reference and Some Empirical Results, in: QUiS 3: Quality in Services Conference - Proceedings, Scheuing, E.E., Edvardsson, B., Lascelles, D., Little, C.H. (Hrsg.), New York 1994, S. 361-378.

Nyquist, J.D., Bitner, M.J., Booms, B.H., Identifying Communication Difficulties in the Service Encounter: A Critical Incident Approach, in: The Service Encounter: Managing Employee/Customer Interaction in Service Businesses, Czepiel, J.A., Solomon, M.R., Surprenant, C.F. (Hrsg.), Lexington 1985, S. 195-212.

Ölander, F., Consumer Satisfaction - A Sceptic's View, in: Conceptualization and Measurement of Consumer Satisfaction and Dissatisfaction, Hunt, H.K. (Hrsg.), Cambridge 1977, S. 409-452.

Oliver, R.L., A Cognitive Model of the Antecedents and Consequences of Satisfaction Decisions, in: JoMR, Vol. 17, November 1980, S. 460-469.

Oliver, R.L., Measurement and Evaluation of Satisfaction Processes in Retail Settings, in: JoR, Vol. 57, No. 3, 1981, S. 25-48.

Oliver, R.L., A Conceptual Model of Service Quality and Service Satisfaction: Compatible Goals, Different Concepts, in: Advances in Service Marketing and Management: Research and Practice, Swartz, T.A., Bowen, D.E., Brown, St.W. (Hrsg.), 2. Aufl., Greenwich, London 1993, S. 65-85.

Oliver, R.L., DeSarbo, W.S., Response Determinants in Satisfaction Judgements, in: JoCR, Vol. 14, March 1988, S. 495-507.

Opp, K.-D., Methodologie der Sozialwissenschaften: Einführung in Probleme ihrer Theorienbildung, Reinbeck 1970.

Parasuraman, A., Zeithaml, A., Berry, L.L., SERVQUAL: A Multiple-Item Scale for Measuring Consumer Perceptions of Service Quality, in: JoR, Vol. 64 , No. 1,1988 , S. $12-40$.

Parasuraman, A., Zeithaml, V.A., Berry, L.L., Reassessment of Expectations as a Comparison Standard in Measuring Service Quality: Implications for Further Research, in: JoM, Vol. 58, January 1994, S. 111-124. 
Peter, P.J., Construct Validity: A Review of Basic Issues and Marketing Practices, in: JoMR, Vol. 18, May 1981, S. 133-145.

Peterson, R.A., Wilson, W.R., Measuring Customer Satisfaction: Fact and Artifact, in: JAMS, Vol. 20, No. 1, 1992, S. 61-71.

Pirath, C., Die Grundlagen der Verkehrswirtschaft, 2. Aufl., Berlin, Göttingen, Heidelberg 1949.

Pompl, W., Luftverkehr: Eine ökonomische Einführung, 2. Aufl., Berlin u.a. 1991.

Pompl, W., Touristikmanagement 2: Qualitäts-, Produkt-, Preismanagement, Berlin u.a. 1996.

Rahn, T., Der Hochgeschwindigkeitszug InterCityExpress der DB, in: DBB, Jg. 67, H. 5, 1991, S. 537-542.

Reichheld, F.F., Sasser, W.E., Zero-Migration: Dienstleister im Sog der Qualitätsrevolution, in: HM, Jg. 13, H. 4, 1991, S. 108-116.

Renoux, Y., Consumer Dissatisfaction and Public Policy, in: Public Policy and Marketing Practices, Allvine, F.C. (Hrsg.), Chicago 1973, S. 53-65.

Richins, M.L., Bloch, P.H., Post-Purchase Product Satisfaction: Incorporating the Effects of Involvement and Time, in: JoBR, Vol. 23, No. 2, 1991, S. 145-158.

Riemer, M., Beschwerdemanagement, Frankfurt am Main, New York 1986.

Runow, H., Zur Theorie und Messung der Verbraucherzufriedenheit, Frankfurt am Main 1982.

Rust, R.T., Oliver, R.L., Service Quality: Insights and Managerial Implications from the Frontier, in: Service Quality: New Directions in Theory and Practice, Rust, R.T., Oliver, R.L. (Hrsg.), Thousand Oaks u.a. 1994, S. 1-19.

Rust, R.T., Zahorik, A.J., Keiningham, T.L., Return on Quality (ROQ): Making Service Quality Financially Accountable, Marketing Science Institute (Report No. 94-106), Cambridge, Mass. 1994.

Schanz, G., Zwei Arten von Empirismus, in: ZfbF, Jg. 27, 1975, S. 324-326.

Scharitzer, D., Dienstleistungsqualität - Kundenzufriedenheit, Wien 1994.

Scherhorn, G., Die Funktionsfähigkeit von Konsumgütermärkten, in: Marktpsychologie als Sozialwissenschaft, Irle, M. (Hrsg.), Göttingen 1983, S. 45-150. 
Schmidt, F.K., Das Verkehrsangebot „Bahn“, in: Tourismus-Management: Tourismus-Marketing und Fremdenverkehrsplanung, Haedrich, G., Kaspar, C., Kleinert, H., Klemm, K. (Hrsg.), Berlin, New York 1983, S. $149-162$.

Schmidt, R., Marktorientierte Konzeptfindung für langlebige Gebrauchsgüter: Messung und QFD-gestützte Umsetzung von Kundenanforderungen und Kundenurteilen, Wiesbaden 1996.

Schmitz, G., Qualitätsmanagement im Privatkundengeschäft von Banken: Konzeption und aufbauorganisatorische Verankerung, Wiesbaden 1996.

Schneider, J., Die Privatisierung der Deutschen Bundes- und Reichsbahn: institutioneller Rahmen - Wertkettenorientiertes Synergiekonzept Analyse der Infrastrukturgesellschaft, Wiesbaden 1995.

Schörcher, U., Marketing im Luftverkehr, in: Tourismus-Management: Tourismus-Marketing und Fremdenverkehrsplanung, Haedrich, G., Kaspar, C., Kleinert, H., Klemm, K. (Hrsg.), Berlin, New York 1983, S. 131-147.

Schörcher, U., Buchholz, R., Qualitätsmanagement in der Luftfahrt, in: Handbuch Qualitätsmanagement, Masing, W. (Hrsg.), 3. Aufl., München, Wien 1994, S. 833-848.

Schütze, R., Kundenzufriedenheit: After-Sales-Marketing auf industriellen Märkten, Wiesbaden 1992.

Sebastian, K.-H., Paffrath, R., Lauszus, D., Runneboon, T., Messung von Kundenzufriedenheit bei industriellen Dienstleistungen, in: Kundenzufriedenheit: Konzepte, Methoden, Erfahrungen, Simon, $H_{\text {., }}$ Homburg, Ch. (Hrsg.), Wiesbaden 1995, S. 341-365.

Shostack, G.L., Designing Services that Deliver, in: HBR, Vol. 62, January/ February 1984, S. 133-139.

Shostack, G.L., How to Design a Service, in: EJoM, Vol. 18, No. 1, 1984, S. 49-63.

Shostack, G.L., Planning the Service Encounter, in: The Service Encounter: Managing Employee/Customer Interaction in Service Businesses, Czepiel, J.A., Solomon, M.R., Surprenant, C.F. (Hrsg.), Lexington 1985, S. 243-253.

Shostack, G.L., Service Positioning through Structural Change, in: JoM, Vol. 51, January 1987, S. 34-43. 
Shostack, G.L., Understanding Services Through Blueprinting, in: Advances in Service Marketing and Management: Research and Practice, Swartz, T.A., Bowen, D.E., Brown, St.W. (Hrsg.), Greenwich, London 1992, S. 75-90.

Silvestro, R., Fitzgerald, L., Johnston, R., Voss, Ch., Towards a Classification of Service Processes, in: IJoSIM, Vol. 3, No. 3, 1992, S. 62-75.

Simon, H., Homburg, Ch., Kundenzufriedenheit als strategischer Erfolgsfaktor: Einführende Überlegungen, in: Kundenzufriedenheit: Konzepte, Methoden, Erfahrungen, Simon, H., Homburg, Ch. (Hrsg.), Wiesbaden 1995, S. 15-27.

Smith, R.A., Houston, M.J., Script-Based Evaluations of Satisfaction with Services, in: Emerging Perspectives on Services Marketing, Proceedings Series, AMA, Berry, L.L., Shostack, G.L., Upah, G.D. (Hrsg.), Chicago 1983, S. 59-62.

Solomon, M.R., Surprenant, C., Czepiel, J.A., Gutman, E.G., A Role Theory Perspective on Dyadic Interactions: The Service Encounter, in: JoM, Vol. 49, Winter 1985, S. 99-111.

Spreng, R.A., Singh, A.K., An Empirical Assessment of the Servqual Scale and the Relationship Between Service Quality and Satisfaction, in: AMA, Summer 1993, S. 1-6.

Stabenau, H., Verkehrsbetriebslehre, 3. Aufl., Düsseldorf 1994.

Stauss, B., Beschwerdepolitik als Instrument des Dienstleistungsmarketing, in: JdAV, Jg. 35, H. 1, 1989, S. 41-62.

Stauss, B., Service-Qualität als strategischer Erfolgsfaktor, in: Erfolg durch Service-Qualität, Stauss, B. (Hrsg.), München 1991, S. 7-36.

Stauss, B., Dienstleister und die vierte Dimension, in: HM, Jg. 13, H. 2, 1991 , S. 81-89.

Stauss, B., Total Quality Management und Marketing, in: Marketing ZFP, Jg. 16, H. 3, 1994, S. 149-159.

Stauss, B., Der Einsatz der "Critical Incident Technique" im Dienstleistungsmarketing, in: Kundennähe realisieren, Tomczak, T., Belz, Ch. (Hrsg.), St. Gallen 1994, S. 233-250.

Stauss, B., "Augenblicke der Wahrheit" in der Dienstleistungserstellung, in: Dienstleistungsqualität: Konzepte, Methoden, Erfahrungen, Bruhn, M., Stauss, B. (Hrsg.), 2. Aufl., Wiesbaden 1995, S. 379-399. 
Stauss, B., Kundenprozeßorientiertes Qualitätsmanagement im Dienstleistungsbereich, in: Total Quality Management II, Preßmar, D.B. (Hrsg.), Wiesbaden 1995, S. 25-50.

Stauss, B., Friege, Ch., Zehn Lektionen in TQM, in: HBM, H. 2, 1996, S. 20-32.

Stauss, B., Hentschel, B., Verfahren der Problementdeckung und -analyse im Qualitätsmanagement von Dienstleistungsunternehmen, in: JdAV, Jg. 36, H. 3, 1990, S. 232-259.

Stauss, B., Hentschel, B., Dienstleistungsqualität, in: WiSt, Jg. 20, H. 5, 1991, S. 238-244.

Stauss, B., Hentschel, B., Messung von Kundenzufriedenheit - Merkmals- oder ereignisorientierte Beurteilung von Dienstleistungsqualität, in: M\&M, Jg. 36, H. 3, 1992, S. 115-122.

Stauss, B., Neuhaus, P., Das Qualitative Zufriedenheitsmodell (QZM), Diskussionsbeiträge der Wirtschaftswissenschaftlichen Fakultät Ingolstadt Nr. 66, Ingolstadt 1995.

Stauss, B., Seidel, W., Prozessuale Zufriedenheitsermittlung und Zufriedenheitsdynamik bei Dienstleistungen, in: Kundenzufriedenheit: Konzepte, Methoden, Erfahrungen, Simon, H., Homburg, Ch. (Hrsg.), Wiesbaden 1995, S. 179-203.

Stauss, B., Seidel, W., Beschwerdemanagement: Fehler vermeiden, Leistung verbessern, Kunden binden, München, Wien 1996.

Stauss, B., Weinlich, B., Die Sequentielle Ereignismethode - ein Instrument der prozeßorientierten Messung von Dienstleistungsqualität, in: Der Markt, Jg. 35, H. 1, 1996, S. 49-58.

Stiftung Warentest (Hrsg.), Service der Bahn - Zügig informiert, zuviel kassiert, in: Test, H. 5, 1995, S. 80-83.

Strandvik, T., Liljander, V., A Comparison of Episode Performance and Relationship Performance for a Discrete Service, in: Dienstleistungsmarketing: Konzeptionen und Anwendungen, Kleinaltenkamp, M. (Hrsg.), Wiesbaden 1995, S. 111-139.

Taylor, Sh., Waiting for Service: The Relationship Between Delays and Evaluation of Services, in: JoM, Vol. 58, April 1994, S. 56-69.

Taylor, Sh., Claxton, J.D., Delays and the Dynamics of Service Evaluations, in: JAMS, Vol. 22, No. 3, 1994, S. 254-264.

Taylor, St.A., Baker, Th., An Assessment of the Relationship Between Service Quality and Customer Satisfaction in the Formation of Consumers' Purchase Intention, in: JoR, Vol. 70, No. 2, 1994, S. 163-178.

Andreas Siefke and Universität Münster - 978-3-631-75103-9 
Trommsdorff, V., Konsumentenverhalten, 2. Aufl., Stuttgart, Berlin, Köln 1993.

Tse, D.K., Wilton, P.C., Models of Consumer Satisfaction Formation: An Extension, in: JoMR, Vol. 24, May 1988, S. 204-212.

Tulving, E., How many Memory Systems are there?, in: American Psychologist, Vol. 40 , No. 4,1985 , S. $385-398$.

Ungefug, H.-G., Bahnservice - KKV gesucht, in: WiWo, Jg. 49, H. 22, 1995 , S. 84-88.

Voss, C.A., Where should Service Quality be measured? Conclusions from a Process Based Model, in: QUiS 2, Quality in Service Conference Selected Papers, Scheuing, E., Gummesson, E., Little, C.H. (Hrsg.), New York 1992, S. 29-34.

Wagner, W., Personenverkehr der Deutschen Bundesbahn, Hannover 1991.

Walker, J.L., Service Encounter Satisfaction: Conceptualized, in: JoSM, Vol. 9, No. 1, 1995, S. 5-14.

Watzlik, S., Die Bedeutung von Involvement und kognitiven Strukturen für das Marketing von Dienstleistungen am Beispiel von Finanzdienstleistungen, in: Dienstleistungsmarketing: Konzeptionen und Anwendungen, Kleinaltenkamp, M. (Hrsg.), Wiesbaden 1995, S. 89-109.

Weigand, W., Planungs- und Prognosemethoden für das Angebot im Hochgeschwindigkeitsverkehr der Zukunft, in: ETR, Jg. 43, H. 5, 1994, S. $279-290$.

Weinberg, P., Das Entscheidungsverhalten der Konsumenten, Paderborn u.a. 1981.

Westerbarkey, P., Methoden zur Messung und Beeinflussung der Dienstleistungsqualität: Feedback- und Anreizsysteme in Beherbergungsunternehmen, Wiesbaden 1996.

Wilken, D., Kriterien der Verkehrsmittelwahl im Personenfernverkehr und ihre Bedeutung in Verkehrsnachfragemodellen, in: IVW, Jg. 26, H. 1, 1974, S. 3-9.

Wittink, D.R., Bayer, L.R., The Measurement Imperative, in: Customer Satisfaction Measurement: A Management Information System for Total Quality, Marr, S.L., Crosby, A. (Hrsg.), Chicago 1993, S. 14-22.

Wold, H., Soft Modeling: The Basic Design and Some Extensions, in: Systems under Indirect Observation: Causality, Structure, Prediction, Jöreskog, K.G., Wold, H. (Hrsg.), 2. Aufl., Amsterdam 1982, S. 1-54. 
Wöllenstein, St., Betriebstypenprofilierung in vertraglichen Vertriebssystemen: Eine Analyse von Einflußfaktoren und Erfolgswirkungen auf der Grundlage eines Vertragshändlersystems im Automobilhandel, Frankfurt am Main u.a. 1996.

Woodruff, R.B., Developing and Applying Consumer Satisfaction Knowledge: Implications for Future Research, in: JoCS/D\&CB, Vol. 6, 1993, S. 1-11.

Woodside, A.G., Frey, L.L., Daly, R.T., Linking Service Quality, Customer Satisfaction, and Behavioral Intention, in: Journal of Health Care Marketing, Vol. 9, No. 4, 1989, S. 5-17.

Woratschek, H., Die Typologie von Dienstleistungen aus informationsökonomischer Sicht, in: Der Markt, Jg. 35, H. 1, 1996, S. 59-71.

Zeithaml, V.A., How Consumer Evaluation Processes Differ between Goods and Services, in: Services Marketing, Lovelock, Ch.H. (Hrsg.), 2. Aufl., Englewood Cliffs, N.J. 1991, S. 39-47.

Zeithaml, V.A., Berry, L.L., Parasuraman, A., The Nature and Determinats of Customer Expectations of Service, in: Managing Services Marketing: Text and Readings, Bateson, J.E.G (Hrsg.), 3. Aufl., Fort Worth u.a. 1995, S. 43-56.

Zeithaml, V.A., Berry, L.L., Parasuraman, A., The Behavioral Consequences of Service Quality, in: JoM, Vol. 60, April 1996, S. 31-46.

Zimmermann, H.-J., Zum Nutzen empirischer Untersuchungen, in: Der praktische Nutzen empirischer Forschung, Witte, E. (Hrsg.), Tübingen 1981, S. 271-303. 
Andreas Siefke and Universität Münster - 978-3-631-75103-9

Downloaded from PubFactory at 01/11/2019 08:18:16AM

via free access 


\section{SCHRIFTEN ZUM MARKETING}

Band 1 Friedrich Wehrle: Strategische Marketingplanung in Warenhäusern. Anwendung der Portfolio-Methode. 1981. 2. Auflage. 1984.

Band 2 Jürgen Althans: Die Übertragbarkeit von Werbekonzeptionen auf internationale Märkte. Analyse und Exploration auf der Grundlage einer Befragung bei europaweit tätigen Werbeagenturen. 1982.

Band 3 Günter Kimmeskamp: Die Rollenbeurteilung von Handelsvertretungen. Eine empirische Untersuchung zur Einschätzung des Dienstleistungsangebotes durch Industrie und Handel. 1982.

Band 4 Manfred Bruhn: Konsumentenzufriedenheit und Beschwerden. Erklärungsansätze und Ergebnisse einer empirischen Untersuchung in ausgewählten Konsumbereichen. 1982.

Band 5 Heribert Meffert (Hrsg.): Kundendienst-Management. Entwicklungsstand und Entscheidungsprobleme der Kundendienstpolitik. 1982.

Band 6 Ralf Becker: Die Beurteilung von Handelsvertretern und Reisenden durch Hersteller und Kunden. Eine empirische Untersuchung zum Vergleich der Funktionen und Leistungen. 1982.

Band 7 Gerd Schnetkamp: Einstellungen und Involvement als Bestimmungsfaktoren des sozialen Verhaltens. Eine empirische Analyse am Beispiel der Organspendebereitschaft in der Bundesrepublik Deutschland. 1982.

Band 8 Stephan Bentz: Kennzahlensysteme zur Erfolgskontrolle des Verkaufs und der MarketingLogistik. Entwicklung und Anwendung in der Konsumgüterindustrie. 1983.

Band 9 Jan Honsel: Das Kaufverhalten im Antiquitätenmarkt. Eine empirische Analyse der Kaufmotive, ihrer Bestimmungsfaktoren und Verhaltenswirkungen. 1984.

\section{SCHRIFTEN ZU MARKETING UND MANAGEMENT}

Band 10 Matthias Krups: Marketing innovativer Dienstleistungen am Beispiel elektronischer Wirtschaftsinformationsdienste. 1985.

Band 11 Bernd Faehsler: Emotionale Grundhaltungen als Einflußfaktoren des Käuferverhaltens. Eine empirische Analyse der Beziehungen zwischen emotionalen Grundhaltungen und ausgewählten Konsumstrukturen. 1986.

Band 12 Ernst-Otto Thiesing: Strategische Marketingplanung in filialisierten Universalbanken. Integrierte Filial- und Kundengruppenstrategien auf der Grundlage erfolgsbeeinflussender Schlüsselfaktoren. 1986.

Band 13 Rainer Landwehr: Standardisierung der internationalen Werbeplanung. Eine Untersuchung der Prozeßstandardisierung am Beispiel der Werbebudgetierung im Automobilmarkt. 1988.

Band 14 Paul-Josef Patt: Strategische Erfolgsfaktoren im Einzelhandel. Eine empirische Analyse am Beispiel des Bekleidungsfachhandels. 1988. 2. Auflage. 1990.

Band 15 Elisabeth Tolle: Der Einfluß ablenkender Tätigkeiten auf die Werbewirkung. Bestimmungsfaktoren der Art und Höhe von Ablenkungseffekten bei Rundfunkspots. 1988.

Band 16 Hanns Ostmeier: Ökologieorientierte Produktinnovationen. Eine empirische Analyse unter besonderer Berücksichtigung ihrer Erfolgseinschätzung. 1990.

Band 17 Bernd Büker: Qualitätsbeurteilung investiver Dienstleistungen. Operationalisierungsansätze an einem empirischen Beispiel zentraler EDV-Dienste. 1991.

Band 18 Kerstin Ch. Monhemius: Umweltbewußtes Kaufverhalten von Konsumenten. Ein Beitrag zur Operationalisierung, Erklärung und Typologie des Verhaltens in der Kaufsituation. 1993. 
Band 19 Uwe Schürmann: Erfolgsfaktoren der Werbung im Produktlebenszyklus. Ein Beitrag zur Werbewirkungsforschung. 1993.

Band 20 Ralf Birkelbach: Qualitätsmanagement in Dienstleistungscentern. Konzeption und typenspezifische Ausgestaltung unter besonderer Berücksichtigung von Verkehrsflughäfen. 1993.

Band 21 Simone Frömbling. Zielgruppenmarketing im Fremdenverkehr von Regionen. Ein Beitrag zur Marktsegmentierung auf der Grundlage von Werten, Motiven und Einstellungen. 1993.

Band 22 Marcus Poggenpohl: Verbundanalyse im Einzelhandel auf der Grundlage von Kundenkarteninformationen. Eine empirische Untersuchung von Verbundbeziehungen zwischen Abteilungen. 1994.

Band 23 Kai Bauche: Segmentierung von Kundendienstleistungen auf investiven Märkten. Dargestellt am Beispiel von Personal Computern. 1994.

Band 24 Ewald Werthmöller: Räumliche Identität als Aufgabenfeld des Städte- und Regionenmarketing. Ein Beitrag zur Fundierung des Placemarketing. 1995.

Band 25 Nicolaus Müller: Marketingstrategien in High-Tech-Märkten. Typologisierung, Ausgestaltungsformen und Einflußfaktoren auf der Grundlage strategischer Gruppen. 1995.

Band 26 Nicolaus Henke: Wettbewerbsvorteile durch Integration von Geschäftsaktivitäten. Ein zeitablaufbezogener wettbewerbsstrategischer Analyseansatz unter besonderer Berücksichtigung des Einsatzes von Kommunikations- und Informationssystemen (KIS). 1995.

Band 27 Kai Laakmann: Value-Added Services als Profilierungsinstrument im Wettbewerb. Analyse, Generierung und Bewertung. 1995.

Band 28 Stephan Wöllenstein: Betriebstypenprofilierung in vertraglichen Vertriebssystemen. Eine Analyse von Einflußfaktoren und Erfolgswirkungen auf der Grundlage eines Vertragshändlersystems im Automobilhandel. 1996.

Band 29 Michael Szeliga: Push und Pull in der Markenpolitik. Ein Beitrag zur modellgestützten Marketingplanung am Beispiel des Reifenmarktes. 1996.

Band 30 Hans-Ulrich Schröder: Globales Produktmanagement. Eine empirische Analyse des Instrumenteeinsatzes in ausgewählten Branchen der Konsumgüterindustrie. 1996.

Band 31 Peter Lensker: Planung und Implementierung standardisierter vs. differenzierter Sortimentsstrategien in Filialbetrieben des Einzelhandels. 1996.

Band 32 Michael H. Ceyp: Ökologieorientierte Profilierung im vertikalen Marketing. Dargestellt am Beispiel der Elektrobranche. 1996.

Band 34 Ralf Ueding: Management und Messebeteiligungen. Identifikation und Erklärung messespezifischer Grundhaltungen auf der Basis einer empirischen Untersuchung. 1998.

Band 35 Andreas Siefke: Zufriedenheit mit Dienstleistungen. Ein phasenorientierter Ansatz zur Operationalisierung und Erklärung der Kundenzufriedenheit im Verkehrsbereich auf empirischer Basis. 1998. 


\section{Der westeuropäische Wirtschaftsraum im globalen Wettbewerb}

\section{Regionale Integration und Standortwettbewerbsfähigkeit}

Frankfurt/M., Berlin, Bern, New York, Paris, Wien, 1997.

406 S., 21 Abb., 30 Tab.

Studien der Bremer Gesellschaft für Wirtschaftsforschung e.V.

Herausgegeben von Alfons Lemper und Rolf W. Stuchtey. Bd. 7

ISBN 3-631-31731-X · br. DM 98.-*

In den vergangenen fünfzig Jahren entwickelte sich Westeuropa zu einem hochintegrierten Wirtschaftsraum, der an die Spitze der Welteinkommenspyramide aufrückte. Daran anknüpfend will die Studie zwei Hauptsachverhalte näher ergründen. Erstens geht sie der Frage nach, welche politischökonomischen „Mechanismen" im Hinblick auf den westeuropäischen Integrationsverlauf identifizierbar sind. Zweitens analysiert sie die relative Attraktivität des westeuropäischen Wirtschaftsstandortes für

unternehmerische Aktivitäten und damit dessen Potential, das erreichte Wohlstandsniveau im globalen Standortwettbewerb um mobile Ressourcen auch zukünftig sichern und möglichst noch anheben zu können.

Aus dem Inhalt: Politisch-ökonomische Entwicklungslinien und -mechanismen der westeuropäischen Integration 1947-1996 - Theorie der relativen Standortattraktivität · Stärken-Schwächen-Profil Westeuropas im globalen Standortwettbewerb - Wirtschaftspolitische Reformempfehlungen · Geoökonomische Megatrends und Weltwirtschaftsordnung

Frankfurt/M - Berlin - Bern - New York - Paris - Wien

Auslieferung: Verlag Peter Lang AG

Jupiterstr. 15, CH-3000 Bern 15

Telefax (004131) 9402131

*inklusive Mehrwertsteuer

Preisänderungen vorbehalten 
Andreas Siefke and Universität Münster - 978-3-631-75103-9

Downloaded from PubFactory at 01/11/2019 08:18:16AM

via free access 